Prepared in cooperation with Alabama Association of Conservation Districts; Alabama Association of Resource Conservation and Development Councils; Alabama Department of Agriculture and Industries; Alabama Department of Conservation and Natural Resources Division of Wildlife and Freshwater Fisheries; Alabama Department of Economic and Community Affairs-Office of Water Resources Division; Alabama Farmers Federation; Alabama Power; Choctawhatchee, Pea and Yellow Rivers Watershed Management Authority; Geological Survey of Alabama; and The University of Alabama-Water Policy and Law Institute

\title{
Low-Flow Frequency and Flow-Duration Characteristics of Selected Alabama Streams Through March 2014
}

\section{Scientific Investigations Report $2017-5083$}

5

U.S. Department of the Interior U.S. Geological Survey 
Cover:

Front: Looking upstream from USGS streamgaging station 02399200 Little River near Blue Pond, Alabama, November 2016. Photograph by Andrew Vanover., USGS.

Back: Pea River at Ariton, Alabama, June 2011. Photograph by Joseph Scott Wallace, USGS. 


\section{Low-Flow Frequency and Flow-Duration Characteristics of Selected Streams in Alabama Through March 2014}

By Toby D. Feaster and Kathryn G. Lee

Prepared in cooperation with Alabama Association of Conservation Districts; Alabama Association of Resource Conservation and Development Councils; Alabama Department of Agriculture and Industries; Alabama Department of Conservation and Natural Resources-Division of Wildlife and Freshwater Fisheries; Alabama Department of Economic and Community Affairs-Office of Water Resources Division; Alabama Farmers Federation; Alabama Power; Choctawhatchee, Pea and Yellow Rivers Watershed Management Authority; Geological Survey of Alabama; and The University of Alabama-Water Policy and Law Institute

Scientific Investigations Report 2017-5083 


\title{
U.S. Department of the Interior \\ RYAN K. ZINKE, Secretary
}

\section{U.S. Geological Survey William H. Werkheiser, Acting Director}

\author{
U.S. Geological Survey, Reston, Virginia: 2017
}

For more information on the USGS - the Federal source for science about the Earth, its natural and living resources, natural hazards, and the environment—visit http://www.usgs.gov or call 1-888-ASK-USGS.

For an overview of USGS information products, including maps, imagery, and publications, visit https://store.usgs.gov.

Any use of trade, firm, or product names is for descriptive purposes only and does not imply endorsement by the U.S. Government.

Although this information product, for the most part, is in the public domain, it also may contain copyrighted materials as noted in the text. Permission to reproduce copyrighted items must be secured from the copyright owner.

Suggested citation:

Feaster, T.D., and Lee, K.G., 2017, Low-flow frequency and flow-duration characteristics of selected streams in Alabama through March 2014: U.S. Geological Survey Scientific Investigations Report 2017-5083, 371 p., https://doi.org/10.3133/sir20175083.

ISSN 2328-0328 (online 


\section{Acknowledgments}

The authors are grateful to the numerous cooperators who helped make this investigation possible. Many of those cooperators have a long-standing partnership with the U.S. Geological Survey (USGS) in our efforts to understand and address the water-resources issues in Alabama. The daily mean flows included in this investigation were computed from data collected at USGS streamgages operated in cooperation with a variety of Federal, State, and local agencies. The authors acknowledge the commitment of those cooperators in understanding the importance of such a long-term data-collection program.

We also acknowledge the commitment and dedicated work of the USGS field-office personnel who collected, processed, and stored the water-resources data needed for the completion of this and many other such investigations. 



\section{Contents}

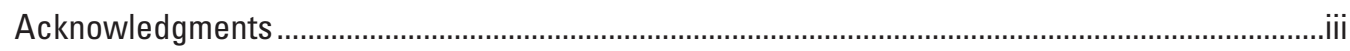

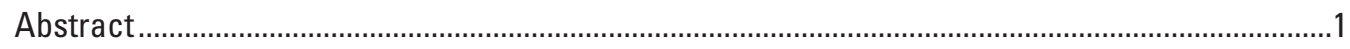

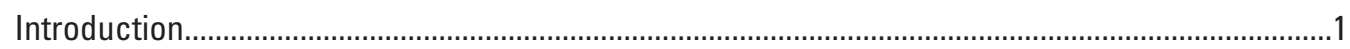

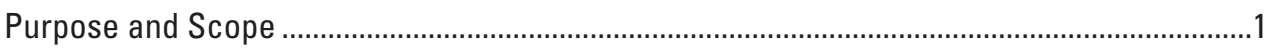

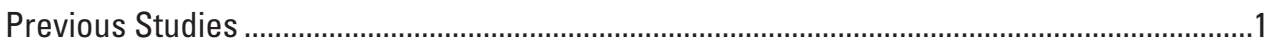

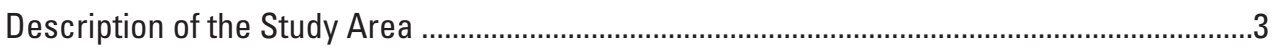

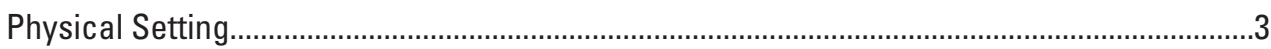

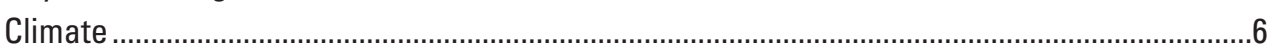

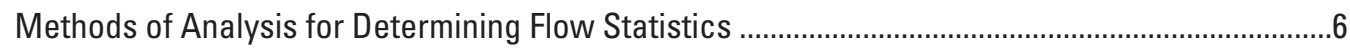

Quality Assurance and Quality Control ..............................................................................

Results of Kendall's Tau Analyses for Unregulated Streamgaging Stations ..................10

Results of Kendall's Tau Analyses for Regulated Streamgaging Stations ......................10

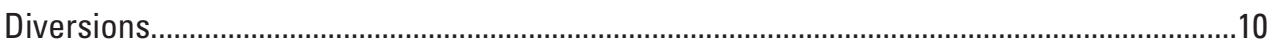

Analytical Approach for Low-Flow Frequency Statistics ..................................................12

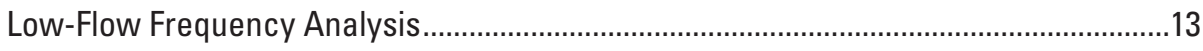

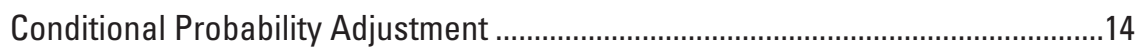

Record-Extension Technique............................................................................... 14

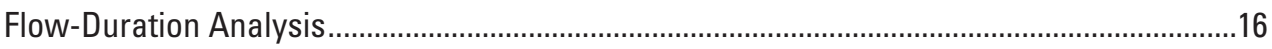

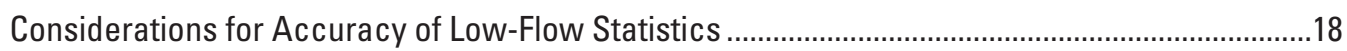

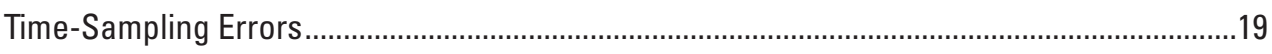

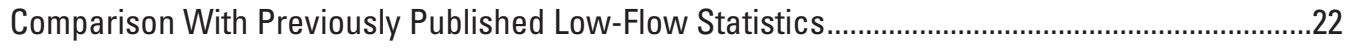

Access to Updated Low-Flow Characteristics Through the StreamStats Application....................22

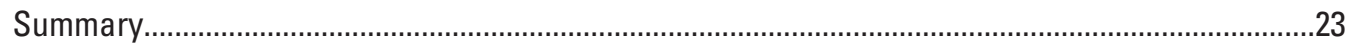

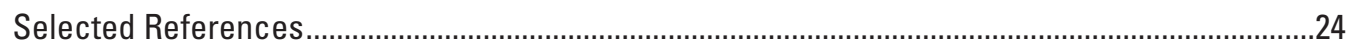

\section{Figures}

1. Map showing the location of U.S. Geological Survey continuous-record streamgages in Alabama and surrounding States included in this investigation .............2

2. Map showing U.S. Environmental Protection Agency Level III ecoregions and major river basins in Alabama.

3. Graphs showing Alabama statewide average annual precipitation for the period 1895 to 2015 and the 10 driest and wettest years for the same period ...................7

4. Map showing average annual precipitation in Alabama, by climate division, for the period 1895 to 2015 and the climate normal period 1981 to 2010

5. Graphs showing various periods of record at U.S. Geological Survey station 02374500, Murder Creek near Evergreen, AL, for which a Kendall's tau trend test was computed for climate years 1938 to 2013, 1989 to 2013, 1954 to 1979, and 1954 to 2013

6. Graph showing comparison of daily mean flow duration curves for U.S. Geological Survey streamgaging stations 02423425, Cahaba River near Cahaba Heights, AL; 02423496, Cahaba River near Hoover, AL; 02423500, Cahaba River near Acton, AL; and 02423555, Cahaba River near Helena, AL 
7. Graph showing example in which the log-Pearson Type III distribution does not adequately fit the annual minimum 1-day flows for regulated U.S. Geological

Survey station 02418500, Tallapoosa River below Tallassee, Alabama.

8. Graphs showing U.S. Geological Survey streamgaging station 02374500 , Murder Creek near Evergreen, AL, low-flow frequency curve for the annual minimum 7-day average streamflow, and annual minimum 7-day average flows by climate year with the 7010 estimate computed from those flows.....

9. Graph showing the MOVE.1 correlation of annual minimum 7-day average flow at U.S. Geological Survey streamgaging station 02361500, Choctawhatchee River near Bellwood, AL, with index station 02361000, Choctawhatchee River near Newton, AL

10. Graph showing flow-duration curve for U.S. Geological Survey streamgaging station 02361000, Choctawhatchee River near Newton, AL, for the periods December 1921 to September 1927 and June 1935 to March 2014

11. Graph showing annual minimum 7-day average streamflow and 7010 estimates at U.S. Geological Survey streamgaging station 02374500, Murder Creek near Evergreen, AL

12. Graph showing annual minimum 7-day average streamflow and 7010 estimates at U.S. Geological Survey streamgaging station 02374500, Murder Creek near Evergreen, AL, for seven 10-year periods: climate years 1938 to 1947, 1948 to 1957, 1958 to 1967,1968 to 1977, 1978 to 1987, 1988 to 1997, and 1998 to 2007.

13. Graphs showing distribution of years of record for the unregulated Alabama stations, and the period of record with the beginning and ending years shown by drainage area.

14. Graph showing annual minimum 7-day average flow at U.S. Geological Survey streamgaging station 02450000, Mulberry Fork near Garden City, AL 23

\section{Tables}

1. U.S. Geological Survey continuous-record streamgaging stations in Alabama and surrounding States included in this investigation.

2. Major river basins in Alabama .

3. The 10 driest and wettest years for the Alabama statewide average annual precipitation from 1895 to 2015 .

4. Results of Kendall's tau statistical test for detection of monotonic trends in the annual minimum 7-day average flow for 210 continuous-record streamgages in Alabama

5. Low-flow statistics for continuous-record streamgaging stations of selected streams in Alabama and surrounding States.

6. Streamgaging stations for which the record was augmented using MOVE.1, index station, additional climate years of record available at the index station, and correlation coefficients for the gaging stations where the record was augmented ......17

7. Differences between the annual minimum 7-day average streamflow with a 10-year recurrence interval in this report and previously published values. 362

8. Summary statistics for the percentage change from the annual minimum 7-day average streamflow presented in this report and those previously published in Atkins and Pearman (1994) for the U.S. Geological Survey streamgaging stations for which additional data were collected since the previous study. 


\section{Conversion Factors}

Inch/Pound to International System of Units

\begin{tabular}{|c|c|c|}
\hline Multiply & By & To obtain \\
\hline \multicolumn{3}{|c|}{ Length } \\
\hline foot $(\mathrm{ft})$ & 0.3048 & meter $(\mathrm{m})$ \\
\hline mile (mi) & 1.609 & kilometer $(\mathrm{km})$ \\
\hline \multicolumn{3}{|c|}{ Area } \\
\hline square mile $\left(\mathrm{mi}^{2}\right)$ & 2.590 & square kilometer $\left(\mathrm{km}^{2}\right)$ \\
\hline \multicolumn{3}{|c|}{ Volume } \\
\hline million gallons (Mgal) & 3,785 & cubic meter $\left(\mathrm{m}^{3}\right)$ \\
\hline cubic foot $\left(\mathrm{ft}^{3}\right)$ & 0.02832 & cubic meter $\left(\mathrm{m}^{3}\right)$ \\
\hline \multicolumn{3}{|c|}{ Flow rate } \\
\hline cubic foot per second $\left(\mathrm{ft}^{3} / \mathrm{s}\right)$ & 0.02832 & cubic meter per second $\left(\mathrm{m}^{3} / \mathrm{s}\right)$ \\
\hline $\begin{array}{l}\text { cubic foot per second per square } \\
\text { mile }\left[\left(\mathrm{ft}^{3} / \mathrm{s}\right) / \mathrm{mi}^{2}\right]\end{array}$ & 0.01093 & $\begin{array}{l}\text { cubic meter per second per } \\
\text { square kilometer }\left[\left(\mathrm{m}^{3} / \mathrm{s}\right) / \mathrm{km}^{2}\right]\end{array}$ \\
\hline million gallons per day $(\mathrm{Mgal} / \mathrm{d})$ & 0.04381 & cubic meter per second $\left(\mathrm{m}^{3} / \mathrm{s}\right)$ \\
\hline
\end{tabular}

Temperature in degrees Fahrenheit $\left({ }^{\circ} \mathrm{F}\right)$ may be converted to degrees Celsius $\left({ }^{\circ} \mathrm{C}\right)$ as follows:

$$
{ }^{\circ} \mathrm{C}=\left({ }^{\circ} \mathrm{F}-32\right) / 1.8 \text {. }
$$

\section{Datum}

Horizontal coordinate information is referenced to the North American Datum of 1927 (NAD 27) or 1983 (NAD 83).

Elevation, as used in this report, refers to distance above the vertical datum. 


\section{Abbreviations}

$\begin{array}{ll}\text { CR } & \text { continuous record } \\ \text { CY } & \text { climate (or climatic) year } \\ \text { EPA } & \text { U.S. Environmental Protection Agency } \\ \text { HUC } & \text { hydrologic unit code } \\ \text { LPIII } & \text { log-Pearson Type III (mathematical technique) } \\ \text { MOVE.1 } & \text { Maintenance of Variance Extension, Type 1 (method) } \\ \text { NWIS } & \text { National Water Information System } \\ \text { OAOC } & \text { quality assurance and quality control } \\ \text { SWSTAT } & \text { Surface-Water Statistics (program) } \\ \text { USGS } & \text { U.S. Geological Survey }\end{array}$




\title{
Low-Flow Frequency and Flow-Duration Characteristics of Selected Streams in Alabama Through March 2014
}

\author{
By Toby D. Feaster and Kathryn G. Lee
}

\begin{abstract}
Low-flow statistics are needed by water-resource engineers, planners, and managers to protect and manage the water resources of Alabama. The accuracy of these statistics is influenced by such factors as length of record and specific hydrologic conditions measured in those records. As such, it is generally recommended that flow statistics be updated about every 10 years to provide improved and representative low-flow characteristics. The previous investigation of low-flow characteristics for Alabama included data through September 1990. Since that time, Alabama has experienced several historic droughts highlighting the need to update the low-flow characteristics at U.S. Geological Survey streamgaging stations. Consequently, this investigation was undertaken in cooperation with a number of State and local agencies to update low-flow frequency and flow-duration statistics at 210 continuous-record streamgaging stations in Alabama and 67 stations from basins that are shared with surrounding States. The flow characteristics were computed on the basis of available data through March 2014.
\end{abstract}

\section{Introduction}

Information about the low-flow characteristics of Alabama streams is vital for water-resource managers engaged in planning, management, and permitting decisions. Having up-to-date information helps inform decisions such as adequate water for consumptive use, water-quality standards, recreation, and aquatic habitat protection (Alabama Department of Environmental Management, 2012). As part of their responsibility to protect public health and aquatic ecosystems, Alabama State regulatory agencies need accurate and representative streamflow statistics to establish applicable water-quality and water-quantity criteria. Historically, lowflow statistics, such as the annual minimum 7-day average flow likely to occur once every 10 years, have been used by water-resource managers and planners as a threshold for applying the chronic aquatic life criteria for determining waste-load allocations for point sources, total maximum daily loads for streams, and the quantity of water that can safely be withdrawn from a particular stream (Alabama Department of Environmental Management, 2012). Droughts that occurred in Alabama during the past few decades have heightened awareness of the need for up-to-date statistics for use in making critical water-resources decisions.

Because of the importance of these applications, it is critical to effectively measure and document base-flow records for use in updating low-flow frequency relations on a regular basis, preferably about every 10 years, and especially after periods of extreme low flow, which have occurred in the Southeast in recent years. Low-flow statistics in Alabama have not been updated on a statewide basis since 1990 (Atkins and Pearman, 1994).

\section{Purpose and Scope}

The purpose of this report is to present updated low-flow statistics at 210 continuous-record (CR) streamgaging stations in Alabama and $67 \mathrm{CR}$ stations from surrounding States that share river basins with Alabama along with flow-duration statistics for daily mean flows (fig. 1; table 1, p. 28). The scope of the report includes both unregulated and regulated streams. Depending on the length of record available at each CR station, the report presents estimates of annual minimum 1 - and 7-day average streamflow with recurrence intervals of $2,5,10,20$, and 50 years. In addition, daily mean flow durations for the 5-, 10-, 25-, 50-, 75-, 90-, and 95-percent probabilities of exceedance are presented for these stations.

\section{Previous Studies}

Previous reports by Pierce $(1959,1967)$, Hayes (1978), Bingham (1981), and Atkins and Pearman (1994) describe the low-flow frequency and flow-duration streamflow data for selected streamgaging stations in Alabama. Pierce (1959) presented low-flow frequency and flow-duration data for Alabama streams using data through 1956. Pierce (1967) provided 7-day low-flow statistics and flow-duration data for 208 continuous-record stations using data through September 30, 1963, and low-flow statistics were provided for 285 partial-record stations. 


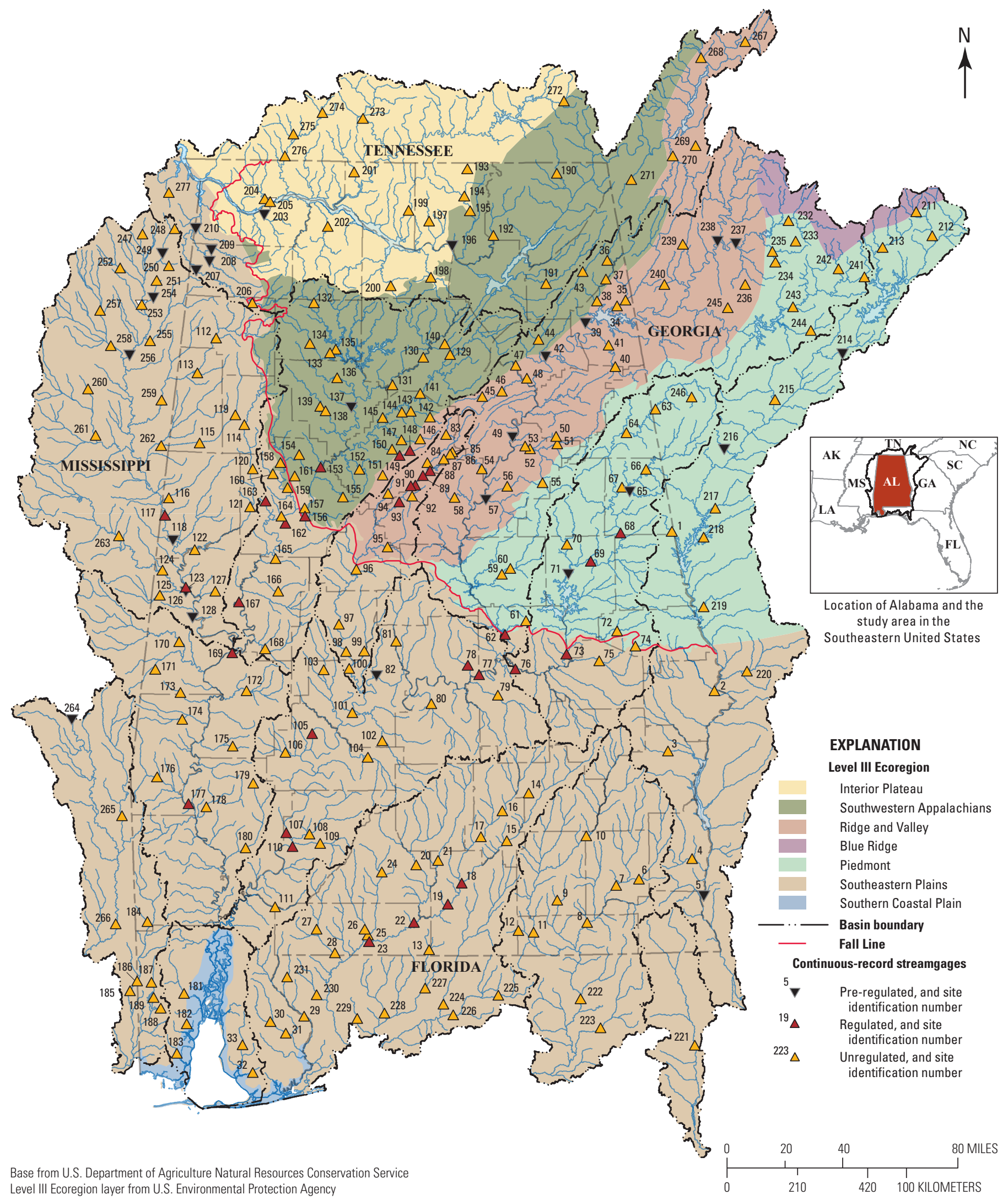

Level III Ecoregion layer from U.S. Environmental Protection Agency

Figure 1. Location of U.S. Geological Survey continuous-record streamgages in Alabama and surrounding States included in this investigation. Pre-regulated sites are currently (2017) regulated but were analyzed for pre-regulation conditions. 
Hayes (1978) presented 7-day low-flow statistics and flow-duration data for 527 locations on 341 Alabama streams. Low-flow characteristics for CR streamgaging stations with less than 10 years of record were estimated on the basis of correlations with CR stations having more than 10 years of record. Also, estimates at partial-record stations were made using correlation methods based on data from CR stations. On the basis of data published by Hayes (1978), Bingham (1981) developed regional low-flow equations to estimate the annual minimum 7-day average streamflow with 2- and 10-year recurrence intervals (7Q2 and 7Q10, respectively) at ungaged locations. The equations were based on geology, drainage area, and mean annual precipitation and were applicable statewide.

The most recent low-flow analysis was completed by Atkins and Pearman (1994) who computed low-flow frequency statistics for the 7Q2 and 7Q10 flows and daily flow-duration characteristics using data through September 30, 1990. In their report, 7Q2 and 7Q10 statistics were computed for $144 \mathrm{CR}$ stations with 10 or more years of record, and correlation methods were used to estimate 7Q2 and 7Q10 values for $81 \mathrm{CR}$ stations with less than 10 years of record. In addition, low-flow frequency estimates were made for 447 partial-record stations using correlation techniques.

\section{Description of the Study Area}

The State of Alabama encompasses 52,420 square miles $\left(\mathrm{mi}^{2}\right)$ in the Southeastern United States (U.S. Geological Survey, 2016). The study area encompasses most of the State with the exception of coastal areas where low flows are tidally influenced. The major river basins in Alabama (fig. 2) are the Alabama, Black Warrior, Cahaba, Chattahoochee, Choctawhatchee, Coosa, Escatawpa, Lower Tombigbee, Mobile Bay, Perdido-Escambia, Tallapoosa, Tennessee, and Upper Tombigbee (table 2; Alabama Department of Environmental Management, 2014). For many of Alabama's major rivers, streamflow is regulated by reservoirs and (or) a system of locks and dams (Ruddy and Hitt, 1990; table 1).

\section{Physical Setting}

The State of Alabama lies within six U.S. Environmental Protection Agency (EPA) level III ecoregions-Piedmont, Southeastern Plains, Ridge and Valley, Southwestern Appalachians, Interior Plateau, and Southern Coastal Plain (fig. 2; U.S. Environmental Protection Agency, 2016). The ecoregions represent areas of general similarity in ecosystems and type, quality, and quantity of environmental resources, and they provide a spatial framework for research, assessment, management, and monitoring of ecosystems and ecosystem components. The ecoregions were determined from an analysis of the spatial patterns and the composition of biotic and abiotic phenomena that include geology, physiography, vegetation, climate, soils, land use, wildlife, and hydrology (Omernik, 1987).

The Southwestern Appalachians ecoregion extends from Kentucky to Alabama and is composed of open, low mountains containing a mosaic of forest and woodland with some cropland and pasture. The eastern boundary of this ecoregion, along the more abrupt escarpment where it meets the Ridge and Valley ecoregion, is relatively smooth and only slightly notched by small, eastward-flowing streams. The Ridge and Valley is composed of roughly parallel ridges and valleys of various widths, heights, and geologic materials. The western boundary is shared with the Interior Plateau ecoregion, which extends from southern Indiana and Ohio to northern Alabama. Elevations in the Interior Plateau are lower than those in the Southwestern Appalachians to the east. Mississippian to Ordovician-age limestone, chert, sandstone, siltstone, and shale compose the landforms of open hills, irregular plains, and tablelands of the Interior Plateau (Omernik, 1987).

The Piedmont ecoregion, which extends from Alabama to New Jersey, is a transitional area between the mountainous ecoregions of the Appalachians to the northwest and the relatively flat coastal plain to the southeast. The Piedmont is a complex mosaic of metamorphic and igneous rocks of the Precambrian and Paleozoic age and contains moderately dissected irregular plains and some hills. The soils tend to be finer textured than those in the coastal plain. The Piedmont was once a largely cultivated region, but much of it has reverted to pine and hardwood woodlands, with increasing conversion to urban and suburban land cover (Omernik, 1987).

The Fall Line, which extends from Alabama to New Jersey, is a geologic feature that separates the higher elevation Interior Plateau, Southwestern Appalachians, Ridge and Valley, and Piedmont ecoregions from the lower elevation Southeastern Plains ecoregion (fig. 2). The Southeastern Plains ecoregion, which extends from Virginia to Louisiana and Tennessee, is composed of irregular plains made up of a mixture of cropland, pasture, woodland, and forest. The sands, silts, and clays of this ecoregion contrast geologically with the older rocks of the Piedmont ecoregion. Elevations and relief in the Southeastern Plains are greater than in the Southern Coastal Plain but generally are less than in much of the Piedmont. Streams in the Southeastern Plains have relatively low gradient and sandy bottoms. The Southern Coastal Plain ecoregion consists of mostly flat plains but is heterogeneous, containing barrier islands, coastal lagoons, marshes, and swampy lowlands along the Gulf and Atlantic coasts. Relative to the Southeastern Plains ecoregion, the Southern Coastal Plain ecoregion is lower in elevation, with less relief and wetter soils (Omernik, 1987). 


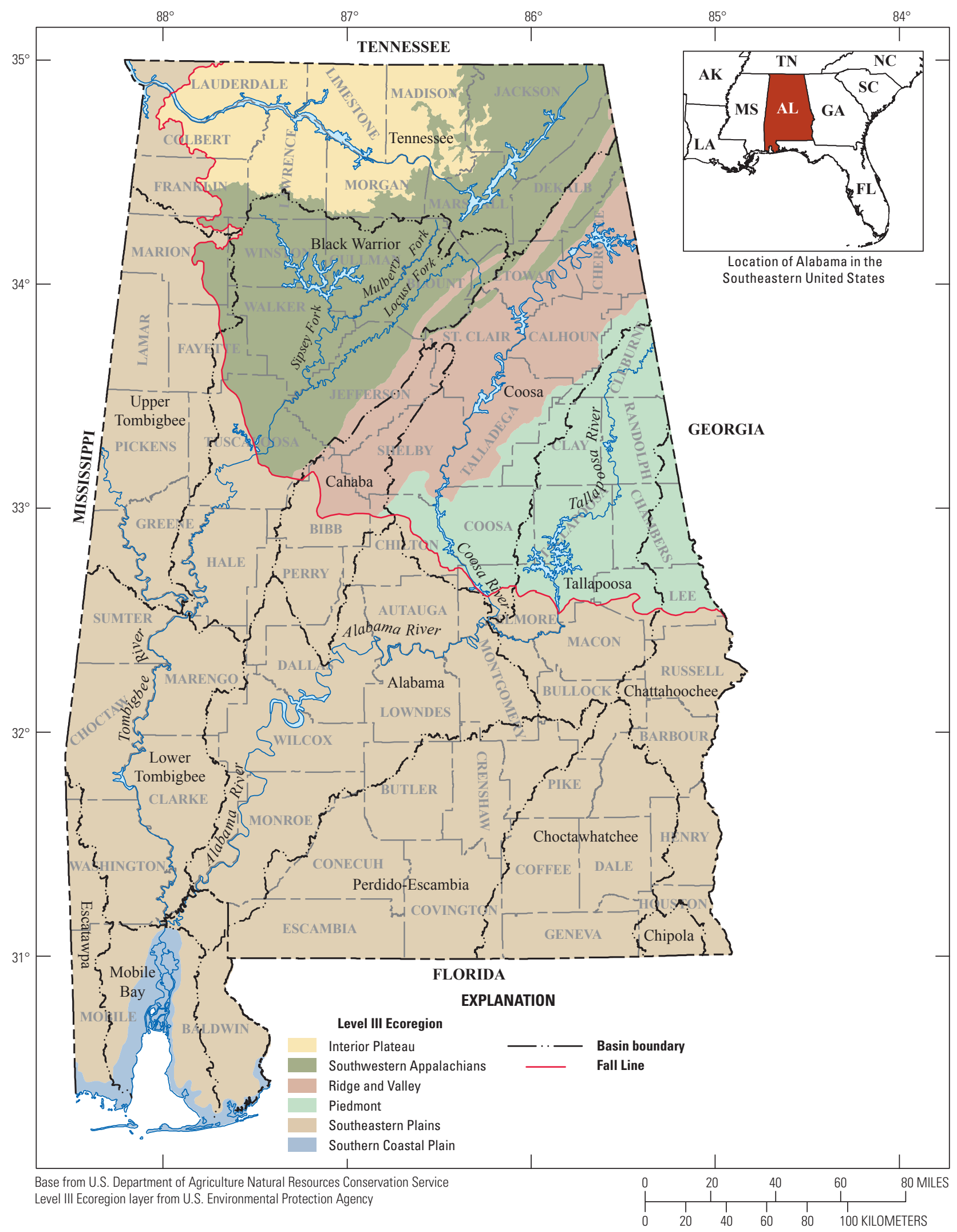

Figure 2. U.S. Environmental Protection Agency Level III ecoregions and major river basins in Alabama (U.S. Environmental Protection Agency, 2016). 
Table 2. Major river basins in Alabama.

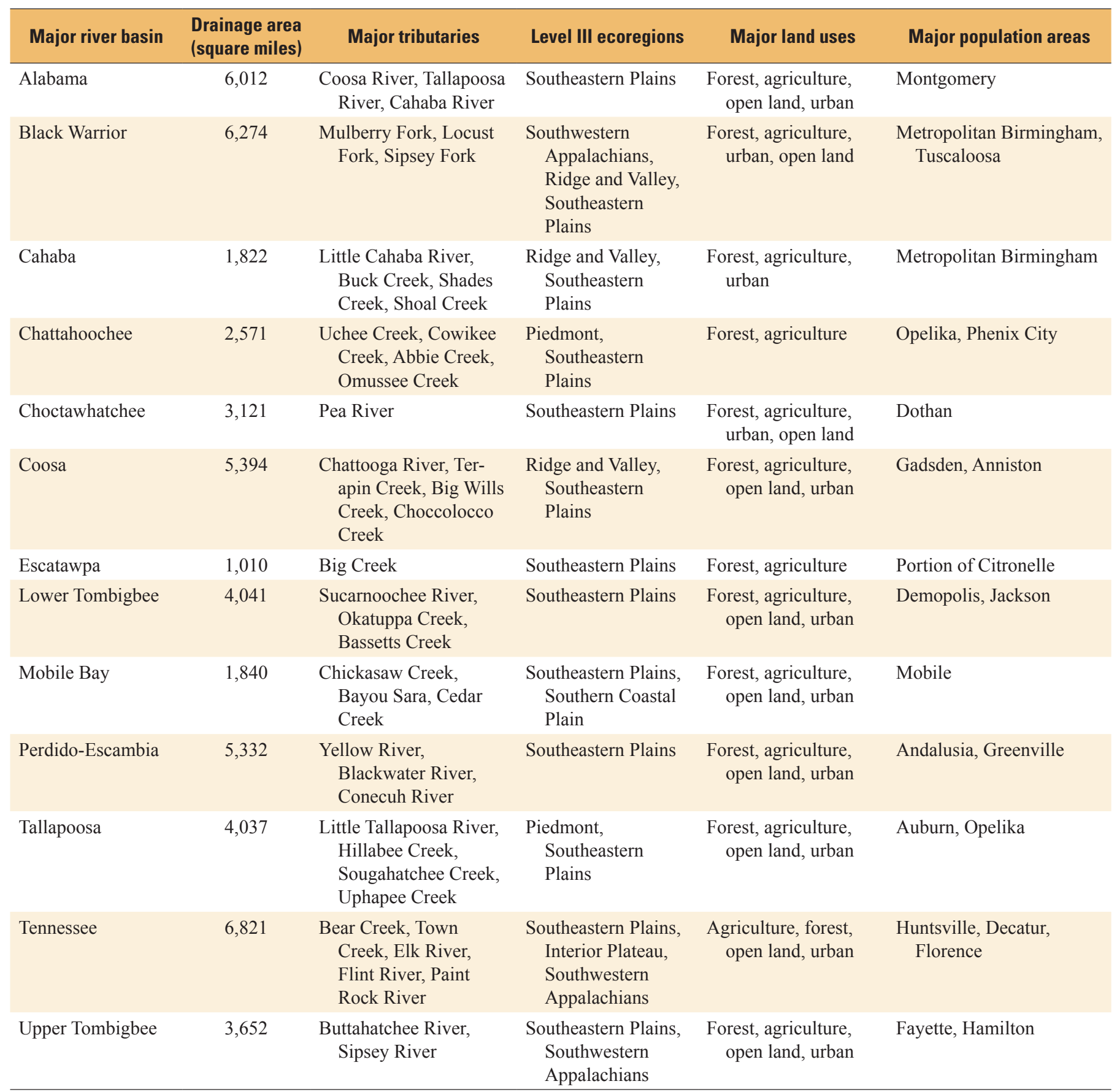




\section{Climate}

The climate of Alabama is characterized by moderate seasonality and is somewhat subtropical near the coast. Summers are typically warm and humid with average high temperatures ranging from 80 to 88 degrees Fahrenheit $\left({ }^{\circ} \mathrm{F}\right)$

(National Oceanic and Atmospheric Administration, 2016a). In the winter, temperatures for most of the State range from 38 to $50^{\circ} \mathrm{F}$ during the evening. The average low winter temperatures range from 29 to $35^{\circ} \mathrm{F}$.

Precipitation amounts and patterns are affected by the Gulf of Mexico and the Appalachian Mountains. The principal sources of precipitation are the Gulf of Mexico and the subtropical Atlantic Ocean with a smaller influence from the Pacific Ocean (Paulson and others, 1991). The average statewide total annual precipitation for Alabama for the period 1895 to 2015 was 55.2 inches (fig. 3A; National Oceanic and Atmospheric Administration, 2016b). Regarding statewide average annual precipitation, the 10 driest and wettest years for the period 1895 to 2015 are listed in table 3 and depicted in figure $3 B$. The second and fourth driest years since 1895 occurred in 2007 and 2000, respectively, highlighting the historic drought periods that occurred since the previous statewide update of low-flow statistics, which included data through September 1990 (Atkins and Pearman, 1994).

A climate normal is defined as the arithmetic average of climatological variables, such as temperature and precipitation, over a 30 -year interval and is useful for placing recent climate conditions into a historical context (National Oceanic and Atmospheric Administration, 2017). For the climate normal period 1981 to 2010 (reflecting precipitation from recent decades), statewide average annual precipitation was 55.7 inches as compared to the statewide average total precipitation of 55.2 inches for the long-term period from
1895 to 2015. Across the eight climate divisions, or regions, in Alabama (National Oceanic and Atmospheric Administration, 2016c), regional differences in average annual precipitation from 1895 to 2015 ranged from 52.8 inches in the Prairie region to 62.2 inches in the Gulf region. For the climate normal period 1981 to 2010 , the average annual precipitation ranged from 53.3 inches in the Prairie region to 63.2 inches in the Gulf region (fig. 4). These comparisons show that the average annual precipitation for the period 1981 to 2010 was relatively consistent with the long-term average annual precipitation for the period 1895 to 2015 and, in most cases, was slightly higher.

\section{Methods of Analysis for Determining Flow Statistics}

Using daily mean streamflow data at USGS streamgages, low-flow statistics can be computed to estimate the magnitude and frequency of low-flow events. Low-flow frequency statistics, as defined in this report, are values of annual minimum daily mean streamflow averaged over designated periods (Riggs, 1972; Note: the use of "average" with respect to the low-flow statistics in this report refers to the arithmetic mean). For example, 7Q10 is one of the most commonly used low-flow statistics and is defined as the annual minimum 7-day average flow with a 10-year recurrence interval (as previously stated). In terms of probability of occurrence, there is a 1 in 10 chance (or 10-percent probability) that the annual minimum 7-day average streamflow in any single year will be equal to or less than the estimated 7Q10 value for a specific location (Riggs, 1968, 1972, 1985).

Table 3. The 10 driest and wettest years for the Alabama statewide average annual precipitation from 1895 to 2015.

\begin{tabular}{ccccccc}
\hline & \multicolumn{2}{c}{ Driest } & & \multicolumn{3}{c}{ Wettest } \\
\cline { 1 - 3 } \cline { 5 - 6 } $\begin{array}{c}\text { Rank from } \\
\text { driest }\end{array}$ & Year & $\begin{array}{c}\text { Statewide average } \\
\text { annual precipitation } \\
\text { (inches) }\end{array}$ & & $\begin{array}{c}\text { Rank from } \\
\text { wettest }\end{array}$ & Year & $\begin{array}{c}\text { Statewide average } \\
\text { annual precipitation } \\
\text { (inches) }\end{array}$ \\
\hline 1 & 1954 & 35.39 & & 1 & 1929 & 75.08 \\
2 & 2007 & 37.87 & & 2 & 1975 & 74.77 \\
3 & 1904 & 40.60 & & 3 & 2009 & 73.78 \\
4 & 2000 & 42.50 & & 4 & 1983 & 70.36 \\
5 & 1931 & 43.84 & & 5 & 1961 & 69.83 \\
6 & 1968 & 45.22 & & 6 & 1979 & 67.92 \\
7 & 1914 & 45.33 & & 7 & 1948 & 67.64 \\
8 & 1921 & 45.44 & & 8 & 1973 & 67.17 \\
9 & 1930 & 45.80 & & 9 & 1912 & 66.56 \\
10 & 1981 & 45.80 & 10 & 1964 & 66.50 \\
\hline
\end{tabular}




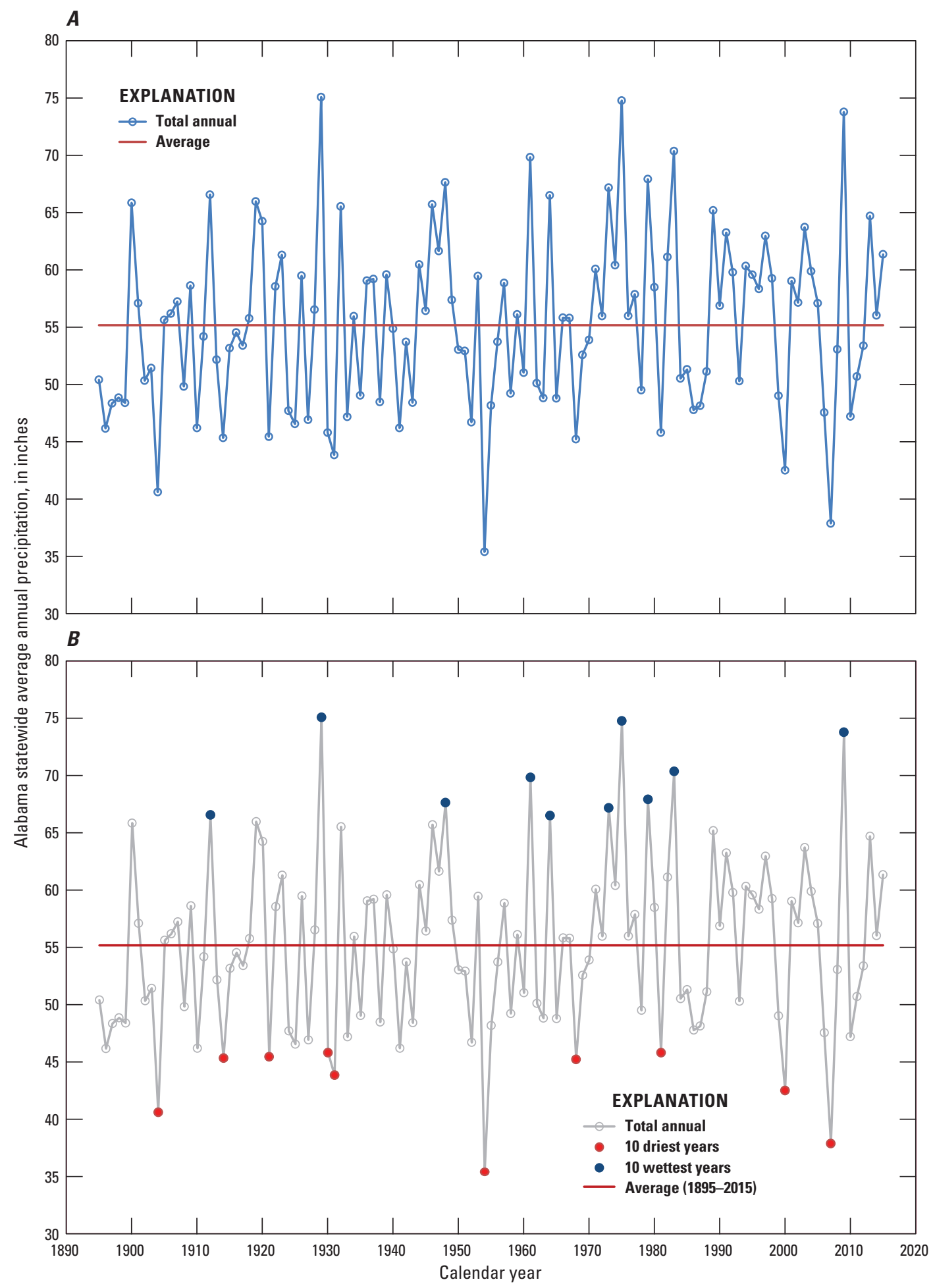

Figure 3. A, Alabama statewide average annual precipitation for the period 1895 to 2015 and $B$, the 10 driest and wettest years for the same period. 


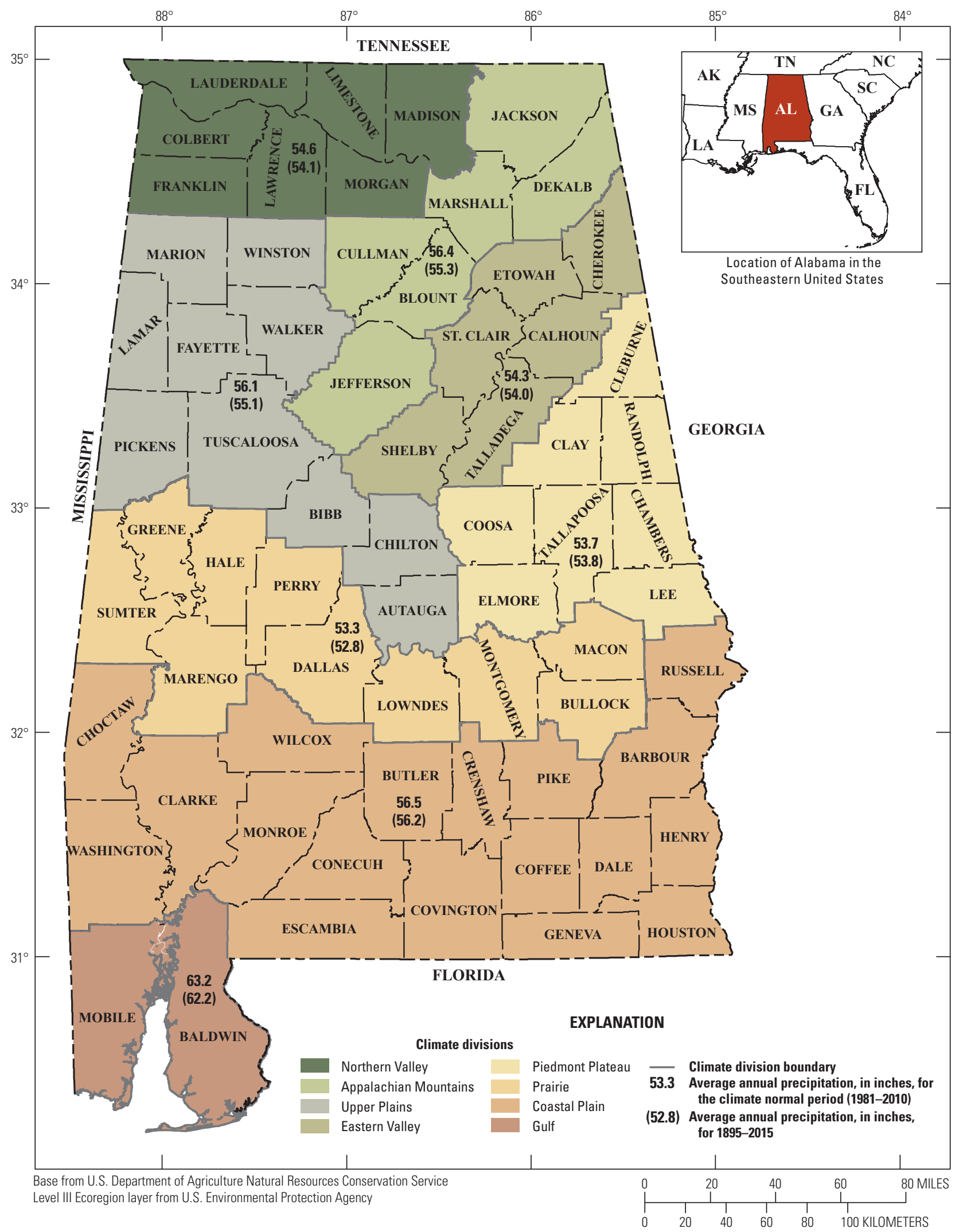

Figure 4. Average annual precipitation in Alabama, by climate division, for the period 1895 to 2015 and the climate normal period 1981 to 2010. 
Flow-duration curves are cumulative frequency curves that show the percentage of time during which specified flows were equaled or exceeded during the period of record analyzed (Searcy, 1959). The flow-duration statistics included in this report were computed using daily mean flows at USGS streamgages. Flow-duration curves can be useful for understanding the broad range of flow characteristics of a gaged stream and can be useful for comparisons with other streams. The USGS WaterWatch website (https://waterwatch.usgs.gov/) uses flow-duration curves to provide a graphical display of current streamflow conditions at real-time streamgages compared to the long-term flowduration statistics at a site of interest.

Daily mean streamflow data for this study were compiled from records available through March 31, 2014, which is the end of the 2013 climate year, and are available through web-based retrievals from the National Water Information System (NWIS; U.S. Geological Survey, 2017). The climate (or climatic) year is a continuous 12-month period that contains a complete annual cycle and is arbitrarily selected for the presentation or analysis of data relative to hydrologic or meteorological phenomena (Langbein and Iseri, 1960). The climate year typically is designated by the calendar year during which most of the selected 12 months occur. For this investigation, the climate year is the 12-month period April 1 through March 31, so the 2013 climate year is the period April 1, 2013, through March 31, 2014. In Alabama, minimum streamflows typically occur in the autumn months of September, October, and November and, therefore, use of the climate year, as selected, prevents the annual low-flow cycle from being artificially placed in separate years.

\section{Quality Assurance and Quality Control}

For the CR streamgaging stations included in this study, quality assurance and quality control (QAQC) analyses were completed for the annual minimum 7-day average streamflow estimates (Feaster and Guimaraes, 2009, 2012, 2014, 2016; Guimaraes and Feaster, 2010). The data at each station were reviewed for homogeneity, which implies relatively stable basin conditions during the period of record. Kendall's tau test was used to assess the homogeneity of the record at each station (Kendall, 1938; Helsel and Hirsch, 2002). Kendall's tau test provides an independent measure of the monotonic relation between the date and annual minimum 7-day average streamflow. The null hypothesis for this test was that there is no monotonic trend between the two variables as determined from the calculated probability value ( $p$-value). The $p$-value estimates the probability of rejecting the null hypothesis if the hypothesis were true. In this study, a trend was considered to be statistically significant when the p-value was less than or equal to 0.05 , meaning that there was less than a 5 -percent chance of obtaining the sample result if the null hypothesis were true. If a trend (nonhomogeneity) was indicated, additional assessments were used to determine if the trend may have been caused by a short-term condition. For example, if the station record began or ended under extreme conditions (excessively wet or dry), the test may indicate a trend, but excluding the extreme events may eliminate the trend. Trends at unregulated stations may result from changes in climate cycles, land use, groundwater pumping, or other influences that may affect groundwater levels. For stations downstream from a major source of regulation, such as a dam, the data were assessed for gross trends, which may indicate a long-term change in the pattern of regulation (William Kirby, U.S. Geological Survey, written commun., June 6, 2005). Additionally, some investigations have shown that substantial urbanization can lead to a reduction in low flows (U.S. Environmental Protection Agency, 2009). Final decisions to include or exclude data from a specific station analysis were made by using professional judgment based on the results of the QAQC analyses and other available information, such as comparisons with other long-term stations.

The QAQC analyses included the use of several computer programs developed to provide both visual and quantitative assessments (SAS Institute, Inc., 1989). The components of the QAQC reviews that were conducted for the CR stations are as follows:

- By climate year, a plot of the median daily mean flow ratios for the weekend (Saturday and Sunday) to the entire week (Sunday through Saturday), and the work week (Monday through Friday) to the entire week. These plots are especially useful for regulated streams and can show if the discharge patterns differ from week days to weekends, which can indicate variations in regulation patterns.

- As noted previously, Kendall's tau test is used to check for trends in the annual minimum 7-day average streamflow data over time.

- Plot of the annual minimum 7-day average streamflow against climate year, which is used along with the Kendall's tau results to assess potential trends.

- Plot of the relation of the ratio of the 10th percentile to the 50th percentile of the average 7-day flows $\left(7 \mathrm{Q}_{10 \%} / 7 \mathrm{Q}_{50 \%}\right)$ against climate year, which is useful for graphically assessing potential trends.

- Plot of the relation of the 50th percentile of the average 7-day flow against climate year. This plot is useful for assessing potential changes in the median average 7-day flow over time.

- Plot of the cumulative annual minimum 7-day average streamflow against climate year. This type of plot is known as a single-mass curve (Bohman and Patterson, 1993). A substantial change in the slope of this relation indicates a change in flow patterns. 
- Single-mass curve plot of the relation of the cumulative $7 \mathrm{Q}_{10 \%} / 7 \mathrm{Q}_{50 \%}$ against climate year. A substantial change in the slope of this relation indicates a change in flow patterns.

- Single-mass curve plot of the relation of the cumulative 50th percentile of the average 7-day flow against climate year. A substantial change in the slope of this relation indicates changes in the median average 7-day flow patterns.

Trend analyses, as described previously, were completed for all stations in the investigation using a p-value of 0.05 to determine significance (Kendall, 1938; Helsel and Hirsch, 2002). For the Kendall's tau trend analysis used in this study, the null hypothesis is that there is no trend in the annual minimum 7-day average flow data. Kendall's tau test is based on enumerating concordant (when both the $\mathrm{x}$ and $\mathrm{y}$ variables increase or decrease) and discordant (when $\mathrm{x}$ increases and $\mathrm{y}$ decreases or $\mathrm{x}$ decreases and $\mathrm{y}$ increases) pairs of $\mathrm{x}$ and $\mathrm{y}$ data (Kendall, 1938). Consequently, when stations have a substantial number of zero flows, causing ties in the pairs of $x$ and $y$ data, interpretation of the trend test can become tenuous at best. For the 277 streamgages analyzed in this investigation, only 12 had 7Q10 values of zero. Other site-specific findings from the QAQC analyses are noted in the Remarks column in table 1.

\section{Results of Kendall's Tau Analyses for Unregulated Streamgaging Stations}

For the annual minimum 7-day average flows at the unregulated streamgaging stations (or stations that are currently (2017) regulated but for which the frequency analysis was based on the unregulated period of record), 139 stations indicated no significant trend, 24 stations indicated a downward trend, and 17 stations indicated an upward trend (table 4, p. 66). Five of the 139 stations (sites 54, 68, 130, 136, and 202) that indicated no significant trend were analyzed for two different periods of record with both periods analyzed indicating no significant trend in the data. Of the 24 stations indicating a downtrend trend, 11 had record lengths less than 30 years. Of the 17 stations indicating and upward trend, 9 had record lengths less than 30 years. The record lengths of the 139 stations that indicated no statistically significant trend ranged from 10 to 85 years with an average of 28 years and a median of 23 years.

As noted previously, interpretations of trend analyses for relatively short records may only reflect a short-term condition and not be representative of an actual long-term change in the system. This is particularly true for relatively short-term records that begin or end in a historically low- or high-flow condition. If these periods include a short-term change and are not reflective of a shift in long-term climate conditions when viewed in terms of longer time frames, any apparent trend may just be part of a much longer term oscillation (Lins and others, 2010). For example, USGS station 02374500, Murder Creek near Evergreen, AL, is one of the long-term, unregulated stations included in this study having a record length of 76 years. Although a linear regression curve, which was included to provide a visual representation of potential trends, shows a slight downward slope for the period of record from climate year (CY) 1938 to 2013, the Kendall's tau analysis indicated no statistically significant trend (table 4; fig. 5A); however, to show how period of record and hydrologic conditions measured in that record can influence the trend assessment, a trend analysis for various subsets of those data was made. For the 25-year period 1989 to 2013, the analysis indicated a downward trend (tau value of -0.35 with a p-value of 0.013 ; fig. $5 B$ ). An analysis of the 26 -year period 1954 to 1979 indicated a statistically significant upward trend (tau value of 0.31 and p-value of less than 0.026; fig. 5C). Yet, a trend analysis of the complete period 1954 to 2013 indicated no significant trend (tau value of -0.096 and $p$-value of 0.28 ; fig. $5 D$ ). Again, these results emphasize the importance of long-term data-collection programs to accurately assess hydrologic trends.

\section{Results of Kendall's Tau Analyses for Regulated Streamgaging Stations}

Interpretations of trend analyses are more complicated for regulated streams. Streamflow at regulated stations also is influenced by changes in climate patterns or basin characteristics; however, those changes can be mitigated, enhanced, or even offset by changes in regulation patterns. Nonetheless, assessments of flow patterns are useful along with other assessments to help determine the appropriateness of a frequency analysis at a regulated station.

Of the 23 stations in which streamflow was influenced by regulated conditions and for which a trend analysis was completed, Kendall's tau results indicated no statistically significant trend at 17 stations, a downward trend at 5 stations, and an upward trend at 1 station (table 4). Regulated flow patterns were determined to be unsuitable for frequency analyses at an additional 7 stations, and, therefore, no trend analysis was completed (table 1). For those stations, exceedance percentiles of annual minimum 7-day average flows and duration of daily mean flows are provided (table 5, p. 78): 02411000, 02418500, 02420000, 02427500, 02429500, 02462500 , and 02462951 .

\section{Diversions}

Diversions from natural streamflow occur for a variety of reasons. Some diversions are the result of water-supply withdrawals, manufacturing processes, point-source discharges, and irrigation. Diversions by manufacturers are sometimes confined to short distances along rivers. Water may be removed from the river channel, passed through the manufacturing plant for use in processing, cooling, or dilution of wastes, and then returned 

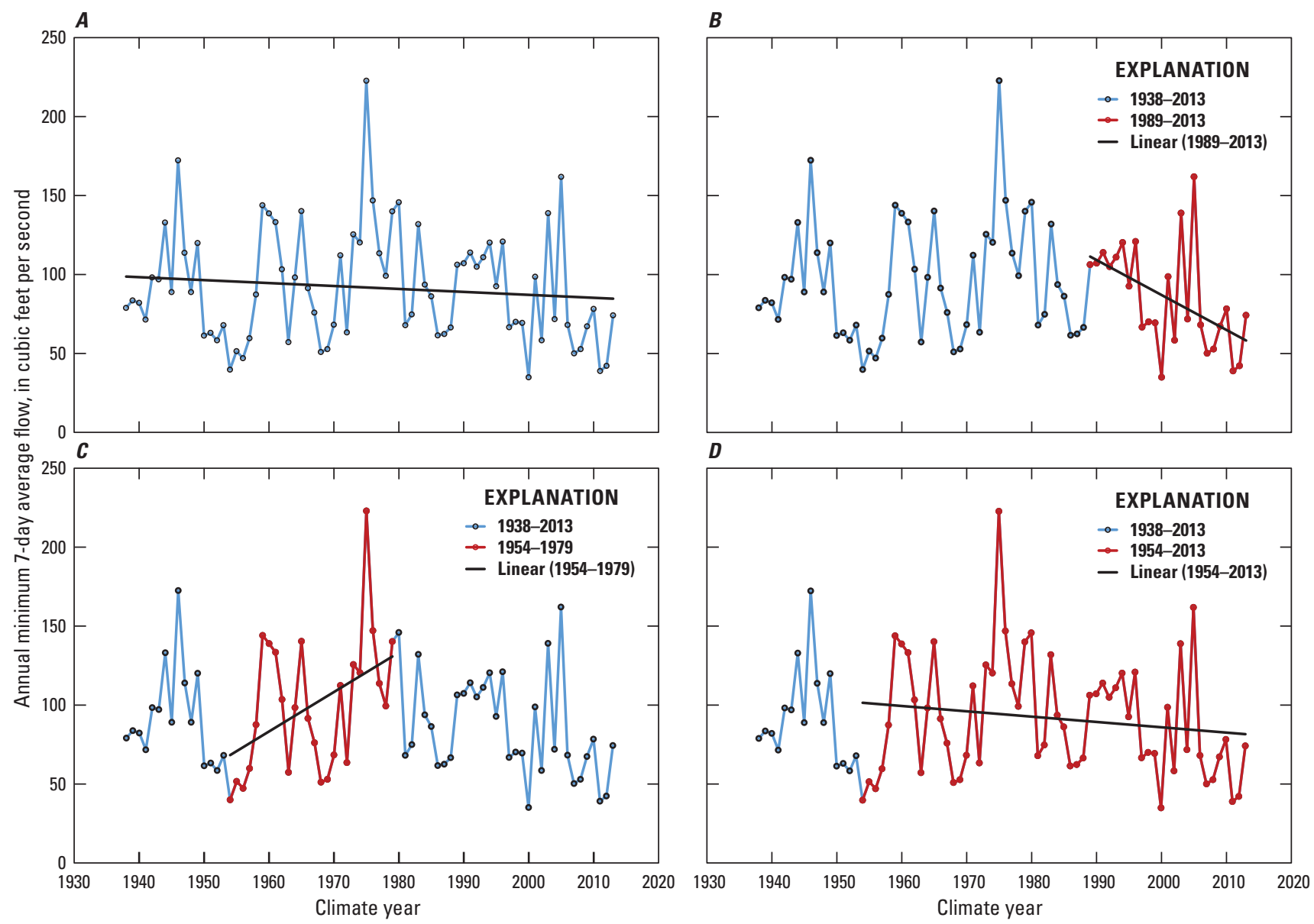

Figure 5. Various periods of record at U.S. Geological Survey station 02374500, Murder Creek near Evergreen, AL, for which a Kendall's tau trend test was computed for climate years A, 1938 to 2013, B, 1989 to 2013, C, 1954 to 1979, and D, 1954 to 2013.

to the river. Therefore, in some cases, consumptive losses from diversions by manufacturers may be negligible (Ries, 1994).

As such, the effects of diversions to the streamflow regime of a river are variable and depend not only on where the diversions occur but also on the final outcome of the diverted water.

Ries (1994) noted that water diverted from a stream or adjacent aquifer for municipal supplies is returned to the basin as effluent from individual septic systems or from wastewatertreatment plants within the basin and generally causes little loss of water to the basin; however, such diversions may affect the temporal pattern of streamflow. Diversions from one basin to another reduce streamflow in the donor basin and increase streamflow in the receiving basin. Diversions between subbasins of a larger basin can substantially affect streamflow in the subbasins, but if consumptive losses are negligible, streamflow in the larger basin may be nearly unaffected. The various diversion scenarios described above indicate that an accurate accounting of all diversions in a basin is generally problematic; therefore, most USGS low-flow analyses are made on the flow data as measured at the station without adjustments for diversions.
For the stations included in this report, determining the extent of any such diversions as noted above was beyond the scope of this investigation. For stations that have operated in the last decade or so, such information may have been included in USGS annual water data reports (https://wdr.water.usgs. gov/). If available, that information is noted in the Remarks section of table 5. In some cases, the QAQC assessments may have indicated the possible influence from some type of anthropogenic diversion or other source and, if so, that also was noted in the Remarks section of table 5 .

As an example of the influence of anthropogenic sources on streamflow, daily mean flow duration curves, standardized by drainage area, for four USGS streamgaging stations on the Cahaba River for the concurrent period July 27, 1996, to March 31, 2014, were compared (fig. 6): stations 02423425, Cahaba River near Cahaba Heights, AL; 02423496, Cahaba River near Hoover, AL; 02423500, Cahaba River near Acton, AL; and 02423555, Cahaba River near Helena, AL (fig. 1). The drainage areas for these stations are 201, 226, 230, and $335 \mathrm{mi}^{2}$, respectively. For high flows, the duration curves indicate that the daily mean flow per square mile is relatively 


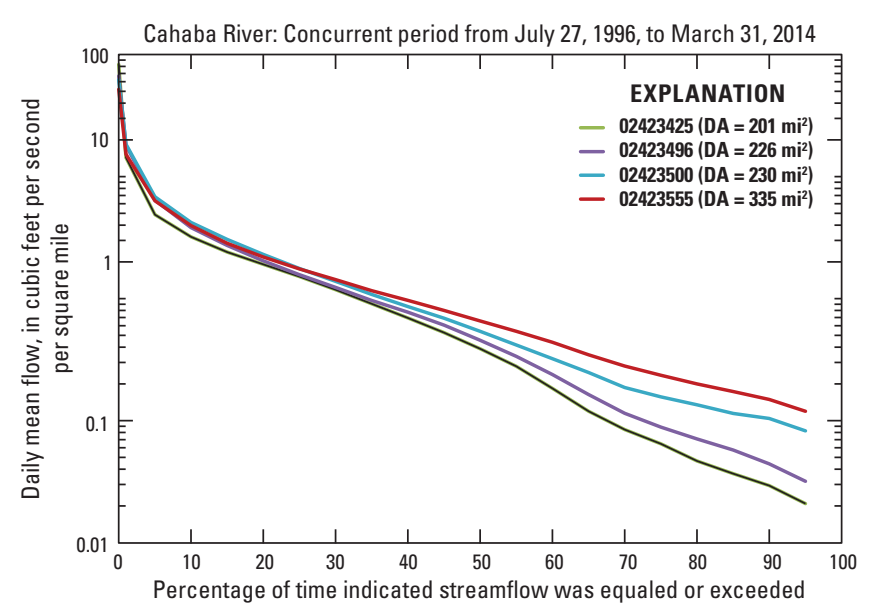

Figure 6. Comparison of daily mean flow duration curves, in cubic feet per second per square mile, for U.S. Geological Survey streamgaging stations 02423425, Cahaba River near Cahaba Heights, AL; 02423496, Cahaba River near Hoover, AL; 02423500, Cahaba River near Acton, AL; and 02423555, Cahaba River near Helena, AL. [DA, drainage area; $\mathrm{mi}^{2}$, square mile]

similar. However, between the 30 and 40 percentile flows, the curves begin to diverge with the slopes for stations 02423500 and 02423555 decreasing, which indicates a source of flow that is not occurring at the upstream gages. The source is likely from wastewater-treatment flow being discharged into the stream downstream from station 02423496. It should also be noted that these four stations are downstream from Lake Purdy, which was completed in 1964 (Ruddy and Hitt, 1990) and which partially regulates the streamflow from Little Cahaba River (U.S. Geological Survey, 2014). In addition, the Birmingham Water Works Board diverts water upstream from station 02423425 .

\section{Analytical Approach for Low-Flow Frequency Statistics}

The statistical analyses of streamflow data at CR stations in this study were generally based on three categories of stations: (1) long-term record stations on unregulated streams, (2) short-term record stations on unregulated streams, and (3) regulated stations. Typically, low-flow statistics are computed at CR stations if at least 10 years of record are available; however, computing the statistics from long-term records is preferred because they are likely to be more representative of a broader range of hydrologic conditions and are better suited for trend assessments and statistical estimates. The USGS typically considers 30 years of streamflow record to designate long-term streamgages (U.S. Geological Survey, 2013).

For stations with short-term records (those which have at least 10 years of record but less than about 30 years), the low-flow statistics can sometimes be improved by using record extensions or augmentation methods based on correlations with nearby long-term gages (Hirsch, 1982). This approach is particularly beneficial if the streamflow data at the short-term record station were collected during an unusually dry, wet, or otherwise unrepresentative period. For this study, streams for which several USGS streamgages are located and include both long-term and short-term gages, assessments were made to determine if suitable correlations exist between the short- and long-term gages. If conditions warranted, the short-term gage record was augmented based on the correlation with the long-term gage.

Although they can be applied to regulated streams if regulated patterns have been determined to be relatively stable for the period being analyzed, the techniques described above for augmenting short-term records are best suited for flows in natural streams and, therefore, were not applied to regulated streams in this investigation. In addition, the low-flow statistics for regulated streams are only relevant for existing regulation patterns and would cease to be applicable if the future regulation patterns were substantially altered.

For this investigation, the annual minimum 1- and 7-day average flows with recurrence intervals of 2, 5, 10, 20, or 50 years were computed using the log-Pearson Type III mathematical technique (LPIII) (Riggs, 1972; Barnes, 1979). The analyses were implemented with Surface-Water Statistics (SWSTAT) version 5.0, a computer program developed by the USGS (Hutchison, 1975; Lumb and others, 1990; Flynn and others, 1995). The SWSTAT program incorporates standard USGS methods for computing low-flow frequency statistics as described by Riggs (1972). In addition to the stand-alone version of SWSTAT, a beta version of the SW Toolbox program also was used in the analysis (Paul Hummel, Aqua Terra Consultants, written commun., September 26, 2014). The SW Toolbox program incorporates the algorithms from SWSTAT into a more user-friendly computer package for computing such statistical analyses. Three basic steps are involved in computing low-flow frequency estimates for CR streamgaging stations using the LPIII method (Riggs, 1972):

1. The low-flow streamflow data for a station are retrieved, compiled, and quality assured including trend assessments.

2. The data are divided into climate years (April 1 to March 31) for the available period of record.

3. The data are then ranked and analyzed for frequency of occurrence.

For CR streamgaging stations on regulated streams, lowflow characteristics also were assessed for long-term trends. If the assessment showed that the regulation patterns had been reasonably consistent and the LPIII distribution provided a reasonable fit of the data, low-flow statistics were computed for that period using similar techniques for the unregulated streamgaging stations (Riggs, 1972). In cases where regulation patterns were shown to be highly variable and (or) where the LPIII distribution did not reasonably represent the data (fig. 7), 


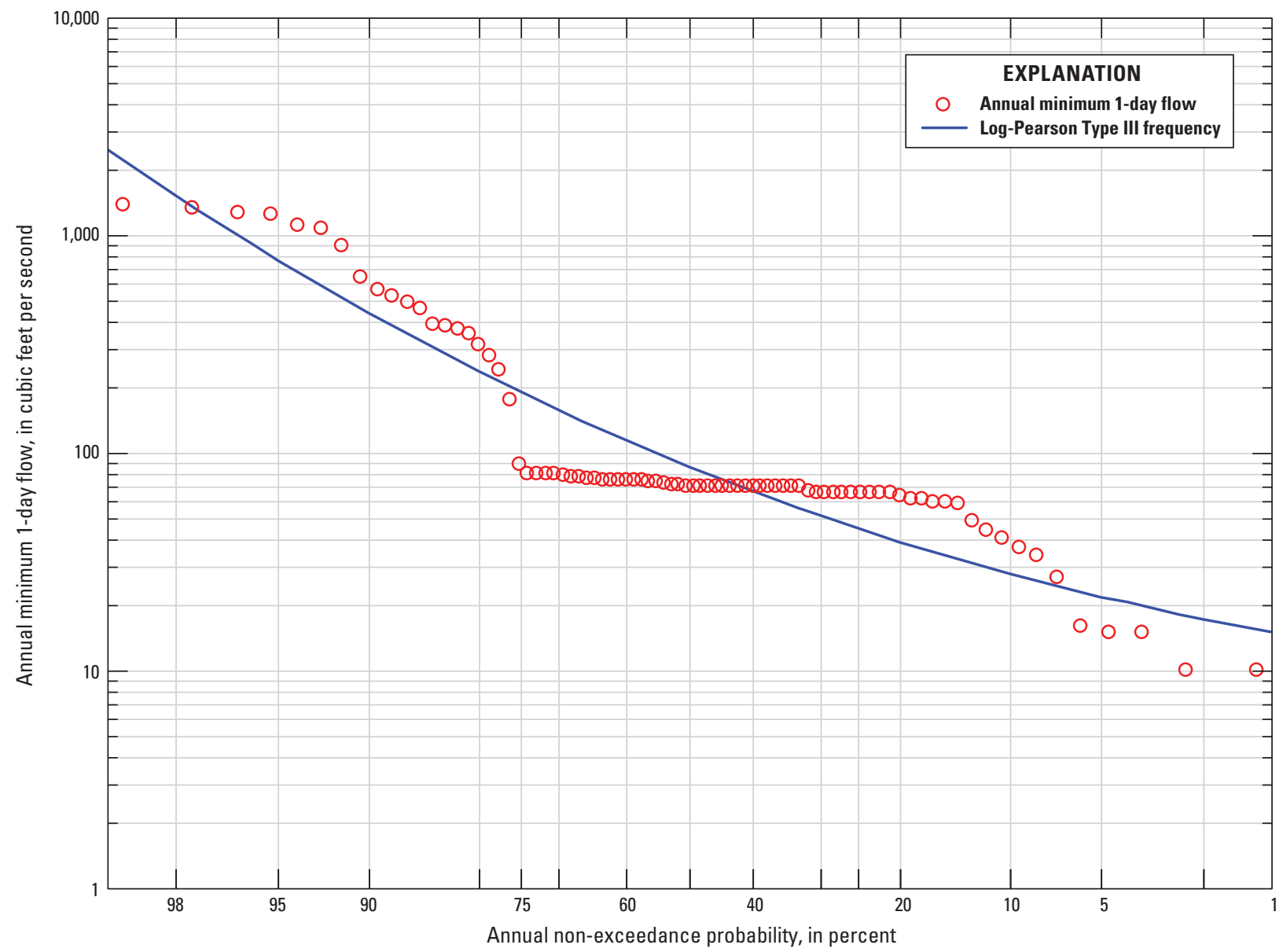

Figure 7. Example in which the log-Pearson Type III distribution does not adequately fit the annual minimum 1-day flows for regulated U.S. Geological Survey station 02418500, Tallapoosa River below Tallassee, Alabama.

tables of exceedance percentiles for consecutive 7-day average flows were generated in place of a frequency analysis for the stations (table 5). These exceedance percentiles should not be considered to be representative of a low-flow frequency but are merely a representation of recorded 7-day average flows. Nonetheless, the data are useful for assessing the flow conditions for the period of record.

\section{Low-Flow Frequency Analysis}

Low-flow frequency statistics at CR streamgaging stations can be computed by fitting a series of annual minimum N-day average streamflow to a known statistical distribution, where $N$ ranges from 1 to 365 . Low-flow frequency statistics for this study were computed by fitting a Pearson Type III distribution to the logarithms (base 10) of the annual minimum 1- and 7-day average streamflows, resulting in a LPIII distribution, and determining the flow estimates for the 2-, 5-, 10-, 20-, and 50-year recurrence intervals, depending on the length of record available at the station. Fitting the distribution required calculating the mean, standard deviation, and skew coefficient of the logarithms of the $\mathrm{N}$-day streamflows. Estimates of the
$\mathrm{N}$-day non-exceedance flows for a specified recurrence interval $T$ were computed by using the following equation:

$$
\log Q_{T}=\bar{X}+K S
$$

where

$Q_{T} \quad$ is the $N$-day low flow, in cubic feet per second, and $T$ is the recurrence interval, in years;

$\bar{X} \quad$ is the mean of the logarithms of the annual minimum $N$-day average streamflow;

$K$ is a frequency factor that is a function of the coefficient of skew at a recurrence interval of $T$, remembering that the low-flow non-exceedance probability is $1 / T$; and

$S \quad$ is the standard deviation of the logarithms of the annual minimum $N$-day average streamflow.

Low-flow statistics typically are presented as a set of non-exceedance probabilities or, alternatively, recurrence intervals with the associated low-flow values. The nonexceedance probability is defined as the probability that a flow 
at a given station will be equal to or less than the associated low-flow value once in a 1-year period and is expressed as a decimal fraction less than 1.0 or as a percentage less than 100 . Recurrence interval is defined as the average interval of years (often referred to as the return period) during which flows at a given station will be equal to or less than the associated low-flow value once. For example, a low-flow value at a given station with a non-exceedance probability of 0.10 indicates that flows at that station have a 10-percent chance of being equal to or less than the low-flow value once in any given year. Recurrence interval and annual non-exceedance probability are the mathematical inverses of one another; therefore, a flow with a non-exceedance probability of 0.10 has a recurrence interval of 1 divided by 0.10 or 10 years. Recurrence intervals, regardless of length, always refer to an average period of time (or years) in which flows at a given station will be equal to or less than the associated low-flow value once. A 10-year recurrence interval does not imply that the low-flow value will have a non-exceedance every 10 years; it does indicate, however, that the average time between recurrences is equal to 10 years. Consequently, an observed interval between a non-exceedance of the 7Q10 may be as short as 1 year or may be considerably longer than 10 years.

The low-flow frequency curve is generated by application of equation 1 to a set of annual minimum $N$-day average flows for a range of specified return periods. Low-flow frequency statistics estimated for recurrence intervals extrapolated substantially outside the number of years of available record can be less reliable (Barnes, 1979). For this study, the following criteria were used to limit the extrapolation of the frequency curves based on the period of record at the station:

1. Low-flow frequency statistics with recurrence intervals of $2,5,10$, and 20 years are provided for stations that have between 10 and 30 years of annual streamflow record; and

2. Low-flow frequency statistics with recurrence intervals of $2,5,10,20$, and 50 years are provided for stations with 30 or more years of annual streamflow record. No data were compiled for recurrence intervals greater than 50 years.

Low-flow statistics were computed for the annual minimum 1and 7-day flows for recurrence intervals of 2, 5, 10, 20, and 50 years, depending on the length of streamflow record available and the criteria noted above. For each station, the frequency curves were reviewed to verify that the LPIII distribution adequately fit the data.

An example of the frequency curve generated using the LPIII curve-fitting procedure is illustrated in figure $8 A$. In figure $8 B$, the annual minimum 7-day average flows computed for USGS station 02374500 for CYs 1938 to 2013 are shown with the 7Q10 estimate from those data. The 7Q10 estimate is based on 76 years of record. Eight of the annual minimum 7-day average flows are about equal to or less than the 7Q10 value and account for about 10 percent of the 76 values. The exceedances do not occur at a regular interval of 10 years, but on average, there was an exceedance about once every 10 years (eight exceedances in the 76-year record). The results of the low-flow frequency analyses are presented in table 5 (p. 78).

\section{Conditional Probability Adjustment}

Zero flows cannot be included in a LPIII distribution because they cannot be transformed logarithmically. When zero flows are part of the $\mathrm{N}$-day flows at a streamgaging station, a conditional probability adjustment can be made in order to estimate the low-flow characteristics (Jennings and Benson, 1969; Tasker, 1987). Additional information on the procedures and guidelines for the conditional probability adjustment can be found in Bulletin 17B of the Hydrology Subcommittee of the Interagency Advisory Committee on Water Data (1982) and in the U.S. Geological Survey Surface Water Branch Technical Memorandum No. 70.07 (Carter, 1969).

To calculate the adjusted probability, a LPIII analysis is done using only the non-zero values. Then, the conditional probability adjustment is made using the following formula:

$$
P_{a d j}=\left(\frac{n}{N}\right) P_{n}+\frac{(N-n)}{N}
$$

where

$$
\begin{aligned}
& P_{a d j} \quad \text { is the adjusted non-exceedance probability; } \\
& P_{n} \quad \text { is the non-exceedance probability for the } \\
& \text { non-zero values; } \\
& n \quad \text { is the number of non-zero values; and } \\
& N \quad \text { is the total number of values. }
\end{aligned}
$$

Using the adjusted probability, $P_{a d j}$, an adjusted frequency factor, $K^{*}$, is obtained for use in equation 1 (Austin and others, 2011).

\section{Record-Extension Technique}

Streamflow statistics may be needed to estimate probabilities of occurrences for periods longer than the actual measured period of record. Consequently, short records that may have been collected during an unusually dry, wet, or otherwise unrepresentative period may not represent the more desirable wider range of potential hydrologic regimes. Under certain conditions, it is possible to extend or augment a short record by using a correlated station having a longer record. The extended record at the short-term record station will better reflect low-flow conditions over a longer period and provide better estimates of low-flow statistics at that station. The record extension can be accomplished as described next.

If a linear relation between the logarithms of the $N$-day flows at a short-term record station is determined to be significantly correlated to a concurrent set of the $\mathrm{N}$-day flows at a long-term record station, or index station, a mathematical record-extension method known as the Maintenance of 


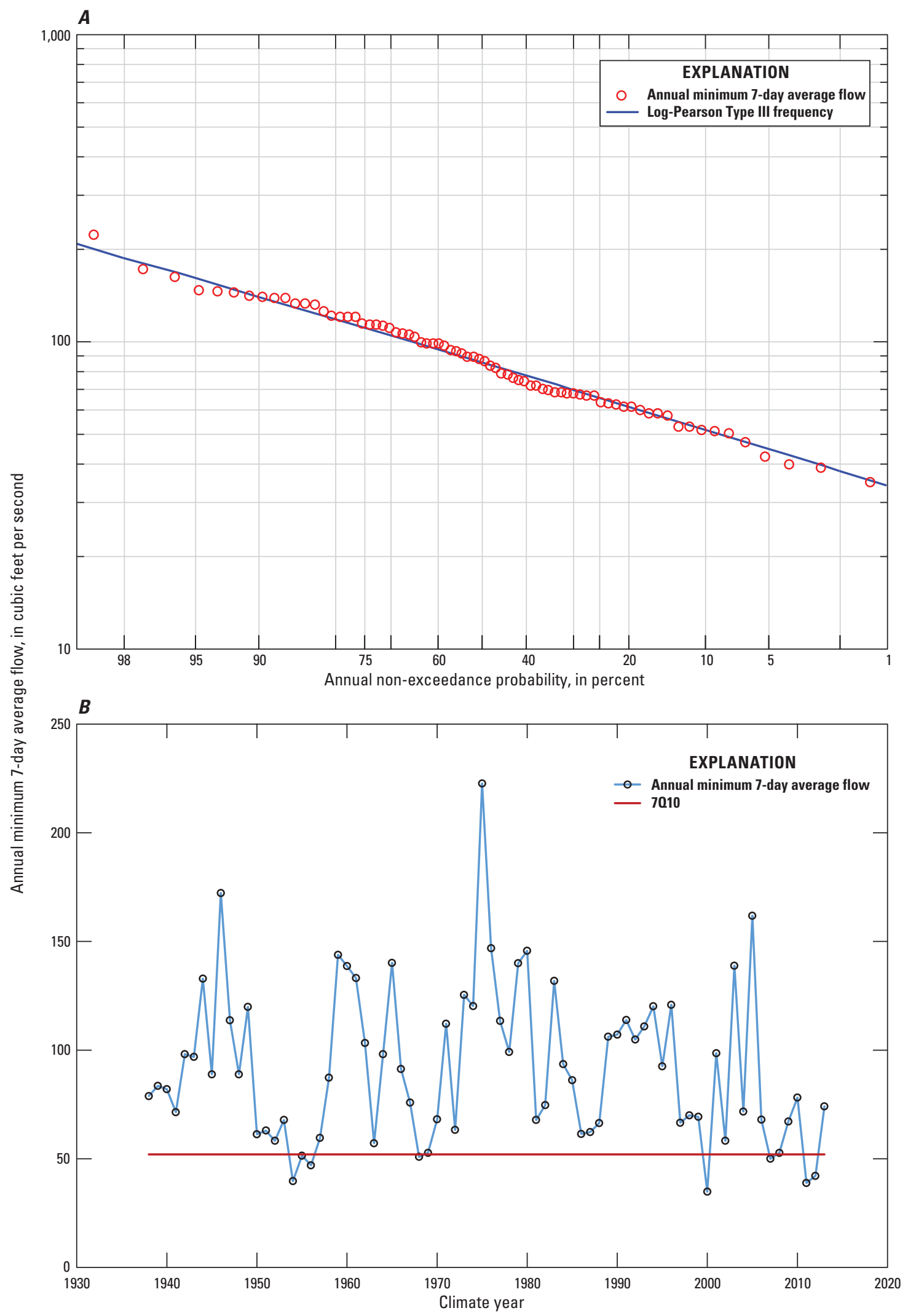

Figure 8. U.S. Geological Survey streamgaging station 02374500, Murder Creek near Evergreen, AL, $A$, low-flow frequency curve for the annual minimum 7-day average streamflow, and $B$, annual minimum 7-day average flows by climate year with the 7010 estimate computed from those flows. 
Variance Extension, Type 1 (MOVE.1) method (Hirsch, 1982) can be used to extend the record at the short-term record station. The MOVE.1 formulation maintains the mean and the variance of the data at the short-term record station and, therefore, allows for the generation of a longer-term set of data that will possess the statistical characteristics of the actual measured data from the short-term record.

The MOVE. 1 equation is

$$
Y_{i}=\bar{Y}+\frac{S_{y}}{S_{x}}\left(X_{i}-\bar{X}\right),
$$

where

$Y_{i} \quad$ is the logarithm of the estimated $N$-day flow at the short-term record station;

$\bar{Y} \quad$ is the mean of the logarithms of $N$-day flows for the concurrent period at the short-term record station;

$S_{y} \quad$ is the standard deviation of the logarithms of $N$-day flows for the concurrent period at the short-term record station;

$S_{x} \quad$ is the standard deviation of the logarithms of $N$-day flows for the concurrent period at the long-term record station or index station;

$X_{i} \quad$ is the logarithm of the flow statistic or observed $N$-day flow at the long-term record station or index station; and

$\bar{X} \quad$ is the mean of the logarithms of the $N$-day flows for the concurrent period at the long-term record station or index station.

For this study, the record augmentations were limited to short-record sites that were located on the same stream as the index station. For an index station to be considered, a minimum of 10 years of concurrent record relative to the short-term station and similar basin geology as the short-term station were necessary. Also, basin sizes were considered (Telis, 1991). The USGS does not have a standard minimum correlation coefficient between concurrent flows for the MOVE.1 technique; however, similar correlation studies have used values ranging from 0.70 to 0.80 (Hydrology Subcommittee of the Interagency Advisory Committee on Water Data, 1982; Stedinger and Thomas, 1985; Ries, 1994; Nielsen, 1999). In addition, if the record at the short-term record station or available index station includes zero flows, record extensions are not recommended because including such values in record-extension techniques has not been adequately tested (Julie Kiang, U.S. Geological Survey Office of Surface Water, written commun., January 26, 2010).
Six CR streamgaging stations met the criteria listed above, and the records were augmented using the MOVE.1 record-extension technique (table 6). Applying the MOVE.1 technique at these six stations allowed for the low-flow statistics at the short-record stations to be enhanced by an additional 48 to 67 years of record. Thus, the augmented lowflow statistics at the short-record stations will reflect a much broader range of hydrologic conditions. An example of the MOVE. 1 augmentation for station 02361500, Choctawhatchee River near Bellwood, AL, using index station 02361000, Choctawhatchee River near Newton, AL, is shown in figure 9.

\section{Flow-Duration Analysis}

Flow-duration analyses estimate the percentage of time that a specified streamflow is equaled or exceeded during a given period (Searcy, 1959). Flow durations are estimated by sorting the daily mean flows for the period of record from the largest value to the smallest value and assigning each streamflow value a rank, from 1 to the largest value. The frequencies of exceedance are then computed using the Weibull formula for computing plotting position (Helsel and Hirsch, 2002):

$$
P=100 *[M /(n+1)],
$$

where

$$
\begin{aligned}
& P \quad \text { is the probability that a given flow will be } \\
& M \quad \text { is the ranked position (dimensionless), and } \\
& n \quad \text { is the number of events for the period of } \\
& \text { record (dimensionless). }
\end{aligned}
$$

Flow-duration curves are a cumulative frequency curve showing the percent of time that specified streamflows have been equaled or exceeded during a given period of record (Searcy, 1959). Yet, if the duration curve is based on a sufficiently long period of record, the statistics can be used as an indicator of probable future conditions. In order to compare flow durations at different stations or in different basins, flow-duration estimates can be normalized by drainage area to represent a streamflow per unit area. Again, it should be noted that the most useful comparisons are those based on similar lengths of record from similar hydrologic periods.

Flow durations for this report are estimated in tabular form for the 5-, 10-, 25-, 50-, 75-, 90-, and 95-percent exceedances (table 5). To be consistent with low-flow statistics, flow durations were computed on the basis of daily mean flows available through the 2013 climate year, which has an end date of March 31, 2014. An example of a flow-duration curve is shown in figure 10 for USGS station 02361000 , Choctawhatchee River near Newton, AL, for the period December 1921 to September 1927 and June 1935 to March 2014. 
Table 6. Streamgaging stations for which record was augmented using MOVE.1, index stations, additional climate years of record available at the index station, and correlation coefficients for the gaging stations where the record was augmented.

[USGS, U.S. Geological Survey; $\mathrm{mi}^{2}$, square mile]

\begin{tabular}{|c|c|c|c|c|c|c|c|c|c|c|c|c|}
\hline \multicolumn{5}{|c|}{ Streamgaging station where record was augmented } & \multicolumn{5}{|c|}{ Index streamgaging stations } & \multirow{2}{*}{$\begin{array}{l}\text { Number of } \\
\text { additional } \\
\text { climate years } \\
\text { of record } \\
\text { available at the } \\
\text { index station }\end{array}$} & \multicolumn{2}{|c|}{$\begin{array}{l}\text { Correlation } \\
\text { coefficient }\end{array}$} \\
\hline $\begin{array}{l}\text { Site } \\
\text { identifi- } \\
\text { cation } \\
\text { number } \\
\text { (fig. 1) }\end{array}$ & $\begin{array}{c}\text { USGS } \\
\text { stream- } \\
\text { gaging } \\
\text { number }\end{array}$ & $\begin{array}{c}\text { USGS } \\
\text { streamgaging } \\
\text { name }\end{array}$ & $\begin{array}{l}\text { Drain- } \\
\text { age } \\
\text { area } \\
\left(\mathrm{mi}^{2}\right)\end{array}$ & Period of record & $\begin{array}{l}\text { Site } \\
\text { identifi- } \\
\text { cation } \\
\text { number } \\
\text { (fig. 1) }\end{array}$ & $\begin{array}{c}\text { USGS } \\
\text { stream- } \\
\text { gaging } \\
\text { number }\end{array}$ & $\begin{array}{c}\text { USGS } \\
\text { streamgaging } \\
\text { name }\end{array}$ & $\begin{array}{l}\text { Drain- } \\
\text { age } \\
\text { area } \\
\left(\mathrm{mi}^{2}\right)\end{array}$ & Period of record & & 1-day & 7-day \\
\hline 8 & 02361500 & $\begin{array}{l}\text { Choctawhatchee } \\
\text { River near } \\
\text { Bellwood, AL }\end{array}$ & 1,280 & $\begin{array}{l}\text { December } 1921 \text { to } \\
\text { October } 1925, \\
\text { December } 2000 \text { to } \\
\text { March } 2014\end{array}$ & 7 & 02361000 & $\begin{array}{l}\text { Choctawhatchee } \\
\text { River near } \\
\text { Newton, AL }\end{array}$ & 686 & $\begin{array}{l}\text { December } 1921 \text { to } \\
\text { September } 1927 \text {, } \\
\text { June } 1935 \text { to } \\
\text { March } 2014\end{array}$ & 67 & 0.95 & 0.97 \\
\hline 25 & 02374700 & $\begin{array}{l}\text { Murder Creek at } \\
\text { State High- } \\
\text { way } 41 \text { at } \\
\text { Brewton, AL }\end{array}$ & 435 & $\begin{array}{r}\text { March } 1999 \text { to } \\
\text { March } 2014\end{array}$ & 24 & 02374500 & $\begin{array}{l}\text { Murder } \\
\text { Creek near } \\
\text { Evergreen, } \\
\text { AL }\end{array}$ & 176 & $\begin{array}{l}\text { October } 1937 \text { to } \\
\text { March } 2014\end{array}$ & 61 & 0.93 & 0.93 \\
\hline 63 & 02411930 & $\begin{array}{l}\text { Tallapoosa } \\
\text { River below } \\
\text { Tallapoosa, } \\
\text { GA }\end{array}$ & 272 & $\begin{array}{l}\text { December } 1999 \text { to } \\
\text { November } 2004 \text {, } \\
\text { October } 2005 \text { to } \\
\text { March } 2014\end{array}$ & 64 & 02412000 & $\begin{array}{l}\text { Tallapoosa } \\
\text { River near } \\
\text { Heflin, AL }\end{array}$ & 448 & $\begin{array}{l}\text { July } 1952 \text { to } \\
\text { March } 2014\end{array}$ & 49 & 0.97 & 0.97 \\
\hline 119 & 02445500 & $\begin{array}{l}\text { Sipsey River at } \\
\text { Fayette, AL }\end{array}$ & 282 & $\begin{array}{l}\text { February } 1939 \text { to } \\
\text { September } 1959\end{array}$ & 121 & 02446500 & $\begin{array}{l}\text { Sipsey } \\
\quad \text { River near } \\
\text { Elrod, AL }\end{array}$ & 528 & $\begin{array}{l}\text { September } 1928 \\
\text { to March } 1932, \\
\text { October } 1939 \text { to } \\
\text { September } 1971 \text {, } \\
\text { October } 1978 \text { to } \\
\text { March } 2014\end{array}$ & 49 & 0.95 & 0.96 \\
\hline 120 & 02446000 & $\begin{array}{c}\text { Sipsey River } \\
\text { at Moores } \\
\text { Bridge, AL }\end{array}$ & 413 & $\begin{array}{l}\text { February } 1939 \text { to } \\
\text { September } 1951\end{array}$ & 121 & 02446500 & $\begin{array}{l}\text { Sipsey } \\
\text { River near } \\
\text { Elrod, AL }\end{array}$ & 528 & $\begin{array}{l}\text { September } 1928 \\
\text { to March } 1932, \\
\text { October } 1939 \text { to } \\
\text { September } 1971 \text {, } \\
\text { October } 1978 \text { to } \\
\text { March } 2014\end{array}$ & 57 & 0.98 & 0.99 \\
\hline 122 & 02447000 & $\begin{array}{l}\text { Sipsey River } \\
\text { near Pleasant } \\
\text { Ridge, AL }\end{array}$ & 769 & $\begin{array}{l}\text { February } 1939 \text { to } \\
\text { September } 1959\end{array}$ & 121 & 02446500 & $\begin{array}{l}\text { Sipsey } \\
\quad \text { River near } \\
\text { Elrod, AL }\end{array}$ & 528 & $\begin{array}{l}\text { September } 1928 \\
\text { to March } 1932, \\
\text { October } 1939 \text { to } \\
\text { September } 1971 \text {, } \\
\text { October } 1978 \text { to } \\
\text { March } 2014\end{array}$ & 48 & 0.97 & 0.98 \\
\hline
\end{tabular}




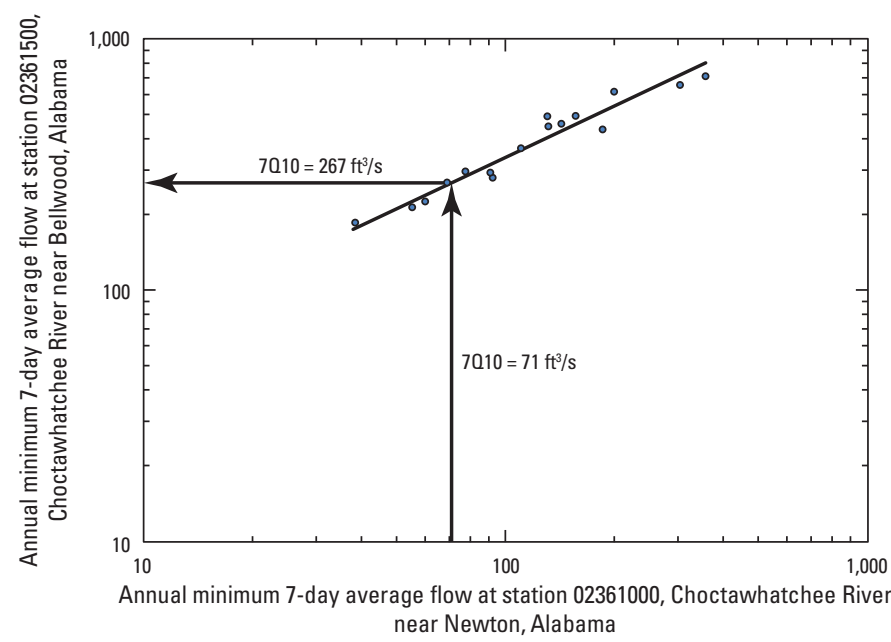

Figure 9. The MOVE.1 correlation of annual minimum 7-day average flow at U.S. Geological Survey streamgaging station 02361500, Choctawhatchee River near Bellwood, AL, with index station 02361000, Choctawhatchee River near Newton, AL. $\left[\mathrm{ft}^{3} / \mathrm{s}\right.$, cubic foot per second]

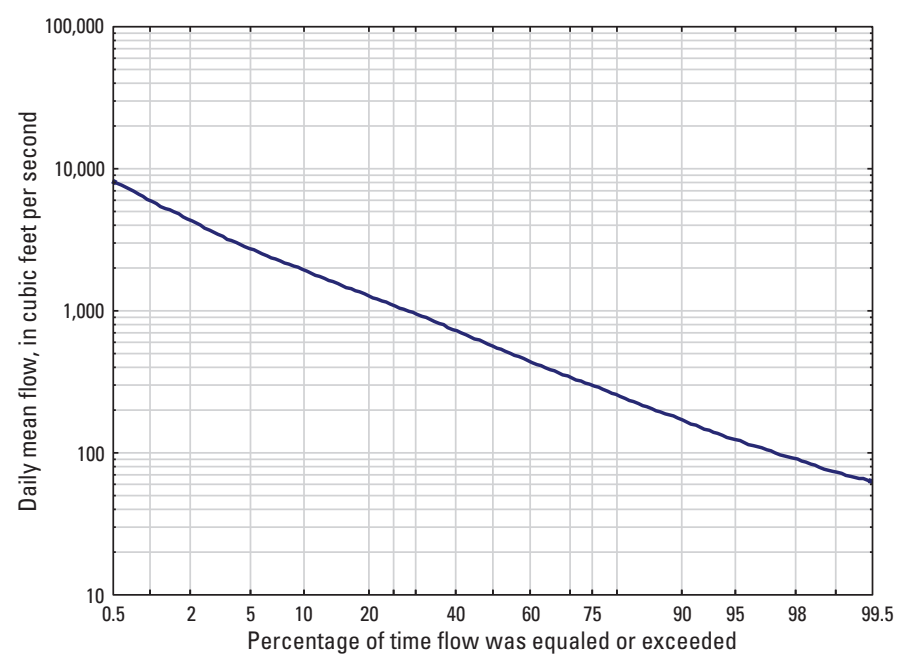

Figure 10. Flow-duration curve for U.S. Geological Survey streamgaging station 02361000, Choctawhatchee River near Newton, AL, for the period December 1921 to September 1927 and June 1935 to March 2014.

\section{Considerations for Accuracy of Low-Flow Statistics}

With respect to streamflow statistics, the period of collected record is considered to be a sample, or small portion of the population, which represents all possible measurements. Statistical results provide inferences about the characteristics of the population on the basis of samples from the population.
For example, statistical measures - such as mean, standard deviation, or skew coefficient — can be described in terms of the sample and then used to make inferences about the population from which the sample was obtained. Statistical values computed from the sample record are estimates of what the value would be if the entire population were known and used to compute the given value. Consequently, the accuracy of low-flow statistics at streamgaging stations is strongly influenced by the length of record (samples from the population) upon which the statistics are based and the hydrologic conditions during the period sampled (Barnes, 1979). The longer the period of record at a streamgaging station that covers a broad range of hydrologic conditions, the more accurate or reflective of long-term conditions the low-flow statistics will be.

Streamflow statistics for short-term records are more sensitive to extreme hydrologic events than those for longterm records. As a result, streamflow statistics, whether high or low, from one 10-year period may differ significantly from another 10-year period (Dalrymple, 1960). Thus, a long-term record is more desirable when computing streamflow statistics. To show the effect that record length and hydrologic conditions have on low-flow statistics, the 7Q10 for USGS station 02374500, Murder Creek near Evergreen, AL, was computed beginning with the first 10 years of record (April 1938-March 1948) and then updated on a 5-year basis through CY 2013. A plot of the annual minimum 7-day average streamflow by climate year for the period of record with the computed 7 Q10 estimates indicates that the 7Q10 for the first 10 years of record, which represents a relatively wet period, was 73 cubic feet per second ( $\mathrm{ft}^{3} / \mathrm{s}$; fig. 11). By CY 1957 and as a result of the drought years in the 1950s, the 7Q10 decreased to $50 \mathrm{ft}^{3} / \mathrm{s}$. The 1970 s tended to be a relatively wet period, and the 7Q10 continued to increase slightly until it peaked at $57 \mathrm{ft}^{3} / \mathrm{s}$ in 1997 . With the dry years that occurred between 2000 and 2013, the 7Q10 decreased slightly to $52 \mathrm{ft}^{3} / \mathrm{s}$ by 2013. The reason the $7 \mathrm{Q} 10$ did not change substantially after CY 1957 but was so variable before that time was that the preceding period from CY 1938 to 1957 included a wide range of hydrologic conditions, such as the relatively wet period in the 1940s and the drought period in the 1950s (fig. 3). In this analysis, the percentage difference between the highest (73 $\mathrm{ft}^{3} / \mathrm{s}$ in CY 1947) and lowest (50 ft $3 / \mathrm{s}$ in CY 1957) 7Q10 is 37 percent, with percentage difference computed as

$$
\text { Percentage Difference }=\frac{\left|x_{1}-x_{2}\right|}{\left(\frac{x_{1}+x_{2}}{2}\right)} \times 100
$$

where

$$
\begin{gathered}
x_{1} \quad \begin{array}{c}
\text { is the highest 7Q10 estimate from the periods } \\
\text { analyzed, and }
\end{array} \\
x_{2} \quad \begin{array}{c}
\text { is the lowest 7Q10 estimate from the periods } \\
\text { analyzed. }
\end{array} \\
\text { To further show the effect of how the 7Q10 can be influ- }
\end{gathered}
$$
enced under varying hydrologic conditions and the substantial 


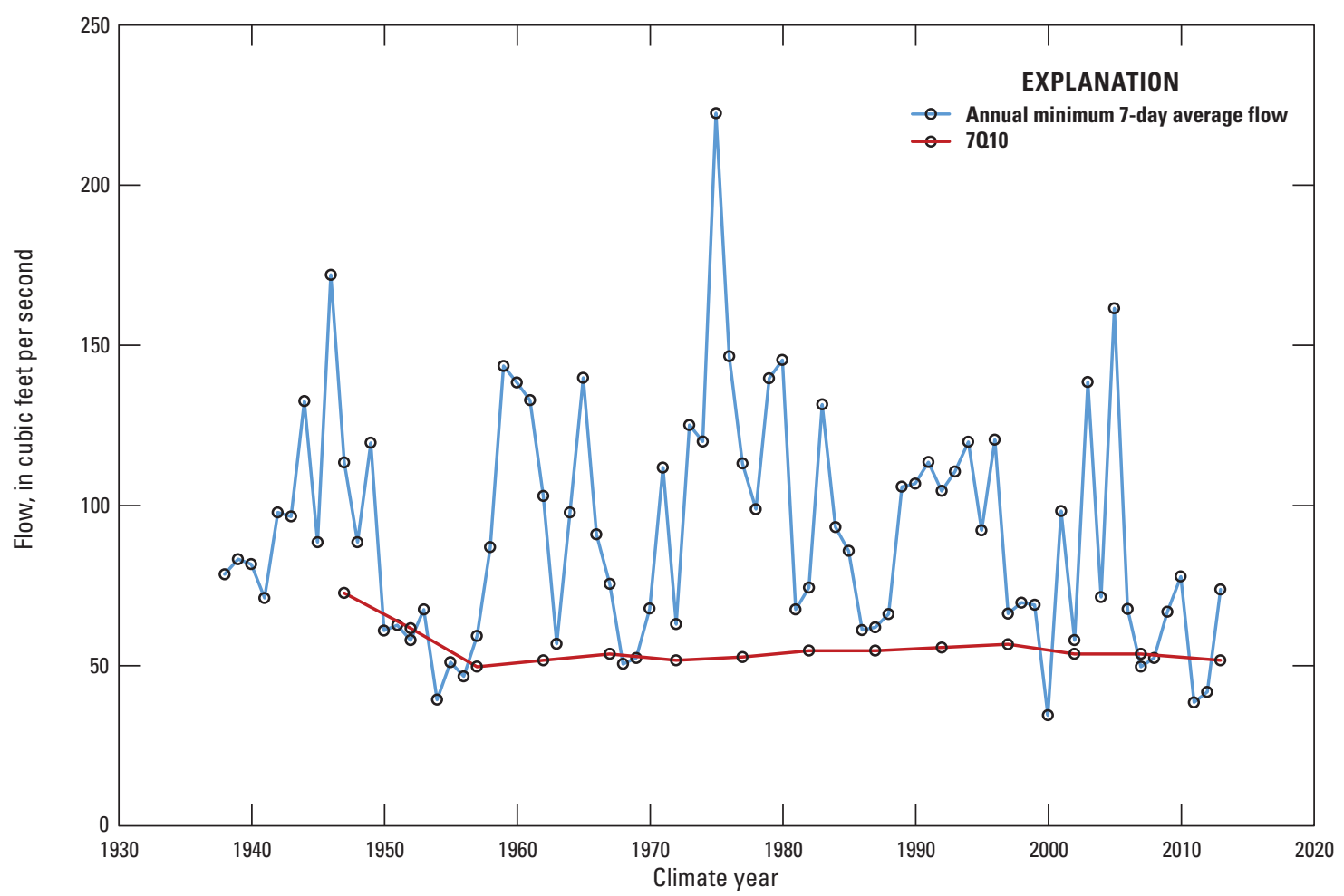

Figure 11. Annual minimum 7-day average streamflow and 7010 estimates at U.S. Geological Survey streamgaging station 02374500, Murder Creek near Evergreen, AL.

influence period of record can have on streamflow statistics, an additional analysis was done using seven different 10-year periods of record at USGS station 02374500 . The comparison was made by computing the $7 \mathrm{Q} 10$ at station 02374500 for the following periods (fig. 12): CY 1938 to 1947, 1948 to 1957, 1958 to 1967,1968 to 1977,1978 to 1987,1988 to 1997 , and 1998 to 2007. For those seven periods, the lowest 7Q10 of $42 \mathrm{ft}^{3} / \mathrm{s}$ was computed for the period CY 1998 to 2007 . The highest 7Q10 of $73 \mathrm{ft}^{3} / \mathrm{s}$ was computed for the periods CY 1938 to 1947 and CY 1988 to 1997 . The percentage difference between the highest and lowest 7Q10 values was 54 percent. As noted in the previous paragraph, the $7 \mathrm{Q} 10$ for the complete period of record from CY 1938 to 2013 was $52 \mathrm{ft}^{3} / \mathrm{s}$. The percentage difference between the 10 -year period with the highest 7Q10 value and the 7Q10 for the complete period of record was 34 percent. The percentage difference between the 10 -year period with the lowest $7 \mathrm{Q} 10$ value and the 7Q10 for the complete period of record was 21 percent.

The previous examples highlight the influence that record length and hydrologic conditions measured in the record, which is related to the period of record measured, have on the low-flow frequency statistics. To provide perspective on these characteristics for the unregulated Alabama stations included in this investigation, the distribution of years of record included in the frequency analysis are shown in figure $13 \mathrm{~A}$, and the period of record with the beginning and ending years plotted by drainage area are shown in figure $13 B$.

\section{Time-Sampling Errors}

As previously discussed, low-flow frequency statistics only provide an estimate of the true (or population) flow characteristic of interest. Uncertainty (or error) in those estimates include a time-sampling error, based on the period of record available for the analysis, and a model error, based on the analytical method being used (Hardison, 1969). There also is uncertainty in the discharge measurements and the analytical methods used for computing the instantaneous values that are based on those measurements (Sauer and Turnipseed, 2010; Turnipseed and Sauer, 2010). Measurement errors are often assumed to be small compared to time-sampling errors; however, for low-flow estimates, that may not always be the case (Telis, 1991).

To provide an estimate of the standard error of the 1- and 7-day low-flow frequency estimates, a time-sampling error was computed for the estimates at each streamgaging station where the particular low-flow statistic was non-zero (table 5). The time-sampling error computations assume that the Pearson Type III distribution adequately fits the logarithms of the 1- and 7-day annual minimum average flows, the measurement error is small compared to the time-sampling error, and the annual low-flow estimates are a reasonable representation of the long-term estimates (Telis, 1991). Although each of these assumptions will not necessarily be valid at all streamgaging stations, the time-sampling errors are useful for general comparisons of accuracy between stations and can be useful for assessing regional variability in the streamflow data. 


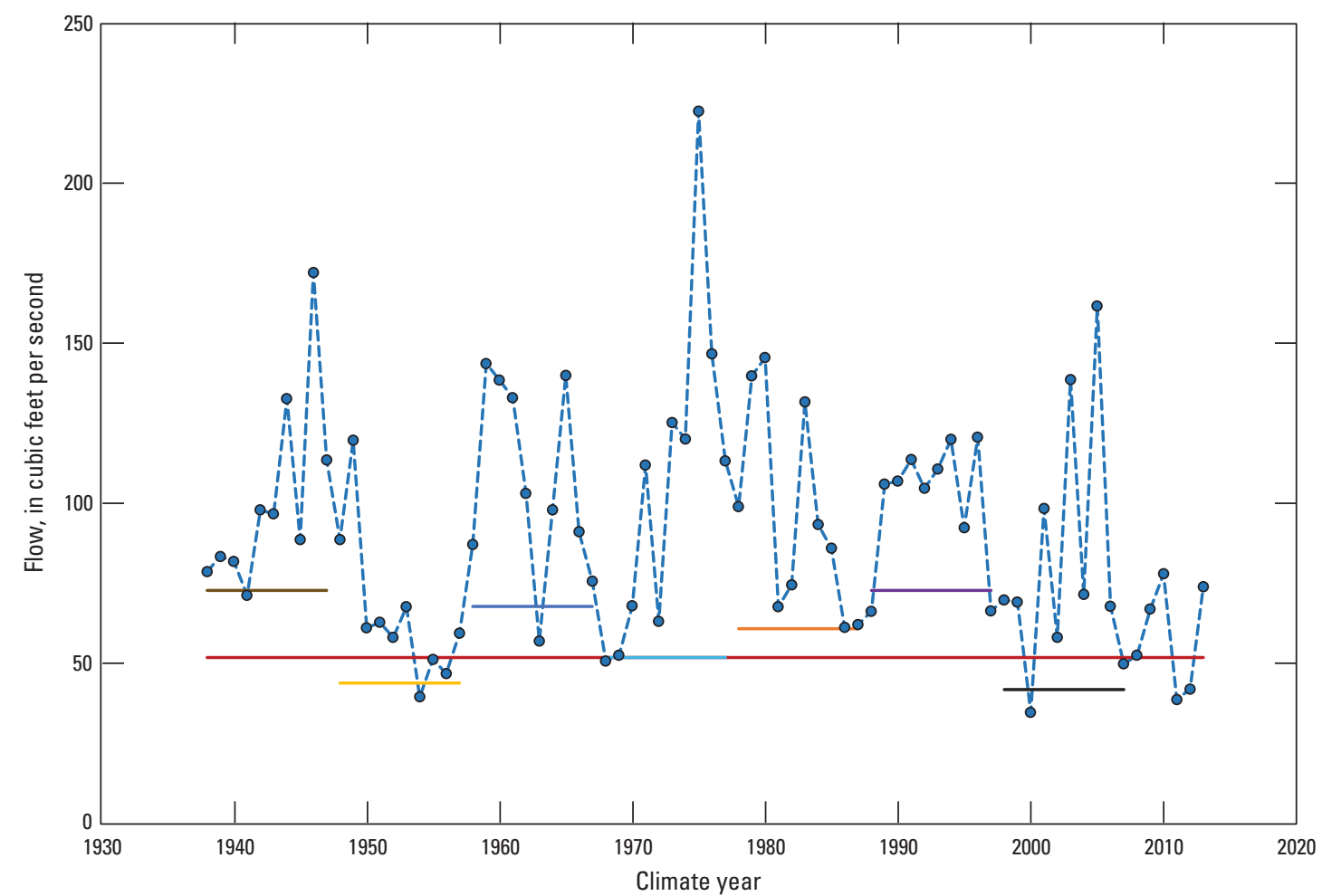

EXPLANATION

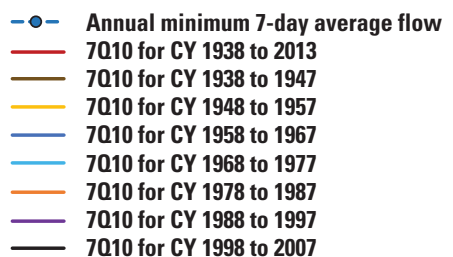

Figure 12. Annual minimum 7-day average streamflow and 7010 estimates at U.S. Geological Survey streamgaging station 02374500, Murder Creek near Evergreen, AL, for seven 10-year periods: climate years 1938 to 1947,1948 to 1957,1958 to 1967,1968 to 1977,1978 to 1987, 1988 to 1997, and 1998 to 2007.

The time-sampling error (SE) is a function of the standard deviation, years of record, and a frequency factor, which is based on the skew of the flow characteristic being used (Kite, 1985). The time-sampling error is defined as

$$
S E=\frac{S}{\sqrt{N}} \delta
$$

where:

$S E \quad$ is the time-sampling error in log units of a low-flow characteristic with a t-year recurrence interval for a continuous record streamgaging station;

$S \quad$ is the standard deviation of the logarithms of the annual minimum 1- or 7-day average flows at the station;

$N \quad$ is the number of years of record of the lowflow series; and $\delta \quad$ is a frequency factor that is a function of the skew of the annual minimum 1- or 7-day average flows at the station and the recurrence interval (Kite, 1985).

The time-sampling errors presented in this report were converted to percentage using the conversion equation

$$
S E(\text { percent })=\left[10^{2.3(S E)^{2}}-1\right]^{0.5}(100) \text {. }
$$

Kite (1985) provided only $\delta$ frequency factors for positive skews from 0.0 to 2.0 for flood-flow exceedances. For low-flow non-exceedances, the $\delta$ frequency factors shown in Kite (1985) would be applicable to the equivalent negative skew. For example, for a skew value of 2.0, the $\delta$ frequency factor shown in Kite (1985) for the 2-year recurrence interval is 1.3913. For low-flow non-exceedances, the skew value for a $\delta$ frequency factor of 1.3913 is -2.0 . The low-flow non-exceedance 

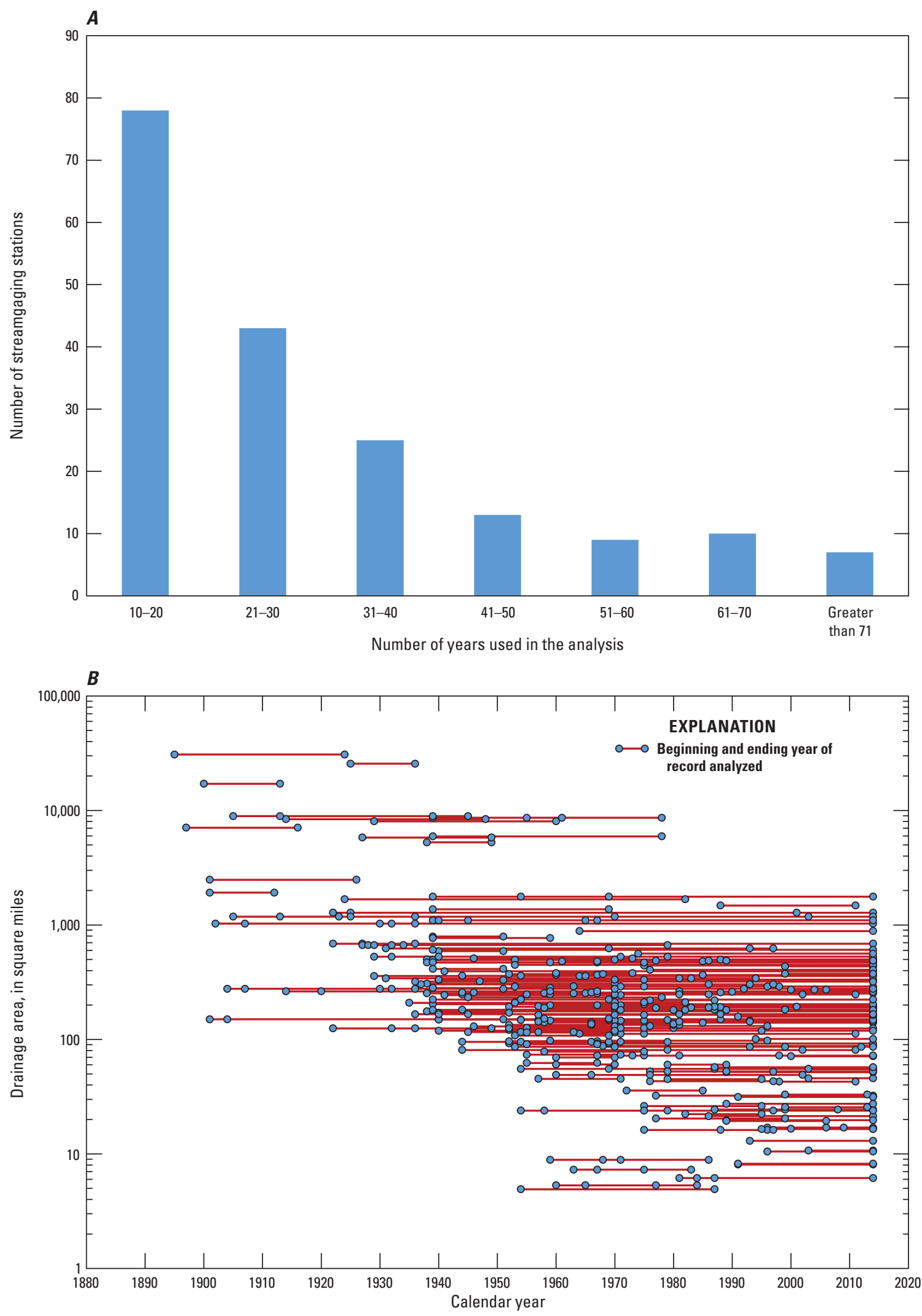

Figure 13. A, Distribution of years of record for the unregulated Alabama stations, and $B$, the period of record with the beginning and ending years shown by drainage area. 
frequency factors for positive skews were not available in Kite (1985) but were obtained from training-class documents for USGS training class Statistical Approach to Surface-Water Hydrologic Analysis (SW-2011-TC), November 1999 (W.O. Thomas, Jr., Michael Baker International, written commun., 2015).

\section{Comparison With Previously Published Low-Flow Statistics}

As previously discussed, low-flow frequency statistics are influenced by length of record, hydrologic regime under which the record was collected, analytical techniques used, and other factors, such as urbanization and diversions. The last systematic update of low-flow statistics in Alabama included data through September 1990 (the 1989 climate year). Since that time, the State has experienced several historic dry periods with 2007 and 2000 having the second and fourth lowest average annual precipitation totals since 1895 (fig. 3B; table 3).

This investigation included 87 stations for which 7Q10 estimates were previously published by Atkins and Pearman (1994), including daily mean flows through CY 1989, and for which additional data were collected. Those previously published 7Q10 values were compared with the current values, and the percentage change was computed as follows:

Percentage change $=[($ current 7Q10 - previous 7Q10) $/$ previous 7Q10] x 100 .

The percentage change indicates the percentage of change from the previously published 7Q10 value (table 7, p. 362). The percentage changes ranged from -61 to 108 percent (table 8 ).

The similarity in the mean difference of -0.2 percent and the median difference of 0.0 indicates that the percentage change data represent a relatively normally distributed dataset. The negative differences for stations indicate that the 7Q10 values decreased, and the positive differences indicate that the 7 Q10 values increased. Of the 87 stations, 42 had negative percentage differences, 38 had positive percentage differences, and 7 had a zero percentage difference. For the station with the largest percentage difference of 108 percent (station 02450000 , Mulberry Fork near Garden City, AL), the QAQC reviews indicated an increase in the annual minimum 7-day average flows after the mid-1960s (fig. 14). It was concluded that the increase was likely due to anthropogenic activities upstream from the station. Consequently, the record was analyzed for two periods: (1) April 1929 to March 1964, and (2) April 1965 to March 2014 (table 5).
Table 8. Summary statistics for the percentage change from the annual minimum 7-day average streamflow presented in this report and those previously published in Atkins and Pearman (1994) for the U.S. Geological Survey streamgaging stations for which additional data were collected since the previous study.

\begin{tabular}{lc}
\hline \multicolumn{1}{c}{ Statistic } & Percentage change \\
\hline Minimum & -61 \\
Mean & -0.2 \\
Maximum & 108 \\
10th percentile & -23 \\
25th percentile & -11 \\
50th percentile (median) & 0.0 \\
75th percentile & 7.6 \\
90th percentile & 27 \\
\hline
\end{tabular}

\section{Access to Updated Low-Flow Characteristics Through the StreamStats Application}

StreamStats is an integrated web-based geographic information system that provides users with access to a variety of analytical tools that are useful for water-resources planning and management, and for engineering design applications, such as design of bridges (Ries and others, 2008). StreamStats allows users to easily obtain streamflow statistics, basin characteristics, and other information for user-selected sites on streams. StreamStats users can choose locations of interest from an interactive map and obtain information for these locations. If a USGS streamgage is selected, the user will be provided with a list of previously published information for the streamgage. The low-flow statistics for Alabama streams provided in table 5 of this report were added to the StreamStatsDB, which is a database accessible to users through the StreamStats application. Instructions for using StreamStats are provided through the links on the StreamStats website at https://water.usgs.gov/ osw/streamstats/index.html. The website also provides links to (1) information about general limitations of the application, (2) other State applications, (3) user instructions, (4) definitions of terms, (5) answers to frequently asked questions, (6) downloadable presentations and other technical information about the application, (7) information that can be accessed only by USGS employees, and (8) contact information. 


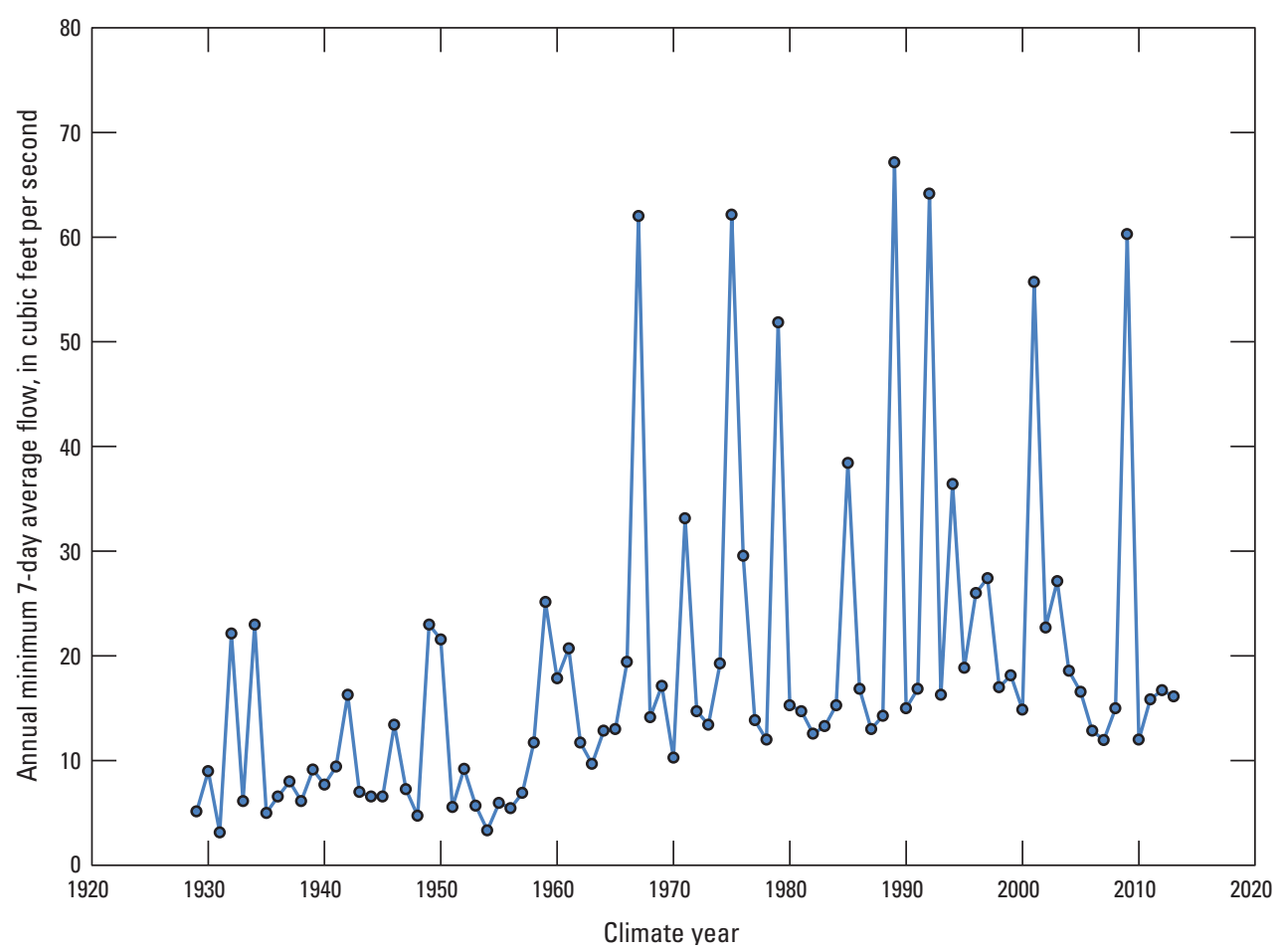

Figure 14. Annual minimum 7-day average flow at U.S. Geological Survey streamgaging station 02450000, Mulberry Fork near Garden City, AL.

\section{Summary}

Alabama water-resource managers need up-to-date low-flow statistics for planning, management, and permitting decisions to help ensure adequate water for consumptive use, water-quality standards, recreation, and aquatic habitat protection. Low-flow statistics for Alabama have not been systematically updated in more than 20 years, which includes a period in which 2 of the 10 driest years of total annual precipitation have occurred since 1895. As such, this report provides a timely update of low-flow frequency and daily duration characteristics for 210 continuous-record streamgaging stations in Alabama and 67 stations from basins adjoining the surrounding States of Florida, Georgia, Mississippi, and Tennessee. Of the 210 Alabama stations, 30 were analyzed for regulated conditions and 19 that are currently (2017) regulated were analyzed for pre-regulation conditions. The characteristics were computed using available data through March 2014. Depending on the length of record available, annual minimum 1- and 7-day average flows with recurrence intervals of $2,5,10,20$, and 50 years are provided in this report. Also, daily flow durations for the 5-, 10-, 25-, 50-, 75-, 90-, and 95-percent probabilities of exceedance are provided. The characteristics are provided for both unregulated and, where appropriate, regulated stations. It was determined that data for six regulated stations were not appropriate to compute the frequency statistics. For those stations, exceedance percentiles of annual minimum 7-day average flows are provided.
Kendall's tau test was used to determine statistical significance of trends in the annual minimum 7-day average flows. For the unregulated stations, including the stations that currently (2017) are regulated but were analyzed for pre-regulation conditions, 139 stations indicated no statistically significant trend, 24 indicated a downward trend, and 17 indicated an upward trend. When analyzing streamflow data for trends, long-term stations are preferable because the trend results from short-term stations can be influenced by shortterm conditions. Of the 24 stations that had a downward trend, 11 had record lengths less than 30 years. Of the 17 stations with an upward trend, 9 had record lengths less than 30 years. The record lengths for the stations indicating no statistically significant trends ranged from 10 to 85 years with an average of 28 years and a median of 23 years. A review of total annual precipitation for Alabama from 1895 to 2015 indicated no substantial changes in the long-term precipitation patterns for the State, which is consistent with the finding that the majority of the USGS stations indicated no statistically significant trends in the annual minimum 7-day average flows.

To highlight the influence that period of record and hydrologic conditions measured in that record can have on low-flow statistics, two assessments were made using long-term data from USGS streamgaging station 02374500 , Murder Creek near Evergreen, AL. The first assessment involved computing the 7Q10 for the first 10 years of record at the gage and then adding the following 5 years of record, recomputing the $7 \mathrm{Q} 10$, and then continuing the process for the complete period of record, which is April 1938 to March 2014. 
The assessment showed that the $7 \mathrm{Q} 10$ varied from a high of 73 cubic feet per second $\left(\mathrm{ft}^{3} / \mathrm{s}\right)$ to a low of $50 \mathrm{ft}^{3} / \mathrm{s}$, which is a difference of 37 percent. The $7 \mathrm{Q} 10$ for the complete period of record was $52 \mathrm{ft}^{3} / \mathrm{s}$. The second assessment used data from the same station but the $7 \mathrm{Q} 10$ analysis was done for seven different periods that included 10 years of record, the minimum length of record typically required for such an analysis. The lowest $7 \mathrm{Q} 10$ value was $42 \mathrm{ft}^{3} / \mathrm{s}$ for the period April 1998 to March 2008, and the highest 7Q10 value was $73 \mathrm{ft}^{3} / \mathrm{s}$ for the period April 1938 to March 1948 and also for the period from April 1988 to March 1997. The difference in the lowest and highest 7Q10 values represents a difference of 54 percent. Consequently, the assessments show the importance that both length and period of record have on low-flow statistics and the reason it is desirable to have long-term records, which will tend to better reflect conditions that might be expected over a longer period of time.

This investigation included 87 Alabama stations for which 7Q10 estimates were previously published, including daily mean flows through climate year 1989, and for which additional data were collected. For those stations, a percentage change between the previous and current $7 \mathrm{Q} 10$ values was computed. The percentage change ranged from -61 to 108 percent with a mean difference of -0.2 percent and a median difference of 0.0 . The closeness of the mean and median percentage change values indicates the data were relatively normally distributed suggesting no dominant pattern of increasing or decreasing 7Q10 estimates for the stations previously analyzed. Of the 87 stations, 42 had negative percentage differences, 38 had positive percentage differences, and 7 had a zero percentage difference.

\section{Selected References}

Alabama Department of Environmental Management, 2012, Water Quality Program, Volume 1, Division 335-6 [revised February 3, 2017]: Alabama Department of Environmental Management, Water Division, accessed March 20, 2013, at http://www.adem.alabama.gov/alEnviroRegLaws/files/ Division6Vol1.pdf.

Alabama Department of Environmental Management, 2014, Integrated water quality monitoring and assessment report: Alabama Department of Environmental Management, Water Division, accessed October 29, 2014, at http://adem.alabama.gov/programs/water/waterforms/ 2014AL-IWQMAR.pdf.

Atkins, J.B., and Pearman, J.L., 1994, Low-flow and flow-duration characteristics of Alabama streams: U.S. Geological Survey Water-Resources Investigations Report 93-4186, 264 p.
Austin, S.H., Krstolic, J.L., and Wiegand, Ute, 2011, Lowflow characteristics of Virginia streams: U.S. Geological Survey Scientific Investigations Report 2011-5143, 122 p. and 9 tables on $\mathrm{CD}$.

Barnes, H.H., Jr., 1979, Low-flow programs: U.S. Geological Survey Surface Water Branch Technical Memorandum No. 79.06, accessed April 26, 2007, at https://water.usgs. gov/admin/memo/SW/sw79.06.html.

Bingham, R.H., 1981, Low-flow characteristics of Alabama streams: U.S. Geological Survey Water-Supply Paper 2083, $27 \mathrm{p}$.

Bohman, Larry, and Patterson, Glenn, 1993, Hydrology assessment, chap. 3 of Marshall, W.D., ed., Assessing change in the Edisto River Basin-An ecological characterization: South Carolina Water Resources Commission Report No. 177, p. 69-83.

Carter, R.W., 1969, Study of surface-water data programsAdjustments for zero-flow items: U.S. Geological Survey Surface Water Branch Technical Memorandum No. 70.07, accessed December 20, 2008, at https://water.usgs.gov/ $\mathrm{admin} / \mathrm{memo} / \mathrm{SW} / \mathrm{sw} 70.07 . \mathrm{html}$.

Dalrymple, Tate, 1960, Flood-frequency analyses, manual of hydrology-Part 3. Flood-flow techniques: U.S. Geological Survey Water-Supply Paper 1543-A, 80 p.

Feaster, T.D., and Guimaraes, W.B., 2009, Low-flow frequency and flow duration of selected South Carolina streams in the Pee Dee River Basin through March 2007 [revised June 22, 2010]: U.S. Geological Survey Open-File Report 2009-1171, 39 p.

Feaster, T.D., and Guimaraes, W.B., 2012, Low-flow frequency and flow duration of selected South Carolina streams in the Saluda, Congaree, and Edisto River Basins through March 2009: U.S. Geological Survey Open-File Report 2012-1253, 53 p. [Also available at https://pubs.usgs.gov/of/2012/1253/.]

Feaster, T.D., and Guimaraes, W.B., 2014, Low-flow frequency and flow duration of selected South Carolina streams in the Catawba-Wateree and Santee River Basins through March 2012: U.S. Geological Survey Open-File Report 2014-1113, 34 p. [Also available at https://doi.org/10.3133/ofr20141113.]

Feaster, T.D., and Guimaraes, W.B., 2016, Low-flow frequency and flow duration of selected South Carolina streams in the Savannah and Salkehatchie River Basins through March 2014 (ver. 1.1, November 2016): U.S. Geological Survey Open-File Report 2016-1102, 62 p. [Also available at https://doi.org/10.3133/ofr20161101.] 
Flynn, K.M., Hummel, P.R., Lumb, A.M., and Kittle, J.L., Jr., 1995, User's manual for ANNIE, version 2, a computer program for interactive hydrologic data management: U.S. Geological Survey Water-Resources Investigations Report 95-4085, 211 p.

Fowler, K.K., and Wilson, J.T., 2015, Low-flow characteristics for selected streams in Indiana: U.S. Geological Survey Scientific Investigations Report 2014-5242, 353 p. [Also available at https://doi.org/10.3133/sir20145242.]

Gotvald, A.J., 2016, Selected low-flow frequency statistics for continuous-record streamgages in Georgia, 2013: U.S. Geological Survey Scientific Investigations Report 2016-5037, 20 p. [Also available at https://doi.org/10.3133/ sir20165037.]

Guimaraes, W.B., and Feaster, T.D., 2010, Low-flow frequency and flow duration of selected South Carolina streams in the Broad River Basin through March 2008: U.S. Geological Survey Open-File Report 2010-1305, 47 p.

Hardison, C.H., 1969, Accuracy of streamflow characteristics: U.S. Geological Survey Professional Paper 650-D, p. D210-D214.

Hayes, E.C., 1978, 7-day low flows and flow duration of Alabama streams through 1973: Geological Survey of Alabama Bulletin 113, 163 p.

Helsel, D.R., and Hirsch, R.M., 2002, Statistical methods in water resources: U.S. Geological Survey Techniques of Water-Resources Investigations, book 4, chap. A3, 522 p. [Also available at https://pubs.usgs.gov/twri/twri4a3/.]

Hirsch, R.M., 1982, A comparison of four record extension techniques: Water Resources Research, v. 18, no. 4, p. 1081-1088.

Hunt, A.M., and Garcia, A.M., 2014, Simulation of natural flows in major river basins in Alabama: U.S. Geological Survey Scientific Investigations Report 2014-5021, 32 p. [Also available at https://doi.org/10.3133/sir20145021.]

Hutchison, N.E., comp., 1975, WATSTORE user's guide, Volume 1, National Water Data Storage and Retrieval System [revised 1984]: U.S. Geological Survey Open-File Report 75-426 [variously paged].

Hydrology Subcommittee of the Interagency Advisory Committee on Water Data, 1982, Guidelines for determining flood frequency: U.S. Geological Survey Bulletin 17B, $183 \mathrm{p}$.

Jennings, M.E., and Benson, M.A., 1969, Frequency curves for annual flood series with some zero events or incomplete data: Water Resources Research, v. 5, no. 1, p. 276-280.

Kendall, M.G., 1938, A new measure of rank correlation: Biometrika, v. 30, no. 1/2, p. 81-93.
Kite, G.W., 1985, Frequency and risk analyses in hydrology: Water Resources Publications, Littleton, Colo., 224 p.

Langbein, W.B., and Iseri, K.T., 1960, General introduction and hydrologic definitions, manual of hydrology-Part 1. General surface-water techniques: U.S. Geological Survey Water-Supply Paper 1541-A, 29 p.

Lins, H.F., Hirsch, R.M., and Kiang, Julie, 2010, Water-The Nations fundamental climate issue, A white paper on the U.S. Geological Survey role and capabilities: U.S. Geological Survey Circular 1347, 9 p. [Also available at https://pubs.usgs.gov/circ/1347/.]

Lumb, A.M., Kittle, J.L., Jr., and Flynn, K.M., 1990, Users manual for ANNIE, a computer program for interactive hydrologic analyses and data management: U.S. Geological Survey Water-Resources Investigations Report 89-4080, $236 \mathrm{p}$.

National Oceanic and Atmospheric Administration, 2016a, Climate of Alabama: National Oceanic and Atmospheric Administration, National Centers of Environmental Information website, accessed June 28, 2016, at http://www.ncdc.noaa.gov/climatenormals/clim60/states/ Clim_AL_01.pdf.

National Oceanic and Atmospheric Administration, 2016b, Climate at a glance: National Oceanic and Atmospheric Administration, National Centers of Environmental Information website, accessed June 28, 2016, at http://www.ncdc.noaa.gov/cag/time-series/us.

National Oceanic and Atmospheric Administration,, 2016c, Climate divisions with counties: National Oceanic and Atmospheric Administration, National Weather Service, Climate Prediction Center website, accessed June 28, 2016, at http://www.cpc.noaa.gov/products/analysis_monitoring/ regional_monitoring/CLIM_DIVS/states_counties_climatedivisions.shtml.

National Oceanic and Atmospheric Administration, 2017, NOAA's 1981-2010 climate normal: National Oceanic and Atmospheric Administration Satellite and Information Service, accessed May 3, 2017, at https://www.ncdc.noaa.gov/oa/climate/normals/usnormals. html.

Nielsen, J.P., 1999, Record extension and streamflow statistics for the Pleasant River, Maine: U.S. Geological Survey Water-Resources Investigations Report 99-4078, 22 p.

Omernik, J.M., 1987, Ecoregions of the conterminous United States: Annals of the Association of American Geographers, v. 77, no. 1, p. 118-125, scale 1:7,500,000. 
Paulson, R.W., Chase, E.B., Roberts, R.S., and Moody, D.W., comps., 1991, National water summary 1988-89, Hydrologic events and floods and droughts: U.S. Geological Survey Water-Supply Paper 2375, 591 p.

Pierce, L.B., 1959, Low-flow and flow-duration data for Alabama streams: Alabama Water Improvement Commission Report, 168 p.

Pierce, L.B., 1967, Seven-day low flows and flow duration of Alabama streams: Geological Survey of Alabama Bulletin 87 , part A, $114 \mathrm{p}$.

Ries, K.G., III, 1994, Estimation of low-flow duration discharges in Massachusetts: U.S Geological Survey Water-Supply Paper 2418, 50 p.

Ries, K.G., III, Guthrie, J.G., Rea, A.H., Steeves, P.A., and Stewart, D.W., 2008, StreamStats: A water resources web application: U.S. Geological Survey Fact Sheet 2008-3067, 6 p. [Also available at https://pubs.usgs.gov/fs/2008/3067/.]

Riggs, H.C., 1968, Frequency curves: U.S. Geological Survey Techniques of Water-Resources Investigations, book 4, chap. A2, $15 \mathrm{p}$.

Riggs, H.C., 1972, Low-flow investigations: U.S. Geological Survey Techniques of Water Resources Investigations, book 4, chap. B1, 18 p.

Riggs, H.C., 1985, Streamflow characteristics: New York, Elsevier, 248 p.

Ruddy, B.C., and Hitt, K.J., 1990, Summary of selected characteristics of large reservoirs in the United States and Puerto Rico, 1988: U.S. Geological Survey Open-File Report 90-163, 295 p.

SAS Institute, Inc., 1989, SAS user's guide-Statistics: Cary, N.C., SAS Institute, Inc., 583 p.

Sauer, V.B., and Turnipseed, D.P., 2010, Stage measurement at gaging stations: U.S. Geological Survey Techniques and Methods, book 3, chap. A7, 45 p. [Also available at https://pubs.usgs.gov/tm/tm3-a7/.]

Searcy, J.K., 1959, Flow-duration curves, manual of hydrology_Part 2. Low-flow techniques: U.S. Geological Survey Water-Supply Paper 1542-A, 33 p.

Stedinger, J.R., and Thomas, W.O., Jr., 1985, Low-flow frequency estimation using base-flow measurements: U.S. Geological Survey Open-File Report 85-95, 22 p.
Tasker, G.D., 1987, A comparison of methods for estimating low flow characteristics of streams: Water Resources Bulletin, v. 23, no. 6, p. 1077-1083.

Telis, P.A., 1991, Low-flow and flow-duration characteristics of Mississippi streams: U.S. Geological Survey WaterResources Investigations Report 90-4087, 214 p.

Turnipseed, D.P., and Sauer, V.B., 2010, Discharge measurements at gaging stations: U.S. Geological Survey Techniques and Methods, book 3, chap. A8, 87 p. [Also available at https://pubs.usgs.gov/tm/tm3-a8/.]

U.S. Environmental Protection Agency, 2009, Polluted runoff (nonpoint source pollution), appendix - case studies: U.S. Environmental Protection Agency website, accessed June 27, 2009, at http://www.epa.gov/owo/nps/urbanize/ appendix.html.

U.S. Environmental Protection Agency, 2016, Ecoregions of the United States: U.S. Environmental Protection Agency website, accessed June 28, 2016, at https://www.epa.gov/ eco-research/ecoregions.

U.S. Geological Survey, 2013, Number of USGS streamgages through time: U.S. Geological Survey National Streamflow Information Program (NSIP) web page, accessed October 22, 2013, at http://water.usgs.gov/nsip/ history 1.html.

U.S. Geological Survey, 2014, Water-resources data for the United States, water year 2013: U.S. Geological Survey Water-Data Report WDR-US-2013, site 02123425, accessed June 28, 2016, at https://wdr.water.usgs.gov/ wy2013/pdfs/02423425.2013.pdf.

U.S. Geological Survey, 2016, How much of your state is wet?: U.S. Geological Survey Water Science School website, accessed June 28, 2016, at https://water.usgs.gov/ edu/wetstates.html.

U.S. Geological Survey, 2017, USGS surface-water data for Alabama, in USGS water data for the Nation: U.S. Geological Survey National Water Information System database, accessed January 17, 2017, at https://doi.org/10.5066/ F7P55KJN. [Site information for Alabama is directly accessible at https://waterdata.usgs.gov/al/nwis/sw/h.] 
Tables 1, 4, 5, and 7 
Table 1. U.S. Geological Survey continuous-record streamgaging stations in Alabama and surrounding States included in this investigation.

[USGS, U.S. Geological Survey; ', degrees; ', minutes; ”, seconds; fťs, cubic foot per second; DA, drainage area; NWISWeb, National Water Information System database; $\mathrm{mi}^{2}$, square mile; QAQC, quality assurance, quality control]

\begin{tabular}{|c|c|c|c|c|c|c|c|c|}
\hline $\begin{array}{c}\text { Site } \\
\text { index } \\
\text { number } \\
\text { (fig. 1) }\end{array}$ & $\begin{array}{l}\text { USGS } \\
\text { station } \\
\text { number }\end{array}$ & Station name & Latitude & Longitude & County & Basin & $\begin{array}{l}\text { Hydrologic } \\
\text { Unit Code }\end{array}$ & $\begin{array}{l}\text { Drainage } \\
\text { area }\left(\mathrm{mi}^{2}\right)\end{array}$ \\
\hline 1 & 02339225 & $\begin{array}{l}\text { Wehadkee Creek below } \\
\text { Rock Mills, AL }\end{array}$ & $33^{\circ} 07^{\prime} 20^{\prime \prime}$ & $85^{\circ} 14^{\prime} 57^{\prime \prime}$ & Randolph & Chattahoochee & 03130002 & 60.2 \\
\hline 2 & 02342500 & $\begin{array}{l}\text { Uchee Creek near Fort } \\
\text { Mitchell, AL }\end{array}$ & $32^{\circ} 19^{\prime} 00^{\prime \prime}$ & $85^{\circ} 00^{\prime} 54^{\prime \prime}$ & Russell & Chattahoochee & 03130003 & 322 \\
\hline 3 & 02342933 & $\begin{array}{l}\text { South Fork Cowikee Creek } \\
\text { near Batesville, AL }\end{array}$ & $32^{\circ} 01^{\prime} 03^{\prime \prime}$ & $85^{\circ} 17^{\prime} 45^{\prime \prime}$ & Barbour & Chattahoochee & 03130003 & 112 \\
\hline 4 & 02343300 & $\begin{array}{l}\text { Abbie Creek near } \\
\text { Haleburg, AL }\end{array}$ & $31^{\circ} 28^{\prime} 24^{\prime \prime}$ & $85^{\circ} 09^{\prime} 45^{\prime \prime}$ & Henry & Chattahoochee & 03130004 & 146 \\
\hline 5 & 02343500 & $\begin{array}{l}\text { Chattahoochee River at } \\
\text { Columbia, AL }\end{array}$ & $31^{\circ} 17^{\prime} 01^{\prime \prime}$ & $85^{\circ} 05^{\prime} 58^{\prime \prime}$ & Houston & Chattahoochee & 03130004 & 8,040 \\
\hline 7 & 02361000 & $\begin{array}{l}\text { Choctawhatchee River near } \\
\text { Newton, AL }\end{array}$ & $31^{\circ} 20^{\prime} 34^{\prime \prime}$ & $85^{\circ} 36^{\prime} 38^{\prime \prime}$ & Dale & Choctawhatchee & 03140201 & 686 \\
\hline 8 & 02361500 & $\begin{array}{l}\text { Choctawhatchee River near } \\
\text { Bellwood, AL }\end{array}$ & $31^{\circ} 09^{\prime} 33^{\prime \prime}$ & $85^{\circ} 47^{\prime} 04^{\prime \prime}$ & Geneva & Choctawhatchee & 03140201 & 1,280 \\
\hline 9 & 02362240 & $\begin{array}{l}\text { Little Double Bridges Creek } \\
\text { near Enterprise, AL }\end{array}$ & $31^{\circ} 16^{\prime} 20^{\prime \prime}$ & $85^{\circ} 57^{\prime} 30^{\prime \prime}$ & Coffee & Choctawhatchee & 03140201 & 21.4 \\
\hline 10 & 02363000 & Pea River near Ariton, AL & $31^{\circ} 35^{\prime} 41^{\prime \prime}$ & $85^{\circ} 46^{\prime} 59^{\prime \prime}$ & Dale & Choctawhatchee & 03140202 & 498 \\
\hline 13 & 02369800 & $\begin{array}{l}\text { Blackwater River near } \\
\text { Bradley, AL }\end{array}$ & $31^{\circ} 01^{\prime} 39^{\prime \prime}$ & $86^{\circ} 42^{\prime} 36^{\prime \prime}$ & Escambia & Blackwater & 03140104 & 87.7 \\
\hline 14 & 02371200 & Indian Creek near Troy, AL & $31^{\circ} 48^{\prime} 50^{\prime \prime}$ & $86^{\circ} 07^{\prime} 15^{\prime \prime}$ & Pike & Conecuh & 03140301 & 8.87 \\
\hline 15 & 02371500 & $\begin{array}{l}\text { Conecuh River at } \\
\text { Brantley, AL }\end{array}$ & $31^{\circ} 34^{\prime} 24^{\prime \prime}$ & $86^{\circ} 15^{\prime} 06^{\prime \prime}$ & Crenshaw & Conecuh & 03140301 & 500 \\
\hline 16 & 02372000 & $\begin{array}{l}\text { Patsaliga Creek at } \\
\text { Lurverne, } \mathrm{AL}\end{array}$ & $31^{\circ} 43^{\prime} 27^{\prime \prime}$ & $86^{\circ} 16^{\prime} 42^{\prime \prime}$ & Crenshaw & Conecuh & 03140302 & 254 \\
\hline 17 & 02372250 & $\begin{array}{l}\text { Patsaliga Creek near } \\
\text { Brantley, } \mathrm{AL}\end{array}$ & $31^{\circ} 35^{\prime} 46^{\prime \prime}$ & $86^{\circ} 24^{\prime} 20^{\prime \prime}$ & Crenshaw & Conecuh & 03140302 & 442 \\
\hline 18 & 02372422 & $\begin{array}{l}\text { Conecuh River below } \\
\text { Point A Dam near River } \\
\text { Falls, AL }\end{array}$ & $31^{\circ} 21^{\prime} 40^{\prime \prime}$ & $86^{\circ} 31^{\prime} 11^{\prime \prime}$ & Covington & Conecuh & 03140301 & 1,273 \\
\hline
\end{tabular}


Table 1. U.S. Geological Survey continuous-record streamgaging stations in Alabama and surrounding States included in this investigation.-Continued

[USGS, U.S. Geological Survey; ', degrees; ', minutes; ”, seconds; ft³/s, cubic foot per second; DA, drainage area; NWISWeb, National Water Information System database; $\mathrm{mi}^{2}$, square mile; QAQC, quality assurance, quality control]

\begin{tabular}{|c|c|c|c|c|c|}
\hline $\begin{array}{l}\text { Site } \\
\text { index } \\
\text { number } \\
\text { (fig. 1) }\end{array}$ & Period of record available & Period of record analyzed & $\begin{array}{l}\text { Number of } \\
\text { climate years } \\
\text { used in } \\
\text { analysis }\end{array}$ & $\begin{array}{l}\text { Flow } \\
\text { condition } \\
\text { for period } \\
\text { analyzed }\end{array}$ & Remarks \\
\hline 1 & October 1978 to January 1990 & April 1979 to March 1989 & 10 & Unregulated & \\
\hline 2 & October 1946 to March 2014 & April 1947 to March 2014 & 67 & Unregulated & \\
\hline 3 & $\begin{array}{c}\text { October } 1963 \text { to September } 1971, \\
\text { October } 1974 \text { to October } 2011\end{array}$ & $\begin{array}{l}\text { April } 1964 \text { to March 1971, } \\
\text { April } 1975 \text { to March } 2011\end{array}$ & 43 & Unregulated & \\
\hline 4 & $\begin{array}{l}\text { October } 1958 \text { to September 1971, } \\
\text { October } 1974 \text { to August } 1993\end{array}$ & $\begin{array}{l}\text { April } 1959 \text { to March } 1971 \text {, } \\
\text { April } 1975 \text { to March } 1993\end{array}$ & 30 & Unregulated & \\
\hline 5 & July 1928 to September 1960 & April 1929 to March 1960 & 31 & ${ }^{1}$ Unregulated & $\begin{array}{l}\text { Since } 1962, \text { site in backwater from } \\
\text { Columbia Lock and Dam. }\end{array}$ \\
\hline 6 & June 1952 to September 1963 & April 1953 to March 1963 & 10 & Unregulated & \\
\hline 7 & $\begin{array}{l}\text { December } 1921 \text { to September 1927, } \\
\text { June } 1935 \text { to March } 2014\end{array}$ & $\begin{array}{l}\text { April } 1922 \text { to March } 1927, \\
\text { April } 1936 \text { to March } 2014\end{array}$ & 83 & Unregulated & \\
\hline 8 & $\begin{array}{l}\text { December } 1921 \text { to October } 1925 \text {, } \\
\text { December } 2000 \text { to March } 2014\end{array}$ & $\begin{array}{l}\text { April } 1922 \text { to March 1925, } \\
\text { April } 2001 \text { to March } 2014\end{array}$ & 16 & Unregulated & $\begin{array}{l}\text { Low-flow frequency estimates } \\
\text { adjusted based on MOVE. } 1 \\
\text { correlation with station } 02361000 \text {, } \\
\text { Choctawhatchee River near } \\
\text { Newton, AL. }\end{array}$ \\
\hline 9 & August 1985 to March 2014 & April 1986 to March 2014 & 28 & Unregulated & \\
\hline 10 & $\begin{array}{c}\text { October } 1938 \text { to September 1970, } \\
\text { October } 1987 \text { to March } 2014\end{array}$ & $\begin{array}{l}\text { April } 1939 \text { to March } 1970, \\
\text { April } 1988 \text { to March } 2014\end{array}$ & 57 & Unregulated & \\
\hline 11 & $\begin{array}{l}\text { September } 1904 \text { to August } 1913 \text {, } \\
\text { October } 1922 \text { to September 1925, } \\
\text { October } 1935 \text { to September 1970, } \\
\text { October } 2002 \text { to March } 2014\end{array}$ & $\begin{array}{l}\text { April } 1905 \text { to March 1913, } \\
\text { April } 1923 \text { to March 1925, } \\
\text { April } 1936 \text { to March 1970, } \\
\text { April } 2003 \text { March } 2014\end{array}$ & 55 & Unregulated & \\
\hline 12 & October 1974 to September 1995 & April 1975 to March 1995 & 20 & Unregulated & \\
\hline 13 & October 1967 to March 2014 & April 1968 to March 2014 & 46 & Unregulated & \\
\hline 14 & $\begin{array}{l}\text { October } 1958 \text { to September } 1968 \text {, } \\
\text { October } 1970 \text { to September } 1986\end{array}$ & $\begin{array}{l}\text { April } 1959 \text { to March } 1968, \\
\text { April } 1971 \text { to March } 1986\end{array}$ & 24 & Unregulated & \\
\hline 15 & October 1937 to March 2014 & April 1938 to March 2014 & 76 & Unregulated & \\
\hline 16 & October 1943 to September 1958 & April 1944 to March 1958 & 14 & Unregulated & \\
\hline 17 & October 1974 to March 2014 & April 1975 to March 2014 & 39 & Unregulated & \\
\hline 18 & June 1999 to March 2013 & April 2000 to March 2013 & 13 & Regulated & $\begin{array}{l}\text { Regulated by Gantt Dam since } 1924 \\
\text { and Point A Reservoir since } 1925 .\end{array}$ \\
\hline
\end{tabular}


Table 1. U.S. Geological Survey continuous-record streamgaging stations in Alabama and surrounding States included in this investigation.-Continued

[USGS, U.S. Geological Survey; ', degrees; ', minutes; ”, seconds; fťs, cubic foot per second; DA, drainage area; NWISWeb, National Water Information System database; $\mathrm{mi}^{2}$, square mile; QAQC, quality assurance, quality control]

\begin{tabular}{|c|c|c|c|c|c|c|c|c|}
\hline $\begin{array}{c}\text { Site } \\
\text { index } \\
\text { number } \\
\text { (fig. 1) }\end{array}$ & $\begin{array}{l}\text { USGS } \\
\text { station } \\
\text { number }\end{array}$ & Station name & Latitude & Longitude & County & Basin & $\begin{array}{l}\text { Hydrologic } \\
\text { Unit Code }\end{array}$ & $\begin{array}{l}\text { Drainage } \\
\text { area }\left(\mathrm{mi}^{2}\right)\end{array}$ \\
\hline 19 & 02372500 & $\begin{array}{l}\text { Conecuh River near } \\
\text { Andalusia, } \mathrm{AL}\end{array}$ & $31^{\circ} 15^{\prime} 19^{\prime \prime}$ & $86^{\circ} 36^{\prime} 01^{\prime \prime}$ & Covington & Conecuh & 03140301 & 1,344 \\
\hline 21 & 02373500 & Pigeon Creek near Thad, AL & $31^{\circ} 28^{\prime} 36^{\prime \prime}$ & $86^{\circ} 39^{\prime} 30^{\prime \prime}$ & Covington & Conecuh & 03140303 & 307 \\
\hline 22 & 02374000 & $\begin{array}{l}\text { Conecuh River near } \\
\text { Brooklyn, AL }\end{array}$ & $31^{\circ} 09^{\prime} 49^{\prime \prime}$ & $86^{\circ} 48^{\prime} 00^{\prime \prime}$ & Escambia & Conecuh & 03140304 & 2,495 \\
\hline 25 & 02374700 & $\begin{array}{l}\text { Murder Creek at State } \\
\text { Highway } 41 \text { near } \\
\text { Brewton, AL }\end{array}$ & $31^{\circ} 06^{\prime} 03^{\prime \prime}$ & $87^{\circ} 04^{\prime} 08^{\prime \prime}$ & Escambia & Conecuh & 03140304 & 435 \\
\hline 26 & 02374745 & $\begin{array}{l}\text { Burnt Corn Creek at } \\
\text { State Highway } 41 \text { near } \\
\text { Brewton, AL }\end{array}$ & $31^{\circ} 07^{\prime} 47^{\prime \prime}$ & $87^{\circ} 05^{\prime} 14^{\prime \prime}$ & Escambia & Conecuh & 03140304 & 182 \\
\hline 27 & 02374950 & $\begin{array}{l}\text { Big Escambia Creek at } \\
\text { Sardine Bridge near } \\
\text { Stanley Crossroads, AL }\end{array}$ & $31^{\circ} 07^{\prime} 46^{\prime \prime}$ & $87^{\circ} 22^{\prime} 14^{\prime \prime}$ & Escambia & Conecuh & 03140305 & 193 \\
\hline 31 & 02377570 & Styx River near Elsanor, AL & $30^{\circ} 36^{\prime} 20^{\prime \prime}$ & $87^{\circ} 32^{\prime} 50^{\prime \prime}$ & Baldwin & Perdido & 03140106 & 192 \\
\hline 32 & 02378300 & $\begin{array}{l}\text { Magnolia River at U.S. } 98 \\
\text { near Foley, AL }\end{array}$ & $30^{\circ} 24^{\prime} 23^{\prime \prime}$ & $87^{\circ} 44^{\prime} 13^{\prime \prime}$ & Baldwin & Mobile & 03160205 & 16.6 \\
\hline 33 & 02378500 & $\begin{array}{l}\text { Fish River near Silver } \\
\text { Hill, AL }\end{array}$ & $30^{\circ} 32^{\prime} 43^{\prime \prime}$ & $87^{\circ} 47^{\prime} 55^{\prime \prime}$ & Baldwin & Mobile & 03160205 & 55.3 \\
\hline 34 & 02398300 & $\begin{array}{c}\text { Chattooga River above } \\
\text { Gaylesville, AL }\end{array}$ & $34^{\circ} 17^{\prime} 25^{\prime \prime}$ & $85^{\circ} 30^{\prime} 33^{\prime \prime}$ & Cherokee & Coosa & 03150105 & 366 \\
\hline 35 & 02398500 & $\begin{array}{c}\text { Chattooga River at } \\
\text { Gaylesville, } \mathrm{AL}\end{array}$ & $34^{\circ} 15^{\prime} 47^{\prime \prime}$ & $85^{\circ} 33^{\prime} 39^{\prime \prime}$ & Cherokee & Coosa & 03150105 & 379 \\
\hline 36 & 02398950 & $\begin{array}{l}\text { West Fork Little River at } \\
\text { Desoto Park near Fort } \\
\text { Payne, AL }\end{array}$ & $34^{\circ} 29^{\prime} 30^{\prime \prime}$ & $85^{\circ} 37^{\prime} 00^{\prime \prime}$ & DeKalb & Coosa & 03150105 & 42.8 \\
\hline 37 & 02399000 & $\begin{array}{l}\text { Little River near } \\
\quad \text { Jamestown, AL }\end{array}$ & $34^{\circ} 23^{\prime} 51^{\prime \prime}$ & $85^{\circ} 37^{\prime} 36^{\prime \prime}$ & Cherokee & Coosa & 03150105 & 125 \\
\hline
\end{tabular}


Table 1. U.S. Geological Survey continuous-record streamgaging stations in Alabama and surrounding States included in this investigation.-Continued

[USGS, U.S. Geological Survey; ' , degrees; ', minutes; ”, seconds; ft³/s, cubic foot per second; DA, drainage area; NWISWeb, National Water Information System database; $\mathrm{mi}^{2}$, square mile; QAQC, quality assurance, quality control]

\begin{tabular}{|c|c|c|c|c|c|}
\hline $\begin{array}{l}\text { Site } \\
\text { index } \\
\text { number } \\
\text { (fig. 1) }\end{array}$ & Period of record available & Period of record analyzed & $\begin{array}{l}\text { Number of } \\
\text { climate years } \\
\text { used in } \\
\text { analysis }\end{array}$ & $\begin{array}{l}\text { Flow } \\
\text { condition } \\
\text { for period } \\
\text { analyzed }\end{array}$ & Remarks \\
\hline 19 & $\begin{array}{l}\text { September } 1904 \text { to December } 1919 \text {, } \\
\text { October } 1929 \text { to September } 1952 \text {, } \\
\text { October } 1965 \text { to September } 1968\end{array}$ & $\begin{array}{l}\text { April } 1930 \text { to March } 1952, \\
\text { April } 1966 \text { to March } 1968\end{array}$ & 24 & Regulated & $\begin{array}{l}\text { Regulated by Gantt Dam since } 1924 \\
\text { and Point A Reservoir since } 1925 . \\
\text { Analysis is for regulation condi- } \\
\text { tions only. }\end{array}$ \\
\hline 20 & $\begin{array}{l}\text { October } 1937 \text { to September } 1967 \text {, } \\
\text { October } 1974 \text { to March } 2014\end{array}$ & $\begin{array}{l}\text { April } 1938 \text { to March } 1967, \\
\text { April } 1975 \text { to March } 2014\end{array}$ & 68 & Unregulated & \\
\hline 21 & October 1937 to September 1970 & April 1938 to March 1970 & 32 & Unregulated & \\
\hline 22 & June 1935 to December 1957 & April 1936 to March 1957 & 21 & Regulated & $\begin{array}{l}\text { Regulated by Gantt Dam since } 1924 \\
\text { and Point A Reservoir since } 1925 .\end{array}$ \\
\hline 23 & April 1999 to March 2014 & April 2000 to March 2014 & 14 & Regulated & $\begin{array}{l}\text { Regulated by Gantt Dam since } 1924 \\
\text { and Point A Reservoir since } 1925 .\end{array}$ \\
\hline 24 & October 1937 to March 2014 & April 1938 to March 2014 & 76 & Unregulated & \\
\hline 25 & March 1999 to March 2014 & April 1999 to March 2014 & 15 & Unregulated & $\begin{array}{l}\text { Low-flow frequency estimates } \\
\text { adjusted based on MOVE. } 1 \\
\text { correlation with station } 02374500 \\
\text { Murder Creek near Evergreen, AL. }\end{array}$ \\
\hline 26 & March 1999 to March 2014 & April 1999 to March 2014 & 15 & Unregulated & \\
\hline 27 & May 2000 to March 2014 & April 2001 to March 2014 & 13 & Unregulated & \\
\hline 28 & January 1939 to December 1951 & April 1940 to March 1951 & 12 & Unregulated & \\
\hline 29 & June 1941 to March 2014 & April 1941 to March 2014 & 72 & Unregulated & \\
\hline 30 & $\begin{array}{l}\text { October } 1951 \text { to September } 1969, \\
\text { October } 1970 \text { to September } 1971\end{array}$ & April 1952 to March 1969 & 17 & Unregulated & \\
\hline 31 & October 1987 to March 2014 & April 1988 to March 2014 & 26 & Unregulated & \\
\hline 32 & July 1999 to March 2014 & April 2000 to March 2014 & 14 & Unregulated & \\
\hline 33 & $\begin{array}{l}\text { July } 1953 \text { to September } 1969 \text {, } \\
\text { October } 1970 \text { to September } 1971 \text {, } \\
\text { November } 1986 \text { to March } 2014\end{array}$ & $\begin{array}{l}\text { April } 1954 \text { to March } 1969 \text {, } \\
\text { April } 1987 \text { to March } 2014\end{array}$ & 42 & Unregulated & \\
\hline 34 & $\begin{array}{c}\text { January } 1959 \text { to September } 1967 \text {, } \\
\text { October } 1984 \text { to March } 2014\end{array}$ & $\begin{array}{l}\text { April } 1960 \text { to March } 1967 \text {, } \\
\text { April } 1985 \text { to March } 2014\end{array}$ & 37 & Unregulated & \\
\hline 35 & June 1937 to September 1960 & April 1937 to March 1960 & 22 & Unregulated & \\
\hline 36 & October 1997 to January 2012 & April 1998 to March 2011 & 13 & Unregulated & \\
\hline 37 & $\begin{array}{l}\text { February } 1922 \text { to March } 1932 \text {, } \\
\text { June } 1935 \text { to September } 1949\end{array}$ & $\begin{array}{l}\text { April } 1922 \text { to March } 1932, \\
\text { April } 1936 \text { to March } 1949\end{array}$ & 23 & Unregulated & \\
\hline
\end{tabular}


Table 1. U.S. Geological Survey continuous-record streamgaging stations in Alabama and surrounding States included in this investigation.-Continued

[USGS, U.S. Geological Survey; ', degrees; ', minutes; ”, seconds; fťs, cubic foot per second; DA, drainage area; NWISWeb, National Water Information System database; $\mathrm{mi}^{2}$, square mile; QAQC, quality assurance, quality control]

\begin{tabular}{|c|c|c|c|c|c|c|c|c|}
\hline $\begin{array}{c}\text { Site } \\
\text { index } \\
\text { number } \\
\text { (fig. 1) }\end{array}$ & $\begin{array}{l}\text { USGS } \\
\text { station } \\
\text { number }\end{array}$ & Station name & Latitude & Longitude & County & Basin & $\begin{array}{l}\text { Hydrologic } \\
\text { Unit Code }\end{array}$ & $\begin{array}{l}\text { Drainage } \\
\text { area }\left(\mathrm{mi}^{2}\right)\end{array}$ \\
\hline 38 & 02399200 & $\begin{array}{l}\text { Little River near Blue } \\
\text { Rond, AL }\end{array}$ & $34^{\circ} 17^{\prime} 20^{\prime \prime}$ & $85^{\circ} 40^{\prime} 50^{\prime \prime}$ & Cherokee & Coosa & 03150105 & 199 \\
\hline 39 & 02399500 & Coosa River at Leesburg, AL & $34^{\circ} 10^{\prime} 36^{\prime \prime}$ & $85^{\circ} 45^{\prime} 14^{\prime \prime}$ & Cherokee & Coosa & 03150105 & 5,270 \\
\hline
\end{tabular}

\begin{tabular}{|c|c|c|c|c|c|c|c|c|}
\hline 40 & 02400000 & $\begin{array}{l}\text { Terrapin Creek near } \\
\text { Piedmont, AL }\end{array}$ & $33^{\circ} 57^{\prime} 23^{\prime \prime}$ & $85^{\circ} 34^{\prime} 38^{\prime \prime}$ & Calhoun & Coosa & 03150105 & 116 \\
\hline 41 & 02400100 & $\begin{array}{l}\text { Terrapin Creek at } \\
\text { Ellisville, } \mathrm{AL}\end{array}$ & $34^{\circ} 03^{\prime} 54^{\prime \prime}$ & $85^{\circ} 36^{\prime} 51^{\prime \prime}$ & Cherokee & Coosa & 03150105 & 252 \\
\hline 42 & 02400500 & Coosa River at Gadsden, AL & $34^{\circ} 00^{\prime} 37^{\prime \prime}$ & $85^{\circ} 59^{\prime} 52^{\prime \prime}$ & Etowah & Coosa & 03150106 & 5805 \\
\hline 43 & 02400680 & $\begin{array}{l}\text { Big Wills Creek at State } \\
\text { Highway } 35 \text { near Fort } \\
\text { Payne, AL }\end{array}$ & $34^{\circ} 26^{\prime} 17^{\prime \prime}$ & $85^{\circ} 46^{\prime} 02^{\prime \prime}$ & DeKalb & Coosa & 03150106 & 55.4 \\
\hline 44 & 02401000 & $\begin{array}{l}\text { Big Wills Creek near Reece } \\
\text { City, AL }\end{array}$ & $34^{\circ} 05^{\prime} 53^{\prime \prime}$ & $86^{\circ} 02^{\prime} 17^{\prime \prime}$ & Etowah & Coosa & 03150106 & 182 \\
\hline 45 & 02401370 & $\begin{array}{l}\text { Big Canoe Creek near } \\
\text { Springville, AL }\end{array}$ & $33^{\circ} 48^{\prime} 49^{\prime \prime}$ & $86^{\circ} 22^{\prime} 54^{\prime \prime}$ & St. Clair & Coosa & 03150106 & 45 \\
\hline 46 & 02401390 & $\begin{array}{l}\text { Big Canoe Creek near } \\
\text { Ashville, AL }\end{array}$ & $33^{\circ} 50^{\prime} 23^{\prime \prime}$ & $86^{\circ} 15^{\prime} 46^{\prime \prime}$ & St. Clair & Coosa & 03150106 & 141 \\
\hline 47 & 02401470 & $\begin{array}{l}\text { Little Canoe Creek near } \\
\text { Steele, AL }\end{array}$ & $33^{\circ} 58^{\prime} 09^{\prime \prime}$ & $86^{\circ} 10^{\prime} 40^{\prime \prime}$ & Etowah & Coosa & 03150106 & 22.3 \\
\hline 48 & 02401500 & $\begin{array}{l}\text { Big Canoe Creek near } \\
\text { Gadsden, AL }\end{array}$ & $33^{\circ} 54^{\prime} 11^{\prime \prime}$ & $86^{\circ} 06^{\prime} 36^{\prime \prime}$ & Etowah & Coosa & 03150106 & 253 \\
\hline 49 & 02402500 & Coosa River at Riverside, $\mathrm{AL}$ & $33^{\circ} 36^{\prime} 20^{\prime \prime}$ & $86^{\circ} 11^{\prime} 57^{\prime \prime}$ & St. Clair & Coosa & 03150106 & 7,069 \\
\hline
\end{tabular}

\begin{tabular}{|c|c|c|c|c|c|c|c|c|}
\hline 50 & 02403500 & $\begin{array}{l}\text { Coldwater Spring near } \\
\text { Anniston, AL }\end{array}$ & $33^{\circ} 36^{\prime} 10^{\prime \prime}$ & $85^{\circ} 55^{\prime} 33^{\prime \prime}$ & Calhoun & Coosa & 03150106 & Indeterminate \\
\hline 51 & 02404000 & $\begin{array}{l}\text { Choccolocco Creek near } \\
\text { Jenifer, AL }\end{array}$ & $33^{\circ} 34^{\prime} 14^{\prime \prime}$ & $85^{\circ} 55^{\prime} 50^{\prime \prime}$ & Talladega & Coosa & 03150106 & 277 \\
\hline 52 & 02404400 & $\begin{array}{l}\text { Choccolocco Creek at } \\
\text { Jackson Shoal near } \\
\text { Lincoln, AL }\end{array}$ & $33^{\circ} 32^{\prime} 54^{\prime \prime}$ & $86^{\circ} 05^{\prime} 49^{\prime \prime}$ & Talladega & Coosa & 03150106 & 481 \\
\hline 53 & 02404500 & $\begin{array}{l}\text { Choccolocco Creek near } \\
\text { Lincoln, AL }\end{array}$ & $33^{\circ} 33^{\prime} 38^{\prime \prime}$ & $86^{\circ} 07^{\prime} 35^{\prime \prime}$ & Talladega & Coosa & 03150106 & 496 \\
\hline
\end{tabular}


Table 1. U.S. Geological Survey continuous-record streamgaging stations in Alabama and surrounding States included in this investigation.-Continued

[USGS, U.S. Geological Survey; ', degrees; ', minutes; ”, seconds; ft³/s, cubic foot per second; DA, drainage area; NWISWeb, National Water Information System database; $\mathrm{mi}^{2}$, square mile; QAQC, quality assurance, quality control]

\begin{tabular}{|c|c|c|c|c|c|}
\hline $\begin{array}{l}\text { Site } \\
\text { index } \\
\text { number } \\
\text { (fig. 1) }\end{array}$ & Period of record available & Period of record analyzed & $\begin{array}{l}\text { Number of } \\
\text { climate years } \\
\text { used in } \\
\text { analysis }\end{array}$ & $\begin{array}{l}\text { Flow } \\
\text { condition } \\
\text { for period } \\
\text { analyzed }\end{array}$ & Remarks \\
\hline 38 & $\begin{array}{c}\text { October } 1958 \text { to September } 1967, \\
\text { October } 1970 \text { to March } 2014\end{array}$ & $\begin{array}{l}\text { April } 1959 \text { to March 1967, } \\
\text { April } 1971 \text { to March } 2014\end{array}$ & 51 & Unregulated & \\
\hline 39 & October 1937 to September 1958 & April 1938 to March 1949 & 11 & ${ }^{1}$ Unregulated & $\begin{array}{l}\text { Since about December 1949, flow } \\
\text { was regulated by Allatoona Lake } \\
\text { and since about April 1961, by } \\
\text { Weiss Lake. }\end{array}$ \\
\hline 40 & $\begin{array}{l}\text { October } 1944 \text { to September } 1954, \\
\text { October } 1956 \text { to September } 1963\end{array}$ & $\begin{array}{l}\text { April } 1945 \text { to March } 1954, \\
\text { April } 1957 \text { to March } 1963\end{array}$ & 15 & Unregulated & \\
\hline 41 & $\begin{array}{c}\text { October } 1962 \text { to September } 1967 \text {, } \\
\text { October } 1980 \text { to March } 2014\end{array}$ & $\begin{array}{l}\text { April } 1963 \text { to March 1967, } \\
\text { April } 1981 \text { to March } 2014\end{array}$ & 37 & Unregulated & \\
\hline 43 & October 2002 to March 2014 & April 2003 to March 2014 & 11 & Unregulated & \\
\hline
\end{tabular}

\begin{tabular}{|c|c|c|c|c|c|}
\hline 44 & $\begin{array}{c}\text { October } 1943 \text { to September 1970, } \\
\text { October } 1986 \text { to March } 2014\end{array}$ & $\begin{array}{l}\text { April } 1944 \text { to March } 1970, \\
\text { April } 1987 \text { to March } 2014\end{array}$ & 53 & Unregulated & \\
\hline 45 & October 1978 to May 1995 & April 1979 to March 1995 & 16 & Unregulated & \\
\hline 46 & October 1965 to March 2014 & April 1966 to March 2014 & 48 & Unregulated & \\
\hline 47 & April 1982 to May 1995 & April 1982 to March 1995 & 13 & Unregulated & \\
\hline 48 & January 1938 to September 1965 & April 1938 to March 1965 & 26 & Unregulated & \\
\hline 49 & October 1896 to September 1916 & April 1897 to March 1916 & 19 & ${ }^{1}$ Unregulated & $\begin{array}{l}\text { Since about December 1949, flow } \\
\text { was regulated by Allatoona Lake } \\
\text { and since about April 1961, by } \\
\text { Weiss Lake. }\end{array}$ \\
\hline 51 & $\begin{array}{l}\text { August } 1903 \text { to February } 1908, \\
\text { October } 1929 \text { to March 1932, } \\
\text { October } 1935 \text { to September } 1970\end{array}$ & $\begin{array}{l}\text { April } 1904 \text { to March 1907, } \\
\text { April } 1930 \text { to March 1932, } \\
\text { April } 1936 \text { to March } 1970\end{array}$ & 39 & Unregulated & \\
\hline 52 & $\begin{array}{c}\text { October } 1960 \text { to September } 1967, \\
\text { October } 1984 \text { to March } 2014\end{array}$ & $\begin{array}{l}\text { April } 1961 \text { to March 1967, } \\
\text { April } 1985 \text { to March } 2014\end{array}$ & 35 & Unregulated & $\begin{array}{l}\text { Potential diversion upstream for } \\
\text { municipal water supply for the } \\
\text { City of Anniston. }\end{array}$ \\
\hline 53 & October 1938 to September 1953 & April 1939 to March 1953 & 14 & Unregulated & $\begin{array}{l}\text { Since about } 1964, \text { site is likely influ- } \\
\text { enced by backwater from Logan } \\
\text { Martin Lake. }\end{array}$ \\
\hline
\end{tabular}


Table 1. U.S. Geological Survey continuous-record streamgaging stations in Alabama and surrounding States included in this investigation.-Continued

[USGS, U.S. Geological Survey; ', degrees; ', minutes; ”, seconds; fťs, cubic foot per second; DA, drainage area; NWISWeb, National Water Information System database; $\mathrm{mi}^{2}$, square mile; QAQC, quality assurance, quality control]

\begin{tabular}{|c|c|c|c|c|c|c|c|c|}
\hline $\begin{array}{c}\text { Site } \\
\text { index } \\
\text { number } \\
\text { (fig. 1) }\end{array}$ & $\begin{array}{l}\text { USGS } \\
\text { station } \\
\text { number }\end{array}$ & Station name & Latitude & Longitude & County & Basin & $\begin{array}{l}\text { Hydrologic } \\
\text { Unit Code }\end{array}$ & $\begin{array}{l}\text { Drainage } \\
\text { area }\left(\mathrm{mi}^{2}\right)\end{array}$ \\
\hline 54 & 02405500 & $\begin{array}{l}\text { Kelly Creek near } \\
\text { Vincent, AL }\end{array}$ & $33^{\circ} 26^{\prime} 51^{\prime \prime}$ & $86^{\circ} 23^{\prime} 13^{\prime \prime}$ & Shelby & Coosa & 03150106 & 193 \\
\hline
\end{tabular}

\begin{tabular}{|c|c|c|c|c|c|c|c|c|}
\hline 55 & 02405800 & $\begin{array}{l}\text { Talladega Creek above } \\
\text { Talladega, AL }\end{array}$ & $33^{\circ} 22^{\prime} 38^{\prime \prime}$ & $86^{\circ} 01^{\prime} 22^{\prime \prime}$ & Talladega & Coosa & 03150106 & 69.6 \\
\hline 56 & 02406500 & $\begin{array}{l}\text { Talladega Creek at } \\
\text { Alpine, AL }\end{array}$ & $33^{\circ} 21^{\prime} 34^{\prime \prime}$ & $86^{\circ} 14^{\prime} 03^{\prime \prime}$ & Talladega & Coosa & 03150106 & 150 \\
\hline 57 & 02407000 & $\begin{array}{l}\text { Coosa River at } \\
\text { Childersburg, AL }\end{array}$ & $33^{\circ} 17^{\prime} 30^{\prime \prime}$ & $86^{\circ} 21^{\prime} 50^{\prime \prime}$ & Shelby & Coosa & 03150107 & 8,392 \\
\hline 58 & 02407500 & Yellowleaf Creek near & $33^{\circ} 18^{\prime} 23^{\prime \prime}$ & $86^{\circ} 33^{\prime} 04^{\prime \prime}$ & Shelby & Coosa & 03150107 & 96.5 \\
\hline 59 & 02408500 & $\begin{array}{l}\text { Hatchet Creek near } \\
\text { Rockford, AL }\end{array}$ & $32^{\circ} 56^{\prime} 42^{\prime \prime}$ & $86^{\circ} 13^{\prime} 06^{\prime \prime}$ & Coosa & Coosa & 03150107 & 233 \\
\hline 60 & 02408540 & $\begin{array}{l}\text { Hatchet Creek below } \\
\text { Rockford, AL }\end{array}$ & $32^{\circ} 55^{\prime} 00^{\prime \prime}$ & $86^{\circ} 16^{\prime} 13^{\prime \prime}$ & Coosa & Coosa & 03150107 & 263 \\
\hline 61 & 02410000 & $\begin{array}{l}\text { Paterson Creek near } \\
\text { Central, AL }\end{array}$ & $32^{\circ} 40^{\prime} 54^{\prime \prime}$ & $86^{\circ} 07^{\prime} 40^{\prime \prime}$ & Elmore & Coosa & 03150107 & 4.91 \\
\hline 62 & 02411000 & $\begin{array}{l}\text { Coosa River at Jordan Dam } \\
\text { near Wetumpka, AL }\end{array}$ & $32^{\circ} 36^{\prime} 50^{\prime \prime}$ & $86^{\circ} 15^{\prime} 18^{\prime \prime}$ & Elmore & Coosa & 03150107 & 10,102 \\
\hline
\end{tabular}

\begin{tabular}{|c|c|c|c|c|c|c|c|c|}
\hline 63 & 02411930 & $\begin{array}{l}\text { Tallapoosa River below } \\
\text { Tallapoosa, GA }\end{array}$ & $33^{\circ} 44^{\prime} 27^{\prime \prime}$ & $85^{\circ} 20^{\prime} 11^{\prime \prime}$ & Haralson & Tallapoosa & 03150108 & 272 \\
\hline 64 & 02412000 & $\begin{array}{l}\text { Tallapoosa River near } \\
\text { Heflin, AL }\end{array}$ & $33^{\circ} 37^{\prime} 22^{\prime \prime}$ & $85^{\circ} 30^{\prime} 48^{\prime \prime}$ & Cleburne & Tallapoosa & 03150108 & 448 \\
\hline
\end{tabular}


Table 1. U.S. Geological Survey continuous-record streamgaging stations in Alabama and surrounding States included in this investigation.-Continued

[USGS, U.S. Geological Survey; ', degrees; ', minutes; ”, seconds; fts/s, cubic foot per second; DA, drainage area; NWISWeb, National Water Information System database; $\mathrm{mi}^{2}$, square mile; QAQC, quality assurance, quality control]

\begin{tabular}{|c|c|c|c|c|c|}
\hline $\begin{array}{c}\text { Site } \\
\text { index } \\
\text { number } \\
\text { (fig. 1) }\end{array}$ & Period of record available & Period of record analyzed & $\begin{array}{c}\text { Number of } \\
\text { climate years } \\
\text { used in } \\
\text { analysis }\end{array}$ & $\begin{array}{l}\text { Flow } \\
\text { condition } \\
\text { for period } \\
\text { analyzed }\end{array}$ & Remarks \\
\hline 54 & $\begin{array}{l}\text { December } 1951 \text { to September } 1970 \text {, } \\
\text { October } 1986 \text { to March } 2014\end{array}$ & April 1952 to March 1970 & 18 & Unregulated & $\begin{array}{l}\text { The period from } 1951 \text { to } 1970 \text { is } \\
\text { likely more representative of natu- } \\
\text { ral conditions than the record from } \\
1986 \text { forward. }\end{array}$ \\
\hline 54 & $\begin{array}{l}\text { December } 1951 \text { to September 1970, } \\
\text { October } 1986 \text { to March } 2014\end{array}$ & April 1987 to March 2014 & 27 & Unregulated & $\begin{array}{l}\text { QAQC reviews indicated that the } \\
\text { streamflow for the period from } \\
1986 \text { to } 2014 \text { is likely more influ- } \\
\text { enced by anthropogenic sources } \\
\text { than the earlier period from } 1952 \\
\text { to } 1970 \text {. }\end{array}$ \\
\hline 55 & June 1959 to September 1970 & April 1960 to March 1970 & 10 & Unregulated & \\
\hline 56 & $\begin{array}{l}\text { August } 1900 \text { to December } 1904 \text {, } \\
\text { October } 1939 \text { to September 1951, } \\
\text { October } 1987 \text { to March } 2014\end{array}$ & $\begin{array}{l}\text { April } 1901 \text { to March 1904, } \\
\text { April } 1940 \text { to March 1951, } \\
\text { April } 1988 \text { to March } 2014\end{array}$ & 40 & Unregulated & \\
\hline 57 & $\begin{array}{c}\text { October } 1913 \text { to September 1978, } \\
\text { June } 2011 \text { to September } 2013\end{array}$ & April 1914 to March 1948 & 35 & ${ }^{1}$ Unregulated & $\begin{array}{l}\text { The period of record analyzed rep- } \\
\text { resents pre-regulated conditions. } \\
\text { Since December 1949, flow was } \\
\text { regulated by Allatoona Lake on } \\
\text { Etowah River, since April } 1961 \text { by } \\
\text { Weiss Lake on Coosa River, since } \\
\text { July } 1964 \text { by Logan Martin Lake } \\
\text { on Coosa River, and since } 1966 \text { by } \\
\text { H. Neely Henry Lake on the Coosa } \\
\text { River. QAQC reviews did not in- } \\
\text { dicate a sufficient length of record } \\
\text { with relatively stable regulation } \\
\text { patterns to warrant frequency } \\
\text { analysis for the regulated period. }\end{array}$ \\
\hline 58 & January 1951 to September 1967 & April 1952 to March 1967 & 16 & Unregulated & \\
\hline 59 & October 1944 to February 1979 & April 1945 to March 1978 & 33 & Unregulated & \\
\hline 60 & October 1980 to March 2014 & April 1981 to March 2014 & 33 & Unregulated & \\
\hline 61 & October 1953 to September 1987 & April 1954 to March 1987 & 33 & Unregulated & \\
\hline 62 & $\begin{array}{l}\text { October } 1912 \text { to September } 1914, \\
\quad \text { October } 1925 \text { to September } 2013\end{array}$ & $\begin{array}{l}\text { April } 1913 \text { to March 1914, } \\
\text { April } 1926 \text { to March } 2013\end{array}$ & 88 & Regulated & $\begin{array}{l}\text { QAQC reviews did not indicate a } \\
\text { sufficient number of years of re- } \\
\text { cord with stable regulation patterns } \\
\text { to warrant a frequency analysis. } \\
\text { Thus, only a plot of annual mini- } \\
\text { mum 7-day average flows will be } \\
\text { published. }\end{array}$ \\
\hline 63 & $\begin{array}{l}\text { December } 1999 \text { to November 2004, } \\
\text { October } 2005 \text { to March } 2014\end{array}$ & $\begin{array}{l}\text { April } 2000 \text { to March 2004, } \\
\text { April } 2006 \text { to March } 2014\end{array}$ & 12 & Unregulated & $\begin{array}{l}\text { MOVE. } 1 \text { analysis using } 02412000 \text { as } \\
\text { the index station. }\end{array}$ \\
\hline 64 & July 1952 to March 2014 & April 1953 to March 2014 & 61 & Unregulated & \\
\hline
\end{tabular}


Table 1. U.S. Geological Survey continuous-record streamgaging stations in Alabama and surrounding States included in this investigation.-Continued

[USGS, U.S. Geological Survey; ', degrees; ', minutes; ”, seconds; fťs, cubic foot per second; DA, drainage area; NWISWeb, National Water Information System database; $\mathrm{mi}^{2}$, square mile; QAQC, quality assurance, quality control]

\begin{tabular}{|c|c|c|c|c|c|c|c|c|}
\hline $\begin{array}{c}\text { Site } \\
\text { index } \\
\text { number } \\
\text { (fig. 1) }\end{array}$ & $\begin{array}{l}\text { USGS } \\
\text { station } \\
\text { number }\end{array}$ & Station name & Latitude & Longitude & County & Basin & $\begin{array}{l}\text { Hydrologic } \\
\text { Unit Code }\end{array}$ & $\begin{array}{l}\text { Drainage } \\
\text { area }\left(\mathrm{mi}^{2}\right)\end{array}$ \\
\hline 65 & 02412500 & $\begin{array}{l}\text { Tallapoosa River near } \\
\text { Ofelia, AL }\end{array}$ & $33^{\circ} 19^{\prime} 34^{\prime \prime}$ & $85^{\circ} 35^{\prime} 31^{\prime \prime}$ & Randolph & Tallapoosa & 03150108 & 792 \\
\hline 66 & 02413300 & $\begin{array}{l}\text { Little Tallapoosa River near } \\
\text { Newell, AL }\end{array}$ & $33^{\circ} 26^{\prime} 14^{\prime \prime}$ & $85^{\circ} 23^{\prime} 57^{\prime \prime}$ & Randolph & Tallapoosa & 03150108 & 406 \\
\hline 67 & 02413500 & $\begin{array}{l}\text { Little Tallapoosa River near } \\
\text { Wedowee, AL }\end{array}$ & $33^{\circ} 20^{\prime} 57^{\prime \prime}$ & $85^{\circ} 32^{\prime} 43^{\prime \prime}$ & Randolph & Tallapoosa & 03150108 & 591 \\
\hline 68 & 02414500 & $\begin{array}{l}\text { Tallapoosa River at } \\
\text { Wadley, AL }\end{array}$ & $33^{\circ} 07^{\prime} 00^{\prime \prime}$ & $85^{\circ} 33^{\prime} 39^{\prime \prime}$ & Randolph & Tallapoosa & 03150109 & 1,675 \\
\hline 68 & 02414500 & $\begin{array}{l}\text { Tallapoosa River at } \\
\text { Wadley, AL }\end{array}$ & $33^{\circ} 07^{\prime} 00^{\prime \prime}$ & $85^{\circ} 33^{\prime} 39^{\prime \prime}$ & Randolph & Tallapoosa & 03150109 & 1,675 \\
\hline 69 & 02414715 & $\begin{array}{l}\text { Tallapoosa River near New } \\
\text { Site, AL }\end{array}$ & $32^{\circ} 58^{\prime} 38^{\prime \prime}$ & $85^{\circ} 44^{\prime} 23^{\prime \prime}$ & Tallapoosa & Tallapoosa & 03150109 & 2,058 \\
\hline 70 & 02415000 & $\begin{array}{l}\text { Hillabee Creek near } \\
\text { Hackneyville, AL }\end{array}$ & $33^{\circ} 03^{\prime} 55^{\prime \prime}$ & $85^{\circ} 52^{\prime} 41^{\prime \prime}$ & Tallapoosa & Tallapoosa & 03150109 & 190 \\
\hline 71 & 02416000 & $\begin{array}{l}\text { Tallapoosa River at } \\
\text { Sturdivant, } \mathrm{AL}\end{array}$ & $32^{\circ} 54^{\prime} 48^{\prime \prime}$ & $85^{\circ} 52^{\prime} 16^{\prime \prime}$ & Tallapoosa & Tallapoosa & 03150109 & 2,480 \\
\hline 72 & 02418230 & $\begin{array}{l}\text { Sougahatchee Creek near } \\
\text { Loachapoka, AL }\end{array}$ & $32^{\circ} 37^{\prime} 36^{\prime \prime}$ & $85^{\circ} 35^{\prime} 17^{\prime \prime}$ & Lee & Tallapoosa & 03150110 & 71.3 \\
\hline 73 & 02418500 & $\begin{array}{l}\text { Tallapoosa River below } \\
\text { Tallassee, AL }\end{array}$ & $32^{\circ} 30^{\prime} 45^{\prime \prime}$ & $85^{\circ} 53^{\prime} 21^{\prime \prime}$ & Tallapoosa & Tallapoosa & 03150110 & 3,328 \\
\hline
\end{tabular}

\begin{tabular}{|c|c|c|c|c|c|c|c|c|}
\hline 74 & 02418760 & $\begin{array}{l}\text { Chewacla Creek at } \\
\text { Chewacla State Park } \\
\text { near Auburn, AL }\end{array}$ & $32^{\circ} 32^{\prime} 53^{\prime \prime}$ & $85^{\circ} 28^{\prime} 50^{\prime \prime}$ & Lee & Tallapoosa & 03150110 & 45.8 \\
\hline 75 & 02419000 & $\begin{array}{c}\text { Uphapee Creek near } \\
\text { Tuskegee, AL }\end{array}$ & $32^{\circ} 28^{\prime} 36^{\prime \prime}$ & $85^{\circ} 41^{\prime} 42^{\prime \prime}$ & Macon & Tallapoosa & 03150110 & 333 \\
\hline 76 & 02419890 & $\begin{array}{c}\text { Tallapoosa River near } \\
\text { Montgomery, AL }\end{array}$ & $32^{\circ} 26^{\prime} 23^{\prime \prime}$ & $86^{\circ} 11^{\prime} 44^{\prime \prime}$ & Montgomery & Tallapoosa & 03150110 & 4,646 \\
\hline
\end{tabular}


Table 1. U.S. Geological Survey continuous-record streamgaging stations in Alabama and surrounding States included in this investigation.-Continued

[USGS, U.S. Geological Survey; ', degrees; ', minutes; ”, seconds; fts/s, cubic foot per second; DA, drainage area; NWISWeb, National Water Information System database; $\mathrm{mi}^{2}$, square mile; QAQC, quality assurance, quality control]

\begin{tabular}{|c|c|c|c|c|c|}
\hline $\begin{array}{l}\text { Site } \\
\text { index } \\
\text { number } \\
\text { (fig. 1) }\end{array}$ & Period of record available & Period of record analyzed & $\begin{array}{l}\text { Number of } \\
\text { climate years } \\
\text { used in } \\
\text { analysis }\end{array}$ & $\begin{array}{l}\text { Flow } \\
\text { condition } \\
\text { for period } \\
\text { analyzed }\end{array}$ & Remarks \\
\hline 65 & January 1939 to December 1951 & April 1939 to March 1951 & 12 & ${ }^{1}$ Unregulated & $\begin{array}{l}\text { Since 1983, in backwater from R.L. } \\
\text { Harris Dam (Lake Wedowee). }\end{array}$ \\
\hline 66 & October 1975 to March 2014 & April 1976 to March 2014 & 38 & Unregulated & \\
\hline 67 & October 1939 to December 1951 & April 1940 to March 1951 & 11 & Unregulated & \\
\hline 68 & October 1923 to March 2014 & April 1924 to March 1982 & 58 & ${ }^{1}$ Unregulated & $\begin{array}{l}\text { Regulated by R.L. Harris Dam since } \\
\quad 1983 .\end{array}$ \\
\hline 68 & October 1923 to March 2014 & April 1983 to March 2014 & 31 & Regulated & $\begin{array}{l}\text { Regulated by R.L. Harris Dam since } \\
1983 .\end{array}$ \\
\hline 69 & November 1985 to March 2014 & April 1986 to March 2014 & 28 & Regulated & $\begin{array}{l}\text { Regulated since about } 1983 \text { by R.L. } \\
\text { Harris Dam. }\end{array}$ \\
\hline 70 & $\begin{array}{l}\text { July } 1952 \text { to September } 1970 \text {, } \\
\text { October } 1985 \text { to March } 2014\end{array}$ & $\begin{array}{l}\text { April } 1953 \text { to March } 1970, \\
\text { April } 1986 \text { to March } 2014\end{array}$ & 45 & Unregulated & \\
\hline 71 & October 1900 to July 1926 & April 1901 to March 1926 & 25 & ${ }^{1}$ Unregulated & $\begin{array}{l}\text { Since } 1926, \text { site in backwater from } \\
\text { Martin Dam. }\end{array}$ \\
\hline 72 & November 1999 to March 2014 & April 2000 to March 2014 & 14 & Unregulated & $\begin{array}{l}\text { Flow includes wastewater-treatment } \\
\text { plant discharges from the City of } \\
\text { Auburn. }\end{array}$ \\
\hline 73 & October 1928 to September 2013 & April 1929 to March 2013 & 84 & Regulated & $\begin{array}{l}\text { Records collected by Alabama Power } \\
\text { Company, under general supervi- } \\
\text { sion of the U.S. Geological Survey, } \\
\text { in connection with a Federal } \\
\text { Energy Regulatory Commission } \\
\text { project. Daily discharge computed } \\
\text { on basis of powerplant records, } \\
\text { flow over spillway, and measured } \\
\text { leakage. Flow regulated by R.L. } \\
\text { Harris Lake (1983), Lake Martin } \\
\text { (1926), other hydroelectric plants, } \\
\text { and small mill dams above station. } \\
\text { Because of substantial influence } \\
\text { from regulation, only exceedance } \\
\text { percentiles of annual 7-day mini- } \\
\text { mum flows and duration of daily } \\
\text { flow are provided. }\end{array}$ \\
\hline 74 & October 2002 to March 2014 & April 2003 to March 2014 & 11 & Unregulated & \\
\hline 75 & $\begin{array}{l}\text { October } 1939 \text { to September } 1970 \text {, } \\
\text { October } 1974 \text { to March } 2014\end{array}$ & $\begin{array}{l}\text { April } 1940 \text { to March 1970, } \\
\text { April } 1975 \text { to March } 2014\end{array}$ & 69 & Unregulated & \\
\hline 76 & October 1995 to March 2014 & April 1996 to March 2014 & 18 & Regulated & $\begin{array}{l}\text { Flow regulated by R.L. Harris Lake } \\
\text { (1983), Lake Martin (1926), other } \\
\text { hydroelectric plants, and small } \\
\text { mill dams above station. }\end{array}$ \\
\hline
\end{tabular}


Table 1. U.S. Geological Survey continuous-record streamgaging stations in Alabama and surrounding States included in this investigation.-Continued

[USGS, U.S. Geological Survey; ', degrees; ', minutes; ”, seconds; fťs, cubic foot per second; DA, drainage area; NWISWeb, National Water Information System database; $\mathrm{mi}^{2}$, square mile; QAQC, quality assurance, quality control]

\begin{tabular}{|c|c|c|c|c|c|c|c|c|}
\hline $\begin{array}{c}\text { Site } \\
\text { index } \\
\text { number } \\
\text { (fig. 1) }\end{array}$ & $\begin{array}{l}\text { USGS } \\
\text { station } \\
\text { number }\end{array}$ & Station name & Latitude & Longitude & County & Basin & $\begin{array}{l}\text { Hydrologic } \\
\text { Unit Code }\end{array}$ & $\begin{array}{l}\text { Drainage } \\
\text { area }\left(\mathrm{mi}^{2}\right)\end{array}$ \\
\hline 77 & 02420000 & $\begin{array}{c}\text { Alabama River near } \\
\text { Montgomery, AL }\end{array}$ & $32^{\circ} 24^{\prime} 41^{\prime \prime}$ & $86^{\circ} 24^{\prime} 30^{\prime \prime}$ & Montgomery & Alabama & 03150201 & 15,087 \\
\hline
\end{tabular}

\begin{tabular}{|c|c|c|c|c|c|c|c|c|}
\hline 78 & 02420500 & $\begin{array}{l}\text { Autauga Creek at } \\
\text { Prattville, AL }\end{array}$ & $32^{\circ} 27^{\prime} 30^{\prime \prime}$ & $86^{\circ} 28^{\prime} 30^{\prime \prime}$ & Autauga & Alabama & 03150201 & 116 \\
\hline 79 & 02421000 & $\begin{array}{r}\text { Catoma Creek near } \\
\text { Montgomery, AL }\end{array}$ & $32^{\circ} 18^{\prime} 26^{\prime \prime}$ & $86^{\circ} 17^{\prime} 58^{\prime \prime}$ & Montgomery & Alabama & 03150201 & 290 \\
\hline 80 & 02422000 & $\begin{array}{l}\text { Big Swamp Creek near } \\
\text { Lowndesboro, AL }\end{array}$ & $32^{\circ} 16^{\prime} 00^{\prime \prime}$ & $86^{\circ} 41^{\prime} 40^{\prime \prime}$ & Lowndes & Alabama & 03150201 & 244 \\
\hline 81 & 02422500 & Mulberry Creek at Jones, AL & $32^{\circ} 34^{\prime} 58^{\prime \prime}$ & $86^{\circ} 54^{\prime} 13^{\prime \prime}$ & Dallas & Alabama & 03150201 & 203 \\
\hline 82 & 02423000 & Alabama River at Selma, AL & $32^{\circ} 24^{\prime} 20^{\prime \prime}$ & $87^{\circ} 01^{\prime} 07^{\prime \prime}$ & Dallas & Alabama & 03150201 & 17,095 \\
\hline 83 & 02423130 & $\begin{array}{l}\text { Cahaba River at } \\
\text { Trussville, AL }\end{array}$ & $33^{\circ} 37^{\prime} 20^{\prime \prime}$ & $86^{\circ} 35^{\prime} 58^{\prime \prime}$ & Jefferson & Cahaba & 03150202 & 19.7 \\
\hline 84 & 02423380 & $\begin{array}{l}\text { Cahaba River near Mountain } \\
\text { Brook, AL }\end{array}$ & $33^{\circ} 28^{\prime} 54^{\prime \prime}$ & $86^{\circ} 42^{\prime} 46^{\prime \prime}$ & Jefferson & Cahaba & 03150202 & 140 \\
\hline 85 & 02423397 & $\begin{array}{l}\text { Little Cahaba River below } \\
\text { Leeds, AL }\end{array}$ & $33^{\circ} 32^{\prime} 04^{\prime \prime}$ & $86^{\circ} 33^{\prime} 45^{\prime \prime}$ & Jefferson & Cahaba & 03150202 & 17 \\
\hline 86 & 02423398 & $\begin{array}{l}\text { Little Cahaba River near } \\
\text { Leeds, AL }\end{array}$ & $33^{\circ} 31^{\prime} 27^{\prime \prime}$ & $86^{\circ} 34^{\prime} 32^{\prime \prime}$ & Jefferson & Cahaba & 03150202 & 19.4 \\
\hline 87 & 02423400 & $\begin{array}{l}\text { Little Cahaba River near } \\
\text { Jefferson Park, AL }\end{array}$ & $33^{\circ} 29^{\prime} 59^{\prime \prime}$ & $86^{\circ} 36^{\prime} 51^{\prime \prime}$ & Jefferson & Cahaba & 03150202 & 24.4 \\
\hline 88 & 02423414 & $\begin{array}{l}\text { Little Cahaba River at } \\
\text { Cahaba Beach Road near } \\
\text { Cahaba Heights, AL }\end{array}$ & $33^{\circ} 26^{\prime} 23^{\prime \prime}$ & $86^{\circ} 41^{\prime} 56^{\prime \prime}$ & Shelby & Cahaba & 03150202 & 47 \\
\hline
\end{tabular}


Table 1. U.S. Geological Survey continuous-record streamgaging stations in Alabama and surrounding States included in this investigation.-Continued

[USGS, U.S. Geological Survey; ', degrees; ', minutes; ”, seconds; ft³/s, cubic foot per second; DA, drainage area; NWISWeb, National Water Information System database; $\mathrm{mi}^{2}$, square mile; QAQC, quality assurance, quality control]

\begin{tabular}{|c|c|c|c|c|c|}
\hline $\begin{array}{l}\text { Site } \\
\text { index } \\
\text { number } \\
\text { (fig. 1) }\end{array}$ & Period of record available & Period of record analyzed & $\begin{array}{l}\text { Number of } \\
\text { climate years } \\
\text { used in } \\
\text { analysis }\end{array}$ & $\begin{array}{l}\text { Flow } \\
\text { condition } \\
\text { for period } \\
\text { analyzed }\end{array}$ & Remarks \\
\hline 77 & $\begin{array}{l}\text { October } 1927 \text { to September } 1990 \text {, } \\
\text { October } 2001 \text { to March } 2014\end{array}$ & April 2002 to March 2014 & 12 & Regulated & $\begin{array}{l}\text { Regulated by dams on the Coosa } \\
\text { and Tallapoosa Rivers since } 1914 \\
\text { with most recent dams completed } \\
\text { in the } 1960 \text { s. The most relatively } \\
\text { stable period of regulation based } \\
\text { on QAQC reviews was used in the } \\
\text { 7-day exceedances and daily dura- } \\
\text { tion analysis. }\end{array}$ \\
\hline 78 & January 1939 to September 1959 & April 1939 to March 1959 & 20 & Regulated & $\begin{array}{l}\text { Since about } 1920 \text {, regulated by Prat- } \\
\text { tville Lake. }\end{array}$ \\
\hline 79 & $\begin{array}{l}\text { July } 1952 \text { to September } 1971 \text {, } \\
\text { October } 1974 \text { to September } 1996 \text {, } \\
\text { October } 1997 \text { to March } 2014\end{array}$ & $\begin{array}{l}\text { April } 1953 \text { to March 1971, } \\
\text { April } 1975 \text { to March 1996, } \\
\text { April } 1998 \text { to March } 2014\end{array}$ & 55 & Unregulated & \\
\hline 80 & October 1940 to September 1971 & April 1941 to March 1971 & 30 & Unregulated & \\
\hline 81 & $\begin{array}{l}\text { October } 1938 \text { to September } 1970 \\
\text { October } 1974 \text { to March } 2014\end{array}$ & $\begin{array}{l}\text { April } 1939 \text { to March 1970, } \\
\text { April } 1975 \text { to March } 2014\end{array}$ & 70 & Unregulated & \\
\hline 82 & $\begin{array}{l}\text { January } 1900 \text { to December } 1913 \text {, } \\
\text { October } 1928 \text { to September } 1970\end{array}$ & April 1900 to March 1913 & 13 & ${ }^{1}$ Unregulated & $\begin{array}{l}\text { Flows regulated by reservoirs on } \\
\text { the Etowah, Coosa, and Tal- } \\
\text { lapoosa Rivers. Based on review } \\
\text { of current streamgages on the } \\
\text { Alabama River, current regulation } \\
\text { does not likely reflect the period } \\
\text { of regulated record available at } \\
\text { station 02423000. Consequently, } \\
\text { 1- and 7-day frequency statistics } \\
\text { were computed for the unregulated } \\
\text { period from April } 1900 \text { to March } \\
\text { 1913, and daily duration statistics } \\
\text { were computed for January } 1900 \\
\text { to March 1913. Also, a plot of the } \\
\text { annual minimum 7-day average } \\
\text { flows for the complete period of } \\
\text { record is provided. }\end{array}$ \\
\hline 83 & October 1988 to March 2014 & April 1989 to March 2014 & 25 & Unregulated & \\
\hline 84 & $\begin{array}{l}\text { October } 1980 \text { to September 1981, } \\
\text { June } 1984 \text { to March } 2014\end{array}$ & April 1985 to March 2014 & 29 & Unregulated & \\
\hline 85 & $\begin{array}{l}\text { June } 1995 \text { to December 2006, } \\
\text { October } 2008 \text { to March } 2014\end{array}$ & $\begin{array}{l}\text { April } 1996 \text { to March 2006, } \\
\text { April } 2009 \text { to March } 2016\end{array}$ & 15 & Unregulated & \\
\hline 86 & $\begin{array}{l}\text { October } 1980 \text { to September } 1981 \\
\text { May } 1988 \text { to December } 2006\end{array}$ & April 1989 to March 2006 & 17 & Unregulated & \\
\hline 87 & $\begin{array}{r}\text { July } 1986 \text { to February } 2000 \text {, } \\
\text { June } 2007 \text { to March } 2014\end{array}$ & $\begin{array}{l}\text { April } 1987 \text { to March 1999, } \\
\text { April } 2008 \text { to March } 2014\end{array}$ & 18 & Unregulated & \\
\hline 88 & August 2003 to March 2014 & April 2004 to March 2014 & 10 & Regulated & $\begin{array}{l}\text { Flow regulated on Little Cahaba } \\
\text { River by Lake Purdy (1929). }\end{array}$ \\
\hline
\end{tabular}


Table 1. U.S. Geological Survey continuous-record streamgaging stations in Alabama and surrounding States included in this investigation.-Continued

[USGS, U.S. Geological Survey; ', degrees; ', minutes; ”, seconds; fťs, cubic foot per second; DA, drainage area; NWISWeb, National Water Information System database; $\mathrm{mi}^{2}$, square mile; QAQC, quality assurance, quality control]

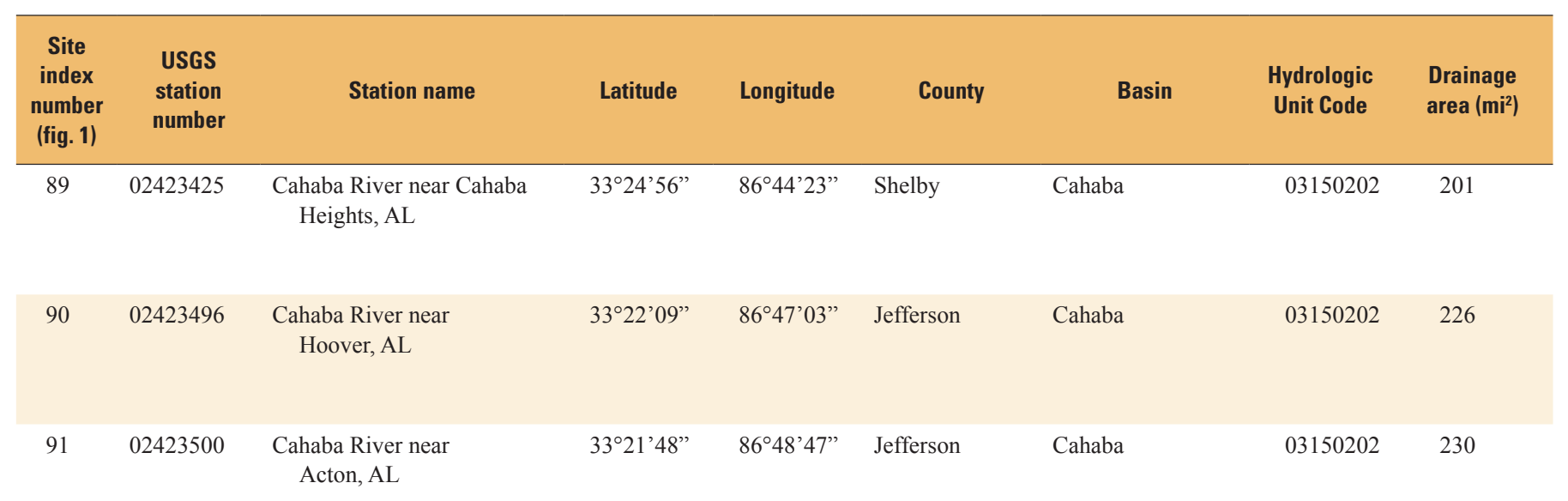

\begin{tabular}{|c|c|c|c|c|c|c|c|c|}
\hline 92 & 0242354750 & $\begin{array}{l}\text { Cahaba Valley Creek at } \\
\text { Cross Creek Road at } \\
\text { Pelham, AL }\end{array}$ & $33^{\circ} 18^{\prime} 48^{\prime \prime}$ & $86^{\circ} 48^{\prime} 23^{\prime \prime}$ & Shelby & Cahaba & 03150202 & 25.6 \\
\hline 93 & 02423555 & $\begin{array}{c}\text { Cahaba River near } \\
\text { Helena, AL }\end{array}$ & $33^{\circ} 17^{\prime} 04^{\prime \prime}$ & $86^{\circ} 52^{\prime} 57^{\prime \prime}$ & Shelby & Cahaba & 03150202 & 335 \\
\hline
\end{tabular}

\begin{tabular}{|c|c|c|c|c|c|c|c|c|}
\hline 94 & 02423630 & $\begin{array}{r}\text { Shades Creek near } \\
\text { Greenwood, AL }\end{array}$ & $33^{\circ} 19^{\prime} 34^{\prime \prime}$ & $86^{\circ} 56^{\prime} 59^{\prime \prime}$ & Jefferson & Cahaba & 03150202 & 72.3 \\
\hline 96 & 02424000 & $\begin{array}{l}\text { Cahaba River at } \\
\text { Centreville, } \mathrm{AL}\end{array}$ & $32^{\circ} 56^{\prime} 42^{\prime \prime}$ & $87^{\circ} 08^{\prime} 21^{\prime \prime}$ & Bibb & Cahaba & 03150202 & 1,027 \\
\hline
\end{tabular}


Table 1. U.S. Geological Survey continuous-record streamgaging stations in Alabama and surrounding States included in this investigation.-Continued

[USGS, U.S. Geological Survey; ' , degrees; ', minutes; ”, seconds; ft³/s, cubic foot per second; DA, drainage area; NWISWeb, National Water Information System database; $\mathrm{mi}^{2}$, square mile; QAQC, quality assurance, quality control]

\begin{tabular}{|c|c|c|c|c|c|}
\hline $\begin{array}{l}\text { Site } \\
\text { index } \\
\text { number } \\
\text { (fig. 1) }\end{array}$ & Period of record available & Period of record analyzed & $\begin{array}{c}\text { Number of } \\
\text { climate years } \\
\text { used in } \\
\text { analysis }\end{array}$ & $\begin{array}{c}\text { Flow } \\
\text { condition } \\
\text { for period } \\
\text { analyzed }\end{array}$ & Remarks \\
\hline 89 & $\begin{array}{l}\text { August } 1975 \text { to September } 1985 \text {, } \\
\text { July } 1996 \text { to March } 2014\end{array}$ & $\begin{array}{l}\text { April } 1976 \text { to March 1985, } \\
\text { April } 1997 \text { to March } 2014\end{array}$ & 26 & Regulated & $\begin{array}{l}\text { Flow regulated on Little Cahaba } \\
\text { River by Lake Purdy (1929); flow } \\
\text { diverted upstream by Birmingham } \\
\text { Water Works. }\end{array}$ \\
\hline 91 & $\begin{array}{c}\text { October } 1938 \text { to September } 1957 \text {, } \\
\text { October } 1983 \text { to March } 2014\end{array}$ & April 1984 to March 2014 & 30 & Regulated & $\begin{array}{l}\text { Flow regulated on Little Cahaba } \\
\text { River by Lake Purdy (1929); flow } \\
\text { diverted upstream by Birmingham } \\
\text { Water Works and also is influenced } \\
\text { by releases from several wastewa- } \\
\text { ter-treatment plants. The period of } \\
\text { analysis was from October } 1983 \\
\text { to March 2014, which was the } \\
\text { most recent period for which data } \\
\text { reviews indicated relatively stable } \\
\text { flow patterns. }\end{array}$ \\
\hline
\end{tabular}

\begin{tabular}{|c|c|c|c|c|c|}
\hline 92 & October 1998 to September 2013 & April 1999 to March 2013 & 14 & Unregulated & \\
\hline 93 & October 1995 to March 2014 & April 1996 to March 2014 & 18 & Regulated & $\begin{array}{l}\text { Flow partly regulated by Lake Purdy } \\
\text { (capacity, } 15,300 \text { acre-feet) on } \\
\text { Little Cahaba River. An average } \\
\text { flow of } 70 \mathrm{ft}^{3} / \mathrm{s} \text { was diverted above } \\
\text { station by Birmingham Water } \\
\text { Works and was not included in re- } \\
\text { cords, except flows of about } 5 \mathrm{ft}^{3} / \mathrm{s} \text {, } \\
\text { which were returned to river above } \\
\text { station. Flows also were influenced } \\
\text { by releases from several upstream } \\
\text { wastewater-treatment plants. }\end{array}$ \\
\hline 94 & $\begin{array}{l}\text { October } 1964 \text { to September } 1965 \text {, } \\
\text { October } 1966 \text { to September } 1973 \text {, } \\
\text { October } 1974 \text { to September 1981, } \\
\text { October } 1997 \text { to March } 2014\end{array}$ & $\begin{array}{l}\text { April } 1967 \text { to March 1973, } \\
\text { April } 1975 \text { to March 1981, } \\
\text { April } 1998 \text { to March } 2014\end{array}$ & 28 & Unregulated & \\
\hline 95 & December 1957 to September 1970 & April 1958 to March 1970 & 12 & Unregulated & \\
\hline 96 & $\begin{array}{l}\text { August } 1901 \text { to February } 1908, \\
\text { May } 1929 \text { to March } 1932, \\
\text { May } 1935 \text { to March } 2014\end{array}$ & $\begin{array}{l}\text { April } 1902 \text { to March 1907, } \\
\text { April } 1930 \text { to March 1932, } \\
\text { April } 1936 \text { to March } 2014\end{array}$ & 85 & Unregulated & $\begin{array}{l}\text { An average flow of } 82 \mathrm{ft}^{3} / \mathrm{s} \text { was } \\
\text { diverted upstream from station by } \\
\text { Birmingham Water Works Board } \\
\text { and was not included in records. } \\
\text { Flow partly regulated by Lake } \\
\text { Purdy (1929) (capacity, 15,300 } \\
\text { acre-feet) on Little Cahaba River } \\
\text { and several wastewater-treatment } \\
\text { plants. At this downstream location, } \\
\text { Lake Purdy is not likely to influ- } \\
\text { ence the flows. }\end{array}$ \\
\hline
\end{tabular}


Table 1. U.S. Geological Survey continuous-record streamgaging stations in Alabama and surrounding States included in this investigation.-Continued

[USGS, U.S. Geological Survey; ', degrees; ', minutes; ”, seconds; fťs, cubic foot per second; DA, drainage area; NWISWeb, National Water Information System database; $\mathrm{mi}^{2}$, square mile; QAQC, quality assurance, quality control]

\begin{tabular}{|c|c|c|c|c|c|c|c|c|}
\hline $\begin{array}{c}\text { Site } \\
\text { index } \\
\text { number } \\
\text { (fig. 1) }\end{array}$ & $\begin{array}{l}\text { USGS } \\
\text { station } \\
\text { number }\end{array}$ & Station name & Latitude & Longitude & County & Basin & $\begin{array}{c}\text { Hydrologic } \\
\text { Unit Code }\end{array}$ & $\begin{array}{l}\text { Drainage } \\
\text { area }\left(\mathrm{mi}^{2}\right)\end{array}$ \\
\hline 97 & 02424500 & Cahaba River at Sprott, $\mathrm{AL}$ & $32^{\circ} 40^{\prime} 05^{\prime \prime}$ & $87^{\circ} 14^{\prime} 30^{\prime \prime}$ & Perry & Cahaba & 03150202 & 1,370 \\
\hline
\end{tabular}

98 02424590

Cahaba River near Suttle, AL $\quad 32^{\circ} 31^{\prime} 45^{\prime \prime}$

$87^{\circ} 11^{\prime} 56^{\prime \prime} \quad$ Perry

Cahaba

$03150202 \quad 1,480$

100

$02424940 \quad$ Oakmulgee Creek near Augustin, AL

$32^{\circ} 31^{\prime} 58^{\prime \prime} \quad 87^{\circ} 05^{\prime} 24^{\prime \prime} \quad$ Dallas

$32^{\circ} 26^{\prime} 38^{\prime \prime}$

02425000

Cahaba River near Marion

Junction, AL

$10102425200 \quad \begin{aligned} & \text { Big Swamp Creek near } \\ & \text { Orrville, AL }\end{aligned}$

$\begin{array}{ccc}02425500 & \text { Cedar Creek at Minter, AL } \\ 103 & 02426000 & \begin{array}{c}\text { Boguechitto Creek near } \\ \text { Browns, AL }\end{array}\end{array}$

$\begin{array}{ccc}104 & 02427250 & \begin{array}{c}\text { Pine Barren Creek near } \\ \text { Snow Hill, AL }\end{array} \\ 105 & 02427500 & \begin{array}{c}\text { Alabama River near Mille } \\ \text { Ferry, AL }\end{array}\end{array}$

$32^{\circ} 13^{\prime} 17^{\prime \prime}$

$32^{\circ} 04^{\prime} 45^{\prime \prime}$

$87^{\circ} 09^{\prime} 48^{\prime \prime}$

(1)

$86^{\circ} 59^{\prime} 02^{\prime \prime}$

Dallas

$87^{\circ} 20^{\prime} 02^{\prime \prime}$

Dallas

$31^{\circ} 59^{\prime} 46^{\prime \prime}$

$87^{\circ} 04^{\prime} 06^{\prime \prime} \quad$ Wilcox

Wilcox

$32^{\circ} 06^{\prime} 52^{\prime \prime}$

87²3'58' Wilcox Ferry, AL

$32^{\circ} 26^{\prime} 18^{\prime \prime}$

(

$32^{\circ} 01^{\prime} 15^{\prime \prime}$

$31^{\circ} 36^{\prime} 30^{\prime \prime}$

\section{7³3’30” Wilcox}

87³3’02” Monroe

Claiborne L\&D near

Monroeville, AL

108

02428500
Big Flat Creek near Fountain, AL


Table 1. U.S. Geological Survey continuous-record streamgaging stations in Alabama and surrounding States included in this investigation.-Continued

[USGS, U.S. Geological Survey; ', degrees; ', minutes; ”, seconds; ft³/s, cubic foot per second; DA, drainage area; NWISWeb, National Water Information System database; $\mathrm{mi}^{2}$, square mile; QAQC, quality assurance, quality control]

\begin{tabular}{|c|c|c|c|c|c|}
\hline $\begin{array}{c}\text { Site } \\
\text { index } \\
\text { number } \\
\text { (fig. 1) }\end{array}$ & Period of record available & Period of record analyzed & $\begin{array}{l}\text { Number of } \\
\text { climate years } \\
\text { used in } \\
\text { analysis }\end{array}$ & $\begin{array}{l}\text { Flow } \\
\text { condition } \\
\text { for period } \\
\text { analyzed }\end{array}$ & Remarks \\
\hline 97 & October 1938 to September 1969 & April 1939 to March 1969 & 30 & Unregulated & $\begin{array}{l}\text { Flow partly regulated by Lake Purdy } \\
\text { (1929) (capacity, 15,300 acre-feet) } \\
\text { on Little Cahaba River. At this } \\
\text { downstream location, Lake Purdy } \\
\text { is not likely to influence the flows. } \\
\text { Flow was diverted upstream from } \\
\text { station by Birmingham Water } \\
\text { Works Board and releases by sev- } \\
\text { eral wastewater-treatment plants. }\end{array}$ \\
\hline 98 & August 1987 to October 2011 & April 1988 to March 2011 & 23 & Unregulated & $\begin{array}{l}\text { Flow partly regulated by Lake Purdy } \\
\text { Reservoir (1929) (capacity, 15,300 } \\
\text { acre-feet) on Little Cahaba River. } \\
\text { At this downstream location, Lake } \\
\text { Purdy is not likely to influence the } \\
\text { flows. Flow is diverted upstream } \\
\text { from station by Birmingham Water } \\
\text { Works Board and releases by sev- } \\
\text { eral wastewater-treatment plants. }\end{array}$ \\
\hline 99 & May 1975 to May 1987 & April 1976 to March 1987 & 11 & Unregulated & \\
\hline 100 & $\begin{array}{l}\text { October } 1938 \text { to September } 1954 \text {, } \\
\text { October } 1968 \text { to March } 2014\end{array}$ & $\begin{array}{l}\text { April } 1939 \text { to March 1954, } \\
\text { April } 1969 \text { to March } 2014\end{array}$ & 60 & Unregulated & $\begin{array}{l}\text { Flow partly regulated by Lake Purdy } \\
\text { (1929) (capacity, 15,300 acre-feet) } \\
\text { on Little Cahaba River. At this } \\
\text { downstream location, Lake Purdy } \\
\text { is not likely to influence the flows. } \\
\text { Flow was diverted upstream from } \\
\text { station by Birmingham Water } \\
\text { Works Board and releases by sev- } \\
\text { eral wastewater-treatment plants. }\end{array}$ \\
\hline 101 & March 1972 to September 1985 & April 1972 to March 1985 & 13 & Unregulated & \\
\hline 102 & $\begin{array}{l}\text { July } 1952 \text { to September } 1970 \text {, } \\
\text { October } 1974 \text { to September } 1982\end{array}$ & $\begin{array}{l}\text { April } 1953 \text { to March } 1970, \\
\text { April } 1975 \text { to March } 1982\end{array}$ & 24 & Unregulated & \\
\hline 103 & $\begin{array}{l}\text { February } 1944 \text { to June } 1954, \\
\quad \text { October } 1965 \text { to September } 1971\end{array}$ & $\begin{array}{l}\text { April } 1944 \text { to March } 1954, \\
\text { April } 1966 \text { to March } 1971\end{array}$ & 15 & Unregulated & \\
\hline 104 & October 1989 to March 2014 & April 1990 to March 2014 & 24 & Unregulated & \\
\hline 105 & October 1937 to September 1954 & October 1937 to September 1954 & 16 & Regulated & $\begin{array}{l}\text { Based on QAQC reviews, only the } \\
\text { non-exceedances of 7-day mini- } \\
\text { mum flows and daily durations } \\
\text { were computed. }\end{array}$ \\
\hline 106 & October 1958 to September 1996 & April 1959 to March 1996 & 37 & Unregulated & \\
\hline 107 & October 1975 to March 2014 & April 1976 to March 2014 & 38 & Regulated & \\
\hline 108 & October 1943 to September 1970 & April 1944 to March 1970 & 26 & Unregulated & \\
\hline
\end{tabular}


Table 1. U.S. Geological Survey continuous-record streamgaging stations in Alabama and surrounding States included in this investigation.-Continued

[USGS, U.S. Geological Survey; ', degrees; ', minutes; ”, seconds; fťs, cubic foot per second; DA, drainage area; NWISWeb, National Water Information System database; $\mathrm{mi}^{2}$, square mile; QAQC, quality assurance, quality control]

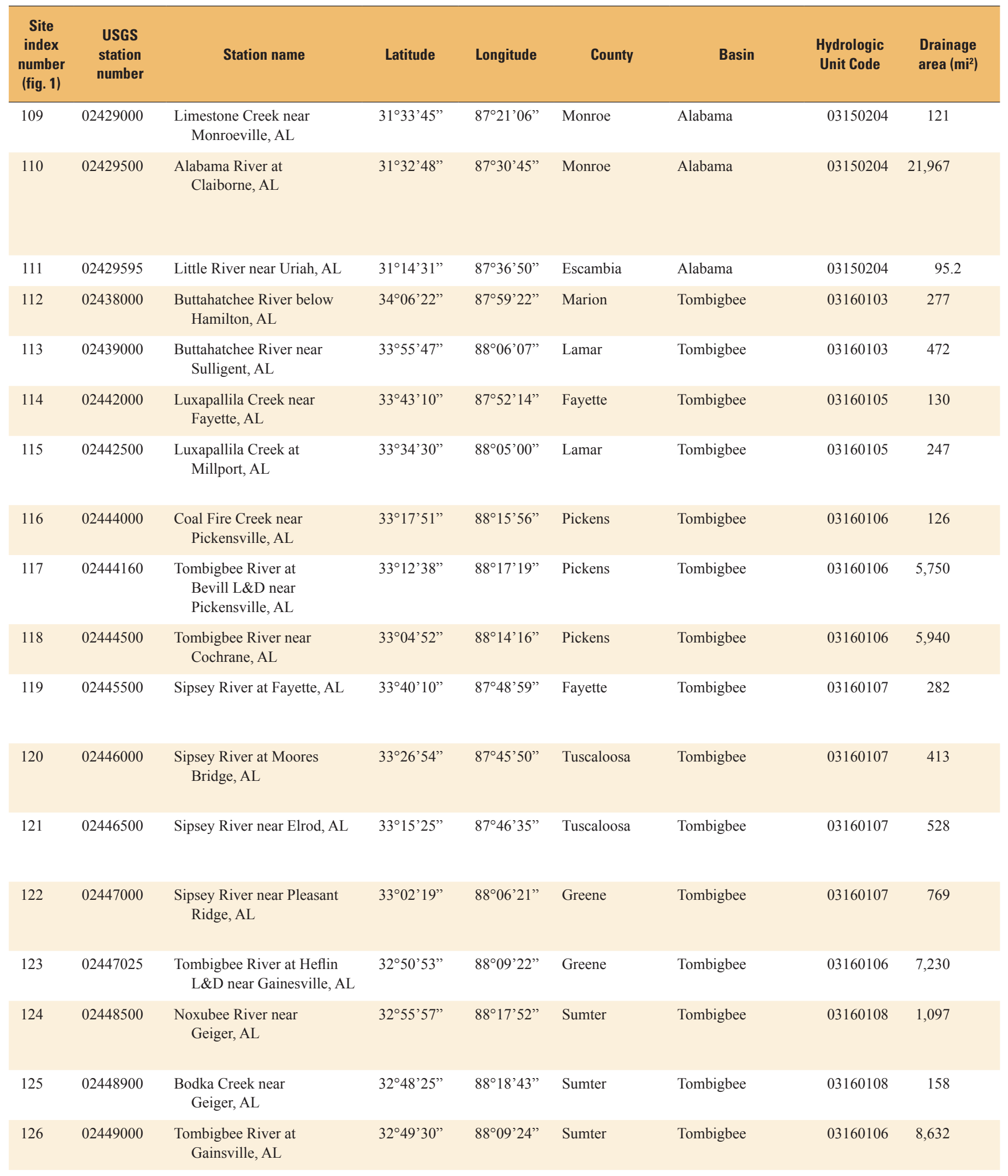


Table 1. U.S. Geological Survey continuous-record streamgaging stations in Alabama and surrounding States included in this investigation.-Continued

[USGS, U.S. Geological Survey; ', degrees; ', minutes; ”, seconds; ft³/s, cubic foot per second; DA, drainage area; NWISWeb, National Water Information System database; $\mathrm{mi}^{2}$, square mile; QAQC, quality assurance, quality control]

\begin{tabular}{|c|c|c|c|c|c|}
\hline $\begin{array}{l}\text { Site } \\
\text { index } \\
\text { number } \\
\text { (fig. 1) }\end{array}$ & Period of record available & Period of record analyzed & $\begin{array}{l}\text { Number of } \\
\text { climate years } \\
\text { used in } \\
\text { analysis }\end{array}$ & $\begin{array}{l}\text { Flow } \\
\text { condition } \\
\text { for period } \\
\text { analyzed }\end{array}$ & Remarks \\
\hline 109 & January 1952 to September 1970 & April 1952 to March 1970 & 18 & Unregulated & \\
\hline 110 & April 1930 to September 1975 & April 1930 to March 1975 & 45 & Regulated & $\begin{array}{l}\text { Only non-exceedances of 7-day mini- } \\
\text { mum flows and daily durations } \\
\text { were computed because current } \\
\text { regulation pattern likely does not } \\
\text { reflect this period of record. }\end{array}$ \\
\hline 111 & October 1968 to September 1979 & April 1969 to March 1979 & 10 & Unregulated & \\
\hline 112 & $\begin{array}{l}\text { October } 1950 \text { to September 1970, } \\
\text { October } 1991 \text { to September } 2014\end{array}$ & $\begin{array}{l}\text { April } 1951 \text { to March 1970, } \\
\text { April } 1992 \text { to March } 2014\end{array}$ & 42 & Unregulated & \\
\hline 113 & March 1939 to September 1959 & April 1939 to March 1959 & 20 & Unregulated & \\
\hline 114 & May 1945 to September 1970 & April 1946 to March 1970 & 24 & Unregulated & \\
\hline 115 & $\begin{array}{l}\text { August } 1954 \text { to September } 1959 \text {, } \\
\text { December } 1980 \text { to September 1986, } \\
\text { October } 2001 \text { to October } 2011\end{array}$ & $\begin{array}{l}\text { April } 1955 \text { to March 1959, } \\
\text { April } 1981 \text { to March 1986, } \\
\text { April } 2002 \text { to March } 2011\end{array}$ & 18 & Unregulated & \\
\hline 116 & $\begin{array}{l}\text { October } 1954 \text { to September 1971, } \\
\text { October } 1974 \text { to September } 1980\end{array}$ & $\begin{array}{l}\text { April } 1955 \text { to March } 1971, \\
\text { April } 1975 \text { to March } 1980\end{array}$ & 21 & Unregulated & \\
\hline 117 & $\begin{array}{c}\text { October } 1980 \text { to September 2009, } \\
\text { October } 2010 \text { to March } 2014\end{array}$ & $\begin{array}{l}\text { April } 1985 \text { to March 2009, } \\
\text { April } 2011 \text { to March } 2014\end{array}$ & 27 & Regulated & $\begin{array}{l}\text { Reflects diversions and regulation } \\
\text { structures since } 1985 .\end{array}$ \\
\hline 118 & October 1938 to March 1978 & April 1939 to March 1978 & 39 & ${ }^{1}$ Unregulated & \\
\hline 119 & February 1939 to September 1959 & April 1939 to March 1959 & 20 & Unregulated & $\begin{array}{l}\text { Low-flow frequency estimates were } \\
\text { adjusted based on MOVE. } 1 \text { cor- } \\
\text { relation with station } 02446500 \text {. }\end{array}$ \\
\hline 120 & February 1939 to September 1951 & April 1939 to March 1951 & 12 & Unregulated & $\begin{array}{l}\text { Low-flow frequency estimates were } \\
\text { adjusted based on MOVE.1 cor- } \\
\text { relation with station } 02446500 \text {. }\end{array}$ \\
\hline 121 & $\begin{array}{l}\text { September } 1928 \text { to March 1932, } \\
\text { October } 1939 \text { to September 1971, } \\
\text { October } 1978 \text { to September } 2014\end{array}$ & $\begin{array}{l}\text { April } 1929 \text { to March 1932, } \\
\text { April } 1940 \text { to March 1971, } \\
\text { April } 1979 \text { to March 2014 }\end{array}$ & 69 & Unregulated & \\
\hline 122 & February 1939 to September 1959 & April 1939 to March1959 & 20 & Unregulated & $\begin{array}{l}\text { Low-flow frequency estimates were } \\
\text { adjusted based on MOVE.1 cor- } \\
\text { relation with station } 02446500 \text {. }\end{array}$ \\
\hline 123 & March 1978 to March 2014 & April 1985 to March 2014 & 29 & Regulated & $\begin{array}{l}\text { Reflects diversions and regulation } \\
\text { structures since } 1985 .\end{array}$ \\
\hline 124 & $\begin{array}{l}\text { March } 1939 \text { to September } 1940 \text {, } \\
\text { August } 1944 \text { to September 1965, } \\
\text { October } 1966 \text { to March } 2014 \text {. }\end{array}$ & $\begin{array}{l}\text { April } 1939 \text { to March 1940, } \\
\text { April } 1945 \text { to March 1965, } \\
\text { April } 1967 \text { to March } 2014\end{array}$ & 67 & Unregulated & \\
\hline 125 & October 1990 to March 2014 & April 1991 to March 2014 & 23 & Unregulated & \\
\hline 126 & $\begin{array}{l}\text { October } 1938 \text { to September } 1955 \\
\text { October } 1960 \text { to September } 1978\end{array}$ & $\begin{array}{l}\text { April } 1939 \text { to March 1955, } \\
\text { April } 1961 \text { to March } 1978\end{array}$ & 33 & ${ }^{1}$ Unregulated & \\
\hline
\end{tabular}


Table 1. U.S. Geological Survey continuous-record streamgaging stations in Alabama and surrounding States included in this investigation.-Continued

[USGS, U.S. Geological Survey; ', degrees; ', minutes; ”, seconds; fťs, cubic foot per second; DA, drainage area; NWISWeb, National Water Information System database; $\mathrm{mi}^{2}$, square mile; QAQC, quality assurance, quality control]

\begin{tabular}{|c|c|c|c|c|c|c|c|c|}
\hline $\begin{array}{c}\text { Site } \\
\text { index } \\
\text { number } \\
\text { (fig. 1) }\end{array}$ & $\begin{array}{l}\text { USGS } \\
\text { station } \\
\text { number }\end{array}$ & Station name & Latitude & Longitude & County & Basin & $\begin{array}{l}\text { Hydrologic } \\
\text { Unit Code }\end{array}$ & $\begin{array}{l}\text { Drainage } \\
\text { area }\left(\mathrm{mi}^{2}\right)\end{array}$ \\
\hline 127 & 02449245 & Brush Creek near Eutaw, AL & $32^{\circ} 49^{\prime} 51^{\prime \prime}$ & $87^{\circ} 58^{\prime} 56^{\prime \prime}$ & Greene & Tombigbee & 03160106 & 43.2 \\
\hline 128 & 02449500 & $\begin{array}{l}\text { Tombigbee River at } \\
\text { Epes, } \mathrm{AL}\end{array}$ & $32^{\circ} 41^{\prime} 41^{\prime \prime}$ & $88^{\circ} 06^{\prime} 53^{\prime \prime}$ & Sumter & Tombigbee & 03160106 & 8,930 \\
\hline 129 & 02449882 & $\begin{array}{l}\text { Blue Springs Creek near } \\
\text { Blountsville, AL }\end{array}$ & $34^{\circ} 04^{\prime} 47^{\prime \prime}$ & $86^{\circ} 36^{\prime} 28^{\prime \prime}$ & Blount & Blackwarrior & 03160109 & 13 \\
\hline 130 & 02450000 & $\begin{array}{l}\text { Mulberry Fork near Garden } \\
\text { City, AL }\end{array}$ & $34^{\circ} 00^{\prime} 43^{\prime \prime}$ & $86^{\circ} 44^{\prime} 12^{\prime \prime}$ & Blount & Blackwarrior & 03160109 & 358 \\
\hline 130 & 02450000 & $\begin{array}{l}\text { Mulberry Fork near Garden } \\
\text { City, AL }\end{array}$ & $34^{\circ} 00^{\prime} 43^{\prime \prime}$ & $86^{\circ} 44^{\prime} 12^{\prime \prime}$ & Blount & Blackwarrior & 03160109 & 358 \\
\hline
\end{tabular}

\begin{tabular}{|c|c|c|c|c|c|c|c|c|}
\hline 131 & 02450180 & $\begin{array}{l}\text { Mulberry Fork near } \\
\text { Arkadelphia, } \mathrm{AL}\end{array}$ & $33^{\circ} 52^{\prime} 19^{\prime \prime}$ & $86^{\circ} 55^{\prime} 20^{\prime \prime}$ & Cullman & Blackwarrior & 03160109 & 487 \\
\hline 132 & 02450250 & $\begin{array}{l}\text { Sipsey Fork near } \\
\text { Grayson, AL }\end{array}$ & $34^{\circ} 17^{\prime} 07^{\prime \prime}$ & $87^{\circ} 23^{\prime} 56^{\prime \prime}$ & Winston & Blackwarrior & 03160110 & 92.1 \\
\hline 133 & 02450500 & $\begin{array}{l}\text { Sipsey Fork near Falls } \\
\quad \text { City, AL }\end{array}$ & $34^{\circ} 03^{\prime} 07^{\prime \prime}$ & $87^{\circ} 16^{\prime} 01^{\prime \prime}$ & Winston & Blackwarrior & 03160110 & 360 \\
\hline 134 & 02450825 & $\begin{array}{l}\text { Clear Creek at New Hope } \\
\text { Church near Poplar } \\
\text { Springs, AL }\end{array}$ & $34^{\circ} 04^{\prime} 52^{\prime \prime}$ & $87^{\circ} 25^{\prime} 22^{\prime \prime}$ & Winston & Blackwarrior & 03160110 & 101 \\
\hline 135 & 02451000 & Clear Creek at Falls City, AL & $34^{\circ} 02^{\prime} 05^{\prime \prime}$ & $87^{\circ} 18^{\prime} 00^{\prime \prime}$ & Winston & Blackwarrior & 03160110 & 149 \\
\hline
\end{tabular}

\begin{tabular}{|c|c|c|c|c|c|c|c|c|}
\hline 136 & 02453000 & $\begin{array}{l}\text { Blackwater Creek near } \\
\text { Manchester, AL }\end{array}$ & $33^{\circ} 54^{\prime} 30^{\prime \prime}$ & $87^{\circ} 15^{\prime} 25^{\prime \prime}$ & Walker & Blackwarrior & 03160109 & 181 \\
\hline 137 & 02453500 & $\begin{array}{l}\text { Mulberry Fork at } \\
\text { Cordova, AL }\end{array}$ & $33^{\circ} 45^{\prime} 27^{\prime \prime}$ & $87^{\circ} 10^{\prime} 13^{\prime \prime}$ & Walker & Blackwarrior & 03160109 & 1,916 \\
\hline
\end{tabular}


Table 1. U.S. Geological Survey continuous-record streamgaging stations in Alabama and surrounding States included in this investigation.-Continued

[USGS, U.S. Geological Survey; ', degrees; ', minutes; ”, seconds; fts/s, cubic foot per second; DA, drainage area; NWISWeb, National Water Information System database; $\mathrm{mi}^{2}$, square mile; QAQC, quality assurance, quality control]

\begin{tabular}{|c|c|c|c|c|c|}
\hline $\begin{array}{l}\text { Site } \\
\text { index } \\
\text { number } \\
\text { (fig. 1) }\end{array}$ & Period of record available & Period of record analyzed & $\begin{array}{l}\text { Number of } \\
\text { climate years } \\
\text { used in } \\
\text { analysis }\end{array}$ & $\begin{array}{l}\text { Flow } \\
\text { condition } \\
\text { for period } \\
\text { analyzed }\end{array}$ & Remarks \\
\hline 127 & June 1975 to September 1997 & April 1976 to March 1997 & 21 & Unregulated & \\
\hline 128 & $\begin{array}{l}\text { January } 1901 \text { to December } 1901 \text {, } \\
\text { January } 1905 \text { to August } 1913 \text {, } \\
\text { October } 1938 \text { to September } 1945\end{array}$ & $\begin{array}{l}\text { April } 1905 \text { to March } 1913, \\
\text { April } 1939 \text { to March } 1945\end{array}$ & 14 & ${ }^{1}$ Unregulated & \\
\hline 129 & October 1992 to March 2014 & April 1993 to March 2014 & 21 & Unregulated & \\
\hline 130 & October 1928 to March 2014 & April 1929 to March 1964 & 35 & Unregulated & $\begin{array}{l}\text { The flow patterns changed in the } \\
\text { mid 1960s with increasing annual } \\
\text { minimum 7-day average flows } \\
\text { likely associated with anthropo- } \\
\text { genic changes in the watershed. } \\
\text { This analysis is for October } 1928 \\
\text { to March 1964, which likely repre- } \\
\text { sents a more natural condition that } \\
\text { the flows after } 1964 \text {. }\end{array}$ \\
\hline 130 & October 1928 to March 2014 & April 1965 to March 2014 & 49 & Unregulated & $\begin{array}{l}\text { The flow patterns changed in the } \\
\text { mid 1960s with increasing annual } \\
\text { minimum 7-day average flows } \\
\text { likely associated with anthropo- } \\
\text { genic changes in the watershed. } \\
\text { This analysis is for April } 1965 \text { to } \\
\text { March } 2014 \text {. }\end{array}$ \\
\hline 131 & $\begin{array}{c}\text { October } 1976 \text { to September } 1986 \text {, } \\
\text { October } 1988 \text { to March } 2014\end{array}$ & $\begin{array}{l}\text { April } 1977 \text { to March 1986, } \\
\text { April } 1989 \text { to March } 2014\end{array}$ & 34 & Unregulated & $\begin{array}{l}\text { Based on findings from station } \\
02450000, \text { the low flows at this } \\
\text { station are likely substantially } \\
\text { influenced by anthropogenic influ- } \\
\text { ences. }\end{array}$ \\
\hline 132 & October 1966 to March 2014 & April 1967 to March 2014 & 47 & Unregulated & \\
\hline 133 & June 1943 to December 1954 & April 1944 to March 1954 & 10 & Unregulated & $\begin{array}{l}\text { Since 1961, site in backwater from } \\
\text { Lewis Smith Dam. }\end{array}$ \\
\hline 134 & $\begin{array}{l}\text { October } 1980 \text { to September } 1981 \text {, } \\
\text { October } 1993 \text { to March } 2014\end{array}$ & April 1994 to March 2014 & 20 & Unregulated & \\
\hline 135 & October 1939 to November 1954 & April 1940 to March 1954 & 14 & Unregulated & $\begin{array}{l}\text { Since 1961, site in backwater from } \\
\text { Lewis Smith Dam. }\end{array}$ \\
\hline 136 & $\begin{array}{l}\text { October } 1938 \text { to September } 1971 \text {, } \\
\text { October } 1979 \text { to September } 1982 \text {, } \\
\text { October } 1988 \text { to March } 2014\end{array}$ & April 1939 to March 1971 & 32 & Unregulated & $\begin{array}{l}\text { QAQC analysis indicated differences } \\
\text { in flow patterns from the early to } \\
\text { latter periods of record. The period } \\
\text { of record analyzed likely repre- } \\
\text { sents more natural conditions than } \\
\text { the period from } 1988 \text { to } 2014 \text {. }\end{array}$ \\
\hline 136 & $\begin{array}{l}\text { October } 1938 \text { to September } 1971 \text {, } \\
\text { October } 1979 \text { to September } 1982 \text {, } \\
\text { October } 1988 \text { to March } 2014\end{array}$ & $\begin{array}{l}\text { April } 1980 \text { to March 1982, } \\
\text { April } 1989 \text { to March } 2014\end{array}$ & 27 & Unregulated & $\begin{array}{l}\text { QAQC analysis indicated differences } \\
\text { in flow patterns from the early to } \\
\text { latter periods of record. The period } \\
\text { of record analyzed likely includes } \\
\text { anthropogenic influences. }\end{array}$ \\
\hline 137 & June 1900 to December 1912 & April 1901 to March 1912 & 11 & ${ }^{1}$ Unregulated & $\begin{array}{l}\text { Since 1961, regulated by Lewis } \\
\text { Smith Dam on the Sipsey Fork. }\end{array}$ \\
\hline
\end{tabular}


Table 1. U.S. Geological Survey continuous-record streamgaging stations in Alabama and surrounding States included in this investigation.-Continued

[USGS, U.S. Geological Survey; ', degrees; ', minutes; ”, seconds; fťs, cubic foot per second; DA, drainage area; NWISWeb, National Water Information System database; $\mathrm{mi}^{2}$, square mile; QAQC, quality assurance, quality control]

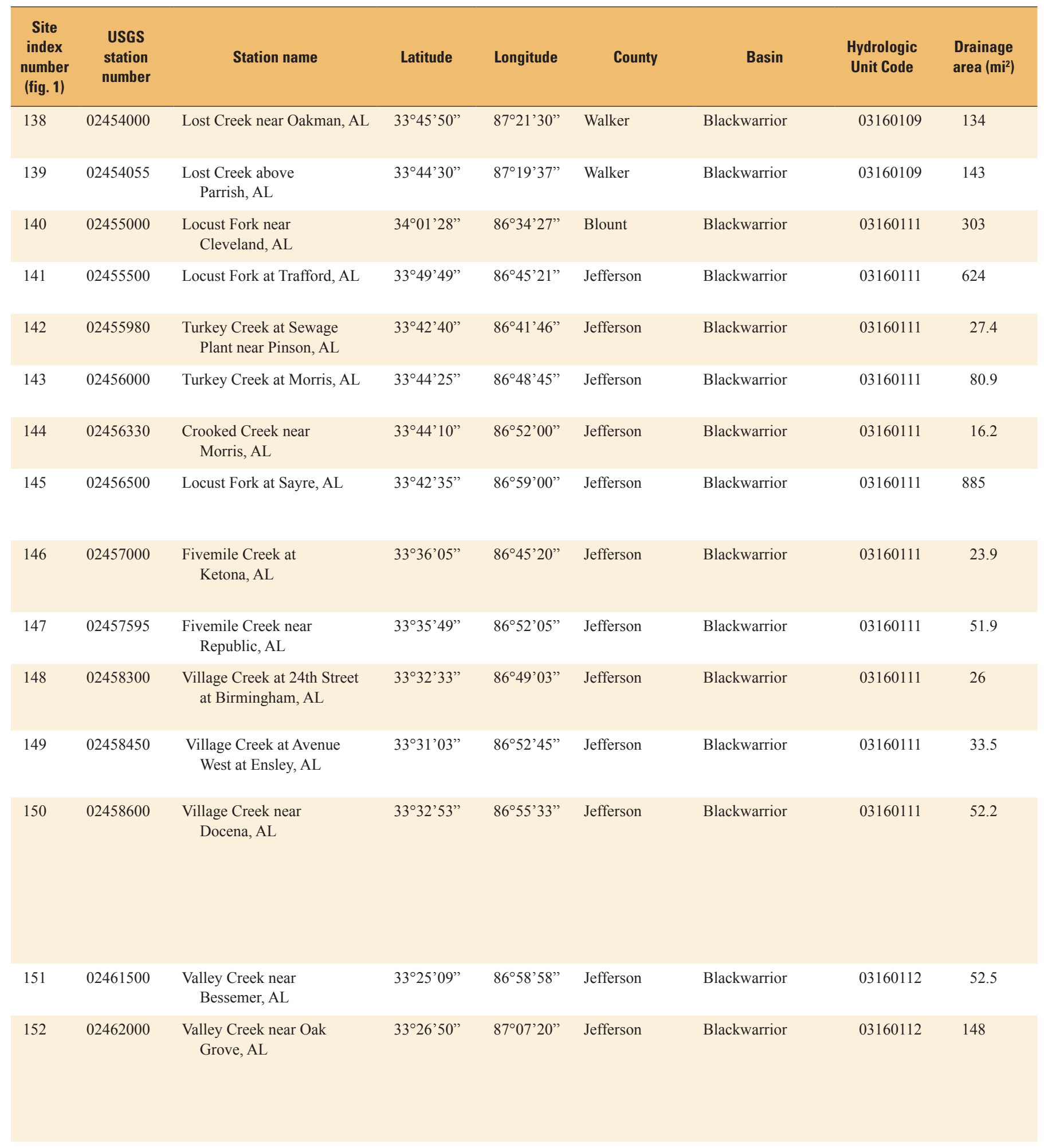


Table 1. U.S. Geological Survey continuous-record streamgaging stations in Alabama and surrounding States included in this investigation.-Continued

[USGS, U.S. Geological Survey; ', degrees; ', minutes; ”, seconds; ft³/s, cubic foot per second; DA, drainage area; NWISWeb, National Water Information System database; $\mathrm{mi}^{2}$, square mile; QAQC, quality assurance, quality control]

\begin{tabular}{|c|c|c|c|c|c|}
\hline $\begin{array}{c}\text { Site } \\
\text { index } \\
\text { number } \\
\text { (fig. 1) }\end{array}$ & Period of record available & Period of record analyzed & $\begin{array}{c}\text { Number of } \\
\text { climate years } \\
\text { used in } \\
\text { analysis }\end{array}$ & $\begin{array}{l}\text { Flow } \\
\text { condition } \\
\text { for period } \\
\text { analyzed }\end{array}$ & Remarks \\
\hline 138 & $\begin{array}{l}\text { October } 1951 \text { to September } 1966, \\
\text { October } 1979 \text { to September } 1981\end{array}$ & $\begin{array}{l}\text { April } 1952 \text { to March } 1966, \\
\text { April } 1980 \text { to March } 1981\end{array}$ & 15 & Unregulated & \\
\hline 139 & October 1992 to March 2014 & April 1993 to March 2014 & 21 & Unregulated & \\
\hline 140 & $\begin{array}{l}\text { December } 1936 \text { to September 1986, } \\
\text { October } 1992 \text { to March } 2014\end{array}$ & $\begin{array}{l}\text { April } 1937 \text { to March 1986, } \\
\text { April } 1993 \text { to March } 2014\end{array}$ & 70 & Unregulated & \\
\hline 141 & $\begin{array}{l}\text { October } 1930 \text { to September } 1969, \\
\text { October } 1992 \text { to September } 1997\end{array}$ & $\begin{array}{l}\text { April } 1931 \text { to March } 1969, \\
\text { April } 1993 \text { to March } 1997\end{array}$ & 42 & Unregulated & \\
\hline 142 & July 1988 to March 2014 & April 1989 to March 2014 & 25 & Unregulated & \\
\hline 143 & $\begin{array}{c}\text { January } 1944 \text { to September } 1979, \\
\text { March } 2002 \text { to October } 2011\end{array}$ & $\begin{array}{l}\text { April } 1944 \text { to March 1979, } \\
\text { April } 2002 \text { to March } 2011\end{array}$ & 42 & Unregulated & \\
\hline 144 & $\begin{array}{r}\text { November } 1975 \text { to September } 1988, \\
\text { October } 1996 \text { to September } 1997\end{array}$ & $\begin{array}{r}\text { November } 1975 \text { to September 1988, } \\
\text { October } 1996 \text { to September } 1997\end{array}$ & 12 & Unregulated & \\
\hline 145 & $\begin{array}{l}\text { October } 1928 \text { to March } 1932, \\
\text { October } 1941 \text { to March } 2014\end{array}$ & April 1964 to March 2014 & 50 & Unregulated & $\begin{array}{l}\text { Early part of record was excluded due } \\
\text { to differences in flow patterns as } \\
\text { indicated in the QAQC analyses. }\end{array}$ \\
\hline 146 & $\begin{array}{l}\text { October } 1953 \text { to September } 1958 \text {, } \\
\text { December } 1974 \text { to September } 1979 \text {, } \\
\text { May } 1996 \text { to March } 2014\end{array}$ & $\begin{array}{l}\text { April } 1954 \text { to March } 1958, \\
\text { April } 1975 \text { to March 1979, } \\
\text { April } 1997 \text { to March } 2014\end{array}$ & 25 & Unregulated & \\
\hline 147 & May 1988 to March 2014 & April 1989 to March 2014 & 25 & Unregulated & \\
\hline 148 & $\begin{array}{l}\text { June } 1988 \text { to December } 2006 \text {, } \\
\text { July } 2008 \text { to September } 2013\end{array}$ & $\begin{array}{l}\text { April } 1989 \text { to March 2006, } \\
\text { April } 2009 \text { to March } 2013\end{array}$ & 21 & Regulated & $\begin{array}{l}\text { Low and medium stages may be } \\
\text { influenced by upstream plants and } \\
\text { mills. }\end{array}$ \\
\hline 149 & $\begin{array}{c}\text { July } 1975 \text { to September } 1979 \text {, } \\
\text { July } 1988 \text { to March } 2014\end{array}$ & $\begin{array}{l}\text { April } 1976 \text { to March } 1979, \\
\text { April } 1989 \text { to March } 2014\end{array}$ & 28 & Regulated & $\begin{array}{l}\text { Low and medium stages may be } \\
\text { influenced by upstream plants and } \\
\text { mills. }\end{array}$ \\
\hline 150 & June 1996 to March 2014 & April 1997 to March 2014 & 17 & Unregulated & $\begin{array}{l}\text { QAQC reviews indicate that flow was } \\
\text { affected by the dam at Bayview } \\
\text { Lake, likely attributed to backwater } \\
\text { issues. Duration curve compari- } \\
\text { son with stations } 02458300 \text { and } \\
02458450 \text { shows this station has } \\
\text { substantially more flow per square } \\
\text { mile at the low end. }\end{array}$ \\
\hline 151 & $\begin{array}{c}\text { May } 1975 \text { to September } 1979, \\
\text { May } 1988 \text { to March } 2014\end{array}$ & $\begin{array}{l}\text { April } 1976 \text { to March } 1979, \\
\text { April } 1989 \text { to March } 2014\end{array}$ & 28 & Unregulated & $\begin{array}{l}\text { Low flows likely were influenced by } \\
\text { upstream discharges. }\end{array}$ \\
\hline 152 & $\begin{array}{l}\text { October } 1953 \text { to September } 1958 \text {, } \\
\text { October } 1964 \text { to September } 1965 \text {, } \\
\text { June } 1978 \text { to March } 2014\end{array}$ & $\begin{array}{l}\text { April } 1954 \text { to March } 1958, \\
\text { April } 1979 \text { to March } 2014\end{array}$ & 39 & Unregulated & $\begin{array}{l}\text { Low streamflows were influenced by } \\
\text { municipal sewage and industrial } \\
\text { wastes with much of the discharge } \\
\text { being water diverted from the } \\
\text { Cahaba River and Blackborne } \\
\text { Fork and from other sources. }\end{array}$ \\
\hline
\end{tabular}


Table 1. U.S. Geological Survey continuous-record streamgaging stations in Alabama and surrounding States included in this investigation.-Continued

[USGS, U.S. Geological Survey; ', degrees; ', minutes; ”, seconds; fťs, cubic foot per second; DA, drainage area; NWISWeb, National Water Information System database; $\mathrm{mi}^{2}$, square mile; QAQC, quality assurance, quality control]

\begin{tabular}{|c|c|c|c|c|c|c|c|c|}
\hline $\begin{array}{c}\text { Site } \\
\text { index } \\
\text { number } \\
\text { (fig. 1) }\end{array}$ & $\begin{array}{l}\text { USGS } \\
\text { station } \\
\text { number }\end{array}$ & Station name & Latitude & Longitude & County & Basin & $\begin{array}{l}\text { Hydrologic } \\
\text { Unit Code }\end{array}$ & $\begin{array}{l}\text { Drainage } \\
\text { area }\left(\mathrm{mi}^{2}\right)\end{array}$ \\
\hline 153 & 02462500 & $\begin{array}{l}\text { Black Warrior River at } \\
\text { Bankhead L\&D near } \\
\text { Bessemer, AL }\end{array}$ & $33^{\circ} 27^{\prime} 30^{\prime \prime}$ & $87^{\circ} 21^{\prime} 15^{\prime \prime}$ & Tuscaloosa & Blackwarrior & 03160112 & 3,981 \\
\hline
\end{tabular}

\begin{tabular}{|c|c|c|c|c|c|c|c|c|}
\hline 154 & 02462600 & $\begin{array}{l}\text { Blue Creek near } \\
\text { Oakman, AL }\end{array}$ & $33^{\circ} 31^{\prime} 17^{\prime \prime}$ & $87^{\circ} 29^{\prime} 07^{\prime \prime}$ & Tuscaloosa & Blackwarrior & 03160112 & 5.32 \\
\hline 155 & 02462800 & $\begin{array}{l}\text { Davis Creek below } \\
\text { Abernant, AL }\end{array}$ & $33^{\circ} 18^{\prime} 30^{\prime \prime}$ & $87^{\circ} 13^{\prime} 10^{\prime \prime}$ & Tuscaloosa & Blackwarrior & 03160112 & 45.3 \\
\hline 156 & 02462951 & $\begin{array}{l}\text { Black Warrior River at Holt } \\
\text { L\&D near Holt, AL }\end{array}$ & $33^{\circ} 15^{\prime} 11^{\prime \prime}$ & $87^{\circ} 26^{\prime} 57^{\prime \prime}$ & Tuscaloosa & Blackwarrior & 03160112 & 4,219 \\
\hline 157 & 02463500 & $\begin{array}{l}\text { Hurricane Creek near } \\
\text { Holt, AL }\end{array}$ & $33^{\circ} 12^{\prime} 40^{\prime \prime}$ & $87^{\circ} 26^{\prime} 51^{\prime \prime}$ & Tuscaloosa & Blackwarrior & 03160112 & 108 \\
\hline 158 & 02464000 & $\begin{array}{l}\text { North River near } \\
\text { Samantha, AL }\end{array}$ & $33^{\circ} 28^{\prime} 45^{\prime \prime}$ & $87^{\circ} 35^{\prime} 50^{\prime \prime}$ & Tuscaloosa & Blackwarrior & 03160112 & 223 \\
\hline 159 & 02464146 & $\begin{array}{c}\text { Turkey Creek near } \\
\text { Tuscaloosa, AL }\end{array}$ & $33^{\circ} 24^{\prime} 48^{\prime \prime}$ & $87^{\circ} 30^{\prime} 38^{\prime \prime}$ & Tuscaloosa & Blackwarrior & 03160112 & 6.16 \\
\hline 161 & 02464500 & $\begin{array}{l}\text { North River (Site B) near } \\
\text { Tuscaloosa, AL }\end{array}$ & $33^{\circ} 21^{\prime} 14^{\prime \prime}$ & $87^{\circ} 33^{\prime} 12^{\prime \prime}$ & Tuscaloosa & Blackwarrior & 03160112 & 372 \\
\hline 162 & 02465000 & $\begin{array}{l}\text { Black Warrior River at } \\
\text { Oliver Lock and Dam at } \\
\text { Northport, AL }\end{array}$ & $33^{\circ} 12^{\prime} 33^{\prime \prime}$ & $87^{\circ} 35^{\prime} 24^{\prime \prime}$ & Tuscaloosa & Blackwarrior & 03160112 & 4,820 \\
\hline 166 & 02465500 & $\begin{array}{l}\text { Fivemile Creek near } \\
\text { Greensboro, AL }\end{array}$ & $32^{\circ} 49^{\prime} 57^{\prime \prime}$ & $87^{\circ} 36^{\prime} 24^{\prime \prime}$ & Hale & Blackwarrior & 03160113 & 73.6 \\
\hline
\end{tabular}


Table 1. U.S. Geological Survey continuous-record streamgaging stations in Alabama and surrounding States included in this investigation.-Continued

[USGS, U.S. Geological Survey; ' , degrees; ', minutes; ”, seconds; ft³/s, cubic foot per second; DA, drainage area; NWISWeb, National Water Information System database; $\mathrm{mi}^{2}$, square mile; QAQC, quality assurance, quality control]

\begin{tabular}{|c|c|c|c|c|c|}
\hline $\begin{array}{c}\text { Site } \\
\text { index } \\
\text { number } \\
\text { (fig. 1) }\end{array}$ & Period of record available & Period of record analyzed & $\begin{array}{c}\text { Number of } \\
\text { climate years } \\
\text { used in } \\
\text { analysis }\end{array}$ & $\begin{array}{c}\text { Flow } \\
\text { condition } \\
\text { for period } \\
\text { analyzed }\end{array}$ & Remarks \\
\hline 153 & $\begin{array}{r}\text { October } 1928 \text { to September } 1936, \\
\text { October } 1976 \text { to February } 2014\end{array}$ & October 1976 to February 2014 & 36 & Regulated & $\begin{array}{l}\text { Flow was regulated since } 1961 \text { by } \\
\text { Lewis Smith Lake. Non-exceed- } \\
\text { ances of 7-day minimum flows } \\
\text { only for regulated period. Flow } \\
\text { period included zero flows. Log- } \\
\text { Pearson type III distribution does } \\
\text { not adequately fit the data due to } \\
\text { regulation. No frequency analysis } \\
\text { was done. Only 7-day exceed- } \\
\text { ances and daily durations were } \\
\text { computed. }\end{array}$ \\
\hline
\end{tabular}

\begin{tabular}{|c|c|c|c|c|c|}
\hline 154 & $\begin{array}{l}\text { June } 1959 \text { to September } 1965 \text {, } \\
\text { October } 1976 \text { to September } 1984\end{array}$ & $\begin{array}{l}\text { April } 1960 \text { to March } 1965, \\
\text { April } 1977 \text { to March } 1984\end{array}$ & 12 & Unregulated & \\
\hline 155 & October 1956 to September 1971 & April 1957 to March 1971 & 14 & Unregulated & \\
\hline 156 & $\begin{array}{l}\text { October } 1976 \text { to November } 2000 \text {, } \\
\text { August } 2001 \text { to September } 2013\end{array}$ & $\begin{array}{l}\text { October } 1976 \text { to November } 2000 \text {, } \\
\text { August } 2002 \text { to September } 2013\end{array}$ & 34 & Regulated & $\begin{array}{l}\text { Flow was regulated by Lewis Smith } \\
\text { Lake and Bankhead Lock and } \\
\text { Dam. Non-exceedances of 7-day } \\
\text { minimum flows and daily dura- } \\
\text { tions only. Zero flows. }\end{array}$ \\
\hline 157 & August 1952 to September 1969 & April 1953 to March 1969 & 16 & Unregulated & \\
\hline 158 & $\begin{array}{l}\text { December } 1938 \text { to September 1954, } \\
\text { October } 1968 \text { to March } 2014\end{array}$ & $\begin{array}{l}\text { April } 1939 \text { to March } 1954, \\
\text { April } 1969 \text { to March } 2014\end{array}$ & 60 & Unregulated & \\
\hline 159 & $\begin{array}{l}\text { February } 1981 \text { to September } 1984 \text {, } \\
\text { October } 1986 \text { to March } 2014\end{array}$ & $\begin{array}{l}\text { April } 1981 \text { to March 1984, } \\
\text { April } 1987 \text { to March } 2014\end{array}$ & 30 & Unregulated & \\
\hline 160 & October 1986 to March 2014 & April 1987 to March 2014 & 27 & Unregulated & \\
\hline 161 & December 1951 to December 1968 & April 1952 to March 1968 & 16 & Unregulated & $\begin{array}{l}\text { Since } 1970 \text {, in backwater from Lake } \\
\text { Tuscaloosa Dam. }\end{array}$ \\
\hline 162 & $\begin{array}{c}\text { January } 1895 \text { to December } 1902 \text {, } \\
\text { August } 1928 \text { to March } 2014\end{array}$ & April 1976 to March 2014 & 38 & Regulated & $\begin{array}{l}\text { Flow was regulated since } 1914 \text { by } \\
\text { Bankhead Lock and Dam on Black } \\
\text { Warrior River, since } 1961 \text { by } \\
\text { Lewis Smith Lake on Sipsey Fork, } \\
\text { and since } 1969 \text { by Holt Lock and } \\
\text { Dam on Black Warrior River. } \\
\text { Period of record analyzed was } \\
\text { April } 1976 \text { to March 2014, which } \\
\text { was the most recent period of } \\
\text { relatively stable regulation. }\end{array}$ \\
\hline 163 & November 1956 to September 1970 & April 1957 to March 1970 & 13 & Regulated & Downstream from Lake Lurleen. \\
\hline 164 & July 2002 to March 2014 & April 2003 to March 2014 & 11 & Unregulated & \\
\hline 165 & October 1976 to March 2014 & April 1977 to March 2014 & 37 & Unregulated & \\
\hline 166 & October 1954 to September 1971 & April 1955 to March 1971 & 16 & Unregulated & \\
\hline
\end{tabular}


Table 1. U.S. Geological Survey continuous-record streamgaging stations in Alabama and surrounding States included in this investigation.-Continued

[USGS, U.S. Geological Survey; ', degrees; ', minutes; ”, seconds; fťs, cubic foot per second; DA, drainage area; NWISWeb, National Water Information System database; $\mathrm{mi}^{2}$, square mile; QAQC, quality assurance, quality control]

\begin{tabular}{|c|c|c|c|c|c|c|c|c|}
\hline $\begin{array}{l}\text { Site } \\
\text { index } \\
\text { number } \\
\text { (fig. 1) }\end{array}$ & $\begin{array}{l}\text { USGS } \\
\text { station } \\
\text { number }\end{array}$ & Station name & Latitude & Longitude & County & Basin & $\begin{array}{l}\text { Hydrologic } \\
\text { Unit Code }\end{array}$ & $\begin{array}{l}\text { Drainage } \\
\text { area }\left(\mathrm{mi}^{2}\right)\end{array}$ \\
\hline 167 & 02466030 & $\begin{array}{l}\text { Black Warrior River at } \\
\text { Selden L\&D near } \\
\text { Eutaw, AL }\end{array}$ & $32^{\circ} 46^{\prime} 40^{\prime \prime}$ & $87^{\circ} 50^{\prime} 26^{\prime \prime}$ & Hale & Blackwarrior & 03160113 & 5,810 \\
\hline 169 & 02467000 & $\begin{array}{l}\text { Tombigbee River at } \\
\text { Demopolis L\&D near } \\
\text { Coatopa, AL }\end{array}$ & $32^{\circ} 31^{\prime} 10^{\prime \prime}$ & $87^{\circ} 52^{\prime} 42^{\prime \prime}$ & Marengo & Tombigbee & 03160201 & 15,385 \\
\hline 170 & 02467500 & $\begin{array}{l}\text { Sucarnoochee River at } \\
\text { Livingston, } \mathrm{AL}\end{array}$ & $32^{\circ} 34^{\prime} 25^{\prime \prime}$ & $88^{\circ} 11^{\prime} 36^{\prime \prime}$ & Sumter & Tombigbee & 03160202 & 607 \\
\hline 173 & 02469000 & $\begin{array}{l}\text { Kinterbish Creek near } \\
\quad \text { York, AL }\end{array}$ & $32^{\circ} 19^{\prime} 00^{\prime \prime}$ & $88^{\circ} 11^{\prime} 00^{\prime \prime}$ & Sumter & Tombigbee & 03160201 & 90.9 \\
\hline 174 & 02469500 & $\begin{array}{l}\text { Tuckabum Creek near } \\
\text { Butler, AL }\end{array}$ & $32^{\circ} 11^{\prime} 00^{\prime \prime}$ & $88^{\circ} 10^{\prime} 12^{\prime \prime}$ & Choctaw & Tombigbee & 03160201 & 115 \\
\hline 175 & 02469550 & $\begin{array}{l}\text { Horse Creek near } \\
\quad \text { Sweetwater, AL }\end{array}$ & $32^{\circ} 02^{\prime} 57^{\prime \prime}$ & $87^{\circ} 52^{\prime} 12^{\prime \prime}$ & Marengo & Tombigbee & 03160201 & 60.4 \\
\hline 176 & 02469700 & $\begin{array}{c}\text { Okatuppa Creek at } \\
\text { Gilbertown, AL }\end{array}$ & $31^{\circ} 53^{\prime} 27^{\prime \prime}$ & $88^{\circ} 18^{\prime} 48^{\prime \prime}$ & Choctaw & Tombigbee & 03160201 & 148 \\
\hline 180 & 02470100 & $\begin{array}{l}\text { Bassett Creek at Walker } \\
\text { Springs, AL }\end{array}$ & $31^{\circ} 32^{\prime} 15^{\prime \prime}$ & $87^{\circ} 47^{\prime} 24^{\prime \prime}$ & Clarke & Tombigbee & 03160203 & 195 \\
\hline 181 & 02471001 & $\begin{array}{l}\text { Chickasaw Creek near } \\
\text { Kushla, AL }\end{array}$ & $30^{\circ} 48^{\prime} 10^{\prime \prime}$ & $88^{\circ} 08^{\prime} 36^{\prime \prime}$ & Mobile & Mobile & 03160204 & 125 \\
\hline 182 & 02471065 & $\begin{array}{l}\text { Montlimar Creek at } \\
\text { U.S. Highway } 90 \text { at } \\
\text { Mobile, AL }\end{array}$ & $30^{\circ} 38^{\prime} 59^{\prime \prime}$ & $88^{\circ} 07^{\prime} 34^{\prime \prime}$ & Mobile & Mobile & 03160205 & 7.28 \\
\hline 183 & 02471078 & $\begin{array}{l}\text { Fowl River at Half-Mile } \\
\quad \text { Road near Laurendine, AL }\end{array}$ & $30^{\circ} 30^{\prime} 02^{\prime \prime}$ & $88^{\circ} 10^{\prime} 53^{\prime \prime}$ & Mobile & Mobile & 03160205 & 16.5 \\
\hline 184 & 02479431 & $\begin{array}{l}\text { Pond Creek near Deer } \\
\text { Park, AL }\end{array}$ & $31^{\circ} 09^{\prime} 39^{\prime \prime}$ & $88^{\circ} 21^{\prime} 43^{\prime \prime}$ & Washington & Escatwapa & 03170008 & 20.4 \\
\hline
\end{tabular}


Table 1. U.S. Geological Survey continuous-record streamgaging stations in Alabama and surrounding States included in this investigation.-Continued

[USGS, U.S. Geological Survey; ', degrees; ', minutes; ”, seconds; ft³/s, cubic foot per second; DA, drainage area; NWISWeb, National Water Information System database; $\mathrm{mi}^{2}$, square mile; QAQC, quality assurance, quality control]

\begin{tabular}{|c|c|c|c|c|c|}
\hline $\begin{array}{l}\text { Site } \\
\text { index } \\
\text { number } \\
\text { (fig. 1) }\end{array}$ & Period of record available & Period of record analyzed & $\begin{array}{l}\text { Number of } \\
\text { climate years } \\
\text { used in } \\
\text { analysis }\end{array}$ & $\begin{array}{l}\text { Flow } \\
\text { condition } \\
\text { for period } \\
\text { analyzed }\end{array}$ & Remarks \\
\hline 167 & October 1976 to March 2014 & April 1977 to March 2014 & 36 & Regulated & \\
\hline 168 & $\begin{array}{l}\text { February } 1940 \text { to September } 1952, \\
\quad \text { October } 1990 \text { to September } 1991\end{array}$ & April 1940 to March 1952 & 12 & Unregulated & \\
\hline 169 & August 1928 to March 2014 & April 1985 to March 2014 & 29 & Regulated & $\begin{array}{l}\text { Reflects diversions and regulation } \\
\text { structures since } 1985 .\end{array}$ \\
\hline 170 & October 1938 to March 2014 & April 1939 to March 2014 & 75 & Unregulated & \\
\hline 171 & August 1954 to September 1967 & April 1955 to March 1967 & 12 & Unregulated & \\
\hline 172 & $\begin{array}{l}\text { January } 1944 \text { to September } 1946, \\
\text { October } 1965 \text { to September } 1988\end{array}$ & $\begin{array}{l}\text { April } 1944 \text { to March 1946, } \\
\text { April } 1966 \text { to March } 1988\end{array}$ & 24 & Unregulated & \\
\hline 173 & August 1954 to September 1967 & April 1955 to March 1967 & 12 & Unregulated & \\
\hline 174 & October 1954 to September 1970 & April 1955 to March 1970 & 15 & Unregulated & \\
\hline 175 & October 1959 to September 1970 & April 1960 to March 1970 & 10 & Unregulated & \\
\hline 176 & October 1956 to September 1969 & April 1957 to March 1969 & 12 & Unregulated & \\
\hline 177 & October 1960 to March 2014 & April 1985 to March 2014 & 28 & Regulated & \\
\hline 178 & $\begin{array}{l}\text { October } 1956 \text { to September } 1970 \\
\text { October } 1974 \text { to September } 2014\end{array}$ & $\begin{array}{l}\text { April } 1957 \text { to March 1970, } \\
\text { April } 1975 \text { to March } 2014\end{array}$ & 52 & Unregulated & \\
\hline 179 & October 1995 to September 2014 & April 1996 to March 2014 & 18 & Unregulated & \\
\hline 180 & October 1956 to September 1970 & April 1957 to March 1970 & 13 & Unregulated & \\
\hline 181 & October 1951 to March 2014 & April 1952 to March 2014 & 62 & Unregulated & $\begin{array}{l}\text { Data from October } 1951 \text { to } \\
\text { September } 1968 \text { are from } \\
\text { 02471000, Chickasaw Creek } \\
\text { near Whistler, AL. }\end{array}$ \\
\hline 182 & $\begin{array}{l}\text { June } 1962 \text { to September } 1967 \text {, } \\
\text { October } 1974 \text { to September } 1983\end{array}$ & $\begin{array}{l}\text { April } 1963 \text { to March 1967, } \\
\text { April } 1975 \text { to March } 1983\end{array}$ & 12 & Unregulated & \\
\hline 183 & March 1995 to March 2014 & April 1995 to March 2014 & 19 & Unregulated & \\
\hline 184 & October 1976 to September 1999 & April 1977 to March 1999 & 22 & Unregulated & \\
\hline
\end{tabular}


Table 1. U.S. Geological Survey continuous-record streamgaging stations in Alabama and surrounding States included in this investigation.-Continued

[USGS, U.S. Geological Survey; ', degrees; ', minutes; ”, seconds; fťs, cubic foot per second; DA, drainage area; NWISWeb, National Water Information System database; $\mathrm{mi}^{2}$, square mile; QAQC, quality assurance, quality control]

\begin{tabular}{|c|c|c|c|c|c|c|c|c|}
\hline $\begin{array}{c}\text { Site } \\
\text { index } \\
\text { number } \\
\text { (fig. 1) }\end{array}$ & $\begin{array}{l}\text { USGS } \\
\text { station } \\
\text { number }\end{array}$ & Station name & Latitude & Longitude & County & Basin & $\begin{array}{l}\text { Hydrologic } \\
\text { Unit Code }\end{array}$ & $\begin{array}{l}\text { Drainage } \\
\text { area }\left(\mathrm{mi}^{2}\right)\end{array}$ \\
\hline 185 & 02479500 & $\begin{array}{l}\text { Escatawapa River near } \\
\text { Wilmer, AL }\end{array}$ & $30^{\circ} 51^{\prime} 44^{\prime \prime}$ & $88^{\circ} 25^{\prime} 04^{\prime \prime}$ & Mobile & Escatwapa & 03170008 & 511 \\
\hline 186 & 02479560 & $\begin{array}{l}\text { Escatawapa River near } \\
\text { Agricola, } \mathrm{AL}\end{array}$ & $30^{\circ} 48^{\prime} 42^{\prime \prime}$ & $88^{\circ} 27^{\prime} 31^{\prime \prime}$ & George, MS & Escatwapa & 03170008 & 562 \\
\hline 187 & 02479945 & $\begin{array}{l}\text { Big Creek at County Road } \\
63 \text { near Wilmer, AL }\end{array}$ & $30^{\circ} 51^{\prime} 21^{\prime \prime}$ & $88^{\circ} 20^{\prime} 02^{\prime \prime}$ & Mobile & Escatwapa & 03170008 & 31.48 \\
\hline 188 & 02479980 & $\begin{array}{l}\text { Crooked Creek near } \\
\text { Fairview, AL }\end{array}$ & $30^{\circ} 46^{\prime} 48^{\prime \prime}$ & $88^{\circ} 19^{\prime} 08^{\prime \prime}$ & Mobile & Escatwapa & 03170008 & 8.08 \\
\hline 189 & 02480002 & $\begin{array}{l}\text { Hamilton Creek at Snow } \\
\text { Road near Semmes, AL }\end{array}$ & $30^{\circ} 43^{\prime} 33^{\prime \prime}$ & $88^{\circ} 16^{\prime} 35^{\prime \prime}$ & Mobile & Escatwapa & 03170008 & 8.22 \\
\hline 192 & 03574500 & $\begin{array}{l}\text { Paint Rock River near } \\
\text { Woodville, AL }\end{array}$ & $34^{\circ} 37^{\prime} 27^{\prime \prime}$ & $86^{\circ} 18^{\prime} 23^{\prime \prime}$ & Jackson & Tennessee & 06030002 & 320 \\
\hline 193 & 0357479650 & $\begin{array}{l}\text { Hester Creek at Buddy } \\
\text { Williamson Road near } \\
\text { Plevna, AL }\end{array}$ & $34^{\circ} 57^{\prime} 39^{\prime \prime}$ & $86^{\circ} 27^{\prime} 49^{\prime \prime}$ & Madison & Tennessee & 06030002 & 33 \\
\hline 194 & 03575000 & Flint River near Chase, $\mathrm{AL}$ & $34^{\circ} 49^{\prime} 22^{\prime \prime}$ & $86^{\circ} 28^{\prime} 59^{\prime \prime}$ & Madison & Tennessee & 06030002 & 342 \\
\hline 195 & 03575100 & $\begin{array}{l}\text { Flint River at } \\
\text { Brownsboro, } \mathrm{AL}\end{array}$ & $34^{\circ} 44^{\prime} 57^{\prime \prime}$ & $86^{\circ} 26^{\prime} 48^{\prime \prime}$ & Madison & Tennessee & 06030002 & 375 \\
\hline 200 & 03576500 & $\begin{array}{c}\text { Flint Creek near } \\
\text { Falkville, } \mathrm{AL}\end{array}$ & $34^{\circ} 22^{\prime} 23^{\prime \prime}$ & $86^{\circ} 56^{\prime} 01^{\prime \prime}$ & Morgan & Tennessee & 06030002 & 86.3 \\
\hline 201 & 03585300 & $\begin{array}{l}\text { Sugar Creek near Good } \\
\text { Springs, AL }\end{array}$ & $34^{\circ} 56^{\prime} 40^{\prime \prime}$ & $87^{\circ} 09^{\prime} 20^{\prime \prime}$ & Limestone & Tennessee & 06030004 & 152 \\
\hline 202 & 03586500 & $\begin{array}{l}\text { Big Nance Creek at } \\
\text { Coutland, AL }\end{array}$ & $34^{\circ} 40^{\prime} 12^{\prime \prime}$ & $87^{\circ} 19^{\prime} 02 ”$ & Lawrence & Tennessee & 06030005 & 166 \\
\hline
\end{tabular}


Table 1. U.S. Geological Survey continuous-record streamgaging stations in Alabama and surrounding States included in this investigation.-Continued

[USGS, U.S. Geological Survey; ', degrees; ', minutes; ”, seconds; ft³/s, cubic foot per second; DA, drainage area; NWISWeb, National Water Information System database; $\mathrm{mi}^{2}$, square mile; QAQC, quality assurance, quality control]

\begin{tabular}{|c|c|c|c|c|c|}
\hline $\begin{array}{c}\text { Site } \\
\text { index } \\
\text { number } \\
\text { (fig. 1) }\end{array}$ & Period of record available & Period of record analyzed & $\begin{array}{c}\text { Number of } \\
\text { climate years } \\
\text { used in } \\
\text { analysis }\end{array}$ & $\begin{array}{l}\text { Flow } \\
\text { condition } \\
\text { for period } \\
\text { analyzed }\end{array}$ & Remarks \\
\hline 185 & October 1945 to September 1973 & April 1946 to March 1973 & 27 & Unregulated & \\
\hline 186 & October 1973 to March 2014 & April 1974 to March 2014 & 40 & Unregulated & \\
\hline 187 & June 1990 to March 2014 & April 1991 to March 2014 & 23 & Unregulated & \\
\hline 188 & June 1990 to March 2014 & April 1991 to March 2014 & 23 & Unregulated & \\
\hline 189 & June 1990 to March 2014 & April 1991 to March 2014 & 23 & Unregulated & \\
\hline 190 & May 1975 to October 1996 & April 1976 to March 1996 & 20 & Unregulated & \\
\hline 191 & October 1957 to September 1980 & April 1958 to March 1980 & 22 & Unregulated & \\
\hline 192 & January 1936 to September 2014 & April 1936 to March 2014 & 78 & Unregulated & \\
\hline 193 & October 1998 to September 2013 & April 1999 to March 2013 & 14 & Unregulated & \\
\hline 194 & $\begin{array}{l}\text { May } 1930 \text { to September } 1981 \text {, } \\
\text { October } 1982 \text { to December } 1994\end{array}$ & $\begin{array}{l}\text { April } 1931 \text { to March 1981, } \\
\text { April } 1983 \text { to March } 1994\end{array}$ & 61 & Unregulated & \\
\hline 195 & October 1998 to September 2014 & April 1999 to March 2014 & 15 & Unregulated & \\
\hline 196 & October 1924 to September 2005 & April 1925 to March 1936 & 11 & ${ }^{1}$ Unregulated & \\
\hline 197 & $\begin{array}{l}\text { October } 1959 \text { to September } 1966 \text {, } \\
\text { October } 1975 \text { to June } 2002\end{array}$ & $\begin{array}{l}\text { April } 1960 \text { to March 1966, } \\
\text { April } 1976 \text { to March } 2002\end{array}$ & 32 & Unregulated & \\
\hline 198 & October 1965 to September 1980 & April 1966 to March 1980 & 14 & Unregulated & \\
\hline 199 & $\begin{array}{l}\text { October } 1939 \text { to September } 1970 \\
\text { October } 1994 \text { to September } 2014\end{array}$ & $\begin{array}{l}\text { April } 1940 \text { to March 1970, } \\
\text { April } 1995 \text { to March } 2014\end{array}$ & 49 & Unregulated & \\
\hline 200 & $\begin{array}{l}\text { August } 1952 \text { to September } 1970, \\
\text { October } 1992 \text { to September 1999, } \\
\text { October } 2011 \text { to September } 2014\end{array}$ & $\begin{array}{l}\text { April } 1953 \text { to March 1970, } \\
\text { April } 1993 \text { to March 1999, } \\
\text { April } 2012 \text { to March } 2014\end{array}$ & 25 & Unregulated & \\
\hline 201 & October 1957 to September 1969 & April 1958 to March 1969 & 11 & Unregulated & \\
\hline 202 & $\begin{array}{l}\text { September } 1935 \text { tp September } 1940 \text {, } \\
\text { April } 1945 \text { to September } 1981 \text {, } \\
\text { March } 1988 \text { to September } 2014\end{array}$ & $\begin{array}{l}\text { April } 1936 \text { to March } 1940, \\
\text { April } 1945 \text { to March } 1981\end{array}$ & 40 & Unregulated & $\begin{array}{l}\text { Based on QAQC reviews, it appears } \\
\text { that low-flow patterns after } 1981 \\
\text { are different than the previous } \\
\text { recocrd, which is likely due to } \\
\text { anthropogenic influences in the } \\
\text { basin. Thus, the period being ana- } \\
\text { lyzed here likely represents more } \\
\text { natural conditions. A separate } \\
\text { analysis was done for the period } \\
\text { after } 1981 .\end{array}$ \\
\hline
\end{tabular}


Table 1. U.S. Geological Survey continuous-record streamgaging stations in Alabama and surrounding States included in this investigation.-Continued

[USGS, U.S. Geological Survey; ', degrees; ', minutes; ”, seconds; fťs, cubic foot per second; DA, drainage area; NWISWeb, National Water Information System database; $\mathrm{mi}^{2}$, square mile; QAQC, quality assurance, quality control]

\begin{tabular}{|c|c|c|c|c|c|c|c|c|}
\hline $\begin{array}{c}\text { Site } \\
\text { index } \\
\text { number } \\
\text { (fig. 1) }\end{array}$ & $\begin{array}{l}\text { USGS } \\
\text { station } \\
\text { number }\end{array}$ & Station name & Latitude & Longitude & County & Basin & $\begin{array}{l}\text { Hydrologic } \\
\text { Unit Code }\end{array}$ & $\begin{array}{l}\text { Drainage } \\
\text { area }\left(\mathrm{mi}^{2}\right)\end{array}$ \\
\hline 202 & 03586500 & $\begin{array}{c}\text { Big Nance Creek at } \\
\text { Coutland, AL }\end{array}$ & $34^{\circ} 40^{\prime} 12^{\prime \prime}$ & $87^{\circ} 19^{\prime} 02^{\prime \prime}$ & Lawrence & Tennessee & 06030005 & 166 \\
\hline
\end{tabular}

\begin{tabular}{|c|c|c|c|c|c|c|c|c|}
\hline 203 & 03589500 & $\begin{array}{c}\text { Tennessee River at } \\
\text { Florence, AL }\end{array}$ & $34^{\circ} 47^{\prime} 13^{\prime \prime}$ & $87^{\circ} 40^{\prime} 12^{\prime \prime}$ & Lauderdale & Tennessee & 06030005 & 30,810 \\
\hline 204 & 03590000 & $\begin{array}{l}\text { Cypress Creek near } \\
\text { Florence, AL }\end{array}$ & $34^{\circ} 48^{\prime} 28^{\prime \prime}$ & $87^{\circ} 42^{\prime} 02^{\prime \prime}$ & Lauderdale & Tennessee & 06030005 & 209 \\
\hline 205 & 03590500 & $\begin{array}{l}\text { Tuscumbia Spring at } \\
\text { Tuscumbia, AL }\end{array}$ & $34^{\circ} 43^{\prime} 45^{\prime \prime}$ & $87^{\circ} 42^{\prime} 15^{\prime \prime}$ & Colbert & Tennessee & 06030005 & Indeterminate \\
\hline 206 & 03591800 & $\begin{array}{l}\text { Bear Creek near } \\
\text { Hackeburg, AL }\end{array}$ & $34^{\circ} 17^{\prime} 01^{\prime \prime}$ & $87^{\circ} 46^{\prime} 26^{\prime \prime}$ & Marion & Tennessee & 06030006 & 143 \\
\hline 207 & 03592000 & $\begin{array}{c}\text { Bear Creek near } \\
\text { Red Bay, AL }\end{array}$ & $34^{\circ} 26^{\prime} 38^{\prime \prime}$ & $88^{\circ} 06^{\prime} 56^{\prime \prime}$ & Franklin & Tennessee & 06030006 & 263 \\
\hline 208 & 03592200 & $\begin{array}{l}\text { Cedar Creek near Pleasant } \\
\text { Site, AL }\end{array}$ & $34^{\circ} 32^{\prime} 56^{\prime \prime}$ & $88^{\circ} 01^{\prime} 09^{\prime \prime}$ & Franklin & Tennessee & 06030006 & 189 \\
\hline 209 & 03592300 & $\begin{array}{l}\text { Little Bear Creek near } \\
\text { Halltown, AL }\end{array}$ & $34^{\circ} 29^{\prime} 19^{\prime \prime}$ & $88^{\circ} 02^{\prime} 07^{\prime \prime}$ & Franklin & Tennessee & 06030006 & 78.2 \\
\hline 210 & 03592500 & Bear Creek at Bishop, AL & $34^{\circ} 39^{\prime} 21^{\prime \prime}$ & $88^{\circ} 07^{\prime} 21^{\prime \prime}$ & Colbert & Tennessee & 06030006 & 667 \\
\hline
\end{tabular}

\begin{tabular}{|c|c|c|c|c|c|c|c|c|}
\hline \multicolumn{9}{|c|}{ Stations from surrounding States } \\
\hline 211 & 02330450 & $\begin{array}{l}\text { Chattahoochee River at } \\
\text { Helen, GA }\end{array}$ & $34^{\circ} 42^{\prime} 03^{\prime \prime}$ & $83^{\circ} 43^{\prime} 44^{\prime \prime}$ & White & Apalachicola & 03130001 & 44.7 \\
\hline 212 & 02331000 & $\begin{array}{l}\text { Chattahoochee River near } \\
\text { Leaf, GA }\end{array}$ & $34^{\circ} 34^{\prime} 37^{\prime \prime}$ & $83^{\circ} 38^{\prime} 09^{\prime \prime}$ & Habersham & Apalachicola & 03130001 & 150 \\
\hline 213 & 02333500 & $\begin{array}{c}\text { Chestatee River near } \\
\text { Dahlonega, GA }\end{array}$ & $34^{\circ} 31^{\prime} 41^{\prime \prime}$ & $83^{\circ} 56^{\prime} 23^{\prime \prime}$ & Lumpkin & Apalachicola & 03130001 & 153 \\
\hline 214 & 02335000 & $\begin{array}{l}\text { Chattahoochee River near } \\
\text { Norcross, GA }\end{array}$ & $33^{\circ} 59^{\prime} 50^{\prime \prime}$ & $84^{\circ} 12^{\prime} 07^{\prime \prime}$ & Gwinnett & Apalachicola & 03130001 & 1,170 \\
\hline 215 & 02337000 & $\begin{array}{c}\text { Sweetwater Creek near } \\
\text { Austell, GA }\end{array}$ & $33^{\circ} 46^{\prime} 35^{\prime \prime}$ & $84^{\circ} 36^{\prime} 56^{\prime \prime}$ & Douglas & Apalachicola & 03130002 & 238 \\
\hline 216 & 02337500 & $\begin{array}{l}\text { Snake Creek near } \\
\text { Whitesburg, GA }\end{array}$ & $33^{\circ} 31^{\prime} 46^{\prime \prime}$ & $84^{\circ} 55^{\prime} 42^{\prime \prime}$ & Carroll & Apalachicola & 03130002 & 35.5 \\
\hline
\end{tabular}


Table 1. U.S. Geological Survey continuous-record streamgaging stations in Alabama and surrounding States included in this investigation.-Continued

[USGS, U.S. Geological Survey; ', degrees; ', minutes; ”, seconds; ft³/s, cubic foot per second; DA, drainage area; NWISWeb, National Water Information System database; $\mathrm{mi}^{2}$, square mile; QAQC, quality assurance, quality control]

\begin{tabular}{|c|c|c|c|c|c|}
\hline $\begin{array}{c}\text { Site } \\
\text { index } \\
\text { number } \\
\text { (fig. 1) }\end{array}$ & Period of record available & Period of record analyzed & $\begin{array}{l}\text { Number of } \\
\text { climate years } \\
\text { used in } \\
\text { analysis }\end{array}$ & $\begin{array}{l}\text { Flow } \\
\text { condition } \\
\text { for period } \\
\text { analyzed }\end{array}$ & Remarks \\
\hline 202 & $\begin{array}{l}\text { September } 1935 \text { tp September } 1940, \\
\text { April } 1945 \text { to September } 1981 \text {, } \\
\text { March } 1988 \text { to September } 2014\end{array}$ & April 1988 to March 2014 & 26 & Unregulated & $\begin{array}{l}\text { Based on QAQC reviews, it appears } \\
\text { that low-flow patterns after } 1981 \\
\text { are different than the previous } \\
\text { record, which is likely due to } \\
\text { anthropogenic influences in the } \\
\text { basin. The period of record used } \\
\text { in this analysis represents current } \\
\text { conditions in the basin reflecting } \\
\text { anthropogenic influences. }\end{array}$ \\
\hline 203 & October 1894 to November 2005 & April 1895 to March 1924 & 29 & ${ }^{1}$ Unregulated & \\
\hline 204 & $\begin{array}{l}\text { June } 1934 \text { to September } 1953 . \\
\text { Monthly discharges for } \\
\text { October } 1933 \text { to May } 1934\end{array}$ & April 1935 to March 1953 & 18 & Unregulated & \\
\hline 205 & $\begin{array}{l}\text { December } 1928 \text { to March 1930, } \\
\quad \text { January } 1956 \text { to September } 1965\end{array}$ & $\begin{array}{l}\text { April } 1929 \text { to March 1930, } \\
\text { April } 1956 \text { to March } 1965\end{array}$ & 11 & Unregulated & $\begin{array}{l}\text { No drainage area in NWISWeb } \\
\text { because the site is a spring. }\end{array}$ \\
\hline 206 & $\begin{array}{l}\text { October } 1956 \text { to September } 1979 \\
\text { October } 1980 \text { to September } 1981\end{array}$ & April 1957 to March 1981 & 22 & Unregulated & \\
\hline 207 & $\begin{array}{l}\text { October } 1913 \text { to May } 1920 \text {, } \\
\text { October } 1958 \text { to September } 1967 \text {, } \\
\text { March } 1969 \text { to September } 1981\end{array}$ & $\begin{array}{l}\text { April } 1914 \text { to March } 1920, \\
\text { April } 1959 \text { to March } 1967\end{array}$ & 14 & ${ }^{1}$ Unregulated & \\
\hline 208 & October 1957 to September 1977 & April 1958 to March 1977 & 19 & ${ }^{1}$ Unregulated & \\
\hline 209 & October 1957 to September 1977 & April 1958 to March 1975 & 17 & ${ }^{1}$ Unregulated & \\
\hline 210 & $\begin{array}{l}\text { October } 1926 \text { to May } 1928, \\
\text { March } 1929 \text { to March } 1932, \\
\text { October } 1933 \text { to September } 1979\end{array}$ & $\begin{array}{l}\text { April } 1927 \text { to March } 1928, \\
\text { April } 1929 \text { to March 1932, } \\
\text { April } 1934 \text { to March } 1979\end{array}$ & 49 & ${ }^{1}$ Unregulated & \\
\hline
\end{tabular}

\begin{tabular}{|c|c|c|c|c|c|}
\hline \multirow[b]{2}{*}{211} & \multicolumn{5}{|c|}{ Stations from surrounding States-Continued } \\
\hline & May 1981 to March 2014 & April 1982 to March 2014 & 32 & Unregulated & \\
\hline 212 & $\begin{array}{c}\text { February } 1940 \text { to September } 1971 \text {, } \\
\text { October } 2008 \text { to March } 2014\end{array}$ & $\begin{array}{l}\text { April } 1940 \text { to March 1971, } \\
\text { April } 2009 \text { to March } 2014\end{array}$ & 36 & Unregulated & $\begin{array}{l}\text { Noted in Gotvald (2016) as poten- } \\
\text { tially being influenced by upstream } \\
\text { diversions. }\end{array}$ \\
\hline 213 & $\begin{array}{l}\text { July } 1929 \text { to January } 1932 \text {, } \\
\text { April } 1940 \text { to March } 2014\end{array}$ & $\begin{array}{l}\text { April } 1930 \text { to March 1931, } \\
\text { April } 1940 \text { to March 2014 }\end{array}$ & 75 & Unregulated & \\
\hline 214 & $\begin{array}{c}\text { January } 1903 \text { to September } 1946 \text {, } \\
\text { October } 1956 \text { to March } 2014\end{array}$ & April 1903 to March 1946 & 43 & ${ }^{1}$ Unregulated & \\
\hline 215 & $\begin{array}{r}\text { May } 1904 \text { to December } 1905 \text {, } \\
\text { March } 1937 \text { to March } 2014\end{array}$ & April 1937 to March 2014 & 77 & Unregulated & $\begin{array}{l}\text { Noted in Gotvald (2016) as poten- } \\
\text { tially being influenced by upstream } \\
\text { diversions. }\end{array}$ \\
\hline 216 & September 1954 to March 2014 & April 1955 to March 2000 & 45 & ${ }^{1}$ Unregulated & $\begin{array}{l}\text { Flow regulated by earthen dam } \\
\text { (Snake Creek Reservoir) upstream } \\
\text { from gage since 2001. Frequency } \\
\text { and duration statistics represent } \\
\text { pre-regulation period. }\end{array}$ \\
\hline
\end{tabular}


Table 1. U.S. Geological Survey continuous-record streamgaging stations in Alabama and surrounding States included in this investigation.-Continued

[USGS, U.S. Geological Survey; ', degrees; ', minutes; ”, seconds; fťs, cubic foot per second; DA, drainage area; NWISWeb, National Water Information System database; $\mathrm{mi}^{2}$, square mile; QAQC, quality assurance, quality control]

\begin{tabular}{|c|c|c|c|c|c|c|c|c|}
\hline $\begin{array}{c}\text { Site } \\
\text { index } \\
\text { number } \\
\text { (fig. 1) }\end{array}$ & $\begin{array}{l}\text { USGS } \\
\text { station } \\
\text { number }\end{array}$ & Station name & Latitude & Longitude & County & Basin & $\begin{array}{l}\text { Hydrologic } \\
\text { Unit Code }\end{array}$ & $\begin{array}{l}\text { Drainage } \\
\text { area }\left(\mathrm{mi}^{2}\right)\end{array}$ \\
\hline 217 & 02338660 & $\begin{array}{l}\text { New River at GA 100, near } \\
\text { Corinth, GA }\end{array}$ & $33^{\circ} 14^{\prime} 07^{\prime \prime}$ & $84^{\circ} 59^{\prime} 16^{\prime \prime}$ & Heard & Apalachicola & 03130002 & 127 \\
\hline 219 & 02340500 & $\begin{array}{l}\text { Mountain Oak Creek near } \\
\text { Hamilton, GA }\end{array}$ & $32^{\circ} 44^{\prime} 28^{\prime \prime}$ & $85^{\circ} 04^{\prime} 08^{\prime \prime}$ & Harris & Apalachicola & 03130002 & 61.7 \\
\hline 220 & 02341800 & $\begin{array}{c}\text { Upatoi Creek near } \\
\text { Columbus, GA }\end{array}$ & $32^{\circ} 24^{\prime} 48^{\prime \prime}$ & $84^{\circ} 49^{\prime} 12^{\prime \prime}$ & Chattahoochee & Apalachicola & 03130003 & 342 \\
\hline
\end{tabular}

\begin{tabular}{|c|c|c|c|c|c|c|c|c|}
\hline 222 & 02365500 & $\begin{array}{l}\text { Choctawhatchee River at } \\
\text { Caryville, FL }\end{array}$ & $30^{\circ} 46^{\prime} 32^{\prime \prime}$ & $85^{\circ} 49^{\prime} 40^{\prime \prime}$ & Holmes & Choctawhatchee & 03140203 & 3,499 \\
\hline 223 & 02366000 & Holmes Creek at Vernon, FL & $30^{\circ} 37^{\prime} 36^{\prime \prime}$ & $85^{\circ} 42^{\prime} 44^{\prime \prime}$ & Washington & Choctawhatchee & 03140203 & 386 \\
\hline 224 & 02368000 & Yellow River at Milligan, FL & $30^{\circ} 45^{\prime} 10^{\prime \prime}$ & $86^{\circ} 37^{\prime} 45^{\prime \prime}$ & Okaloosa & Blackwater & 03140103 & 624 \\
\hline 225 & 02368500 & $\begin{array}{l}\text { Shoal River near Mossy } \\
\text { Head, FL }\end{array}$ & $30^{\circ} 47^{\prime} 45^{\prime \prime}$ & $86^{\circ} 18^{\prime} 25^{\prime \prime}$ & Walton & Blackwater & 03140103 & 123 \\
\hline 226 & 02369000 & $\begin{array}{l}02369000 \text { Shoal River near } \\
\text { Crestview, FL }\end{array}$ & $30^{\circ} 41^{\prime} 50^{\prime \prime}$ & $86^{\circ} 34^{\prime} 15^{\prime \prime}$ & Okaloosa & Blackwater & 03140103 & 474 \\
\hline 227 & 02370000 & $\begin{array}{l}\text { Blackwater River near } \\
\text { Baker, FL }\end{array}$ & $30^{\circ} 50^{\prime} 00^{\prime \prime}$ & $86^{\circ} 44^{\prime} 05^{\prime \prime}$ & Okaloosa & Blackwater & 03140104 & 205 \\
\hline 229 & 02370700 & Pond Creek near Milton, FL & $30^{\circ} 40^{\prime} 50^{\prime \prime}$ & $87^{\circ} 07^{\prime} 55^{\prime \prime}$ & Santa Rosa & Blackwater & 03140104 & 58.7 \\
\hline
\end{tabular}

\begin{tabular}{|c|c|c|c|c|c|c|c|c|}
\hline 230 & 02376000 & $\begin{array}{l}\text { Pine Barren Creek near } \\
\text { Barth, FL }\end{array}$ & $30^{\circ} 47^{\prime} 55^{\prime \prime}$ & $87^{\circ} 22^{\prime} 05^{\prime \prime}$ & Escambia & Conecuh & 03140305 & 75.3 \\
\hline 231 & 02376300 & $\begin{array}{l}\text { Brushy Creek near Walnut } \\
\text { Hill, FL }\end{array}$ & $30^{\circ} 53^{\prime} 21^{\prime \prime}$ & $87^{\circ} 32^{\prime} 24^{\prime \prime}$ & Escambia & Perdido & 03140106 & 49 \\
\hline 232 & 02380500 & $\begin{array}{l}\text { Coosawattee River near } \\
\text { Ellijay, GA }\end{array}$ & $34^{\circ} 40^{\prime} 30^{\prime \prime}$ & $84^{\circ} 30^{\prime} 31^{\prime \prime}$ & Gilmer & Coosa-Tallapoosa & 03150102 & 236 \\
\hline
\end{tabular}


Table 1. U.S. Geological Survey continuous-record streamgaging stations in Alabama and surrounding States included in this investigation.-Continued

[USGS, U.S. Geological Survey; ', degrees; ', minutes; ”, seconds; fts/s, cubic foot per second; DA, drainage area; NWISWeb, National Water Information System database; $\mathrm{mi}^{2}$, square mile; QAQC, quality assurance, quality control]

\begin{tabular}{|c|c|c|c|c|c|}
\hline $\begin{array}{l}\text { Site } \\
\text { index } \\
\text { number } \\
\text { (fig. 1) }\end{array}$ & Period of record available & Period of record analyzed & $\begin{array}{l}\text { Number of } \\
\text { climate years } \\
\text { used in } \\
\text { analysis }\end{array}$ & $\begin{array}{l}\text { Flow } \\
\text { condition } \\
\text { for period } \\
\text { analyzed }\end{array}$ & Remarks \\
\hline 217 & October 1978 to March 2014 & April 1979 to March 2014 & 35 & Unregulated & $\begin{array}{l}\text { Noted in Gotvald (2016) as poten- } \\
\text { tially being influenced by upstream } \\
\text { diversions. }\end{array}$ \\
\hline 218 & January 1951 to March 1971 & April 1951 to March 1971 & 20 & Unregulated & $\begin{array}{l}\text { Inundated by West Point Lake about } \\
1974 .\end{array}$ \\
\hline 219 & December 1943 to September 1971 & $\begin{array}{l}\text { December } 1943 \text { to } \\
\text { September } 1971\end{array}$ & 27 & Unregulated & \\
\hline 220 & April 1968 to March 2014 & April 1968 to March 2014 & 46 & Unregulated & \\
\hline 221 & $\begin{array}{l}\text { December } 1912 \text { to December } 1913 \text {, } \\
\text { October } 1921 \text { to September 1927, } \\
\text { August } 1929 \text { to September } 1931 \text {, } \\
\text { March } 1943 \text { to March } 2014\end{array}$ & $\begin{array}{l}\text { April } 1922 \text { to March 1927, } \\
\text { April } 1930 \text { to March 1931, } \\
\text { April } 1943 \text { to March } 2014\end{array}$ & 77 & Unregulated & \\
\hline 222 & $\begin{array}{l}\text { October } 1929 \text { to March 1995, } \\
\text { October } 1996 \text { to March } 2014\end{array}$ & $\begin{array}{l}\text { April } 1930 \text { to March 1994, } \\
\text { April } 2001 \text { to March } 2014\end{array}$ & 77 & Unregulated & \\
\hline 223 & $\begin{array}{l}\text { April } 1950 \text { to May } 1979 \text {, } \\
\text { October } 2005 \text { to December } 2013\end{array}$ & $\begin{array}{l}\text { April } 1950 \text { to March 1979, } \\
\text { April } 2006 \text { to March } 2013\end{array}$ & 36 & Unregulated & \\
\hline 224 & $\begin{array}{l}\text { August } 1938 \text { to October 1993, } \\
\text { August } 1996 \text { to March } 2014\end{array}$ & $\begin{array}{l}\text { April } 1939 \text { to March 1993, } \\
\text { April } 1997 \text { to March } 2014\end{array}$ & 71 & Unregulated & \\
\hline 225 & $\begin{array}{l}\text { March } 1951 \text { to October } 1978, \\
\text { May } 2000 \text { to September 2002, } \\
\text { October } 2003 \text { to March } 2014\end{array}$ & $\begin{array}{l}\text { April } 1951 \text { to March 1978, } \\
\text { April } 2001 \text { to March 2002, } \\
\text { April } 2004 \text { to March } 2014\end{array}$ & 38 & Unregulated & \\
\hline 226 & August 1938 to March 2014 & April 1939 to March 2014 & 75 & Unregulated & \\
\hline 227 & $\begin{array}{l}\text { April } 1950 \text { to November } 1992, \\
\text { July } 1996 \text { to March } 2014\end{array}$ & $\begin{array}{l}\text { April } 1950 \text { to March 1992, } \\
\text { April } 1997 \text { to March } 2014\end{array}$ & 59 & Unregulated & \\
\hline 228 & $\begin{array}{l}\text { December } 1938 \text { to June } 1979, \\
\text { February } 1980 \text { to April } 1980, \\
\text { July } 1980 \text { to March } 1992, \\
\text { October } 1997 \text { to August } 1999, \\
\text { May } 2000 \text { to February } 2014\end{array}$ & $\begin{array}{l}\text { April } 1939 \text { to March 1979, } \\
\text { April } 1981 \text { to March 1992, } \\
\text { April } 1998 \text { to March 1999, } \\
\text { April } 2001 \text { to March } 2013\end{array}$ & 63 & Unregulated & \\
\hline 229 & $\begin{array}{l}\text { January } 1958 \text { to October } 1978, \\
\text { April } 1979 \text { to July } 1979, \\
\text { November } 1999 \text { to January } 2003 \text {, } \\
\text { March } 2003 \text { to September } 2006\end{array}$ & $\begin{array}{l}\text { April } 1958 \text { to March 1978, } \\
\text { April } 2000 \text { to March 2002, } \\
\text { April } 2003 \text { to March } 2006\end{array}$ & 25 & Unregulated & \\
\hline 230 & October 1952 to September 1994 & April 1953 to March 1994 & 41 & Unregulated & \\
\hline 231 & February 1958 to November 1991 & February 1958 to November 1991 & 33 & Unregulated & \\
\hline 232 & $\begin{array}{l}\text { October } 1938 \text { to December } 1949 \text {, } \\
\text { June } 1963 \text { to March } 2014\end{array}$ & $\begin{array}{l}\text { April } 1939 \text { to March 1949, } \\
\text { April } 1964 \text { to March } 2014\end{array}$ & 60 & Unregulated & $\begin{array}{l}\text { Noted in Gotvald (2016) as poten- } \\
\text { tially being influenced by upstream } \\
\text { diversions. }\end{array}$ \\
\hline 233 & October 1974 to March 2014 & April 1975 to March 2014 & 39 & Unregulated & \\
\hline
\end{tabular}


Table 1. U.S. Geological Survey continuous-record streamgaging stations in Alabama and surrounding States included in this investigation.-Continued

[USGS, U.S. Geological Survey; ', degrees; ', minutes; ”, seconds; fťs, cubic foot per second; DA, drainage area; NWISWeb, National Water Information System database; $\mathrm{mi}^{2}$, square mile; QAQC, quality assurance, quality control]

\begin{tabular}{|c|c|c|c|c|c|c|c|c|}
\hline $\begin{array}{c}\text { Site } \\
\text { index } \\
\text { number } \\
\text { (fig. 1) }\end{array}$ & $\begin{array}{l}\text { USGS } \\
\text { station } \\
\text { number }\end{array}$ & Station name & Latitude & Longitude & County & Basin & $\begin{array}{l}\text { Hydrologic } \\
\text { Unit Code }\end{array}$ & $\begin{array}{l}\text { Drainage } \\
\text { area }\left(\mathrm{mi}^{2}\right)\end{array}$ \\
\hline 234 & 02382000 & $\begin{array}{l}\text { Scarecorn Creek at } \\
\text { Hinton, GA }\end{array}$ & $34^{\circ} 28^{\prime} 04^{\prime \prime}$ & $84^{\circ} 35^{\prime} 30^{\prime \prime}$ & Pickens & Coosa-Tallapoosa & 03150102 & 21.3 \\
\hline 236 & 02383000 & $\begin{array}{r}\text { Rock Creek near } \\
\text { Fairmont, GA }\end{array}$ & $34^{\circ} 21^{\prime} 32^{\prime \prime}$ & $84^{\circ} 46^{\prime} 46^{\prime \prime}$ & Bartow & Coosa-Tallapoosa & 03150102 & 6.17 \\
\hline 237 & 02383500 & $\begin{array}{l}\text { Coosawattee River near Pine } \\
\text { Chapel, GA }\end{array}$ & $34^{\circ} 33^{\prime} 51^{\prime \prime}$ & $84^{\circ} 49^{\prime} 59^{\prime \prime}$ & Gordon & Coosa-Tallapoosa & 03150102 & 831 \\
\hline 239 & 02388000 & $\begin{array}{l}\text { West Armuchee Creek near } \\
\text { Subligna, GA }\end{array}$ & $34^{\circ} 34^{\prime} 04^{\prime \prime}$ & $85^{\circ} 09^{\prime} 16^{\prime \prime}$ & Chattooga & Coosa-Tallapoosa & 03150103 & 36.4 \\
\hline 240 & 02388300 & Heath Creek near Rome, GA & $34^{\circ} 21^{\prime} 57^{\prime \prime}$ & $85^{\circ} 16^{\prime} 17^{\prime \prime}$ & Floyd & Coosa-Tallapoosa & 03150103 & 14.7 \\
\hline 241 & 02389000 & $\begin{array}{l}\text { Etowah River near } \\
\text { Dawsonville, GA }\end{array}$ & $34^{\circ} 22^{\prime} 57^{\prime \prime}$ & $84^{\circ} 03^{\prime} 21^{\prime \prime}$ & Dawson & Coosa-Tallapoosa & 03150104 & 107 \\
\hline 242 & 02390000 & $\begin{array}{c}\text { Amicalola Creek near } \\
\text { Dawsonville, GA }\end{array}$ & $34^{\circ} 25^{\prime} 32^{\prime \prime}$ & $84^{\circ} 12^{\prime} 43^{\prime \prime}$ & Dawson & Coosa-Tallapoosa & 03150104 & 89 \\
\hline 243 & 02392000 & Etowah River at Canton, GA & $34^{\circ} 14^{\prime} 23^{\prime \prime}$ & $84^{\circ} 29^{\prime} 41^{\prime \prime}$ & Cherokee & Coosa-Tallapoosa & 03150104 & 613 \\
\hline 247 & 02429900 & $\begin{array}{l}\text { Big Brown Creek near } \\
\text { Booneville, MS }\end{array}$ & $34^{\circ} 37^{\prime} 29^{\prime \prime}$ & $88^{\circ} 26^{\prime} 42^{\prime \prime}$ & Prentiss & Tombigbee & 03160101 & 27.1 \\
\hline 248 & 02429980 & $\begin{array}{l}\text { Pollard Mill Branch at } \\
\text { Paden, MS }\end{array}$ & $34^{\circ} 39^{\prime} 14^{\prime \prime}$ & $88^{\circ} 14^{\prime} 56^{\prime \prime}$ & Tishomingo & Tombigbee & 03160101 & 2.01 \\
\hline 249 & 02430000 & $\begin{array}{l}\text { Mackeys Creek near } \\
\text { Dennis, MS }\end{array}$ & $34^{\circ} 31^{\prime} 34^{\prime \prime}$ & $88^{\circ} 19^{\prime} 22^{\prime \prime}$ & Tishomingo & Tombigbee & 03160101 & 66.9 \\
\hline 250 & 02430085 & $\begin{array}{l}\text { Red Bud Creek near Moores } \\
\text { Mill, MS }\end{array}$ & $34^{\circ} 28^{\prime} 00^{\prime \prime}$ & $88^{\circ} 17^{\prime} 01^{\prime \prime}$ & Tishomingo & Tombigbee & 03160101 & 15.7 \\
\hline 251 & 02430615 & $\begin{array}{r}\text { Mud Creek near } \\
\text { Fairview, MS }\end{array}$ & $34^{\circ} 23^{\prime} 33^{\prime \prime}$ & $88^{\circ} 21^{\prime} 18^{\prime \prime}$ & Itawamba & Tombigbee & 03160101 & 11.1 \\
\hline 252 & 02430680 & $\begin{array}{l}\text { Twentymile Creek near } \\
\text { Guntown, MS }\end{array}$ & $34^{\circ} 27^{\prime} 10^{\prime \prime}$ & $88^{\circ} 34^{\prime} 38^{\prime \prime}$ & Lee & Tombigbee & 03160101 & 131 \\
\hline
\end{tabular}


Table 1. U.S. Geological Survey continuous-record streamgaging stations in Alabama and surrounding States included in this investigation.-Continued

[USGS, U.S. Geological Survey; ', degrees; ', minutes; ”, seconds; ft³/s, cubic foot per second; DA, drainage area; NWISWeb, National Water Information System database; $\mathrm{mi}^{2}$, square mile; QAQC, quality assurance, quality control]

\begin{tabular}{|c|c|c|c|c|c|}
\hline $\begin{array}{c}\text { Site } \\
\text { index } \\
\text { number } \\
\text { (fig. 1) }\end{array}$ & Period of record available & Period of record analyzed & $\begin{array}{l}\text { Number of } \\
\text { climate years } \\
\text { used in } \\
\text { analysis }\end{array}$ & $\begin{array}{l}\text { Flow } \\
\text { condition } \\
\text { for period } \\
\text { analyzed }\end{array}$ & Remarks \\
\hline 234 & $\begin{array}{l}\text { April } 1939 \text { to December } 1942, \\
\text { May } 1959 \text { to September } 1974, \\
\text { August } 1986 \text { to April } 1991\end{array}$ & $\begin{array}{l}\text { April } 1939 \text { to March 1942, } \\
\text { April } 1960 \text { to March 1974, } \\
\text { April } 1981 \text { to March 1991 }\end{array}$ & 21 & Unregulated & \\
\hline 235 & November 1973 to March 2014 & April 1974 to March 2014 & 40 & Unregulated & \\
\hline 236 & October 1951 to September 1974 & April 1952 to March 1974 & 22 & Unregulated & \\
\hline 237 & November 1938 to March 2014 & April 1939 to March 1973 & 34 & ${ }^{1}$ Unregulated & $\begin{array}{l}\text { Pre-regulation period (currently } \\
\text { regulated by Carters Lake and re- } \\
\text { regulation dam). }\end{array}$ \\
\hline 238 & November 1892 to March 2014 & April 1893 to March 1973 & 80 & ${ }^{1}$ Unregulated & $\begin{array}{l}\text { Pre-regulation period (currently } \\
\text { regulated by Carters Lake and re- } \\
\text { regulation dam). }\end{array}$ \\
\hline 239 & $\begin{array}{l}\text { April } 1939 \text { to June } 1940 \text {, } \\
\quad \text { May } 1960 \text { to September } 1981\end{array}$ & $\begin{array}{l}\text { April } 1939 \text { to March } 1940, \\
\text { April } 1961 \text { to March } 1981\end{array}$ & 21 & Unregulated & \\
\hline 240 & May 1968 to September 1989 & April 1969 to March 1989 & 20 & Unregulated & \\
\hline 241 & March 1940 to September 1976 & April 1940 to March 1976 & 36 & Unregulated & \\
\hline 242 & $\begin{array}{l}\text { April } 1939 \text { to May 1952, } \\
\text { September } 2005 \text { to March } 2014\end{array}$ & $\begin{array}{l}\text { April } 1939 \text { to March 1952, } \\
\text { April } 2006 \text { to March } 2014\end{array}$ & 21 & Unregulated & \\
\hline 243 & $\begin{array}{l}\text { October } 1896 \text { to September } 1905 \text {, } \\
\text { October } 1936 \text { to March } 2014\end{array}$ & $\begin{array}{l}\text { April } 1897 \text { to March } 1905, \\
\text { April } 1937 \text { to March } 2014\end{array}$ & 85 & Unregulated & $\begin{array}{l}\text { Noted in Gotvard (2016) as poten- } \\
\text { tially being influenced by upstream } \\
\text { diversions. }\end{array}$ \\
\hline 244 & January 1947 to September 1976 & April 1947 to March 1976 & 29 & Unregulated & $\begin{array}{l}\text { Noted in Gotvard (2016) as poten- } \\
\text { tially being influenced by upstream } \\
\text { diversions. }\end{array}$ \\
\hline 245 & May 1980 to March 2014 & April 1981 to March 2014 & 33 & Unregulated & \\
\hline 246 & June 1959 to September 1985 & April 1960 to March 1985 & 25 & Unregulated & \\
\hline 247 & June 1973 to September 2003 & April 1974 to March 2003 & 29 & Unregulated & \\
\hline 248 & October 1972 to May 2004 & April 1973 to March 2004 & 31 & Unregulated & \\
\hline 249 & October 1937 to October 1979 & April 1938 to March 1973 & 35 & ${ }^{1}$ Unregulated & $\begin{array}{l}\text { Site regulated by Tennessee-Tom- } \\
\text { bigbee Waterway since } 1975 \text { but } \\
\text { some documents note construction } \\
\text { began about } 1973 \text {. }\end{array}$ \\
\hline 250 & June 1975 to March 2014 & April 1976 to March 2014 & 38 & Unregulated & \\
\hline 251 & June 1975 to December 2011 & April 1976 to March 2011 & 35 & Unregulated & \\
\hline 252 & October 1982 to March 2014 & April 1983 to March 2014 & 31 & Unregulated & \\
\hline
\end{tabular}


Table 1. U.S. Geological Survey continuous-record streamgaging stations in Alabama and surrounding States included in this investigation.-Continued

[USGS, U.S. Geological Survey; ', degrees; ', minutes; ”, seconds; fťs, cubic foot per second; DA, drainage area; NWISWeb, National Water Information System database; $\mathrm{mi}^{2}$, square mile; QAQC, quality assurance, quality control]

\begin{tabular}{|c|c|c|c|c|c|c|c|c|}
\hline $\begin{array}{c}\text { Site } \\
\text { index } \\
\text { number } \\
\text { (fig. 1) }\end{array}$ & $\begin{array}{c}\text { USGS } \\
\text { station } \\
\text { number }\end{array}$ & Station name & Latitude & Longitude & County & Basin & $\begin{array}{l}\text { Hydrologic } \\
\text { Unit Code }\end{array}$ & $\begin{array}{l}\text { Drainage } \\
\text { area }\left(\mathrm{mi}^{2}\right)\end{array}$ \\
\hline 253 & 02430880 & $\begin{array}{l}\text { Cummings Creek near } \\
\text { Fulton, MS }\end{array}$ & $34^{\circ} 18^{\prime} 16^{\prime \prime}$ & $88^{\circ} 22^{\prime} 16^{\prime \prime}$ & Itawamba & Tombigbee & 03160101 & 19.1 \\
\hline 255 & 02433000 & $\begin{array}{l}\text { Bull Mountain Creek near } \\
\text { Smithville, MS }\end{array}$ & $34^{\circ} 05^{\prime} 18^{\prime \prime}$ & $88^{\circ} 23^{\prime} 26^{\prime \prime}$ & Itawamba & Tombigbee & 03160101 & 336 \\
\hline 256 & 02433500 & $\begin{array}{l}\text { Tombigbee River at } \\
\text { Bigbee, MS }\end{array}$ & $34^{\circ} 00^{\prime} 41^{\prime \prime}$ & $88^{\circ} 30^{\prime} 49^{\prime \prime}$ & Monroe & Tombigbee & 03160101 & 1,226 \\
\hline 258 & 02436500 & $\begin{array}{r}\text { Town Creek near } \\
\text { Nettleton, MS }\end{array}$ & 3403'33”' & $88^{\circ} 37^{\prime} 41^{\prime \prime}$ & Monroe & Tombigbee & 03160102 & 620 \\
\hline 259 & 02439400 & $\begin{array}{l}\text { Buttahatchee River near } \\
\text { Aberdeen, MS }\end{array}$ & $33^{\circ} 47^{\prime} 24^{\prime \prime}$ & $88^{\circ} 18^{\prime} 55^{\prime \prime}$ & Monroe & Tombigbee & 03160103 & 798 \\
\hline 260 & 02440000 & $\begin{array}{l}\text { Chuquatonchee Creek near } \\
\text { Egypt, MS }\end{array}$ & $33^{\circ} 50^{\prime} 24^{\prime \prime}$ & $88^{\circ} 45^{\prime} 43^{\prime \prime}$ & Chickasaw & Tombigbee & 03160104 & 167 \\
\hline 261 & 02440500 & $\begin{array}{l}\text { Chuquatonchee Creek near } \\
\text { West Point, MS }\end{array}$ & $33^{\circ} 36^{\prime} 26^{\prime \prime}$ & $88^{\circ} 42^{\prime} 33^{\prime \prime}$ & Clay & Tombigbee & 03160104 & 505 \\
\hline
\end{tabular}

\begin{tabular}{|c|c|c|c|c|c|c|c|c|}
\hline 264 & 02477000 & $\begin{array}{l}\text { Chickasawhay River at } \\
\text { Enterprise, MS }\end{array}$ & $32^{\circ} 10^{\prime} 33^{\prime \prime}$ & $88^{\circ} 49^{\prime} 11^{\prime \prime}$ & Clarke & Pascagoula & 03170002 & 918 \\
\hline
\end{tabular}


Table 1. U.S. Geological Survey continuous-record streamgaging stations in Alabama and surrounding States included in this investigation.-Continued

[USGS, U.S. Geological Survey; ', degrees; ', minutes; ”, seconds; ft³/s, cubic foot per second; DA, drainage area; NWISWeb, National Water Information System database; $\mathrm{mi}^{2}$, square mile; QAQC, quality assurance, quality control]

\begin{tabular}{|ccccc}
\hline $\begin{array}{c}\text { Site } \\
\text { index } \\
\text { number } \\
\text { (fig. 1) }\end{array}$ & Period of record available & Period of record analyzed & $\begin{array}{c}\text { Number of } \\
\text { climate years } \\
\text { used in } \\
\text { analysis }\end{array}$ & $\begin{array}{c}\text { Flow } \\
\text { condition } \\
\text { for period } \\
\text { analyzed }\end{array}$ \\
\hline 253 & July 1975 to March 2014 & April 1976 to March 2014 & 38 & Unregulated \\
\hline
\end{tabular}

\begin{tabular}{|c|c|c|c|c|c|}
\hline 254 & October 1928 to March 2014 & April 1929 to March 1984 & 55 & ${ }^{1}$ Unregulated & $\begin{array}{l}\text { Since about } 1985 \text {, flows influenced } \\
\text { by upstream diversions and regula- } \\
\text { tion. }\end{array}$ \\
\hline 255 & October 1940 to September 1984 & April 1941 to March 1984 & 43 & Unregulated & \\
\hline 256 & $\begin{array}{l}\text { October } 1944 \text { to September 1946, } \\
\text { October } 1947 \text { to September 1954, } \\
\text { October } 1963 \text { to September 1998, } \\
\text { October } 2001 \text { to March } 2014 .\end{array}$ & $\begin{array}{l}\text { April } 1945 \text { to March 1946, } \\
\text { April } 1948 \text { to March 1954, } \\
\text { April } 1964 \text { to March } 1984 .\end{array}$ & 27 & ${ }^{1}$ Unregulated & $\begin{array}{l}\text { Since about } 1985 \text {, flows influenced } \\
\text { by upstream diversions and regula- } \\
\text { tion. }\end{array}$ \\
\hline 257 & October 1970 to September 2003 & April 1971 to March 2003 & 32 & Unregulated & \\
\hline 258 & $\begin{array}{l}\text { October } 1939 \text { to September 1988, } \\
\text { June } 1989 \text { to March } 2014\end{array}$ & $\begin{array}{l}\text { April } 1940 \text { to March 1988, } \\
\text { April } 1990 \text { to March } 2014\end{array}$ & 71 & Unregulated & \\
\hline 259 & July 1966 to March 2014 & April 1967 to March 2014 & 47 & Unregulated & \\
\hline 261 & $\begin{array}{l}\text { October } 1943 \text { to September } 1946 \text {, } \\
\text { October } 1947 \text { to September 1973, } \\
\text { April } 1996 \text { to September } 2004\end{array}$ & $\begin{array}{l}\text { April } 1944 \text { to March 1946, } \\
\text { April } 1948 \text { to March 1973, } \\
\text { April } 1997 \text { to March 2004 }\end{array}$ & 34 & Unregulated & \\
\hline 262 & $\begin{array}{l}\text { October } 1943 \text { to September } 1947, \\
\text { October } 1949 \text { to October } 1977, \\
\text { November } 1987 \text { to August } 1989\end{array}$ & $\begin{array}{l}\text { April } 1944 \text { to March } 1947, \\
\text { April } 1950 \text { to March } 1977\end{array}$ & 30 & Unregulated & \\
\hline 263 & $\begin{array}{l}\text { August } 1928 \text { to September } \\
\text { 1932, September } 1938 \text { to } \\
\text { September } 1989 \text {, October } 1990 \\
\text { to March } 2014\end{array}$ & $\begin{array}{l}\text { April } 1929 \text { to March 1932, } \\
\text { April } 1939 \text { to March 1989, } \\
\text { April } 1991 \text { to March 2014 }\end{array}$ & 76 & Unregulated & $\begin{array}{l}\text { Telis (1991) indicates that approxi- } \\
\text { mately } 22 \text { percent of the basin has } \\
\text { been regulated by Bluff Lake since } \\
\text { 1950. QAQC reviews of Google } \\
\text { Earth and USGS NWISWeb maps } \\
\text { indicate that estimate may be } \\
\text { high. Bluff Lake is on a tributary } \\
\text { to Noxubee River, is far up in the } \\
\text { basin, and is, therefore, not likely } \\
\text { to substantially influence flows at } \\
\text { this gage. }\end{array}$ \\
\hline 265 & January 1972 to September 2013 & April 1972 to March 2013 & 41 & Unregulated & \\
\hline
\end{tabular}


Table 1. U.S. Geological Survey continuous-record streamgaging stations in Alabama and surrounding States included in this investigation.-Continued

[USGS, U.S. Geological Survey; ', degrees; ', minutes; ”, seconds; fťs, cubic foot per second; DA, drainage area; NWISWeb, National Water Information System database; $\mathrm{mi}^{2}$, square mile; QAQC, quality assurance, quality control]

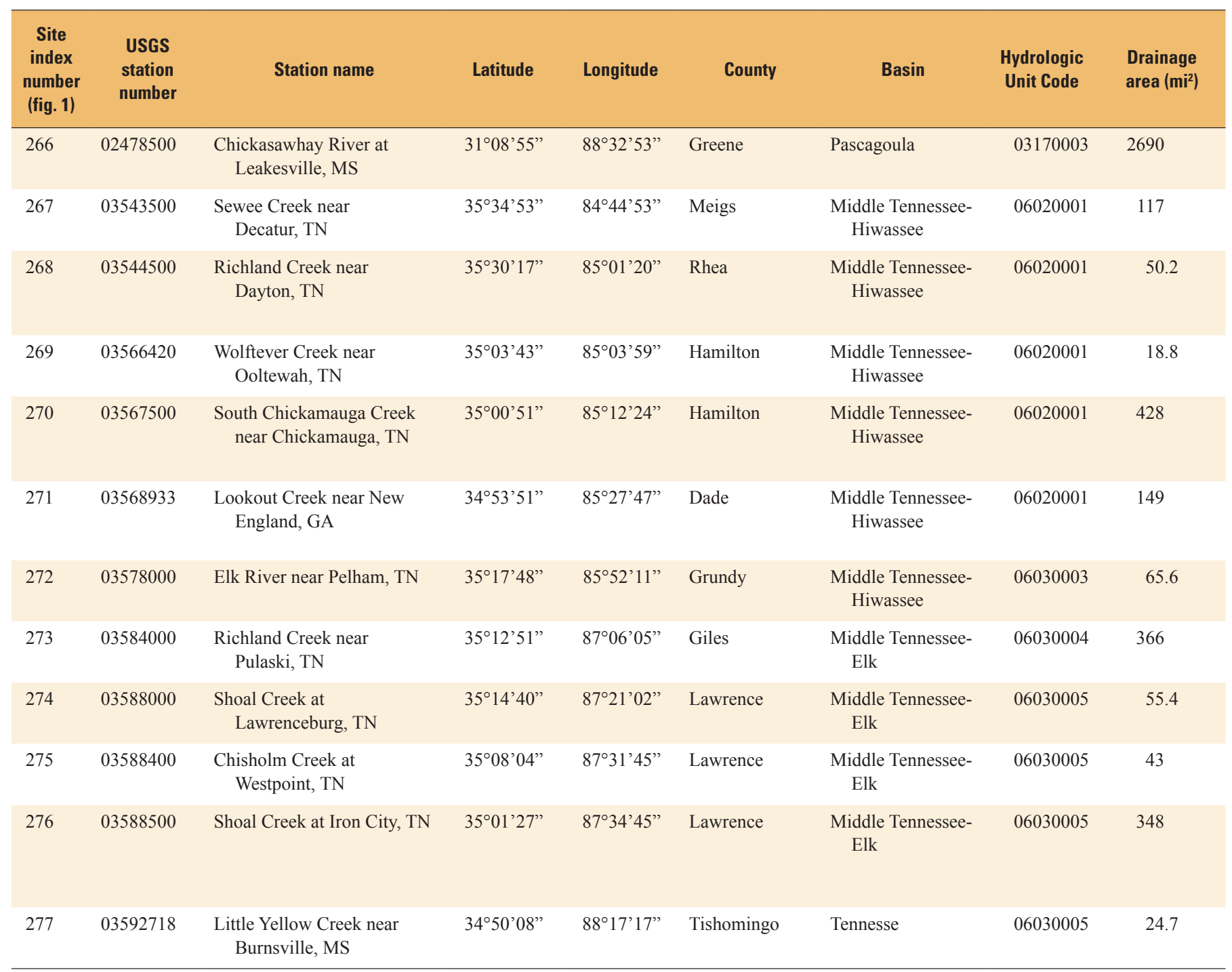

${ }^{1}$ Site is currently (2017) regulated, but low-flow frequency statistics were based on pre-regulation data. 
Table 1. U.S. Geological Survey continuous-record streamgaging stations in Alabama and surrounding States included in this investigation.-Continued

[USGS, U.S. Geological Survey; ', degrees; ', minutes; ”, seconds; fts/s, cubic foot per second; DA, drainage area; NWISWeb, National Water Information System database; $\mathrm{mi}^{2}$, square mile; QAQC, quality assurance, quality control]

\begin{tabular}{|c|c|c|c|c|c|}
\hline $\begin{array}{c}\text { Site } \\
\text { index } \\
\text { number } \\
\text { (fig. 1) }\end{array}$ & Period of record available & Period of record analyzed & $\begin{array}{l}\text { Number of } \\
\text { climate years } \\
\text { used in } \\
\text { analysis }\end{array}$ & $\begin{array}{l}\text { Flow } \\
\text { condition } \\
\text { for period } \\
\text { analyzed }\end{array}$ & Remarks \\
\hline 266 & September 1938 to March 2014 & April 1939 to March 2014 & 75 & Unregulated & \\
\hline 267 & $\begin{array}{c}\text { June } 1934 \text { to November 1994, } \\
\text { April } 2012 \text { to March } 2014\end{array}$ & $\begin{array}{l}\text { April } 1935 \text { to March 1994, } \\
\text { April } 2012 \text { to March } 2014\end{array}$ & 61 & Unregulated & \\
\hline 268 & $\begin{array}{l}\text { July } 1927 \text { to September } 1931 \text {, } \\
\text { July } 1934 \text { to September } 1955 \text {, } \\
\text { July } 1979 \text { to December } 1981\end{array}$ & $\begin{array}{l}\text { April } 1928 \text { to March } 1931 \text {, } \\
\text { April } 1935 \text { to March 1955, } \\
\text { April } 1980 \text { to March } 1981\end{array}$ & 24 & Unregulated & \\
\hline 269 & February 1964 to September 1989 & April 1964 to March 1989 & 25 & Unregulated & \\
\hline 270 & $\begin{array}{l}\text { October } 1928 \text { to September } 1978 \text {, } \\
\text { October } 1980 \text { to September 1994, } \\
\text { May } 2012 \text { to March } 2014\end{array}$ & $\begin{array}{l}\text { April } 1929 \text { to March 1978, } \\
\text { April } 1981 \text { to March 1994, } \\
\text { April } 2013 \text { to March } 2014\end{array}$ & 63 & Unregulated & \\
\hline 271 & August 1979 to March 2014 & April 1980 to March 2014 & 34 & Unregulated & $\begin{array}{l}\text { Noted in Gotvald (2016) as poten- } \\
\text { tially being influenced by upstream } \\
\text { diversions. }\end{array}$ \\
\hline 272 & $\begin{array}{l}\text { December } 1951 \text { to December 1987, } \\
\text { November } 2000 \text { to March } 2014\end{array}$ & $\begin{array}{l}\text { April } 1952 \text { to March 1987, } \\
\text { April } 2001 \text { to March } 2014\end{array}$ & 48 & Unregulated & \\
\hline 273 & May 1934 to October 1975 & April 1935 to March 1975 & 40 & Unregulated & \\
\hline 274 & $\begin{array}{l}\text { July } 1932 \text { to March 1934, March } 1967 \\
\text { to November } 1991\end{array}$ & $\begin{array}{l}\text { April } 1933 \text { to March } 1934, \\
\text { April } 1967 \text { to March } 1991\end{array}$ & 25 & Unregulated & \\
\hline 275 & August 1962 to December 1987 & April 1963 to March 1987 & 24 & Unregulated & \\
\hline 276 & $\begin{array}{l}\text { July } 1925 \text { to September } 1994, \\
\text { October } 2000 \text { to March } 2014\end{array}$ & $\begin{array}{l}\text { April } 1951 \text { to March 1994, } \\
\text { April } 2001 \text { to March } 2014\end{array}$ & 56 & Unregulated & $\begin{array}{l}\text { From U.S. Geological Survey (2014), } \\
\text { "Prior to January 1951, diurnal } \\
\text { fluctuation at low flow caused by } \\
\text { powerplant near Lawrenceburg." }\end{array}$ \\
\hline 277 & May 1973 to March 2013 & April 1974 to March 2012 & 38 & Unregulated & \\
\hline
\end{tabular}


Table 4. Results of Kendall's tau statistical test for detection of monotonic trends in the annual minimum 7-day average flow for 210 continuous-record streamgages in

\section{Alabama.}

$\left[\mathrm{mi}^{2}\right.$, square mile; - , no trend analysis was done for this station; $<$, less than]

\begin{tabular}{|c|c|c|c|c|c|c|c|c|c|}
\hline $\begin{array}{l}\text { Site } \\
\text { index } \\
\text { number } \\
\text { (fig. 1) }\end{array}$ & $\begin{array}{c}\text { USGS } \\
\text { station } \\
\text { number }\end{array}$ & Station name & $\begin{array}{l}\text { Regulation } \\
\text { status }\end{array}$ & $\begin{array}{l}\text { Drainage area } \\
\qquad\left(\mathrm{mi}^{2}\right)\end{array}$ & Period of record analyzed & $\begin{array}{l}\text { Number } \\
\text { of climate } \\
\text { years used in } \\
\text { analysis }\end{array}$ & $\begin{array}{l}\text { Kendall's } \\
\text { tau }\end{array}$ & P-value & Trend direction \\
\hline 1 & 02339225 & Wehadkee Creek below Rock Mills, AL & Unregulated & 60.2 & April 1979 to March 1989 & 10 & -0.200 & 0.47 & Not significant \\
\hline 2 & 02342500 & Uchee Creek near Fort Mitchell, AL & Unregulated & 322 & April 1947 to March 2014 & 67 & -0.220 & 0.01 & Downward \\
\hline 3 & 02342933 & $\begin{array}{l}\text { South Fork Cowikee Creek near } \\
\text { Batesville, AL }\end{array}$ & Unregulated & 112 & $\begin{array}{l}\text { April } 1964 \text { to March } 1971, \\
\text { April } 1975 \text { to March } 2011\end{array}$ & 43 & -0.020 & 0.06 & Not significant \\
\hline 4 & 02343300 & Abbie Creek near Haleburg, AL & Unregulated & 146 & $\begin{array}{l}\text { April } 1959 \text { to March } 1971, \\
\text { April } 1975 \text { to March } 1993\end{array}$ & 30 & -0.290 & 0.02 & Downward \\
\hline 5 & 02343500 & Chattahoochee River at Columbia, AL & ${ }^{1}$ Unregulated & 8,040 & April 1929 to March 1960 & 31 & -0.049 & 0.71 & Not significant \\
\hline 6 & 02360500 & $\begin{array}{l}\text { East Fork Choctawhatchee River near } \\
\text { Midland City, AL }\end{array}$ & Unregulated & 291 & April 1953 to March 1963 & 10 & 0.690 & 0.01 & Upward \\
\hline 7 & 02361000 & Choctawhatchee River near Newton, AL & Unregulated & 686 & $\begin{array}{l}\text { April } 1922 \text { to March 1927, } \\
\text { April } 1936 \text { to March } 2014\end{array}$ & 83 & -0.270 & 0.00 & Downward \\
\hline 8 & 02361500 & Choctawhatchee River near Bellwood, AL & Unregulated & 1,280 & $\begin{array}{l}\text { April } 1922 \text { to March } 1925 \text {, } \\
\text { April } 2001 \text { to March } 2014\end{array}$ & 16 & -0.320 & 0.09 & Not significant \\
\hline 9 & 02362240 & $\begin{array}{l}\text { Little Double Bridges Creek near } \\
\text { Enterprise, AL }\end{array}$ & Unregulated & 21.4 & April 1986 to March 2014 & 28 & 0.032 & 0.83 & Not significant \\
\hline 10 & 02363000 & Pea River near Ariton, AL & Unregulated & 498 & $\begin{array}{l}\text { April } 1939 \text { to March } 1970, \\
\text { April } 1988 \text { to March } 2014\end{array}$ & 57 & -0.270 & 0.00 & Downward \\
\hline 11 & 02364500 & Pea River near Samson, AL & Unregulated & 1,182 & $\begin{array}{l}\text { April } 1905 \text { to March } 1913, \\
\text { April } 1923 \text { to March 1925, } \\
\text { April } 1936 \text { to March 1970, } \\
\text { April } 2003 \text { March } 2014\end{array}$ & 55 & -0.300 & 0.00 & Downward \\
\hline 12 & 02364570 & Panther Creek near Hacoda, AL & Unregulated & 26.2 & April 1975 to March 1995 & 20 & -0.160 & 0.35 & Not significant \\
\hline 13 & 02369800 & Blackwater River near Bradley, AL & Unregulated & 87.7 & April 1968 to March 2014 & 46 & -0.160 & 0.12 & Not significant \\
\hline 14 & 02371200 & Indian Creek near Troy, AL & Unregulated & 8.87 & $\begin{array}{l}\text { April } 1959 \text { to March } 1968, \\
\text { April } 1971 \text { to March } 1986\end{array}$ & 24 & -0.250 & 0.09 & Not significant \\
\hline 15 & 02371500 & Conecuh River at Brantley, AL & Unregulated & 500 & April 1938 to March 2014 & 76 & -0.320 & $<0.0001$ & Downward \\
\hline 16 & 02372000 & Patsaliga Creek at Lurverne, AL & Unregulated & 254 & April 1944 to March 1958 & 14 & -0.520 & 0.01 & Downward \\
\hline 17 & 02372250 & Patsaliga Creek near Brantley, AL & Unregulated & 442 & April 1975 to March 2014 & 39 & -0.290 & 0.01 & Downward \\
\hline 18 & 02372422 & $\begin{array}{l}\text { Conecuh River below Point A Dam near } \\
\text { River Falls, AL }\end{array}$ & Regulated & 1,273 & April 2000 to March 2013 & 13 & -0.180 & 0.39 & Not significant \\
\hline
\end{tabular}


Table 4. Results of Kendall's tau statistical test for detection of monotonic trends in the annual minimum 7-day average flow for 210 continuous-record streamgages in Alabama.-Continued

$\left[\mathrm{mi}^{2}\right.$, square mile; - , no trend analysis was done for this station; $<$, less than $]$

\begin{tabular}{|c|c|c|c|c|c|c|c|c|c|}
\hline $\begin{array}{c}\text { Site } \\
\text { index } \\
\text { number } \\
\text { (fig. 1) }\end{array}$ & $\begin{array}{l}\text { USGS } \\
\text { station } \\
\text { number }\end{array}$ & Station name & $\begin{array}{l}\text { Regulation } \\
\text { status }\end{array}$ & $\begin{array}{l}\text { Drainage area } \\
\qquad\left(\mathrm{mi}^{2}\right)\end{array}$ & Period of record analyzed & $\begin{array}{l}\text { Number } \\
\text { of climate } \\
\text { years used in } \\
\text { analysis }\end{array}$ & $\begin{array}{l}\text { Kendall's } \\
\text { tau }\end{array}$ & P-value & Trend direction \\
\hline 19 & 02372500 & Conecuh River near Andalusia, AL & Regulated & 1,344 & $\begin{array}{l}\text { April } 1930 \text { to March } 1952, \\
\text { April } 1966 \text { to March } 1968\end{array}$ & 24 & 0.120 & 0.43 & Not significant \\
\hline 20 & 02373000 & Sepulga River near McKenzie, AL & Unregulated & 470 & $\begin{array}{l}\text { April } 1938 \text { to March } 1967, \\
\text { April } 1975 \text { to March } 2014\end{array}$ & 68 & -0.120 & 0.16 & Not significant \\
\hline 21 & 02373500 & Pigeon Creek near Thad, AL & Unregulated & 307 & April 1938 to March 1970 & 32 & -0.250 & 0.04 & Downward \\
\hline 22 & 02374000 & Conecuh River near Brooklyn, AL & Regulated & 2,495 & April 1936 to March 1957 & 21 & -0.340 & 0.03 & Downward \\
\hline 23 & 02374250 & $\begin{array}{l}\text { Conecuh River at State Highway } 41 \text { near } \\
\text { Brewton, AL }\end{array}$ & Regulated & 2,661 & April 2000 to March 2014 & 14 & -0.033 & 0.87 & Not significant \\
\hline 24 & 02374500 & Murder Creek near Evergreen, AL & Unregulated & 176 & April 1938 to March 2014 & 76 & -0.078 & 0.32 & Not significant \\
\hline 25 & 02374700 & $\begin{array}{l}\text { Murder Creek at State Highway } 41 \text { near } \\
\text { Brewton, AL }\end{array}$ & Unregulated & 435 & April 1999 to March 2014 & 15 & -0.260 & 0.18 & Not significant \\
\hline 26 & 02374745 & $\begin{array}{l}\text { Burnt Corn Creek at State Highway } 41 \\
\text { near Brewton, AL }\end{array}$ & Unregulated & 182 & April 1999 to March 2014 & 15 & -0.240 & 0.22 & Not significant \\
\hline 27 & 02374950 & $\begin{array}{l}\text { Big Escambia Creek at Sardine Bridge near } \\
\text { Stanley Crossroads, AL }\end{array}$ & Unregulated & 193 & April 2001 to March 2014 & 13 & -0.260 & 0.22 & Not significant \\
\hline 28 & 02375000 & Big Escambia Creek at Flomaton, AL & Unregulated & 330 & April 1940 to March 1951 & 12 & 0.360 & 0.10 & Not significant \\
\hline 29 & 02376500 & Perdido River at Barrineau Park, FL & Unregulated & 394 & April 1941 to March 2014 & 72 & -0.150 & 0.07 & Not significant \\
\hline 30 & 02377500 & Styx River near Loxley, AL & Unregulated & 92.2 & April 1952 to March 1969 & 17 & -0.022 & 0.90 & Not significant \\
\hline 31 & 02377570 & Styx River near Elsanor, AL & Unregulated & 192 & April 1988 to March 2014 & 26 & -0.300 & 0.03 & Downward \\
\hline 32 & 02378300 & Magnolia River at U.S. 98 near Foley, AL & Unregulated & 16.6 & April 2000 to March 2014 & 14 & 0.420 & 0.04 & Upward \\
\hline 33 & 02378500 & Fish River near Silver Hill, AL & Unregulated & 55.3 & $\begin{array}{l}\text { April } 1954 \text { to March } 1969, \\
\text { April } 1987 \text { to March } 2014\end{array}$ & 42 & -0.170 & 0.11 & Not significant \\
\hline 34 & 02398300 & Chattooga River above Gaylesville, $\mathrm{AL}$ & Unregulated & 366 & $\begin{array}{l}\text { April } 1960 \text { to March 1967, } \\
\text { April } 1985 \text { to March } 2014\end{array}$ & 37 & -0.080 & 0.49 & Not significant \\
\hline 35 & 02398500 & Chattooga River at Gaylesville, AL & Unregulated & 379 & April 1937 to March 1960 & 22 & 0.220 & 0.15 & Not significant \\
\hline 36 & 02398950 & $\begin{array}{l}\text { West Fork Little River at Desoto Park near } \\
\text { Fort Payne, AL }\end{array}$ & Unregulated & 42.8 & April 1998 to March 2011 & 13 & -0.077 & 0.71 & Not significant \\
\hline 37 & 02399000 & Little River near Jamestown, AL & Unregulated & 125 & $\begin{array}{l}\text { April } 1922 \text { to March } 1932, \\
\text { April } 1936 \text { to March } 1949\end{array}$ & 23 & 0.230 & 0.12 & Not significant \\
\hline
\end{tabular}


Table 4. Results of Kendall's tau statistical test for detection of monotonic trends in the annual minimum 7-day average flow for 210 continuous-record streamgages in

Alabama.-Continued

$\left[\mathrm{mi}^{2}\right.$, square mile; - , no trend analysis was done for this station; $<$, less than]

\begin{tabular}{|c|c|c|c|c|c|c|c|c|c|}
\hline $\begin{array}{c}\text { Site } \\
\text { index } \\
\text { number } \\
\text { (fig. 1) }\end{array}$ & $\begin{array}{l}\text { USGS } \\
\text { station } \\
\text { number }\end{array}$ & Station name & $\begin{array}{l}\text { Regulation } \\
\text { status }\end{array}$ & $\begin{array}{c}\text { Drainage area } \\
\left(\mathrm{mi}^{2}\right)\end{array}$ & Period of record analyzed & $\begin{array}{c}\text { Number } \\
\text { of climate } \\
\text { years used in } \\
\text { analysis }\end{array}$ & $\begin{array}{l}\text { Kendall's } \\
\text { tau }\end{array}$ & P-value & Trend direction \\
\hline 38 & 02399200 & Little River near Blue Rond, AL & Unregulated & 199 & $\begin{array}{l}\text { April } 1959 \text { to March } 1967, \\
\text { April } 1971 \text { to March } 2014\end{array}$ & 51 & -0.023 & 0.81 & Not significant \\
\hline 39 & 02399500 & Coosa River at Leesburg, $\mathrm{AL}$ & ${ }^{1}$ Unregulated & 5,270 & April 1938 to March 1949 & 11 & 0.130 & 0.59 & Not significant \\
\hline 40 & 02400000 & Terrapin Creek near Piedmont, AL & Unregulated & 116 & $\begin{array}{l}\text { April } 1945 \text { to March } 1954, \\
\text { April } 1957 \text { to March } 1963\end{array}$ & 15 & -0.120 & 0.52 & Not significant \\
\hline 41 & 02400100 & Terrapin Creek at Ellisville, AL & Unregulated & 252 & $\begin{array}{l}\text { April } 1963 \text { to March } 1967, \\
\text { April } 1981 \text { to March } 2014\end{array}$ & 37 & -0.210 & 0.07 & Not significant \\
\hline 42 & 02400500 & Coosa River at Gadsden, $\mathrm{AL}$ & ${ }^{1}$ Unregulated & 5805 & April 1927 to March 1949 & 22 & -0.087 & 0.57 & Not significant \\
\hline 43 & 02400680 & $\begin{array}{l}\text { Big Wills Creek at State Highway } 35 \text { near } \\
\text { Fort Payne, AL }\end{array}$ & Unregulated & 55.4 & April 2003 to March 2014 & 11 & -0.220 & 0.35 & Not significant \\
\hline 44 & 02401000 & Big Wills Creek near Reece City, AL & Unregulated & 182 & $\begin{array}{l}\text { April } 1944 \text { to March } 1970, \\
\text { April } 1987 \text { to March } 2014\end{array}$ & 53 & 0.075 & 0.43 & Not significant \\
\hline 45 & 02401370 & Big Canoe Creek near Springville, AL & Unregulated & 45 & April 1979 to March 1995 & 16 & 0.050 & 0.79 & Not significant \\
\hline 46 & 02401390 & Big Canoe Creek near Ashville, AL & Unregulated & 141 & April 1966 to March 2014 & 48 & -0.016 & 0.87 & Not significant \\
\hline 47 & 02401470 & Little Canoe Creek near Steele, AL & Unregulated & 22.3 & April 1982 to March 1995 & 13 & 0.051 & 0.81 & Not significant \\
\hline 48 & 02401500 & Big Canoe Creek near Gadsden, AL & Unregulated & 253 & April 1938 to March 1965 & 26 & 0.099 & 0.48 & Not significant \\
\hline 49 & 02402500 & Coosa River at Riverside, $\mathrm{AL}$ & ${ }^{1}$ Unregulated & 7,069 & April 1897 to March 1916 & 19 & -0.064 & 0.70 & Not significant \\
\hline 50 & 02403500 & Coldwater Spring near Anniston, AL & Unregulated & Indeterminate & April 1957 to March 1996 & 39 & -0.020 & 0.86 & Not significant \\
\hline 51 & 02404000 & Choccolocco Creek near Jenifer, AL & Unregulated & 277 & $\begin{array}{l}\text { April } 1904 \text { to March 1907, } \\
\text { April } 1930 \text { to March 1932, } \\
\text { and April } 1936 \text { to March } 1970\end{array}$ & 39 & 0.240 & 0.03 & Upward \\
\hline 52 & 02404400 & $\begin{array}{l}\text { Choccolocco Creek at Jackson Shoal near } \\
\text { Lincoln, AL }\end{array}$ & Unregulated & 481 & $\begin{array}{l}\text { April } 1961 \text { to March } 1967, \\
\text { April } 1985 \text { to March } 2014\end{array}$ & 35 & -0.082 & 0.49 & Not significant \\
\hline 53 & 02404500 & Choccolocco Creek near Lincoln, AL & Unregulated & 496 & April 1939 to March 1953 & 14 & 0.160 & 0.41 & Not significant \\
\hline 54 & 02405500 & Kelly Creek near Vincent, AL & Unregulated & 193 & April 1952 to March 1970 & 18 & 0.290 & 0.09 & Not significant \\
\hline 54 & 02405500 & Kelly Creek near Vincent, AL & Unregulated & 193 & April 1987 to March 2014 & 27 & 0.220 & 0.11 & Not significant \\
\hline 55 & 02405800 & Talladega Creek above Talladega, AL & Unregulated & 69.6 & April 1960 to March 1970 & 10 & 0.330 & 0.18 & Not significant \\
\hline
\end{tabular}


Table 4. Results of Kendall's tau statistical test for detection of monotonic trends in the annual minimum 7-day average flow for 210 continuous-record streamgages in Alabama.-Continued

$\left[\mathrm{mi}^{2}\right.$, square mile; - , no trend analysis was done for this station; $<$, less than $]$

\begin{tabular}{|c|c|c|c|c|c|c|c|c|c|}
\hline $\begin{array}{l}\text { Site } \\
\text { index } \\
\text { number } \\
\text { (fig. 1) }\end{array}$ & $\begin{array}{c}\text { USGS } \\
\text { station } \\
\text { number }\end{array}$ & Station name & $\begin{array}{l}\text { Regulation } \\
\text { status }\end{array}$ & $\begin{array}{l}\text { Drainage area } \\
\left(\mathrm{mi}^{2}\right)\end{array}$ & Period of record analyzed & $\begin{array}{l}\text { Number } \\
\text { of climate } \\
\text { years used in } \\
\text { analysis }\end{array}$ & $\begin{array}{l}\text { Kendall's } \\
\text { tau }\end{array}$ & P-value & Trend direction \\
\hline 56 & 02406500 & Talladega Creek at Alpine, $\mathrm{AL}$ & Unregulated & 150 & $\begin{array}{l}\text { April } 1901 \text { to March 1904, } \\
\text { April } 1940 \text { to March 1951, } \\
\text { April } 1988 \text { to March } 2014\end{array}$ & 40 & -0.160 & 0.16 & Not significant \\
\hline 57 & 02407000 & Coosa River at Childersburg, AL & ${ }^{1}$ Unregulated & 8,392 & April 1914 to March 1948 & 35 & -0.270 & 0.02 & Downward \\
\hline 58 & 02407500 & Yellowleaf Creek near Wilsonville, AL & Unregulated & 96.5 & April 1952 to March 1967 & 16 & 0.300 & 0.10 & Not significant \\
\hline 59 & 02408500 & Hatchet Creek near Rockford, AL & Unregulated & 233 & April 1945 to March 1978 & 33 & 0.200 & 0.10 & Not significant \\
\hline 60 & 02408540 & Hatchet Creek below Rockford, AL & Unregulated & 263 & April 1981 to March 2014 & 33 & -0.190 & 0.12 & Not significant \\
\hline 61 & 02410000 & Paterson Creek near Central, AL & Unregulated & 4.91 & April 1954 to March 1987 & 33 & 0.160 & 0.19 & Not significant \\
\hline 62 & 02411000 & $\begin{array}{l}\text { Coosa River at Jordan Dam near } \\
\text { Wetumpka, AL }\end{array}$ & Regulated & 10,102 & - & - & - & - & - \\
\hline 63 & 02411930 & Tallapoosa River below Tallapoosa, GA & Unregulated & 272 & $\begin{array}{l}\text { April } 2000 \text { to March 2004, } \\
\text { April } 2006 \text { to March } 2014\end{array}$ & 12 & -0.120 & 0.58 & Not significant \\
\hline 64 & 02412000 & Tallapoosa River near Heflin, AL & Unregulated & 448 & April 1953 to March 2014 & 61 & 0.140 & 0.25 & Not significant \\
\hline 65 & 02412500 & Tallapoosa River near Ofelia, AL & ${ }^{1}$ Unregulated & 792 & April 1939 to March 1951 & 12 & 0.120 & 0.58 & Not significant \\
\hline 66 & 02413300 & Little Tallapoosa River near Newell, AL & Unregulated & 406 & April 1976 to March 2014 & 38 & -0.230 & 0.04 & Downward \\
\hline 67 & 02413500 & Little Tallapoosa River near Wedowee, AL & Unregulated & 591 & April 1940 to March 1951 & 11 & 0.310 & 0.19 & Not significant \\
\hline 68 & 02414500 & Tallapoosa River at Wadley, AL & ${ }^{1}$ Unregulated & 1,675 & April 1924 to March 1982 & 58 & 0.160 & 0.08 & Not significant \\
\hline 68 & 02414500 & Tallapoosa River at Wadley, AL & Regulated & 1,675 & April 1983 to March 2014 & 31 & 0.095 & 0.45 & Not significant \\
\hline 69 & 02414715 & Tallapoosa River near New Site, AL & Regulated & 2,058 & April 1986 to March 2014 & 28 & -0.026 & 0.84 & Not significant \\
\hline 70 & 02415000 & Hillabee Creek near Hackneyville, AL & Unregulated & 190 & $\begin{array}{l}\text { April } 1953 \text { to March } 1970, \\
\text { April } 1986 \text { to March } 2014\end{array}$ & 45 & -0.140 & 0.17 & Not significant \\
\hline 71 & 02416000 & Tallapoosa River at Sturdivant, AL & Unregulated & 2,480 & April 1901 to March 1926 & 25 & -0.270 & 0.06 & Not significant \\
\hline 72 & 02418230 & Sougahatchee Creek near Loachapoka, AL & Unregulated & 71.3 & April 2000 to March 2014 & 14 & -0.240 & 0.23 & Not significant \\
\hline 73 & 02418500 & Tallapoosa River below Tallassee, AL & Regulated & 3,328 & - & - & - & - & - \\
\hline 74 & 02418760 & $\begin{array}{l}\text { Chewacla Creek at Chewacla State Park } \\
\text { near Auburn, AL }\end{array}$ & Unregulated & 45.8 & April 2003 to March 2014 & 11 & -0.420 & 0.07 & Not significant \\
\hline 75 & 02419000 & Uphapee Creek near Tuskegee, AL & Unregulated & 333 & $\begin{array}{l}\text { April } 1940 \text { to March } 1970, \\
\quad \text { April } 1975 \text { to March } 2014\end{array}$ & 69 & 0.072 & 0.38 & Not significant \\
\hline
\end{tabular}


Table 4. Results of Kendall's tau statistical test for detection of monotonic trends in the annual minimum 7-day average flow for 210 continuous-record streamgages in

Alabama.-Continued

$\left[\mathrm{mi}^{2}\right.$, square mile; - , no trend analysis was done for this station; $<$, less than]

\begin{tabular}{|c|c|c|c|c|c|c|c|c|c|}
\hline $\begin{array}{l}\text { Site } \\
\text { index } \\
\text { number } \\
\text { (fig. 1) }\end{array}$ & $\begin{array}{l}\text { USGS } \\
\text { station } \\
\text { number }\end{array}$ & Station name & $\begin{array}{l}\text { Regulation } \\
\text { status }\end{array}$ & $\begin{array}{c}\text { Drainage area } \\
\qquad\left(\mathrm{mi}^{2}\right)\end{array}$ & Period of record analyzed & $\begin{array}{l}\text { Number } \\
\text { of climate } \\
\text { years used in } \\
\text { analysis }\end{array}$ & $\begin{array}{c}\text { Kendall's } \\
\text { tau }\end{array}$ & P-value & Trend direction \\
\hline 76 & 02419890 & Tallapoosa River near Montgomery, AL & Regulated & 4,646 & April 1996 to March 2014 & 18 & -0.370 & 0.03 & Downward \\
\hline 77 & 02420000 & Alabama River near Montgomery, AL & Regulated & 15,087 & - & - & - & - & - \\
\hline 78 & 02420500 & Autauga Creek at Prattville, $\mathrm{AL}$ & Regulated & 116 & April 1939 to March 1959 & 20 & -0.450 & 0.01 & Downward \\
\hline 79 & 02421000 & Catoma Creek near Montgomery, AL & Unregulated & 290 & $\begin{array}{l}\text { April } 1953 \text { to March 1971, } \\
\text { April } 1975 \text { to March 1996, } \\
\text { April } 1998 \text { to March } 2014\end{array}$ & 55 & 0.200 & 0.04 & Upward \\
\hline 80 & 02422000 & Big Swamp Creek near Lowndesboro, AL & Unregulated & 244 & April 1941 to March 1971 & 30 & -0.049 & 0.71 & Not significant \\
\hline 81 & 02422500 & Mulberry Creek at Jones, AL & Unregulated & 203 & $\begin{array}{l}\text { April } 1939 \text { to March } 1970 \\
\text { April } 1975 \text { to March } 2014\end{array}$ & 70 & -0.210 & 0.01 & Downward \\
\hline 82 & 02423000 & Alabama River at Selma, AL & ${ }^{1}$ Unregulated & 17,095 & April 1900 to March 1913 & 13 & -0.130 & 0.54 & Not significant \\
\hline 83 & 02423130 & Cahaba River at Trussville, $\mathrm{AL}$ & Unregulated & 19.7 & April 1989 to March 2014 & 25 & -0.220 & 0.13 & Not significant \\
\hline 84 & 02423380 & Cahaba River near Mountain Brook, AL & Unregulated & 140 & April 1985 to March 2014 & 29 & -0.003 & 0.98 & Not significant \\
\hline 85 & 02423397 & Little Cahaba River below Leeds, AL & Unregulated & 17 & $\begin{array}{l}\text { April } 1996 \text { to March 2006, } \\
\text { April } 2009 \text { to March } 2016\end{array}$ & 15 & -0.220 & 0.26 & Not significant \\
\hline 86 & 02423398 & Little Cahaba River near Leeds, AL & Unregulated & 19.4 & April 1989 to March 2006 & 17 & 0.029 & 0.87 & Not significant \\
\hline 87 & 02423400 & $\begin{array}{l}\text { Little Cahaba River near Jefferson } \\
\text { Park, AL }\end{array}$ & Unregulated & 24.4 & $\begin{array}{l}\text { April } 1987 \text { to March } 1999 \text {, } \\
\text { April } 2008 \text { to March } 2014\end{array}$ & 18 & 0.150 & 0.38 & Not significant \\
\hline 88 & 02423414 & $\begin{array}{l}\text { Little Cahaba River at Cahaba Beach Road } \\
\text { near Cahaba Heights, AL }\end{array}$ & Regulated & 47 & April 2004 to March 2014 & 10 & 0.470 & 0.06 & Not significant \\
\hline 89 & 02423425 & Cahaba River near Cahaba Heights, AL & Regulated & 201 & $\begin{array}{l}\text { April } 1976 \text { to March } 1985, \\
\text { April } 1997 \text { to March } 2014\end{array}$ & 26 & 0.220 & 0.12 & Not significant \\
\hline 90 & 02423496 & Cahaba River near Hoover, AL & Regulated & 226 & April 1989 to March 2014 & 25 & 0.230 & 0.10 & Not significant \\
\hline 91 & 02423500 & Cahaba River near Acton, AL & Regulated & 230 & April 1984 to March 2014 & 30 & 0.400 & 0.002 & Upward \\
\hline 92 & 0242354750 & $\begin{array}{l}\text { Cahaba Valley Creek at Cross Creek Road } \\
\text { at Pelham, AL }\end{array}$ & Unregulated & 25.6 & April 1999 to March 2013 & 14 & 0.190 & 0.35 & Not significant \\
\hline 93 & 02423555 & Cahaba River near Helena, AL & Regulated & 335 & April 1996 to March 2014 & 18 & -0.059 & 0.73 & Not significant \\
\hline 94 & 02423630 & Shades Creek near Greenwood, AL & Unregulated & 72.3 & $\begin{array}{l}\text { April } 1967 \text { to March } 1973 \text {, } \\
\text { April } 1975 \text { to March 1981, } \\
\text { April } 1998 \text { to March } 2014\end{array}$ & 28 & -0.510 & 0.0001 & Downward \\
\hline
\end{tabular}


Table 4. Results of Kendall's tau statistical test for detection of monotonic trends in the annual minimum 7-day average flow for 210 continuous-record streamgages in Alabama.-Continued

$\left[\mathrm{mi}^{2}\right.$, square mile; - , no trend analysis was done for this station; $<$, less than $]$

\begin{tabular}{|c|c|c|c|c|c|c|c|c|c|}
\hline $\begin{array}{c}\text { Site } \\
\text { index } \\
\text { number } \\
\text { (fig. 1) }\end{array}$ & $\begin{array}{l}\text { USGS } \\
\text { station } \\
\text { number }\end{array}$ & Station name & $\begin{array}{l}\text { Regulation } \\
\text { status }\end{array}$ & $\begin{array}{c}\text { Drainage area } \\
\qquad\left(\mathrm{mi}^{2}\right)\end{array}$ & Period of record analyzed & $\begin{array}{c}\text { Number } \\
\text { of climate } \\
\text { years used in } \\
\text { analysis }\end{array}$ & $\begin{array}{c}\text { Kendall's } \\
\text { tau }\end{array}$ & P-value & Trend direction \\
\hline 95 & 02423800 & Little Cahaba River near Brierfield, AL & Unregulated & 147 & April 1958 to March 1970 & 12 & 0.091 & 0.68 & Not significant \\
\hline 96 & 02424000 & Cahaba River at Centreville, $\mathrm{AL}$ & Unregulated & 1,027 & $\begin{array}{l}\text { April } 1902 \text { to March } 1907, \\
\text { April } 1930 \text { to March 1932, } \\
\text { April } 1936 \text { to March 2014 }\end{array}$ & 85 & 0.070 & 0.34 & Not significant \\
\hline 97 & 02424500 & Cahaba River at Sprott, AL & Unregulated & 1,370 & April 1939 to March 1969 & 30 & -0.270 & 0.03 & Downward \\
\hline 98 & 02424590 & Cahaba River near Suttle, AL & Unregulated & 1,480 & April 1988 to March 2011 & 23 & -0.099 & 0.51 & Not significant \\
\hline 99 & 02424940 & Oakmulgee Creek near Augustin, AL & Unregulated & 220 & April 1976 to March 1987 & 11 & -0.240 & 0.31 & Not significant \\
\hline 100 & 02425000 & Cahaba River near Marion Junction, AL & Unregulated & 1,766 & $\begin{array}{l}\text { April } 1939 \text { to March } 1954, \\
\text { April } 1969 \text { to March } 2014\end{array}$ & 60 & -0.130 & 0.13 & Not significant \\
\hline 101 & 02425200 & Big Swamp Creek near Orrville, AL & Unregulated & 35.8 & April 1972 to March 1985 & 13 & -0.150 & 0.46 & Not significant \\
\hline 102 & 02425500 & Cedar Creek at Minter, $\mathrm{AL}$ & Unregulated & 211 & $\begin{array}{l}\text { April } 1953 \text { to March } 1970, \\
\text { April } 1975 \text { to March } 1982\end{array}$ & 24 & 0.260 & 0.07 & Not significant \\
\hline 103 & 02426000 & Boguechitto Creek near Browns, AL & Unregulated & 95.4 & $\begin{array}{l}\text { April } 1944 \text { to March } 1954, \\
\text { April } 1966 \text { to March } 1971\end{array}$ & 15 & -0.450 & 0.02 & Downward \\
\hline 104 & 02427250 & Pine Barren Creek near Snow Hill, AL & Unregulated & 261 & April 1990 to March 2014 & 24 & -0.250 & 0.09 & Not significant \\
\hline 105 & 02427500 & Alabama River near Millers Ferry, AL & Regulated & 20,637 & - & - & - & - & - \\
\hline 106 & 02427700 & Turkey Creek at Kimbrough, AL & Unregulated & 97.5 & April 1959 to March 1996 & 37 & -0.075 & 0.51 & Not significant \\
\hline 107 & 02428400 & $\begin{array}{l}\text { Alabama River at Claiborne L\&D near } \\
\text { Monroeville, AL }\end{array}$ & Regulated & 21,473 & April 1976 to March 2014 & 38 & -0.280 & 0.01 & Downward \\
\hline 108 & 02428500 & Big Flat Creek near Fountain, AL & Unregulated & 247 & April 1944 to March 1970 & 26 & -0.170 & 0.22 & Not significant \\
\hline 109 & 02429000 & Limestone Creek near Monroeville, AL & Unregulated & 121 & April 1952 to March 1970 & 18 & 0.079 & 0.65 & Not significant \\
\hline 110 & 02429500 & Alabama River at Claiborne, $\mathrm{AL}$ & Regulated & 21,967 & - & - & - & - & - \\
\hline 111 & 02429595 & Little River near Uriah, AL & Unregulated & 95.2 & April 1969 to March 1979 & 10 & 0.470 & 0.06 & Not significant \\
\hline 112 & 02438000 & Buttahatchee River below Hamilton, AL & Unregulated & 277 & $\begin{array}{l}\text { April } 1951 \text { to March 1970, } \\
\text { April } 1992 \text { to March } 2014\end{array}$ & 42 & 0.290 & 0.01 & Upward \\
\hline 113 & 02439000 & Buttahatchee River near Sulligent, AL & Unregulated & 472 & April 1939 to March 1959 & 20 & -0.210 & 0.19 & Not significant \\
\hline 114 & 02442000 & Luxapallila Creek near Fayette, AL & Unregulated & 130 & April 1946 to March 1970 & 24 & -0.030 & 0.86 & Not significant \\
\hline
\end{tabular}


Table 4. Results of Kendall's tau statistical test for detection of monotonic trends in the annual minimum 7-day average flow for 210 continuous-record streamgages in

Alabama.-Continued

$\left[\mathrm{mi}^{2}\right.$, square mile; - , no trend analysis was done for this station; $<$, less than]

\begin{tabular}{|c|c|c|c|c|c|c|c|c|c|}
\hline $\begin{array}{c}\text { Site } \\
\text { index } \\
\text { number } \\
\text { (fig. 1) }\end{array}$ & $\begin{array}{l}\text { USGS } \\
\text { station } \\
\text { number }\end{array}$ & Station name & $\begin{array}{l}\text { Regulation } \\
\text { status }\end{array}$ & $\begin{array}{l}\text { Drainage area } \\
\qquad\left(\mathbf{m i}^{2}\right)\end{array}$ & Period of record analyzed & $\begin{array}{l}\text { Number } \\
\text { of climate } \\
\text { years used in } \\
\text { analysis }\end{array}$ & $\begin{array}{c}\text { Kendall's } \\
\text { tau }\end{array}$ & P-value & Trend direction \\
\hline 115 & 02442500 & Luxapallila Creek at Millport, AL & Unregulated & 247 & $\begin{array}{l}\text { April } 1955 \text { to March } 1959, \\
\text { April } 1981 \text { to March 1986, } \\
\text { April } 2002 \text { to March 2011 }\end{array}$ & 18 & 0.300 & 0.08 & Not significant \\
\hline 116 & 02444000 & Coal Fire Creek near Pickensville, AL & Unregulated & 126 & $\begin{array}{l}\text { April } 1955 \text { to March 1971, } \\
\text { April } 1975 \text { to March } 1980\end{array}$ & 21 & 0.440 & 0.01 & Upward \\
\hline 117 & 02444160 & $\begin{array}{l}\text { Tombigbee River at Bevill L\&D near } \\
\text { Pickensville, AL }\end{array}$ & Regulated & 5,750 & $\begin{array}{l}\text { April } 1985 \text { to March } 2009 \text {, } \\
\text { April } 2011 \text { to March } 2014\end{array}$ & 27 & -0.031 & 0.82 & Not significant \\
\hline 118 & 02444500 & Tombigbee River near Cochrane, AL & ${ }^{1}$ Unregulated & 5,940 & April 1939 to March 1978 & 39 & 0.390 & 0.00 & Upward \\
\hline 119 & 02445500 & Sipsey River at Fayette, AL & Unregulated & 282 & April 1939 to March 1959 & 20 & -0.280 & 0.08 & Not significant \\
\hline 120 & 02446000 & Sipsey River at Moores Bridge, $\mathrm{AL}$ & Unregulated & 413 & April 1939 to March 1951 & 12 & 0.230 & 0.30 & Not significant \\
\hline 121 & 02446500 & Sipsey River near Elrod, AL & Unregulated & 528 & $\begin{array}{l}\text { April } 1929 \text { to March 1932, } \\
\text { April } 1940 \text { to March 1971, } \\
\text { April } 1979 \text { to March 2014 }\end{array}$ & 69 & 0.240 & 0.00 & Upward \\
\hline 122 & 02447000 & Sipsey River near Pleasant Ridge, AL & Unregulated & 769 & April 1939 to March1959 & 20 & -0.370 & 0.02 & Downward \\
\hline 123 & 02447025 & $\begin{array}{l}\text { Tombigbee River at Heflin L\&D near } \\
\text { Gainesville, AL }\end{array}$ & Regulated & 7,230 & April 1985 to March 2014 & 29 & -0.094 & 0.48 & Not significant \\
\hline 124 & 02448500 & Noxubee River near Geiger, AL & Unregulated & 1,097 & $\begin{array}{l}\text { April } 1939 \text { to March } 1940, \\
\text { April } 1945 \text { to March 1965, } \\
\text { April } 1967 \text { to March } 2014\end{array}$ & 68 & 0.180 & 0.03 & Upward \\
\hline 125 & 02448900 & Bodka Creek near Geiger, AL & Unregulated & 158 & April 1991 to March 2014 & 23 & 0.000 & 1.00 & Not significant \\
\hline 126 & 02449000 & Tombigbee River at Gainsville, AL & ${ }^{1}$ Unregulated & 8,632 & $\begin{array}{l}\text { April } 1939 \text { to March } 1955 \text {, } \\
\text { April } 1961 \text { to March } 1978\end{array}$ & 33 & 0.460 & 0.00 & Upward \\
\hline 127 & 02449245 & Brush Creek near Eutaw, AL & Unregulated & 43.2 & April 1976 to March 1997 & 21 & -0.070 & 0.67 & Not significant \\
\hline 128 & 02449500 & Tombigbee River at Epes, AL & ${ }^{1}$ Unregulated & 8,930 & $\begin{array}{l}\text { April } 1905 \text { to March } 1913, \\
\text { April } 1939 \text { to March } 1945\end{array}$ & 14 & -0.270 & 0.19 & Not significant \\
\hline 129 & 02449882 & Blue Springs Creek near Blountsville, AL & Unregulated & 13 & April 1993 to March 2014 & 21 & -0.270 & 0.09 & Not significant \\
\hline 130 & 02450000 & Mulberry Fork near Garden City, AL & Unregulated & 358 & April 1929 to March 1964 & 35 & 0.150 & 0.20 & Not significant \\
\hline 130 & 02450000 & Mulberry Fork near Garden City, AL & Unregulated & 358 & April 1965 to March 2014 & 49 & 0.031 & 0.76 & Not significant \\
\hline 131 & 02450180 & Mulberry Fork near Arkadelphia, AL & Unregulated & 487 & $\begin{array}{l}\text { April } 1977 \text { to March } 1986, \\
\text { April } 1989 \text { to March } 2014\end{array}$ & 34 & 0.038 & 0.76 & Not significant \\
\hline
\end{tabular}


Table 4. Results of Kendall's tau statistical test for detection of monotonic trends in the annual minimum 7-day average flow for 210 continuous-record streamgages in Alabama.-Continued

$\left[\mathrm{mi}^{2}\right.$, square mile; - , no trend analysis was done for this station; $<$, less than $]$

\begin{tabular}{|c|c|c|c|c|c|c|c|c|c|}
\hline $\begin{array}{c}\text { Site } \\
\text { index } \\
\text { number } \\
\text { (fig. 1) }\end{array}$ & $\begin{array}{l}\text { USGS } \\
\text { station } \\
\text { number }\end{array}$ & Station name & $\begin{array}{l}\text { Regulation } \\
\text { status }\end{array}$ & $\begin{array}{l}\text { Drainage area } \\
\qquad\left(\mathrm{mi}^{2}\right)\end{array}$ & Period of record analyzed & $\begin{array}{l}\text { Number } \\
\text { of climate } \\
\text { years used in } \\
\text { analysis }\end{array}$ & $\begin{array}{c}\text { Kendall's } \\
\text { tau }\end{array}$ & P-value & Trend direction \\
\hline 132 & 02450250 & Sipsey Fork near Grayson, AL & Unregulated & 92.1 & April 1967 to March 2014 & 47 & -0.034 & 0.73 & Not significant \\
\hline 133 & 02450500 & Sipsey Fork near Falls City, AL & Unregulated & 360 & April 1944 to March 1954 & 10 & -0.290 & 0.24 & Not significant \\
\hline 134 & 02450825 & $\begin{array}{l}\text { Clear Creek at New Hope Church near } \\
\text { Poplar Springs, AL }\end{array}$ & Unregulated & 101 & April 1994 to March 2014 & 20 & -0.100 & 0.54 & Not significant \\
\hline 135 & 02451000 & Clear Creek at Falls City, AL & Unregulated & 149 & April 1940 to March 1954 & 14 & 0.033 & 0.87 & Not significant \\
\hline 136 & 02453000 & Blackwater Creek near Manchester, AL & Unregulated & 181 & April 1939 to March 1971 & 32 & -0.010 & 0.94 & Not significant \\
\hline 136 & 02453000 & Blackwater Creek near Manchester, AL & Unregulated & 181 & $\begin{array}{l}\text { April } 1980 \text { to March } 1982, \\
\text { April } 1989 \text { to March } 2014\end{array}$ & 27 & 0.140 & 0.11 & Not significant \\
\hline 137 & 02453500 & Mulberry Fork at Cordova, AL & ${ }^{1}$ Unregulated & 1,916 & April 1901 to March 1912 & 11 & -0.055 & 0.82 & Not significant \\
\hline 138 & 02454000 & Lost Creek near Oakman, AL & Unregulated & 134 & $\begin{array}{l}\text { April } 1952 \text { to March } 1966, \\
\text { April } 1980 \text { to March } 1981\end{array}$ & 15 & 0.110 & 0.55 & Not significant \\
\hline 139 & 02454055 & Lost Creek above Parrish, AL & Unregulated & 143 & April 1993 to March 2014 & 21 & 0.057 & 0.72 & Not significant \\
\hline 140 & 02455000 & Locust Fork near Cleveland, AL & Unregulated & 303 & $\begin{array}{l}\text { April } 1937 \text { to March } 1986, \\
\text { April } 1993 \text { to March } 2014\end{array}$ & 70 & 0.072 & 0.38 & Not significant \\
\hline 141 & 02455500 & Locust Fork at Trafford, AL & Unregulated & 624 & $\begin{array}{l}\text { April } 1931 \text { to March } 1969, \\
\text { April } 1993 \text { to March } 1997\end{array}$ & 42 & 0.110 & 0.32 & Not significant \\
\hline 142 & 02455980 & $\begin{array}{l}\text { Turkey Creek at Sewage Plant near } \\
\text { Pinson, AL }\end{array}$ & Unregulated & 27.4 & April 1989 to March 2014 & 25 & -0.180 & 0.21 & Not significant \\
\hline 143 & 02456000 & Turkey Creek at Morris, AL & Unregulated & 80.9 & $\begin{array}{l}\text { April } 1944 \text { to March 1979, } \\
\text { April } 2002 \text { to March } 2011\end{array}$ & 42 & 0.460 & $<0.0001$ & Not significant \\
\hline 144 & 02456330 & Crooked Creek near Morris, AL & Unregulated & 16.2 & $\begin{array}{l}\text { November } 1975 \text { to } \\
\text { September } 1988 \text {, October } 1996 \\
\text { to September } 1997\end{array}$ & 12 & -0.450 & 0.04 & Downward \\
\hline 145 & 02456500 & Locust Fork at Sayre, AL & Unregulated & 885 & April 1964 to March 2014 & 50 & -0.021 & 0.83 & Not significant \\
\hline 146 & 02457000 & Fivemile Creek at Ketona, AL & Unregulated & 23.9 & $\begin{array}{l}\text { April } 1954 \text { to March 1958, } \\
\text { April } 1975 \text { to March 1979, } \\
\text { April } 1997 \text { to March 2014 }\end{array}$ & 25 & -0.020 & 0.89 & Not significant \\
\hline 147 & 02457595 & Fivemile Creek near Republic, AL & Unregulated & 51.9 & April 1989 to March 2014 & 25 & 0.034 & 0.82 & Not significant \\
\hline 148 & 02458300 & $\begin{array}{l}\text { Village Creek at 24th Street at } \\
\text { Birmingham, AL }\end{array}$ & Regulated & 26 & $\begin{array}{l}\text { April } 1989 \text { to March 2006, } \\
\text { April } 2009 \text { to March } 2013\end{array}$ & 21 & 0.260 & 0.10 & Not significant \\
\hline
\end{tabular}


Table 4. Results of Kendall's tau statistical test for detection of monotonic trends in the annual minimum 7-day average flow for 210 continuous-record streamgages in

Alabama.-Continued

$\left[\mathrm{mi}^{2}\right.$, square mile; - , no trend analysis was done for this station; $<$, less than]

\begin{tabular}{|c|c|c|c|c|c|c|c|c|c|}
\hline $\begin{array}{c}\text { Site } \\
\text { index } \\
\text { number } \\
\text { (fig. 1) }\end{array}$ & $\begin{array}{c}\text { USGS } \\
\text { station } \\
\text { number }\end{array}$ & Station name & $\begin{array}{l}\text { Regulation } \\
\text { status }\end{array}$ & $\begin{array}{l}\text { Drainage area } \\
\qquad\left(\mathrm{mi}^{2}\right)\end{array}$ & Period of record analyzed & $\begin{array}{l}\text { Number } \\
\text { of climate } \\
\text { years used in } \\
\text { analysis }\end{array}$ & $\begin{array}{c}\text { Kendall's } \\
\text { tau }\end{array}$ & P-value & Trend direction \\
\hline 149 & 02458450 & $\begin{array}{l}\text { Village Creek at Avenue West at } \\
\text { Ensley, AL }\end{array}$ & Regulated & 33.5 & $\begin{array}{l}\text { April } 1976 \text { to March 1979, } \\
\text { April } 1989 \text { to March } 2014\end{array}$ & 28 & -0.220 & 0.10 & Not significant \\
\hline 150 & 02458600 & Village Creek near Docena, AL & Unregulated & 52.2 & April 1997 to March 2014 & 17 & -0.350 & 0.05 & Not significant \\
\hline 151 & 02461500 & Valley Creek near Bessemer, AL & Unregulated & 52.5 & $\begin{array}{l}\text { April } 1976 \text { to March } 1979, \\
\text { April } 1989 \text { to March } 2014\end{array}$ & 28 & 0.021 & 0.87 & Not significant \\
\hline 152 & 02462000 & Valley Creek near Oak Grove, AL & Unregulated & 148 & $\begin{array}{l}\text { April } 1954 \text { to March } 1958, \\
\text { April } 1979 \text { to March } 2014\end{array}$ & 39 & 0.001 & 0.99 & Not significant \\
\hline 153 & 02462500 & $\begin{array}{l}\text { Black Warrior River at Bankhead L\&D } \\
\text { near Bessemer, AL }\end{array}$ & Regulated & 3,981 & - & - & - & - & - \\
\hline 154 & 02462600 & Blue Creek near Oakman, AL & Unregulated & 5.32 & $\begin{array}{l}\text { April } 1960 \text { to March } 1965, \\
\text { April } 1977 \text { to March } 1984\end{array}$ & 12 & 0.530 & 0.03 & Upward \\
\hline 155 & 02462800 & Davis Creek below Abernant, $\mathrm{AL}$ & Unregulated & 45.3 & April 1957 to March 1971 & 14 & -0.120 & 0.55 & Not significant \\
\hline 156 & 02462951 & $\begin{array}{l}\text { Black Warrior River at Holt L\&D near } \\
\text { Holt, AL }\end{array}$ & Regulated & 4,219 & - & - & - & - & - \\
\hline 157 & 02463500 & Hurricane Creek near Holt, AL & Unregulated & 108 & April 1953 to March 1969 & 16 & 0.380 & 0.04 & Upward \\
\hline 158 & 02464000 & North River near Samantha, AL & Unregulated & 223 & $\begin{array}{l}\text { April } 1939 \text { to March } 1954, \\
\text { April } 1969 \text { to March } 2014\end{array}$ & 60 & -0.025 & 0.78 & Not significant \\
\hline 159 & 02464146 & Turkey Creek near Tuscaloosa, AL & Unregulated & 6.16 & $\begin{array}{l}\text { April } 1981 \text { to March } 1984, \\
\text { April } 1987 \text { to March } 2014\end{array}$ & 30 & 0.021 & 0.87 & Not significant \\
\hline 160 & 02464360 & $\begin{array}{l}\text { Binion Creek below Gin Creek near } \\
\text { Samantha, AL }\end{array}$ & Unregulated & 57.2 & April 1987 to March 2014 & 27 & -0.170 & 0.21 & Not significant \\
\hline 161 & 02464500 & North River (Site B) near Tuscaloosa, AL & Unregulated & 372 & April 1952 to March 1968 & 16 & 0.430 & 0.02 & Upward \\
\hline 162 & 02465000 & $\begin{array}{l}\text { Black Warrior River at Oliver Lock and } \\
\text { Dam at Northport, AL }\end{array}$ & Regulated & 4,820 & April 1976 to March 2014 & 38 & -0.330 & 0.00 & Downward \\
\hline 163 & 02465200 & Lake Creek near Northport, AL & Regulated & 3.71 & April 1957 to March 1970 & 13 & -0.039 & 0.85 & Not significant \\
\hline 164 & 02465292 & $\begin{array}{l}\text { Cribbs Mill Creek at WW Plant at } \\
\text { Tuscaloosa, AL }\end{array}$ & Unregulated & 10.7 & April 2003 to March 2014 & 11 & 0.270 & 0.24 & Not significant \\
\hline 165 & 02465493 & Elliotts Creek at Moundville, AL & Unregulated & 32.3 & April 1977 to March 2014 & 37 & -0.220 & 0.05 & Not significant \\
\hline 166 & 02465500 & Fivemile Creek near Greensboro, AL & Unregulated & 73.6 & April 1955 to March 1971 & 16 & 0.220 & 0.24 & Not significant \\
\hline
\end{tabular}


Table 4. Results of Kendall's tau statistical test for detection of monotonic trends in the annual minimum 7-day average flow for 210 continuous-record streamgages in Alabama.-Continued

$\left[\mathrm{mi}^{2}\right.$, square mile; - , no trend analysis was done for this station; $<$, less than $]$

\begin{tabular}{|c|c|c|c|c|c|c|c|c|c|}
\hline $\begin{array}{c}\text { Site } \\
\text { index } \\
\text { number } \\
\text { (fig. 1) }\end{array}$ & $\begin{array}{c}\text { USGS } \\
\text { station } \\
\text { number }\end{array}$ & Station name & $\begin{array}{l}\text { Regulation } \\
\text { status }\end{array}$ & $\begin{array}{l}\text { Drainage area } \\
\qquad\left(\mathrm{mi}^{2}\right)\end{array}$ & Period of record analyzed & $\begin{array}{l}\text { Number } \\
\text { of climate } \\
\text { years used in } \\
\text { analysis }\end{array}$ & $\begin{array}{l}\text { Kendall's } \\
\text { tau }\end{array}$ & P-value & Trend direction \\
\hline 167 & 02466030 & $\begin{array}{l}\text { Black Warrior River at Selden L\&D near } \\
\text { Eutaw, AL }\end{array}$ & Regulated & 5,810 & April 1977 to March 2014 & 36 & -0.096 & 0.40 & Not significant \\
\hline 168 & 02466500 & Big Prairie Creek near Gallion, AL & Unregulated & 171 & April 1940 to March 1952 & 12 & 0.180 & 0.41 & Not significant \\
\hline 169 & 02467000 & $\begin{array}{l}\text { Tombigbee River at Demopolis L\&D near } \\
\text { Coatopa, AL }\end{array}$ & Regulated & 15,385 & April 1985 to March 2014 & 29 & -0.130 & 0.34 & Not significant \\
\hline 170 & 02467500 & Sucarnoochee River at Livingston, $\mathrm{AL}$ & Unregulated & 607 & April 1939 to March 2014 & 75 & 0.020 & 0.76 & Not significant \\
\hline 171 & 02468000 & Alamuchee Creek near Cuba, AL & Unregulated & 62.3 & April 1955 to March 1967 & 12 & 0.240 & 0.27 & Not significant \\
\hline 172 & 02468500 & Chickasaw Bogue near Linden, AL & Unregulated & 257 & $\begin{array}{l}\text { April } 1944 \text { to March } 1946, \\
\text { April } 1966 \text { to March } 1988\end{array}$ & 24 & 0.150 & 0.30 & Not significant \\
\hline 173 & 02469000 & Kinterbish Creek near York, AL & Unregulated & 90.9 & April 1955 to March 1967 & 12 & 0.360 & 0.10 & Not significant \\
\hline 174 & 02469500 & Tuckabum Creek near Butler, AL & Unregulated & 115 & April 1955 to March 1970 & 15 & 0.020 & 0.92 & Not significant \\
\hline 175 & 02469550 & Horse Creek near Sweetwater, AL & Unregulated & 60.4 & April 1960 to March 1970 & 10 & -0.470 & 0.06 & Not significant \\
\hline 176 & 02469700 & Okatuppa Creek at Gilbertown, AL & Unregulated & 148 & April 1957 to March 1969 & 12 & -0.270 & 0.22 & Not significant \\
\hline 177 & 02469761 & $\begin{array}{l}\text { Tombigbee River at Coffeeville L\&D near } \\
\text { Coffeeville, } \mathrm{AL}\end{array}$ & Regulated & 18,417 & April 1985 to March 2014 & 28 & -0.044 & 0.74 & Not significant \\
\hline 178 & 02469800 & Satilpa Creek near Coffeeville, AL & Unregulated & 164 & $\begin{array}{l}\text { April } 1957 \text { to March } 1970, \\
\text { April } 1975 \text { to March } 2014\end{array}$ & 52 & -0.140 & 0.14 & Not significant \\
\hline 179 & 02470072 & $\begin{array}{l}\text { Bassett Creek at U.S. Highway } 43 \text { near } \\
\text { Thomasville, AL }\end{array}$ & Unregulated & 10.5 & April 1996 to March 2014 & 18 & 0.050 & 0.76 & Not significant \\
\hline 180 & 02470100 & Bassett Creek at Walker Springs, AL & Unregulated & 195 & April 1957 to March 1970 & 13 & -0.560 & 0.01 & Downward \\
\hline 181 & 02471001 & Chickasaw Creek near Kushla, AL & Unregulated & 125 & April 1952 to March 2014 & 62 & -0.110 & 0.20 & Not significant \\
\hline 182 & 02471065 & $\begin{array}{l}\text { Montlimar Creek at U.S. Highway } 90 \text { at } \\
\text { Mobile, AL }\end{array}$ & Unregulated & 7.28 & $\begin{array}{l}\text { April } 1963 \text { to March 1967, } \\
\text { April } 1975 \text { to March } 1983\end{array}$ & 12 & 0.520 & 0.02 & Upward \\
\hline 183 & 02471078 & $\begin{array}{l}\text { Fowl River at Half-Mile Road near } \\
\text { Laurendine, } \mathrm{AL}\end{array}$ & Unregulated & 16.5 & April 1995 to March 2014 & 19 & -0.350 & 0.04 & Downward \\
\hline 184 & 02479431 & Pond Creek near Deer Park, AL & Unregulated & 20.4 & April 1977 to March 1999 & 22 & -0.091 & 0.55 & Not significant \\
\hline 185 & 02479500 & Escatawapa River near Wilmer, AL & Unregulated & 511 & April 1946 to March 1973 & 27 & -0.190 & 0.18 & Not significant \\
\hline 186 & 02479560 & Escatawapa River near Agricola, AL & Unregulated & 562 & April 1974 to March 2014 & 40 & -0.300 & 0.01 & Downward \\
\hline
\end{tabular}


Table 4. Results of Kendall's tau statistical test for detection of monotonic trends in the annual minimum 7-day average flow for 210 continuous-record streamgages in

Alabama.-Continued

$\left[\mathrm{mi}^{2}\right.$, square mile; - , no trend analysis was done for this station; $<$, less than]

\begin{tabular}{|c|c|c|c|c|c|c|c|c|c|}
\hline $\begin{array}{c}\text { Site } \\
\text { index } \\
\text { number } \\
\text { (fig. 1) }\end{array}$ & $\begin{array}{l}\text { USGS } \\
\text { station } \\
\text { number }\end{array}$ & Station name & $\begin{array}{l}\text { Regulation } \\
\text { status }\end{array}$ & $\begin{array}{l}\text { Drainage area } \\
\qquad\left(\mathrm{mi}^{2}\right)\end{array}$ & Period of record analyzed & $\begin{array}{l}\text { Number } \\
\text { of climate } \\
\text { years used in } \\
\text { analysis }\end{array}$ & $\begin{array}{l}\text { Kendall's } \\
\text { tau }\end{array}$ & P-value & Trend direction \\
\hline 187 & 02479945 & $\begin{array}{l}\text { Big Creek at County Road } 63 \text { near } \\
\text { Wilmer, AL }\end{array}$ & Unregulated & 31.48 & April 1991 to March 2014 & 23 & -0.150 & 0.33 & Not significant \\
\hline 188 & 02479980 & Crooked Creek near Fairview, AL & Unregulated & 8.08 & April 1991 to March 2014 & 23 & -0.260 & 0.08 & Not significant \\
\hline 189 & 02480002 & $\begin{array}{l}\text { Hamilton Creek at Snow Road near } \\
\text { Semmes, AL }\end{array}$ & Unregulated & 8.22 & April 1991 to March 2014 & 23 & -0.330 & 0.03 & Downward \\
\hline 190 & 03572110 & Crow Creek at Bass, $\mathrm{AL}$ & Unregulated & 131 & April 1976 to March 1996 & 20 & 0.040 & 0.80 & Not significant \\
\hline 191 & 03572900 & Town Creek near Geraldine, AL & Unregulated & 141 & April 1958 to March 1980 & 22 & 0.160 & 0.28 & Not significant \\
\hline 192 & 03574500 & Paint Rock River near Woodville, AL & Unregulated & 320 & April 1936 to March 2014 & 78 & 0.090 & 0.26 & Not significant \\
\hline 193 & 0357479650 & $\begin{array}{l}\text { Hester Creek at Buddy Williamson Road } \\
\text { near Plevna, AL }\end{array}$ & Unregulated & 33 & April 1999 to March 2013 & 14 & -0.540 & 0.01 & Downward \\
\hline 194 & 03575000 & Flint River near Chase, $\mathrm{AL}$ & Unregulated & 342 & $\begin{array}{l}\text { April } 1931 \text { to March } 1981, \\
\text { April } 1983 \text { to March } 1994\end{array}$ & 61 & 0.150 & 0.09 & Not significant \\
\hline 195 & 03575100 & Flint River at Brownsboro, AL & Unregulated & 375 & April 1999 to March 2014 & 15 & -0.030 & 0.88 & Not significant \\
\hline 196 & 03575500 & Tennessee River at Whitesburg, AL & ${ }^{1}$ Unregulated & 25,610 & April 1925 to March 1936 & 11 & 0.020 & 0.94 & Not significant \\
\hline 197 & 03575830 & Indian Creek near Madison, AL & Unregulated & 49 & $\begin{array}{l}\text { April } 1960 \text { to March } 1966, \\
\text { April } 1976 \text { to March } 2002\end{array}$ & 32 & -0.110 & 0.39 & Not significant \\
\hline 198 & 03576148 & Cotaco Creek at Florette, $\mathrm{AL}$ & Unregulated & 136 & April 1966 to March 1980 & 14 & -0.010 & 0.96 & Not significant \\
\hline 199 & 03576250 & Limestone Creek near Athens, AL & Unregulated & 119 & $\begin{array}{l}\text { April } 1940 \text { to March 1970, } \\
\text { April } 1995 \text { to March } 2014\end{array}$ & 49 & 0.080 & 0.44 & Not significant \\
\hline 200 & 03576500 & Flint Creek near Falkville, AL & Unregulated & 86.3 & $\begin{array}{l}\text { April } 1953 \text { to March 1970, } \\
\text { April } 1993 \text { to March 1999, } \\
\text { April } 2012 \text { to March 2014 }\end{array}$ & 25 & 0.340 & 0.02 & Upward \\
\hline 201 & 03585300 & Sugar Creek near Good Springs, AL & Unregulated & 152 & April 1958 to March 1969. & 11 & 0.160 & 0.48 & Not significant \\
\hline 202 & 03586500 & Big Nance Creek at Coutland, AL & Unregulated & 166 & $\begin{array}{l}\text { April } 1936 \text { to March } 1940, \\
\text { April } 1945 \text { to March } 1981\end{array}$ & 40 & 0.200 & 0.07 & Not significant \\
\hline 202 & 03586500 & Big Nance Creek at Coutland, AL & Unregulated & 166 & April 1988 to March 2014 & 26 & -0.160 & 0.27 & Not significant \\
\hline 203 & 03589500 & Tennessee River at Florence, AL & ${ }^{1}$ Unregulated & 30,810 & April 1895 to March 1924 & 29 & -0.090 & 0.48 & Not significant \\
\hline 204 & 03590000 & Cypress Creek near Florence, AL & Unregulated & 209 & April 1935 to March 1953 & 18 & 0.370 & 0.03 & Upward \\
\hline
\end{tabular}


Table 4. Results of Kendall's tau statistical test for detection of monotonic trends in the annual minimum 7-day average flow for 210 continuous-record streamgages in Alabama.-Continued

$\left[\mathrm{mi}^{2}\right.$, square mile; - , no trend analysis was done for this station; $<$, less than $]$

\begin{tabular}{|c|c|c|c|c|c|c|c|c|c|}
\hline $\begin{array}{c}\text { Site } \\
\text { index } \\
\text { number } \\
\text { (fig. 1) }\end{array}$ & $\begin{array}{l}\text { USGS } \\
\text { station } \\
\text { number }\end{array}$ & Station name & $\begin{array}{l}\text { Regulation } \\
\text { status }\end{array}$ & $\begin{array}{c}\text { Drainage area } \\
\qquad\left(\mathrm{mi}^{2}\right)\end{array}$ & Period of record analyzed & $\begin{array}{l}\text { Number } \\
\text { of climate } \\
\text { years used in } \\
\text { analysis }\end{array}$ & $\begin{array}{l}\text { Kendall's } \\
\text { tau }\end{array}$ & P-value & Trend direction \\
\hline 205 & 03590500 & Tuscumbia Spring at Tuscumbia, AL & Unregulated & Indeterminate & $\begin{array}{l}\text { April } 1929 \text { to March } 1930, \\
\text { April } 1956 \text { to March } 1965\end{array}$ & 11 & -0.450 & 0.05 & Downward \\
\hline 206 & 03591800 & Bear Creek near Hackeburg, AL & Unregulated & 143 & April 1957 to March 1981 & 22 & 0.250 & 0.11 & Not significant \\
\hline 207 & 03592000 & Bear Creek near Red Bay, AL & ${ }^{1}$ Unregulated & 263 & $\begin{array}{l}\text { April } 1914 \text { to March } 1920, \\
\text { April } 1959 \text { to March } 1967\end{array}$ & 14 & 0.030 & 0.87 & Not significant \\
\hline 208 & 03592200 & Cedar Creek near Pleasant Site, AL & Unregulated & 189 & April 1958 to March 1977 & 19 & 0.190 & 0.25 & Not significant \\
\hline 209 & 03592300 & Little Bear Creek near Halltown, AL & ${ }^{1}$ Unregulated & 78.2 & April 1958 to March 1975 & 17 & 0.290 & 0.09 & Not significant \\
\hline 210 & 03592500 & Bear Creek at Bishop, AL & ${ }^{1}$ Unregulated & 667 & $\begin{array}{l}\text { April } 1927 \text { to March 1928, } \\
\text { April } 1929 \text { to March 1932, } \\
\text { April } 1934 \text { to March 1979 }\end{array}$ & 49 & 0.230 & 0.02 & Upward \\
\hline
\end{tabular}

'Site is currently (2017) regulated, but low-flow frequency statistics were based on pre-regulation data. 
Table 5. Low-flow statistics for continuous-record streamgaging stations of selected streams in Alabama and surrounding States.

[lat, latitude; long, longitude; ft, foot; mi, mile; $\mathrm{mi}^{2}$, square mile; $\mathrm{ft}^{3}$, cubic foot; USGS, U.S. Geological Survey; $\mathrm{ft}^{3} / \mathrm{s}$, cubic foot per second; water year, the 12-month period from October 1 through September 30 and designated by the year in which the period ends]

Notes: The station low-flow statistics are presented in the following pages in numerical order by station number. See figure 1 for location of the streamgaging stations. 
STATION NAME AND NUMBER—02339225 Wehadkee Creek below Rock Mills, AL

LOCATION.-Lat $33^{\circ} 07^{\prime} 20^{\prime \prime}$, long $85^{\circ} 14^{\prime} 7^{\prime \prime}$ referenced to North American Datum of 1927, Randolph County, AL, Hydrologic Unit 03130002, on county road, 0.7 mi downstream from Little Wehadkee Creek, 2.1 mi upstream from Guss Creek, and 3.5 mi southeast of Rock Mills.

DRAINAGE AREA. $-60.2 \mathrm{mi}^{2}$.

PERIOD OF RECORD.-October 1978 to January 1990.

PERIOD OF ANALYSIS.-_April 1979 to March 1989.

NUMBER OF CLIMATE YEARS IN FREQUENCY ANALYSIS.-10

\section{REMARKS.-}

\begin{tabular}{|c|c|c|c|c|}
\hline \multirow[t]{2}{*}{$\begin{array}{l}\text { Recurrence } \\
\text { intervals } \\
\text { (years) }\end{array}$} & \multicolumn{2}{|c|}{$\begin{array}{l}\text { Lowest average flow for } \\
\text { indicated number of } \\
\text { consecutive days } \\
\text { (cubic feet per second) }\end{array}$} & \multicolumn{2}{|c|}{$\begin{array}{l}\text { Time-sampling error } \\
\text { (in percent) }\end{array}$} \\
\hline & 1 & 7 & 1 & 7 \\
\hline 2 & 9.0 & 10 & 27 & 27 \\
\hline 5 & 4.6 & 5.2 & 34 & 34 \\
\hline 10 & 3.1 & 3.4 & 46 & 46 \\
\hline 20 & 2.1 & 2.3 & 67 & 68 \\
\hline
\end{tabular}

\section{DURATION OF DAILY FLOW}

Flow equaled or exceeded for indicated percentage of time (cubic feet per second)

\begin{tabular}{|c|c|c|c|c|c|c|c|}
\hline Percentage & 5 & 10 & 25 & 50 & 75 & 90 & 95 \\
\hline Flow & 275 & 166 & 91 & 48 & 27 & 15 & 9.6 \\
\hline
\end{tabular}


STATION NAME AND NUMBER—02342500 Uchee Creek near Fort Mitchell, AL

LOCATION.--Lat $32^{\circ} 19^{\prime} 00^{\prime \prime}$, long $85^{\circ} 00^{\prime} 54^{\prime \prime}$ referenced to North American Datum of 1927, Russell County, AL, Hydrologic Unit 03130003, at bridge on State Highway 165, 2 mi south of Fort Mitchell, 4.8 mi downstream of Little Uchee Creek, and 5.3 mi upstream of mouth.

DRAINAGE AREA.-322 $\mathrm{mi}^{2}$.

PERIOD OF RECORD.- October 1946 to March 2014.

PERIOD OF ANALYSIS.-April 1947 to March 2014.

NUMBER OF CLIMATE YEARS IN FREQUENCY ANALYSIS. - 67

REMARKS.-

\begin{tabular}{|c|c|c|c|c|}
\hline $\begin{array}{l}\text { Recurrence } \\
\text { intervals } \\
\text { (years) }\end{array}$ & \multicolumn{2}{|c|}{$\begin{array}{l}\text { Lowest average flow for } \\
\text { indicated number of } \\
\text { consecutive days } \\
\text { (cubic feet per second) }\end{array}$} & \multicolumn{2}{|c|}{$\begin{array}{l}\text { Time-sampling error } \\
\text { (in percent) }\end{array}$} \\
\hline & 1 & 7 & 1 & 7 \\
\hline 2 & 16 & 17 & 12 & 11 \\
\hline 5 & 7.7 & 8.0 & 15 & 14 \\
\hline 10 & 4.8 & 5.0 & 20 & 19 \\
\hline 20 & 3.1 & 3.2 & 28 & 27 \\
\hline 50 & 1.8 & 1.9 & 43 & 40 \\
\hline
\end{tabular}

\section{DURATION OF DAILY FLOW}

Flow equaled or exceeded for indicated percentage of time (cubic feet per second)

\begin{tabular}{|c|c|c|c|c|c|c|c|}
\hline Percentage & 5 & 10 & 25 & 50 & 75 & 90 & 95 \\
\hline Flow & 1,470 & 921 & 443 & 167 & 53 & 23 & 14 \\
\hline
\end{tabular}


STATION NAME AND NUMBER—02342933 South Fork Cowikee Creek near Batesville, AL

LOCATION.-Lat $32^{\circ} 01^{\prime} 03^{\prime \prime}$, long $85^{\circ} 17^{\prime} 45^{\prime \prime}$ referenced to North American Datum of 1927, Barbour County, AL, Hydrologic Unit 03130003, on left bank at downstream side of bridge on county road, 0.1 mi downstream of Bear Creek, 1.2 mi northeast of Batesville, 11.2 mi northwest of Eufaula, and 13.0 mi upstream of mouth.

DRAINAGE AREA. - $112 \mathrm{mi}^{2}$.

PERIOD OF RECORD.- October 1963 to September 1971, October 1974 to October 2011.

PERIOD OF ANALYSIS.-April 1964 to March 1971, April 1975 to March 2011.

NUMBER OF CLIMATE YEARS IN FREQUENCY ANALYSIS.-43

\section{REMARKS.-}

\begin{tabular}{|c|c|c|c|c|}
\hline $\begin{array}{c}\text { Recurrence } \\
\text { intervals } \\
\text { (years) }\end{array}$ & \multicolumn{2}{|c|}{$\begin{array}{c}\text { Lowest average flow for } \\
\text { indicated number of } \\
\text { consecutive days } \\
\text { (cubic feet per second) }\end{array}$} & \multicolumn{2}{|c|}{$\begin{array}{c}\text { Time-sampling error } \\
\text { (in percent) }\end{array}$} \\
\hline & 1 & 7 & 1 & 7 \\
\hline 2 & 1.9 & 2.4 & 19 & 20 \\
\hline 5 & 0.73 & 0.95 & 22 & 25 \\
\hline 10 & 0.42 & 0.57 & 28 & 32 \\
\hline 20 & 0.26 & 0.37 & 36 & 43 \\
\hline 50 & 0.15 & 0.23 & 49 & \\
\hline
\end{tabular}

\begin{tabular}{|c|c|c|c|c|c|c|c|}
\hline \multicolumn{7}{|c|}{ DURATION OF DAILY FLOW } \\
\hline \multicolumn{7}{|c|}{ Flow equaled or exceeded for indicated percentage of time } \\
(cubic feet per second)
\end{tabular}


STATION NAME AND NUMBER—02343300 Abbie Creek near Haleburg, AL

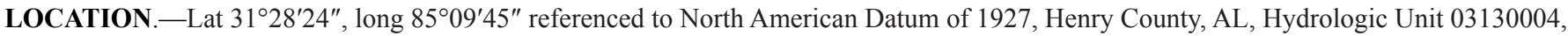
on State Highway 95, 1.2 mi upstream from Peterman Creek, 4.5 mi northwest of Haleburg, 7.8 mi upstream from mouth, and 9 mi southeast of Abbeville.

DRAINAGE AREA. - $146 \mathrm{mi}^{2}$.

PERIOD OF RECORD.- October 1958 to September 1971, October 1974 to August 1993.

PERIOD OF ANALYSIS.-April 1959 to March 1971, April 1975 to March 1993.

NUMBER OF CLIMATE YEARS IN FREQUENCY ANALYSIS.-30

REMARKS.-

\begin{tabular}{|c|c|c|c|c|}
\hline $\begin{array}{l}\text { Recurrence } \\
\text { intervals } \\
\text { (years) }\end{array}$ & \multicolumn{2}{|c|}{$\begin{array}{l}\text { Lowest average flow for } \\
\text { indicated number of } \\
\text { consecutive days } \\
\text { (cubic feet per second) }\end{array}$} & \multicolumn{2}{|c|}{$\begin{array}{l}\text { Time-sampling error } \\
\text { (in percent) }\end{array}$} \\
\hline & 1 & 7 & 1 & 7 \\
\hline 2 & 37 & 41 & 10 & 10 \\
\hline 5 & 24 & 27 & 12 & 11 \\
\hline 10 & 18 & 21 & 15 & 13 \\
\hline 20 & 15 & 17 & 20 & 16 \\
\hline 50 & 11 & 14 & 27 & 22 \\
\hline
\end{tabular}

\begin{tabular}{|c|c|c|c|c|c|c|c|c|}
\hline \multicolumn{1}{|c|}{ DURATION OF DAILY FLOW } \\
\hline \multicolumn{7}{|c|}{ Flow equaled or exceeded for indicated percentage of time } \\
(cubic feet per second)
\end{tabular}


STATION NAME AND NUMBER—02343500 Chattahoochee River at Columbia, AL

LOCATION.-Lat $31^{\circ} 17^{\prime} 01^{\prime \prime}$, long $85^{\circ} 05^{\prime} 58^{\prime \prime}$ referenced to North American Datum of 1927, Houston County, AL, Hydrologic Unit 03130004, at State Highway 52, half a mile upstream from Omussee Creek and half a mile east of Columbia.

DRAINAGE AREA. $-8,040 \mathrm{mi}^{2}$.

PERIOD OF RECORD.- July 1928 to September 1960.

PERIOD OF ANALYSIS.-April 1929 to March 1960.

NUMBER OF CLIMATE YEARS IN FREQUENCY ANALYSIS.-31

REMARKS.-Since 1962, site in backwater from Columbia Lock and Dam. Results represent pre-regulation conditions.

\begin{tabular}{|c|c|c|c|c|}
\hline \multirow[t]{2}{*}{$\begin{array}{l}\text { Recurrence } \\
\text { intervals } \\
\text { (years) }\end{array}$} & \multicolumn{2}{|c|}{$\begin{array}{l}\text { Lowest average flow for } \\
\text { indicated number of } \\
\text { consecutive days } \\
\text { (cubic feet per second) }\end{array}$} & \multicolumn{2}{|c|}{$\begin{array}{l}\text { Time-sampling error } \\
\text { (in percent) }\end{array}$} \\
\hline & 1 & 7 & 1 & 7 \\
\hline 2 & 2,540 & 2,870 & 7 & 7 \\
\hline 5 & 1,890 & 2,070 & 8 & 8 \\
\hline 10 & 1,610 & 1,730 & 10 & 11 \\
\hline 20 & 1,390 & 1,480 & 12 & 14 \\
\hline 50 & 1,180 & 1,230 & 16 & 18 \\
\hline
\end{tabular}

\section{DURATION OF DAILY FLOW}

Flow equaled or exceeded for indicated percentage of time (cubic feet per second)

\begin{tabular}{|c|c|c|c|c|c|c|c|}
\hline Percentage & 5 & 10 & 25 & 50 & 75 & 90 & 95 \\
\hline Flow & 29,400 & 19,800 & 11,800 & 7,320 & 4,610 & 3,180 & 2,570 \\
\hline
\end{tabular}


STATION NAME AND NUMBER—02360500 East Fork Choctawhatchee River near Midland City, AL

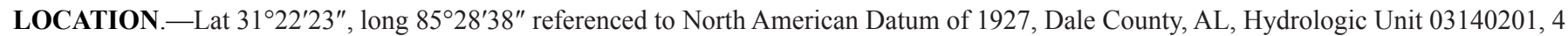
mi upstream from West Fork Choctawhatchee River and 4 mi north of Midland City.

DRAINAGE AREA.-291 $\mathrm{mi}^{2}$.

PERIOD OF RECORD.- June 1952 to September 1963.

PERIOD OF ANALYSIS.-April 1953 to March 1963.

NUMBER OF CLIMATE YEARS IN FREQUENCY ANALYSIS.-10

REMARKS.-

\begin{tabular}{|c|c|c|c|c|}
\hline $\begin{array}{c}\text { Recurrence } \\
\text { intervals } \\
\text { (years) }\end{array}$ & \multicolumn{2}{|c|}{$\begin{array}{c}\text { Lowest average flow for } \\
\text { indicated number of } \\
\text { consecutive days } \\
\text { (cubic feet per second) }\end{array}$} & \multicolumn{2}{|c|}{$\begin{array}{c}\text { Time-sampling error } \\
\text { (in percent) }\end{array}$} \\
\hline \multicolumn{7}{|c|}{1} & 7 & 1 & 7 \\
\hline 2 & 54 & 59 & 14 & 15 \\
\hline 5 & 38 & 40 & 17 & 24 \\
\hline 10 & 31 & 32 & 23 & 32 \\
\hline 20 & 25 & 27 & 30 & \\
\hline
\end{tabular}

\begin{tabular}{|c|c|c|c|c|c|}
\hline \multicolumn{1}{|c|}{ DURATION OF DAILY FLOW } \\
\hline \multicolumn{7}{|c|}{ Flow equaled or exceeded for indicated percentage of time } \\
(cubic feet per second)
\end{tabular}


STATION NAME AND NUMBER—02361000 Choctawhatchee River near Newton, AL

LOCATION.-Lat $31^{\circ} 20^{\prime} 34^{\prime \prime}$, long 85³6'38" referenced to North American Datum of 1927, Dale County, AL, Hydrologic Unit 03140201, on left bank at downstream side of bridge on State Highway 123, $100 \mathrm{ft}$ upstream of abandoned mill dam, 1,800 ft upstream of Hurricane Creek, $0.8 \mathrm{mi}$ north of Newton, $1 \mathrm{mi}$ downstream of Atlantic Coast Line Railroad bridge, and at mile 133.0.

DRAINAGE AREA. $-686 \mathrm{mi}^{2}$.

PERIOD OF RECORD.-December 1921 to September 1927, June 1935 to March 2014.

PERIOD OF ANALYSIS.-April 1922 to March 1927, April 1936 to March 2014.

NUMBER OF CLIMATE YEARS IN FREQUENCY ANALYSIS.- -83

\section{REMARKS.-}

\begin{tabular}{|c|c|c|c|c|}
\hline $\begin{array}{c}\text { Recurrence } \\
\text { intervals } \\
\text { (years) }\end{array}$ & \multicolumn{2}{|c|}{$\begin{array}{c}\text { Lowest average flow for } \\
\text { indicated number of } \\
\text { consecutive days } \\
\text { (cubic feet per second) }\end{array}$} & \multicolumn{2}{|c|}{$\begin{array}{c}\text { Time-sampling error } \\
\text { (in percent) }\end{array}$} \\
\hline & 1 & 7 & 1 & 7 \\
\hline 2 & 127 & 144 & 6 & 7 \\
\hline 5 & 80 & 91 & 7 & 8 \\
\hline 10 & 63 & 71 & 9 & 10 \\
\hline 20 & 51 & 58 & 11 & 14 \\
\hline 50 & 41 & 46 & 14 & \\
\hline
\end{tabular}

\begin{tabular}{|c|c|c|c|c|c|c|c|}
\hline \multicolumn{7}{|c|}{ DURATION OF DAILY FLOW } \\
\hline \multicolumn{7}{|c|}{$\begin{array}{c}\text { Flow equaled or exceeded for indicated percentage of time } \\
\text { (cubic feet per second) }\end{array}$} \\
\hline Percentage & 5 & 10 & 25 & 50 & 75 & 90 & 95 \\
\hline Flow & 2,710 & 1,920 & 1,090 & 562 & 297 & 170 & 123 \\
\hline
\end{tabular}


STATION NAME AND NUMBER—02361500 Choctawhatchee River near Bellwood, AL

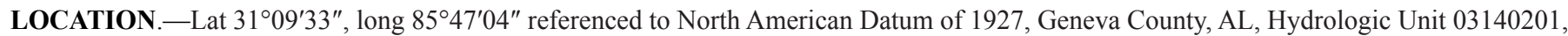
at bridge on County Road 45, 0.1 mi downstream of Wilkerson Creek, 1 mi southeast of Bellwood, 4.0 mi downstream of Claybank Creek, and at river mile 109.3.

DRAINAGE AREA.- $-1,280 \mathrm{mi}^{2}$.

PERIOD OF RECORD.--December 1921 to October 1925, December 2000 to March 2014.

PERIOD OF ANALYSIS.-April 1922 to March 1925, April 2001 to March 2014.

NUMBER OF CLIMATE YEARS IN FREQUENCY ANALYSIS.-16

REMARKS.-Frequency statistics were computed based on a MOVE.1 correlation with station 02361000 Choctawhatchee River near Newton, AL, which has a period of record from December 1921 to September 1927, June 1935 to March 2014.

\begin{tabular}{|c|c|c|c|c|}
\hline $\begin{array}{l}\text { Recurrence } \\
\text { intervals } \\
\text { (years) }\end{array}$ & \multicolumn{2}{|c|}{$\begin{array}{l}\text { Lowest average flow for } \\
\text { indicated number of } \\
\text { consecutive days } \\
\text { (cubic feet per second) }\end{array}$} & \multicolumn{2}{|c|}{$\begin{array}{l}\text { Time-sampling error } \\
\text { (in percent) }\end{array}$} \\
\hline & 1 & 7 & 1 & 7 \\
\hline 2 & 393 & 432 & 11 & 11 \\
\hline 5 & 291 & 316 & 13 & 12 \\
\hline 10 & 249 & 267 & 16 & 15 \\
\hline 20 & 217 & 233 & 20 & 18 \\
\hline 50 & 189 & 199 & 27 & 24 \\
\hline
\end{tabular}

\section{DURATION OF DAILY FLOW}

Flow equaled or exceeded for indicated percentage of time (cubic feet per second)

\begin{tabular}{|c|c|c|c|c|c|c|c|}
\hline Percentage & 5 & 10 & 25 & 50 & 75 & 90 & 95 \\
\hline Flow & 4,670 & 3,320 & 1,830 & 1,040 & 595 & 373 & 300 \\
\hline
\end{tabular}


STATION NAME AND NUMBER—02362240 Little Double Bridges Creek near Enterprise, AL

LOCATION.-Lat $31^{\circ} 16^{\prime} 20^{\prime \prime}$, long 85 57'30" referenced to North American Datum of 1927, Coffee County, AL, Hydrologic Unit 03140201, near right bank on downstream side of bridge on County Road 18, 8.4 mi southwest of Enterprise.

DRAINAGE AREA. $-21.4 \mathrm{mi}^{2}$.

PERIOD OF RECORD.--August 1985 to March 2014.

PERIOD OF ANALYSIS.-April 1986 to March 2014.

NUMBER OF CLIMATE YEARS IN FREQUENCY ANALYSIS.-28

REMARKS.-

\begin{tabular}{|c|c|c|c|c|}
\hline $\begin{array}{c}\text { Recurrence } \\
\text { intervals } \\
\text { (years) }\end{array}$ & \multicolumn{2}{|c|}{$\begin{array}{c}\text { Lowest average flow for } \\
\text { indicated number of } \\
\text { consecutive days } \\
\text { (cubic feet per second) }\end{array}$} & \multicolumn{2}{|c|}{$\begin{array}{c}\text { Time-sampling error } \\
\text { (in percent) }\end{array}$} \\
\hline \multicolumn{5}{|c|}{} \\
1 & 5.9 & 7 & 1 & 7 \\
\hline 2 & 3.4 & 4.1 & 13 & 16 \\
\hline 5 & 2.4 & 2.9 & 16 & 22 \\
\hline 10 & 1.8 & 2.2 & 30 & 30 \\
\hline 20 & & & & \\
\hline
\end{tabular}

\section{DURATION OF DAILY FLOW}

Flow equaled or exceeded for indicated percentage of time (cubic feet per second)

\begin{tabular}{|c|c|c|c|c|c|c|c|}
\hline Percentage & 5 & 10 & 25 & 50 & 75 & 90 & 95 \\
\hline Flow & 89 & 57 & 34 & 21 & 13 & 8.3 & 6.1 \\
\hline
\end{tabular}


STATION NAME AND NUMBER—02363000 Pea River near Ariton, AL

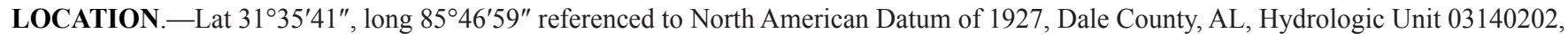
in SW $1 / 4$ sec. 7, T. 7 N., R. 23 E., Dale County, on left bank at downstream side of abandoned bridge and about 20 feet upstream of bridge on U.S. Highway 231, 2.2 miles downstream of Bryors Mill Creek, 2.8 miles downstream of Atlantic Coastline Railroad bridge, 3.5 miles west of Ariton, and at mile 92.5 .

DRAINAGE AREA. $-498 \mathrm{mi}^{2}$.

PERIOD OF RECORD.-October 1938 to September 1970, October 1987 to March 2014.

PERIOD OF ANALYSIS.-April 1939 to March 1970, April 1988 to March 2014.

NUMBER OF CLIMATE YEARS IN FREQUENCY ANALYSIS.—-57

REMARKS. -

\begin{tabular}{|c|c|c|c|c|}
\hline $\begin{array}{c}\text { Recurrence } \\
\text { intervals } \\
\text { (years) }\end{array}$ & \multicolumn{2}{|c|}{$\begin{array}{l}\text { Lowest average flow for } \\
\text { indicated number of } \\
\text { consecutive days } \\
\text { (cubic feet per second) }\end{array}$} & \multicolumn{2}{|c|}{$\begin{array}{l}\text { Time-sampling error } \\
\text { (in percent) }\end{array}$} \\
\hline & 1 & 7 & 1 & 7 \\
\hline 2 & 27 & 31 & 11 & 11 \\
\hline 5 & 14 & 16 & 13 & 13 \\
\hline 10 & 9.5 & 11 & 16 & 16 \\
\hline 20 & 6.9 & 7.9 & 21 & 20 \\
\hline 50 & 4.7 & 5.4 & 28 & 27 \\
\hline
\end{tabular}

\section{DURATION OF DAILY FLOW}

Flow equaled or exceeded for indicated percentage of time (cubic feet per second)

\begin{tabular}{|c|c|c|c|c|c|c|c|}
\hline Percentage & 5 & 10 & 25 & 50 & 75 & 90 & 95 \\
\hline Flow & 1,970 & 1,350 & 701 & 281 & 98 & 39 & 23 \\
\hline
\end{tabular}


STATION NAME AND NUMBER—02364500 Pea River near Samson, AL

LOCATION.-Lat $31^{\circ} 06^{\prime} 45^{\prime \prime}$, long $86^{\circ} 05^{\prime} 58^{\prime \prime}$ referenced to North American Datum of 1927, Geneva County, AL, Hydrologic Unit 03140202, at Alabama Highway 52 bridge, $500 \mathrm{ft}$ downstream of Boyenton Creek, 6.5 mi upstream of Flat Creek, and 3 mi north of Samson.

DRAINAGE AREA.- $-1,182 \mathrm{mi}^{2}$.

PERIOD OF RECORD.-September 1904 to August 1913, October 1922 to September 1925, October 1935 to September 1970 , October 2002 to March 2014.

PERIOD OF ANALYSIS.-April 1905 to March 1913, April 1923 to March 1925, April 1936 to March 1970, April 2003 to March 2014.

NUMBER OF CLIMATE YEARS IN FREQUENCY ANALYSIS.- 55

REMARKS.-

\begin{tabular}{|c|c|c|c|c|}
\hline $\begin{array}{c}\text { Recurrence } \\
\text { intervals } \\
\text { (years) }\end{array}$ & \multicolumn{2}{|c|}{$\begin{array}{c}\text { Lowest average flow for } \\
\text { indicated number of } \\
\text { consecutive days } \\
\text { (cubic feet per second) }\end{array}$} & \multicolumn{2}{|c|}{$\begin{array}{c}\text { Time-sampling error } \\
\text { (in percent) }\end{array}$} \\
\hline & 1 & 7 & 1 & 7 \\
\hline 2 & 200 & 240 & 9 & 10 \\
\hline 5 & 119 & 146 & 10 & 13 \\
\hline 10 & 88 & 109 & 13 & 16 \\
\hline 20 & 68 & 85 & 17 & 22 \\
\hline 50 & 50 & 64 & 23 & \\
\hline
\end{tabular}

\section{DURATION OF DAILY FLOW}

Flow equaled or exceeded for indicated percentage of time (cubic feet per second)

\begin{tabular}{|c|c|c|c|c|c|c|c|}
\hline Percentage & 5 & 10 & 25 & 50 & 75 & 90 & 95 \\
\hline Flow & 5,410 & 3,680 & 1,970 & 967 & 476 & 272 & 190 \\
\hline
\end{tabular}


STATION NAME AND NUMBER—02364570 Panther Creek near Hacoda, AL

LOCATION.-Lat $31^{\circ} 07^{\prime} 15^{\prime \prime}$, long $86^{\circ} 11^{\prime} 13^{\prime \prime}$ referenced to North American Datum of 1927, Geneva County, AL, Hydrologic Unit 03140202, 5 mi northwest of Hacoda.

DRAINAGE AREA. $-26.2 \mathrm{mi}^{2}$.

PERIOD OF RECORD.-October 1974 to September 1995.

PERIOD OF ANALYSIS.-April 1975 to March 1995.

NUMBER OF CLIMATE YEARS IN FREQUENCY ANALYSIS.—-20

REMARKS.-

\begin{tabular}{|c|c|c|c|c|}
\hline $\begin{array}{c}\text { Recurrence } \\
\text { intervals } \\
\text { (years) }\end{array}$ & \multicolumn{2}{|c|}{$\begin{array}{c}\text { Lowest average flow for } \\
\text { indicated number of } \\
\text { consecutive days } \\
\text { (cubic feet per second) }\end{array}$} & \multicolumn{2}{|c|}{$\begin{array}{c}\text { Time-sampling error } \\
\text { (in percent) }\end{array}$} \\
\hline \multicolumn{5}{|c|}{} \\
\hline & 1 & 7 & 1 & 7 \\
\hline 2 & 1.8 & 2.1 & 13 & 16 \\
\hline 5 & 1.1 & 1.3 & 15 & 19 \\
\hline 10 & 0.86 & 0.98 & 19 & 24 \\
\hline 20 & 0.69 & 0.78 & 24 & \\
\hline
\end{tabular}

\begin{tabular}{|c|c|c|c|c|c|c|c|}
\hline \multicolumn{1}{|c|}{ DURATION OF DAILY FLOW } \\
\hline \multicolumn{1}{|c|}{ Flow equaled or exceeded for indicated percentage of time } \\
(cubic feet per second) \\
\hline Percentage & 5 & 10 & 25 & 50 & 75 & 90 & 95 \\
\hline Flow & 150 & 91 & 48 & 19 & 6.7 & 3.0 & 2.1 \\
\hline
\end{tabular}


STATION NAME AND NUMBER—02369800 Blackwater River near Bradley, AL

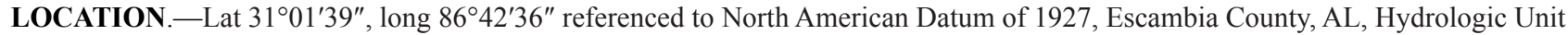
03140104, in Conecuh National Forest, near left bank on downstream side of bridge on county road, and 1 mi east of Bradley.

DRAINAGE AREA. $-87.7 \mathrm{mi}^{2}$.

PERIOD OF RECORD.-October 1967 to March 2014.

PERIOD OF ANALYSIS.-April 1968 to March 2014.

NUMBER OF CLIMATE YEARS IN FREQUENCY ANALYSIS. - 46

REMARKS.-

\begin{tabular}{|c|c|c|c|c|}
\hline $\begin{array}{l}\text { Recurrence } \\
\text { intervals } \\
\text { (years) }\end{array}$ & \multicolumn{2}{|c|}{$\begin{array}{l}\text { Lowest average flow for } \\
\text { indicated number of } \\
\text { consecutive days } \\
\text { (cubic feet per second) }\end{array}$} & \multicolumn{2}{|c|}{$\begin{array}{l}\text { Time-sampling erro } \\
\text { (in percent) }\end{array}$} \\
\hline & 1 & 7 & 1 & 7 \\
\hline 2 & 32 & 34 & 5 & 5 \\
\hline 5 & 25 & 26 & 5 & 5 \\
\hline 10 & 22 & 23 & 6 & 6 \\
\hline 20 & 19 & 21 & 8 & 7 \\
\hline 50 & 17 & 19 & 10 & 9 \\
\hline
\end{tabular}

\begin{tabular}{|c|c|c|c|c|c|c|c|}
\hline \multicolumn{1}{|c|}{ DURATION OF DAILY FLOW } \\
\hline \multicolumn{7}{|c|}{ Flow equaled or exceeded for indicated percentage of time } \\
(cubic feet per second)
\end{tabular}


STATION NAME AND NUMBER—02371200 Indian Creek near Troy, AL

LOCATION.-Lat $31^{\circ} 48^{\prime} 50^{\prime \prime}$, long $86^{\circ} 07^{\prime} 15^{\prime \prime}$ referenced to North American Datum of 1927, Pike County, AL, Hydrologic Unit 03140301, on U.S. Highway 29, 3.5 mi upstream from mouth, and 9 mi west of Troy.

DRAINAGE AREA. $-8.87 \mathrm{mi}^{2}$.

PERIOD OF RECORD.- October 1958 to September 1968, October 1970 to September 1986.

PERIOD OF ANALYSIS.-April 1959 to March 1968, April 1971 to March 1986.

NUMBER OF CLIMATE YEARS IN FREQUENCY ANALYSIS.—-24

REMARKS.-

\begin{tabular}{|c|c|c|c|c|}
\hline $\begin{array}{l}\text { Recurrence } \\
\text { intervals } \\
\text { (years) }\end{array}$ & \multicolumn{2}{|c|}{$\begin{array}{l}\text { Lowest average flow for } \\
\text { indicated number of } \\
\text { consecutive days } \\
\text { (cubic feet per second) }\end{array}$} & \multicolumn{2}{|c|}{$\begin{array}{l}\text { Time-sampling error } \\
\text { (in percent) }\end{array}$} \\
\hline & 1 & 7 & 1 & 7 \\
\hline 2 & 1.3 & 1.5 & 19 & 18 \\
\hline 5 & 0.64 & 0.74 & 23 & 22 \\
\hline 10 & 0.42 & 0.50 & 30 & 27 \\
\hline 20 & 0.28 & 0.35 & 40 & 35 \\
\hline
\end{tabular}

\begin{tabular}{|c|c|c|c|c|c|c|c|}
\hline \multicolumn{1}{|c|}{ DURATION OF DAILY FLOW } \\
\hline \multicolumn{1}{|c|}{ Flow equaled or exceeded for indicated percentage of time } \\
(cubic feet per second) \\
\hline Percentage & 5 & 10 & 25 & 50 & 75 & 90 & 95 \\
\hline Flow & 39 & 25 & 14 & 7.1 & 3.4 & 1.6 & 0.94 \\
\hline
\end{tabular}


STATION NAME AND NUMBER—02371500 Conecuh River at Brantley, AL

LOCATION.-Lat $31^{\circ} 34^{\prime} 24^{\prime \prime}$, long $86^{\circ} 15^{\prime} 06^{\prime \prime}$ referenced to North American Datum of 1927, Crenshaw County, AL, Hydrologic Unit 03140301, on left bank $10 \mathrm{ft}$ upstream of bridge on U.S. Highway 331 and State Highway 52, 0.5 mi downstream of Moody Mill Creek, 0.8 mi southeast of Brantley, and at river mile 112.3.

DRAINAGE AREA. - $500 \mathrm{mi}^{2}$.

PERIOD OF RECORD.- October 1937 to March 2014.

PERIOD OF ANALYSIS.-April 1938 to March 2014.

NUMBER OF CLIMATE YEARS IN FREQUENCY ANALYSIS.-76

\section{REMARKS.-}

\begin{tabular}{|c|c|c|c|c|}
\hline $\begin{array}{c}\text { Recurrence } \\
\text { intervals } \\
\text { (years) }\end{array}$ & \multicolumn{2}{|c|}{$\begin{array}{c}\text { Lowest average flow for } \\
\text { indicated number of } \\
\text { consecutive days } \\
\text { (cubic feet per second) }\end{array}$} & \multicolumn{2}{|c|}{$\begin{array}{c}\text { Time-sampling error } \\
\text { (in percent) }\end{array}$} \\
\hline & 1 & 7 & 1 & 7 \\
\hline 2 & 51 & 55 & 7 & 7 \\
\hline 5 & 32 & 34 & 7 & 9 \\
\hline 10 & 25 & 27 & 8 & 10 \\
\hline 20 & 21 & 22 & 10 & 13 \\
\hline 50 & 17 & 18 & 13 & \\
\hline
\end{tabular}

\begin{tabular}{|c|c|c|c|c|c|c|c|}
\hline \multicolumn{1}{|c|}{ DURATION OF DAILY FLOW } \\
\hline \multicolumn{7}{|c|}{ Flow equaled or exceeded for indicated percentage of time } \\
(cubic feet per second) \\
\hline Percentage & 5 & 10 & 25 & 50 & 75 & 90 & 95 \\
\hline Flow & 2,100 & 1,450 & 784 & 317 & 125 & 64 & 44 \\
\hline
\end{tabular}


STATION NAME AND NUMBER—02372000 Patsaliga Creek at Luverne, AL

LOCATION.-Lat $31^{\circ} 43^{\prime} 27^{\prime \prime}$, long $86^{\circ} 16^{\prime} 42^{\prime \prime}$ referenced to North American Datum of 1927, Crenshaw County, AL, Hydrologic Unit 03140302, on U.S. Highway 331, 1 mi northwest of Luverne, and 3 mi downstream from Pond Creek.

DRAINAGE AREA.-254 $\mathrm{mi}^{2}$.

PERIOD OF RECORD.- October 1943 to September 1958.

PERIOD OF ANALYSIS.-April 1944 to March 1958.

NUMBER OF CLIMATE YEARS IN FREQUENCY ANALYSIS.-14

REMARKS.-

\begin{tabular}{|c|c|c|c|c|}
\hline $\begin{array}{c}\text { Recurrence } \\
\text { intervals } \\
\text { (years) }\end{array}$ & \multicolumn{2}{|c|}{$\begin{array}{c}\text { Lowest average flow for } \\
\text { indicated number of } \\
\text { consecutive days } \\
\text { (cubic feet per second) }\end{array}$} & \multicolumn{2}{|c|}{$\begin{array}{c}\text { Time-sampling error } \\
\text { (in percent) }\end{array}$} \\
\hline \multicolumn{7}{|c|}{1} & 7 & 1 & 7 \\
\hline 2 & 22 & 25 & 26 & 26 \\
\hline 5 & 10 & 12 & 29 & 34 \\
\hline 10 & 7.0 & 8.0 & 34 & 43 \\
\hline 20 & 5.0 & 5.8 & 42 & 28 \\
\hline
\end{tabular}

\begin{tabular}{|c|c|c|c|c|c|c|c|c|}
\hline \multicolumn{1}{|c|}{ DURATION OF DAILY FLOW } \\
\hline \multicolumn{1}{|c|}{ Flow equaled or exceeded for indicated percentage of time } \\
(cubic feet per second) \\
\hline Percentage & 5 & 10 & 25 & 50 & 75 & 90 & 95 \\
\hline Flow & 1,220 & 819 & 414 & 170 & 66 & 32 & 20 \\
\hline
\end{tabular}


STATION NAME AND NUMBER—02372250 Patsaliga Creek near Brantley, AL

LOCATION.-Lat $31^{\circ} 35^{\prime} 46^{\prime \prime}$, long 86 24'20" referenced to North American Datum of 1927, Crenshaw County, AL, Hydrologic Unit 03140302, near left bank on downstream side of bridge on State Highway 106, 3.0 mi north of Leon, and 10.9 mi northwest of Brantley.

DRAINAGE AREA. $-442 \mathrm{mi}^{2}$.

PERIOD OF RECORD.-October 1974 to March 2014.

PERIOD OF ANALYSIS.-April 1975 to March 2014.

NUMBER OF CLIMATE YEARS IN FREQUENCY ANALYSIS.-39

REMARKS.-

\begin{tabular}{|c|c|c|c|c|}
\hline $\begin{array}{c}\text { Recurrence } \\
\text { intervals } \\
\text { (years) }\end{array}$ & \multicolumn{2}{|c|}{$\begin{array}{c}\text { Lowest average flow for } \\
\text { indicated number of } \\
\text { consecutive days } \\
\text { (cubic feet per second) }\end{array}$} & \multicolumn{2}{|c|}{$\begin{array}{c}\text { Time-sampling error } \\
\text { (in percent) }\end{array}$} \\
\hline \multicolumn{5}{|c|}{} \\
\hline & 4 & 7 & 1 & 7 \\
\hline 2 & 25 & 49 & 12 & 13 \\
\hline 5 & 19 & 20 & 13 & 15 \\
\hline 10 & 14 & 16 & 16 & 18 \\
\hline 20 & 11 & 12 & 20 & 24 \\
\hline 50 &
\end{tabular}

\begin{tabular}{|c|c|c|c|c|c|c|c|}
\hline \multicolumn{1}{|c|}{ DURATION OF DAILY FLOW } \\
\hline \multicolumn{7}{|c|}{ Flow equaled or exceeded for indicated percentage of time } \\
(cubic feet per second)
\end{tabular}


STATION NAME AND NUMBER—02372422 Conecuh River below Point A Dam near River Falls, AL

LOCATION.-Lat $31^{\circ} 21^{\prime} 40^{\prime \prime}$, long $86^{\circ} 31^{\prime} 11^{\prime \prime}$ referenced to North American Datum of 1927, Covington County, AL, Hydrologic Unit 03140301, on right bank wingwall of Point A Dam, 1.65 miles upstream of U.S. Highway 84 bridge, 1.4 miles northeast of River Falls, 4 miles northwest of Andalusia, and at river mile 79.50.

DRAINAGE AREA.-1,273 $\mathrm{mi}^{2}$.

PERIOD OF RECORD.- June 1999 to March 2013.

PERIOD OF ANALYSIS.-April 2000 to March 2013.

NUMBER OF CLIMATE YEARS IN FREQUENCY ANALYSIS.-13

REMARKS.- - Regulated by Gantt Dam since 1924 and Point A Reservoir since 1925.

\begin{tabular}{|c|c|c|c|c|}
\hline $\begin{array}{c}\text { Recurrence } \\
\text { intervals } \\
\text { (years) }\end{array}$ & \multicolumn{2}{|c|}{$\begin{array}{c}\text { Lowest average flow for } \\
\text { indicated number of } \\
\text { consecutive days } \\
\text { ccubic feet per second) }\end{array}$} & \multicolumn{2}{|c|}{$\begin{array}{c}\text { Time-sampling error } \\
\text { (in percent) }\end{array}$} \\
\hline \multicolumn{2}{|c|}{1} & 7 & 1 & 7 \\
\hline 2 & 54 & 89 & 20 & 27 \\
\hline 5 & 32 & 43 & 20 & 31 \\
\hline 10 & 24 & 30 & 23 & 38 \\
\hline 20 & 20 & 23 & 28 & 28 \\
\hline
\end{tabular}

\section{DURATION OF DAILY FLOW}

Flow equaled or exceeded for indicated percentage of time (cubic feet per second)

\begin{tabular}{|c|c|c|c|c|c|c|c|}
\hline Percentage & 5 & 10 & 25 & 50 & 75 & 90 & 95 \\
\hline Flow & 5,030 & 3,100 & 1,530 & 692 & 240 & 80 & 54 \\
\hline
\end{tabular}


STATION NAME AND NUMBER—02372500 Conecuh River near Andalusia, AL

LOCATION.-Lat $31^{\circ} 15^{\prime} 19^{\prime \prime}$, long $86^{\circ} 36^{\prime} 01^{\prime \prime}$ referenced to North American Datum of 1927, Covington County, AL, Hydrologic Unit 03140301, on county road, 0.5 mi upstream from Simmons Mill Creek, and 7.5 mi southwest of Andalusia..

DRAINAGE AREA.- $-1,344 \mathrm{mi}^{2}$.

PERIOD OF RECORD.-September 1904 to December 1919, October 1929 to September 1952, October 1965 to September 1968.

PERIOD OF ANALYSIS.-April 1930 to March 1952, April 1966 to March 1968.

NUMBER OF CLIMATE YEARS IN FREQUENCY ANALYSIS. - 24

REMARKS.- - Regulated by Gantt Dam since 1924 and Point A Reservoir since 1925. Only the data from the regulated period were included in the analysis.

\begin{tabular}{|c|c|c|c|c|}
\hline $\begin{array}{c}\text { Recurrence } \\
\text { intervals } \\
\text { (years) }\end{array}$ & \multicolumn{2}{|c|}{$\begin{array}{c}\text { Lowest average flow for } \\
\text { indicated number of } \\
\text { consecutive days } \\
\text { (cubic feet per second) }\end{array}$} & \multicolumn{2}{|c|}{$\begin{array}{c}\text { Time-sampling error } \\
\text { (in percent) }\end{array}$} \\
\hline \multicolumn{5}{|c|}{} \\
1 & 110 & 7 & 1 & 7 \\
\hline 2 & 74 & 156 & 11 & 12 \\
\hline 5 & 60 & 127 & 13 & 12 \\
\hline 10 & 51 & 109 & 15 & 15 \\
\hline 20 & & & & \\
\hline
\end{tabular}

\section{DURATION OF DAILY FLOW}

Flow equaled or exceeded for indicated percentage of time (cubic feet per second)

\begin{tabular}{|c|c|c|c|c|c|c|c|}
\hline Percentage & 5 & 10 & 25 & 50 & 75 & 90 & 95 \\
\hline Flow & 6,040 & 4,160 & 2,280 & 1,130 & 579 & 305 & 212 \\
\hline
\end{tabular}


STATION NAME AND NUMBER—02373000 Sepulga River near McKenzie, AL

LOCATION.-Lat $31^{\circ} 27^{\prime} 13^{\prime \prime}$, long 86 $47^{\prime} 13^{\prime \prime}$ referenced to North American Datum of 1927, Conecuh County, AL, Hydrologic Unit 03140303, on left bank $100 \mathrm{ft}$ downstream of bridge on U.S. Highway 31, 0.38 mi upstream of Old Town Creek, 2.5 mi upstream of Piney Woods Creek, $5.5 \mathrm{mi}$ downstream of Persimmon Creek, and $7 \mathrm{mi}$ southwest of McKenzie.

DRAINAGE AREA. $-470 \mathrm{mi}^{2}$.

PERIOD OF RECORD.- October 1937 to September 1967, October 1974 to March 2014.

PERIOD OF ANALYSIS.-April 1938 to March 1967, April 1975 to March 2014.

NUMBER OF CLIMATE YEARS IN FREQUENCY ANALYSIS. - 68

\section{REMARKS.-}

\begin{tabular}{|c|c|c|c|c|}
\hline $\begin{array}{c}\text { Recurrence } \\
\text { intervals } \\
\text { (years) }\end{array}$ & \multicolumn{2}{|c|}{$\begin{array}{c}\text { Lowest average flow for } \\
\text { indicated number of } \\
\text { consecutive days } \\
\text { (cubic feet per second) }\end{array}$} & \multicolumn{2}{|c|}{$\begin{array}{c}\text { Time-sampling error } \\
\text { (in percent) }\end{array}$} \\
\hline & 1 & 7 & 1 & 7 \\
\hline 2 & 23 & 26 & 10 & 10 \\
\hline 5 & 12 & 13 & 12 & 14 \\
\hline 10 & 8.2 & 9.4 & 15 & 18 \\
\hline 20 & 5.9 & 6.9 & 19 & 24 \\
\hline 50 & 4.0 & 4.8 & 26 & \\
\hline
\end{tabular}

\begin{tabular}{|c|c|c|c|c|c|c|c|}
\hline \multicolumn{1}{|c|}{ DURATION OF DAILY FLOW } \\
\hline \multicolumn{7}{|c|}{ Flow equaled or exceeded for indicated percentage of time } \\
(cubic feet per second)
\end{tabular}


STATION NAME AND NUMBER—02373500 Pigeon Creek near Thad, AL

LOCATION.-Lat $31^{\circ} 28^{\prime} 36^{\prime \prime}$, long 86 39'30" referenced to North American Datum of 1927, Covington County, AL, Hydrologic Unit 03140303, downstream from State Highway 55, 2 mi southeast of Thad, 5.5 mi southeast of McKenzie.

DRAINAGE AREA. - -307 $\mathrm{mi}^{2}$.

PERIOD OF RECORD.- October 1937 to September 1970.

PERIOD OF ANALYSIS.-April 1938 to March 1970.

NUMBER OF CLIMATE YEARS IN FREQUENCY ANALYSIS.-32

REMARKS.-

\begin{tabular}{|c|c|c|c|c|}
\hline $\begin{array}{l}\text { Recurrence } \\
\text { intervals } \\
\text { (years) }\end{array}$ & \multicolumn{2}{|c|}{$\begin{array}{l}\text { Lowest average flow for } \\
\text { indicated number of } \\
\text { consecutive days } \\
\text { (cubic feet per second) }\end{array}$} & \multicolumn{2}{|c|}{$\begin{array}{l}\text { Time-sampling error } \\
\text { (in percent) }\end{array}$} \\
\hline & 1 & 7 & 1 & 7 \\
\hline 2 & 37 & 41 & 11 & 10 \\
\hline 5 & 22 & 26 & 13 & 12 \\
\hline 10 & 16 & 20 & 18 & 15 \\
\hline 20 & 13 & 16 & 23 & 19 \\
\hline 50 & 9.1 & 12 & 33 & 26 \\
\hline
\end{tabular}

\section{DURATION OF DAILY FLOW}

Flow equaled or exceeded for indicated percentage of time (cubic feet per second)

\begin{tabular}{|c|c|c|c|c|c|c|c|}
\hline Percentage & 5 & 10 & 25 & 50 & 75 & 90 & 95 \\
\hline Flow & 1,490 & 967 & 477 & 195 & 84 & 50 & 38 \\
\hline
\end{tabular}


STATION NAME AND NUMBER—02374000 Conecuh River near Brooklyn, AL

LOCATION.-Lat $31^{\circ} 09^{\prime} 49^{\prime \prime}$, long $86^{\circ} 48^{\prime} 00^{\prime \prime}$ referenced to North American Datum of 1927, Escambia County, AL, Hydrologic Unit 03140304, on U.S. Highway 29, 3 mi downstream from Sepulga River, and 7 mi southwest of Brooklyn.

DRAINAGE AREA.-2,495 $\mathrm{mi}^{2}$.

PERIOD OF RECORD.- June 1935 to December 1957.

PERIOD OF ANALYSIS.-April 1936 to March 1957.

NUMBER OF CLIMATE YEARS IN FREQUENCY ANALYSIS.-21

REMARKS.-Regulated by Gantt Lake since 1924 and by Point A Reservoir since 1925.

\begin{tabular}{|c|c|c|c|c|}
\hline \multirow[t]{2}{*}{$\begin{array}{l}\text { Recurrence } \\
\text { intervals } \\
\text { (years) }\end{array}$} & \multicolumn{2}{|c|}{$\begin{array}{l}\text { Lowest average flow for } \\
\text { indicated number of } \\
\text { consecutive days } \\
\text { (cubic feet per second) }\end{array}$} & \multicolumn{2}{|c|}{$\begin{array}{l}\text { Time-sampling error } \\
\text { (in percent) }\end{array}$} \\
\hline & 1 & 7 & 1 & 7 \\
\hline 2 & 360 & 515 & 11 & 13 \\
\hline 5 & 248 & 321 & 11 & 15 \\
\hline 10 & 205 & 249 & 13 & 18 \\
\hline 20 & 176 & 202 & 16 & 22 \\
\hline
\end{tabular}

\begin{tabular}{|c|c|c|c|c|c|c|c|c|}
\hline \multicolumn{1}{|c|}{ DURATION OF DAILY FLOW } \\
\hline \multicolumn{1}{|c|}{ Flow equaled or exceeded for indicated percentage of time } \\
(cubic feet per second) \\
\hline Percentage & 5 & 10 & 25 & 50 & 75 & 90 & 95 \\
\hline Flow & 11,800 & 8,020 & 4,180 & 1,990 & 982 & 575 & 440 \\
\hline
\end{tabular}


STATION NAME AND NUMBER—02374250 Conecuh River at State Highway 41 near Brewton, AL

LOCATION.-Lat $31^{\circ} 04^{\prime} 01^{\prime \prime}$, long $87^{\circ} 03^{\prime} 42^{\prime \prime}$ referenced to North American Datum of 1927, Escambia County, AL, Hydrologic Unit 03140304, 100 feet downstream of State Highway 41 bridge, 1.0 mile south of East Brewton, 2.7 miles upstream of Murder Creek, at river mile 10.1 .

DRAINAGE AREA. - $2,661 \mathrm{mi}^{2}$.

PERIOD OF RECORD.-April 1999 to March 2014.

PERIOD OF ANALYSIS.-April 2000 to March 2014.

NUMBER OF CLIMATE YEARS IN FREQUENCY ANALYSIS.-14

REMARKS.- Regulated by Gantt Lake since 1924 and by Point A Reservoir since 1925.

\begin{tabular}{|c|c|c|c|c|}
\hline $\begin{array}{c}\text { Recurrence } \\
\text { intervals } \\
\text { (years) }\end{array}$ & \multicolumn{2}{|c|}{$\begin{array}{c}\text { Lowest average flow for } \\
\text { indicated number of } \\
\text { consecutive days } \\
\text { (cubic feet per second) }\end{array}$} & \multicolumn{2}{|c|}{$\begin{array}{c}\text { Time-sampling error } \\
\text { (in percent) }\end{array}$} \\
\hline & 1 & 7 & 1 & 7 \\
\hline 2 & 449 & 489 & 17 & 17 \\
\hline 5 & 286 & 304 & 16 & 19 \\
\hline 10 & 231 & 242 & 17 & 23 \\
\hline 20 & 196 & 202 & 21 & \\
\hline
\end{tabular}

\begin{tabular}{|c|c|c|c|c|c|c|c|c|}
\hline \multicolumn{1}{|c|}{ DURATION OF DAILY FLOW } \\
\hline \multicolumn{7}{|c|}{ Flow equaled or exceeded for indicated percentage of time } \\
(cubic feet per second)
\end{tabular}


STATION NAME AND NUMBER—02374500 Murder Creek near Evergreen, AL

LOCATION.-Lat $31^{\circ} 25^{\prime} 06^{\prime \prime}$, long 86 59'12" referenced to North American Datum of 1927, Conecuh County, AL, Hydrologic Unit 03140304, on left bank 30 feet upstream of U.S. Highway 31 bridge, 1 mi upstream of Louisville \& Nashville Railroad bridge, and 2.5 mi southwest of Evergreen, and at river mile 35.6.

DRAINAGE AREA. $-176 \mathrm{mi}^{2}$.

PERIOD OF RECORD.-October 1937 to March 2014.

PERIOD OF ANALYSIS.-April 1938 to March 2014.

NUMBER OF CLIMATE YEARS IN FREQUENCY ANALYSIS.-76

\section{REMARKS.-}

\begin{tabular}{|c|c|c|c|c|}
\hline $\begin{array}{c}\text { Recurrence } \\
\text { intervals } \\
\text { (years) }\end{array}$ & \multicolumn{2}{|c|}{$\begin{array}{l}\text { Lowest average flow for } \\
\text { indicated number of } \\
\text { consecutive days } \\
\text { (cubic feet per second) }\end{array}$} & \multicolumn{2}{|c|}{$\begin{array}{l}\text { Time-sampling error } \\
\text { (in percent) }\end{array}$} \\
\hline & 1 & 7 & 1 & 7 \\
\hline 2 & 81 & 85 & 5 & 5 \\
\hline 5 & 58 & 61 & 5 & 5 \\
\hline 10 & 49 & 52 & 6 & 6 \\
\hline 20 & 42 & 45 & 8 & 8 \\
\hline 50 & 36 & 38 & 10 & 10 \\
\hline
\end{tabular}

\section{DURATION OF DAILY FLOW}

Flow equaled or exceeded for indicated percentage of time (cubic feet per second)

\begin{tabular}{|c|c|c|c|c|c|c|c|}
\hline Percentage & 5 & 10 & 25 & 50 & 75 & 90 & 95 \\
\hline Flow & 736 & 508 & 310 & 190 & 123 & 86 & 70 \\
\hline
\end{tabular}


STATION NAME AND NUMBER—02374700 Murder Creek at Brewton, AL

LOCATION.-Lat $31^{\circ} 06^{\prime} 03^{\prime \prime}$, long $87^{\circ} 04^{\prime} 08^{\prime \prime}$ referenced to North American Datum of 1927 , Escambia County, AL, Hydrologic Unit 03140304, near right bank at bridge on U.S. Highway 29 and State Highway 41, 0.5 mi upstream of Burnt Corn Creek, 3.6 mi upstream of Conecuh River and at river mile 3.6.

DRAINAGE AREA. $-435 \mathrm{mi}^{2}$.

PERIOD OF RECORD.-March 1999 to March 2014.

PERIOD OF ANALYSIS.-April 1999 to March 2014.

NUMBER OF CLIMATE YEARS IN FREQUENCY ANALYSIS. - 15

REMARKS.-Low-flow frequency estimates adjusted based on MOVE.1 correlation with station 02374500 Murder Creek near Evergreen, AL, using the period of record from October 1937 to March 2014.

\begin{tabular}{|c|c|c|c|c|}
\hline $\begin{array}{l}\text { Recurrence } \\
\text { intervals } \\
\text { (years) }\end{array}$ & \multicolumn{2}{|c|}{$\begin{array}{l}\text { Lowest average flow for } \\
\text { indicated number of } \\
\text { consecutive days } \\
\text { (cubic feet per second) }\end{array}$} & \multicolumn{2}{|c|}{$\begin{array}{l}\text { Time-sampling erro } \\
\text { (in percent) }\end{array}$} \\
\hline & 1 & 7 & 1 & 7 \\
\hline 2 & 178 & 185 & 11 & 11 \\
\hline 5 & 132 & 139 & 9 & 10 \\
\hline 10 & 114 & 121 & 10 & 10 \\
\hline 20 & 99 & 107 & 12 & 12 \\
\hline 50 & 87 & 92 & 17 & 17 \\
\hline
\end{tabular}

\begin{tabular}{|c|c|c|c|c|c|c|c|c|}
\hline \multicolumn{1}{|c|}{ DURATION OF DAILY FLOW } \\
\hline \multicolumn{7}{|c|}{ Flow equaled or exceeded for indicated percentage of time } \\
(cubic feet per second)
\end{tabular}


STATION NAME AND NUMBER—02374745 Burnt Corn Creek at State Highway 41 near Brewton, AL

LOCATION.-Lat $31^{\circ} 07^{\prime} 47^{\prime \prime}$, long $87^{\circ} 05^{\prime} 14^{\prime \prime}$ referenced to North American Datum of 1927 , Escambia County, AL, Hydrologic Unit 03140304, near right bank at bridge on State Highway 41, 1.8 mi northwest of Brewton, 3.2 mi upstream of Murder Creek, and at river mile 3.2 .

DRAINAGE AREA.- $-182 \mathrm{mi}^{2}$.

PERIOD OF RECORD.-March 1999 to March 2014.

PERIOD OF ANALYSIS.-April 1999 to March 2014.

NUMBER OF CLIMATE YEARS IN FREQUENCY ANALYSIS.-15

\section{REMARKS.-}

\begin{tabular}{|c|c|c|c|c|}
\hline $\begin{array}{l}\text { Recurrence } \\
\text { intervals } \\
\text { (years) }\end{array}$ & \multicolumn{2}{|c|}{$\begin{array}{l}\text { Lowest average flow for } \\
\text { indicated number of } \\
\text { consecutive days } \\
\text { (cubic feet per second) }\end{array}$} & \multicolumn{2}{|c|}{$\begin{array}{l}\text { Time-sampling error } \\
\text { (in percent) }\end{array}$} \\
\hline & 1 & 7 & 1 & 7 \\
\hline 2 & 25 & 27 & 17 & 17 \\
\hline 5 & 15 & 17 & 17 & 17 \\
\hline 10 & 12 & 13 & 19 & 19 \\
\hline 20 & 9.8 & 11 & 23 & 23 \\
\hline
\end{tabular}

\begin{tabular}{|c|c|c|c|c|c|c|c|}
\hline \multicolumn{8}{|c|}{ DURATION OF DAILY FLOW } \\
\hline \multicolumn{8}{|c|}{$\begin{array}{l}\text { Flow equaled or exceeded for indicated percentage of time } \\
\text { (cubic feet per second) }\end{array}$} \\
\hline Percentage & 5 & 10 & 25 & 50 & 75 & 90 & 95 \\
\hline Flow & 853 & 573 & 277 & 133 & 60 & 31 & 23 \\
\hline
\end{tabular}


STATION NAME AND NUMBER—02374950 Big Escambia Creek at Sardine Bridge near Stanley Crossroads, AL

LOCATION.-Lat $31^{\circ} 07^{\prime} 46^{\prime \prime}$, long 87²2'14" referenced to North American Datum of 1927, Escambia County, AL, Hydrologic Unit 03140305, on upstream side of Sardine Bridge on County Road 27, 2 mi west of Stanley Crossroads, 13 mi northwest of Flomaton.

DRAINAGE AREA. $-193 \mathrm{mi}^{2}$.

PERIOD OF RECORD.--May 2000 to March 2014.

PERIOD OF ANALYSIS.-April 2001 to March 2014.

NUMBER OF CLIMATE YEARS IN FREQUENCY ANALYSIS.-13

REMARKS.-

\begin{tabular}{|c|c|c|c|c|}
\hline \multirow[t]{2}{*}{$\begin{array}{l}\text { Recurrence } \\
\text { intervals } \\
\text { (years) }\end{array}$} & \multicolumn{2}{|c|}{$\begin{array}{l}\text { Lowest average flow for } \\
\text { indicated number of } \\
\text { consecutive days } \\
\text { (cubic feet per second) }\end{array}$} & \multicolumn{2}{|c|}{$\begin{array}{l}\text { Time-sampling erro } \\
\text { (in percent) }\end{array}$} \\
\hline & 1 & 7 & 1 & 7 \\
\hline 2 & 71 & 74 & 12 & 12 \\
\hline 5 & 52 & 54 & 12 & 12 \\
\hline 10 & 44 & 46 & 13 & 13 \\
\hline 20 & 39 & 41 & 16 & 16 \\
\hline
\end{tabular}

\section{DURATION OF DAILY FLOW}

Flow equaled or exceeded for indicated percentage of time (cubic feet per second)

\begin{tabular}{|c|c|c|c|c|c|c|c|}
\hline Percentage & 5 & 10 & 25 & 50 & 75 & 90 & 95 \\
\hline Flow & 804 & 536 & 296 & 173 & 101 & 73 & 59 \\
\hline
\end{tabular}


STATION NAME AND NUMBER—02375000 Big Escambia Creek at Flomaton, AL

LOCATION.-Lat $31^{\circ} 00^{\prime} 38^{\prime \prime}$, long $87^{\circ} 15^{\prime} 46^{\prime \prime}$ referenced to North American Datum of 1927, Escambia County, AL, Hydrologic Unit 03140305, on U.S. Highway 31 at north edge of Flomaton, 1.5 mi upstream from Alabama-Florida stateline, and 4 mi upstream from mouth.

DRAINAGE AREA. $-330 \mathrm{mi}^{2}$.

PERIOD OF RECORD.- - June 1939 to December 1951.

PERIOD OF ANALYSIS.-April 1939 to March 1951.

NUMBER OF CLIMATE YEARS IN FREQUENCY ANALYSIS.-12

REMARKS.-

\begin{tabular}{|c|c|c|c|c|}
\hline $\begin{array}{l}\text { Recurrence } \\
\text { intervals } \\
\text { (years) }\end{array}$ & \multicolumn{2}{|c|}{$\begin{array}{l}\text { Lowest average flow for } \\
\text { indicated number of } \\
\text { consecutive days } \\
\text { (cubic feet per second) }\end{array}$} & \multicolumn{2}{|c|}{$\begin{array}{l}\text { Time-sampling error } \\
\text { (in percent) }\end{array}$} \\
\hline & 1 & 7 & 1 & 7 \\
\hline 2 & 232 & 240 & 7 & 6 \\
\hline 5 & 192 & 200 & 8 & 8 \\
\hline 10 & 171 & 180 & 11 & 10 \\
\hline 20 & 154 & 164 & 15 & 13 \\
\hline
\end{tabular}

\section{DURATION OF DAILY FLOW}

Flow equaled or exceeded for indicated percentage of time (cubic feet per second)

\begin{tabular}{|c|c|c|c|c|c|c|c|}
\hline Percentage & 5 & 10 & 25 & 50 & 75 & 90 & 95 \\
\hline Flow & 1,920 & 1,210 & 663 & 417 & 305 & 248 & 222 \\
\hline
\end{tabular}


STATION NAME AND NUMBER—02376500 Perdido River at Barrineau Park, FL

LOCATION.-Lat 3041'25", long 87²6'25" referenced to North American Datum of 1927, Baldwin County, AL, Hydrologic Unit 03140106, on right bank $25 \mathrm{ft}$ downstream of bridge on county road, 1,000 ft downstream of Alligator Creek, $0.5 \mathrm{mi}$ southwest of Barrineau Park, and at mile 28.0.

DRAINAGE AREA. $-394 \mathrm{mi}^{2}$.

PERIOD OF RECORD.--June 1941 to March 2014.

PERIOD OF ANALYSIS.-April 1942 to March 2014.

NUMBER OF CLIMATE YEARS IN FREQUENCY ANALYSIS.—72

REMARKS.-

\begin{tabular}{|c|c|c|c|c|}
\hline \multirow[t]{2}{*}{$\begin{array}{l}\text { Recurrence } \\
\text { intervals } \\
\text { (years) }\end{array}$} & \multicolumn{2}{|c|}{$\begin{array}{l}\text { Lowest average flow for } \\
\text { indicated number of } \\
\text { consecutive days } \\
\text { (cubic feet per second) }\end{array}$} & \multicolumn{2}{|c|}{$\begin{array}{l}\text { Time-sampling error } \\
\text { (in percent) }\end{array}$} \\
\hline & 1 & 7 & 1 & 7 \\
\hline 2 & 267 & 277 & 3 & 3 \\
\hline 5 & 223 & 231 & 3 & 3 \\
\hline 10 & 202 & 209 & 4 & 4 \\
\hline 20 & 187 & 193 & 4 & 4 \\
\hline 50 & 171 & 176 & 6 & 6 \\
\hline
\end{tabular}

DURATION OF DAILY FLOW

Flow equaled or exceeded for indicated percentage of time (cubic feet per second)

\begin{tabular}{|c|c|c|c|c|c|c|c|}
\hline Percentage & 5 & 10 & 25 & 50 & 75 & 90 & 95 \\
\hline Flow & 2,100 & 1,410 & 800 & 497 & 356 & 284 & 251 \\
\hline
\end{tabular}


STATION NAME AND NUMBER—02377500 Styx River near Loxley, AL

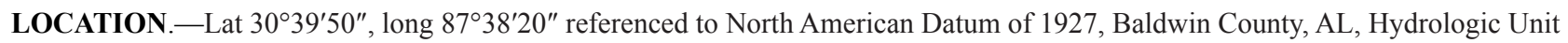
03140106, on county road, 2 mi upstream from Hollinger Creek, and 7 mi northeast of Loxley.

DRAINAGE AREA. $-92.2 \mathrm{mi}^{2}$.

PERIOD OF RECORD.-October 1951 to September 1969, October 1970 to September 1971.

PERIOD OF ANALYSIS.-April 1952 to March 1969.

NUMBER OF CLIMATE YEARS IN FREQUENCY ANALYSIS.-17

REMARKS.-

\begin{tabular}{|c|c|c|c|c|}
\hline $\begin{array}{l}\text { Recurrence } \\
\text { intervals } \\
\text { (years) }\end{array}$ & \multicolumn{2}{|c|}{$\begin{array}{l}\text { Lowest average flow for } \\
\text { indicated number of } \\
\text { consecutive days } \\
\text { (cubic feet per second) }\end{array}$} & \multicolumn{2}{|c|}{$\begin{array}{l}\text { Time-sampling error } \\
\text { (in percent) }\end{array}$} \\
\hline & 1 & 7 & 1 & 7 \\
\hline 2 & 27 & 30 & 12 & 12 \\
\hline 5 & 19 & 21 & 11 & 10 \\
\hline 10 & 17 & 18 & 11 & 11 \\
\hline 20 & 15 & 16 & 14 & 14 \\
\hline
\end{tabular}

\section{DURATION OF DAILY FLOW}

Flow equaled or exceeded for indicated percentage of time (cubic feet per second)

\begin{tabular}{|c|c|c|c|c|c|c|c|}
\hline Percentage & 5 & 10 & 25 & 50 & 75 & 90 & 95 \\
\hline Flow & 538 & 371 & 188 & 90 & 53 & 35 & 28 \\
\hline
\end{tabular}


STATION NAME AND NUMBER—02377570 Styx River near Elsanor, AL

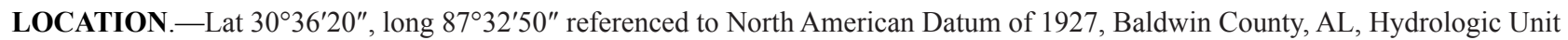
03140106, near left bank on downstream side of bridge on County Road 87, 0.2 mi downstream of Cowpen Creek, 5 mi northeast of Elsanor, and 11.4 mi upstream of mouth.

DRAINAGE AREA.-192 $\mathrm{mi}^{2}$.

PERIOD OF RECORD.- October 1987 to March 2014.

PERIOD OF ANALYSIS.-April 1988 to March 2014.

NUMBER OF CLIMATE YEARS IN FREQUENCY ANALYSIS.-26

\section{REMARKS.-}

\begin{tabular}{|c|c|c|c|c|}
\hline $\begin{array}{c}\text { Recurrence } \\
\text { intervals } \\
\text { (years) }\end{array}$ & \multicolumn{2}{|c|}{$\begin{array}{c}\text { Lowest average flow for } \\
\text { indicated number of } \\
\text { consecutive days } \\
\text { (cubic feet per second) }\end{array}$} & \multicolumn{2}{|c|}{$\begin{array}{c}\text { Time-sampling error } \\
\text { (in percent) }\end{array}$} \\
\hline \multicolumn{7}{|c|}{1} & 7 & 1 & 7 \\
\hline 2 & 113 & 117 & 5 & 6 \\
\hline 5 & 91 & 95 & 6 & 7 \\
\hline 10 & 82 & 85 & 7 & 8 \\
\hline 20 & 75 & 78 & 8 & 5 \\
\hline
\end{tabular}

\begin{tabular}{|c|c|c|c|c|c|c|c|}
\hline \multicolumn{7}{|c|}{ DURATION OF DAILY FLOW } \\
\hline \multicolumn{7}{|c|}{ Flow equaled or exceeded for indicated percentage of time } \\
(cubic feet per second)
\end{tabular}


STATION NAME AND NUMBER—02378300 Magnolia River at U.S. Highway 98 near Foley, AL

LOCATION.-Lat 30²4'23", long 8744'13" referenced to North American Datum of 1927, Baldwin County, AL, Hydrologic Unit 03160205, at bridge on U.S. Highway 98,3 mi west of Foley.

DRAINAGE AREA.- $-16.6 \mathrm{mi}^{2}$.

PERIOD OF RECORD.- July 1999 to March 2014.

PERIOD OF ANALYSIS.-April 2000 to March 2014.

NUMBER OF CLIMATE YEARS IN FREQUENCY ANALYSIS.-14

REMARKS.-

\begin{tabular}{|c|c|c|c|c|}
\hline $\begin{array}{c}\text { Recurrence } \\
\text { intervals } \\
\text { (years) }\end{array}$ & \multicolumn{2}{|c|}{$\begin{array}{c}\text { Lowest average flow for } \\
\text { indicated number of } \\
\text { consecutive days } \\
\text { (cubic feet per second) }\end{array}$} & \multicolumn{2}{|c|}{$\begin{array}{c}\text { Time-sampling error } \\
\text { (in percent) }\end{array}$} \\
\hline \multicolumn{7}{|c|}{1} & 7 & 1 & 7 \\
\hline 2 & 13 & 14 & 7 & 8 \\
\hline 5 & 11 & 11 & 9 & 10 \\
\hline 10 & 9.5 & 10 & 11 & 12 \\
\hline 20 & 8.5 & 9.3 & 14 & 7 \\
\hline
\end{tabular}

\begin{tabular}{|c|c|c|c|c|c|c|c|}
\hline \multicolumn{7}{|c|}{ DURATION OF DAILY FLOW } \\
\hline \multicolumn{7}{|c|}{ Flow equaled or exceeded for indicated percentage of time } \\
(cubic feet per second) \\
\hline Percentage & 5 & 10 & 25 & 50 & 75 & 90 & 95 \\
\hline Flow & 95 & 50 & 29 & 21 & 17 & 13 & 12 \\
\hline
\end{tabular}


STATION NAME AND NUMBER—02378500 Fish River near Silver Hill, AL

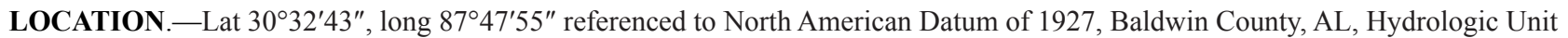
03160205, near mid-channel on upstream side of bridge on State Highway 104, 0.2 mi downstream of Caney Branch, 0.5 mi upstream of Perone Branch, 2.8 mi west of Silver Hill, and 12 mi upstream of mouth.

DRAINAGE AREA. $-55.3 \mathrm{mi}^{2}$.

PERIOD OF RECORD.- July 1953 to September 1969, October 1970 to September 1971, November 1986 to March 2014.

PERIOD OF ANALYSIS.-April 1954 to March 1969, April 1987 to March 2014.

NUMBER OF CLIMATE YEARS IN FREQUENCY ANALYSIS. - 42

REMARKS.-

\begin{tabular}{|c|c|c|c|c|}
\hline $\begin{array}{l}\text { Recurrence } \\
\text { intervals } \\
\text { (years) }\end{array}$ & \multicolumn{2}{|c|}{$\begin{array}{l}\text { Lowest average flow for } \\
\text { indicated number of } \\
\text { consecutive days } \\
\text { (cubic feet per second) }\end{array}$} & \multicolumn{2}{|c|}{$\begin{array}{l}\text { Time-sampling error } \\
\text { (in percent) }\end{array}$} \\
\hline & 1 & 7 & 1 & 7 \\
\hline 2 & 52 & 54 & 4 & 4 \\
\hline 5 & 43 & 44 & 4 & 4 \\
\hline 10 & 38 & 39 & 5 & 5 \\
\hline 20 & 35 & 36 & 7 & 7 \\
\hline 50 & 31 & 32 & 9 & 9 \\
\hline
\end{tabular}

\section{DURATION OF DAILY FLOW}

Flow equaled or exceeded for indicated percentage of time (cubic feet per second)

\begin{tabular}{|c|c|c|c|c|c|c|c|}
\hline Percentage & 5 & 10 & 25 & 50 & 75 & 90 & 95 \\
\hline Flow & 244 & 168 & 107 & 79 & 63 & 51 & 46 \\
\hline
\end{tabular}


STATION NAME AND NUMBER—02398300 Chattooga River above Gaylesville, AL

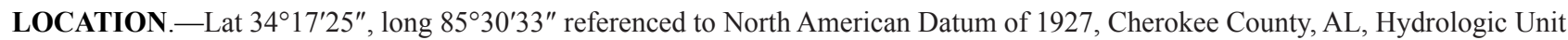
03150105, on left bank $10 \mathrm{ft}$ upstream from bridge on county road, $600 \mathrm{ft}$ downstream from Mills Creek, $3.5 \mathrm{mi}$ northeast of Gaylesville, and 20.1 mi upstream from mouth.

DRAINAGE AREA. $-366 \mathrm{mi}^{2}$.

PERIOD OF RECORD._-January 1959 to September 1967, October 1984 to March 2014.

PERIOD OF ANALYSIS.-April 1959 to March 1967, April 1985 to March 2014.

NUMBER OF CLIMATE YEARS IN FREQUENCY ANALYSIS.-37

REMARKS.-

\begin{tabular}{|c|c|c|c|c|}
\hline $\begin{array}{c}\text { Recurrence } \\
\text { intervals } \\
\text { (years) }\end{array}$ & \multicolumn{2}{|c|}{$\begin{array}{c}\text { Lowest average flow for } \\
\text { indicated number of } \\
\text { consecutive days } \\
\text { (cubic feet per second) }\end{array}$} & \multicolumn{2}{|c|}{$\begin{array}{c}\text { Time-sampling error } \\
\text { (in percent) }\end{array}$} \\
\hline & 1 & 7 & 1 & 7 \\
\hline 2 & 122 & 127 & 5 & 5 \\
\hline 5 & 98 & 103 & 5 & 6 \\
\hline 10 & 87 & 92 & 6 & 7 \\
\hline 20 & 78 & 84 & 8 & 9 \\
\hline 50 & 70 & 76 & 11 & 5 \\
\hline
\end{tabular}

\begin{tabular}{|c|c|c|c|c|c|c|c|}
\hline \multicolumn{1}{|c|}{ DURATION OF DAILY FLOW } \\
\hline \multicolumn{7}{|c|}{ Flow equaled or exceeded for indicated percentage of time } \\
(cubic feet per second)
\end{tabular}


STATION NAME AND NUMBER—02398500 Chattooga River at Gaylesville, AL

LOCATION.-Lat 34¹5'47", long 85³3'39" referenced to North American Datum of 1927, Cherokee County, AL, Hydrologic Unit 03150105, on State Highway 35, 0.2 mi southwest of Gaylesville, and 9 mi upstream from Little River.

DRAINAGE AREA. - $379 \mathrm{mi}^{2}$.

PERIOD OF RECORD.--June 1937 to September 1960.

PERIOD OF ANALYSIS.-April 1938 to March 1960.

NUMBER OF CLIMATE YEARS IN FREQUENCY ANALYSIS.-22

REMARKS.-

\begin{tabular}{|c|c|c|c|c|}
\hline $\begin{array}{c}\text { Recurrence } \\
\text { intervals } \\
\text { (years) }\end{array}$ & \multicolumn{2}{|c|}{$\begin{array}{c}\text { Lowest average flow for } \\
\text { indicated number of } \\
\text { consecutive days } \\
\text { (cubic feet per second) }\end{array}$} & \multicolumn{2}{|c|}{$\begin{array}{c}\text { Time-sampling error } \\
\text { (in percent) }\end{array}$} \\
\hline \multicolumn{7}{|c|}{1} & 7 & 1 & 7 \\
\hline 2 & 109 & 121 & 7 & 5 \\
\hline 5 & 89 & 99 & 5 & 6 \\
\hline 10 & 82 & 91 & 6 & 7 \\
\hline 20 & 77 & 85 & 7 & 7 \\
\hline
\end{tabular}

\begin{tabular}{|c|c|c|c|c|c|c|c|c|}
\hline \multicolumn{1}{|c|}{ DURATION OF DAILY FLOW } \\
\hline \multicolumn{7}{|c|}{ Flow equaled or exceeded for indicated percentage of time } \\
(cubic feet per second)
\end{tabular}


STATION NAME AND NUMBER—02398950 West Fork Little River at Desoto Park near Fort Payne, AL

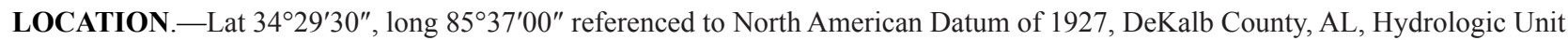
03150105, on right bank just off DeSoto Parkway near Park Headquarters, 8 mi northeast of Fort Payne.

DRAINAGE AREA. $-42.8 \mathrm{mi}^{2}$.

PERIOD OF RECORD.- October 1997 to January 2012.

PERIOD OF ANALYSIS.-April 1998 to March 2011.

NUMBER OF CLIMATE YEARS IN FREQUENCY ANALYSIS.-13

REMARKS.-

\begin{tabular}{|c|c|c|c|c|}
\hline $\begin{array}{l}\text { Recurrence } \\
\text { intervals } \\
\text { (years) }\end{array}$ & \multicolumn{2}{|c|}{$\begin{array}{l}\text { Lowest average flow for } \\
\text { indicated number of } \\
\text { consecutive days } \\
\text { (cubic feet per second) }\end{array}$} & \multicolumn{2}{|c|}{$\begin{array}{l}\text { Time-sampling erro } \\
\text { (in percent) }\end{array}$} \\
\hline & 1 & 7 & 1 & 7 \\
\hline 2 & 0.13 & 0.18 & 35 & 38 \\
\hline 5 & 0.05 & 0.06 & 44 & 48 \\
\hline 10 & 0.03 & 0.03 & 59 & 65 \\
\hline 20 & 0.02 & 0.02 & 83 & 94 \\
\hline
\end{tabular}

\begin{tabular}{|c|c|c|c|c|c|c|c|c|}
\hline \multicolumn{1}{|c|}{ DURATION OF DAILY FLOW } \\
\hline \multicolumn{1}{|c|}{ Flow equaled or exceeded for indicated percentage of time } \\
(cubic feet per second)
\end{tabular}


STATION NAME AND NUMBER—02399000 Little River near Jamestown, AL

LOCATION.--Lat 34²3'51", long 85³7'36" referenced to North American Datum of 1927, Cherokee County, AL, Hydrologic Unit 03150105, at site of former highway bridge, 0.2 mi upstream from Yellow Creek, 0.3 mi upstream from present highway bridge, and 2.5 west of Jamestown.

DRAINAGE AREA. $-125 \mathrm{mi}^{2}$.

PERIOD OF RECORD.-February 1922 to March 1932, June 1935 to September 1949.

PERIOD OF ANALYSIS.-April 1922 to March 1932, April 1936 to March 1949.

NUMBER OF CLIMATE YEARS IN FREQUENCY ANALYSIS.-23

\section{REMARKS.-}

\begin{tabular}{|c|c|c|c|c|}
\hline $\begin{array}{c}\text { Recurrence } \\
\text { intervals } \\
\text { (years) }\end{array}$ & \multicolumn{2}{|c|}{$\begin{array}{c}\text { Lowest average flow for } \\
\text { indicated number of } \\
\text { consecutive days } \\
\text { (cubic feet per second) }\end{array}$} & \multicolumn{2}{|c|}{$\begin{array}{c}\text { Time-sampling error } \\
\text { (in percent) }\end{array}$} \\
\hline \multicolumn{7}{|c|}{1} & 7 & 1 & 7 \\
\hline 2 & 0.42 & 0.54 & 20 & 19 \\
\hline 5 & 0.17 & 0.22 & 19 & -- \\
\hline 10 & 0.00 & 0.00 & -- & -- \\
\hline 20 & 0.00 & 0.00 & -- & \\
\hline
\end{tabular}

\section{DURATION OF DAILY FLOW}

Flow equaled or exceeded for indicated percentage of time (cubic feet per second)

\begin{tabular}{|c|c|c|c|c|c|c|c|}
\hline Percentage & 5 & 10 & 25 & 50 & 75 & 90 & 95 \\
\hline Flow & 1,060 & 655 & 296 & 74 & 7.9 & 1.4 & 0.5 \\
\hline
\end{tabular}


STATION NAME AND NUMBER—02399200 Little River near Blue Pond, AL

LOCATION.--Lat $34^{\circ} 17^{\prime} 20^{\prime \prime}$, long 8540'50" referenced to North American Datum of 1927, Cherokee County, AL, Hydrologic Unit 03150105, on right bank at Canyon Mouth Park, 0.9 mi upstream from State Highway 176, 2.5 mi upstream from Wolf Creek, 4.2 mi northeast of Blue Pond, and 7.5 miles upstream from mouth.

DRAINAGE AREA.-199 $\mathrm{mi}^{2}$.

PERIOD OF RECORD.- October 1958 to September 1967, October 1970 to March 2014.

PERIOD OF ANALYSIS.-April 1959 to March 1967, April 1971 to March 2014.

NUMBER OF CLIMATE YEARS IN FREQUENCY ANALYSIS.- - 51

REMARKS.-

\begin{tabular}{|c|c|c|c|c|}
\hline $\begin{array}{c}\text { Recurrence } \\
\text { intervals } \\
\text { (years) }\end{array}$ & \multicolumn{2}{|c|}{$\begin{array}{c}\text { Lowest average flow for } \\
\text { indicated number of } \\
\text { consecutive days } \\
\text { (cubic feet per second) }\end{array}$} & \multicolumn{2}{|c|}{$\begin{array}{c}\text { Time-sampling error } \\
\text { (in percent) }\end{array}$} \\
\hline & 1 & 7 & 1 & 7 \\
\hline 2 & 1.9 & 2.3 & 17 & 17 \\
\hline 5 & 0.73 & 0.90 & 18 & 21 \\
\hline 10 & 0.45 & 0.56 & 21 & 25 \\
\hline 20 & 0.31 & 0.38 & 25 & 33 \\
\hline 50 & 0.20 & 0.25 & 33 & \\
\hline
\end{tabular}

\begin{tabular}{|c|c|c|c|c|c|c|c|}
\hline \multicolumn{1}{|c|}{ DURATION OF DAILY FLOW } \\
\hline \multicolumn{7}{|c|}{ Flow equaled or exceeded for indicated percentage of time } \\
(cubic feet per second)
\end{tabular}


STATION NAME AND NUMBER—02399500 Coosa River at Leesburg, AL

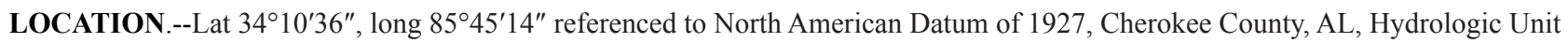
03150105, on U.S. Highway 411, 1 mi east of Leesburg, 4 mi downstream from Yellow Creek, and at mile 226.1.

DRAINAGE AREA. $-5,270 \mathrm{mi}^{2}$.

PERIOD OF RECORD.- October 1937 to September 1958.

PERIOD OF ANALYSIS.-April 1938 to March 1949.

NUMBER OF CLIMATE YEARS IN FREQUENCY ANALYSIS.-11

REMARKS.- - Since about December 1949, flow regulated by Allatoona Reservoir and since about April 1961, by Weiss Reservoir. The regulated period of record was not of sufficient length to warrant low-flow frequency analyses. Consequently, low-flow characteristics were estimated for pre-regulated conditions only. However, a plot of the annual minimum 7-day average flows for the complete period of record is provided.

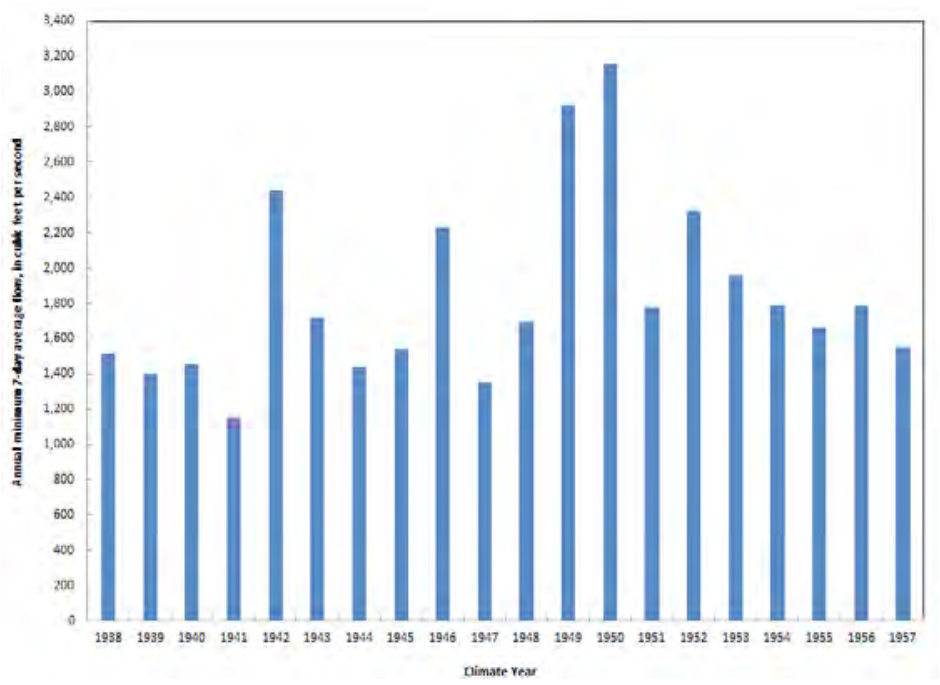

\begin{tabular}{|c|c|c|c|c|}
\hline \multirow[t]{2}{*}{$\begin{array}{l}\text { Recurrence } \\
\text { intervals } \\
\text { (years) }\end{array}$} & \multicolumn{2}{|c|}{$\begin{array}{l}\text { Lowest average flow for } \\
\text { indicated number of } \\
\text { consecutive days } \\
\text { (cubic feet per second) }\end{array}$} & \multicolumn{2}{|c|}{$\begin{array}{l}\text { Time-sampling error } \\
\text { (in percent) }\end{array}$} \\
\hline & 1 & 7 & 1 & 7 \\
\hline 2 & 1,510 & 1,540 & 6 & 8 \\
\hline 5 & 1,310 & 1,320 & 6 & 6 \\
\hline 10 & 1,220 & 1,240 & 6 & 6 \\
\hline 20 & 1,170 & 1,190 & 7 & 8 \\
\hline
\end{tabular}

\begin{tabular}{|c|c|c|c|c|c|c|c|c|}
\hline \multicolumn{1}{|c|}{ DURATION OF DAILY FLOW } \\
\hline \multicolumn{1}{|c|}{ Flow equaled or exceeded for indicated percentage of time } \\
(cubic feet per second)
\end{tabular}


STATION NAME AND NUMBER—02400000 Terrapin Creek near Piedmont, AL

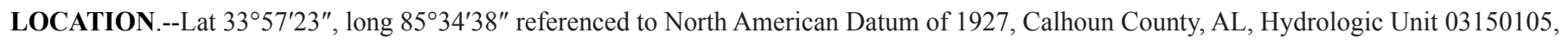
on U.S. Highway 278 and State Highway 74, 0.5 mi upstream from Ladiga Creek, and 3 mi northeast of Piedmont.

DRAINAGE AREA.- $-116 \mathrm{mi}^{2}$.

PERIOD OF RECORD.- October 1944 to September 1954, October 1956 to September 1963.

PERIOD OF ANALYSIS.-April 1945 to March 1954, April 1957 to March 1963.

NUMBER OF CLIMATE YEARS IN FREQUENCY ANALYSIS.-15

REMARKS.-

\begin{tabular}{|c|c|c|c|c|}
\hline $\begin{array}{c}\text { Recurrence } \\
\text { intervals } \\
\text { (years) }\end{array}$ & \multicolumn{2}{|c|}{$\begin{array}{c}\text { Lowest average flow for } \\
\text { indicated number of } \\
\text { consecutive days } \\
\text { (cubic feet per second) }\end{array}$} & \multicolumn{2}{|c|}{$\begin{array}{c}\text { Time-sampling error } \\
\text { (in percent) }\end{array}$} \\
\hline \multicolumn{5}{|c|}{} \\
\hline & 6.6 & 7 & 1 & 7 \\
\hline 2 & 4.3 & 4.9 & 14 & 17 \\
\hline 5 & 3.4 & 3.8 & 16 & 20 \\
\hline 10 & 2.8 & 3.1 & 25 & 25 \\
\hline 20 & & & & \\
\hline
\end{tabular}

\begin{tabular}{|c|c|c|c|c|c|c|c|c|}
\hline \multicolumn{1}{|c|}{ DURATION OF DAILY FLOW } \\
\hline \multicolumn{1}{|c|}{ Flow equaled or exceeded for indicated percentage of time } \\
(cubic feet per second) \\
\hline Percentage & 5 & 10 & 25 & 50 & 75 & 90 & 95 \\
\hline Flow & 514 & 311 & 158 & 66 & 28 & 14 & 9.8 \\
\hline
\end{tabular}


STATION NAME AND NUMBER—02400100 Terrapin Creek at Ellisville, AL

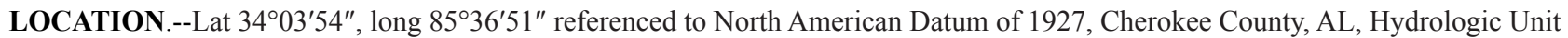
03150105, on right bank $40 \mathrm{ft}$ downstream from bridge on State Highway 9, 0.2 mi southwest of Ellisville, and 6.7 miles upstream from mouth.

DRAINAGE AREA. $-252 \mathrm{mi}^{2}$.

PERIOD OF RECORD.- October 1962 to September 1967, October 1980 to March 2014.

PERIOD OF ANALYSIS.-April 1963 to March 1967, April 1981 to March 2014.

NUMBER OF CLIMATE YEARS IN FREQUENCY ANALYSIS. - 37

\section{REMARKS.-}

\begin{tabular}{|c|c|c|c|c|}
\hline \multirow[t]{2}{*}{$\begin{array}{l}\text { Recurrence } \\
\text { intervals } \\
\text { (years) }\end{array}$} & \multicolumn{2}{|c|}{$\begin{array}{l}\text { Lowest average flow for } \\
\text { indicated number of } \\
\text { consecutive days } \\
\text { (cubic feet per second) }\end{array}$} & \multicolumn{2}{|c|}{$\begin{array}{l}\text { Time-sampling error } \\
\text { (in percent) }\end{array}$} \\
\hline & 1 & 7 & 1 & 7 \\
\hline 2 & 83 & 86 & 5 & 5 \\
\hline 5 & 64 & 66 & 6 & 6 \\
\hline 10 & 55 & 57 & 8 & 8 \\
\hline 20 & 48 & 51 & 10 & 10 \\
\hline 50 & 42 & 44 & 14 & 13 \\
\hline
\end{tabular}

\begin{tabular}{|c|c|c|c|c|c|c|c|}
\hline \multicolumn{1}{|c|}{ DURATION OF DAILY FLOW } \\
\hline \multicolumn{7}{|c|}{ Flow equaled or exceeded for indicated percentage of time } \\
(cubic feet per second)
\end{tabular}


STATION NAME AND NUMBER—02400500 Coosa River at Gadsden, AL

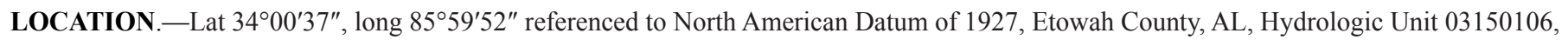
on Forrest Avenue in Gadsden, 1.5 mi upstream from Big Wills Creek, and at mile 174.8.

DRAINAGE AREA. $-5,805 \mathrm{mi}^{2}$.

PERIOD OF RECORD.- October 1926 to September 1976.

PERIOD OF ANALYSIS.-April 1927 to March 1949.

NUMBER OF CLIMATE YEARS IN FREQUENCY ANALYSIS._-22

REMARKS.-Since about December 1949, flow regulated by Allatoona Lake and since about April 1961, by Weiss Lake. The period of record for which regulation patterns appeared to be relatively stable was not of sufficient length to warrant low-flow frequency analyses. Consequently, low-flow characteristics were estimated for pre-regulated conditions only. However, a plot of the annual minimum 7-day average flows for the complete period of record is provided.

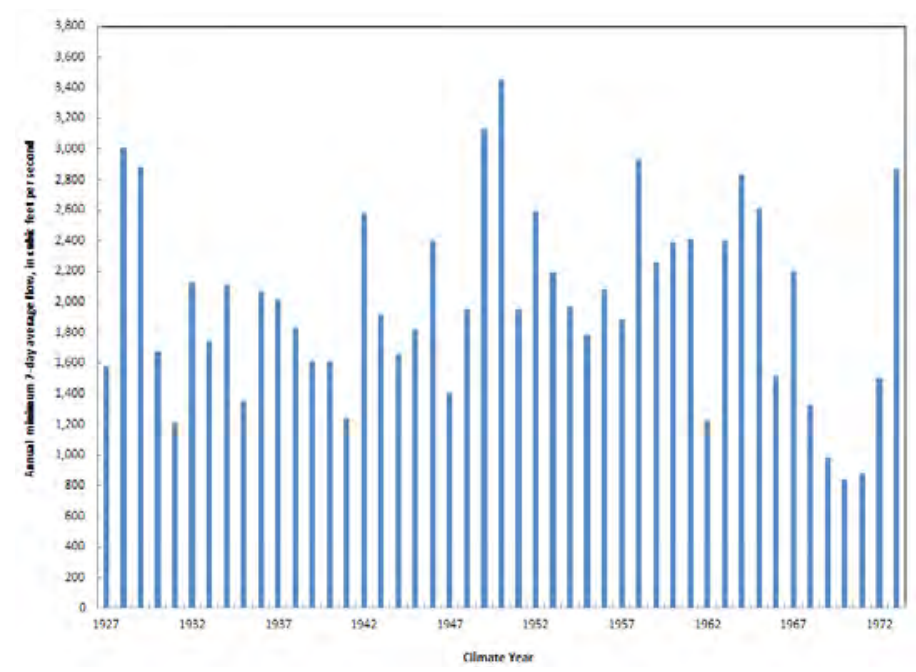

\begin{tabular}{|c|c|c|c|c|}
\hline $\begin{array}{c}\text { Recurrence } \\
\text { intervals } \\
\text { (years) }\end{array}$ & \multicolumn{2}{|c|}{$\begin{array}{c}\text { Lowest average flow for } \\
\text { indicated number of } \\
\text { consecutive days } \\
\text { (cubic feet per second) }\end{array}$} & \multicolumn{2}{|c|}{$\begin{array}{c}\text { Time-sampling error } \\
\text { (in percent) }\end{array}$} \\
\hline \multicolumn{7}{|c|}{1} & 7 & 1 & 6 \\
\hline 2 & 1,770 & 1,830 & 6 & 6 \\
\hline 5 & 1,460 & 1,500 & 6 & 6 \\
\hline 10 & 1,330 & 1,360 & 6 & 8 \\
\hline 20 & 1,240 & 1,250 & 7 & 6 \\
\hline
\end{tabular}

\begin{tabular}{|c|c|c|c|c|c|c|c|c|}
\hline \multicolumn{1}{|c|}{ DURATION OF DAILY FLOW } \\
\hline \multicolumn{7}{|c|}{ Flow equaled or exceeded for indicated percentage of time } \\
(cubic feet per second)
\end{tabular}


STATION NAME AND NUMBER—02400680 Big Wills Creek at State Highway 35 near Fort Payne, AL

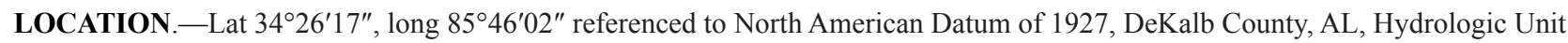
03150106, on downstream side of State Highway 35 bridge, about 2 miles west of Fort Payne.

DRAINAGE AREA. $-55.4 \mathrm{mi}^{2}$.

PERIOD OF RECORD.-October 2002 to March 2014.

PERIOD OF ANALYSIS.-April 2003 to March 2014.

NUMBER OF CLIMATE YEARS IN FREQUENCY ANALYSIS.-11

REMARKS.-

\begin{tabular}{|c|c|c|c|c|}
\hline $\begin{array}{c}\text { Recurrence } \\
\text { intervals } \\
\text { (years) }\end{array}$ & \multicolumn{2}{|c|}{$\begin{array}{c}\text { Lowest average flow for } \\
\text { indicated number of } \\
\text { consecutive days } \\
\text { (cubic feet per second) }\end{array}$} & \multicolumn{2}{|c|}{$\begin{array}{c}\text { Time-sampling error } \\
\text { (in percent) }\end{array}$} \\
\hline \multicolumn{2}{|c|}{1} & 7 & 1 & 7 \\
\hline 2 & 10 & 11 & 10 & 8 \\
\hline 5 & 8.1 & 8.6 & 8 & 8 \\
\hline 10 & 7.5 & 7.8 & 8 & 11 \\
\hline 20 & 7.0 & 7.3 & 11 & \\
\hline
\end{tabular}

\begin{tabular}{|c|c|c|c|c|c|c|c|c|}
\hline \multicolumn{1}{|c|}{ DURATION OF DAILY FLOW } \\
\hline \multicolumn{7}{|c|}{ Flow equaled or exceeded for indicated percentage of time } \\
(cubic feet per second) \\
\hline Percentage & 5 & 10 & 25 & 50 & 75 & 90 & 95 \\
\hline Flow & 235 & 153 & 83 & 41 & 18 & 12 & 9.9 \\
\hline
\end{tabular}


STATION NAME AND NUMBER—02401000 Big Wills Creek near Reece City, AL

LOCATION.-Lat 3405'53", long 8602'17" referenced to North American Datum of 1927, Etowah County, AL, Hydrologic Unit 03150106, near right bank on upstream side of bridge on county road, 1 mi upstream from Fisher Creek, 1.8 mi northwest of Reece City, and at mile 25.0.

DRAINAGE AREA.- $-182 \mathrm{mi}^{2}$.

PERIOD OF RECORD.- October 1943 to September 1970, October 1986 to March 2014.

PERIOD OF ANALYSIS.-April 1944 to March 1970, April 1987 to March 2014.

NUMBER OF CLIMATE YEARS IN FREQUENCY ANALYSIS.—-53

REMARKS.-Prior to October 1986 published as Big Wills Creek “near Crudup”.

\begin{tabular}{|c|c|c|c|c|}
\hline $\begin{array}{c}\text { Recurrence } \\
\text { intervals } \\
\text { (years) }\end{array}$ & \multicolumn{2}{|c|}{$\begin{array}{c}\text { Lowest average flow for } \\
\text { indicated number of } \\
\text { consecutive days } \\
\text { (cubic feet per second) }\end{array}$} & \multicolumn{2}{|c|}{$\begin{array}{c}\text { Time-sampling error } \\
\text { (in percent) }\end{array}$} \\
\hline & 1 & 7 & 1 & 4 \\
\hline 2 & 49 & 51 & 5 & 5 \\
\hline 5 & 38 & 39 & 5 & 6 \\
\hline 10 & 32 & 34 & 7 & 8 \\
\hline 20 & 28 & 30 & 9 & 10 \\
\hline 50 & 24 & 26 & 13 & 4 \\
\hline
\end{tabular}

\begin{tabular}{|c|c|c|c|c|c|c|c|c|}
\hline \multicolumn{1}{|c|}{ DURATION OF DAILY FLOW } \\
\hline \multicolumn{2}{|c|}{ Flow equaled or exceeded for indicated percentage of time } \\
(cubic feet per second)
\end{tabular}


STATION NAME AND NUMBER—02401370 Big Canoe Creek near Springville, AL

LOCATION.-Lat 3348'49", long 86²2'54" referenced to North American Datum of 1927, St. Clair County, AL, Hydrologic Unit 03150106, on U.S. Highway 11, 1 mi west of Caldwell, 4 mi northwest of Springville, and 37.0 mi upstream from mouth.

DRAINAGE AREA. $-45.0 \mathrm{mi}^{2}$.

PERIOD OF RECORD.- October 1978 to May 1995.

PERIOD OF ANALYSIS.-April 1979 to March 1995.

NUMBER OF CLIMATE YEARS IN FREQUENCY ANALYSIS.-16

REMARKS.-

\begin{tabular}{|c|c|c|c|c|}
\hline $\begin{array}{c}\text { Recurrence } \\
\text { intervals } \\
\text { (years) }\end{array}$ & \multicolumn{2}{|c|}{$\begin{array}{c}\text { Lowest average flow for } \\
\text { indicated number of } \\
\text { consecutive days } \\
\text { (cubic feet per second) }\end{array}$} & \multicolumn{2}{|c|}{$\begin{array}{c}\text { Time-sampling error } \\
\text { (in percent) }\end{array}$} \\
\hline \multicolumn{7}{|c|}{1} & 7 & 1 & 7 \\
\hline 2 & 7.1 & 7.5 & 7 & 7 \\
\hline 5 & 5.7 & 6.1 & 7 & 7 \\
\hline 10 & 5.2 & 5.5 & 8 & 9 \\
\hline 20 & 4.8 & 5.2 & 10 & 7 \\
\hline
\end{tabular}

\begin{tabular}{|c|c|c|c|c|c|c|c|}
\hline \multicolumn{1}{|c|}{ DURATION OF DAILY FLOW } \\
\hline \multicolumn{7}{|c|}{ Flow equaled or exceeded for indicated percentage of time } \\
(cubic feet per second)
\end{tabular}


STATION NAME AND NUMBER—02401390 Big Canoe Creek at Ashville, AL

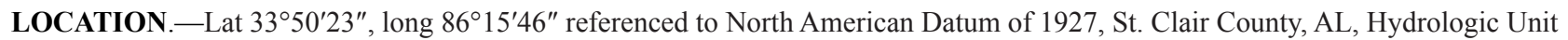
03150106, on downstream side of bridge on U.S. Highway 231, 0.5 mi west-northwest of Ashville, 1.7 mi downstream from Muckleroy Creek, and 22.3 mi upstream from mouth.

DRAINAGE AREA.-141 $\mathrm{mi}^{2}$.

PERIOD OF RECORD.- October 1965 to March 2014.

PERIOD OF ANALYSIS.-April 1966 to March 2014.

NUMBER OF CLIMATE YEARS IN FREQUENCY ANALYSIS.-48

REMARKS.-

\begin{tabular}{|c|c|c|c|c|}
\hline $\begin{array}{c}\text { Recurrence } \\
\text { intervals } \\
\text { (years) }\end{array}$ & \multicolumn{2}{|c|}{$\begin{array}{c}\text { Lowest average flow for } \\
\text { indicated number of } \\
\text { consecutive days } \\
\text { (cubic feet per second) }\end{array}$} & \multicolumn{2}{|c|}{$\begin{array}{c}\text { Time-sampling error } \\
\text { (in percent) }\end{array}$} \\
\hline & 1 & 7 & 1 & 7 \\
\hline 2 & 18 & 19 & 5 & 6 \\
\hline 5 & 13 & 14 & 6 & 8 \\
\hline 10 & 11 & 12 & 8 & 10 \\
\hline 20 & 9.5 & 10 & 10 & 14 \\
\hline 50 & 7.9 & 8.5 & 14 & \\
\hline
\end{tabular}

\begin{tabular}{|c|c|c|c|c|c|c|c|}
\hline \multicolumn{1}{|c|}{ DURATION OF DAILY FLOW } \\
\hline \multicolumn{7}{|c|}{ Flow equaled or exceeded for indicated percentage of time } \\
(cubic feet per second)
\end{tabular}


STATION NAME AND NUMBER—02401470 Little Canoe Creek near Steele, AL

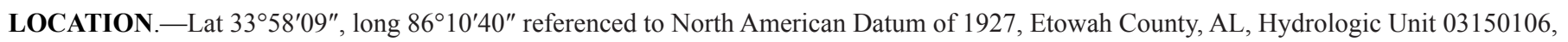
on U.S. Highway 11, $2.3 \mathrm{mi}$ north of Steele, $7.2 \mathrm{mi}$ upstream from mouth.

DRAINAGE AREA. $-22.3 \mathrm{mi}^{2}$.

PERIOD OF RECORD.-April 1982 to May 1995.

PERIOD OF ANALYSIS.-April 1982 to March 1995.

NUMBER OF CLIMATE YEARS IN FREQUENCY ANALYSIS.-13

REMARKS.-

\begin{tabular}{|c|c|c|c|c|}
\hline $\begin{array}{c}\text { Recurrence } \\
\text { intervals } \\
\text { (years) }\end{array}$ & \multicolumn{2}{|c|}{$\begin{array}{c}\text { Lowest average flow for } \\
\text { indicated number of } \\
\text { consecutive days } \\
\text { (cubic feet per second) }\end{array}$} & \multicolumn{2}{|c|}{$\begin{array}{c}\text { Time-sampling error } \\
\text { (in percent) }\end{array}$} \\
\hline \multicolumn{7}{|c|}{} & 1 & 7 & 1 & 7 \\
\hline 2 & 2.4 & 2.8 & 15 & 17 \\
\hline 5 & 1.6 & 1.9 & 17 & 21 \\
\hline 10 & 1.2 & 1.5 & 21 & 27 \\
\hline 20 & 1.0 & 1.2 & 26 & \\
\hline
\end{tabular}

\begin{tabular}{|c|c|c|c|c|c|c|c|}
\hline \multicolumn{1}{|c|}{ DURATION OF DAILY FLOW } \\
\hline \multicolumn{7}{|c|}{ Flow equaled or exceeded for indicated percentage of time } \\
(cubic feet per second)
\end{tabular}


STATION NAME AND NUMBER—02401500 Big Canoe Creek near Gadsden, AL

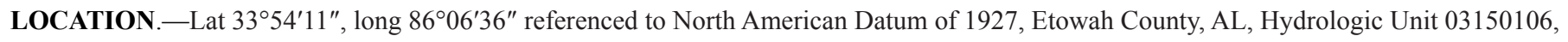
on U.S. Highway 411, $400 \mathrm{ft}$ downstream from Rock Creek, 5 mi upstream from mouth, and 10 mi southwest of Gadsden.

DRAINAGE AREA.-253 $\mathrm{mi}^{2}$.

PERIOD OF RECORD._-January 1938 to September 1965.

PERIOD OF ANALYSIS.-April 1938 to March 1965.

NUMBER OF CLIMATE YEARS IN FREQUENCY ANALYSIS.—-26

REMARKS.-

\begin{tabular}{|c|c|c|c|c|}
\hline $\begin{array}{c}\text { Recurrence } \\
\text { intervals } \\
\text { (years) }\end{array}$ & \multicolumn{2}{|c|}{$\begin{array}{c}\text { Lowest average flow for } \\
\text { indicated number of } \\
\text { consecutive days } \\
\text { (cubic feet per second) }\end{array}$} & \multicolumn{2}{|c|}{$\begin{array}{c}\text { Time-sampling error } \\
\text { (in percent) }\end{array}$} \\
\hline \multicolumn{7}{|c|}{1} & 7 & 1 & 7 \\
\hline 2 & 19 & 20 & 8 & 8 \\
\hline 5 & 14 & 15 & 9 & 10 \\
\hline 10 & 11 & 13 & 10 & 12 \\
\hline 20 & 10 & 11 & 13 & \\
\hline
\end{tabular}

\begin{tabular}{|c|c|c|c|c|c|c|c|c|}
\hline \multicolumn{1}{|c|}{ DURATION OF DAILY FLOW } \\
\hline \multicolumn{1}{|c|}{ Flow equaled or exceeded for indicated percentage of time } \\
(cubic feet per second) \\
\hline Percentage & 5 & 10 & 25 & 50 & 75 & 90 & 95 \\
\hline Flow & 1,950 & 1,060 & 431 & 122 & 43 & 24 & 19 \\
\hline
\end{tabular}


STATION NAME AND NUMBER—02402500 Coosa River at Riverside, AL

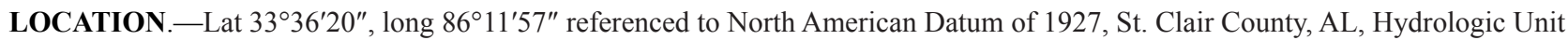
03150106, 1 mi upstream from Blue Eye Creek, 4 mi downstream from dam at Lock 4, and 7 mi upstream from Choccolocco Creek.

DRAINAGE AREA. $-7,069 \mathrm{mi}^{2}$.

PERIOD OF RECORD.- October 1896 to September 1916.

PERIOD OF ANALYSIS.-April 1897 to March 1916.

NUMBER OF CLIMATE YEARS IN FREQUENCY ANALYSIS.-19

REMARKS.-Since about December 1949, flow regulated by Allatoona Lake and since about April 1961, by Weiss Lake. The period of record analyzed represents pre-regulated conditions.

\begin{tabular}{|c|c|c|c|c|}
\hline \multirow[t]{2}{*}{$\begin{array}{l}\text { Recurrence } \\
\text { intervals } \\
\text { (years) }\end{array}$} & \multicolumn{2}{|c|}{$\begin{array}{l}\text { Lowest average flow for } \\
\text { indicated number of } \\
\text { consecutive days } \\
\text { (cubic feet per second) }\end{array}$} & \multicolumn{2}{|c|}{$\begin{array}{l}\text { Time-sampling error } \\
\text { (in percent) }\end{array}$} \\
\hline & 1 & 7 & 1 & 7 \\
\hline 2 & 2,350 & 2,480 & 8 & 8 \\
\hline 5 & 1,810 & 1,880 & 8 & 9 \\
\hline 10 & 1,570 & 1,610 & 10 & 11 \\
\hline 20 & 1,400 & 1,420 & 13 & 14 \\
\hline
\end{tabular}

\section{DURATION OF DAILY FLOW}

Flow equaled or exceeded for indicated percentage of time (cubic feet per second)

\begin{tabular}{|c|c|c|c|c|c|c|c|}
\hline Percentage & 5 & 10 & 25 & 50 & 75 & 90 & 95 \\
\hline Flow & 42,200 & 28,800 & 13,800 & 7,060 & 3,890 & 2,720 & 2,260 \\
\hline
\end{tabular}


STATION NAME AND NUMBER—02403500 Coldwater Spring near Anniston, AL

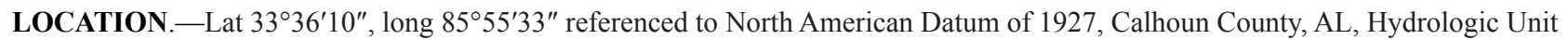
03150106, in pool of Coldwater Spring, $200 \mathrm{ft}$ upstream from Coldwater Creek, 2 mi upstream from Choccolocco Creek, and 7 mi southwest of Anniston.

DRAINAGE AREA.--Indeterminate

PERIOD OF RECORD.-April 1957 to September 1996.

PERIOD OF ANALYSIS.-April 1957 to March 1996.

NUMBER OF CLIMATE YEARS IN FREQUENCY ANALYSIS. - 39

REMARKS.-Atkins and Pearman (1994) noted that flows include about $20 \mathrm{ft}^{3} / \mathrm{s}$ diverted by city of Anniston for water supply. Drainage area not determined due to streamflow originating from a spring.

\begin{tabular}{|c|c|c|c|c|}
\hline $\begin{array}{l}\text { Recurrence } \\
\text { intervals } \\
\text { (years) }\end{array}$ & \multicolumn{2}{|c|}{$\begin{array}{l}\text { Lowest average flow for } \\
\text { indicated number of } \\
\text { consecutive days } \\
\text { (cubic feet per second) }\end{array}$} & \multicolumn{2}{|c|}{$\begin{array}{l}\text { Time-sampling error } \\
\text { (in percent) }\end{array}$} \\
\hline & 1 & 7 & 1 & 7 \\
\hline 2 & 40 & 41 & 2 & 2 \\
\hline 5 & 36 & 37 & 3 & 3 \\
\hline 10 & 34 & 35 & 4 & 3 \\
\hline 20 & 32 & 33 & 5 & 5 \\
\hline 50 & 29 & 31 & 8 & 7 \\
\hline
\end{tabular}

\begin{tabular}{|c|c|c|c|c|c|c|c|}
\hline \multicolumn{8}{|c|}{ DURATION OF DAILY FLOW } \\
\hline \multicolumn{8}{|c|}{$\begin{array}{l}\text { Flow equaled or exceeded for indicated percentage of time } \\
\text { (cubic feet per second) }\end{array}$} \\
\hline Percentage & 5 & 10 & 25 & 50 & 75 & 90 & 95 \\
\hline Flow & 59 & 56 & 52 & 48 & 43 & 39 & 36 \\
\hline
\end{tabular}


STATION NAME AND NUMBER—02404000 Choccolocco Creek near Jenifer, AL

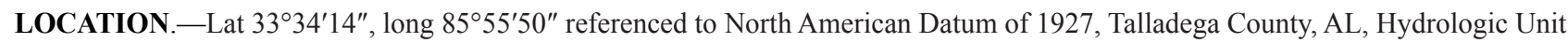
03150106, 0.8 mi upstream from Salt Creek, and 1.5 mi north of Jenifer.

DRAINAGE AREA. $-277 \mathrm{mi}^{2}$.

PERIOD OF RECORD.-August 1903 to February 1908, October 1929 to March 1932, and October 1935 to September 1970.

PERIOD OF ANALYSIS.-April 1904 to March 1907, April 1930 to March 1932, and April 1936 to March 1970.

NUMBER OF CLIMATE YEARS IN FREQUENCY ANALYSIS.-39

REMARKS.-

\begin{tabular}{|c|c|c|c|c|}
\hline $\begin{array}{c}\text { Recurrence } \\
\text { intervals } \\
\text { (years) }\end{array}$ & \multicolumn{2}{|c|}{$\begin{array}{c}\text { Lowest average flow for } \\
\text { indicated number of } \\
\text { consecutive days } \\
\text { (cubic feet per second) }\end{array}$} & \multicolumn{2}{|c|}{$\begin{array}{c}\text { Time-sampling error } \\
\text { (in percent) }\end{array}$} \\
\hline \multicolumn{5}{|c|}{} \\
\hline & 88 & 7 & 1 & 5 \\
\hline 2 & 67 & 70 & 6 & 6 \\
\hline 5 & 56 & 61 & 9 & 7 \\
\hline 10 & 48 & 55 & 13 & 9 \\
\hline 20 & 40 & 48 & 18 & 11 \\
\hline 50 &
\end{tabular}

\begin{tabular}{|c|c|c|c|c|c|c|c|c|}
\hline \multicolumn{7}{|c|}{ DURATION OF DAILY FLOW } \\
\hline \multicolumn{7}{|c|}{ Flow equaled or exceeded for indicated percentage of time } \\
(cubic feet per second)
\end{tabular}


STATION NAME AND NUMBER—02404400 Choccolocco Creek at Jackson Shoal near Lincoln, AL

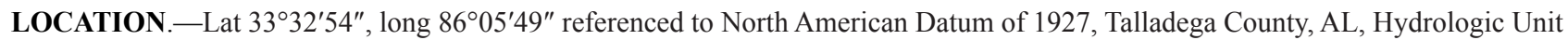
03150106, on left bank at foot of Jackson Shoals, $50 \mathrm{ft}$ upstream from Alabama Power Company Jackson Shoals transformer station, $900 \mathrm{ft}$ upstream from highway bridge, $1.8 \mathrm{mi}$ downstream from Eastaboga Creek, and $4.5 \mathrm{mi}$ southeast of Lincoln.

DRAINAGE AREA. - $-481 \mathrm{mi}^{2}$.

PERIOD OF RECORD.- October 1960 to September 1967, October 1984 to March 2014.

PERIOD OF ANALYSIS.-April 1961 to March 1967, April 1985 to March 2014.

NUMBER OF CLIMATE YEARS IN FREQUENCY ANALYSIS.—-35

REMARKS.-Potential diversion upstream for municipal water supply for city of Anniston.

\begin{tabular}{|c|c|c|c|c|}
\hline $\begin{array}{c}\text { Recurrence } \\
\text { intervals } \\
\text { (years) }\end{array}$ & \multicolumn{2}{|c|}{$\begin{array}{c}\text { Lowest average flow for } \\
\text { indicated number of } \\
\text { consecutive days } \\
\text { (cubic feet per second) }\end{array}$} & \multicolumn{2}{|c|}{$\begin{array}{c}\text { Time-sampling error } \\
\text { (in percent) }\end{array}$} \\
\hline & 1 & 7 & 1 & 5 \\
\hline 2 & 171 & 177 & 5 & 7 \\
\hline 5 & 132 & 136 & 7 & 8 \\
\hline 10 & 113 & 117 & 8 & 11 \\
\hline 20 & 99 & 102 & 11 & 15 \\
\hline 50 & 84 & 88 & 15 & 7 \\
\hline
\end{tabular}

\begin{tabular}{|c|c|c|c|c|c|c|c|}
\hline \multicolumn{1}{|c|}{ DURATION OF DAILY FLOW } \\
\hline \multicolumn{7}{|c|}{ Flow equaled or exceeded for indicated percentage of time } \\
(cubic feet per second)
\end{tabular}


STATION NAME AND NUMBER—02404500 Choccolocco Creek near Lincoln, AL

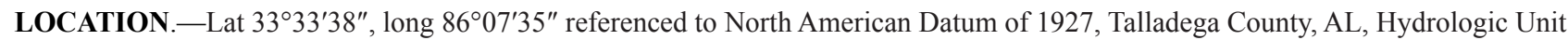
03150106, on State Highway 77, 4 mi south of Lincoln, 6 mi upstream from mouth, and 8 mi north of Talladega.

DRAINAGE AREA. - $496 \mathrm{mi}^{2}$.

PERIOD OF RECORD.- October 1938 to September 1953.

PERIOD OF ANALYSIS.-April 1939 to March 1953.

NUMBER OF CLIMATE YEARS IN FREQUENCY ANALYSIS.—14

REMARKS.-

\begin{tabular}{|c|c|c|c|c|}
\hline $\begin{array}{c}\text { Recurrence } \\
\text { intervals } \\
\text { (years) }\end{array}$ & \multicolumn{2}{|c|}{$\begin{array}{c}\text { Lowest average flow for } \\
\text { indicated number of } \\
\text { consecutive days } \\
\text { (cubic feet per second) }\end{array}$} & \multicolumn{2}{|c|}{$\begin{array}{c}\text { Time-sampling error } \\
\text { (in percent) }\end{array}$} \\
\hline \multicolumn{7}{|c|}{1} & 7 & 1 & 7 \\
\hline 2 & 173 & 177 & 3 & 3 \\
\hline 5 & 159 & 163 & 3 & 4 \\
\hline 10 & 153 & 156 & 3 & 4 \\
\hline 20 & 148 & 150 & 4 & 3 \\
\hline
\end{tabular}

\begin{tabular}{|c|c|c|c|c|c|c|c|c|}
\hline \multicolumn{1}{|c|}{ DURATION OF DAILY FLOW } \\
\hline \multicolumn{7}{|c|}{ Flow equaled or exceeded for indicated percentage of time } \\
(cubic feet per second) \\
\hline Percentage & 5 & 10 & 25 & 50 & 75 & 90 & 95 \\
\hline Flow & 2,200 & 1,380 & 741 & 374 & 241 & 193 & 180 \\
\hline
\end{tabular}


STATION NAME AND NUMBER—02405500 Kelly Creek near Vincent, AL

LOCATION.-Lat 3326'51", long 86²3'13" referenced to North American Datum of 1927, Shelby County, AL, Hydrologic Unit 03150106, on downstream side of left pier of bridge on U.S. Highway 231, 1.5 mi downstream of Little Creek, 4.2 mi north of Vincent, and 5 mi upstream of mouth.

DRAINAGE AREA.-193 $\mathrm{mi}^{2}$.

PERIOD OF RECORD.-December 1951 to September 1970, October 1986 to March 2014.

PERIOD OF ANALYSIS.-April 1952 to March 1970.

NUMBER OF CLIMATE YEARS IN FREQUENCY ANALYSIS.-18

REMARKS.-This period of record analyzed likely represents a more natural condition than the period from October 1986 to March 2014.

\begin{tabular}{|c|c|c|c|c|}
\hline $\begin{array}{c}\text { Recurrence } \\
\text { intervals } \\
\text { (years) }\end{array}$ & \multicolumn{2}{|c|}{$\begin{array}{c}\text { Lowest average flow for } \\
\text { indicated number of } \\
\text { consecutive days } \\
\text { (cubic feet per second) }\end{array}$} & \multicolumn{2}{|c|}{$\begin{array}{c}\text { Time-sampling error } \\
\text { (in percent) }\end{array}$} \\
\hline \multicolumn{7}{|c|}{1} & 7 & 1 & 7 \\
\hline 2 & 3.7 & 4.1 & 13 & 14 \\
\hline 5 & 2.4 & 2.6 & 13 & 16 \\
\hline 10 & 2.0 & 2.1 & 14 & 19 \\
\hline 20 & 1.7 & 1.8 & 17 & 14 \\
\hline
\end{tabular}

\section{DURATION OF DAILY FLOW}

Flow equaled or exceeded for indicated percentage of time (cubic feet per second)

\begin{tabular}{|c|c|c|c|c|c|c|c|}
\hline Percentage & 5 & 10 & 25 & 50 & 75 & 90 & 95 \\
\hline Flow & 1,230 & 784 & 327 & 79 & 16 & 5.9 & 3.7 \\
\hline
\end{tabular}


STATION NAME AND NUMBER—02405500 Kelly Creek near Vincent, AL

LOCATION.-Lat 3326'51", long 86²3'13" referenced to North American Datum of 1927, Shelby County, AL, Hydrologic Unit 03150106, on downstream side of left pier of bridge on U.S. Highway 231, 1.5 mi downstream of Little Creek, 4.2 mi north of Vincent, and 5 mi upstream of mouth.

DRAINAGE AREA. - $193 \mathrm{mi}^{2}$.

PERIOD OF RECORD.--December 1951 to September 1970, October 1986 to March 2014.

PERIOD OF ANALYSIS.-April 1987 to March 2014.

NUMBER OF CLIMATE YEARS IN FREQUENCY ANALYSIS.—27

REMARKS.-QAQC reviews indicated that the streamflow record included for this period of analysis is likely influenced by anthropogenic sources.

\begin{tabular}{|c|c|c|c|c|}
\hline $\begin{array}{c}\text { Recurrence } \\
\text { intervals } \\
\text { (years) }\end{array}$ & \multicolumn{2}{|c|}{$\begin{array}{c}\text { Lowest average flow for } \\
\text { indicated number of } \\
\text { consecutive days } \\
\text { (cubic feet per second) }\end{array}$} & \multicolumn{2}{|c|}{$\begin{array}{c}\text { Time-sampling error } \\
\text { (in percent) }\end{array}$} \\
\hline \multicolumn{2}{|c|}{1} & 7 & 1 & 7 \\
\hline 2 & 5.4 & 6.1 & 17 & 20 \\
\hline 5 & 2.7 & 3.0 & 20 & 25 \\
\hline 10 & 1.8 & 2.0 & 25 & 32 \\
\hline 20 & 1.3 & 1.5 & 32 & \\
\hline
\end{tabular}

\begin{tabular}{|c|c|c|c|c|c|c|c|c|}
\hline \multicolumn{7}{|c|}{ DURATION OF DAILY FLOW } \\
\hline \multicolumn{7}{|c|}{ Flow equaled or exceeded for indicated percentage of time } \\
(cubic feet per second)
\end{tabular}


STATION NAME AND NUMBER—02405800 Talladega Creek above Talladega, AL

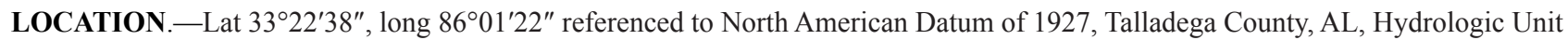
03150106, right bank $300 \mathrm{ft}$ upstream from Mump Creek, 0.5 mi upstream from bridge on State Highway 77, and 6 mi southeast of Talladega.

DRAINAGE AREA. $-69.6 \mathrm{mi}^{2}$.

PERIOD OF RECORD.- June 1959 to September 1970.

PERIOD OF ANALYSIS.-April 1960 to March 1970.

NUMBER OF CLIMATE YEARS IN FREQUENCY ANALYSIS.—10

REMARKS.-

\begin{tabular}{|c|c|c|c|c|}
\hline $\begin{array}{c}\text { Recurrence } \\
\text { intervals } \\
\text { (years) }\end{array}$ & \multicolumn{2}{|c|}{$\begin{array}{c}\text { Lowest average flow for } \\
\text { indicated number of } \\
\text { consecutive days } \\
\text { (cubic feet per second) }\end{array}$} & \multicolumn{2}{|c|}{$\begin{array}{c}\text { Time-sampling error } \\
\text { (in percent) }\end{array}$} \\
\hline \multicolumn{7}{|c|}{1} & 7 & 1 & 7 \\
\hline 2 & 13 & 14 & 14 & 13 \\
\hline 5 & 9.3 & 10 & 14 & 15 \\
\hline 10 & 8.0 & 9.0 & 15 & 18 \\
\hline 20 & 7.0 & 7.9 & 18 & \\
\hline
\end{tabular}

\begin{tabular}{|c|c|c|c|c|c|c|c|}
\hline \multicolumn{1}{|c|}{ DURATION OF DAILY FLOW } \\
\hline \multicolumn{1}{|c|}{ Flow equaled or exceeded for indicated percentage of time } \\
(cubic feet per second) \\
\hline Percentage & 5 & 10 & 25 & 50 & 75 & 90 & 95 \\
\hline Flow & 302 & 200 & 114 & 55 & 28 & 18 & 14 \\
\hline
\end{tabular}


STATION NAME AND NUMBER—02406500 Talladega Creek at Alpine, AL

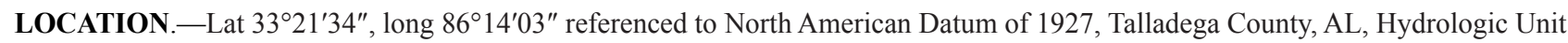
03150106, at bridge on County Road 207, 1 mi north of Alpine, 9 mi southwest of Talladega, and 11.0 mi upstream of mouth.

DRAINAGE AREA. $-150 \mathrm{mi}^{2}$.

PERIOD OF RECORD.-August 1900 to December 1904, October 1939 to September 1951, October 1987 to March 2014.

PERIOD OF ANALYSIS.-April 1901 to March 1904, April 1940 to March 1951, April 1988 to March 2014.

NUMBER OF CLIMATE YEARS IN FREQUENCY ANALYSIS.—40

REMARKS.-

\begin{tabular}{|c|c|c|c|c|}
\hline $\begin{array}{c}\text { Recurrence } \\
\text { intervals } \\
\text { (years) }\end{array}$ & \multicolumn{2}{|c|}{$\begin{array}{c}\text { Lowest average flow for } \\
\text { indicated number of } \\
\text { consecutive days } \\
\text { (cubic feet per second) }\end{array}$} & \multicolumn{2}{|c|}{$\begin{array}{c}\text { Time-sampling error } \\
\text { (in percent) }\end{array}$} \\
\hline \multicolumn{7}{|c|}{} & 1 & 7 & 1 & 5 \\
\hline 2 & 61 & 63 & 5 & 6 \\
\hline 5 & 48 & 49 & 6 & 7 \\
\hline 10 & 42 & 43 & 7 & 9 \\
\hline 20 & 37 & 38 & 8 & 12 \\
\hline 50 & 33 & 33 & 11 & \\
\hline
\end{tabular}

\begin{tabular}{|c|c|c|c|c|c|c|c|c|}
\hline \multicolumn{7}{|c|}{ DURATION OF DAILY FLOW } \\
\hline \multicolumn{7}{|c|}{ Flow equaled or exceeded for indicated percentage of time } \\
(cubic feet per second) \\
\hline Percentage & 5 & 10 & 25 & 50 & 75 & 90 & 95 \\
\hline Flow & 612 & 441 & 263 & 141 & 88 & 64 & 51 \\
\hline
\end{tabular}


STATION NAME AND NUMBER—02407000 Coosa River at Childersburg, AL

LOCATION.-Lat $33^{\circ} 17^{\prime} 30^{\prime \prime}$, long 86²1'50" referenced to North American Datum of 1927, Shelby County, AL, Hydrologic Unit 03150107, near right bank on downstream side of Central of Georgia Railway bridge, $700 \mathrm{ft}$ upstream of bridge on State Highway $38,0.5 \mathrm{mi}$ downstream of Tallasseehatchee Creek, 1 mi northwest of Childersburg, and at mile 86.3.

DRAINAGE AREA.- $8,392 \mathrm{mi}^{2}$.

PERIOD OF RECORD.- October 1913 to September 1978, June 2011 to September 2013.

PERIOD OF ANALYSIS.-April 1914 to March 1948.

\section{NUMBER OF CLIMATE YEARS IN FREQUENCY ANALYSIS.-35}

REMARKS.-The period of record analyzed represents pre-regulated conditions. Since December 1949 flow regulated by Allatoona Lake on Etowah River, since April 1961 by Weiss Lake on Coosa River, since July 1964 by Logan-Martin Lake on Coosa River, and since 1966 by H. Neely Henry Lake on the Coosa River. QAQC reviews did not indicate a sufficient length of record with relatively stable regulation patterns to warrant frequency analysis for the regulated period.

\begin{tabular}{|c|c|c|c|c|}
\hline $\begin{array}{l}\text { Recurrence } \\
\text { intervals } \\
\text { (years) }\end{array}$ & \multicolumn{2}{|c|}{$\begin{array}{l}\text { Lowest average flow for } \\
\text { indicated number of } \\
\text { consecutive days } \\
\text { (cubic feet per second) }\end{array}$} & \multicolumn{2}{|c|}{$\begin{array}{l}\text { Time-sampling error } \\
\text { (in percent) }\end{array}$} \\
\hline & 1 & 7 & 1 & 7 \\
\hline 2 & 2,660 & 2,760 & 5 & 5 \\
\hline 5 & 2,120 & 2,190 & 6 & 6 \\
\hline 10 & 1,860 & 1,910 & 7 & 8 \\
\hline 20 & 1,660 & 1,690 & 10 & 10 \\
\hline 50 & 1,450 & 1,470 & 13 & 14 \\
\hline
\end{tabular}

\section{DURATION OF DAILY FLOW}

Flow equaled or exceeded for indicated percentage of time (cubic feet per second)

\begin{tabular}{|c|c|c|c|c|c|c|c|}
\hline Percentage & 5 & 10 & 25 & 50 & 75 & 90 & 95 \\
\hline Flow & 50,900 & 34,400 & 15,400 & 7,560 & 4,440 & 3,110 & 2,690 \\
\hline
\end{tabular}


STATION NAME AND NUMBER—02407500 Yellowleaf Creek near Wilsonville, AL

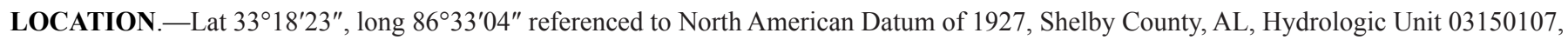
on county road, 3.5 mi south of U.S. Highway 280, 4 mi upstream from Muddy Prong, and 6 mi northwest of Wilsonville.

DRAINAGE AREA. $-96.5 \mathrm{mi}^{2}$.

PERIOD OF RECORD.--June 1951 to September 1967.

PERIOD OF ANALYSIS.-April 1952 to March 1967.

NUMBER OF CLIMATE YEARS IN FREQUENCY ANALYSIS.—16

REMARKS.-

\begin{tabular}{|c|c|c|c|c|}
\hline $\begin{array}{c}\text { Recurrence } \\
\text { intervals } \\
\text { (years) }\end{array}$ & \multicolumn{2}{|c|}{$\begin{array}{c}\text { Lowest average flow for } \\
\text { indicated number of } \\
\text { consecutive days } \\
\text { (cubic feet per second) }\end{array}$} & \multicolumn{2}{|c|}{$\begin{array}{c}\text { Time-sampling error } \\
\text { (in percent) }\end{array}$} \\
\hline \multicolumn{7}{|c|}{1} & 7 & 1 & 7 \\
\hline 2 & 0.33 & 0.46 & 31 & 62 \\
\hline 5 & 0.04 & 0.06 & 36 & -- \\
\hline 10 & 0.00 & 0.00 & -- & -- \\
\hline 20 & 0.00 & 0.00 & -- & 46 \\
\hline
\end{tabular}

\begin{tabular}{|c|c|c|c|c|c|c|c|}
\hline \multicolumn{1}{|c|}{ DURATION OF DAILY FLOW } \\
\hline \multicolumn{7}{|c|}{ Flow equaled or exceeded for indicated percentage of time } \\
(cubic feet per second)
\end{tabular}


STATION NAME AND NUMBER—02408500 Hatchet Creek near Rockford, AL

LOCATION.-Lat 32 56'42", long 86 $13^{\prime} 06^{\prime \prime}$ referenced to North American Datum of 1927, Coosa County, AL, Hydrologic Unit 03150107, on county road, 1 mi downstream from U.S. Highway 231, 1.5 mi downstream from Socapatoy Creek, and 4 mi north of Rockford.

DRAINAGE AREA.-233 $\mathrm{mi}^{2}$.

PERIOD OF RECORD.- October 1944 to February 1979.

PERIOD OF ANALYSIS.-April 1945 to March 1978.

NUMBER OF CLIMATE YEARS IN FREQUENCY ANALYSIS.—33

REMARKS.-

\begin{tabular}{|c|c|c|c|c|}
\hline \multirow[t]{2}{*}{$\begin{array}{l}\text { Recurrence } \\
\text { intervals } \\
\text { (years) }\end{array}$} & \multicolumn{2}{|c|}{$\begin{array}{l}\text { Lowest average flow for } \\
\text { indicated number of } \\
\text { consecutive days } \\
\text { (cubic feet per second) }\end{array}$} & \multicolumn{2}{|c|}{$\begin{array}{l}\text { Time-sampling error } \\
\text { (in percent) }\end{array}$} \\
\hline & 1 & 7 & 1 & 7 \\
\hline 2 & 45 & 51 & 10 & 11 \\
\hline 5 & 28 & 31 & 12 & 13 \\
\hline 10 & 21 & 23 & 16 & 17 \\
\hline 20 & 16 & 18 & 21 & 23 \\
\hline 50 & 12 & 13 & 29 & 32 \\
\hline
\end{tabular}

\begin{tabular}{|c|c|c|c|c|c|c|c|c|}
\hline \multicolumn{7}{|c|}{ DURATION OF DAILY FLOW } \\
\hline \multicolumn{1}{|c|}{ Flow equaled or exceeded for indicated percentage of time } \\
(cubic feet per second)
\end{tabular}


STATION NAME AND NUMBER—02408540 Hatchet Creek below Rockford, AL

LOCATION.-Lat $32^{\circ} 55^{\prime} 00^{\prime \prime}$, long $86^{\circ} 16^{\prime} 13^{\prime \prime}$ referenced to North American Datum of 1927, Coosa County, AL, Hydrologic Unit 03150107, on downstream side of pier near right bank of bridge on County Road 18, 2.1 mi downstream of Jack Creek, and 4 mi northwest of Rockford.

DRAINAGE AREA. $-263 \mathrm{mi}^{2}$.

PERIOD OF RECORD.-October 1980 to March 2014.

PERIOD OF ANALYSIS.-April 1981 to March 2014.

NUMBER OF CLIMATE YEARS IN FREQUENCY ANALYSIS.—33

REMARKS.-

\begin{tabular}{|c|c|c|c|c|}
\hline $\begin{array}{c}\text { Recurrence } \\
\text { intervals } \\
\text { (years) }\end{array}$ & \multicolumn{2}{|c|}{$\begin{array}{c}\text { Lowest average flow for } \\
\text { indicated number of } \\
\text { consecutive days } \\
\text { (cubic feet per second) }\end{array}$} & \multicolumn{2}{|c|}{$\begin{array}{c}\text { Time-sampling error } \\
\text { (in percent) }\end{array}$} \\
\hline \multicolumn{5}{|c|}{} \\
\hline & 35 & 7 & 1 & 79 \\
\hline 2 & 15 & 17 & 19 & 23 \\
\hline 5 & 8.6 & 9.9 & 24 & 32 \\
\hline 10 & 5.3 & 6.1 & 45 & 45 \\
\hline 20 & 2.9 & 3.4 & 68 & 69 \\
\hline 50 &
\end{tabular}

\begin{tabular}{|c|c|c|c|c|c|c|c|}
\hline \multicolumn{1}{|c|}{ DURATION OF DAILY FLOW } \\
\hline \multicolumn{1}{|c|}{ Flow equaled or exceeded for indicated percentage of time } \\
(cubic feet per second)
\end{tabular}


STATION NAME AND NUMBER—02410000 Paterson Creek near Central, AL

LOCATION.-Lat $32^{\circ} 40^{\prime} 54^{\prime \prime}$, long $86^{\circ} 07^{\prime} 40^{\prime \prime}$ referenced to North American Datum of 1927, Elmore County, AL, Hydrologic Unit 03150107, on county road, 2 mi west of Central, and 11 mi northeast of Wetumpka.

DRAINAGE AREA. - $4.91 \mathrm{mi}^{2}$.

PERIOD OF RECORD.-October 1953 to September 1987.

PERIOD OF ANALYSIS.-April 1954 to March 1987.

NUMBER OF CLIMATE YEARS IN FREQUENCY ANALYSIS.—33

REMARKS.-

\begin{tabular}{|c|c|c|c|c|}
\hline \multirow[t]{2}{*}{$\begin{array}{l}\text { Recurrence } \\
\text { intervals } \\
\text { (years) }\end{array}$} & \multicolumn{2}{|c|}{$\begin{array}{l}\text { Lowest average flow for } \\
\text { indicated number of } \\
\text { consecutive days } \\
\text { (cubic feet per second) }\end{array}$} & \multicolumn{2}{|c|}{$\begin{array}{l}\text { Time-sampling error } \\
\text { (in percent) }\end{array}$} \\
\hline & 1 & 7 & 1 & 7 \\
\hline 2 & 0.54 & 0.66 & 24 & 22 \\
\hline 5 & 0.18 & 0.24 & 30 & 28 \\
\hline 10 & 0.09 & 0.13 & 40 & 37 \\
\hline 20 & 0.05 & 0.08 & 56 & 51 \\
\hline 50 & 0.02 & 0.04 & 86 & 78 \\
\hline
\end{tabular}

\section{DURATION OF DAILY FLOW}

Flow equaled or exceeded for indicated percentage of time (cubic feet per second)

\begin{tabular}{|c|c|c|c|c|c|c|c|}
\hline Percentage & 5 & 10 & 25 & 50 & 75 & 90 & 95 \\
\hline Flow & 20 & 13 & 7.6 & 3.9 & 1.9 & 1.0 & 0.63 \\
\hline
\end{tabular}


STATION NAME AND NUMBER—02411000 Coosa River at Jordan Dam near Wetumpka, AL

LOCATION.-Lat $32^{\circ} 36^{\prime} 50^{\prime \prime}$, long $86^{\circ} 15^{\prime} 18^{\prime \prime}$ referenced to North American Datum of 1927, Elmore County, AL, Hydrologic Unit 03150107, on right bank $0.5 \mathrm{mi}$ downstream of Jordan Dam, 4 mi upstream of Corn Creek, 5.5 mi northwest of Wetumpka, and at river mile 18.6.

DRAINAGE AREA. $-10,102 \mathrm{mi}^{2}$.

PERIOD OF RECORD.- October 1912 to September 1914, October 1925 to November 1925, February 1926 to September 2013.

PERIOD OF ANALYSIS. - April 1975 to March 2013.

REMARKS.-Because of the various patterns of regulation, only a plot of the annual minimum 7-day average flows are being provided. On the Coosa River, flow has been regulated since 1914 by Lay Dam, since 1923 by Mitchell Dam, since 1929 by Jordan Dam, since 1961 by Weiss Dam, since 1964 by Logan-Martin Dam, since 1966 by H. Neely Henry Dam, and since 1967 by Walter Bouldin Dam. Since 1949, flow regulated by Allatoona Lake on Etowah River and since 1974, flow regulated by Carter Lake on the Coosawattee River. The QAQC reviews indicated that the regulation patterns were not suitable for a frequency analysis. Thus, only the 7-day exceedance percentiles and daily durations for climate year 1975 forward, which is the period for which all major dams and reservoirs were in place, were computed.

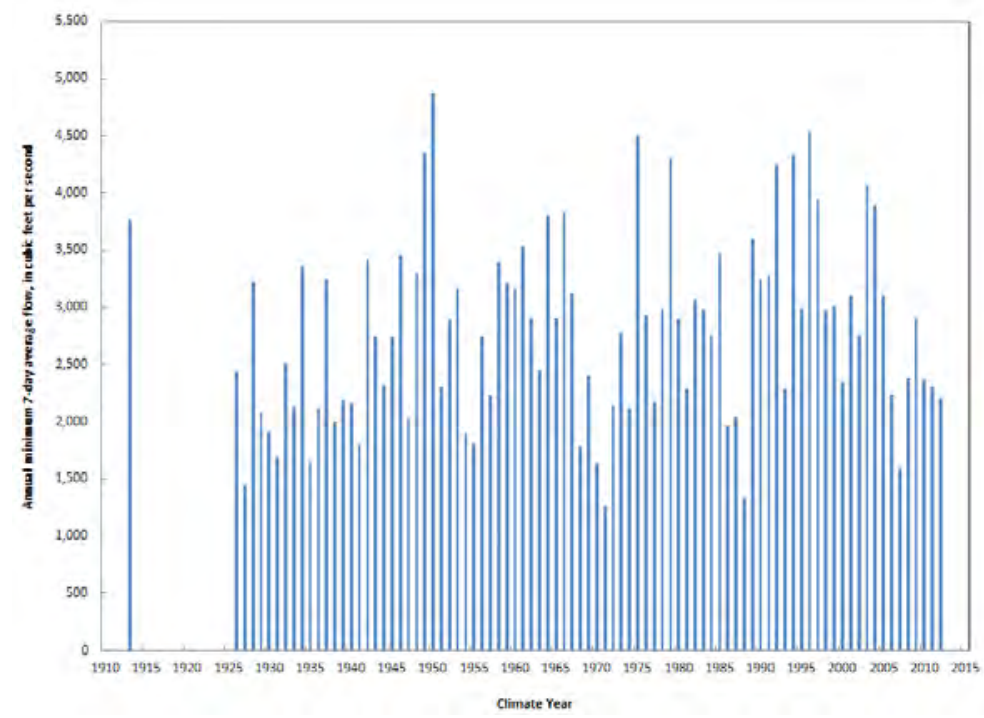

\begin{tabular}{|l|c|c|c|c|c|c|c|c|c|c|}
\hline \multicolumn{1}{|c|}{ EXCEEDANCE PERCENTILES OF ANNUAL MINIMUM 7-DAY AVERAGE FLOWS } \\
\hline Annual minimum 7-day average flow exceeded for indicated percentiles (cubic feet per second) \\
\hline Percentile & 10 & 20 & 30 & 40 & 50 & 60 & 70 & 80 & 90 \\
\hline Flow & 4,300 & 3,900 & 3,260 & 3,030 & 2,980 & 2,840 & 2,360 & 2.280 & 2,030 \\
\hline
\end{tabular}

\begin{tabular}{|l|c|c|c|c|c|c|c|}
\hline \multicolumn{7}{|c|}{ DURATION OF DAILY FLOW } \\
\hline Flow equaled or exceeded for indicated percentage of time (cubic feet per second) \\
\hline Percentage & 5 & 10 & 25 & 50 & 75 & 90 & 95 \\
\hline Flow & 52,500 & 37,700 & 19,000 & 9,520 & 5,160 & 2,950 & 2,010 \\
\hline
\end{tabular}


STATION NAME AND NUMBER—02411930 Tallapoosa River below Tallapoosa, GA

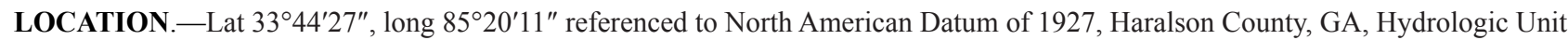
03150108, at bridge on U.S. Highway 78, 0.4 mi upstream from Walker Creek, and 2.7 mi west of Tallapoosa, Georgia, and at mile 216.5.

DRAINAGE AREA.- $-272 \mathrm{mi}^{2}$.

PERIOD OF RECORD.-December 1999 to November 2004, October 2005 to March 2014.

PERIOD OF ANALYSIS.-April 2000 to March 2004, April 2006 to March 2014.

NUMBER OF CLIMATE YEARS IN FREQUENCY ANALYSIS.-12

REMARKS.-Frequency statistics were computed based on a MOVE.1 correlation with station 02412000, Tallapoosa River near Heflin, AL, which has a period of record from July 1952 to March 2014.

\begin{tabular}{|c|c|c|c|c|}
\hline \multirow[t]{2}{*}{$\begin{array}{l}\text { Recurrence } \\
\text { intervals } \\
\text { (years) }\end{array}$} & \multicolumn{2}{|c|}{$\begin{array}{l}\text { Lowest average flow for } \\
\text { indicated number of } \\
\text { consecutive days } \\
\text { (cubic feet per second) }\end{array}$} & \multicolumn{2}{|c|}{$\begin{array}{l}\text { Time-sampling error } \\
\text { (in percent) }\end{array}$} \\
\hline & 1 & 7 & 1 & 7 \\
\hline 2 & 38 & 44 & 43 & 41 \\
\hline 5 & 15 & 18 & 44 & 43 \\
\hline 10 & 8.3 & 10 & 51 & 50 \\
\hline 20 & 4.6 & 6.2 & 63 & 62 \\
\hline 50 & 2.5 & 3.1 & 87 & 86 \\
\hline
\end{tabular}

\begin{tabular}{|c|c|c|c|c|c|c|c|}
\hline \multicolumn{7}{|c|}{ DURATION OF DAILY FLOW } \\
\hline \multicolumn{7}{|c|}{ Flow equaled or exceeded for indicated percentage of time } \\
(cubic feet per second)
\end{tabular}


STATION NAME AND NUMBER—02412000 Tallapoosa River near Heflin, AL

LOCATION.-Lat 3337'22", long 85³0'48" referenced to North American Datum of 1927, Cleburne County, AL, Hydrologic Unit 03150108, on right bank $5 \mathrm{ft}$ downstream from County Road 18 bridge, 2.2 mi upstream from Cane Creek, 4 mi southeast of Heflin, and at mile 186.8.

DRAINAGE AREA. $-448 \mathrm{mi}^{2}$.

PERIOD OF RECORD.- July 1952 to March 2014.

PERIOD OF ANALYSIS.-April 1953 to March 2014.

NUMBER OF CLIMATE YEARS IN FREQUENCY ANALYSIS.-61

REMARKS.-

\begin{tabular}{|c|c|c|c|c|}
\hline $\begin{array}{c}\text { Recurrence } \\
\text { intervals } \\
\text { (years) }\end{array}$ & \multicolumn{2}{|c|}{$\begin{array}{c}\text { Lowest average flow for } \\
\text { indicated number of } \\
\text { consecutive days } \\
\text { (cubic feet per second) }\end{array}$} & \multicolumn{2}{|c|}{$\begin{array}{c}\text { Time-sampling error } \\
\text { (in percent) }\end{array}$} \\
\hline & 1 & 7 & 1 & 7 \\
\hline 2 & 76 & 83 & 13 & 13 \\
\hline 5 & 33 & 37 & 17 & 23 \\
\hline 10 & 20 & 22 & 22 & 32 \\
\hline 20 & 12 & 14 & 32 & 49 \\
\hline 50 & 7.0 & 7.5 & 48 & \\
\hline
\end{tabular}

\begin{tabular}{|c|c|c|c|c|c|c|c|}
\hline \multicolumn{1}{|c|}{ DURATION OF DAILY FLOW } \\
\hline \multicolumn{7}{|c|}{$\begin{array}{c}\text { Flow equaled or exceeded for indicated percentage of time } \\
\text { (cubic feet per second) }\end{array}$} \\
\hline Percentage & 5 & 10 & 25 & 50 & 75 & 90 & 95 \\
\hline Flow & 1,860 & 1,210 & 728 & 387 & 189 & 94 & 56 \\
\hline
\end{tabular}


STATION NAME AND NUMBER—02412500 Tallapoosa River near Ofelia, AL

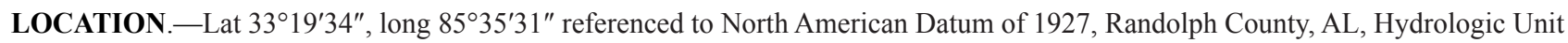
03150108, 1 mi northeast of Ofelia, 1.5 mi upstream from Little Tallapoosa River, and 9 mi east of Lineville.

DRAINAGE AREA.- $-792 \mathrm{mi}^{2}$.

PERIOD OF RECORD.-January 1939 to December 1951.

PERIOD OF ANALYSIS.-April 1939 to March 1951.

NUMBER OF CLIMATE YEARS IN FREQUENCY ANALYSIS.—12

REMARKS.-Frequency statistics represent pre-regulation period.

\begin{tabular}{|c|c|c|c|c|}
\hline $\begin{array}{c}\text { Recurrence } \\
\text { intervals } \\
\text { (years) }\end{array}$ & \multicolumn{2}{|c|}{$\begin{array}{c}\text { Lowest average flow for } \\
\text { indicated number of } \\
\text { consecutive days } \\
\text { (cubic feet per second) }\end{array}$} & \multicolumn{2}{|c|}{$\begin{array}{c}\text { Time-sampling error } \\
\text { (in percent) }\end{array}$} \\
\hline \multicolumn{7}{|c|}{1} & 7 & 1 & 7 \\
\hline 2 & 180 & 211 & 17 & 20 \\
\hline 5 & 114 & 144 & 22 & 27 \\
\hline 10 & 85 & 110 & 30 & 40 \\
\hline 20 & 64 & 86 & 45 & \\
\hline
\end{tabular}

\section{DURATION OF DAILY FLOW}

Flow equaled or exceeded for indicated percentage of time (cubic feet per second)

\begin{tabular}{|c|c|c|c|c|c|c|c|}
\hline Percentage & 5 & 10 & 25 & 50 & 75 & 90 & 95 \\
\hline Flow & 3,590 & 2,220 & 1,240 & 689 & 400 & 267 & 218 \\
\hline
\end{tabular}


STATION NAME AND NUMBER—02413300 Little Tallapoosa River near Newell, AL

LOCATION.-Lat 3326'14", long 85²3'57" referenced to North American Datum of 1927, Randolph County, AL, Hydrologic Unit 03150108, near right bank on downstream side of bridge on County Highway 82, 1.0 mi upstream of Cut Nose Creek, and 2.0 mi east of Newell.

DRAINAGE AREA. $-406 \mathrm{mi}^{2}$.

PERIOD OF RECORD.- October 1975 to March 2014.

PERIOD OF ANALYSIS.-A April 1976 to March 2014.

NUMBER OF CLIMATE YEARS IN FREQUENCY ANALYSIS.—38

REMARKS.-

\begin{tabular}{|c|c|c|c|c|}
\hline $\begin{array}{c}\text { Recurrence } \\
\text { intervals } \\
\text { (years) }\end{array}$ & \multicolumn{2}{|c|}{$\begin{array}{c}\text { Lowest average flow for } \\
\text { indicated number of } \\
\text { consecutive days } \\
\text { (cubic feet per second) }\end{array}$} & \multicolumn{2}{|c|}{$\begin{array}{c}\text { Time-sampling error } \\
\text { (in percent) }\end{array}$} \\
\hline & 1 & 7 & 1 & 7 \\
\hline 2 & 61 & 70 & 18 & 24 \\
\hline 5 & 25 & 29 & 24 & 32 \\
\hline 10 & 14 & 17 & 32 & 46 \\
\hline 20 & 8.5 & 9.8 & 46 & 75 \\
\hline 50 & 4.4 & 5.1 & 74 & \\
\hline
\end{tabular}

\begin{tabular}{|c|c|c|c|c|c|c|c|}
\hline \multicolumn{1}{|c|}{ DURATION OF DAILY FLOW } \\
\hline \multicolumn{7}{|c|}{$\begin{array}{c}\text { Flow equaled or exceeded for indicated percentage of time } \\
\text { (cubic feet per second) }\end{array}$} \\
\hline Percentage & 5 & 10 & 25 & 50 & 75 & 90 & 95 \\
\hline Flow & 1,900 & 1,220 & 640 & 337 & 158 & 76 & 49 \\
\hline
\end{tabular}


STATION NAME AND NUMBER—02413500 Little Tallapoosa River near Wedowee, AL

LOCATION.-Lat $33^{\circ} 20^{\prime} 57^{\prime \prime}$, long $85^{\circ} 32^{\prime} 43^{\prime \prime}$ referenced to North American Datum of 1927, Randolph County, AL, Hydrologic Unit 03150108, 4.5 mi northwest of Wedowee and 5.5 mi upstream from mouth.

DRAINAGE AREA.- $-591 \mathrm{mi}^{2}$.

PERIOD OF RECORD.-October 1939 to December 1951.

PERIOD OF ANALYSIS.-April 1940 to March 1951.

NUMBER OF CLIMATE YEARS IN FREQUENCY ANALYSIS.—-11

REMARKS.-

\begin{tabular}{|c|c|c|c|c|}
\hline $\begin{array}{l}\text { Recurrence } \\
\text { intervals } \\
\text { (years) }\end{array}$ & \multicolumn{2}{|c|}{$\begin{array}{l}\text { Lowest average flow for } \\
\text { indicated number of } \\
\text { consecutive days } \\
\text { (cubic feet per second) }\end{array}$} & \multicolumn{2}{|c|}{$\begin{array}{l}\text { Time-sampling erro } \\
\text { (in percent) }\end{array}$} \\
\hline & 1 & 7 & 1 & 7 \\
\hline 2 & 149 & 168 & 17 & 15 \\
\hline 5 & 97 & 114 & 22 & 20 \\
\hline 10 & 73 & 88 & 30 & 27 \\
\hline 20 & 55 & 69 & 45 & 40 \\
\hline
\end{tabular}

\begin{tabular}{|c|c|c|c|c|c|c|c|}
\hline \multicolumn{1}{|c|}{ DURATION OF DAILY FLOW } \\
\hline \multicolumn{1}{|c|}{ Flow equaled or exceeded for indicated percentage of time } \\
(cubic feet per second)
\end{tabular}


STATION NAME AND NUMBER—02414500 Tallapoosa River at Wadley, AL (Pre-regulation)

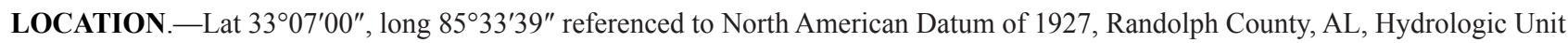
03150109, on left bank $50 \mathrm{ft}$ upstream of bridge on State Highway 22, 1 mi downstream of Beaver Dam Creek, and at mile 125.3.

DRAINAGE AREA.-1,675 $\mathrm{mi}^{2}$.

PERIOD OF RECORD.-October 1923 to March 2014.

PERIOD OF ANALYSIS.-April 1924 to March 1982.

\section{NUMBER OF CLIMATE YEARS IN FREQUENCY ANALYSIS.—58}

REMARKS.-The complete period of record at this station is from October 1923 to March 2014; however, results below represent the unregulated period from October 1923 to March 1982. According to the U.S. Army Corps of Engineers, filling of the reservoir (R.L. Harris Dam) began in October 1982 (accessed on June 9, 2014, at http://www.sam.usace.army.mil/Portals/46/docs/planning_environmental/act/docs/New/ ACT\%20Master\%20Manual_March\%2013.pdf).

\begin{tabular}{|c|c|c|c|c|}
\hline $\begin{array}{l}\text { Recurrence } \\
\text { intervals } \\
\text { (years) }\end{array}$ & \multicolumn{2}{|c|}{$\begin{array}{l}\text { Lowest average flow for } \\
\text { indicated number of } \\
\text { consecutive days } \\
\text { (cubic feet per second) }\end{array}$} & \multicolumn{2}{|c|}{$\begin{array}{l}\text { Time-sampling error } \\
\text { (in percent) }\end{array}$} \\
\hline & 1 & 7 & 1 & 7 \\
\hline 2 & 342 & 387 & 9 & 9 \\
\hline 5 & 200 & 228 & 11 & 11 \\
\hline 10 & 142 & 162 & 15 & 15 \\
\hline 20 & 104 & 119 & 21 & 21 \\
\hline 50 & 71 & 81 & 31 & 32 \\
\hline
\end{tabular}

\begin{tabular}{|cccccccc|}
\hline \multicolumn{7}{|c|}{ DURATION OF DAILY FLOW } \\
\hline \multicolumn{7}{|c|}{$\begin{array}{c}\text { Flow equaled or exceeded for indicated percentage of time } \\
\text { (cubic feet per second) }\end{array}$} \\
\hline Percentage & 5 & 10 & 25 & 50 & 75 & 90 & 95 \\
\hline Flow & 7,820 & 5,060 & 2,930 & 1,610 & 870 & 528 & 387 \\
\hline
\end{tabular}


STATION NAME AND NUMBER—02414500 Tallapoosa River at Wadley, AL (Regulated)

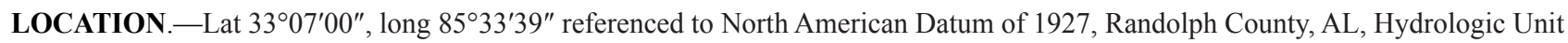
03150109, on left bank $50 \mathrm{ft}$ upstream of bridge on State Highway 22, 1 mi downstream of Beaver Dam Creek, and at mile 125.3.

DRAINAGE AREA.-1,675 $\mathrm{mi}^{2}$.

PERIOD OF RECORD.- October 1923 to March 2014.

PERIOD OF ANALYSIS.-April 1983 to March 2014.

NUMBER OF CLIMATE YEARS IN FREQUENCY ANALYSIS.—31

REMARKS.-The complete period of record at this station is from October 1923 to March 2014; however, results below represent the regulated period from April 1983 to March 2014. According to the U.S. Army Corps of Engineers, filling of the reservoir (R.L. Harris Dam) began in October 1982 and the pool reached the minimum power guide curve in December 1982 (accessed on June 9, 2014, at http://www.sam.usace. army.mil/Portals/46/docs/planning_environmental/act/docs/New/ACT\%20Master\%20Manual_March\%2013.pdf). Frequency analysis results for the annual minimum 1-day flows were not included due to a trend in the data and the log-Pearson Type III distribution not providing a satisifactory fit to the data.

\begin{tabular}{|c|c|c|c|c|}
\hline $\begin{array}{c}\text { Recurrence } \\
\text { intervals } \\
\text { (years) }\end{array}$ & \multicolumn{2}{|c|}{$\begin{array}{c}\text { Lowest average flow for } \\
\text { indicated number of } \\
\text { consecutive days } \\
\text { (cubic feet per second) }\end{array}$} & \multicolumn{2}{|c|}{$\begin{array}{c}\text { Time-sampling error } \\
\text { (in percent) }\end{array}$} \\
\hline & 1 & 7 & 1 & 10 \\
\hline 2 & -- & 230 & -- & 9 \\
\hline 5 & -- & 157 & -- & 10 \\
\hline 10 & -- & 130 & -- & 12 \\
\hline 20 & -- & 113 & -- & 16 \\
\hline 50 & -- & 97 & -- & \\
\hline
\end{tabular}

\begin{tabular}{|c|c|c|c|c|c|c|c|}
\hline \multicolumn{1}{|c|}{ DURATION OF DAILY FLOW } \\
\hline \multicolumn{7}{|c|}{$\begin{array}{c}\text { Flow equaled or exceeded for indicated percentage of time } \\
\text { (cubic feet per second) }\end{array}$} \\
\hline Percentage & 5 & 10 & 25 & 50 & 75 & 90 & 95 \\
\hline Flow & 8,080 & 5,350 & 3,130 & 1,600 & 459 & 222 & 170 \\
\hline
\end{tabular}


STATION NAME AND NUMBER—02414715 Tallapoosa River near New Site, AL

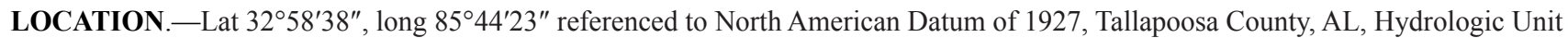
03150109, on right bank $100 \mathrm{ft}$ downstream of bridge on State Highway 49, 1 mi upstream of Emuckfaw Creek, 5 mi south of New Site, and at river mile 95.9 .

DRAINAGE AREA.-2,058 $\mathrm{mi}^{2}$.

PERIOD OF RECORD.--November 1985 to March 2014.

PERIOD OF ANALYSIS.-April 1986 to March 2014.

NUMBER OF CLIMATE YEARS IN FREQUENCY ANALYSIS.—-28

REMARKS.-Since about 1983, regulated by R.L. Harris Dam.

\begin{tabular}{|c|c|c|c|c|}
\hline \multirow[t]{2}{*}{$\begin{array}{l}\text { Recurrence } \\
\text { intervals } \\
\text { (years) }\end{array}$} & \multicolumn{2}{|c|}{$\begin{array}{l}\text { Lowest average flow for } \\
\text { indicated number of } \\
\text { consecutive days } \\
\text { (cubic feet per second) }\end{array}$} & \multicolumn{2}{|c|}{$\begin{array}{l}\text { Time-sampling error } \\
\text { (in percent) }\end{array}$} \\
\hline & 1 & 7 & 1 & 7 \\
\hline 2 & 224 & 354 & 10 & 12 \\
\hline 5 & 151 & 221 & 10 & 12 \\
\hline 10 & 124 & 176 & 11 & 13 \\
\hline 20 & 106 & 147 & 13 & 16 \\
\hline
\end{tabular}

\section{DURATION OF DAILY FLOW}

Flow equaled or exceeded for indicated percentage of time (cubic feet per second)

\begin{tabular}{|c|c|c|c|c|c|c|c|}
\hline Percentage & 5 & 10 & 25 & 50 & 75 & 90 & 95 \\
\hline Flow & 9,150 & 6,350 & 3,790 & 2,110 & 800 & 336 & 260 \\
\hline
\end{tabular}


STATION NAME AND NUMBER—02415000 Hillabee Creek near Hackneyville, AL

LOCATION.-Lat $33^{\circ} 03^{\prime} 55^{\prime \prime}$, long $85^{\circ} 52^{\prime} 41^{\prime \prime}$ referenced to North American Datum of 1927, Tallapoosa County, AL, Hydrologic Unit 03150109, on left bank, about $800 \mathrm{ft}$. downstream of county road bridge, 1 mi downstream of Enitachopco Creek, 4 mi upstream of Hackney Creek, and 3 mi east of Hackneyville.

DRAINAGE AREA. $-190 \mathrm{mi}^{2}$.

PERIOD OF RECORD.- July 1952 to September 1970, October 1985 to March 2014.

PERIOD OF ANALYSIS.-April 1953 to March 1970, April 1986 to March 2014.

NUMBER OF CLIMATE YEARS IN FREQUENCY ANALYSIS.—44

REMARKS.-

\begin{tabular}{|c|c|c|c|c|}
\hline $\begin{array}{c}\text { Recurrence } \\
\text { intervals } \\
\text { (years) }\end{array}$ & \multicolumn{2}{|c|}{$\begin{array}{c}\text { Lowest average flow for } \\
\text { indicated number of } \\
\text { consecutive days } \\
\text { (cubic feet per second) }\end{array}$} & \multicolumn{2}{|c|}{$\begin{array}{c}\text { Time-sampling error } \\
\text { (in percent) }\end{array}$} \\
\hline & 1 & 7 & 1 & 7 \\
\hline 2 & 26 & 30 & 15 & 15 \\
\hline 5 & 12 & 14 & 18 & 24 \\
\hline 10 & 7.4 & 8.5 & 24 & 34 \\
\hline 20 & 4.8 & 5.5 & 34 & 50 \\
\hline 50 & 2.8 & 3.2 & 50 & \\
\hline
\end{tabular}

\begin{tabular}{|c|c|c|c|c|c|c|c|}
\hline \multicolumn{1}{|c|}{ DURATION OF DAILY FLOW } \\
\hline \multicolumn{7}{|c|}{ Flow equaled or exceeded for indicated percentage of time } \\
(cubic feet per second)
\end{tabular}


STATION NAME AND NUMBER—02416000 Tallapoosa River at Sturdivant, AL

LOCATION.-Lat 32 54'48", long 85 52'16" referenced to North American Datum of 1927, Tallapoosa County, AL, Hydrologic Unit 03150109, 5 mi downstream from Hillabee Creek and 5 mi southeast of Alexander City.

DRAINAGE AREA.-2,480 $\mathrm{mi}^{2}$.

PERIOD OF RECORD.- October 1900 to July 1926.

PERIOD OF ANALYSIS.-April 1901 to March 1926.

NUMBER OF CLIMATE YEARS IN FREQUENCY ANALYSIS.—25

REMARKS.-Since 1926, site in backwater from Martin Dam. Results represent pre-regulation conditions.

\begin{tabular}{|c|c|c|c|c|}
\hline $\begin{array}{c}\text { Recurrence } \\
\text { intervals } \\
\text { (years) }\end{array}$ & \multicolumn{2}{|c|}{$\begin{array}{c}\text { Lowest average flow for } \\
\text { indicated number of } \\
\text { consecutive days } \\
\text { (cubic feet per second) }\end{array}$} & \multicolumn{2}{|c|}{$\begin{array}{c}\text { Time-sampling error } \\
\text { (in percent) }\end{array}$} \\
\hline \multicolumn{7}{|c|}{1} & 7 & 1 & 7 \\
\hline 2 & 567 & 640 & 15 & 15 \\
\hline 5 & 320 & 363 & 19 & 26 \\
\hline 10 & 222 & 250 & 25 & 37 \\
\hline 20 & 158 & 176 & 36 & 19 \\
\hline
\end{tabular}

\begin{tabular}{|c|c|c|c|c|c|c|c|}
\hline \multicolumn{7}{|c|}{ DURATION OF DAILY FLOW } \\
\hline \multicolumn{7}{|c|}{$\begin{array}{c}\text { Flow equaled or exceeded for indicated percentage of time } \\
\text { (cubic feet per second) }\end{array}$} \\
\hline Percentage & 5 & 10 & 25 & 50 & 75 & 90 & 95 \\
\hline Flow & 12,100 & 7,540 & 4,400 & 2,520 & 1,400 & 855 & 640 \\
\hline
\end{tabular}


STATION NAME AND NUMBER—02418230 Sougahatchee Creek near Loachapoka, AL

LOCATION._Lat 32³7'36", long 85³5'17" referenced to North American Datum of 1927, Lee County, AL, Hydrologic Unit 03150110, on downstream side of bridge on County Road 188, 1 mi upstream of Loblocklee Creek, and 2 mi north of Loachapoka.

DRAINAGE AREA. $-71.3 \mathrm{mi}^{2}$.

PERIOD OF RECORD.-November 1999 to March 2014.

PERIOD OF ANALYSIS.-April 2000 to March 2014.

NUMBER OF CLIMATE YEARS IN FREQUENCY ANALYSIS.-14

REMARKS.-Flow includes wastewater treatment plant discharges from City of Auburn.

\begin{tabular}{|c|c|c|c|c|}
\hline $\begin{array}{l}\text { Recurrence } \\
\text { intervals } \\
\text { (years) }\end{array}$ & \multicolumn{2}{|c|}{$\begin{array}{l}\text { Lowest average flow for } \\
\text { indicated number of } \\
\text { consecutive days } \\
\text { (cubic feet per second) }\end{array}$} & \multicolumn{2}{|c|}{$\begin{array}{l}\text { Time-sampling error } \\
\text { (in percent) }\end{array}$} \\
\hline & 1 & 7 & 1 & 7 \\
\hline 2 & 8.7 & 10 & 18 & 17 \\
\hline 5 & 5.6 & 6.4 & 15 & 15 \\
\hline 10 & 4.6 & 5.2 & 16 & 16 \\
\hline 20 & 4.0 & 4.5 & 20 & 20 \\
\hline
\end{tabular}

\begin{tabular}{|c|c|c|c|c|c|c|c|}
\hline \multicolumn{1}{|c|}{ DURATION OF DAILY FLOW } \\
\hline \multicolumn{1}{|c|}{ Flow equaled or exceeded for indicated percentage of time } \\
(cubic feet per second)
\end{tabular}


STATION NAME AND NUMBER—02418500 Tallapoosa River below Tallassee, AL

LOCATION.-Lat 32 $30^{\prime} 45^{\prime \prime}$, long 85 53'21" referenced to North American Datum of 1927, Tallapoosa County, AL, Hydrologic Unit 03150110, on left bank, 1.5 mi downstream of Benjamin Fitzpatrick Highway bridge and Thurlow Dam at Tallassee, 3.5 mi upstream of Uphapee Creek, and at river mile 48.1 .

DRAINAGE AREA. - $-3,328 \mathrm{mi}^{2}$.

PERIOD OF RECORD.- October 1928 to September 2013.

PERIOD OF ANALYSIS.-April 1929 to March 2013.

REMARKS.-Records collected by Alabama Power Company, under general supervision of U.S. Geological Survey, in connection with a Federal Energy Regulatory Commission project. Daily discharge computed on basis of powerplant records, flow over spillway, and measured leakage. Flow regulated by R.L. Harris Lake (1983), Lake Martin (1926), other hydroelectric plants, and small mill dams above station. Because of substantial influence from regulation, only exceedance percentiles of annual 7-day minimum flows and duration of daily flow are being provided.

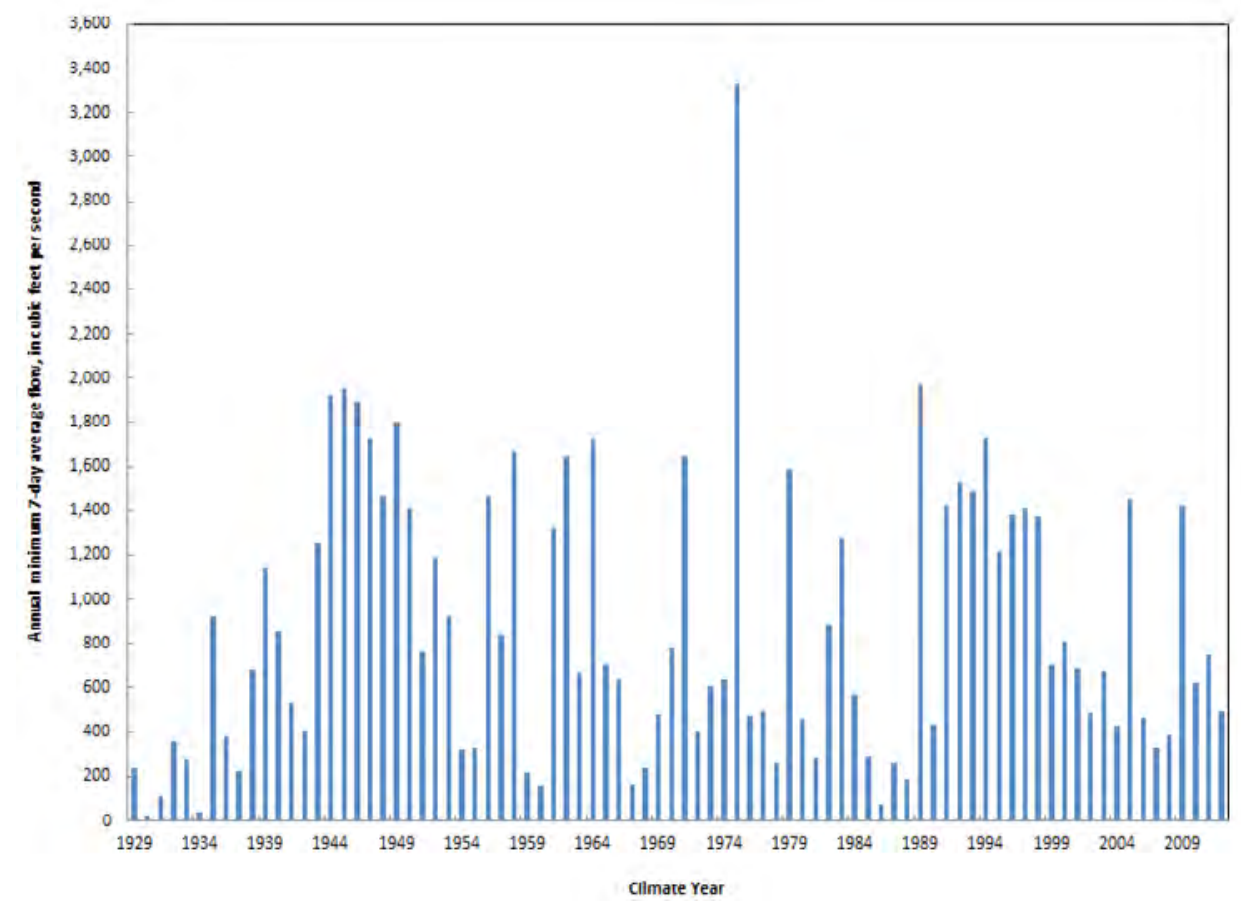

\begin{tabular}{|c|c|c|c|c|c|c|c|c|c|c|}
\hline \multicolumn{1}{|c|}{ EXCEEDANCE PERCENTILES OF ANNUAL MINIMUM 7-DAY AVERAGE FLOWS } \\
\hline \multicolumn{1}{|c|}{ Annual minimum 7-day average flow exceeded for indicated percentiles (cubic feet per second) } \\
\hline Percentile & 10 & 20 & 30 & 40 & 50 & 60 & 70 & 80 & 90 \\
\hline Flow & 1,720 & 1,460 & 1,300 & 924 & 640 & 531 & 433 & 318 & 219 \\
\hline
\end{tabular}

\begin{tabular}{|c|c|c|c|c|c|c|c|}
\hline \multicolumn{8}{|c|}{ DURATION OF DAILY FLOW } \\
\hline \multicolumn{8}{|c|}{$\begin{array}{l}\text { Flow equaled or exceeded for indicated percentage of time } \\
\text { (cubic feet per second) }\end{array}$} \\
\hline Percentage & 5 & 10 & 25 & 50 & 75 & 90 & 95 \\
\hline Flow & 10,700 & 9,260 & 6,160 & 3,840 & 1,640 & 501 & 80 \\
\hline
\end{tabular}


STATION NAME AND NUMBER—02418760 Chewacla Creek at Chewacla State Park near Auburn, AL

LOCATION.-Lat 32 $32^{\prime} 53^{\prime \prime}$, long $85^{\circ} 28^{\prime} 50^{\prime \prime}$ referenced to North American Datum of 1927, Lee County, AL, Hydrologic Unit 03150110, at abandoned bridge in Chewacla State Park, 0.2 mi downstream of Moores Mill Creek, downstream of Lake Ogletree, and 4 mi south of Auburn.

DRAINAGE AREA. $-45.8 \mathrm{mi}^{2}$.

PERIOD OF RECORD.- October 2002 to March 2014.

PERIOD OF ANALYSIS.-April 2003 to March 2014.

NUMBER OF CLIMATE YEARS IN FREQUENCY ANALYSIS.—-11

REMARKS.-Downstream of Lake Ogletree.

\begin{tabular}{|c|c|c|c|c|}
\hline \multirow[t]{2}{*}{$\begin{array}{l}\text { Recurrence } \\
\text { intervals } \\
\text { (years) }\end{array}$} & \multicolumn{2}{|c|}{$\begin{array}{l}\text { Lowest average flow for } \\
\text { indicated number of } \\
\text { consecutive days } \\
\text { (cubic feet per second) }\end{array}$} & \multicolumn{2}{|c|}{$\begin{array}{l}\text { Time-sampling error } \\
\text { (in percent) }\end{array}$} \\
\hline & 1 & 7 & 1 & 7 \\
\hline 2 & 1.3 & 3.4 & 55 & 42 \\
\hline 5 & 0.35 & 1.2 & 59 & 52 \\
\hline 10 & 0.18 & 0.62 & 71 & 70 \\
\hline 20 & 0.10 & 0.35 & 91 & 100 \\
\hline
\end{tabular}

\begin{tabular}{|c|c|c|c|c|c|c|c|}
\hline \multicolumn{7}{|c|}{ DURATION OF DAILY FLOW } \\
\hline \multicolumn{7}{|c|}{ Flow equaled or exceeded for indicated percentage of time } \\
(cubic feet per second)
\end{tabular}


STATION NAME AND NUMBER—02419000 Uphapee Creek near Tuskegee, AL

LOCATION.-Lat 32²8'36", long 8541'42" referenced to North American Datum of 1927, Macon County, AL, Hydrologic Unit 03150110, on left bank at downstream side of bridge on State Highway 81, 1 mi upstream of Red Creek, 1.2 mi upstream of Western Railway of Alabama bridge, and $4 \mathrm{mi}$ north of Tuskegee.

DRAINAGE AREA. - $333 \mathrm{mi}^{2}$.

PERIOD OF RECORD.- October 1939 to September 1970, October 1974 to March 2014.

PERIOD OF ANALYSIS.-April 1940 to March 1970, April 1975 to March 2014.

NUMBER OF CLIMATE YEARS IN FREQUENCY ANALYSIS.—69

REMARKS.-

\begin{tabular}{|c|c|c|c|c|}
\hline \multirow[t]{2}{*}{$\begin{array}{l}\text { Recurrence } \\
\text { intervals } \\
\text { (years) }\end{array}$} & \multicolumn{2}{|c|}{$\begin{array}{l}\text { Lowest average flow for } \\
\text { indicated number of } \\
\text { consecutive days } \\
\text { (cubic feet per second) }\end{array}$} & \multicolumn{2}{|c|}{$\begin{array}{l}\text { Time-sampling error } \\
\text { (in percent) }\end{array}$} \\
\hline & 1 & 7 & 1 & 7 \\
\hline 2 & 16 & 18 & 11 & 10 \\
\hline 5 & 8.0 & 9.4 & 14 & 13 \\
\hline 10 & 5.0 & 6.2 & 19 & 17 \\
\hline 20 & 3.3 & 4.3 & 28 & 23 \\
\hline 50 & 1.9 & 2.7 & 43 & 34 \\
\hline
\end{tabular}

\begin{tabular}{|c|c|c|c|c|c|c|c|}
\hline \multicolumn{1}{|c|}{ DURATION OF DAILY FLOW } \\
\hline \multicolumn{7}{|c|}{ Flow equaled or exceeded for indicated percentage of time } \\
(cubic feet per second) \\
\hline Percentage & 5 & 10 & 25 & 50 & 75 & 90 & 95 \\
\hline Flow & 1,530 & 905 & 400 & 144 & 47 & 23 & 16 \\
\hline
\end{tabular}


STATION NAME AND NUMBER—02419890 Tallapoosa River near Montgomery, AL

LOCATION.-Lat $32^{\circ} 26^{\prime} 23^{\prime \prime}$, long $86^{\circ} 11^{\prime} 44^{\prime \prime}$ referenced to North American Datum of 1927, Montgomery County, AL, Hydrologic Unit 03150110, on left bank at the Clarence T. Perry (City of Montgomery) water purification plant, 2.5 mi upstream of U.S. Highway 231 bridge, 4 mi northeast of Montgomery, and at river mile 12.8 .

DRAINAGE AREA. - $4,646 \mathrm{mi}^{2}$.

PERIOD OF RECORD.--October 1995 to March 2014.

PERIOD OF ANALYSIS.-April 1996 to March 2014.

\section{NUMBER OF CLIMATE YEARS IN FREQUENCY ANALYSIS.—-18}

REMARKS.-Daily discharges can be affected by backwater from the Alabama River. Flow is regulated by Harris Reservoir, Lake Martin, and other hydroelectric plants above station including Yates and Thurlow Dams.

\begin{tabular}{|c|c|c|c|c|}
\hline $\begin{array}{c}\text { Recurrence } \\
\text { intervals } \\
\text { (years) }\end{array}$ & \multicolumn{2}{|c|}{$\begin{array}{c}\text { Lowest average flow for } \\
\text { indicated number of } \\
\text { consecutive days } \\
\text { ccubic feet per second) }\end{array}$} & \multicolumn{2}{|c|}{$\begin{array}{c}\text { Time-sampling error } \\
\text { (in percent) }\end{array}$} \\
\hline \multicolumn{7}{|c|}{1} & 7 & 1 & 7 \\
\hline 2 & 922 & 1,220 & 14 & 16 \\
\hline 5 & 577 & 776 & 18 & 22 \\
\hline 10 & 429 & 592 & 24 & 29 \\
\hline 20 & 326 & 464 & 34 & \\
\hline
\end{tabular}

\begin{tabular}{|c|c|c|c|c|c|c|c|}
\hline \multicolumn{7}{|c|}{ DURATION OF DAILY FLOW } \\
\hline \multicolumn{7}{|c|}{$\begin{array}{c}\text { Flow equaled or exceeded for indicated percentage of time } \\
\text { (cubic feet per second) }\end{array}$} \\
\hline Percentage & 5 & 10 & 25 & 50 & 75 & 90 & 95 \\
\hline Flow & 19,600 & 14,400 & 6,580 & 3,070 & 1,730 & 1,240 & 865 \\
\hline
\end{tabular}


STATION NAME AND NUMBER—02420000 Alabama River near Montgomery, AL

LOCATION.-Lat $32^{\circ} 24^{\prime} 41^{\prime \prime}$, long $86^{\circ} 24^{\prime} 30^{\prime \prime}$ referenced to North American Datum of 1927, Montgomery County, AL, Hydrologic Unit 03150201, on pier near midstream of bridge on U.S. Highway 31, 4 mi upstream of Autauga Creek, 6 mi northwest of Montgomery, and at river mile 287.6.

DRAINAGE AREA. $-15,087 \mathrm{mi}^{2}$.

PERIOD OF RECORD.- October 1927 to September 1990, October 2001 to March 2014.

PERIOD OF ANALYSIS. - April 2002 to March 2014.

REMARKS.-Flows regulated by reservoirs on the Etowah, Coosa, and Tallapoosa Rivers. Reviews indicate various patterns of regulation for the complete period of record. Thus, the exceedance percentiles and duration of daily flow data were computed using the most recent period of relatively stable regulation.

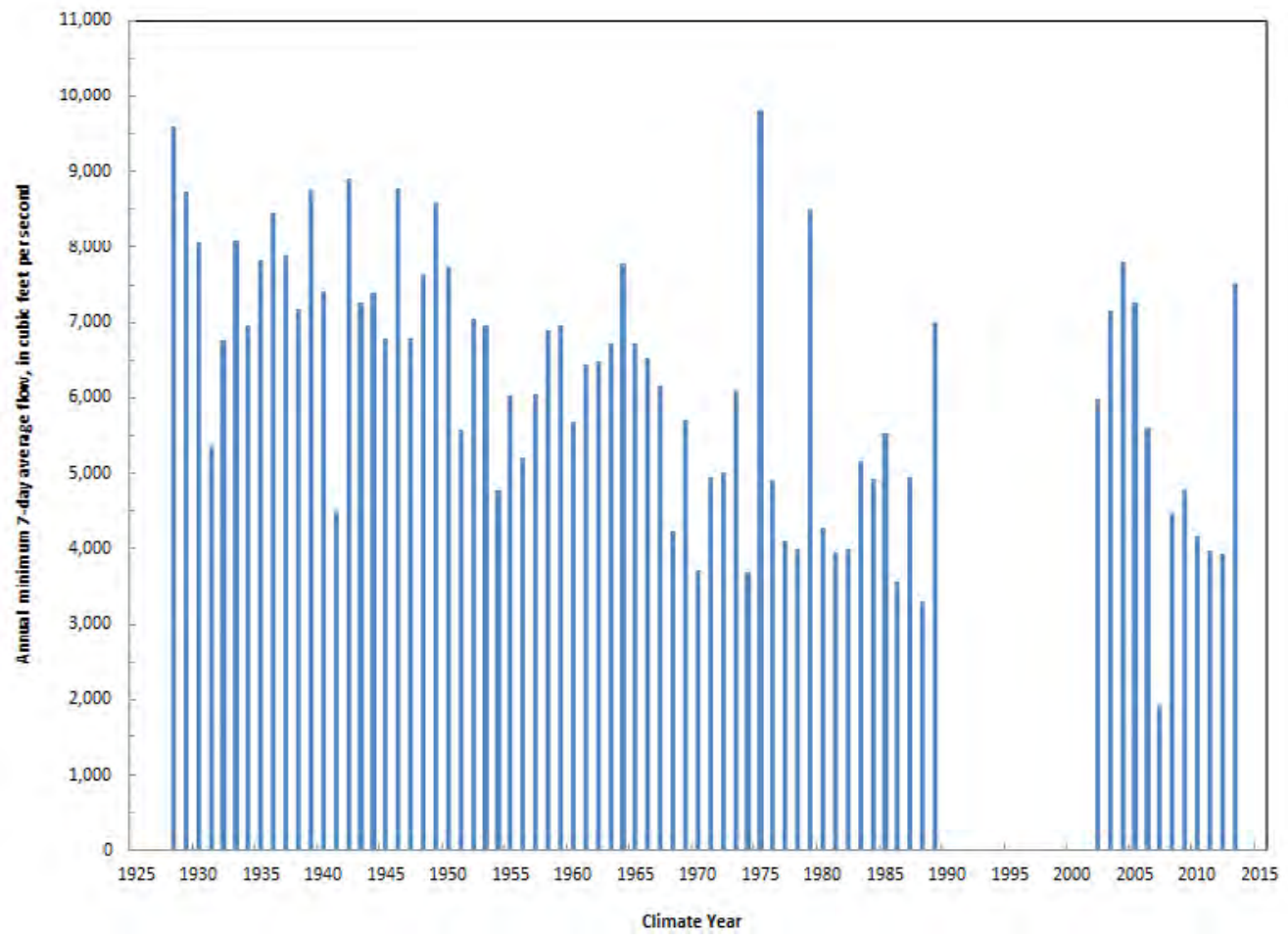

\begin{tabular}{|c|c|c|c|c|c|c|c|c|c|}
\hline \multicolumn{10}{|c|}{ EXCEEDANCE PERCENTILES OF ANNUAL MINIMUM 7-DAY AVERAGE FLOWS } \\
\hline \multicolumn{10}{|c|}{ Annual minimum 7-day average flow exceeded for indicated percentiles (cubic feet per second) } \\
\hline Percentile & 10 & 20 & 30 & 40 & 50 & 60 & 70 & 80 & 90 \\
\hline Flow & 7,710 & 7,360 & 7,170 & 5,900 & 5,190 & 4,540 & 4,160 & 3,950 & 2,520 \\
\hline
\end{tabular}

\begin{tabular}{|c|c|c|c|c|c|c|c|}
\hline \multicolumn{7}{|c|}{ DURATION OF DAILY FLOW } \\
\hline \multicolumn{7}{|c|}{ Flow equaled or exceeded for indicated percentage of time } \\
(cubic feet per second)
\end{tabular}


STATION NAME AND NUMBER—02420500 Autauga Creek at Prattville, AL

LOCATION.-Lat $32^{\circ} 27^{\prime} 30^{\prime \prime}$, long $86^{\circ} 28^{\prime} 30^{\prime \prime}$ referenced to North American Datum of 1927, Autauga County, AL, Hydrologic Unit 03150201, $25 \mathrm{ft}$ upstream from Bridge Street in Prattville, $500 \mathrm{ft}$ downstream from dam, and 5 mi upstream from mouth.

DRAINAGE AREA.-116 $\mathrm{mi}^{2}$.

PERIOD OF RECORD.—-January 1939 to September 1959.

PERIOD OF ANALYSIS.-April 1939 to March 1959.

NUMBER OF CLIMATE YEARS IN FREQUENCY ANALYSIS.—-20

REMARKS.- - Since about 1920, flows regulated by Prattville Lake.

\begin{tabular}{|c|c|c|c|c|}
\hline \multirow[t]{2}{*}{$\begin{array}{l}\text { Recurrence } \\
\text { intervals } \\
\text { (years) }\end{array}$} & \multicolumn{2}{|c|}{$\begin{array}{l}\text { Lowest average flow for } \\
\text { indicated number of } \\
\text { consecutive days } \\
\text { (cubic feet per second) }\end{array}$} & \multicolumn{2}{|c|}{$\begin{array}{l}\text { Time-sampling error } \\
\text { (in percent) }\end{array}$} \\
\hline & 1 & 7 & 1 & 7 \\
\hline 2 & 62 & 72 & 8 & 8 \\
\hline 5 & 47 & 55 & 9 & 9 \\
\hline 10 & 40 & 47 & 11 & 11 \\
\hline 20 & 35 & 41 & 13 & 14 \\
\hline
\end{tabular}

\section{DURATION OF DAILY FLOW}

Flow equaled or exceeded for indicated percentage of time (cubic feet per second)

\begin{tabular}{|c|c|c|c|c|c|c|c|}
\hline Percentage & 5 & 10 & 25 & 50 & 75 & 90 & 95 \\
\hline Flow & 410 & 320 & 216 & 145 & 101 & 76 & 63 \\
\hline
\end{tabular}


STATION NAME AND NUMBER—02421000 Catoma Creek near Montgomery, AL

LOCATION. - Lat $32^{\circ} 18^{\prime} 26^{\prime \prime}$, long $86^{\circ} 17^{\prime} 58^{\prime \prime}$ referenced to North American Datum of 1927, Montgomery County, AL, Hydrologic Unit 03150201, on right bank on downstream side of bridge on old U.S. Highway 331, 5 mi south of Montgomery, and at mile 16.1.

DRAINAGE AREA. $-290 \mathrm{mi}^{2}$.

PERIOD OF RECORD._-July 1952 to September 1971, October 1974 to September 1996, October 1997 to March 2014.

PERIOD OF ANALYSIS.-April 1953 to March 1971, April 1975 to March 1996, April 1998 to March 2014.

NUMBER OF CLIMATE YEARS IN FREQUENCY ANALYSIS.—55

REMARKS.-

\begin{tabular}{|c|c|c|c|c|}
\hline $\begin{array}{c}\text { Recurrence } \\
\text { intervals } \\
\text { (years) }\end{array}$ & \multicolumn{2}{|c|}{$\begin{array}{c}\text { Lowest average flow for } \\
\text { indicated number of } \\
\text { consecutive days } \\
\text { (cubic feet per second) }\end{array}$} & \multicolumn{2}{|c|}{$\begin{array}{c}\text { Time-sampling error } \\
\text { (in percent) }\end{array}$} \\
\hline \multicolumn{7}{|c|}{1} & 7 & 1 & 7 \\
\hline 2 & 0.52 & 0.79 & 21 & 25 \\
\hline 5 & 0.09 & 0.21 & 26 & 32 \\
\hline 10 & 0.00 & 0.07 & -- & -- \\
\hline 20 & 0.00 & 0.00 & -- & -- \\
\hline 50 & 0.00 & 0.00 & -- & \\
\hline
\end{tabular}

\section{DURATION OF DAILY FLOW}

Flow equaled or exceeded for indicated percentage of time (cubic feet per second)

\begin{tabular}{|c|c|c|c|c|c|c|c|}
\hline Percentage & 5 & 10 & 25 & 50 & 75 & 90 & 95 \\
\hline Flow & 1,740 & 826 & 174 & 40 & 7.2 & 1.7 & 0.68 \\
\hline
\end{tabular}


STATION NAME AND NUMBER—02422000 Big Swamp Creek near Lowndesboro, AL

LOCATION.-Lat $32^{\circ} 16^{\prime} 00^{\prime \prime}$, long 86 $41^{\prime} 40^{\prime \prime}$ referenced to North American Datum of 1927, Lowndes County, AL, Hydrologic Unit 03150201, on U.S. Highway 80, 1 mi downstream from Panther Creek, 5 mi west of Lowndesboro, and 12 upstream from mouth.

DRAINAGE AREA. $-244 \mathrm{mi}^{2}$.

PERIOD OF RECORD.- - October 1940 to September 1971.

PERIOD OF ANALYSIS.-April 1941 to March 1971.

NUMBER OF CLIMATE YEARS IN FREQUENCY ANALYSIS.—30

REMARKS.-

\begin{tabular}{|c|c|c|c|c|}
\hline $\begin{array}{c}\text { Recurrence } \\
\text { intervals } \\
\text { (years) }\end{array}$ & \multicolumn{2}{|c|}{$\begin{array}{c}\text { Lowest average flow for } \\
\text { indicated number of } \\
\text { consecutive days } \\
\text { (cubic feet per second) }\end{array}$} & \multicolumn{2}{|c|}{$\begin{array}{c}\text { Time-sampling error } \\
\text { (in percent) }\end{array}$} \\
\hline \multicolumn{7}{|c|}{1} & 7 & 1 & 7 \\
\hline 2 & 0.11 & 0.19 & 26 & -- \\
\hline 5 & 0.00 & 0.00 & -- & -- \\
\hline 10 & 0.00 & 0.00 & -- & -- \\
\hline 20 & 0.00 & 0.00 & -- & -- \\
\hline 50 & 0.00 & 0.00 & -- & \\
\hline
\end{tabular}

\section{DURATION OF DAILY FLOW}

Flow equaled or exceeded for indicated percentage of time (cubic feet per second)

\begin{tabular}{|c|c|c|c|c|c|c|c|}
\hline Percentage & 5 & 10 & 25 & 50 & 75 & 90 & 95 \\
\hline Flow & 1,370 & 768 & 154 & 20 & 2.0 & 0.4 & 0.1 \\
\hline
\end{tabular}


STATION NAME AND NUMBER—02422500 Mulberry Creek at Jones, AL

LOCATION.-Lat 32³4'58", long 8654'13" referenced to North American Datum of 1927, Dallas County, AL, Hydrologic Unit 03150201, on right bank $75 \mathrm{ft}$ downstream of highway bridge, 0.4 mi west of Jones, 6 mi upstream of Buck Creek, and 11 mi upstream of mouth.

DRAINAGE AREA. $-203 \mathrm{mi}^{2}$.

PERIOD OF RECORD.- October 1938 to September 1970, October 1974 to March 2014.

PERIOD OF ANALYSIS.-April 1939 to March 1970, April 1975 to March 2014.

NUMBER OF CLIMATE YEARS IN FREQUENCY ANALYSIS.—70

\section{REMARKS.-}

\begin{tabular}{|c|c|c|c|c|}
\hline $\begin{array}{l}\text { Recurrence } \\
\text { intervals } \\
\text { (years) }\end{array}$ & \multicolumn{2}{|c|}{$\begin{array}{l}\text { Lowest average flow for } \\
\text { indicated number of } \\
\text { consecutive days } \\
\text { (cubic feet per second) }\end{array}$} & \multicolumn{2}{|c|}{$\begin{array}{l}\text { Time-sampling error } \\
\text { (in percent) }\end{array}$} \\
\hline & 1 & 7 & 1 & 7 \\
\hline 2 & 57 & 60 & 5 & 4 \\
\hline 5 & 42 & 45 & 5 & 5 \\
\hline 10 & 35 & 38 & 7 & 7 \\
\hline 20 & 30 & 32 & 9 & 9 \\
\hline 50 & 25 & 27 & 12 & 12 \\
\hline
\end{tabular}

\section{DURATION OF DAILY FLOW}

Flow equaled or exceeded for indicated percentage of time (cubic feet per second)

\begin{tabular}{|c|c|c|c|c|c|c|c|}
\hline Percentage & 5 & 10 & 25 & 50 & 75 & 90 & 95 \\
\hline Flow & 891 & 586 & 316 & 160 & 94 & 68 & 56 \\
\hline
\end{tabular}


STATION NAME AND NUMBER—02423000 Alabama River at Selma, AL

LOCATION.-Lat $32^{\circ} 24^{\prime} 20^{\prime \prime}$, long $87^{\circ} 01^{\prime} 07^{\prime \prime}$ referenced to North American Datum of 1927, Dallas County, AL, Hydrologic Unit 03150201, at first pier from right bank of Edmund Pettus Bridge on U.S. Highway 80 in Selma, 1 mi upstream from Valley Creek, and at river mile 214.8.

DRAINAGE AREA. $-17,095 \mathrm{mi}^{2}$.

PERIOD OF RECORD._-January 1900 to December 1913, October 1928 to September 1970.

PERIOD OF ANALYSIS. — April 1900 to March 1913.

\section{NUMBER OF CLIMATE YEARS IN FREQUENCY ANALYSIS.—-13}

REMARKS.-Flows regulated by reservoirs on the Etowah, Coosa, and Tallapoosa Rivers. Based on review of current streamgages on the Alabama River, current regulation does not likely reflect the period of regulated record available at 02423000. Consequently, 1- and 7-day frequency statistics were computed for the unregulated period from April 1900 to March 1913 and daily duration statistics were computed for January 1900 to March 1913. Also, a plot of the annual minimum 7-day average flows for the complete period of record is provided.

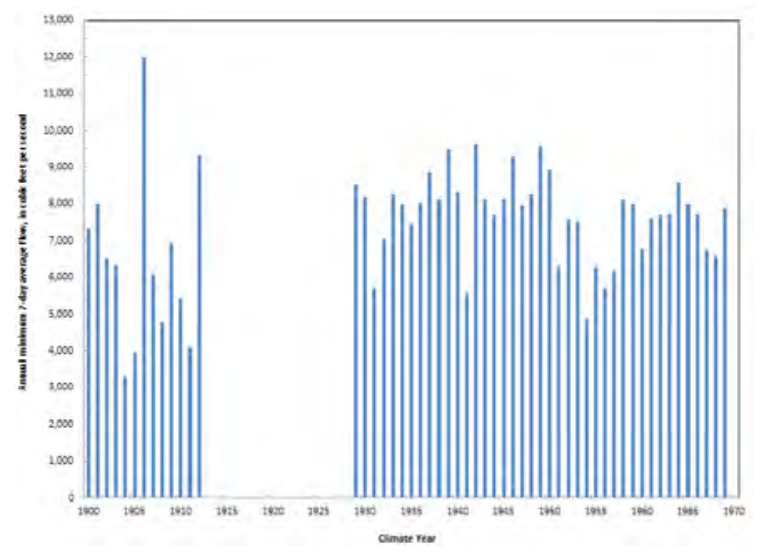

\begin{tabular}{|c|c|c|c|c|}
\hline $\begin{array}{c}\text { Recurrence } \\
\text { intervals } \\
\text { (years) }\end{array}$ & \multicolumn{2}{|c|}{$\begin{array}{c}\text { Lowest average flow for } \\
\text { indicated number of } \\
\text { consecutive days } \\
\text { (cubic feet per second) }\end{array}$} & \multicolumn{2}{|c|}{$\begin{array}{c}\text { Time-sampling error } \\
\text { (in percent) }\end{array}$} \\
\hline & 1 & 7 & 1 & 11 \\
\hline 2 & 5,940 & 6,070 & 11 & 12 \\
\hline 5 & 4,390 & 4,490 & 12 & 14 \\
\hline 10 & 3,750 & 3,840 & 14 & 16 \\
\hline 20 & 3,290 & 3,380 & 17 & \\
\hline
\end{tabular}

\begin{tabular}{|c|c|c|c|c|c|c|c|}
\hline \multicolumn{1}{|c|}{ DURATION OF DAILY FLOW } \\
\hline \multicolumn{1}{|c|}{ Flow equaled or exceeded for indicated percentage of time } \\
(cubic feet per second)
\end{tabular}


STATION NAME AND NUMBER—02423130 Cahaba River at Trussville, AL

LOCATION.-Lat 3337'20", long 86 $35^{\prime} 58^{\prime \prime}$ referenced to North American Datum of 1927, Jefferson County, AL, Hydrologic Unit 03150202, at center of walk bridge $300 \mathrm{ft}$ upstream of U.S. Highway 11 bridge, $0.5 \mathrm{mi}$ east of Trussville, 8.6 mi upstream from Big Black Creek, and at mile 182.3.

DRAINAGE AREA. $-19.7 \mathrm{mi}^{2}$.

PERIOD OF RECORD.- October 1988 to March 2014.

PERIOD OF ANALYSIS.-April 1989 to March 2014.

NUMBER OF CLIMATE YEARS IN FREQUENCY ANALYSIS.—-25

REMARKS.-

\begin{tabular}{|c|c|c|c|c|}
\hline $\begin{array}{c}\text { Recurrence } \\
\text { intervals } \\
\text { (years) }\end{array}$ & \multicolumn{2}{|c|}{$\begin{array}{c}\text { Lowest average flow for } \\
\text { indicated number of } \\
\text { consecutive days } \\
\text { (cubic feet per second) }\end{array}$} & \multicolumn{2}{|c|}{$\begin{array}{c}\text { Time-sampling error } \\
\text { (in percent) }\end{array}$} \\
\hline \multicolumn{7}{|c|}{1} & 7 & 1 & 7 \\
\hline 2 & 0.04 & 0.10 & 54 & -- \\
\hline 5 & 0.00 & 0.00 & -- & -- \\
\hline 10 & 0.00 & 0.00 & -- & -- \\
\hline 20 & 0.00 & 0.00 & -- & - \\
\hline
\end{tabular}

\begin{tabular}{|c|c|c|c|c|c|c|c|}
\hline \multicolumn{7}{|c|}{ DURATION OF DAILY FLOW } \\
\hline \multicolumn{7}{|c|}{ Flow equaled or exceeded for indicated percentage of time } \\
(cubic feet per second)
\end{tabular}


STATION NAME AND NUMBER—02423380 Cahaba River near Mountain Brook, AL

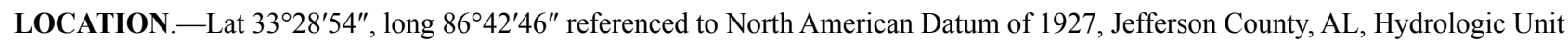
03150202, on downstream side bridge on county road, 0.1 mi upstream from Fuller Creek, 3.5 mi east of Mountain Brook, 5.4 mi upstream from Little Cahaba River, and at mi 153.6.

DRAINAGE AREA. $-140 \mathrm{mi}^{2}$.

PERIOD OF RECORD.- October 1980 to September 1981, June 1984 to March 2014.

PERIOD OF ANALYSIS.-April 1985 to March 2014.

NUMBER OF CLIMATE YEARS IN FREQUENCY ANALYSIS.—29

\section{REMARKS.-}

\begin{tabular}{|c|c|c|c|c|}
\hline $\begin{array}{l}\text { Recurrence } \\
\text { intervals } \\
\text { (years) }\end{array}$ & \multicolumn{2}{|c|}{$\begin{array}{l}\text { Lowest average flow for } \\
\text { indicated number of } \\
\text { consecutive days } \\
\text { (cubic feet per second) }\end{array}$} & \multicolumn{2}{|c|}{$\begin{array}{l}\text { Time-sampling error } \\
\text { (in percent) }\end{array}$} \\
\hline & 1 & 7 & 1 & 7 \\
\hline 2 & 8.2 & 9.7 & 13 & 13 \\
\hline 5 & 4.8 & 5.8 & 14 & 13 \\
\hline 10 & 3.6 & 4.5 & 17 & 15 \\
\hline 20 & 2.8 & 3.6 & 20 & 18 \\
\hline
\end{tabular}

\begin{tabular}{|c|c|c|c|c|c|c|c|}
\hline \multicolumn{7}{|c|}{ DURATION OF DAILY FLOW } \\
\hline \multicolumn{7}{|c|}{ Flow equaled or exceeded for indicated percentage of time } \\
(cubic feet per second)
\end{tabular}


STATION NAME AND NUMBER—02423397 Little Cahaba River below Leeds, AL

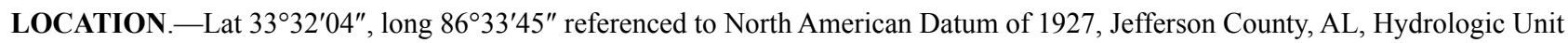
03150202, on left bank, $0.1 \mathrm{mi}$ downstream of Dry Branch, 0.5 mi southwest of Leeds, and 13.8 mi upstream from mouth.

DRAINAGE AREA. $-17.0 \mathrm{mi}^{2}$.

PERIOD OF RECORD.- -June 1995 to December 2006, October 2008 to March 2014.

PERIOD OF ANALYSIS.-April 1996 to March 2006, April 2009 to March 2014.

NUMBER OF CLIMATE YEARS IN FREQUENCY ANALYSIS.—15

\section{REMARKS.-}

\begin{tabular}{|c|c|c|c|c|}
\hline \multirow[t]{2}{*}{$\begin{array}{l}\text { Recurrence } \\
\text { intervals } \\
\text { (years) }\end{array}$} & \multicolumn{2}{|c|}{$\begin{array}{l}\text { Lowest average flow for } \\
\text { indicated number of } \\
\text { consecutive days } \\
\text { (cubic feet per second) }\end{array}$} & \multicolumn{2}{|c|}{$\begin{array}{l}\text { Time-sampling error } \\
\text { (in percent) }\end{array}$} \\
\hline & 1 & 7 & 1 & 7 \\
\hline 2 & 5.2 & 6.1 & 7 & 7 \\
\hline 5 & 4.2 & 4.9 & 9 & 8 \\
\hline 10 & 3.7 & 4.3 & 11 & 11 \\
\hline 20 & 3.3 & 3.8 & 14 & 14 \\
\hline
\end{tabular}

\section{DURATION OF DAILY FLOW}

Flow equaled or exceeded for indicated percentage of time (cubic feet per second)

\begin{tabular}{|c|c|c|c|c|c|c|c|}
\hline Percentage & 5 & 10 & 25 & 50 & 75 & 90 & 95 \\
\hline Flow & 104 & 66 & 35 & 18 & 10 & 7.1 & 6.0 \\
\hline
\end{tabular}


STATION NAME AND NUMBER—02423398 Little Cahaba River near Leeds, AL

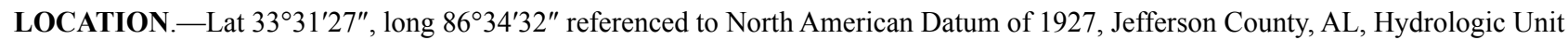
03150202, on left bank on downstream side bridge on county road, 1.2 mi downstream from Dry Branch, 2.0 mi southwest of Leeds, and 12.8 mi upstream from mouth.

DRAINAGE AREA.- $-19.4 \mathrm{mi}^{2}$.

PERIOD OF RECORD.- October 1980 to September 1981, May 1988 to December 2006.

PERIOD OF ANALYSIS.-April 1989 to March 2006.

NUMBER OF CLIMATE YEARS IN FREQUENCY ANALYSIS.—17

REMARKS.-Potential influence from waste water treatment plant discharges.

\begin{tabular}{|c|c|c|c|c|}
\hline $\begin{array}{l}\text { Recurrence } \\
\text { intervals } \\
\text { (years) }\end{array}$ & \multicolumn{2}{|c|}{$\begin{array}{l}\text { Lowest average flow for } \\
\text { indicated number of } \\
\text { consecutive days } \\
\text { (cubic feet per second) }\end{array}$} & \multicolumn{2}{|c|}{$\begin{array}{l}\text { Time-sampling error } \\
\text { (in percent) }\end{array}$} \\
\hline & 1 & 7 & 1 & 7 \\
\hline 2 & 7.1 & 7.9 & 6 & 5 \\
\hline 5 & 5.8 & 6.6 & 8 & 7 \\
\hline 10 & 5.1 & 5.9 & 10 & 9 \\
\hline 20 & 4.6 & 5.4 & 14 & 11 \\
\hline
\end{tabular}

\begin{tabular}{|c|c|c|c|c|c|c|c|}
\hline \multicolumn{8}{|c|}{ DURATION OF DAILY FLOW } \\
\hline \multicolumn{8}{|c|}{$\begin{array}{l}\text { Flow equaled or exceeded for indicated percentage of time } \\
\text { (cubic feet per second) }\end{array}$} \\
\hline Percentage & 5 & 10 & 25 & 50 & 75 & 90 & 95 \\
\hline Flow & 116 & 72 & 37 & 20 & 12 & 8.5 & 7.4 \\
\hline
\end{tabular}


STATION NAME AND NUMBER—02423400 Little Cahaba River near Jefferson Park, AL

LOCATION.-Lat 3329'59", long 86³6'51" referenced to North American Datum of 1927, Jefferson County, AL, Hydrologic Unit 03150202, on left upstream side of bridge on county road, 0.5 mi west of Highway 119, 0.7 mi upstream of Lake Purdy, 3.3 mi southwest of Jefferson Park, and 5.1 mi southeast of Leeds.

DRAINAGE AREA. $-24.4 \mathrm{mi}^{2}$.

PERIOD OF RECORD.- July 1986 to February 2000, June 2007 to March 2014.

PERIOD OF ANALYSIS.-April 1987 to March 1999, April 2008 to March 2014.

NUMBER OF CLIMATE YEARS IN FREQUENCY ANALYSIS.-18

REMARKS.-

\begin{tabular}{|c|c|c|c|c|}
\hline $\begin{array}{l}\text { Recurrence } \\
\text { intervals } \\
\text { (years) }\end{array}$ & \multicolumn{2}{|c|}{$\begin{array}{l}\text { Lowest average flow for } \\
\text { indicated number of } \\
\text { consecutive days } \\
\text { (cubic feet per second) }\end{array}$} & \multicolumn{2}{|c|}{$\begin{array}{l}\text { Time-sampling error } \\
\text { (in percent) }\end{array}$} \\
\hline & 1 & 7 & 1 & 7 \\
\hline 2 & 7.1 & 7.8 & 10 & 8 \\
\hline 5 & 5.1 & 5.8 & 13 & 10 \\
\hline 10 & 4.1 & 4.9 & 17 & 13 \\
\hline 20 & 3.4 & 4.2 & 24 & 18 \\
\hline
\end{tabular}

\begin{tabular}{|c|c|c|c|c|c|c|c|}
\hline \multicolumn{7}{|c|}{ DURATION OF DAILY FLOW } \\
\hline \multicolumn{7}{|c|}{ Flow equaled or exceeded for indicated percentage of time } \\
(cubic feet per second)
\end{tabular}


STATION NAME AND NUMBER—02423414 Little Cahaba River at Cahaba Beach Road near Cahaba Heights, AL

LOCATION.-Lat 3326'23", long 86 41'56" referenced to North American Datum of 1927, Shelby County, AL, Hydrologic Unit 03150202, on right bank, $1.3 \mathrm{mi}$ from mouth, $1.5 \mathrm{mi}$ southeast of Cahaba Heights, and $3.9 \mathrm{mi}$ southwest of Lake Purdy dam.

DRAINAGE AREA. $-47.0 \mathrm{mi}^{2}$.

PERIOD OF RECORD.-August 2003 to March 2014.

PERIOD OF ANALYSIS.-April 2004 to March 2014.

NUMBER OF CLIMATE YEARS IN FREQUENCY ANALYSIS.-10

REMARKS.-Since about 1929, flow regulated on Little Cahaba River by Lake Purdy.

\begin{tabular}{|c|c|c|c|c|}
\hline $\begin{array}{c}\text { Recurrence } \\
\text { intervals } \\
\text { (years) }\end{array}$ & \multicolumn{2}{|c|}{$\begin{array}{c}\text { Lowest average flow for } \\
\text { indicated number of } \\
\text { consecutive days } \\
\text { (cubic feet per second) }\end{array}$} & \multicolumn{2}{|c|}{$\begin{array}{c}\text { Time-sampling error } \\
\text { (in percent) }\end{array}$} \\
\hline \multicolumn{7}{|c|}{1} & 7 & 1 & 24 \\
\hline 2 & 4.0 & 7.2 & 21 & 26 \\
\hline 5 & 2.3 & 4.0 & 25 & 32 \\
\hline 10 & 1.7 & 2.9 & 32 & 41 \\
\hline 20 & 1.3 & 2.3 & 41 & \\
\hline
\end{tabular}

\section{DURATION OF DAILY FLOW}

Flow equaled or exceeded for indicated percentage of time (cubic feet per second)

\begin{tabular}{|c|c|c|c|c|c|c|c|}
\hline Percentage & 5 & 10 & 25 & 50 & 75 & 90 & 95 \\
\hline Flow & 255 & 165 & 88 & 59 & 40 & 16 & 8.9 \\
\hline
\end{tabular}


STATION NAME AND NUMBER—02423425 Cahaba River near Cahaba Heights, AL

LOCATION.-Lat 3324'56", long 8644'23" referenced to North American Datum of 1927, Shelby County, AL, Hydrologic Unit 03150202, on left bank $700 \mathrm{ft}$ upstream from bridge on county road, $2.5 \mathrm{mi}$ upstream from Little Shades Creek, 3.0 mi south of Cahaba Heights, $3.4 \mathrm{mi}$ downstream from Little Cahaba River, and at river mile 144.9.

DRAINAGE AREA. - $201 \mathrm{mi}^{2}$.

PERIOD OF RECORD.-August 1975 to September 1985, July 1996 to March 2014.

PERIOD OF ANALYSIS.-April 1976 to March 1985, April 1997 to March 2014.

NUMBER OF CLIMATE YEARS IN FREQUENCY ANALYSIS.-26

REMARKS.-An average of $82 \mathrm{ft}^{3} / \mathrm{s}$ is diverted above station by Birmingham Water Works Board, and is not included in records. Since about 1929, flow partly regulated by Lake Purdy (capacity, 15,300 acre-ft) on Little Cahaba River.

\begin{tabular}{|c|c|c|c|c|}
\hline $\begin{array}{c}\text { Recurrence } \\
\text { intervals } \\
\text { (years) }\end{array}$ & \multicolumn{2}{|c|}{$\begin{array}{c}\text { Lowest average flow for } \\
\text { indicated number of } \\
\text { consecutive days } \\
\text { (cubic feet per second) }\end{array}$} & \multicolumn{2}{|c|}{$\begin{array}{c}\text { Time-sampling error } \\
\text { (in percent) }\end{array}$} \\
\hline \multicolumn{2}{|c|}{1} & 7 & 1 & 7 \\
\hline 2 & 2.6 & 4.0 & 23 & 38 \\
\hline 5 & 1.2 & 1.7 & 34 & 47 \\
\hline 10 & 0.66 & 0.89 & 42 & 75 \\
\hline 20 & 0.36 & 0.46 & 67 & \\
\hline
\end{tabular}

\begin{tabular}{|c|c|c|c|c|c|c|c|}
\hline \multicolumn{1}{|c|}{ DURATION OF DAILY FLOW } \\
\hline \multicolumn{7}{|c|}{ Flow equaled or exceeded for indicated percentage of time } \\
(cubic feet per second) \\
\hline Percentage & 5 & 10 & 25 & 50 & 75 & 90 & 95 \\
\hline Flow & 1,080 & 681 & 301 & 76 & 12 & 4.8 & 3.3 \\
\hline
\end{tabular}


STATION NAME AND NUMBER—02423496 Cahaba River near Hoover, AL

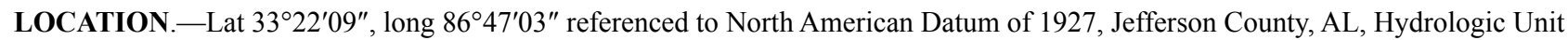
03150202, upstream of Cahaba River Sewage Treatment Plant, 1.1 mi upstream from U.S. Highway 31, 2.1 mi upstream from station 02423500 , and at mile 138.9 .

DRAINAGE AREA. $-226 \mathrm{mi}^{2}$.

PERIOD OF RECORD.--May 1988 to March 2014.

PERIOD OF ANALYSIS.-April 1989 to March 2014.

NUMBER OF CLIMATE YEARS IN FREQUENCY ANALYSIS.—25

REMARKS.-Since about 1929, flow partly regulated by Lake Purdy on Little Cahaba River. Flow diverted upstream by Birmingham Water Works.

\begin{tabular}{|c|c|c|c|c|}
\hline $\begin{array}{l}\text { Recurrence } \\
\text { intervals } \\
\text { (years) }\end{array}$ & \multicolumn{2}{|c|}{$\begin{array}{l}\text { Lowest average flow for } \\
\text { indicated number of } \\
\text { consecutive days } \\
\text { (cubic feet per second) }\end{array}$} & \multicolumn{2}{|c|}{$\begin{array}{l}\text { Time-sampling error } \\
\text { (in percent) }\end{array}$} \\
\hline & 1 & 7 & 1 & 7 \\
\hline 2 & 5.9 & 6.7 & 12 & 13 \\
\hline 5 & 3.6 & 4.0 & 15 & 16 \\
\hline 10 & 2.7 & 3.0 & 20 & 20 \\
\hline 20 & 2.1 & 2.3 & 27 & 27 \\
\hline
\end{tabular}

\begin{tabular}{|c|c|c|c|c|c|c|c|}
\hline \multicolumn{1}{|c|}{ DURATION OF DAILY FLOW } \\
\hline \multicolumn{1}{|c|}{ Flow equaled or exceeded for indicated percentage of time } \\
(cubic feet per second) \\
\hline Percentage & 5 & 10 & 25 & 50 & 75 & 90 & 95 \\
\hline Flow & 1,470 & 852 & 349 & 101 & 18 & 9.1 & 6.1 \\
\hline
\end{tabular}


STATION NAME AND NUMBER—02423500 Cahaba River near Acton, AL

LOCATION.-Lat 332 $1^{\prime} 48^{\prime \prime}$, long 86 $48^{\prime} 47^{\prime \prime}$ referenced to North American Datum of 1927, Jefferson County, AL, Hydrologic Unit 03150202, on right bank at downstream side of highway bridge (Bains Bridge), 0.5 mi upstream from Patton Creek, 1 mi downstream from U.S. Highway 31, 1 mi northwest of Acton, $16 \mathrm{mi}$ south of Birmingham, and at mile 136.8.

DRAINAGE AREA. $-230 \mathrm{mi}^{2}$.

PERIOD OF RECORD.- October 1938 to September 1957, October 1983 to March 2014.

PERIOD OF ANALYSIS.-April 1984 to March 2014.

NUMBER OF CLIMATE YEARS IN FREQUENCY ANALYSIS.-30

REMARKS.-Flow is diverted above station by Birmingham Water Works Board, and is not included in record except about $15 \mathrm{ft}^{3} / \mathrm{s}$, which is returned to river above station, and also is influenced by releases from several wastewater treatment plants along the river. Flow partly regulated by Lake Purdy (capacity, 15,300 acre-ft) on Little Cahaba River. The period of analysis includes the most recent period of record for which data reviews indicated relatively stable flow patterns.

\begin{tabular}{|c|c|c|c|c|}
\hline $\begin{array}{l}\text { Recurrence } \\
\text { intervals } \\
\text { (years) }\end{array}$ & \multicolumn{2}{|c|}{$\begin{array}{l}\text { Lowest average flow for } \\
\text { indicated number of } \\
\text { consecutive days } \\
\text { (cubic feet per second) }\end{array}$} & \multicolumn{2}{|c|}{$\begin{array}{l}\text { Time-sampling error } \\
\text { (in percent) }\end{array}$} \\
\hline & 1 & 7 & 1 & 7 \\
\hline 2 & 16 & 18 & 12 & 11 \\
\hline 5 & 10 & 11 & 17 & 15 \\
\hline 10 & 7.2 & 8.2 & 22 & 20 \\
\hline 20 & 5.2 & 6.0 & 33 & 30 \\
\hline 50 & 3.4 & 4.1 & 56 & 49 \\
\hline
\end{tabular}

\section{DURATION OF DAILY FLOW}

Flow equaled or exceeded for indicated percentage of time (cubic feet per second)

\begin{tabular}{|c|c|c|c|c|c|c|c|}
\hline Percentage & 5 & 10 & 25 & 50 & 75 & 90 & 95 \\
\hline Flow & 1,490 & 892 & 370 & 109 & 30 & 19 & 15 \\
\hline
\end{tabular}


STATION NAME AND NUMBER—0242354750 Cahaba Valley Creek at Cross Creek Road at Pelham, AL

LOCATION.-Lat $33^{\circ} 18^{\prime} 48^{\prime \prime}$, long 86 $48^{\prime 2} 3^{\prime \prime}$ referenced to North American Datum of 1927, Shelby County, AL, Hydrologic Unit 03150202, on upstream side of Cross Creek Road at Pelham.

DRAINAGE AREA. $-25.6 \mathrm{mi}^{2}$.

PERIOD OF RECORD.- October 1998 to September 2013.

PERIOD OF ANALYSIS.-April 1999 to March 2013.

NUMBER OF CLIMATE YEARS IN FREQUENCY ANALYSIS.-14

REMARKS.-

\begin{tabular}{|c|c|c|c|c|}
\hline $\begin{array}{l}\text { Recurrence } \\
\text { intervals } \\
\text { (years) }\end{array}$ & \multicolumn{2}{|c|}{$\begin{array}{l}\text { Lowest average flow for } \\
\text { indicated number of } \\
\text { consecutive days } \\
\text { (cubic feet per second) }\end{array}$} & \multicolumn{2}{|c|}{$\begin{array}{l}\text { Time-sampling error } \\
\text { (in percent) }\end{array}$} \\
\hline & 1 & 7 & 1 & 7 \\
\hline 2 & 6.0 & 6.8 & 11 & 10 \\
\hline 5 & 4.4 & 4.9 & 13 & 13 \\
\hline 10 & 3.6 & 4.1 & 16 & 16 \\
\hline 20 & 3.1 & 3.5 & 21 & 21 \\
\hline
\end{tabular}

\section{DURATION OF DAILY FLOW}

Flow equaled or exceeded for indicated percentage of time (cubic feet per second)

\begin{tabular}{|c|c|c|c|c|c|c|c|}
\hline Percentage & 5 & 10 & 25 & 50 & 75 & 90 & 95 \\
\hline Flow & 143 & 90 & 44 & 21 & 11 & 7.3 & 5.9 \\
\hline
\end{tabular}


STATION NAME AND NUMBER—02423555 Cahaba River near Helena, AL

LOCATION.-Lat 3317'04", long 8652'57" referenced to North American Datum of 1927, Shelby County, AL, Hydrologic Unit 03150202, on right bank at downstream side of bridge, $0.9 \mathrm{mi}$ above Trigger Creek, $3 \mathrm{mi}$ west of Helena, and at mile 127.1.

DRAINAGE AREA. $-335 \mathrm{mi}^{2}$.

PERIOD OF RECORD.- October 1995 to March 2014.

PERIOD OF ANALYSIS.--April 1996 to March 2014.

\section{NUMBER OF CLIMATE YEARS IN FREQUENCY ANALYSIS.- 18}

REMARKS.-Since about 1929, flow partly regulated by Lake Purdy on Little Cahaba River. An average of $70 \mathrm{ft}^{3} / \mathrm{s}$ is diverted above station by Birmingham Water Works Company and is not included in records, except about $5 \mathrm{ft}^{3} / \mathrm{s}$, which is returned to river above station. Flows also are influenced by releases from several upstream wastewater treatment plants.

\begin{tabular}{|c|c|c|c|c|}
\hline $\begin{array}{c}\text { Recurrence } \\
\text { intervals } \\
\text { (years) }\end{array}$ & \multicolumn{2}{|c|}{$\begin{array}{c}\text { Lowest average flow for } \\
\text { indicated number of } \\
\text { consecutive days } \\
\text { (cubic feet per second) }\end{array}$} & \multicolumn{2}{|c|}{$\begin{array}{c}\text { Time-sampling error } \\
\text { (in percent) }\end{array}$} \\
\hline \multicolumn{2}{|c|}{1} & 7 & 1 & 7 \\
\hline 2 & 39 & 43 & 7 & 8 \\
\hline 5 & 31 & 34 & 9 & 11 \\
\hline 10 & 26 & 30 & 13 & 15 \\
\hline 20 & 23 & 26 & 18 & \\
\hline
\end{tabular}

\begin{tabular}{|c|c|c|c|c|c|c|c|c|}
\hline \multicolumn{1}{|c|}{ DURATION OF DAILY FLOW } \\
\hline \multicolumn{7}{|c|}{ Flow equaled or exceeded for indicated percentage of time } \\
(cubic feet per second) \\
\hline Percentage & 5 & 10 & 25 & 50 & 75 & 90 & 95 \\
\hline Flow & 2,160 & 1,340 & 597 & 226 & 80 & 51 & 40 \\
\hline
\end{tabular}


STATION NAME AND NUMBER—02423630 Shades Creek near Greenwood, AL

LOCATION.-Lat 3319'34", long 8656'59" referenced to North American Datum of 1927, Jefferson County, AL, Hydrologic Unit 03150202, near left bank on downstream side of bridge on county road, 1.4 mi southwest of Greenwood, 5.5 mi south of Bessemer, and at mile 20.8 .

DRAINAGE AREA. $-72.3 \mathrm{mi}^{2}$.

PERIOD OF RECORD.- October 1964 to September 1965, October 1966 to September 1973, October 1974 to September 1981 , October 1997 to March 2014.

PERIOD OF ANALYSIS.-April 1967 to March 1973, April 1975 to March 1981, April 1998 to March 2014.

NUMBER OF CLIMATE YEARS IN FREQUENCY ANALYSIS.—-28

REMARKS.-

\begin{tabular}{|c|c|c|c|c|}
\hline $\begin{array}{c}\text { Recurrence } \\
\text { intervals } \\
\text { (years) }\end{array}$ & \multicolumn{2}{|c|}{$\begin{array}{c}\text { Lowest average flow for } \\
\text { indicated number of } \\
\text { consecutive days } \\
\text { (cubic feet per second) }\end{array}$} & \multicolumn{2}{|c|}{$\begin{array}{c}\text { Time-sampling error } \\
\text { (in percent) }\end{array}$} \\
\hline \multicolumn{5}{|c|}{} \\
\hline & 1 & 7 & 1 & 7 \\
\hline 2 & 8.9 & 10 & 22 & 26 \\
\hline 5 & 3.6 & 4.3 & 28 & 35 \\
\hline 10 & 2.1 & 2.5 & 37 & 51 \\
\hline 20 & 1.2 & 1.5 & 54 & \\
\hline
\end{tabular}

\begin{tabular}{|c|c|c|c|c|c|c|c|}
\hline \multicolumn{8}{|c|}{ DURATION OF DAILY FLOW } \\
\hline \multicolumn{8}{|c|}{$\begin{array}{l}\text { Flow equaled or exceeded for indicated percentage of time } \\
\text { (cubic feet per second) }\end{array}$} \\
\hline Percentage & 5 & 10 & 25 & 50 & 75 & 90 & 95 \\
\hline Flow & 518 & 296 & 122 & 50 & 25 & 12 & 7.3 \\
\hline
\end{tabular}


STATION NAME AND NUMBER—02423800 Little Cahaba River near Brierfield, AL

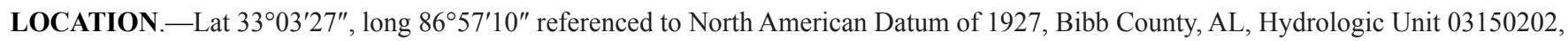
on county road 33, 1.8 mi downstream from Mahan Creek, and 3 mi northwest of Brierfield.

DRAINAGE AREA. $-147 \mathrm{mi}^{2}$.

PERIOD OF RECORD.-December 1957 to September 1970.

PERIOD OF ANALYSIS.-April 1958 to March 1970.

NUMBER OF CLIMATE YEARS IN FREQUENCY ANALYSIS.—12

REMARKS.-

\begin{tabular}{|c|c|c|c|c|}
\hline $\begin{array}{l}\text { Recurrence } \\
\text { intervals } \\
\text { (years) }\end{array}$ & \multicolumn{2}{|c|}{$\begin{array}{l}\text { Lowest average flow for } \\
\text { indicated number of } \\
\text { consecutive days } \\
\text { (cubic feet per second) }\end{array}$} & \multicolumn{2}{|c|}{$\begin{array}{l}\text { Time-sampling error } \\
\text { (in percent) }\end{array}$} \\
\hline & 1 & 7 & 1 & 7 \\
\hline 2 & 49 & 51 & 6 & 5 \\
\hline 5 & 43 & 45 & 4 & 5 \\
\hline 10 & 41 & 43 & 5 & 5 \\
\hline 20 & 40 & 41 & 6 & 6 \\
\hline
\end{tabular}

\section{DURATION OF DAILY FLOW}

Flow equaled or exceeded for indicated percentage of time (cubic feet per second)

\begin{tabular}{|c|c|c|c|c|c|c|c|}
\hline Percentage & 5 & 10 & 25 & 50 & 75 & 90 & 95 \\
\hline Flow & 535 & 352 & 192 & 106 & 71 & 57 & 51 \\
\hline
\end{tabular}


STATION NAME AND NUMBER—02424000 Cahaba River at Centreville, AL

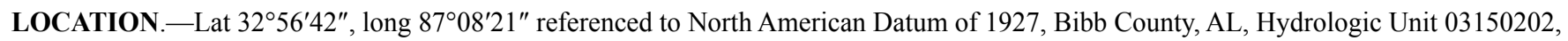
on left bank $60 \mathrm{ft}$ downstream from U.S. Highway 82 bridge, 0.2 mi west of Centreville, 2.5 mi upstream from Sandy Creek, and at mile 81.2 .

DRAINAGE AREA.-1,027 $\mathrm{mi}^{2}$.

PERIOD OF RECORD.-August 1901 to February 1908, May 1929 to March 1932, May 1935 to March 2014.

PERIOD OF ANALYSIS.-April 1902 to March 1907, April 1930 to March 1932, April 1936 to March 2014.

\section{NUMBER OF CLIMATE YEARS IN FREQUENCY ANALYSIS.—85}

REMARKS.-Since about 1929, flow partly regulated by Lake Purdy (capacity, 15,300 acre-ft) on Little Cahaba River; however, that regulation likely does not substantial influence flows at this location. An average of $82 \mathrm{ft}^{3} / \mathrm{s}$ is diverted upstream from station by Birmingham Water Works Board, and is not included in records. Flow is diverted upstream from station by Birmingham Water Works Board and releases by several wastewater treatment plants.

\begin{tabular}{|c|c|c|c|c|}
\hline $\begin{array}{c}\text { Recurrence } \\
\text { intervals } \\
\text { (years) }\end{array}$ & \multicolumn{2}{|c|}{$\begin{array}{c}\text { Lowest average flow for } \\
\text { indicated number of } \\
\text { consecutive days } \\
\text { (cubic feet per second) }\end{array}$} & \multicolumn{2}{|c|}{$\begin{array}{c}\text { Time-sampling error } \\
\text { (in percent) }\end{array}$} \\
\hline & 1 & 7 & 1 & 4 \\
\hline 2 & 206 & 215 & 4 & 4 \\
\hline 5 & 158 & 167 & 4 & 5 \\
\hline 10 & 137 & 145 & 5 & 6 \\
\hline 20 & 121 & 129 & 6 & 8 \\
\hline 50 & 105 & 112 & 9 & 4 \\
\hline
\end{tabular}

\begin{tabular}{|c|c|c|c|c|c|c|c|c|}
\hline \multicolumn{1}{|c|}{ DURATION OF DAILY FLOW } \\
\hline \multicolumn{1}{|c|}{ Flow equaled or exceeded for indicated percentage of time } \\
(cubic feet per second)
\end{tabular}


STATION NAME AND NUMBER—02424500 Cahaba River at Sprott, AL

LOCATION.--Lat $32^{\circ} 40^{\prime} 05^{\prime \prime}$, long $87^{\circ} 14^{\prime} 30^{\prime \prime}$ referenced to North American Datum of 1927, Perry County, AL, Hydrologic Unit 03150202 , on State Highway 14 and 183, 0.5 mi upstream from Goose Creek, 1 mi west of Sprott, 5.5 mi northeast of Marion, and at mile 47.6.

DRAINAGE AREA. $-1,370 \mathrm{mi}^{2}$.

PERIOD OF RECORD.- October 1938 to September 1969.

PERIOD OF ANALYSIS.--April 1939 to March 1969.

\section{NUMBER OF CLIMATE YEARS IN FREQUENCY ANALYSIS.—30}

REMARKS.-Flow is diverted upstream from station by Birmingham Water Works Board and releases by several wastewater treatment plants.

\begin{tabular}{|c|c|c|c|c|}
\hline $\begin{array}{c}\text { Recurrence } \\
\text { intervals } \\
\text { (years) }\end{array}$ & \multicolumn{2}{|c|}{$\begin{array}{c}\text { Lowest average flow for } \\
\text { indicated number of } \\
\text { consecutive days } \\
\text { (cubic feet per second) }\end{array}$} & \multicolumn{2}{|c|}{$\begin{array}{c}\text { Time-sampling error } \\
\text { (in percent) }\end{array}$} \\
\hline \multicolumn{7}{|c|}{1} & 7 & 1 & 7 \\
\hline 2 & 307 & 317 & 4 & 4 \\
\hline 5 & 256 & 266 & 5 & 5 \\
\hline 10 & 234 & 243 & 5 & 6 \\
\hline 20 & 217 & 226 & 6 & 8 \\
\hline 50 & 201 & 209 & 8 & 4 \\
\hline
\end{tabular}

\section{DURATION OF DAILY FLOW}

Flow equaled or exceeded for indicated percentage of time (cubic feet per second)

\begin{tabular}{|c|c|c|c|c|c|c|c|}
\hline Percentage & 5 & 10 & 25 & 50 & 75 & 90 & 95 \\
\hline Flow & 7,080 & 4,260 & 2,070 & 916 & 492 & 350 & 311 \\
\hline
\end{tabular}


STATION NAME AND NUMBER—02424590 Cahaba River near Suttle, AL

LOCATION.--Lat $32^{\circ} 31^{\prime} 45^{\prime \prime}$, long $87^{\circ} 11^{\prime} 56^{\prime \prime}$ referenced to North American Datum of 1927, Perry County, AL, Hydrologic Unit 03150202, at bridge on County Road 6,1.2 mi west of Suttle, 11 mi southeast of Marion, and 31.0 mi upstream of mouth.

DRAINAGE AREA.-1,480 $\mathrm{mi}^{2}$.

PERIOD OF RECORD.- - August 1987 to October 2011.

PERIOD OF ANALYSIS.-April 1988 to March 2011.

\section{NUMBER OF CLIMATE YEARS IN FREQUENCY ANALYSIS.—-23}

REMARKS.-Flow is diverted upstream from station by Birmingham Water Works Board and releases by several wastewater treatment plants.

\begin{tabular}{|c|c|c|c|c|}
\hline $\begin{array}{c}\text { Recurrence } \\
\text { intervals } \\
\text { (years) }\end{array}$ & \multicolumn{2}{|c|}{$\begin{array}{c}\text { Lowest average flow for } \\
\text { indicated number of } \\
\text { consecutive days } \\
\text { (cubic feet per second) }\end{array}$} & \multicolumn{2}{|c|}{$\begin{array}{c}\text { Time-sampling error } \\
\text { (in percent) }\end{array}$} \\
\hline \multicolumn{7}{|c|}{1} & 7 & 1 & 7 \\
\hline 2 & 379 & 399 & 6 & 8 \\
\hline 5 & 296 & 309 & 7 & 10 \\
\hline 10 & 258 & 267 & 9 & 13 \\
\hline 20 & 229 & 235 & 12 & 7 \\
\hline
\end{tabular}

\begin{tabular}{|c|c|c|c|c|c|c|c|}
\hline \multicolumn{7}{|c|}{ DURATION OF DAILY FLOW } \\
\hline \multicolumn{7}{|c|}{ Flow equaled or exceeded for indicated percentage of time } \\
(cubic feet per second)
\end{tabular}


STATION NAME AND NUMBER—02424940 Oakmulgee Creek near Augustin, AL

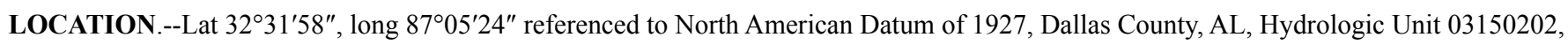
on State Highway 219, 2 mi north of Augustin.

DRAINAGE AREA. $-220 \mathrm{mi}^{2}$.

PERIOD OF RECORD.--May 1975 to May 1987.

PERIOD OF ANALYSIS.-April 1976 to March 1987.

NUMBER OF CLIMATE YEARS IN FREQUENCY ANALYSIS.—11

REMARKS.-

\begin{tabular}{|c|c|c|c|c|}
\hline $\begin{array}{l}\text { Recurrence } \\
\text { intervals } \\
\text { (years) }\end{array}$ & \multicolumn{2}{|c|}{$\begin{array}{l}\text { Lowest average flow for } \\
\text { indicated number of } \\
\text { consecutive days } \\
\text { (cubic feet per second) }\end{array}$} & \multicolumn{2}{|c|}{$\begin{array}{l}\text { Time-sampling error } \\
\text { (in percent) }\end{array}$} \\
\hline & 1 & 7 & 1 & 7 \\
\hline 2 & 27 & 29 & 14 & 14 \\
\hline 5 & 19 & 20 & 18 & 17 \\
\hline 10 & 15 & 16 & 23 & 22 \\
\hline 20 & 12 & 13 & 32 & 29 \\
\hline
\end{tabular}

DURATION OF DAILY FLOW

Flow equaled or exceeded for indicated percentage of time (cubic feet per second)

\begin{tabular}{|c|c|c|c|c|c|c|c|}
\hline Percentage & 5 & 10 & 25 & 50 & 75 & 90 & 95 \\
\hline Flow & 1,040 & 699 & 352 & 158 & 69 & 40 & 31 \\
\hline
\end{tabular}


STATION NAME AND NUMBER—02425000 Cahaba River near Marion Junction, AL

LOCATION.--Lat $32^{\circ} 26^{\prime} 38^{\prime \prime}$, long 87¹0'49" referenced to North American Datum of 1927, Dallas County, AL, Hydrologic Unit 03150202, on upstream side of upstream bridge on U.S. Highway 80, 3.8 mi downstream of Oakmulgee Creek, 3.5 mi east of Marion Junction, and 21.4 mi upstream of mouth.

DRAINAGE AREA.-1,766 $\mathrm{mi}^{2}$.

PERIOD OF RECORD.- October 1938 to September 1954, October 1968 to March 2014.

PERIOD OF ANALYSIS.-April 1939 to March 1954, April 1969 to March 2014.

NUMBER OF CLIMATE YEARS IN FREQUENCY ANALYSIS.-60

\section{REMARKS.-}

\begin{tabular}{|c|c|c|c|c|}
\hline $\begin{array}{c}\text { Recurrence } \\
\text { intervals } \\
\text { (years) }\end{array}$ & \multicolumn{2}{|c|}{$\begin{array}{l}\text { Lowest average flow for } \\
\text { indicated number of } \\
\text { consecutive days } \\
\text { (cubic feet per second) }\end{array}$} & \multicolumn{2}{|c|}{$\begin{array}{l}\text { Time-sampling error } \\
\text { (in percent) }\end{array}$} \\
\hline & 1 & 7 & 1 & 7 \\
\hline 2 & 422 & 440 & 4 & 4 \\
\hline 5 & 332 & 344 & 4 & 4 \\
\hline 10 & 292 & 303 & 5 & 5 \\
\hline 20 & 263 & 272 & 6 & 6 \\
\hline 50 & 234 & 241 & 8 & 8 \\
\hline
\end{tabular}

\section{DURATION OF DAILY FLOW}

Flow equaled or exceeded for indicated percentage of time (cubic feet per second)

\begin{tabular}{|c|c|c|c|c|c|c|c|}
\hline Percentage & 5 & 10 & 25 & 50 & 75 & 90 & 95 \\
\hline Flow & 10,200 & 6,300 & 3,020 & 1,390 & 721 & 494 & 399 \\
\hline
\end{tabular}


STATION NAME AND NUMBER—02425200 Big Swamp Creek near Orrville, AL

LOCATION.--Lat $32^{\circ} 13^{\prime} 17^{\prime \prime}$, long $87^{\circ} 09^{\prime} 48^{\prime \prime}$ referenced to North American Datum of 1927, Dallas County, AL, Hydrologic Unit 03150203, $20 \mathrm{ft}$ upstream from county road, $3 \mathrm{mi}$ upstream from mouth, and $9.8 \mathrm{mi}$ southeast of Orrville.

DRAINAGE AREA.-35.8 $\mathrm{mi}^{2}$.

PERIOD OF RECORD.- March 1972 to September 1985.

PERIOD OF ANALYSIS.--April 1972 to March 1985.

NUMBER OF CLIMATE YEARS IN FREQUENCY ANALYSIS.—13

\section{REMARKS.-}

\begin{tabular}{|c|c|c|c|c|}
\hline \multirow[t]{2}{*}{$\begin{array}{l}\text { Recurrence } \\
\text { intervals } \\
\text { (years) }\end{array}$} & \multicolumn{2}{|c|}{$\begin{array}{l}\text { Lowest average flow for } \\
\text { indicated number of } \\
\text { consecutive days } \\
\text { (cubic feet per second) }\end{array}$} & \multicolumn{2}{|c|}{$\begin{array}{l}\text { Time-sampling error } \\
\text { (in percent) }\end{array}$} \\
\hline & 1 & 7 & 1 & 7 \\
\hline 2 & 1.7 & 1.9 & 35 & 31 \\
\hline 5 & 0.80 & 0.92 & 63 & 46 \\
\hline 10 & 0.43 & 0.54 & 66 & 57 \\
\hline 20 & 0.22 & 0.31 & 111 & 94 \\
\hline
\end{tabular}

\section{DURATION OF DAILY FLOW}

Flow equaled or exceeded for indicated percentage of time (cubic feet per second)

\begin{tabular}{|c|c|c|c|c|c|c|c|}
\hline Percentage & 5 & 10 & 25 & 50 & 75 & 90 & 95 \\
\hline Flow & 209 & 117 & 42 & 9.8 & 3.2 & 1.7 & 1.3 \\
\hline
\end{tabular}


STATION NAME AND NUMBER—02425500 Cedar Creek at Minter, AL

LOCATION.--Lat $32^{\circ} 04^{\prime} 45^{\prime \prime}$, long 86 59'02" referenced to North American Datum of 1927, Dallas County, AL, Hydrologic Unit 03150203, on county road, $0.2 \mathrm{mi}$ downstream from Snake Creek, $0.5 \mathrm{mi}$ east of Minter, and $4 \mathrm{mi}$ upstream from Dry Cedar Creek.

DRAINAGE AREA. $-211 \mathrm{mi}^{2}$.

PERIOD OF RECORD.- July 1952 to September 1970, October 1974 to September 1982.

PERIOD OF ANALYSIS.-April 1953 to March 1970, April 1975 to March 1982.

NUMBER OF CLIMATE YEARS IN FREQUENCY ANALYSIS.—-24

REMARKS.-

\begin{tabular}{|c|c|c|c|c|}
\hline $\begin{array}{l}\text { Recurrence } \\
\text { intervals } \\
\text { (years) }\end{array}$ & \multicolumn{2}{|c|}{$\begin{array}{l}\text { Lowest average flow for } \\
\text { indicated number of } \\
\text { consecutive days } \\
\text { (cubic feet per second) }\end{array}$} & \multicolumn{2}{|c|}{$\begin{array}{l}\text { Time-sampling error } \\
\text { (in percent) }\end{array}$} \\
\hline & 1 & 7 & 1 & 7 \\
\hline 2 & 6.5 & 7.2 & 32 & 26 \\
\hline 5 & 2.0 & 2.6 & 42 & 32 \\
\hline 10 & 0.90 & 1.4 & 59 & 43 \\
\hline 20 & 0.43 & 0.83 & 91 & 61 \\
\hline
\end{tabular}

\section{DURATION OF DAILY FLOW}

Flow equaled or exceeded for indicated percentage of time (cubic feet per second)

\begin{tabular}{|c|c|c|c|c|c|c|c|}
\hline Percentage & 5 & 10 & 25 & 50 & 75 & 90 & 95 \\
\hline Flow & 980 & 460 & 172 & 57 & 22 & 9.9 & 5.7 \\
\hline
\end{tabular}


STATION NAME AND NUMBER—02426000 Boguechitto Creek near Browns, AL

LOCATION.--Lat $32^{\circ} 26^{\prime} 18^{\prime \prime}$, long $87^{\circ} 20^{\prime} 02^{\prime \prime}$ referenced to North American Datum of 1927, Dallas County, AL, Hydrologic Unit 03150203, on U.S. Highway 80, 0.3 mi upstream from Southern Railway bridge, 2 mi east of Browns, and 2.5 mi downstream from Washington Creek.

DRAINAGE AREA. $-95.4 \mathrm{mi}^{2}$.

PERIOD OF RECORD.-February 1944 to June 1954, October 1965 to September 1971.

PERIOD OF ANALYSIS.-April 1944 to March 1954, April 1966 to March 1971.

NUMBER OF CLIMATE YEARS IN FREQUENCY ANALYSIS.—-18

REMARKS.-

\begin{tabular}{|c|c|c|c|c|}
\hline $\begin{array}{c}\text { Recurrence } \\
\text { intervals } \\
\text { (years) }\end{array}$ & \multicolumn{2}{|c|}{$\begin{array}{c}\text { Lowest average flow for } \\
\text { indicated number of } \\
\text { consecutive days } \\
\text { (cubic feet per second) }\end{array}$} & \multicolumn{2}{|c|}{$\begin{array}{c}\text { Time-sampling error } \\
\text { (in percent) }\end{array}$} \\
\hline \multicolumn{7}{|c|}{1} & 7 & 1 & 7 \\
\hline 2 & 0.15 & 0.27 & 55 & -- \\
\hline 5 & 0.00 & 0.00 & -- & -- \\
\hline 10 & 0.00 & 0.00 & -- & -- \\
\hline 20 & 0.00 & 0.00 & -- & - \\
\hline
\end{tabular}

DURATION OF DAILY FLOW

Flow equaled or exceeded for indicated percentage of time (cubic feet per second)

\begin{tabular}{|c|c|c|c|c|c|c|c|}
\hline Percentage & 5 & 10 & 25 & 50 & 75 & 90 & 95 \\
\hline Flow & 598 & 290 & 95 & 31 & 8.1 & 1.5 & 0.2 \\
\hline
\end{tabular}


STATION NAME AND NUMBER—02427250 Pine Barren Creek near Snow Hill, AL

LOCATION.--Lat $31^{\circ} 59^{\prime} 46^{\prime \prime}$, long 8704'06" referenced to North American Datum of 1927, Wilcox County, AL, Hydrologic Unit 03150203, at bridge on State Highway 21, 4 mi west of Snow Hill.

DRAINAGE AREA. $-261 \mathrm{mi}^{2}$.

PERIOD OF RECORD.- October 1989 to March 2014.

PERIOD OF ANALYSIS.-April 1990 to March 2014.

NUMBER OF CLIMATE YEARS IN FREQUENCY ANALYSIS.—-24

REMARKS.-

\begin{tabular}{|c|c|c|c|c|}
\hline $\begin{array}{l}\text { Recurrence } \\
\text { intervals } \\
\text { (years) }\end{array}$ & \multicolumn{2}{|c|}{$\begin{array}{l}\text { Lowest average flow for } \\
\text { indicated number of } \\
\text { consecutive days } \\
\text { (cubic feet per second) }\end{array}$} & \multicolumn{2}{|c|}{$\begin{array}{l}\text { Time-sampling error } \\
\text { (in percent) }\end{array}$} \\
\hline & 1 & 7 & 1 & 7 \\
\hline 2 & 26 & 28 & 10 & 10 \\
\hline 5 & 18 & 19 & 12 & 12 \\
\hline 10 & 14 & 15 & 15 & 15 \\
\hline 20 & 11 & 12 & 20 & 20 \\
\hline
\end{tabular}

\section{DURATION OF DAILY FLOW}

Flow equaled or exceeded for indicated percentage of time (cubic feet per second)

\begin{tabular}{|c|c|c|c|c|c|c|c|}
\hline Percentage & 5 & 10 & 25 & 50 & 75 & 90 & 95 \\
\hline Flow & 1,330 & 632 & 258 & 110 & 52 & 31 & 24 \\
\hline
\end{tabular}


STATION NAME AND NUMBER—02427500 Alabama River near Millers Ferry, AL

LOCATION.--Lat $32^{\circ} 06^{\prime} 52^{\prime \prime}$, long $87^{\circ} 23^{\prime} 58^{\prime \prime}$ referenced to North American Datum of 1927, Wilcox County, AL, Hydrologic Unit 03150203, on State Highway 28, just downstream from Praire Creek, and 2.25 mi northwest of Millers Ferry.

DRAINAGE AREA. $-20,637 \mathrm{mi}^{2}$.

PERIOD OF RECORD.- October 1937 to September 1954.

PERIOD OF ANALYSIS.-October 1937 to September 1954.

REMARKS.-Flows regulated by reservoirs on the Etowah, Coosa, and Tallapoosa Rivers. Based on review of current streamgages on the Alabama River, current regulation patterns do not likely reflect the period of regulated record available at 02427500 .

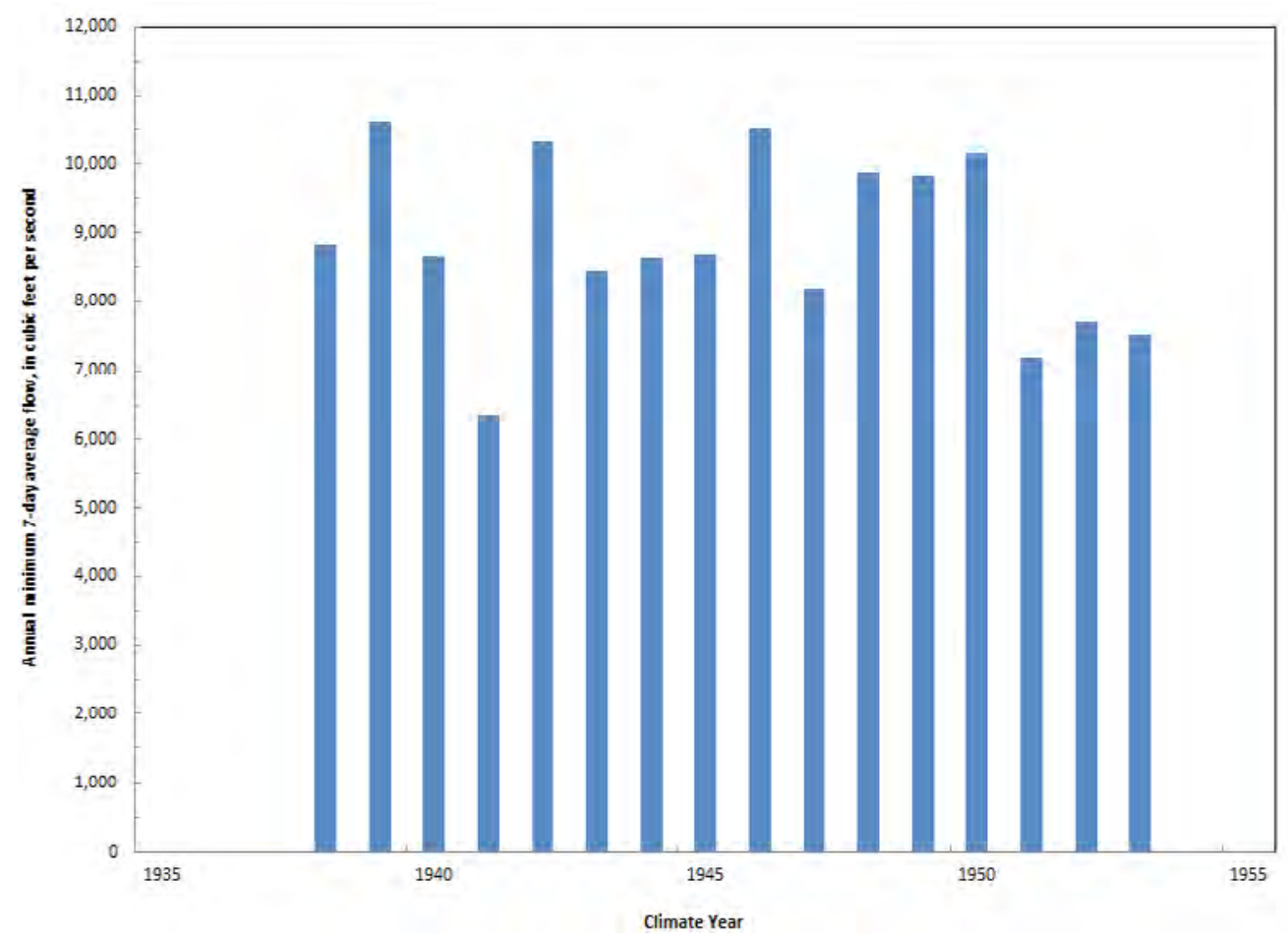

\begin{tabular}{|} 
EXCEEDANCE PERCENTILES OF ANNUAL MINIMUM 7-DAY AVERAGE FLOWS \\
\hline \multicolumn{1}{|c|}{ Annual minimum 7-day average flow exceeded for indicated percentiles (cubic feet per second) } \\
\hline Percentile & 10 & 20 & 30 & 40 & 50 & 60 & 70 & 80 & 90 \\
\hline Flow & 10,600 & 10,300 & 9,890 & 9,030 & 8,670 & 8,600 & 8,210 & 7,600 & 6,930 \\
\hline
\end{tabular}

\begin{tabular}{|c|c|c|c|c|c|c|c|}
\hline \multicolumn{1}{|c|}{ DURATION OF DAILY FLOW } \\
\hline \multicolumn{7}{|c|}{ Flow equaled or exceeded for indicated percentage of time } \\
(cubic feet per second)
\end{tabular}


STATION NAME AND NUMBER—02427700 Turkey Creek at Kimbrough, AL

LOCATION.-Lat $32^{\circ} 01^{\prime} 15^{\prime \prime}$, long $87^{\circ} 33^{\prime} 30^{\prime \prime}$ referenced to North American Datum of 1927, Wilcox County, AL, Hydrologic Unit 03150203, on county road, 0.6 mi downstream from State Highway 5, 1 mi south of Kimbrough, 2 mi upstream from mouth, and 6 mi upstream from Alabama River.

DRAINAGE AREA. $-97.5 \mathrm{mi}^{2}$.

PERIOD OF RECORD.- October 1958 to September 1996.

PERIOD OF ANALYSIS.-April 1959 to March 1996.

NUMBER OF CLIMATE YEARS IN FREQUENCY ANALYSIS.—37

\section{REMARKS.-}

\begin{tabular}{|c|c|c|c|c|}
\hline $\begin{array}{c}\text { Recurrence } \\
\text { intervals } \\
\text { (years) }\end{array}$ & \multicolumn{2}{|c|}{$\begin{array}{l}\text { Lowest average flow for } \\
\text { indicated number of } \\
\text { consecutive days } \\
\text { (cubic feet per second) }\end{array}$} & \multicolumn{2}{|c|}{$\begin{array}{l}\text { Time-sampling error } \\
\text { (in percent) }\end{array}$} \\
\hline & 1 & 7 & 1 & 7 \\
\hline 2 & 2.0 & 2.4 & 20 & 18 \\
\hline 5 & 0.75 & 1.0 & 26 & 22 \\
\hline 10 & 0.40 & 0.62 & 36 & 29 \\
\hline 20 & 0.23 & 0.38 & 52 & 41 \\
\hline 50 & 0.11 & 0.21 & 85 & 62 \\
\hline
\end{tabular}

\section{DURATION OF DAILY FLOW}

Flow equaled or exceeded for indicated percentage of time (cubic feet per second)

\begin{tabular}{|c|c|c|c|c|c|c|c|}
\hline Percentage & 5 & 10 & 25 & 50 & 75 & 90 & 95 \\
\hline Flow & 530 & 262 & 103 & 33 & 9.3 & 4.3 & 2.7 \\
\hline
\end{tabular}


STATION NAME AND NUMBER—02428400 Alabama River at Clairborne Lock and Dam near Monroeville, AL

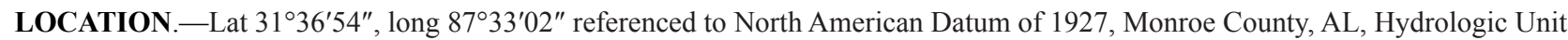
03150204, at control house and downstream end of lock, 3.5 miles upstream of Flat Creek, 3.8 miles downstream of Silver Creek, 15 miles northwest of Monroeville, and at river mile 81.9.

DRAINAGE AREA. $-21,473 \mathrm{mi}^{2}$.

PERIOD OF RECORD.- October 1975 to March 2014.

PERIOD OF ANALYSIS.-April 1976 to March 2014.

NUMBER OF CLIMATE YEARS IN FREQUENCY ANALYSIS.—38

REMARKS.-Flows regulated by reservoirs on the Etowah, Coosa, Tallapoosa and Alabama Rivers.

\begin{tabular}{|c|c|c|c|c|}
\hline $\begin{array}{l}\text { Recurrence } \\
\text { intervals } \\
\text { (years) }\end{array}$ & \multicolumn{2}{|c|}{$\begin{array}{l}\text { Lowest average flow for } \\
\text { indicated number of } \\
\text { consecutive days } \\
\text { (cubic feet per second) }\end{array}$} & \multicolumn{2}{|c|}{$\begin{array}{l}\text { Time-sampling error } \\
\text { (in percent) }\end{array}$} \\
\hline & 1 & 7 & 1 & 7 \\
\hline 2 & 5,530 & 6,430 & 7 & 5 \\
\hline 5 & 3,930 & 4,940 & 9 & 6 \\
\hline 10 & 3,150 & 4,230 & 12 & 8 \\
\hline 20 & 2,560 & 3,690 & 18 & 11 \\
\hline 50 & 1,980 & 3,140 & 27 & 15 \\
\hline
\end{tabular}

DURATION OF DAILY FLOW

Flow equaled or exceeded for indicated percentage of time (cubic feet per second)

\begin{tabular}{|c|c|c|c|c|c|c|c|}
\hline Percentage & 5 & 10 & 25 & 50 & 75 & 90 & 95 \\
\hline Flow & 104,000 & 81,200 & 39,000 & 16,900 & 9,010 & 6,520 & 5,530 \\
\hline
\end{tabular}


STATION NAME AND NUMBER—02428500 Big Flat Creek near Fountain, AL

LOCATION.-Lat $31^{\circ} 36^{\prime} 30^{\prime \prime}$, long $87^{\circ} 24^{\prime} 45^{\prime \prime}$ referenced to North American Datum of 1927, Monroe County, AL, Hydrologic Unit 03150204, on State Highway 41, 1 mi northwest of Fountain, 2 mi upstream from Bradley Mill Creek, 8 mi upstream from mouth, and 8 mi northwest of Monroeville.

DRAINAGE AREA. $-247 \mathrm{mi}^{2}$.

PERIOD OF RECORD.- October 1943 to September 1970.

PERIOD OF ANALYSIS.-April 1944 to March 1970.

NUMBER OF CLIMATE YEARS IN FREQUENCY ANALYSIS.—26

\section{REMARKS.-}

\begin{tabular}{|c|c|c|c|c|}
\hline $\begin{array}{l}\text { Recurrence } \\
\text { intervals } \\
\text { (years) }\end{array}$ & \multicolumn{2}{|c|}{$\begin{array}{l}\text { Lowest average flow for } \\
\text { indicated number of } \\
\text { consecutive days } \\
\text { (cubic feet per second) }\end{array}$} & \multicolumn{2}{|c|}{$\begin{array}{l}\text { Time-sampling error } \\
\text { (in percent) }\end{array}$} \\
\hline & 1 & 7 & 1 & 7 \\
\hline 2 & 6.0 & 7.0 & 25 & 23 \\
\hline 5 & 2.2 & 2.7 & 31 & 29 \\
\hline 10 & 1.2 & 1.5 & 42 & 39 \\
\hline 20 & 0.67 & 0.90 & 60 & 54 \\
\hline
\end{tabular}

\begin{tabular}{|c|c|c|c|c|c|c|c|c|}
\hline \multicolumn{7}{|c|}{ DURATION OF DAILY FLOW } \\
\hline \multicolumn{7}{|c|}{ Flow equaled or exceeded for indicated percentage of time } \\
(cubic feet per second)
\end{tabular}


STATION NAME AND NUMBER—02429000 Limestone Creek near Monroeville, AL

LOCATION.-Lat $31^{\circ} 33^{\prime} 45^{\prime \prime}$, long 87²1'06" referenced to North American Datum of 1927, Monroe County, AL, Hydrologic Unit 03150204, on State Highway 41, 3 mi northwest of Monroeville, and 10 mi upstream from mouth.

DRAINAGE AREA. $-121 \mathrm{mi}^{2}$.

PERIOD OF RECORD.-_January 1952 to September 1970.

PERIOD OF ANALYSIS.--April 1952 to March 1970.

NUMBER OF CLIMATE YEARS IN FREQUENCY ANALYSIS._-18

\section{REMARKS.-}

\begin{tabular}{|c|c|c|c|c|}
\hline $\begin{array}{l}\text { Recurrence } \\
\text { intervals } \\
\text { (years) }\end{array}$ & \multicolumn{2}{|c|}{$\begin{array}{l}\text { Lowest average flow for } \\
\text { indicated number of } \\
\text { consecutive days } \\
\text { (cubic feet per second) }\end{array}$} & \multicolumn{2}{|c|}{$\begin{array}{l}\text { Time-sampling error } \\
\text { (in percent) }\end{array}$} \\
\hline & 1 & 7 & 1 & 7 \\
\hline 2 & 22 & 24 & 12 & 12 \\
\hline 5 & 15 & 16 & 14 & 14 \\
\hline 10 & 12 & 13 & 17 & 17 \\
\hline 20 & 9.7 & 11 & 21 & 21 \\
\hline
\end{tabular}

\section{DURATION OF DAILY FLOW}

Flow equaled or exceeded for indicated percentage of time (cubic feet per second)

\begin{tabular}{|c|c|c|c|c|c|c|c|}
\hline Percentage & 5 & 10 & 25 & 50 & 75 & 90 & 95 \\
\hline Flow & 443 & 286 & 154 & 77 & 42 & 27 & 20 \\
\hline
\end{tabular}


STATION NAME AND NUMBER—02429500 Alabama River at Clairborne, AL

LOCATION.--Lat $31^{\circ} 32^{\prime} 48^{\prime \prime}$, long 87³0'45" referenced to North American Datum of 1927, Monroe County, AL, Hydrologic Unit 03150204, on U.S. Highway 84 at Clairborne, 0.5 mi downstream from Limestone Creek, 12 mi west of Monroeville, and at river mile 76.1.

DRAINAGE AREA. $-21,967 \mathrm{mi}^{2}$.

PERIOD OF RECORD.-April 1930 to September 1975.

PERIOD OF ANALYSIS.- - April 1930 to September 1975.

REMARKS.-Flows regulated by reservoirs on the Etowah, Coosa, Tallapoosa and Alabama Rivers. Based on review of current streamgages on the Alabama River, current regulation patterns do not likely reflect the period of regulated record available at 02429500.

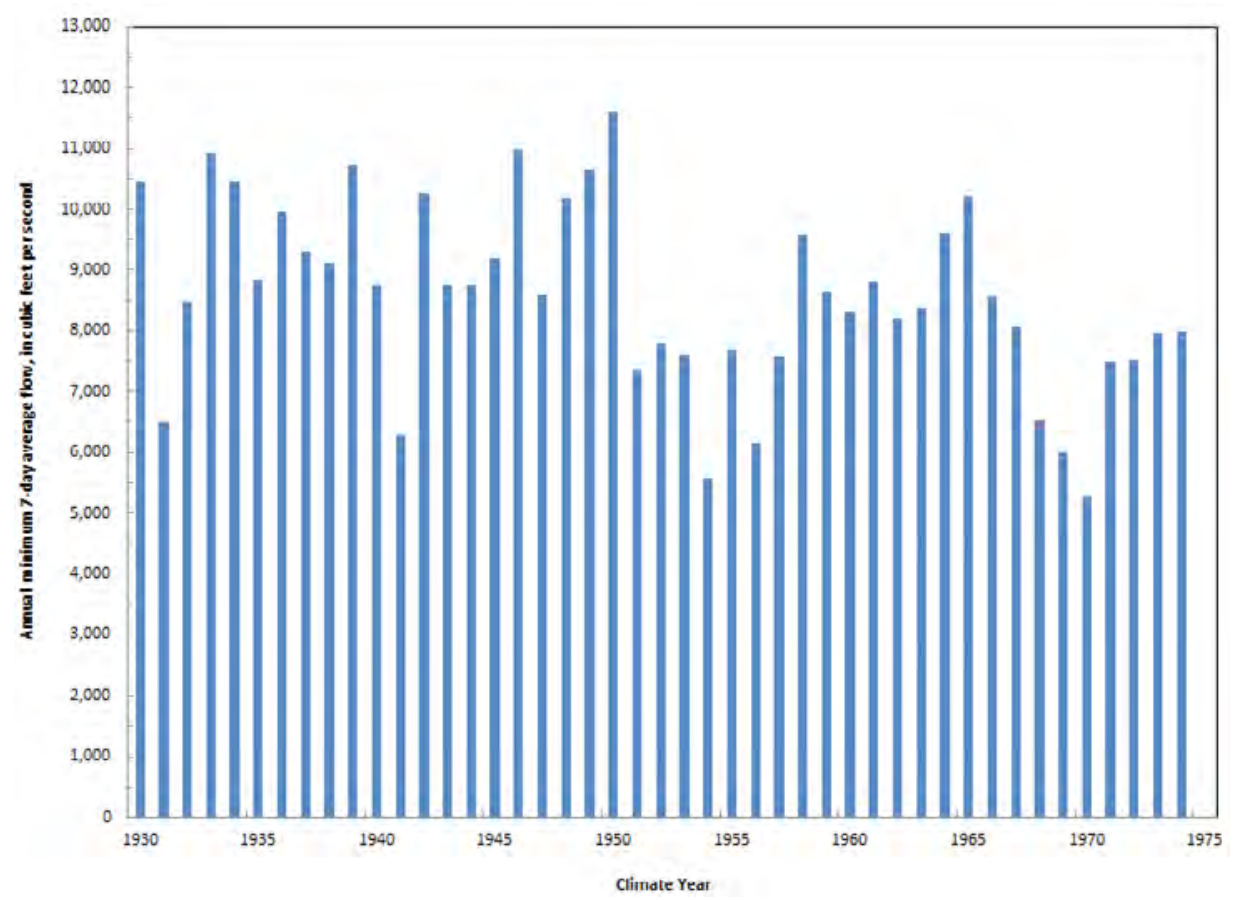

\begin{tabular}{|c|c|c|c|c|c|c|c|c|c|}
\hline \multicolumn{10}{|c|}{ EXCEEDANCE PERCENTILES OF ANNUAL MINIMUM 7-DAY AVERAGE FLOWS } \\
\hline \multicolumn{10}{|c|}{ Annual minimum 7-day average flow exceeded for indicated percentiles (cubic feet per second) } \\
\hline Percentile & 10 & 20 & 30 & 40 & 50 & 60 & 70 & 80 & 90 \\
\hline Flow & 10,700 & 10,200 & 9,350 & 8,780 & 8,610 & 8,250 & 7,770 & 7,490 & 6,220 \\
\hline
\end{tabular}

\begin{tabular}{|c|c|c|c|c|c|c|c|}
\hline \multicolumn{1}{|c|}{ DURATION OF DAILY FLOW } \\
\hline \multicolumn{7}{|c|}{ Flow equaled or exceeded for indicated percentage of time } \\
(cubic feet per second) \\
\hline Percentage & 5 & 10 & 25 & 50 & 75 & 90 & 95 \\
\hline Flow & 102,000 & 77,500 & 38,400 & 19,100 & 12,500 & 9,700 & 8,450 \\
\hline
\end{tabular}


STATION NAME AND NUMBER—02429595 Little River near Uriah, AL

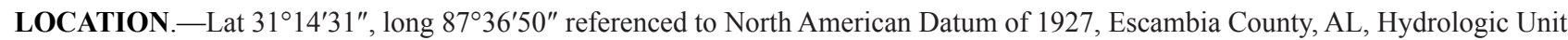
03150204, on county road, 7 mi northwest of McCullough.

DRAINAGE AREA. $-99.2 \mathrm{mi}^{2}$.

PERIOD OF RECORD.- October 1968 to September 1979.

PERIOD OF ANALYSIS.--April 1969 to March 1979.

NUMBER OF CLIMATE YEARS IN FREQUENCY ANALYSIS.-10

\section{REMARKS.-}

\begin{tabular}{|c|c|c|c|c|}
\hline $\begin{array}{l}\text { Recurrence } \\
\text { intervals } \\
\text { (years) }\end{array}$ & \multicolumn{2}{|c|}{$\begin{array}{l}\text { Lowest average flow for } \\
\text { indicated number of } \\
\text { consecutive days } \\
\text { (cubic feet per second) }\end{array}$} & \multicolumn{2}{|c|}{$\begin{array}{l}\text { Time-sampling error } \\
\text { (in percent) }\end{array}$} \\
\hline & 1 & 7 & 1 & 7 \\
\hline 2 & 62 & 64 & 9 & 9 \\
\hline 5 & 51 & 54 & 9 & 7 \\
\hline 10 & 46 & 50 & 10 & 7 \\
\hline 20 & 42 & 48 & 12 & 10 \\
\hline
\end{tabular}

\section{DURATION OF DAILY FLOW}

Flow equaled or exceeded for indicated percentage of time (cubic feet per second)

\begin{tabular}{|c|c|c|c|c|c|c|c|}
\hline Percentage & 5 & 10 & 25 & 50 & 75 & 90 & 95 \\
\hline Flow & 445 & 320 & 196 & 132 & 90 & 70 & 64 \\
\hline
\end{tabular}


STATION NAME AND NUMBER—02438000 Buttahatchee River below Hamilton, AL

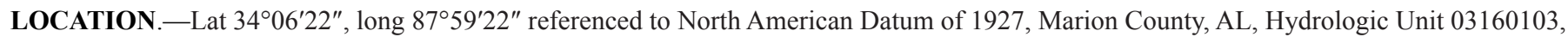
near right bank on downstream side of bridge on Military Street, 0.5 mi downstream from Woods Creek, 2 mi south of Hamilton, and at mile 82.6 .

DRAINAGE AREA. $-277 \mathrm{mi}^{2}$.

PERIOD OF RECORD.-October 1950 to September 1970, October 1991 to March 2014.

PERIOD OF ANALYSIS.-April 1951 to March 1970, April 1992 to March 2014.

NUMBER OF CLIMATE YEARS IN FREQUENCY ANALYSIS.-42

REMARKS.-

\begin{tabular}{|c|c|c|c|c|}
\hline $\begin{array}{l}\text { Recurrence } \\
\text { intervals } \\
\text { (years) }\end{array}$ & \multicolumn{2}{|c|}{$\begin{array}{l}\text { Lowest average flow for } \\
\text { indicated number of } \\
\text { consecutive days } \\
\text { (cubic feet per second) }\end{array}$} & \multicolumn{2}{|c|}{$\begin{array}{l}\text { Time-sampling error } \\
\text { (in percent) }\end{array}$} \\
\hline & 1 & 7 & 1 & 7 \\
\hline 2 & 50 & 54 & 7 & 7 \\
\hline 5 & 35 & 38 & 8 & 9 \\
\hline 10 & 28 & 30 & 11 & 11 \\
\hline 20 & 25 & 25 & 14 & 14 \\
\hline 50 & 19 & 20 & 19 & 20 \\
\hline
\end{tabular}

\begin{tabular}{|c|c|c|c|c|c|c|c|c|}
\hline \multicolumn{1}{|c|}{ DURATION OF DAILY FLOW } \\
\hline \multicolumn{7}{|c|}{ Flow equaled or exceeded for indicated percentage of time } \\
(cubic feet per second)
\end{tabular}


STATION NAME AND NUMBER—02439000 Buttahatchee River near Sulligent, AL

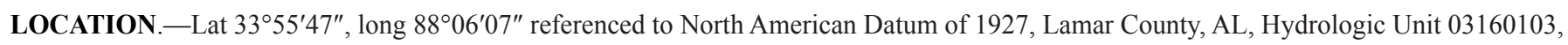
on State Highway 17, 1 mi upstream from Bogue Creek, 1.5 mi northwest of Sulligent, and 2 mi downstream from Beaver Creek.

DRAINAGE AREA. $-472 \mathrm{mi}^{2}$.

PERIOD OF RECORD.-March 1939 to September 1959.

PERIOD OF ANALYSIS.-April 1939 to March 1959.

NUMBER OF CLIMATE YEARS IN FREQUENCY ANALYSIS.-20

REMARKS.-

\begin{tabular}{|c|c|c|c|c|}
\hline $\begin{array}{c}\text { Recurrence } \\
\text { intervals } \\
\text { (years) }\end{array}$ & \multicolumn{2}{|c|}{$\begin{array}{c}\text { Lowest average flow for } \\
\text { indicated number of } \\
\text { consecutive days } \\
\text { (cubic feet per second) }\end{array}$} & \multicolumn{2}{|c|}{$\begin{array}{c}\text { Time-sampling error } \\
\text { (in percent) }\end{array}$} \\
\hline \multicolumn{7}{|c|}{1} & 7 & 1 & 7 \\
\hline 2 & 74 & 78 & 9 & 10 \\
\hline 5 & 53 & 56 & 10 & 12 \\
\hline 10 & 44 & 48 & 13 & 15 \\
\hline 20 & 38 & 41 & 16 & 9 \\
\hline
\end{tabular}

\begin{tabular}{|c|c|c|c|c|c|c|c|c|}
\hline \multicolumn{1}{|c|}{ DURATION OF DAILY FLOW } \\
\hline \multicolumn{7}{|c|}{ Flow equaled or exceeded for indicated percentage of time } \\
(cubic feet per second)
\end{tabular}


STATION NAME AND NUMBER—02442000 Luxapallila Creek near Fayette, AL

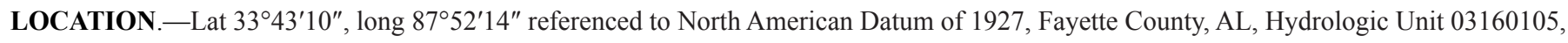
on State Highway 18 and 2 mi northwest of Fayette.

DRAINAGE AREA. $-130 \mathrm{mi}^{2}$.

PERIOD OF RECORD.-May 1945 to September 1970.

PERIOD OF ANALYSIS.-April 1946 to March 1970.

NUMBER OF CLIMATE YEARS IN FREQUENCY ANALYSIS.—24

REMARKS.-

\begin{tabular}{|c|c|c|c|c|}
\hline $\begin{array}{c}\text { Recurrence } \\
\text { intervals } \\
\text { (years) }\end{array}$ & \multicolumn{2}{|c|}{$\begin{array}{c}\text { Lowest average flow for } \\
\text { indicated number of } \\
\text { consecutive days } \\
\text { (cubic feet per second) }\end{array}$} & \multicolumn{2}{|c|}{$\begin{array}{c}\text { Time-sampling error } \\
\text { (in percent) }\end{array}$} \\
\hline \multicolumn{7}{|c|}{1} & 7 & 1 & 7 \\
\hline 2 & 41 & 44 & 5 & 6 \\
\hline 5 & 34 & 37 & 6 & 8 \\
\hline 10 & 30 & 33 & 8 & 10 \\
\hline 20 & 27 & 30 & 10 & 5 \\
\hline
\end{tabular}

\begin{tabular}{|c|c|c|c|c|c|c|c|}
\hline \multicolumn{8}{|c|}{ DURATION OF DAILY FLOW } \\
\hline \multicolumn{8}{|c|}{$\begin{array}{l}\text { Flow equaled or exceeded for indicated percentage of time } \\
\text { (cubic feet per second) }\end{array}$} \\
\hline Percentage & 5 & 10 & 25 & 50 & 75 & 90 & 95 \\
\hline Flow & 610 & 382 & 196 & 100 & 64 & 51 & 44 \\
\hline
\end{tabular}


STATION NAME AND NUMBER—02442500 Luxapallila Creek at Millport, AL

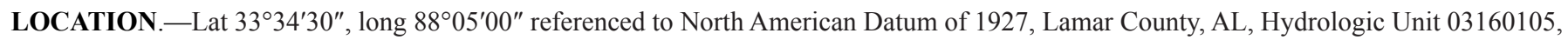
near left bank on downstream side of bridge on State Highway 17, 0.20 mi downstream from Driver Creek, 1.0 mi north of Millport, and at mile 31.6.

DRAINAGE AREA. $-247 \mathrm{mi}^{2}$.

PERIOD OF RECORD.-August 1954 to September 1959, December 1980 to September 1986, and October 2001 to October 2011.

PERIOD OF ANALYSIS.-April 1955 to March 1959, April 1981 to March 1986, and April 2002 to March 2011.

NUMBER OF CLIMATE YEARS IN FREQUENCY ANALYSIS.-18

REMARKS.-

\begin{tabular}{|c|c|c|c|c|}
\hline $\begin{array}{l}\text { Recurrence } \\
\text { intervals } \\
\text { (years) }\end{array}$ & \multicolumn{2}{|c|}{$\begin{array}{l}\text { Lowest average flow for } \\
\text { indicated number of } \\
\text { consecutive days } \\
\text { (cubic feet per second) }\end{array}$} & \multicolumn{2}{|c|}{$\begin{array}{l}\text { Time-sampling error } \\
\text { (in percent) }\end{array}$} \\
\hline & 1 & 7 & 1 & 7 \\
\hline 2 & 69 & 74 & 8 & 8 \\
\hline 5 & 53 & 56 & 9 & 10 \\
\hline 10 & 45 & 48 & 12 & 12 \\
\hline 20 & 40 & 42 & 15 & 16 \\
\hline
\end{tabular}

\begin{tabular}{|c|c|c|c|c|c|c|c|}
\hline \multicolumn{7}{|c|}{ DURATION OF DAILY FLOW } \\
\hline \multicolumn{7}{|c|}{ Flow equaled or exceeded for indicated percentage of time } \\
(cubic feet per second)
\end{tabular}


STATION NAME AND NUMBER—02444000 Coal Fire Creek near Pickensville, AL

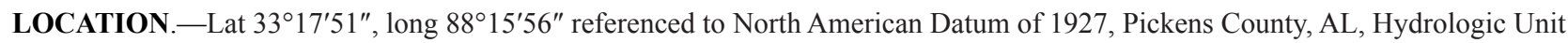
03160106, on State Highway 14, 4.5 mi north of Pickensville, and at mile 4.5.

DRAINAGE AREA.-126 mi

PERIOD OF RECORD.- October 1954 to September 1971, October 1974 to September 1980

PERIOD OF ANALYSIS.-April 1955 to March 1971, April 1975 to March 1980.

NUMBER OF CLIMATE YEARS IN FREQUENCY ANALYSIS.-21

REMARKS.- -

\begin{tabular}{|c|c|c|c|c|}
\hline $\begin{array}{l}\text { Recurrence } \\
\text { intervals } \\
\text { (years) }\end{array}$ & \multicolumn{2}{|c|}{$\begin{array}{l}\text { Lowest average flow for } \\
\text { indicated number of } \\
\text { consecutive days } \\
\text { (cubic feet per second) }\end{array}$} & \multicolumn{2}{|c|}{$\begin{array}{l}\text { Time-sampling error } \\
\text { (in percent) }\end{array}$} \\
\hline & 1 & 7 & 1 & 7 \\
\hline 2 & 13 & 14 & 15 & 12 \\
\hline 5 & 7.6 & 8.7 & 19 & 15 \\
\hline 10 & 5.4 & 6.7 & 26 & 20 \\
\hline 20 & 3.9 & 5.2 & 38 & 27 \\
\hline
\end{tabular}

\section{DURATION OF DAILY FLOW}

Flow equaled or exceeded for indicated percentage of time (cubic feet per second)

\begin{tabular}{|c|c|c|c|c|c|c|c|}
\hline Percentage & 5 & 10 & 25 & 50 & 75 & 90 & 95 \\
\hline Flow & 625 & 416 & 197 & 78 & 32 & 19 & 14 \\
\hline
\end{tabular}


STATION NAME AND NUMBER—02444160 Tombigbee River at Bevill Lock and Dam near Pickensville, AL

LOCATION.-Lat $33^{\circ} 12^{\prime} 38^{\prime \prime}$, long 88 $17^{\prime} 19^{\prime \prime}$ referenced to North American Datum of 1927, Pickens County, AL, Hydrologic Unit 03160106, near left bank at dam, 2 mi southwest of Pickensville, 10 mi northwest of Aliceville, and at river mile 287.7.

DRAINAGE AREA. $-5,750 \mathrm{mi}^{2}$.

PERIOD OF RECORD.- October 1980 to September 2009, October 2010 to March 2014.

PERIOD OF ANALYSIS.-April 1985 to March 2009, April 2011 to March 2014

NUMBER OF CLIMATE YEARS IN FREQUENCY ANALYSIS.-27

REMARKS.- - Period of record analyzed includes all current regulation structures. Records since January 16, 1985, include diversions from Tennessee River basin through Bay Springs lock on Tennessee-Tombigbee Waterway. Regulated by Jamie Whitten Lock and Dam (1983), G.V. Montgomery Lock and Dam (1984), John Ranking Lock and Dam (1984), Fulton Lock and Dam (1981), Glover Wilkins Lock and Dam (1981), Amory Lock and Dam (1979), Aberdeen Lock and Dam (1981), John C. Stennis Lock and Dam (1978), and Tom Bevill Lock and Dam (1978).

\begin{tabular}{|c|c|c|c|c|}
\hline $\begin{array}{l}\text { Recurrence } \\
\text { intervals } \\
\text { (years) }\end{array}$ & \multicolumn{2}{|c|}{$\begin{array}{l}\text { Lowest average flow for } \\
\text { indicated number of } \\
\text { consecutive days } \\
\text { (cubic feet per second) }\end{array}$} & \multicolumn{2}{|c|}{$\begin{array}{l}\text { Time-sampling error } \\
\text { (in percent) }\end{array}$} \\
\hline & 1 & 7 & 1 & 7 \\
\hline 2 & 518 & 736 & 5 & 7 \\
\hline 5 & 418 & 555 & 6 & 7 \\
\hline 10 & 368 & 482 & 8 & 8 \\
\hline 20 & 330 & 431 & 10 & 10 \\
\hline
\end{tabular}

\begin{tabular}{|c|c|c|c|c|c|c|c|c|}
\hline \multicolumn{1}{|c|}{ DURATION OF DAILY FLOW } \\
\hline \multicolumn{7}{|c|}{ Flow equaled or exceeded for indicated percentage of time } \\
(cubic feet per second) \\
\hline Percentage & 5 & 10 & 25 & 50 & 75 & 90 & 95 \\
\hline Flow & 34,300 & 21,700 & 9,300 & 3,810 & 1,610 & 791 & 674 \\
\hline
\end{tabular}


STATION NAME AND NUMBER—02444500 Tombigbee River near Cochrane, AL

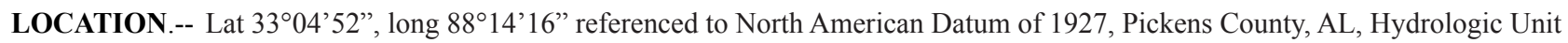
03160106, on State Highway 17, 1.2 mi northeast of Cochrane, 2.2 mi downstream from Boguechitto Creek, 7 mi southwest of Aliceville, and at mile 271.4.

DRAINAGE AREA. $-5,940 \mathrm{mi}^{2}$.

PERIOD OF RECORD.- - October 1938 to March 1978.

PERIOD OF ANALYSIS.-April 1939 to March 1978.

\section{NUMBER OF CLIMATE YEARS IN FREQUENCY ANALYSIS.-39}

REMARKS.-Period of record is for pre-regulation conditions. Currently regulated by Jamie Whitten Lock and Dam (1983), G.V. Montgomery Lock and Dam (1984), John Ranking Lock and Dam (1984), Fulton Lock and Dam (1981), Glover Wilkins Lock and Dam (1981), Amory Lock and Dam (1979), Aberdeen Lock and Dam (1981), John C. Stennis Lock and Dam (1978), and Tom Bevill Lock and Dam (1978). Since January 16, 1985, streamflows include diversions from Tennessee River basin through Bay Springs lock on Tennessee-Tombigbee Waterway.

\begin{tabular}{|c|c|c|c|c|}
\hline $\begin{array}{c}\text { Recurrence } \\
\text { intervals } \\
\text { (years) }\end{array}$ & \multicolumn{2}{|c|}{$\begin{array}{c}\text { Lowest average flow for } \\
\text { indicated number of } \\
\text { consecutive days } \\
\text { (cubic feet per second) }\end{array}$} & \multicolumn{2}{|c|}{$\begin{array}{c}\text { Time-sampling error } \\
\text { (in percent) }\end{array}$} \\
\hline & 1 & 7 & 1 & 7 \\
\hline 2 & 607 & 634 & 7 & 9 \\
\hline 5 & 418 & 435 & 9 & 11 \\
\hline 10 & 338 & 353 & 11 & 14 \\
\hline 20 & 281 & 295 & 15 & 19 \\
\hline 50 & 225 & 239 & 20 & \\
\hline
\end{tabular}

\section{DURATION OF DAILY FLOW}

Flow equaled or exceeded for indicated percentage of time (cubic feet per second)

\begin{tabular}{|c|c|c|c|c|c|c|c|}
\hline Percentage & 5 & 10 & 25 & 50 & 75 & 90 & 95 \\
\hline Flow & 35,000 & 25,000 & 10,500 & 3,320 & 1,300 & 735 & 565 \\
\hline
\end{tabular}


STATION NAME AND NUMBER—02445500 Sispey River at Fayette, AL

LOCATION.-- Lat 3340'10”, long 8748'59” referenced to North American Datum of 1927, Fayette County, AL, Hydrologic Unit 03160107, 1 mi southeast of Fayette, and $1.5 \mathrm{mi}$ downstream from Southern Railway bridge.

DRAINAGE AREA. $-282 \mathrm{mi}^{2}$.

PERIOD OF RECORD.-February 1939 to September 1959.

PERIOD OF ANALYSIS.-April 1939 to March 1959.

NUMBER OF CLIMATE YEARS IN FREQUENCY ANALYSIS. - 20

REMARKS.-Frequency statistics were computed based on a MOVE.1 correlation with station 02446500 Sipsey River near Elrod, AL, which has a period of record from September 1928 to March 1932, October 1939 to September 1971, October 1978 to March 2014, which represents 69 climatic years.

\begin{tabular}{|c|c|c|c|c|}
\hline $\begin{array}{l}\text { Recurrence } \\
\text { intervals } \\
\text { (years) }\end{array}$ & \multicolumn{2}{|c|}{$\begin{array}{l}\text { Lowest average flow for } \\
\text { indicated number of } \\
\text { consecutive days } \\
\text { (cubic feet per second) }\end{array}$} & \multicolumn{2}{|c|}{$\begin{array}{l}\text { Time-sampling erro } \\
\text { (in percent) }\end{array}$} \\
\hline & 1 & 7 & 1 & 7 \\
\hline 2 & 27 & 30 & 14 & 13 \\
\hline 5 & 18 & 20 & 16 & 15 \\
\hline 10 & 14 & 16 & 19 & 18 \\
\hline 20 & 11 & 13 & 24 & 22 \\
\hline 50 & 8.7 & 10 & 31 & 29 \\
\hline
\end{tabular}

\section{DURATION OF DAILY FLOW}

Flow equaled or exceeded for indicated percentage of time (cubic feet per second)

\begin{tabular}{|c|c|c|c|c|c|c|c|}
\hline Percentage & 5 & 10 & 25 & 50 & 75 & 90 & 95 \\
\hline Flow & 1,700 & 1,070 & 453 & 131 & 55 & 30 & 22 \\
\hline
\end{tabular}


STATION NAME AND NUMBER—02446000 Sispey River at Moores Bridge, AL

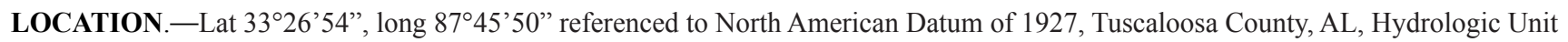
03160107, 1 mi east of Moores Bridge, and 6 mi downstream from Bear Creek.

DRAINAGE AREA. $-413 \mathrm{mi}^{2}$.

PERIOD OF RECORD.-February 1939 to September 1951.

PERIOD OF ANALYSIS.-April 1939 to March 1951.

\section{NUMBER OF CLIMATE YEARS IN FREQUENCY ANALYSIS. - 12}

REMARKS.-Frequency statistics were computed based on a MOVE.1 correlation with station 02446500 Sipsey River near Elrod, AL, which has a period of record from September 1928 to March 1932, October 1939 to September 1971, October 1978 to March 2014, which represents 69 climatic years.

\begin{tabular}{|c|c|c|c|c|}
\hline $\begin{array}{l}\text { Recurrence } \\
\text { intervals } \\
\text { (years) }\end{array}$ & \multicolumn{2}{|c|}{$\begin{array}{l}\text { Lowest average flow for } \\
\text { indicated number of } \\
\text { consecutive days } \\
\text { (cubic feet per second) }\end{array}$} & \multicolumn{2}{|c|}{$\begin{array}{l}\text { Time-sampling error } \\
\text { (in percent) }\end{array}$} \\
\hline & 1 & 7 & 1 & 7 \\
\hline 2 & 46 & 48 & 11 & 12 \\
\hline 5 & 30 & 31 & 11 & 11 \\
\hline 10 & 24 & 25 & 11 & 11 \\
\hline 20 & 19 & 20 & 14 & 14 \\
\hline 50 & 15 & 16 & 18 & 19 \\
\hline
\end{tabular}

\begin{tabular}{|c|c|c|c|c|c|c|c|}
\hline \multicolumn{8}{|c|}{ DURATION OF DAILY FLOW } \\
\hline \multicolumn{8}{|c|}{$\begin{array}{l}\text { Flow equaled or exceeded for indicated percentage of time } \\
\text { (cubic feet per second) }\end{array}$} \\
\hline Percentage & 5 & 10 & 25 & 50 & 75 & 90 & 95 \\
\hline Flow & 2,410 & 1,720 & 847 & 250 & 105 & 61 & 50 \\
\hline
\end{tabular}


STATION NAME AND NUMBER—02446500 Sispey River near Elrod, AL

LOCATION.-Lat $33^{\circ} 15^{\prime} 25^{\prime \prime}$, long $87^{\circ} 46^{\prime} 35^{\prime \prime}$ referenced to North American Datum of 1927, Tuscaloosa County, AL, Hydrologic Unit 03160107, on left bank at downstream side of bridge on State Highway 140, 0.2 mi upstream from Gulf, Mobile \& Ohio Railroad bridge, 1.0 mi east of Elrod, 2.0 mi downstream from Box Creek, and at mile 50.7.

DRAINAGE AREA. $-528 \mathrm{mi}^{2}$.

PERIOD OF RECORD.-September 1928 to March 1932, October 1939 to September 1971, October 1978 to March 2014.

PERIOD OF ANALYSIS.-April 1929 to March 1932, April 1940 to March 1971, April 1979 to March 2014.

NUMBER OF CLIMATE YEARS IN FREQUENCY ANALYSIS. - 69

\section{REMARKS.-}

\begin{tabular}{|c|c|c|c|c|}
\hline $\begin{array}{l}\text { Recurrence } \\
\text { intervals } \\
\text { (years) }\end{array}$ & \multicolumn{2}{|c|}{$\begin{array}{l}\text { Lowest average flow for } \\
\text { indicated number of } \\
\text { consecutive days } \\
\text { (cubic feet per second) }\end{array}$} & \multicolumn{2}{|c|}{$\begin{array}{l}\text { Time-sampling error } \\
\text { (in percent) }\end{array}$} \\
\hline & 1 & 7 & 1 & 7 \\
\hline 2 & 59 & 63 & 6 & 7 \\
\hline 5 & 38 & 40 & 8 & 7 \\
\hline 10 & 30 & 32 & 10 & 9 \\
\hline 20 & 24 & 26 & 12 & 12 \\
\hline 50 & 19 & 20 & 16 & 16 \\
\hline
\end{tabular}

\section{DURATION OF DAILY FLOW}

Flow equaled or exceeded for indicated percentage of time (cubic feet per second)

\begin{tabular}{|c|c|c|c|c|c|c|c|}
\hline Percentage & 5 & 10 & 25 & 50 & 75 & 90 & 95 \\
\hline Flow & 2,900 & 1,980 & 1,010 & 388 & 146 & 78 & 58 \\
\hline
\end{tabular}


STATION NAME AND NUMBER—02447000 Sispey River near Pleasant Ridge, AL

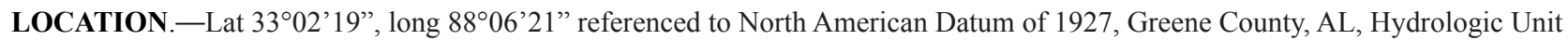
03160107, on State Highway 40, $450 \mathrm{ft}$ downstream from Hughes Creek, 2.5 mi northwest of Pleasant Ridge, 6 mi upstream from mouth, and 6 mi south of Aliceville.

DRAINAGE AREA. $-769 \mathrm{mi}^{2}$.

PERIOD OF RECORD.-February 1939 to September 1959.

PERIOD OF ANALYSIS.-April 1939 to March 1959.

NUMBER OF CLIMATE YEARS IN FREQUENCY ANALYSIS. - 20

REMARKS.-Frequency statistics were computed based on a MOVE.1 correlation with station 02446500 Sipsey River near Elrod, AL, which has a period of record from September 1928 to March 1932, October 1939 to September 1971, October 1978 to March 2014, which represents 69 climatic years.

\begin{tabular}{|c|c|c|c|c|}
\hline \multirow[t]{2}{*}{$\begin{array}{l}\text { Recurrence } \\
\text { intervals } \\
\text { (years) }\end{array}$} & \multicolumn{2}{|c|}{$\begin{array}{l}\text { Lowest average flow for } \\
\text { indicated number of } \\
\text { consecutive days } \\
\text { (cubic feet per second) }\end{array}$} & \multicolumn{2}{|c|}{$\begin{array}{l}\text { Time-sampling error } \\
\text { (in percent) }\end{array}$} \\
\hline & 1 & 7 & 1 & 7 \\
\hline 2 & 78 & 82 & 15 & 15 \\
\hline 5 & 48 & 51 & 18 & 17 \\
\hline 10 & 37 & 40 & 23 & 21 \\
\hline 20 & 29 & 32 & 29 & 27 \\
\hline 50 & 22 & 24 & 41 & 37 \\
\hline
\end{tabular}

\begin{tabular}{|c|c|c|c|c|c|c|c|c|}
\hline \multicolumn{1}{|c|}{ DURATION OF DAILY FLOW } \\
\hline \multicolumn{1}{|c|}{ Flow equaled or exceeded for indicated percentage of time } \\
(cubic feet per second) \\
\hline Percentage & 5 & 10 & 25 & 50 & 75 & 90 & 95 \\
\hline Flow & 3,840 & 2,740 & 1,500 & 480 & 166 & 87 & 62 \\
\hline
\end{tabular}


STATION NAME AND NUMBER—02447025 Tombigbee River at Heflin Lock and Dam near Gainesville, AL

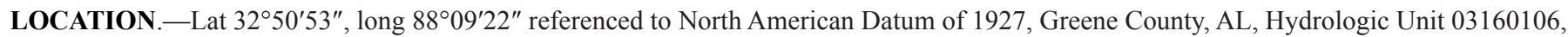
in $\mathrm{N}^{1} / 2$ sec. 36, T.22 N., R.2 W., on left bank end of dam, 1.0 mile downstream from Turkey Paw Branch, 1.8 miles north of Gainesville, 2.4 mi upstream from Noxubee River, and at river mile 238.6.

DRAINAGE AREA. - $7,230 \mathrm{mi}^{2}$.

PERIOD OF RECORD.-March 1978 to March 2014.

PERIOD OF ANALYSIS.-April 1985 to March 2014

NUMBER OF CLIMATE YEARS IN FREQUENCY ANALYSIS.—-29

REMARKS.-Period of record analyzed includes all current regulation structures. Records since January 16, 1985, include diversions from Tennessee River basin through Bay Springs lock on Tennessee-Tombigbee Waterway. Regulated by Jamie Whitten Lock and Dam (1983), G.V. Montgomery Lock and Dam (1984), John Ranking Lock and Dam (1984), Fulton Lock and Dam (1981), Glover Wilkins Lock and Dam (1981), Amory Lock and Dam (1979), Aberdeen Lock and Dam (1981), John C. Stennis Lock and Dam (1978), and Tom Bevill Lock and Dam (1978).

\begin{tabular}{|c|c|c|c|c|}
\hline $\begin{array}{c}\text { Recurrence } \\
\text { intervals } \\
\text { (years) }\end{array}$ & \multicolumn{2}{|c|}{$\begin{array}{c}\text { Lowest average flow for } \\
\text { indicated number of } \\
\text { consecutive days } \\
\text { (cubic feet per second) }\end{array}$} & \multicolumn{2}{|c|}{$\begin{array}{c}\text { Time-sampling error } \\
\text { (in percent) }\end{array}$} \\
\hline \multicolumn{7}{|c|}{1} & 7 & 1 & 7 \\
\hline 2 & 391 & 664 & 7 & 10 \\
\hline 5 & 285 & 455 & 9 & 12 \\
\hline 10 & 237 & 372 & 11 & 14 \\
\hline 20 & 201 & 315 & 15 & 9 \\
\hline
\end{tabular}

\begin{tabular}{|c|c|c|c|c|c|c|c|}
\hline \multicolumn{7}{|c|}{ DURATION OF DAILY FLOW } \\
\hline \multicolumn{7}{|c|}{ Flow equaled or exceeded for indicated percentage of time } \\
(cubic feet per second)
\end{tabular}


STATION NAME AND NUMBER—02448500 Noxubee River near Geiger, AL

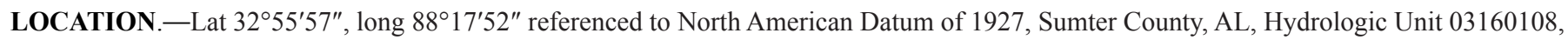
near right bank on downstream side of bridge on State Highway 17, 0.1 mi upstream from Woodward Creek, 2.1 mi upstream from St.LouisSan Francisco Railroad bridge, 5 mi north of Geiger, and at mile 16.9.

DRAINAGE AREA.-1,097 $\mathrm{mi}^{2}$.

PERIOD OF RECORD.-March 1939 to September 1940, August 1944 to September 1965, October 1966 to March 2014.

PERIOD OF ANALYSIS.-April 1939 to March 1940, April 1945 to March 1965, April 1967 to March 2014.

NUMBER OF CLIMATE YEARS IN FREQUENCY ANALYSIS. - 67

\section{REMARKS.-}

\begin{tabular}{|c|c|c|c|c|}
\hline $\begin{array}{c}\text { Recurrence } \\
\text { intervals } \\
\text { (years) }\end{array}$ & \multicolumn{2}{|c|}{$\begin{array}{l}\text { Lowest average flow for } \\
\text { indicated number of } \\
\text { consecutive days } \\
\text { (cubic feet per second) }\end{array}$} & \multicolumn{2}{|c|}{$\begin{array}{l}\text { Time-sampling error } \\
\text { (in percent) }\end{array}$} \\
\hline & 1 & 7 & 1 & 7 \\
\hline 2 & 65 & 69 & 5 & 6 \\
\hline 5 & 45 & 48 & 6 & 6 \\
\hline 10 & 37 & 39 & 8 & 8 \\
\hline 20 & 31 & 33 & 10 & 10 \\
\hline 50 & 25 & 27 & 14 & 13 \\
\hline
\end{tabular}

\section{DURATION OF DAILY FLOW}

Flow equaled or exceeded for indicated percentage of time (cubic feet per second)

\begin{tabular}{|c|c|c|c|c|c|c|c|}
\hline Percentage & 5 & 10 & 25 & 50 & 75 & 90 & 95 \\
\hline Flow & 7,460 & 4,910 & 1,510 & 359 & 130 & 78 & 63 \\
\hline
\end{tabular}


STATION NAME AND NUMBER—02448900 Bodka Creek near Geiger, AL

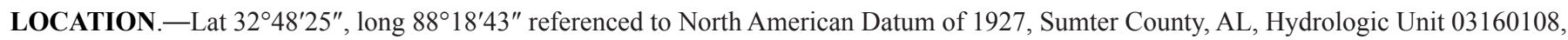
at right bank on downstream side of State Highway 17, 1.6 mi downstream from Tifallili Creek, 4.2 mi north of Geiger, and 9.2 mi upstream from mouth.

DRAINAGE AREA. $-158 \mathrm{mi}^{2}$.

PERIOD OF RECORD.- October 1990 to March 2014.

PERIOD OF ANALYSIS.-April 1991 to March 2014.

NUMBER OF CLIMATE YEARS IN FREQUENCY ANALYSIS.—-23

REMARKS.-Zero flows

\begin{tabular}{|c|c|c|c|c|}
\hline $\begin{array}{c}\text { Recurrence } \\
\text { intervals } \\
\text { (years) }\end{array}$ & \multicolumn{2}{|c|}{$\begin{array}{c}\text { Lowest average flow for } \\
\text { indicated number of } \\
\text { consecutive days } \\
\text { (cubic feet per second) }\end{array}$} & \multicolumn{2}{|c|}{$\begin{array}{c}\text { Time-sampling error } \\
\text { (in percent) }\end{array}$} \\
\hline \multicolumn{7}{|c|}{1} & 7 & 1 & 7 \\
\hline 2 & 0.01 & 0.05 & 33 & -- \\
\hline 5 & 0.00 & 0.00 & -- & -- \\
\hline 10 & 0.00 & 0.00 & -- & -- \\
\hline 20 & 0.00 & 0.00 & -- & \\
\hline
\end{tabular}

\begin{tabular}{|c|c|c|c|c|c|c|c|}
\hline \multicolumn{7}{|c|}{ DURATION OF DAILY FLOW } \\
\hline \multicolumn{7}{|c|}{ Flow equaled or exceeded for indicated percentage of time } \\
(cubic feet per second)
\end{tabular}


STATION NAME AND NUMBER—02449000 Tombigbee River at Gainseville, AL

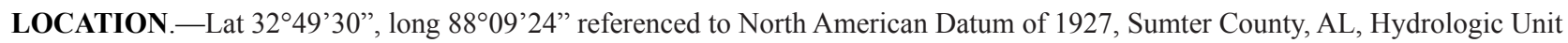
03160106, on State Highway 39 at Gainesville, 2 mi downstream from Noxubee River, and at mile 234.4.

DRAINAGE AREA. $-8,632 \mathrm{mi}^{2}$.

PERIOD OF RECORD.- October 1938 to September 1955, October 1960 to March 1978.

PERIOD OF ANALYSIS.-April 1939 to March 1955, April 1961 to March 1978.

\section{NUMBER OF CLIMATE YEARS IN FREQUENCY ANALYSIS. - 33}

REMARKS.-Period of record is for pre-regulation conditions. Currently regulated by Jamie Whitten Lock and Dam (1983), G.V. Montgomery Lock and Dam (1984), John Ranking Lock and Dam (1984), Fulton Lock and Dam (1981), Glover Wilkins Lock and Dam (1981), Amory Lock and Dam (1979), Aberdeen Lock and Dam (1981), John C. Stennis Lock and Dam (1978), Tom Bevill Lock and Dam (1978), and Howell Heflin Lock and Dam (1977). Since January 16, 1985, streamflows include diversions from Tennessee River basin through Bay Springs lock on Tennessee-Tombigbee Waterway.

\begin{tabular}{|c|c|c|c|c|}
\hline $\begin{array}{l}\text { Recurrence } \\
\text { intervals } \\
\text { (years) }\end{array}$ & \multicolumn{2}{|c|}{$\begin{array}{l}\text { Lowest average flow for } \\
\text { indicated number of } \\
\text { consecutive days } \\
\text { (cubic feet per second) }\end{array}$} & \multicolumn{2}{|c|}{$\begin{array}{l}\text { Time-sampling error } \\
\text { (in percent) }\end{array}$} \\
\hline & 1 & 7 & 1 & 7 \\
\hline 2 & 925 & 985 & 9 & 9 \\
\hline 5 & 619 & 646 & 11 & 11 \\
\hline 10 & 488 & 504 & 14 & 14 \\
\hline 20 & 395 & 405 & 18 & 19 \\
\hline 50 & 306 & 311 & 26 & 27 \\
\hline
\end{tabular}

\begin{tabular}{|c|c|c|c|c|c|c|c|}
\hline \multicolumn{1}{|c|}{ DURATION OF DAILY FLOW } \\
\hline \multicolumn{1}{|c|}{ Flow equaled or exceeded for indicated percentage of time } \\
(cubic feet per second)
\end{tabular}


STATION NAME AND NUMBER—02449245 Brush Creek near Eutaw, AL

LOCATION.-Lat 32 49'51”, long 8758'56” referenced to North American Datum of 1927, Greene County, AL, Hydrologic Unit 03160106, on county highway, 1.3 mi downstream from Pippan Creek, 2.2 mi upstream from Dry Creek, 5.5 mi west of Eutaw, and 7.2 mi upstream from mouth.

DRAINAGE AREA. $-43.2 \mathrm{mi}^{2}$.

PERIOD OF RECORD.- - June 1975 to September 1997.

PERIOD OF ANALYSIS.-April 1976 to March 1997.

NUMBER OF CLIMATE YEARS IN FREQUENCY ANALYSIS.-21

REMARKS.-

\begin{tabular}{|c|c|c|c|c|}
\hline $\begin{array}{c}\text { Recurrence } \\
\text { intervals } \\
\text { (years) }\end{array}$ & \multicolumn{2}{|c|}{$\begin{array}{c}\text { Lowest average flow for } \\
\text { indicated number of } \\
\text { consecutive days } \\
\text { (cubic feet per second) }\end{array}$} & \multicolumn{2}{|c|}{$\begin{array}{c}\text { Time-sampling error } \\
\text { (in percent) }\end{array}$} \\
\hline \multicolumn{7}{|c|}{1} & 7 & 1 & 7 \\
\hline 2 & 0.18 & 0.24 & 29 & 32 \\
\hline 5 & 0.06 & 0.08 & 33 & 39 \\
\hline 10 & 0.04 & 0.05 & 41 & 50 \\
\hline 20 & 0.02 & 0.03 & 53 & 32 \\
\hline
\end{tabular}

\begin{tabular}{|c|c|c|c|c|c|c|c|}
\hline \multicolumn{7}{|c|}{ DURATION OF DAILY FLOW } \\
\hline \multicolumn{7}{|c|}{ Flow equaled or exceeded for indicated percentage of time } \\
(cubic feet per second)
\end{tabular}


STATION NAME AND NUMBER—02449500 Tombigbee River at Epes, AL

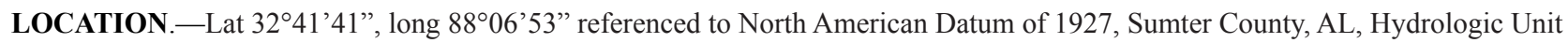
03160106, on U.S. Highway 11, 0.5 mi northeast of Epes, and 0.6 mi downstream from Jones and Factory Creeks.

DRAINAGE AREA. $-8,930 \mathrm{mi}^{2}$.

PERIOD OF RECORD._-January 1901 to December 1901, January 1905 and August 1913, October 1938 to September 1945.

PERIOD OF ANALYSIS.-April 1905 to March 1913, April 1939 to March 1945.

\section{NUMBER OF CLIMATE YEARS IN FREQUENCY ANALYSIS. - 14}

REMARKS.-Period of record is for pre-regulation conditions. Currently regulated by Jamie Whitten Lock and Dam (1983), G.V. Montgomery Lock and Dam (1984), John Ranking Lock and Dam (1984), Fulton Lock and Dam (1981), Glover Wilkins Lock and Dam (1981), Amory Lock and Dam (1979), Aberdeen Lock and Dam (1981), John C. Stennis Lock and Dam (1978), Tom Bevill Lock and Dam (1978), and Howell Heflin Lock and Dam (1977). Since January 16, 1985, streamflows include diversions from Tennessee River basin through Bay Springs lock on Tennessee-Tombigbee Waterway.

\begin{tabular}{|c|c|c|c|c|}
\hline $\begin{array}{l}\text { Recurrence } \\
\text { intervals } \\
\text { (years) }\end{array}$ & \multicolumn{2}{|c|}{$\begin{array}{l}\text { Lowest average flow for } \\
\text { indicated number of } \\
\text { consecutive days } \\
\text { (cubic feet per second) }\end{array}$} & \multicolumn{2}{|c|}{$\begin{array}{l}\text { Time-sampling error } \\
\text { (in percent) }\end{array}$} \\
\hline & 1 & 7 & 1 & 7 \\
\hline 2 & 679 & 700 & 10 & 11 \\
\hline 5 & 541 & 559 & 7 & 7 \\
\hline 10 & 493 & 512 & 8 & 8 \\
\hline 20 & 461 & 482 & 11 & 12 \\
\hline
\end{tabular}

\begin{tabular}{|c|c|c|c|c|c|c|c|}
\hline \multicolumn{7}{|c|}{ DURATION OF DAILY FLOW } \\
\hline \multicolumn{7}{|c|}{ Flow equaled or exceeded for indicated percentage of time } \\
(cubic feet per second) \\
\hline Percentage & 5 & 10 & 25 & 50 & 75 & 90 & 95 \\
\hline Flow & 39,600 & 31,300 & 13,100 & 4,010 & 1,620 & 921 & 770 \\
\hline
\end{tabular}


STATION NAME AND NUMBER—02449882 Blue Springs Creek near Blountsville, AL

LOCATION.-Lat 3404'47”, long 86³6'28” referenced to North American Datum of 1927, Blount County, AL, Hydrologic Unit 03160109, on left bank 300 feet above sewage treatment plant outfall, 1 mi southwest of Blountsville.

DRAINAGE AREA. $-13.0 \mathrm{mi}^{2}$.

PERIOD OF RECORD.-October 1992 to March 2014.

PERIOD OF ANALYSIS.-April 1993 to March 2014.

NUMBER OF CLIMATE YEARS IN FREQUENCY ANALYSIS.—-21

REMARKS.-

\begin{tabular}{|c|c|c|c|c|}
\hline $\begin{array}{c}\text { Recurrence } \\
\text { intervals } \\
\text { (years) }\end{array}$ & \multicolumn{2}{|c|}{$\begin{array}{c}\text { Lowest average flow for } \\
\text { indicated number of } \\
\text { consecutive days } \\
\text { (cubic feet per second) }\end{array}$} & \multicolumn{2}{|c|}{$\begin{array}{c}\text { Time-sampling error } \\
\text { (in percent) }\end{array}$} \\
\hline \multicolumn{7}{|c|}{1} & 7 & 1 & 7 \\
\hline 2 & 0.45 & 0.56 & 26 & 34 \\
\hline 5 & 0.10 & 0.18 & 33 & 46 \\
\hline 10 & 0.00 & 0.08 & -- & 66 \\
\hline 20 & 0.00 & 0.01 & -- & \\
\hline
\end{tabular}

\section{DURATION OF DAILY FLOW}

Flow equaled or exceeded for indicated percentage of time (cubic feet per second)

\begin{tabular}{|c|c|c|c|c|c|c|c|}
\hline Percentage & 5 & 10 & 25 & 50 & 75 & 90 & 95 \\
\hline Flow & 57 & 36 & 18 & 6.6 & 1.9 & 0.73 & 0.41 \\
\hline
\end{tabular}


STATION NAME AND NUMBER—02450000 Mulberry Fork near Garden City, AL

LOCATION.-Lat 3300'43", long 8644'12" referenced to North American Datum of 1927, Blount County, AL, Hydrologic Unit 03160109, on downstream side of bridge on county road, 0.7 mi east of Garden City 1.6 mi upstream of Louisville \& Nashville Railroad bridge, 3.7 mi downstream from Mud Creek.

DRAINAGE AREA. $-358 \mathrm{mi}^{2}$.

PERIOD OF RECORD.-October 1928 to March 2014.

PERIOD OF ANALYSIS.-April 1929 to March 1964.

NUMBER OF CLIMATE YEARS IN FREQUENCY ANALYSES.-35

REMARKS.- The QAQC analysis indicated differences in low-flow patterns between the earlier and latter periods of record. Thus, a separate frequency and duration analysis was done for the two periods. This period likely includes more natural conditions.

\begin{tabular}{|c|c|c|c|c|}
\hline $\begin{array}{l}\text { Recurrence } \\
\text { intervals } \\
\text { (years) }\end{array}$ & \multicolumn{2}{|c|}{$\begin{array}{l}\text { Lowest average flow for } \\
\text { indicated number of } \\
\text { consecutive days } \\
\text { (cubic feet per second) }\end{array}$} & \multicolumn{2}{|c|}{$\begin{array}{l}\text { Time-sampling error } \\
\text { (in percent) }\end{array}$} \\
\hline & 1 & 7 & 1 & 7 \\
\hline 2 & 7.6 & 8.5 & 10 & 11 \\
\hline 5 & 5.0 & 5.4 & 9 & 10 \\
\hline 10 & 4.1 & 4.4 & 10 & 11 \\
\hline 20 & 3.5 & 3.7 & 12 & 13 \\
\hline 50 & 3.0 & 3.1 & 16 & 17 \\
\hline
\end{tabular}

\begin{tabular}{|c|c|c|c|c|c|c|c|}
\hline \multicolumn{8}{|c|}{ DURATION OF DAILY FLOW } \\
\hline \multicolumn{8}{|c|}{$\begin{array}{l}\text { Flow equaled or exceeded for indicated percentage of time } \\
\text { (cubic feet per second) }\end{array}$} \\
\hline Percentage & 5 & 10 & 25 & 50 & 75 & 90 & 95 \\
\hline Flow & 2,430 & 1,520 & 700 & 201 & 44 & 13 & 9 \\
\hline
\end{tabular}


STATION NAME AND NUMBER—02450000 Mulberry Fork near Garden City, AL

LOCATION.-Lat 3300'43", long 8644'12" referenced to North American Datum of 1927, Blount County, AL, Hydrologic Unit 03160109, on downstream side of bridge on county road, $0.7 \mathrm{mi}$ east of Garden City $1.6 \mathrm{mi}$ upstream of Louisville \& Nashville Railroad bridge, 3.7 mi downstream from Mud Creek.

DRAINAGE AREA. $-358 \mathrm{mi}^{2}$.

PERIOD OF RECORD.-October 1928 to March 2014.

PERIOD OF ANALYSIS.-April 1965 to March 2014.

NUMBER OF CLIMATE YEARS IN FREQUENCY ANALYSES.-49

REMARKS.-The QAQC analysis indicated differences in low-flow patterns between the earlier and latter periods of record. Thus, a separate frequency and duration analysis was done for the two periods. This period likely includes anthropogenic influences.

\begin{tabular}{|c|c|c|c|c|}
\hline $\begin{array}{l}\text { Recurrence } \\
\text { intervals } \\
\text { (years) }\end{array}$ & \multicolumn{2}{|c|}{$\begin{array}{l}\text { Lowest average flow for } \\
\text { indicated number of } \\
\text { consecutive days } \\
\text { (cubic feet per second) }\end{array}$} & \multicolumn{2}{|c|}{$\begin{array}{l}\text { Time-sampling erro } \\
\text { (in percent) }\end{array}$} \\
\hline & 1 & 7 & 1 & 7 \\
\hline 2 & 16 & 19 & 8 & 9 \\
\hline 5 & 12 & 13 & 6 & 6 \\
\hline 10 & 10 & 11 & 6 & 7 \\
\hline 20 & 9.4 & 10 & 9 & 10 \\
\hline 50 & 8.7 & 9.6 & 13 & 15 \\
\hline
\end{tabular}

\begin{tabular}{|c|c|c|c|c|c|c|c|c|}
\hline \multicolumn{7}{|c|}{ DURATION OF DAILY FLOW } \\
\hline \multicolumn{7}{|c|}{ Flow equaled or exceeded for indicated percentage of time } \\
(cubic feet per second)
\end{tabular}


STATION NAME AND NUMBER—02450180 Mulberry Fork near Arkadelphia, AL

LOCATION.-Lat 3352'20", long 86 55'24" referenced to North American Datum of 1927, Cullman County, AL, Hydrologic Unit 03160109, on the upstream side of bridge, $4.1 \mathrm{mi}$ south of Arkadelphia, and at mile 58.6.

DRAINAGE AREA. $-487 \mathrm{mi}^{2}$.

PERIOD OF RECORD.- October 1976 to September 1986, October 1988 to March 2014.

PERIOD OF ANALYSIS.-April 1977 to March 1986, April 1989 to March 2014.

NUMBER OF CLIMATE YEARS IN FREQUENCY ANALYSIS.-34

REMARKS.-Likely influence from anthropogenic sources based on analysis from station 02450000.

\begin{tabular}{|c|c|c|c|c|}
\hline $\begin{array}{c}\text { Recurrence } \\
\text { intervals } \\
\text { (years) }\end{array}$ & \multicolumn{2}{|c|}{$\begin{array}{c}\text { Lowest average flow for } \\
\text { indicated number of } \\
\text { consecutive days } \\
\text { (cubic feet per second) }\end{array}$} & \multicolumn{2}{|c|}{$\begin{array}{c}\text { Time-sampling error } \\
\text { (in percent) }\end{array}$} \\
\hline & 1 & 7 & 1 & 7 \\
\hline 2 & 20 & 23 & 10 & 8 \\
\hline 5 & 14 & 16 & 8 & 9 \\
\hline 10 & 12 & 13 & 8 & 12 \\
\hline 20 & 11 & 12 & 11 & 17 \\
\hline 50 & 9.7 & 11 & 15 & 11 \\
\hline
\end{tabular}

\section{DURATION OF DAILY FLOW}

Flow equaled or exceeded for indicated percentage of time (cubic feet per second)

\begin{tabular}{|c|c|c|c|c|c|c|c|}
\hline Percentage & 5 & 10 & 25 & 50 & 75 & 90 & 95 \\
\hline Flow & 3,180 & 2,000 & 953 & 344 & 78 & 29 & 22 \\
\hline
\end{tabular}


STATION NAME AND NUMBER—02450250 Sipsey Fork near Grayson, AL

LOCATION.-Lat $34^{\circ} 17^{\prime} 07^{\prime \prime}$, long 87²3'56" referenced to North American Datum of 1927, Winston County, AL, Hydrologic Unit 03160110, Bankhead National Forest, at downstream side of bridge on Cranal Road, 0.5 mi downstream from Borden Creek, 4.5 mi west of Grayson, 14 mi northeast of Haleyville, and 64.1 miles upstream from mouth.

DRAINAGE AREA. $-92.1 \mathrm{mi}^{2}$.

PERIOD OF RECORD.- October 1966 to March 2014.

PERIOD OF ANALYSIS.-April 1967 to March 2014.

NUMBER OF CLIMATE YEARS IN FREQUENCY ANALYSIS.—47

\section{REMARKS.-}

\begin{tabular}{|c|c|c|c|c|}
\hline \multirow[t]{2}{*}{$\begin{array}{l}\text { Recurrence } \\
\text { intervals } \\
\text { (years) }\end{array}$} & \multicolumn{2}{|c|}{$\begin{array}{l}\text { Lowest average flow for } \\
\text { indicated number of } \\
\text { consecutive days } \\
\text { (cubic feet per second) }\end{array}$} & \multicolumn{2}{|c|}{$\begin{array}{l}\text { Time-sampling error } \\
\text { (in percent) }\end{array}$} \\
\hline & 1 & 7 & 1 & 7 \\
\hline 2 & 3.5 & 3.9 & 10 & 10 \\
\hline 5 & 2.0 & 2.3 & 12 & 11 \\
\hline 10 & 1.4 & 1.7 & 15 & 14 \\
\hline 20 & 1.1 & 1.3 & 20 & 17 \\
\hline 50 & 0.77 & 1.0 & 27 & 22 \\
\hline
\end{tabular}

\section{DURATION OF DAILY FLOW}

Flow equaled or exceeded for indicated percentage of time (cubic feet per second)

\begin{tabular}{|c|c|c|c|c|c|c|c|}
\hline Percentage & 5 & 10 & 25 & 50 & 75 & 90 & 95 \\
\hline Flow & 633 & 366 & 151 & 51 & 13 & 5.9 & 3.9 \\
\hline
\end{tabular}


STATION NAME AND NUMBER—02450500 Sipsey Fork near Falls City, AL

LOCATION.-Lat $34^{\circ} 03^{\prime} 07^{\prime \prime}$, long $87^{\circ} 16^{\prime} 01^{\prime \prime}$ referenced to North American Datum of 1927, Winston County, AL, Hydrologic Unit 03160110, 1.2 mi downstream from Brushy Creek, 1.8 mi north of Falls City, and 2.2 mi upstream from Clear Creek.

DRAINAGE AREA. $-360 \mathrm{mi}^{2}$.

PERIOD OF RECORD.- - June 1943 to December 1954.

PERIOD OF ANALYSIS.-April 1944 to March 1954.

\section{NUMBER OF CLIMATE YEARS IN FREQUENCY ANALYSIS.-10}

REMARKS.-Since 1961, site in backwater from Lewis Smith Dam.

\begin{tabular}{|c|c|c|c|c|}
\hline $\begin{array}{c}\text { Recurrence } \\
\text { intervals } \\
\text { (years) }\end{array}$ & \multicolumn{2}{|c|}{$\begin{array}{c}\text { Lowest average flow for } \\
\text { indicated number of } \\
\text { consecutive days } \\
\text { (cubic feet per second) }\end{array}$} & \multicolumn{2}{|c|}{$\begin{array}{c}\text { Time-sampling error } \\
\text { (in percent) }\end{array}$} \\
\hline \multicolumn{5}{|c|}{} \\
\hline
\end{tabular}

\section{DURATION OF DAILY FLOW}

Flow equaled or exceeded for indicated percentage of time (cubic feet per second)

\begin{tabular}{|c|c|c|c|c|c|c|c|}
\hline Percentage & 5 & 10 & 25 & 50 & 75 & 90 & 95 \\
\hline Flow & 2,320 & 1,440 & 571 & 142 & 47 & 23 & 17 \\
\hline
\end{tabular}


STATION NAME AND NUMBER—02450825 Clear Creek at New Hope Church near Poplar Springs, AL

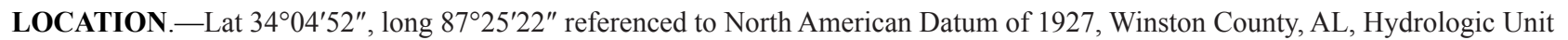
03160110, on upstream side of bridge on county road at New Hope Church, 4.5 mi northeast of Poplar Spring, and 6.1 mi southeast of Double Springs.

DRAINAGE AREA.- $-101 \mathrm{mi}^{2}$.

PERIOD OF RECORD.- October 1980 to September 1981, October 1993 to March 2014.

PERIOD OF ANALYSIS.-April 1994 to March 2014.

NUMBER OF CLIMATE YEARS IN FREQUENCY ANALYSIS.—-20

REMARKS.-

\begin{tabular}{|c|c|c|c|c|}
\hline $\begin{array}{l}\text { Recurrence } \\
\text { intervals } \\
\text { (years) }\end{array}$ & \multicolumn{2}{|c|}{$\begin{array}{l}\text { Lowest average flow for } \\
\text { indicated number of } \\
\text { consecutive days } \\
\text { (cubic feet per second) }\end{array}$} & \multicolumn{2}{|c|}{$\begin{array}{l}\text { Time-sampling error } \\
\text { (in percent) }\end{array}$} \\
\hline & 1 & 7 & 1 & 7 \\
\hline 2 & 19 & 21 & 12 & 12 \\
\hline 5 & 13 & 14 & 13 & 13 \\
\hline 10 & 10 & 11 & 16 & 16 \\
\hline 20 & 8.4 & 9.3 & 20 & 19 \\
\hline
\end{tabular}

\begin{tabular}{|c|c|c|c|c|c|c|c|}
\hline \multicolumn{8}{|c|}{ DURATION OF DAILY FLOW } \\
\hline \multicolumn{8}{|c|}{$\begin{array}{l}\text { Flow equaled or exceeded for indicated percentage of time } \\
\text { (cubic feet per second) }\end{array}$} \\
\hline Percentage & 5 & 10 & 25 & 50 & 75 & 90 & 95 \\
\hline Flow & 581 & 401 & 210 & 101 & 48 & 27 & 20 \\
\hline
\end{tabular}


STATION NAME AND NUMBER—02451000 Clear Creek at Falls City, AL

LOCATION.-Lat $34^{\circ} 02^{\prime} 05^{\prime \prime}$, long $87^{\circ} 18^{\prime} 00^{\prime \prime}$ referenced to North American Datum of 1927, Winston County, AL, Hydrologic Unit 03160110, $15 \mathrm{ft}$ downstream from highway bridge, 0.2 mi upstream from Clear Creek Falls, 0.5 mi south of Falls City, and 2 mi upstream from mouth.

DRAINAGE AREA.-149 $\mathrm{mi}^{2}$.

PERIOD OF RECORD.-October 1939 to November 1954.

PERIOD OF ANALYSIS.-April 1940 to March 1954.

NUMBER OF CLIMATE YEARS IN FREQUENCY ANALYSIS._-14

REMARKS.-Since 1961, site in backwater from Lewis Smith Dam.

\begin{tabular}{|c|c|c|c|c|}
\hline $\begin{array}{l}\text { Recurrence } \\
\text { intervals } \\
\text { (years) }\end{array}$ & \multicolumn{2}{|c|}{$\begin{array}{l}\text { Lowest average flow for } \\
\text { indicated number of } \\
\text { consecutive days } \\
\text { (cubic feet per second) }\end{array}$} & \multicolumn{2}{|c|}{$\begin{array}{l}\text { Time-sampling error } \\
\text { (in percent) }\end{array}$} \\
\hline & 1 & 7 & 1 & 7 \\
\hline 2 & 21 & 23 & 12 & 11 \\
\hline 5 & 15 & 17 & 10 & 10 \\
\hline 10 & 14 & 15 & 10 & 10 \\
\hline 20 & 12 & 14 & 13 & 12 \\
\hline
\end{tabular}

\begin{tabular}{|c|c|c|c|c|c|c|c|}
\hline \multicolumn{8}{|c|}{ DURATION OF DAILY FLOW } \\
\hline \multicolumn{8}{|c|}{$\begin{array}{l}\text { Flow equaled or exceeded for indicated percentage of time } \\
\text { (cubic feet per second) }\end{array}$} \\
\hline Percentage & 5 & 10 & 25 & 50 & 75 & 90 & 95 \\
\hline Flow & 881 & 525 & 250 & 96 & 44 & 27 & 23 \\
\hline
\end{tabular}


STATION NAME AND NUMBER—02453000 Blackwater Creek near Manchester, AL

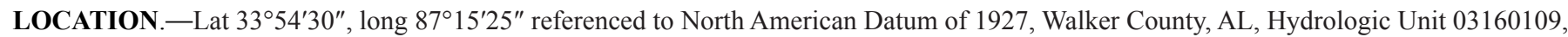
on right bank $100 \mathrm{ft}$ downstream from State Highway 257, 0.2 mi downstream from small unnamed tributary, 2 mi east of Manchester, and 5.5 mi north of Jasper.

DRAINAGE AREA. $-181 \mathrm{mi}^{2}$.

PERIOD OF RECORD.- October 1938 to September 1971, October 1979 to September 1982, October 1988 to March 2014.

PERIOD OF ANALYSIS.-April 1939 to March 1971.

NUMBER OF CLIMATE YEARS IN FREQUENCY ANALYSIS.-32

REMARKS.-The QAQC analysis indicated differences in low-flow patterns between the earlier and latter periods of record. Thus, a separate frequency and duration analysis was done for the two periods. This period likely represents a more natural condition.

\begin{tabular}{|c|c|c|c|c|}
\hline $\begin{array}{l}\text { Recurrence } \\
\text { intervals } \\
\text { (years) }\end{array}$ & \multicolumn{2}{|c|}{$\begin{array}{l}\text { Lowest average flow for } \\
\text { indicated number of } \\
\text { consecutive days } \\
\text { (cubic feet per second) }\end{array}$} & \multicolumn{2}{|c|}{$\begin{array}{l}\text { Time-sampling error } \\
\text { (in percent) }\end{array}$} \\
\hline & 1 & 7 & 1 & 7 \\
\hline 2 & 7.8 & 9.8 & 13 & 13 \\
\hline 5 & 4.3 & 5.4 & 15 & 16 \\
\hline 10 & 3.1 & 3.8 & 19 & 20 \\
\hline 20 & 2.3 & 2.8 & 23 & 27 \\
\hline 50 & 1.7 & 2.0 & 31 & 37 \\
\hline
\end{tabular}

\begin{tabular}{|c|c|c|c|c|c|c|c|c|}
\hline \multicolumn{7}{|c|}{ DURATION OF DAILY FLOW } \\
\hline \multicolumn{7}{|c|}{ Flow equaled or exceeded for indicated percentage of time } \\
(cubic feet per second)
\end{tabular}


STATION NAME AND NUMBER—02453000 Blackwater Creek near Manchester, AL

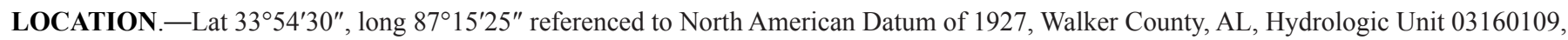
on right bank $100 \mathrm{ft}$ downstream from State Highway 257, 0.2 mi downstream from small unnamed tributary, 2 mi east of Manchester, and 5.5 mi north of Jasper.

DRAINAGE AREA.- $-181 \mathrm{mi}^{2}$.

PERIOD OF RECORD.-October 1938 to September 1971, October 1979 to September 1982, October 1988 to March 2014.

PERIOD OF ANALYSIS.-April 1980 to March 1982, April 1989 to March 2014.

NUMBER OF CLIMATE YEARS IN FREQUENCY ANALYSIS._-27

REMARKS.-The QAQC analysis indicated differences in low-flow patterns between the earlier and latter periods of record. Thus, a separate frequency and duration analysis was done for the two periods. This period likely includes anthropogenic influences.

\begin{tabular}{|c|c|c|c|c|}
\hline $\begin{array}{c}\text { Recurrence } \\
\text { intervals } \\
\text { (years) }\end{array}$ & \multicolumn{2}{|c|}{$\begin{array}{c}\text { Lowest average flow for } \\
\text { indicated number of } \\
\text { consecutive days } \\
\text { (cubic feet per second) }\end{array}$} & \multicolumn{2}{|c|}{$\begin{array}{c}\text { Time-sampling error } \\
\text { (in percent) }\end{array}$} \\
\hline \multicolumn{2}{|c|}{1} & 7 & 1 & 7 \\
\hline 2 & 12 & 15 & 19 & 20 \\
\hline 5 & 5.6 & 7.2 & 24 & 25 \\
\hline 10 & 3.4 & 4.9 & 32 & 33 \\
\hline 20 & 2.2 & 3.5 & 44 & \\
\hline
\end{tabular}

\begin{tabular}{|c|c|c|c|c|c|c|c|}
\hline \multicolumn{1}{|c|}{ DURATION OF DAILY FLOW } \\
\hline \multicolumn{1}{|c|}{ Flow equaled or exceeded for indicated percentage of time } \\
(cubic feet per second) \\
\hline Percentage & 5 & 10 & 25 & 50 & 75 & 90 & 95 \\
\hline Flow & 1,420 & 864 & 383 & 152 & 50 & 21 & 13 \\
\hline
\end{tabular}


STATION NAME AND NUMBER—02453500 Mulberry Fork at Cordova, AL

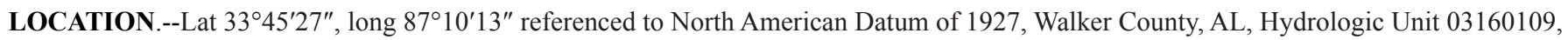
just downstream from Cane Creek, 1 mi east of Cordova, and 12 mi downstream from Sipsey Fork.

DRAINAGE AREA.-1,916 $\mathrm{mi}^{2}$.

PERIOD OF RECORD.- June 1900 to December 1912, November 2009 to September 2013.

PERIOD OF ANALYSIS.--April 1901 to March 1912.

NUMBER OF CLIMATE YEARS IN FREQUENCY ANALYSIS.-11

REMARKS.- - Since 1960, flow affected by Lewis Smith Lakeand Bankhead Lock and Dam on Black Warrior River. Duration of daily flow analysis represents data from June 1900 to December 1912.

\begin{tabular}{|c|c|c|c|c|}
\hline $\begin{array}{c}\text { Recurrence } \\
\text { intervals } \\
\text { (years) }\end{array}$ & \multicolumn{2}{|c|}{$\begin{array}{c}\text { Lowest average flow for } \\
\text { indicated number of } \\
\text { consecutive days } \\
\text { (cubic feet per second) }\end{array}$} & \multicolumn{2}{|c|}{$\begin{array}{c}\text { Time-sampling error } \\
\text { (in percent) }\end{array}$} \\
\hline \multicolumn{2}{|c|}{1} & 7 & 1 & 7 \\
\hline 2 & 74 & 87 & 23 & 24 \\
\hline 5 & 41 & 50 & 26 & 31 \\
\hline 10 & 29 & 37 & 33 & 40 \\
\hline 20 & 22 & 28 & 43 & \\
\hline
\end{tabular}

\begin{tabular}{|c|c|c|c|c|c|c|c|c|}
\hline \multicolumn{1}{|c|}{ DURATION OF DAILY FLOW } \\
\hline \multicolumn{7}{|c|}{ Flow equaled or exceeded for indicated percentage of time } \\
(cubic feet per second)
\end{tabular}


STATION NAME AND NUMBER—02454000 Lost Creek near Oakman, AL

LOCATION.-Lat $33^{\circ} 45^{\prime} 50^{\prime \prime}$, long $87^{\circ} 21^{\prime} 30^{\prime \prime}$ referenced to North American Datum of 1927, Walker County, AL, Hydrologic Unit 03160109, on State Highway 69, 0.2 mi upstream from Wolf Branch, 0.8 mi downstream from Pumpkin Creek, 4.0 mi northeast of Oakman, 6.5 mi southwest of Jasper, and at mile 24.8 .

DRAINAGE AREA. - $134 \mathrm{mi}^{2}$.

PERIOD OF RECORD.- October 1951 to September 1966, October 1979 to September 1981.

PERIOD OF ANALYSIS.-April 1952 to March 1966, April 1980 to March 1981.

NUMBER OF CLIMATE YEARS IN FREQUENCY ANALYSIS.—15

\section{REMARKS.-}

\begin{tabular}{|c|c|c|c|c|}
\hline $\begin{array}{c}\text { Recurrence } \\
\text { intervals } \\
\text { (years) }\end{array}$ & \multicolumn{2}{|c|}{$\begin{array}{c}\text { Lowest average flow for } \\
\text { indicated number of } \\
\text { consecutive days } \\
\text { (cubic feet per second) }\end{array}$} & \multicolumn{2}{|c|}{$\begin{array}{c}\text { Time-sampling error } \\
\text { (in percent) }\end{array}$} \\
\hline \multicolumn{7}{|c|}{1} & 7 & 1 & 79 \\
\hline 2 & 0.36 & 0.59 & 40 & 42 \\
\hline 5 & 0.09 & 0.16 & 37 & 50 \\
\hline 10 & 0.00 & 0.06 & -- & -- \\
\hline 20 & 0.00 & 0.00 & -- & 39 \\
\hline
\end{tabular}

\begin{tabular}{|c|c|c|c|c|c|c|c|}
\hline \multicolumn{7}{|c|}{ DURATION OF DAILY FLOW } \\
\hline \multicolumn{7}{|c|}{ Flow equaled or exceeded for indicated percentage of time } \\
(cubic feet per second)
\end{tabular}


STATION NAME AND NUMBER—02454055 Lost Creek above Parrish, AL

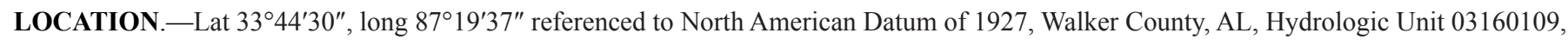
on downstream side of bridge on Browns Bridge Road, 1 mi north of County Road 20 (East), 3 mi northwest of Parrish, 3.4 mi west of Oakman, and $16.8 \mathrm{mi}$ above mouth.

DRAINAGE AREA. $-143 \mathrm{mi}^{2}$.

PERIOD OF RECORD.- October 1992 to March 2014.

PERIOD OF ANALYSIS.-April 1993 to March 2014.

NUMBER OF CLIMATE YEARS IN FREQUENCY ANALYSIS.—-21

REMARKS.-

\begin{tabular}{|c|c|c|c|c|}
\hline $\begin{array}{l}\text { Recurrence } \\
\text { intervals } \\
\text { (years) }\end{array}$ & \multicolumn{2}{|c|}{$\begin{array}{l}\text { Lowest average flow for } \\
\text { indicated number of } \\
\text { consecutive days } \\
\text { (cubic feet per second) }\end{array}$} & \multicolumn{2}{|c|}{$\begin{array}{l}\text { Time-sampling error } \\
\text { (in percent) }\end{array}$} \\
\hline & 1 & 7 & 1 & 7 \\
\hline 2 & 13 & 15 & 17 & 16 \\
\hline 5 & 7.0 & 8.5 & 20 & 19 \\
\hline 10 & 4.9 & 6.1 & 26 & 25 \\
\hline 20 & 3.6 & 4.5 & 34 & 33 \\
\hline
\end{tabular}

\begin{tabular}{|c|c|c|c|c|c|c|c|}
\hline \multicolumn{8}{|c|}{ DURATION OF DAILY FLOW } \\
\hline \multicolumn{8}{|c|}{$\begin{array}{l}\text { Flow equaled or exceeded for indicated percentage of time } \\
\text { (cubic feet per second) }\end{array}$} \\
\hline Percentage & 5 & 10 & 25 & 50 & 75 & 90 & 95 \\
\hline Flow & 977 & 576 & 281 & 111 & 39 & 18 & 12 \\
\hline
\end{tabular}


STATION NAME AND NUMBER—02455000 Locust Fork near Cleveland, AL

LOCATION.-Lat 3401'28", long 86³4'27" referenced to North American Datum of 1927, Blount County, AL, Hydrologic Unit 03160111, on left bank $200 \mathrm{ft}$ upstream from bridge on U.S. Highway 231, 2.5 mi downstream from Graves Creek, 3 mi north of Cleveland, and at mile 98.6.

DRAINAGE AREA. $-303 \mathrm{mi}^{2}$.

PERIOD OF RECORD.-December 1936 to September 1986, October 1992 to March 2014.

PERIOD OF ANALYSIS.-April 1937 to March 1986, April 1993 to March 2014.

NUMBER OF CLIMATE YEARS IN FREQUENCY ANALYSIS. - 70

REMARKS.-Possible flow influences from anthropogenic sources.

\begin{tabular}{|c|c|c|c|c|}
\hline $\begin{array}{c}\text { Recurrence } \\
\text { intervals } \\
\text { (years) }\end{array}$ & \multicolumn{2}{|c|}{$\begin{array}{l}\text { Lowest average flow for } \\
\text { indicated number of } \\
\text { consecutive days } \\
\text { (cubic feet per second) }\end{array}$} & \multicolumn{2}{|c|}{$\begin{array}{l}\text { Time-sampling error } \\
\text { (in percent) }\end{array}$} \\
\hline & 1 & 7 & 1 & 7 \\
\hline 2 & 11 & 12 & 8 & 8 \\
\hline 5 & 6.5 & 7.2 & 8 & 8 \\
\hline 10 & 5.1 & 5.6 & 9 & 9 \\
\hline 20 & 4.1 & 4.6 & 11 & 11 \\
\hline 50 & 3.3 & 3.7 & 15 & 14 \\
\hline
\end{tabular}

\section{DURATION OF DAILY FLOW}

Flow equaled or exceeded for indicated percentage of time (cubic feet per second)

\begin{tabular}{|c|c|c|c|c|c|c|c|}
\hline Percentage & 5 & 10 & 25 & 50 & 75 & 90 & 95 \\
\hline Flow & 2,000 & 1,260 & 583 & 190 & 42 & 17 & 12 \\
\hline
\end{tabular}


STATION NAME AND NUMBER—02455500 Locust Fork at Trafford, AL

LOCATION.-Lat 3349'49", long 8645'21" referenced to North American Datum of 1927, Jefferson County, AL, Hydrologic Unit 03160111, 0.8 mi northwest of Trafford, 1.5 mi east of Coaldale, 2.8 mi upstream from Gurley Creek, and at mile 67.4.

DRAINAGE AREA. $-624 \mathrm{mi}^{2}$.

PERIOD OF RECORD.-October 1930 to September 1969, October 1992 to September 1997.

PERIOD OF ANALYSIS.-April 1931 to March 1969, April 1993 to March 1997.

NUMBER OF CLIMATE YEARS IN FREQUENCY ANALYSIS.-42

REMARKS.-

\begin{tabular}{|c|c|c|c|c|}
\hline $\begin{array}{l}\text { Recurrence } \\
\text { intervals } \\
\text { (years) }\end{array}$ & \multicolumn{2}{|c|}{$\begin{array}{l}\text { Lowest average flow for } \\
\text { indicated number of } \\
\text { consecutive days } \\
\text { (cubic feet per second) }\end{array}$} & \multicolumn{2}{|c|}{$\begin{array}{l}\text { Time-sampling error } \\
\text { (in percent) }\end{array}$} \\
\hline & 1 & 7 & 1 & 7 \\
\hline 2 & 24 & 28 & 8 & 8 \\
\hline 5 & 16 & 19 & 9 & 9 \\
\hline 10 & 13 & 15 & 10 & 10 \\
\hline 20 & 11 & 13 & 13 & 12 \\
\hline 50 & 8.8 & 11 & 16 & 16 \\
\hline
\end{tabular}

\section{DURATION OF DAILY FLOW}

Flow equaled or exceeded for indicated percentage of time (cubic feet per second)

\begin{tabular}{|c|c|c|c|c|c|c|c|}
\hline Percentage & 5 & 10 & 25 & 50 & 75 & 90 & 95 \\
\hline Flow & 3,790 & 2,390 & 1,090 & 324 & 99 & 41 & 30 \\
\hline
\end{tabular}


STATION NAME AND NUMBER—02455980 Turkey Creek at Sewage Plant near Pinson, AL

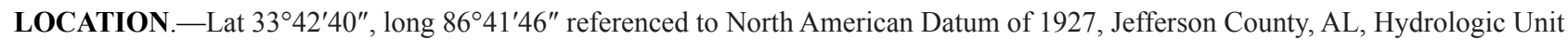
03160111, on left bank, $400 \mathrm{ft}$ upstream from Turkey Creek Wastewater Treatment Plant effluent, 1.8 mi northwest of Pinson, and 7 mi southeast of Morris.

DRAINAGE AREA.- $-27.4 \mathrm{mi}^{2}$.

PERIOD OF RECORD.- July 1988 to March 2014.

PERIOD OF ANALYSIS.-April 1989 to March 2014.

NUMBER OF CLIMATE YEARS IN FREQUENCY ANALYSIS.—-25

\section{REMARKS.-}

\begin{tabular}{|c|c|c|c|c|}
\hline $\begin{array}{l}\text { Recurrence } \\
\text { intervals } \\
\text { (years) }\end{array}$ & \multicolumn{2}{|c|}{$\begin{array}{l}\text { Lowest average flow for } \\
\text { indicated number of } \\
\text { consecutive days } \\
\text { (cubic feet per second) }\end{array}$} & \multicolumn{2}{|c|}{$\begin{array}{l}\text { Time-sampling error } \\
\text { (in percent) }\end{array}$} \\
\hline & 1 & 7 & 1 & 7 \\
\hline 2 & 12 & 13 & 6 & 7 \\
\hline 5 & 9.4 & 10 & 8 & 8 \\
\hline 10 & 8.1 & 8.5 & 10 & 11 \\
\hline 20 & 7.0 & 7.3 & 14 & 15 \\
\hline
\end{tabular}

\begin{tabular}{|c|c|c|c|c|c|c|c|}
\hline \multicolumn{8}{|c|}{ DURATION OF DAILY FLOW } \\
\hline \multicolumn{8}{|c|}{$\begin{array}{l}\text { Flow equaled or exceeded for indicated percentage of time } \\
\text { (cubic feet per second) }\end{array}$} \\
\hline Percentage & 5 & 10 & 25 & 50 & 75 & 90 & 95 \\
\hline Flow & 134 & 83 & 47 & 30 & 19 & 14 & 11 \\
\hline
\end{tabular}


STATION NAME AND NUMBER—02456000 Turkey Creek at Morris, AL

LOCATION.-Lat 3344'25", long 8648'45" referenced to North American Datum of 1927, Jefferson County, AL, Hydrologic Unit 03160111, on downstream side of pier of bridge on county road (former U.S. Highway 31), at Morris, 0.8 mi downstream from Cunningham Creek, at mile 4.0.

DRAINAGE AREA. $-80.9 \mathrm{mi}^{2}$.

PERIOD OF RECORD.- January 1944 to September 1979, March 2002 to October 2011.

PERIOD OF ANALYSIS.-April 1944 to March 1979, April 2002 to March 2011.

NUMBER OF CLIMATE YEARS IN FREQUENCY ANALYSIS.-42

REMARKS.-QAQC assessments indicate that the latter record (from around the early 1970s) is likely influenced to some unknown degree by upstream wastewater treatment plant discharges.

\begin{tabular}{|c|c|c|c|c|}
\hline $\begin{array}{l}\text { Recurrence } \\
\text { intervals } \\
\text { (years) }\end{array}$ & \multicolumn{2}{|c|}{$\begin{array}{l}\text { Lowest average flow for } \\
\text { indicated number of } \\
\text { consecutive days } \\
\text { (cubic feet per second) }\end{array}$} & \multicolumn{2}{|c|}{$\begin{array}{l}\text { Time-sampling error } \\
\text { (in percent) }\end{array}$} \\
\hline & 1 & 7 & 1 & 7 \\
\hline 2 & 13 & 15 & 5 & 5 \\
\hline 5 & 10 & 11 & 5 & 5 \\
\hline 10 & 9.3 & 10 & 6 & 5 \\
\hline 20 & 8.4 & 9.4 & 7 & 6 \\
\hline 50 & 7.6 & 8.6 & 9 & 8 \\
\hline
\end{tabular}

\begin{tabular}{|c|c|c|c|c|c|c|c|}
\hline \multicolumn{8}{|c|}{ DURATION OF DAILY FLOW } \\
\hline \multicolumn{8}{|c|}{$\begin{array}{l}\text { Flow equaled or exceeded for indicated percentage of time } \\
\text { (cubic feet per second) }\end{array}$} \\
\hline Percentage & 5 & 10 & 25 & 50 & 75 & 90 & 95 \\
\hline Flow & 451 & 270 & 132 & 55 & 25 & 17 & 14 \\
\hline
\end{tabular}


STATION NAME AND NUMBER—02456330 Crooked Creek near Morris, AL

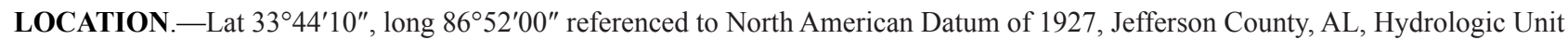
03160111, $100 \mathrm{ft}$ downstream from county road, $2 \mathrm{mi}$ southwest of Sardis, 3.2 mi west-southwest of Morris, and $3.6 \mathrm{mi}$ above mouth.

DRAINAGE AREA.- $-16.2 \mathrm{mi}^{2}$.

PERIOD OF RECORD.--November 1975 to September 1988, October 1996 to September 1997.

PERIOD OF ANALYSIS.-April 1976 to March 1988.

NUMBER OF CLIMATE YEARS IN FREQUENCY ANALYSIS.-12

REMARKS.-

\begin{tabular}{|c|c|c|c|c|}
\hline \multirow[t]{2}{*}{$\begin{array}{l}\text { Recurrence } \\
\text { intervals } \\
\text { (years) }\end{array}$} & \multicolumn{2}{|c|}{$\begin{array}{l}\text { Lowest average flow for } \\
\text { indicated number of } \\
\text { consecutive days } \\
\text { (cubic feet per second) }\end{array}$} & \multicolumn{2}{|c|}{$\begin{array}{l}\text { Time-sampling error } \\
\text { (in percent) }\end{array}$} \\
\hline & 1 & 7 & 1 & 7 \\
\hline 2 & 0.15 & 0.19 & 27 & 25 \\
\hline 5 & 0.08 & 0.10 & 27 & 25 \\
\hline 10 & 0.06 & 0.07 & 30 & 28 \\
\hline 20 & 0.04 & 0.06 & 37 & 33 \\
\hline
\end{tabular}

\section{DURATION OF DAILY FLOW}

Flow equaled or exceeded for indicated percentage of time (cubic feet per second)

\begin{tabular}{|c|c|c|c|c|c|c|c|}
\hline Percentage & 5 & 10 & 25 & 50 & 75 & 90 & 95 \\
\hline Flow & 98 & 54 & 24 & 6.5 & 0.92 & 0.31 & 0.17 \\
\hline
\end{tabular}


STATION NAME AND NUMBER—02456500 Locust Fork at Sayre, AL

LOCATION.-Lat 3342'35", long 86 59'00" referenced to North American Datum of 1927, Jefferson County, AL, Hydrologic Unit 03160111, on left bank $150 \mathrm{ft}$ upstream from bridge on county road at Sayre, $1.5 \mathrm{mi}$ downstream from Camp Creek, and at mile 33.9.

DRAINAGE AREA. $-885 \mathrm{mi}^{2}$.

PERIOD OF RECORD.- October 1928 to March 1932, October 1941 to March 2014.

PERIOD OF ANALYSIS.--April 1964 to March 2014.

\section{NUMBER OF CLIMATE YEARS IN FREQUENCY ANALYSIS. - 50}

REMARKS.-From QAQC assessments, analysis period established based on most recent period of relatively similar flow patterns (possible influence from upstream anthropogenic sources).

\begin{tabular}{|c|c|c|c|c|}
\hline $\begin{array}{c}\text { Recurrence } \\
\text { intervals } \\
\text { (years) }\end{array}$ & \multicolumn{2}{|c|}{$\begin{array}{c}\text { Lowest average flow for } \\
\text { indicated number of } \\
\text { consecutive days } \\
\text { (cubic feet per second) }\end{array}$} & \multicolumn{2}{|c|}{$\begin{array}{c}\text { Time-sampling error } \\
\text { (in percent) }\end{array}$} \\
\hline \multicolumn{7}{|c|}{1} & 7 & 1 & 7 \\
\hline 2 & 63 & 70 & 7 & 8 \\
\hline 5 & 43 & 47 & 8 & 9 \\
\hline 10 & 35 & 38 & 9 & 12 \\
\hline 20 & 29 & 32 & 12 & 15 \\
\hline 50 & 24 & 26 & 15 & 7 \\
\hline
\end{tabular}

\section{DURATION OF DAILY FLOW}

Flow equaled or exceeded for indicated percentage of time (cubic feet per second)

\begin{tabular}{|c|c|c|c|c|c|c|c|}
\hline Percentage & 5 & 10 & 25 & 50 & 75 & 90 & 95 \\
\hline Flow & 5,560 & 3,500 & 1,650 & 610 & 188 & 92 & 69 \\
\hline
\end{tabular}


STATION NAME AND NUMBER—02457000 Fivemile Creek at Ketona, AL

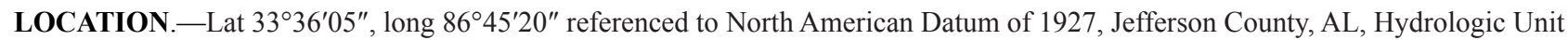
03160111, near center of stream on downstream side of foot bridge at Ketona, 0.6 mi downstream from Barton Branch, 0.9 mi downstream from Tarrant Spring Branch, 2 mi north of Tarrant City, and at mile 35.2.

DRAINAGE AREA.-23.9 $\mathrm{mi}^{2}$.

PERIOD OF RECORD.- October 1953 to September 1958, December 1974 to September 1979, May 1996 to March 2014.

PERIOD OF ANALYSIS.-April 1954 to March 1958, April 1975 to March 1979, April 1997 to March 2014.

NUMBER OF CLIMATE YEARS IN FREQUENCY ANALYSIS. - 25

\section{REMARKS.-}

\begin{tabular}{|c|c|c|c|c|}
\hline $\begin{array}{l}\text { Recurrence } \\
\text { intervals } \\
\text { (years) }\end{array}$ & \multicolumn{2}{|c|}{$\begin{array}{l}\text { Lowest average flow for } \\
\text { indicated number of } \\
\text { consecutive days } \\
\text { (cubic feet per second) }\end{array}$} & \multicolumn{2}{|c|}{$\begin{array}{l}\text { Time-sampling error } \\
\text { (in percent) }\end{array}$} \\
\hline & 1 & 7 & 1 & 7 \\
\hline 2 & 7.3 & 7.7 & 7 & 7 \\
\hline 5 & 5.5 & 5.8 & 9 & 9 \\
\hline 10 & 4.6 & 4.8 & 12 & 12 \\
\hline 20 & 3.9 & 4.1 & 16 & 16 \\
\hline
\end{tabular}

\begin{tabular}{|c|c|c|c|c|c|c|c|}
\hline \multicolumn{8}{|c|}{ DURATION OF DAILY FLOW } \\
\hline \multicolumn{8}{|c|}{$\begin{array}{l}\text { Flow equaled or exceeded for indicated percentage of time } \\
\text { (cubic feet per second) }\end{array}$} \\
\hline Percentage & 5 & 10 & 25 & 50 & 75 & 90 & 95 \\
\hline Flow & 114 & 65 & 34 & 19 & 11 & 8.0 & 6.8 \\
\hline
\end{tabular}


STATION NAME AND NUMBER—02457595 Fivemile Creek near Republic, AL

LOCATION.-Lat 333' 49", long 8652’05” referenced to North American Datum of 1927, Jefferson County, AL, Hydrologic Unit 03160111, on left bank 1,000 ft upstream from Fivemile Creek Wastewater Treatment Plant, 1.1 mi downstream from Coalburg Road bridge, 1.2 mi southeast of Republic, and at mile 24.8 .

DRAINAGE AREA. $-51.9 \mathrm{mi}^{2}$.

PERIOD OF RECORD.--May 1988 to March 2014.

PERIOD OF ANALYSIS.-April 1989 to March 2014.

NUMBER OF CLIMATE YEARS IN FREQUENCY ANALYSIS.—-25

REMARKS.-

\begin{tabular}{|c|c|c|c|c|}
\hline $\begin{array}{l}\text { Recurrence } \\
\text { intervals } \\
\text { (years) }\end{array}$ & \multicolumn{2}{|c|}{$\begin{array}{l}\text { Lowest average flow for } \\
\text { indicated number of } \\
\text { consecutive days } \\
\text { (cubic feet per second) }\end{array}$} & \multicolumn{2}{|c|}{$\begin{array}{l}\text { Time-sampling error } \\
\text { (in percent) }\end{array}$} \\
\hline & 1 & 7 & 1 & 7 \\
\hline 2 & 18 & 20 & 4 & 4 \\
\hline 5 & 15 & 17 & 4 & 4 \\
\hline 10 & 14 & 15 & 5 & 5 \\
\hline 20 & 13 & 14 & 6 & 6 \\
\hline
\end{tabular}

\begin{tabular}{|c|c|c|c|c|c|c|c|}
\hline \multicolumn{7}{|c|}{ DURATION OF DAILY FLOW } \\
\hline \multicolumn{7}{|c|}{ Flow equaled or exceeded for indicated percentage of time } \\
(cubic feet per second)
\end{tabular}


STATION NAME AND NUMBER-02458300 Village Creek at $24^{\text {th }}$ Street at Birmingham, AL

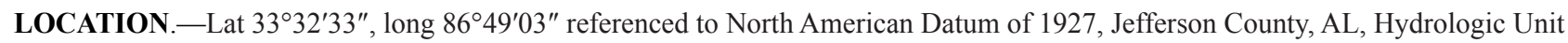
03160111, on right upstream wingwall of 24th Street bridge in Birmingham, and at mile 33.0.

DRAINAGE AREA. $-26.0 \mathrm{mi}^{2}$.

PERIOD OF RECORD._-June 1988 to December 2006, July 2008 to September 2013.

PERIOD OF ANALYSIS.-April 1989 to March 2006, April 2009 to March 2013.

NUMBER OF CLIMATE YEARS IN FREQUENCY ANALYSIS.-21

REMARKS.-Low and medium stages may be influenced by upstream plants and mills.

\begin{tabular}{|c|c|c|c|c|}
\hline $\begin{array}{l}\text { Recurrence } \\
\text { intervals } \\
\text { (years) }\end{array}$ & \multicolumn{2}{|c|}{$\begin{array}{l}\text { Lowest average flow for } \\
\text { indicated number of } \\
\text { consecutive days } \\
\text { (cubic feet per second) }\end{array}$} & \multicolumn{2}{|c|}{$\begin{array}{l}\text { Time-sampling error } \\
\text { (in percent) }\end{array}$} \\
\hline & 1 & 7 & 1 & 7 \\
\hline 2 & 9.0 & 9.8 & 5 & 6 \\
\hline 5 & 7.9 & 8.8 & 3 & 3 \\
\hline 10 & 7.6 & 8.6 & 4 & 5 \\
\hline 20 & 7.3 & 8.4 & 6 & 9 \\
\hline
\end{tabular}

\section{DURATION OF DAILY FLOW}

Flow equaled or exceeded for indicated percentage of time (cubic feet per second)

\begin{tabular}{|c|c|c|c|c|c|c|c|}
\hline Percentage & 5 & 10 & 25 & 50 & 75 & 90 & 95 \\
\hline Flow & 201 & 109 & 43 & 24 & 16 & 12 & 11 \\
\hline
\end{tabular}


STATION NAME AND NUMBER—02458450 Village Creek at Ave. W at Ensley, AL

LOCATION.-Lat 3331'03", long 86 52'45" referenced to North American Datum of 1927, Jefferson County, AL, Hydrologic Unit 03160111, near left bank on downstream side of Ave. W bridge over Village Creek, 5.7 mi upstream of Bay View Lake, 0.55 mi north of Interstate 59 in Ensley, and at mile 28.7.

DRAINAGE AREA. $-33.5 \mathrm{mi}^{2}$.

PERIOD OF RECORD.- July 1975 to September 1979, July 1988 to March 2014.

PERIOD OF ANALYSIS.-April 1976 to March 1979, April 1989 to March 2014.

NUMBER OF CLIMATE YEARS IN FREQUENCY ANALYSIS.—-28

REMARKS.- - Low and medium stages may be influenced by upstream plants and mills.

\begin{tabular}{|c|c|c|c|c|}
\hline $\begin{array}{c}\text { Recurrence } \\
\text { intervals } \\
\text { (years) }\end{array}$ & \multicolumn{2}{|c|}{$\begin{array}{c}\text { Lowest average flow for } \\
\text { indicated number of } \\
\text { consecutive days } \\
\text { (cubic feet per second) }\end{array}$} & \multicolumn{2}{|c|}{$\begin{array}{c}\text { Time-sampling error } \\
\text { (in percent) }\end{array}$} \\
\hline \multicolumn{7}{|c|}{} & 1 & 7 & 1 & 7 \\
\hline 2 & 15 & 18 & 6 & 6 \\
\hline 5 & 12 & 14 & 7 & 8 \\
\hline 10 & 10 & 12 & 9 & 9 \\
\hline 20 & 9.0 & 11 & 12 & 6 \\
\hline
\end{tabular}

\begin{tabular}{|c|c|c|c|c|c|c|c|c|}
\hline \multicolumn{7}{|c|}{ DURATION OF DAILY FLOW } \\
\hline \multicolumn{7}{|c|}{ Flow equaled or exceeded for indicated percentage of time } \\
(cubic feet per second)
\end{tabular}


STATION NAME AND NUMBER—02458600 Village Creek near Docena, AL

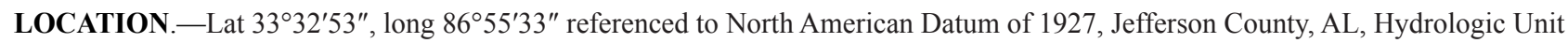
03160111, near right bank on downstream side of bridge on Minor Parkway, 0.5 mi south of Docena, and at mile 23.0.

DRAINAGE AREA. $-52.2 \mathrm{mi}^{2}$.

PERIOD OF RECORD.- - June 1996 to March 2014.

PERIOD OF ANALYSIS.-April 1997 to March 2014.

NUMBER OF CLIMATE YEARS IN FREQUENCY ANALYSIS. - 17

REMARKS.-QAQC reviews indicate that flow affected by dam at Bayview Lake, which is likely backwater issues. Duration curve comparison with stations 02458300 and 02458450 show this station has more flow per square mile for lower flows.

\begin{tabular}{|c|c|c|c|c|}
\hline $\begin{array}{c}\text { Recurrence } \\
\text { intervals } \\
\text { (years) }\end{array}$ & \multicolumn{2}{|c|}{$\begin{array}{l}\text { Lowest average flow for } \\
\text { indicated number of } \\
\text { consecutive days } \\
\text { (cubic feet per second) }\end{array}$} & \multicolumn{2}{|c|}{$\begin{array}{l}\text { Time-sampling error } \\
\text { (in percent) }\end{array}$} \\
\hline & 1 & 7 & 1 & 7 \\
\hline 2 & 45 & 52 & 10 & 9 \\
\hline 5 & 32 & 38 & 12 & 11 \\
\hline 10 & 27 & 32 & 15 & 15 \\
\hline 20 & 23 & 27 & 18 & 19 \\
\hline
\end{tabular}

\begin{tabular}{|c|c|c|c|c|c|c|c|}
\hline \multicolumn{8}{|c|}{ DURATION OF DAILY FLOW } \\
\hline \multicolumn{8}{|c|}{$\begin{array}{l}\text { Flow equaled or exceeded for indicated percentage of time } \\
\text { (cubic feet per second) }\end{array}$} \\
\hline Percentage & 5 & 10 & 25 & 50 & 75 & 90 & 95 \\
\hline Flow & 450 & 286 & 148 & 99 & 74 & 55 & 45 \\
\hline
\end{tabular}


STATION NAME AND NUMBER—02461500 Valley Creek near Bessemer, AL

LOCATION.-Lat 3325'09", long 86 $58^{\prime} 58^{\prime \prime}$ referenced to North American Datum of 1927, Jefferson County, AL, Hydrologic Unit 03160112, near left bank on downstream side of 19th Street bridge, 1.0 mi downstream from Opossum Creek, 2.0 mi west of Bessemer, and at mile 41.38 .

DRAINAGE AREA. $-52.5 \mathrm{mi}^{2}$.

PERIOD OF RECORD.--May 1975 to September 1979, May 1988 to March 2014.

PERIOD OF ANALYSIS.-April 1976 to March 1979, April 1989 to March 2014.

NUMBER OF CLIMATE YEARS IN FREQUENCY ANALYSIS.—-28

REMARKS.- - Low streamflows likely influenced by municipal sewage and industrial wastes with much of the discharge being water diverted from the Cahaba River and Blackborne Fork and from other sources.

\begin{tabular}{|c|c|c|c|c|}
\hline $\begin{array}{c}\text { Recurrence } \\
\text { intervals } \\
\text { (years) }\end{array}$ & \multicolumn{2}{|c|}{$\begin{array}{c}\text { Lowest average flow for } \\
\text { indicated number of } \\
\text { consecutive days } \\
\text { (cubic feet per second) }\end{array}$} & \multicolumn{2}{|c|}{$\begin{array}{c}\text { Time-sampling error } \\
\text { (in percent) }\end{array}$} \\
\hline \multicolumn{2}{|c|}{1} & 7 & 1 & 5 \\
\hline 2 & 31 & 37 & 5 & 3 \\
\hline 5 & 27 & 32 & 4 & 4 \\
\hline 10 & 25 & 30 & 4 & 5 \\
\hline 20 & 24 & 29 & 5 & 5 \\
\hline
\end{tabular}

\begin{tabular}{|c|c|c|c|c|c|c|c|}
\hline \multicolumn{1}{|c|}{ DURATION OF DAILY FLOW } \\
\hline \multicolumn{7}{|c|}{ Flow equaled or exceeded for indicated percentage of time } \\
(cubic feet per second)
\end{tabular}


STATION NAME AND NUMBER—02462000 Valley Creek near Oak Grove, AL

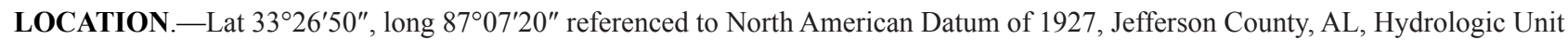
03160112, near center of span on downstream side of highway bridge, 1,000 ft downstream from Raccoon Branch, 1.5 mi east of Oak Grove, $10.5 \mathrm{mi}$ west of Bessemer, and $18.2 \mathrm{mi}$ upstream from mouth.

DRAINAGE AREA. $-148 \mathrm{mi}^{2}$.

PERIOD OF RECORD.- October 1953 to September 1958, October 1964 to September 1965, June 1978 to March 2014.

PERIOD OF ANALYSIS.-April 1954 to March 1958, April 1979 to March 2014.

NUMBER OF CLIMATE YEARS IN FREQUENCY ANALYSIS.—39

REMARKS.- - Low streamflows likely influenced by municipal sewage and industrial wastes with much of the discharge being water diverted from the Cahaba River and Blackborne Fork and from other sources.

\begin{tabular}{|c|c|c|c|c|}
\hline $\begin{array}{l}\text { Recurrence } \\
\text { intervals } \\
\text { (years) }\end{array}$ & \multicolumn{2}{|c|}{$\begin{array}{l}\text { Lowest average flow for } \\
\text { indicated number of } \\
\text { consecutive days } \\
\text { (cubic feet per second) }\end{array}$} & \multicolumn{2}{|c|}{$\begin{array}{l}\text { Time-sampling error } \\
\text { (in percent) }\end{array}$} \\
\hline & 1 & 7 & 1 & 7 \\
\hline 2 & 72 & 79 & 5 & 4 \\
\hline 5 & 56 & 65 & 6 & 5 \\
\hline 10 & 48 & 58 & 8 & 6 \\
\hline 20 & 42 & 52 & 12 & 8 \\
\hline 50 & 35 & 46 & 17 & 11 \\
\hline
\end{tabular}

\begin{tabular}{|c|c|c|c|c|c|c|c|}
\hline \multicolumn{8}{|c|}{ DURATION OF DAILY FLOW } \\
\hline \multicolumn{8}{|c|}{$\begin{array}{l}\text { Flow equaled or exceeded for indicated percentage of time } \\
\text { (cubic feet per second) }\end{array}$} \\
\hline Percentage & 5 & 10 & 25 & 50 & 75 & 90 & 95 \\
\hline Flow & 885 & 594 & 330 & 183 & 116 & 89 & 79 \\
\hline
\end{tabular}


STATION NAME AND NUMBER—02462500 Black Warrior River at Bankhead Lock and Dam near Bessemer, AL

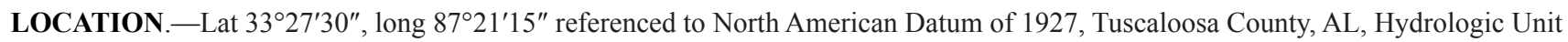
03160112, at abandoned lock wall $300 \mathrm{ft}$ above dam, 1.9 mi downstream from Big Yellow Creek, 23 mi northwest of Bessemer, and at mile 153.6.

DRAINAGE AREA.-3,981 $\mathrm{mi}^{2}$.

PERIOD OF RECORD.- October 1928 to September 1936, October 1976 to February 2014.

PERIOD OF ANALYSIS.-April 1977 to March 2012 (flow duration October 1976 to February 2014).

REMARKS.-Flows regulated since 1961 by Lewis Smith Lake. Regulation patterns were such that the data were not adequately represented by the log Pearson Type III distribution. Therefore, no frequency analysis was done.

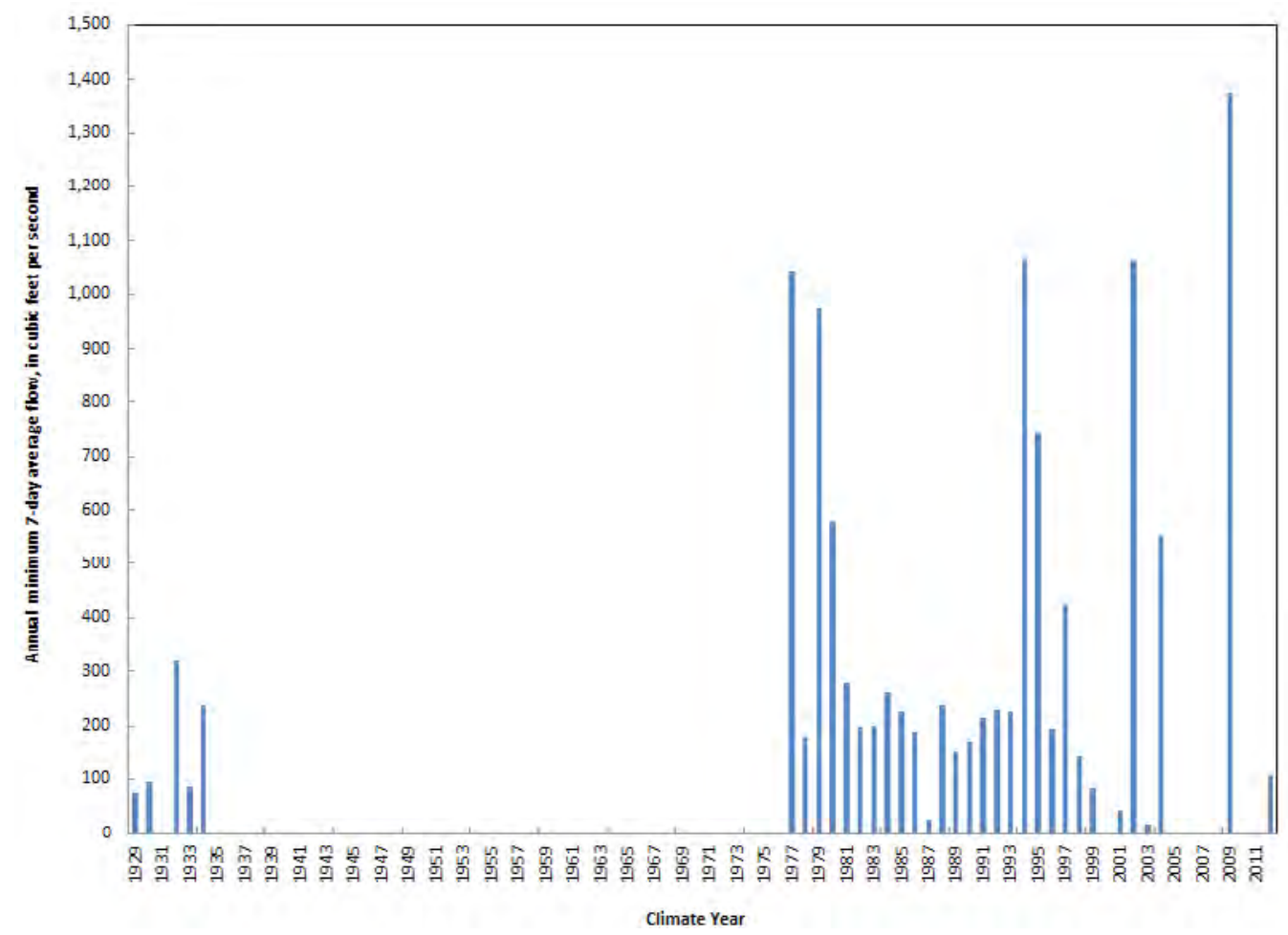

\begin{tabular}{|c|c|c|c|c|c|c|c|c|c|}
\hline \multicolumn{1}{|c|}{ EXCEEDANCE PERCENTILES OF ANNUAL MINIMUM 7-DAY AVERAGE FLOWS } \\
\hline Annual minimum 7-day average flow exceeded for indicated percentiles (cubic feet per second) \\
\hline Percentile & 10 & 20 & 30 & 40 & 50 & 60 & 70 & 80 & 90 \\
\hline Flow & 1,050 & 568 & 259 & 226 & 196 & 167 & 86 & 6.5 & 0 \\
\hline
\end{tabular}

\begin{tabular}{|c|c|c|c|c|c|c|c|}
\hline \multicolumn{1}{|c|}{ DURATION OF DAILY FLOW } \\
\hline \multicolumn{7}{|c|}{ Flow equaled or exceeded for indicated percentage of time } \\
(cubic feet per second) \\
\hline Percentage & 5 & 10 & 25 & 50 & 75 & 90 & 95 \\
\hline Flow & 23,300 & 14,600 & 8,300 & 3,200 & 1,380 & 240 & 0 \\
\hline
\end{tabular}


STATION NAME AND NUMBER—02462600 Blue Creek near Oakman, AL

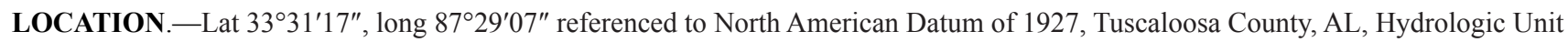
03160112, on State Highway 69, 1.5 mi southwest of Wiley, 2 mi upstream from McDuff Spring Branch, 12.6 mi upstream from mouth, and 14 mi southwest of Oakman.

DRAINAGE AREA. $-5.32 \mathrm{mi}^{2}$.

PERIOD OF RECORD.- June 1959 to September 1965, October 1976 to September 1984.

PERIOD OF ANALYSIS.-April 1960 to March 1965, April 1977 to March 1984.

NUMBER OF CLIMATE YEARS IN FREQUENCY ANALYSIS.-12

REMARKS.-

\begin{tabular}{|c|c|c|c|c|}
\hline $\begin{array}{c}\text { Recurrence } \\
\text { intervals } \\
\text { (years) }\end{array}$ & \multicolumn{2}{|c|}{$\begin{array}{c}\text { Lowest average flow for } \\
\text { indicated number of } \\
\text { consecutive days } \\
\text { (cubic feet per second) }\end{array}$} & \multicolumn{2}{|c|}{$\begin{array}{c}\text { Time-sampling error } \\
\text { (in percent) }\end{array}$} \\
\hline \multicolumn{7}{|c|}{1} & 7 & 1 & 7 \\
\hline 2 & 0.00 & 0.00 & -- & -- \\
\hline 5 & 0.00 & 0.00 & -- & -- \\
\hline 10 & 0.00 & 0.00 & -- & -- \\
\hline 20 & 0.00 & 0.00 & -- & - \\
\hline
\end{tabular}

\begin{tabular}{|c|c|c|c|c|c|c|c|c|}
\hline \multicolumn{7}{|c|}{ DURATION OF DAILY FLOW } \\
\hline \multicolumn{7}{|c|}{ Flow equaled or exceeded for indicated percentage of time } \\
(cubic feet per second)
\end{tabular}


STATION NAME AND NUMBER—02462800 Davis Creek below Abernant, AL

LOCATION.-Lat $33^{\circ} 18^{\prime} 30^{\prime \prime}$, long $87^{\circ} 13^{\prime} 10^{\prime \prime}$ referenced to North American Datum of 1927, Tuscaloosa County, AL, Hydrologic Unit 03160112, on county road, 0.2 mi downstream from Lye Branch, 0.6 mi downstream from Texas Creek, 2 mi northwest of Abernant, and 2.8 mi downstream from Rockcastle Creek.

DRAINAGE AREA. $-45.3 \mathrm{mi}^{2}$.

PERIOD OF RECORD.- October 1956 to September 1971.

PERIOD OF ANALYSIS.-April 1957 to March 1971.

NUMBER OF CLIMATE YEARS IN FREQUENCY ANALYSIS.-14

REMARKS.-

\begin{tabular}{|c|c|c|c|c|}
\hline $\begin{array}{c}\text { Recurrence } \\
\text { intervals } \\
\text { (years) }\end{array}$ & \multicolumn{2}{|c|}{$\begin{array}{c}\text { Lowest average flow for } \\
\text { indicated number of } \\
\text { consecutive days } \\
\text { (cubic feet per second) }\end{array}$} & \multicolumn{2}{|c|}{$\begin{array}{c}\text { Time-sampling error } \\
\text { (in percent) }\end{array}$} \\
\hline \multicolumn{7}{|c|}{1} & 7 & 1 & 7 \\
\hline 2 & 0.64 & 0.90 & 22 & 29 \\
\hline 5 & 0.33 & 0.38 & 21 & 36 \\
\hline 10 & 0.21 & 0.23 & 23 & 48 \\
\hline 20 & 0.00 & 0.14 & -- & 68 \\
\hline
\end{tabular}

\begin{tabular}{|c|c|c|c|c|c|c|c|}
\hline \multicolumn{7}{|c|}{ DURATION OF DAILY FLOW } \\
\hline \multicolumn{7}{|c|}{ Flow equaled or exceeded for indicated percentage of time } \\
(cubic feet per second)
\end{tabular}


STATION NAME AND NUMBER—02462951 Black Warrior River at Holt Lock and Dam near Holt, AL

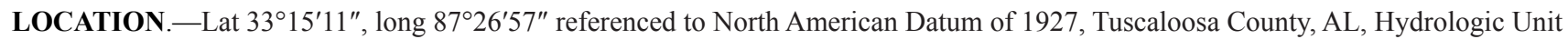
03160112, on left bank $50 \mathrm{ft}$ upstream from lock and dam, 0.1 mi downstream from Jim Mack Branch, 0.7 mi upstream from Hurricane Creek, 2.0 mi northeast of Holt, 3.2 mi upstream from North River, and at mile 135.1.

DRAINAGE AREA.-4,219 $\mathrm{mi}^{2}$.

PERIOD OF RECORD.- October 1976 to November 2000, August 2001 to September 2013.

PERIOD OF ANALYSIS.-April 1977 to March 2000, April 2002 to March 2013.

REMARKS.-Flows regulated by Lewis Smith Lake (1961) and Bankhead Lock and Dam (1975). QAQC reviews indicated that regulation patterns were such that a frequency analysis of the annual 1- and 7-day average minimum flows was not warranted.

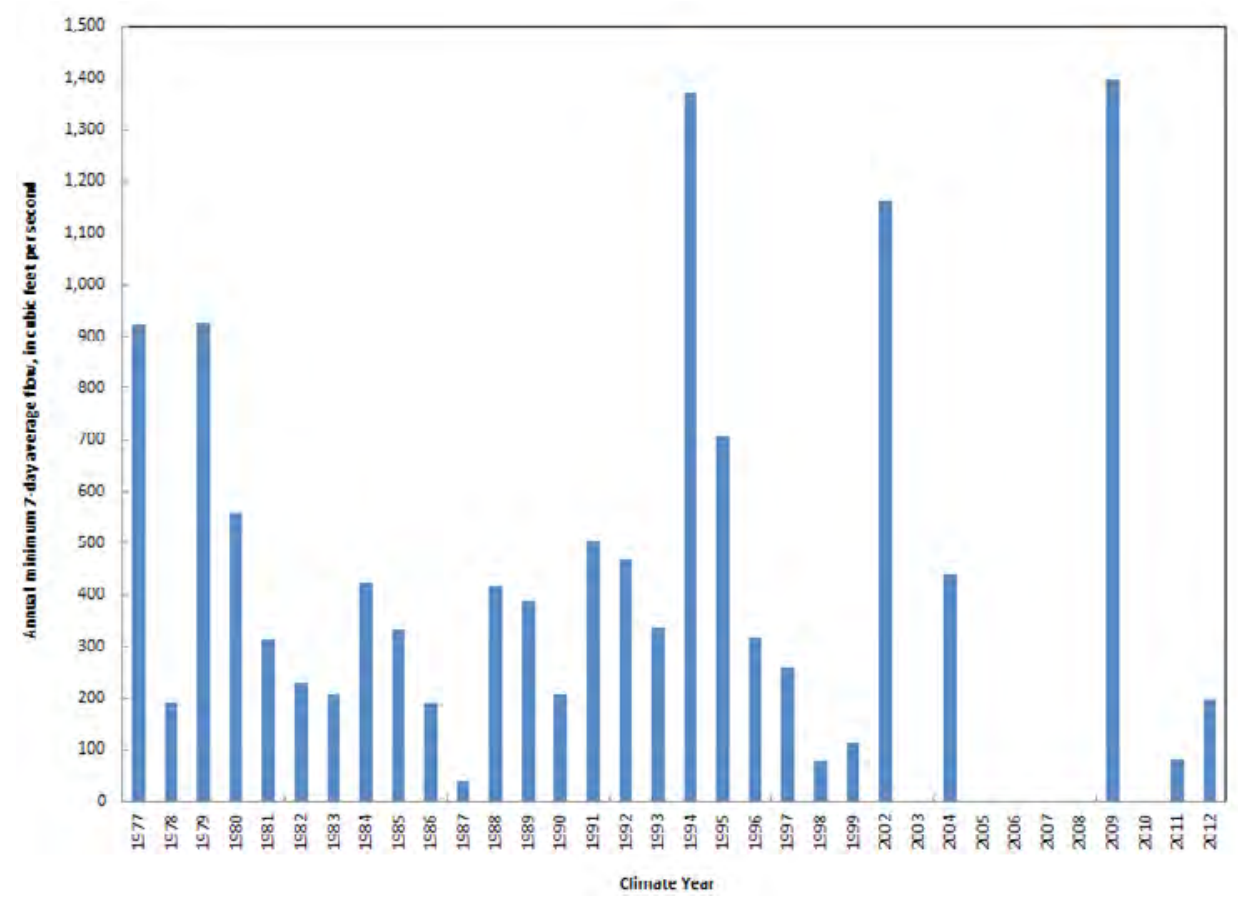

\begin{tabular}{|} 
EXCEEDANCE PERCENTILES OF ANNUAL MINIMUM 7-DAY AVERAGE FLOWS \\
\begin{tabular}{|c|c|c|c|c|c|c|c|c|c|}
\hline \multicolumn{1}{|c|}{ Annual minimum 7-day average flow exceeded for indicated percentiles (cubic feet per second) } \\
\hline Percentile & 10 & 20 & 30 & 40 & 50 & 60 & 70 & 80 & 90 \\
\hline Flow & 1,050 & 559 & 431 & 336 & 286 & 207 & 154 & 41 & 0 \\
\hline
\end{tabular}
\end{tabular}

\begin{tabular}{|c|c|c|c|c|c|c|c|}
\hline \multicolumn{1}{|c|}{ DURATION OF DAILY FLOW } \\
\hline \multicolumn{7}{|c|}{ Flow equaled or exceeded for indicated percentage of time } \\
(cubic feet per second)
\end{tabular}


STATION NAME AND NUMBER—02463500 Hurricane Creek near Holt, AL

LOCATION.-Lat $33^{\circ} 12^{\prime} 40^{\prime \prime}$, long $87^{\circ} 26^{\prime} 5^{\prime \prime}$ referenced to North American Datum of 1927, Tuscaloosa County, AL, Hydrologic Unit 03160112, on State Highway 116, 0.5 mi downstream from Cottondale Creek, 2.8 mi southeast of Holt, and at mile 7.1.

DRAINAGE AREA. $-108 \mathrm{mi}^{2}$.

PERIOD OF RECORD.--August 1952 to September 1969.

PERIOD OF ANALYSIS.-April 1953 to March 1969.

NUMBER OF CLIMATE YEARS IN FREQUENCY ANALYSIS.-16

REMARKS.-

\begin{tabular}{|c|c|c|c|c|}
\hline $\begin{array}{l}\text { Recurrence } \\
\text { intervals } \\
\text { (years) }\end{array}$ & \multicolumn{2}{|c|}{$\begin{array}{l}\text { Lowest average flow for } \\
\text { indicated number of } \\
\text { consecutive days } \\
\text { (cubic feet per second) }\end{array}$} & \multicolumn{2}{|c|}{$\begin{array}{l}\text { Time-sampling error } \\
\text { (in percent) }\end{array}$} \\
\hline & 1 & 7 & 1 & 7 \\
\hline 2 & 5.4 & 6.1 & 15 & 15 \\
\hline 5 & 3.4 & 3.8 & 17 & 17 \\
\hline 10 & 2.6 & 3.0 & 21 & 20 \\
\hline 20 & 2.1 & 2.4 & 27 & 25 \\
\hline
\end{tabular}

\section{DURATION OF DAILY FLOW}

Flow equaled or exceeded for indicated percentage of time (cubic feet per second)

\begin{tabular}{|c|c|c|c|c|c|c|c|}
\hline Percentage & 5 & 10 & 25 & 50 & 75 & 90 & 95 \\
\hline Flow & 510 & 312 & 146 & 48 & 18 & 9.3 & 6.8 \\
\hline
\end{tabular}


STATION NAME AND NUMBER—02464000 North River near Samantha, AL

LOCATION.-Lat $33^{\circ} 28^{\prime} 45^{\prime \prime}$, long $87^{\circ} 35^{\prime} 50^{\prime \prime}$ referenced to North American Datum of 1927, Tuscaloosa County, AL, Hydrologic Unit 03160112, on upstream side of bridge on county road, 1.2 mi upstream of Cripple Creek, 4 mi north of Samantha, and at mile 36.9.

DRAINAGE AREA. $-223 \mathrm{mi}^{2}$.

PERIOD OF RECORD.-December 1938 to September 1954, October 1968 to March 2014.

PERIOD OF ANALYSIS.-April 1939 to March 1954, April 1969 to March 2014.

NUMBER OF CLIMATE YEARS IN FREQUENCY ANALYSIS.—60

REMARKS.-

\begin{tabular}{|c|c|c|c|c|}
\hline $\begin{array}{l}\text { Recurrence } \\
\text { intervals } \\
\text { (years) }\end{array}$ & \multicolumn{2}{|c|}{$\begin{array}{l}\text { Lowest average flow for } \\
\text { indicated number of } \\
\text { consecutive days } \\
\text { (cubic feet per second) }\end{array}$} & \multicolumn{2}{|c|}{$\begin{array}{l}\text { Time-sampling error } \\
\text { (in percent) }\end{array}$} \\
\hline & 1 & 7 & 1 & 7 \\
\hline 2 & 5.9 & 7.1 & 14 & 13 \\
\hline 5 & 2.5 & 3.1 & 17 & 16 \\
\hline 10 & 1.5 & 1.9 & 22 & 21 \\
\hline 20 & 0.93 & 1.2 & 30 & 28 \\
\hline 50 & 0.53 & 0.74 & 43 & 40 \\
\hline
\end{tabular}

\section{DURATION OF DAILY FLOW}

Flow equaled or exceeded for indicated percentage of time (cubic feet per second)

\begin{tabular}{|c|c|c|c|c|c|c|c|}
\hline Percentage & 5 & 10 & 25 & 50 & 75 & 90 & 95 \\
\hline Flow & 1,540 & 868 & 357 & 117 & 29 & 10 & 5.7 \\
\hline
\end{tabular}


STATION NAME AND NUMBER—02464146 Turkey Creek near Tuscaloosa, AL

LOCATION.-Lat $33^{\circ} 24^{\prime} 48^{\prime \prime}$, long $87^{\circ} 30^{\prime} 38^{\prime \prime}$ referenced to North American Datum of 1927, Tuscaloosa County, AL, Hydrologic Unit 03160112, on left bank 1,400 ft downstream from State Highway 69, 1.1 mi upstream from Long Creek, 4.7 mi upstream from mouth, 5.5 mi east of Samantha, and $14 \mathrm{mi}$ north of Tuscaloosa.

DRAINAGE AREA. $-6.16 \mathrm{mi}^{2}$.

PERIOD OF RECORD.-February 1981 to September 1984, October 1986 to March 2014.

PERIOD OF ANALYSIS.-April 1981 to March 1984, April 1987 to March 2014.

NUMBER OF CLIMATE YEARS IN FREQUENCY ANALYSIS.—30

\section{REMARKS.-}

\begin{tabular}{|c|c|c|c|c|}
\hline \multirow[t]{2}{*}{$\begin{array}{l}\text { Recurrence } \\
\text { intervals } \\
\text { (years) }\end{array}$} & \multicolumn{2}{|c|}{$\begin{array}{l}\text { Lowest average flow for } \\
\text { indicated number of } \\
\text { consecutive days } \\
\text { (cubic feet per second) }\end{array}$} & \multicolumn{2}{|c|}{$\begin{array}{l}\text { Time-sampling error } \\
\text { (in percent) }\end{array}$} \\
\hline & 1 & 7 & 1 & 7 \\
\hline 2 & 1.2 & 1.3 & 9 & 9 \\
\hline 5 & 0.83 & 0.91 & 11 & 10 \\
\hline 10 & 0.66 & 0.74 & 14 & 12 \\
\hline 20 & 0.54 & 0.62 & 17 & 15 \\
\hline 50 & 0.43 & 0.51 & 23 & 20 \\
\hline
\end{tabular}

\section{DURATION OF DAILY FLOW}

Flow equaled or exceeded for indicated percentage of time (cubic feet per second)

\begin{tabular}{|c|c|c|c|c|c|c|c|}
\hline Percentage & 5 & 10 & 25 & 50 & 75 & 90 & 95 \\
\hline Flow & 33 & 20 & 10 & 4.9 & 2.5 & 1.5 & 1.2 \\
\hline
\end{tabular}


STATION NAME AND NUMBER—02464360 Binion Creek below Gin Creek near Samantha, AL

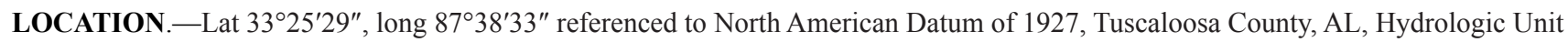
03160112, at county road $30 \mathrm{ft}$ downstream from Gin Creek, $1.0 \mathrm{mi}$ downstream from Wolf Creek, and 2.2 mi west of Samantha.

DRAINAGE AREA. $-57.2 \mathrm{mi}^{2}$.

PERIOD OF RECORD.- October 1986 to March 2014.

PERIOD OF ANALYSIS.-April 1987 to March 2014.

NUMBER OF CLIMATE YEARS IN FREQUENCY ANALYSIS.—27

REMARKS.-

\begin{tabular}{|c|c|c|c|c|}
\hline $\begin{array}{l}\text { Recurrence } \\
\text { intervals } \\
\text { (years) }\end{array}$ & \multicolumn{2}{|c|}{$\begin{array}{l}\text { Lowest average flow for } \\
\text { indicated number of } \\
\text { consecutive days } \\
\text { (cubic feet per second) }\end{array}$} & \multicolumn{2}{|c|}{$\begin{array}{l}\text { Time-sampling error } \\
\text { (in percent) }\end{array}$} \\
\hline & 1 & 7 & 1 & 7 \\
\hline 2 & 14 & 15 & 8 & 8 \\
\hline 5 & 9.8 & 11 & 10 & 10 \\
\hline 10 & 8.0 & 8.6 & 13 & 13 \\
\hline 20 & 6.7 & 7.2 & 17 & 17 \\
\hline
\end{tabular}

\section{DURATION OF DAILY FLOW}

Flow equaled or exceeded for indicated percentage of time (cubic feet per second)

\begin{tabular}{|c|c|c|c|c|c|c|c|}
\hline Percentage & 5 & 10 & 25 & 50 & 75 & 90 & 95 \\
\hline Flow & 283 & 166 & 85 & 47 & 28 & 17 & 13 \\
\hline
\end{tabular}


STATION NAME AND NUMBER—02464500 North River (Site B) near Tuscaloosa, AL

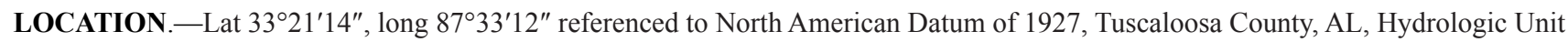
03160112, on State Highway 69, 1,000 ft upstream from Tierce Creek and 10 mi north of Tuscaloosa.

DRAINAGE AREA. $-372 \mathrm{mi}^{2}$.

PERIOD OF RECORD.--December 1951 to December 1968.

PERIOD OF ANALYSIS.-April 1952 to March 1968.

NUMBER OF CLIMATE YEARS IN FREQUENCY ANALYSIS.—16

REMARKS.- Since 1970, site in backwater from Lake Tuscaloosa.

\begin{tabular}{|c|c|c|c|c|}
\hline $\begin{array}{l}\text { Recurrence } \\
\text { intervals } \\
\text { (years) }\end{array}$ & \multicolumn{2}{|c|}{$\begin{array}{l}\text { Lowest average flow for } \\
\text { indicated number of } \\
\text { consecutive days } \\
\text { (cubic feet per second) }\end{array}$} & \multicolumn{2}{|c|}{$\begin{array}{l}\text { Time-sampling error } \\
\text { (in percent) }\end{array}$} \\
\hline & 1 & 7 & 1 & 7 \\
\hline 2 & 20 & 23 & 11 & 11 \\
\hline 5 & 14 & 16 & 13 & 13 \\
\hline 10 & 11 & 13 & 17 & 17 \\
\hline 20 & 9.5 & 11 & 22 & 23 \\
\hline
\end{tabular}

\section{DURATION OF DAILY FLOW}

Flow equaled or exceeded for indicated percentage of time (cubic feet per second)

\begin{tabular}{|c|c|c|c|c|c|c|c|}
\hline Percentage & 5 & 10 & 25 & 50 & 75 & 90 & 95 \\
\hline Flow & 2,020 & 1,210 & 520 & 167 & 65 & 35 & 25 \\
\hline
\end{tabular}


STATION NAME AND NUMBER—02465000 Black Warrior River at Oliver Lock and Dam at Northport, AL

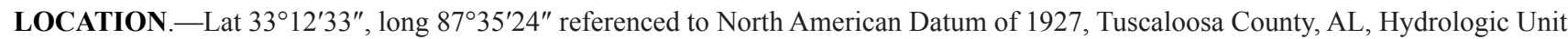
03160112, on upstream guidewall of "new" Oliver Lock and Dam, 0.5 mi upstream from Mill Creek, 0.9 mi downstream from Illinois Central Gulf Railroad bridge, $1.7 \mathrm{mi}$ downstream from Two Mile Creek, $5.9 \mathrm{mi}$ downstream from North River, and at mile 125.9.

DRAINAGE AREA. $-4,820 \mathrm{mi}^{2}$.

PERIOD OF RECORD.—January 1895 to December 1902, August 1928 to March 2014.

PERIOD OF ANALYSIS.-April 1976 to March 2014.

NUMBER OF CLIMATE YEARS IN FREQUENCY ANALYSIS.-38

REMARKS.-Flow regulated since 1914 by Bankhead Lock and Dam on Black Warrior River (with upgrades in 1975),since 1961 by Lewis Smith Lake on Sipsey Fork, and since 1969 by Holt Lock and Dam on Black Warrior River. Lake Tuscaloosa on the North River was completed around 1971. Period of record analyzed was April 1976 to March 2014, which was the most recent period of relatively stable regulation.

\begin{tabular}{|c|c|c|c|c|}
\hline $\begin{array}{c}\text { Recurrence } \\
\text { intervals } \\
\text { (years) }\end{array}$ & \multicolumn{2}{|c|}{$\begin{array}{c}\text { Lowest average flow for } \\
\text { indicated number of } \\
\text { consecutive days } \\
\text { (cubic feet per second) }\end{array}$} & \multicolumn{2}{|c|}{$\begin{array}{c}\text { Time-sampling error } \\
\text { (in percent) }\end{array}$} \\
\hline & 1 & 7 & 1 & 73 \\
\hline 2 & 149 & 429 & 12 & 15 \\
\hline 5 & 81 & 223 & 16 & 19 \\
\hline 10 & 55 & 156 & 21 & 24 \\
\hline 20 & 39 & 115 & 30 & 32 \\
\hline 50 & 25 & 81 & 45 & \\
\hline
\end{tabular}

\begin{tabular}{|c|c|c|c|c|c|c|c|}
\hline \multicolumn{1}{|c|}{ DURATION OF DAILY FLOW } \\
\hline \multicolumn{1}{|c|}{ Flow equaled or exceeded for indicated percentage of time } \\
(cubic feet per second) \\
\hline Percentage & 5 & 10 & 25 & 50 & 75 & 90 & 95 \\
\hline Flow & 27,900 & 18,500 & 10,400 & 4,160 & 1,900 & 527 & 277 \\
\hline
\end{tabular}


STATION NAME AND NUMBER—02465200 Lake Creek near Northport, AL

LOCATION.-Lat $33^{\circ} 17^{\prime} 10^{\prime \prime}$, long $87^{\circ} 41^{\prime} 00^{\prime \prime}$ referenced to North American Datum of 1927, Tuscaloosa County, AL, Hydrologic Unit 03160113, $300 \mathrm{ft}$ downstream from dam, and 9 mi northwest of Northport.

DRAINAGE AREA.-3.71 $\mathrm{mi}^{2}$.

PERIOD OF RECORD.-November 1956 to September 1970.

PERIOD OF ANALYSIS.--April 1957 to March 1970.

NUMBER OF CLIMATE YEARS IN FREQUENCY ANALYSIS.—13

REMARKS.-Since 1954, regulated by Lake Lurleen.

\begin{tabular}{|c|c|c|c|c|}
\hline $\begin{array}{c}\text { Recurrence } \\
\text { intervals } \\
\text { (years) }\end{array}$ & \multicolumn{2}{|c|}{$\begin{array}{c}\text { Lowest average flow for } \\
\text { indicated number of } \\
\text { consecutive days } \\
\text { (cubic feet per second) }\end{array}$} & \multicolumn{2}{|c|}{$\begin{array}{c}\text { Time-sampling error } \\
\text { (in percent) }\end{array}$} \\
\hline \multicolumn{7}{|c|}{} & 1 & 7 & 1 & 7 \\
\hline 2 & 1.1 & 1.3 & 22 & 27 \\
\hline 5 & 0.50 & 0.66 & 28 & 37 \\
\hline 10 & 0.21 & 0.28 & 39 & -- \\
\hline 20 & 0.00 & 0.00 & -- & \\
\hline
\end{tabular}

\section{DURATION OF DAILY FLOW}

Flow equaled or exceeded for indicated percentage of time (cubic feet per second)

\begin{tabular}{|c|c|c|c|c|c|c|c|}
\hline Percentage & 5 & 10 & 25 & 50 & 75 & 90 & 95 \\
\hline Flow & 19 & 13 & 8.1 & 5.0 & 3.2 & 2.0 & 1.4 \\
\hline
\end{tabular}


STATION NAME AND NUMBER—02465292 Cribbs Mill Creek at Wastewater Plant at Tuscaloosa, AL

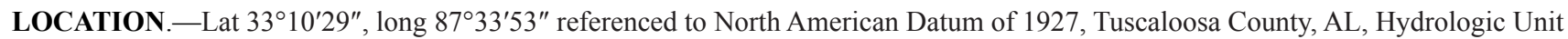
03160113, on right bank, at Hillard R. Fletcher Sewage Treatment Plant, 0.1 mi downstream of Kauloosa Avenue, 2 mi south of Tuscaloosa, at mile 2.5.

DRAINAGE AREA.- $-10.7 \mathrm{mi}^{2}$.

PERIOD OF RECORD.- July 2002 to March 2014.

PERIOD OF ANALYSIS.-April 2003 to March 2014.

NUMBER OF CLIMATE YEARS IN FREQUENCY ANALYSIS.-11

REMARKS.-

\begin{tabular}{|c|c|c|c|c|}
\hline $\begin{array}{c}\text { Recurrence } \\
\text { intervals } \\
\text { (years) }\end{array}$ & \multicolumn{2}{|c|}{$\begin{array}{l}\text { Lowest average flow for } \\
\text { indicated number of } \\
\text { consecutive days } \\
\text { (cubic feet per second) }\end{array}$} & \multicolumn{2}{|c|}{$\begin{array}{l}\text { Time-sampling error } \\
\text { (in percent) }\end{array}$} \\
\hline & 1 & 7 & 1 & 7 \\
\hline 2 & 5.3 & 5.8 & 9 & 9 \\
\hline 5 & 4.3 & 4.5 & 10 & 10 \\
\hline 10 & 3.8 & 4.0 & 12 & 13 \\
\hline 20 & 3.4 & 3.5 & 15 & 16 \\
\hline
\end{tabular}

\begin{tabular}{|c|c|c|c|c|c|c|c|}
\hline \multicolumn{7}{|c|}{ DURATION OF DAILY FLOW } \\
\hline \multicolumn{7}{|c|}{ Flow equaled or exceeded for indicated percentage of time } \\
(cubic feet per second)
\end{tabular}


STATION NAME AND NUMBER—02465493 Elliotts Creek at Moundville, AL

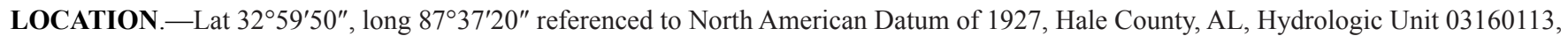
on downstream side of bridge on State Highway 69 at Moundville, 2.5 mi upstream from Southern Railway bridge, and 6.6 mi upstream from mouth.

DRAINAGE AREA. $-32.3 \mathrm{mi}^{2}$.

PERIOD OF RECORD.- October 1976 to March 2014.

PERIOD OF ANALYSIS.-April 1977 to March 2014.

NUMBER OF CLIMATE YEARS IN FREQUENCY ANALYSIS.—37

REMARKS.-

\begin{tabular}{|c|c|c|c|c|}
\hline \multirow[t]{2}{*}{$\begin{array}{l}\text { Recurrence } \\
\text { intervals } \\
\text { (years) }\end{array}$} & \multicolumn{2}{|c|}{$\begin{array}{l}\text { Lowest average flow for } \\
\text { indicated number of } \\
\text { consecutive days } \\
\text { (cubic feet per second) }\end{array}$} & \multicolumn{2}{|c|}{$\begin{array}{l}\text { Time-sampling error } \\
\text { (in percent) }\end{array}$} \\
\hline & 1 & 7 & 1 & 7 \\
\hline 2 & 12 & 13 & 6 & 5 \\
\hline 5 & 9.2 & 9.7 & 7 & 6 \\
\hline 10 & 7.8 & 8.4 & 9 & 8 \\
\hline 20 & 6.7 & 7.4 & 12 & 10 \\
\hline 50 & 5.6 & 6.4 & 17 & 14 \\
\hline
\end{tabular}

\section{DURATION OF DAILY FLOW}

Flow equaled or exceeded for indicated percentage of time (cubic feet per second)

\begin{tabular}{|c|c|c|c|c|c|c|c|}
\hline Percentage & 5 & 10 & 25 & 50 & 75 & 90 & 95 \\
\hline Flow & 106 & 72 & 43 & 27 & 18 & 14 & 12 \\
\hline
\end{tabular}


STATION NAME AND NUMBER—02465500 Fivemile Creek near Greensboro, AL

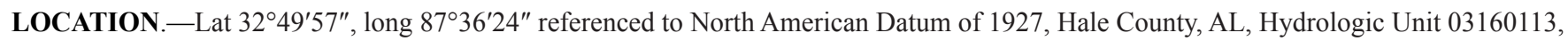
on State Highway 69, 8.5 mi north of Greensboro, and 12 mi upstream from mouth.

DRAINAGE AREA. $-73.6 \mathrm{mi}^{2}$.

PERIOD OF RECORD.- - October 1954 to September 1971.

PERIOD OF ANALYSIS.-April 1955 to March 1971.

NUMBER OF CLIMATE YEARS IN FREQUENCY ANALYSIS.—16

REMARKS.-

\begin{tabular}{|c|c|c|c|c|}
\hline $\begin{array}{l}\text { Recurrence } \\
\text { intervals } \\
\text { (years) }\end{array}$ & \multicolumn{2}{|c|}{$\begin{array}{l}\text { Lowest average flow for } \\
\text { indicated number of } \\
\text { consecutive days } \\
\text { (cubic feet per second) }\end{array}$} & \multicolumn{2}{|c|}{$\begin{array}{l}\text { Time-sampling error } \\
\text { (in percent) }\end{array}$} \\
\hline & 1 & 7 & 1 & 7 \\
\hline 2 & 2.4 & 3.1 & 18 & 16 \\
\hline 5 & 1.4 & 2.0 & 22 & 22 \\
\hline 10 & 0.95 & 1.4 & 30 & 29 \\
\hline 20 & 0.69 & 1.0 & 43 & 44 \\
\hline
\end{tabular}

\section{DURATION OF DAILY FLOW}

Flow equaled or exceeded for indicated percentage of time (cubic feet per second)

\begin{tabular}{|c|c|c|c|c|c|c|c|}
\hline Percentage & 5 & 10 & 25 & 50 & 75 & 90 & 95 \\
\hline Flow & 240 & 159 & 72 & 25 & 9.2 & 5.0 & 3.8 \\
\hline
\end{tabular}


STATION NAME AND NUMBER—02466030 Black Warrior River at Selden Lock and Dam near Eutaw, AL

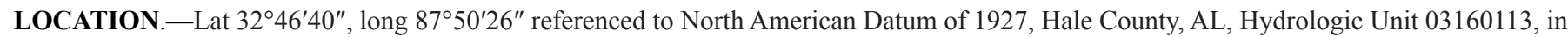
SE $1 / 4$ sec. 24, T.21 N.,R.2 E., on left bank at Armistead I. Selden Lock and Dam, 1.2 mi upstream of White Creek, 5.0 mi southeast of Eutaw, and at river mile 49.6 .

DRAINAGE AREA. $-5,810 \mathrm{mi}^{2}$.

PERIOD OF RECORD.- October 1976 to March 2014.

PERIOD OF ANALYSIS.-April 1977 to March 2014.

NUMBER OF CLIMATE YEARS IN FREQUENCY ANALYSIS.-36

REMARKS.-Flow regulated by Lewis Smith Lake, Bankhead Lock and Dam, Holt Lock and Dam, and Oliver Lock and Dam.

\begin{tabular}{|c|c|c|c|c|}
\hline $\begin{array}{l}\text { Recurrence } \\
\text { intervals } \\
\text { (years) }\end{array}$ & \multicolumn{2}{|c|}{$\begin{array}{l}\text { Lowest average flow for } \\
\text { indicated number of } \\
\text { consecutive days } \\
\text { (cubic feet per second) }\end{array}$} & \multicolumn{2}{|c|}{$\begin{array}{l}\text { Time-sampling error } \\
\text { (in percent) }\end{array}$} \\
\hline & 1 & 7 & 1 & 7 \\
\hline 2 & 783 & 967 & 6 & 8 \\
\hline 5 & 582 & 687 & 8 & 8 \\
\hline 10 & 483 & 580 & 10 & 9 \\
\hline 20 & 408 & 507 & 14 & 10 \\
\hline 50 & 331 & 438 & 21 & 14 \\
\hline
\end{tabular}

DURATION OF DAILY FLOW

Flow equaled or exceeded for indicated percentage of time (cubic feet per second)

\begin{tabular}{|c|c|c|c|c|c|c|c|}
\hline Percentage & 5 & 10 & 25 & 50 & 75 & 90 & 95 \\
\hline Flow & 33,500 & 22,900 & 11,900 & 5,100 & 2,330 & 1,060 & 887 \\
\hline
\end{tabular}


STATION NAME AND NUMBER—02466500 Big Prairie Creek near Gallion, AL

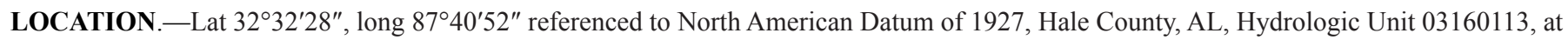
State Highway 69, 4 mi upstream from Little Prairie Creek, and 4 mi northwest of Gallion.

DRAINAGE AREA.-171 $\mathrm{mi}^{2}$.

PERIOD OF RECORD.-February 1940 to September 1952, October 1990 to September 1991.

PERIOD OF ANALYSIS.-April 1940 to March 1952.

NUMBER OF CLIMATE YEARS IN FREQUENCY ANALYSIS.-12

REMARKS.-

\begin{tabular}{|c|c|c|c|c|}
\hline $\begin{array}{l}\text { Recurrence } \\
\text { intervals } \\
\text { (years) }\end{array}$ & \multicolumn{2}{|c|}{$\begin{array}{l}\text { Lowest average flow for } \\
\text { indicated number of } \\
\text { consecutive days } \\
\text { (cubic feet per second) }\end{array}$} & \multicolumn{2}{|c|}{$\begin{array}{l}\text { Time-sampling error } \\
\text { (in percent) }\end{array}$} \\
\hline & 1 & 7 & 1 & 7 \\
\hline 2 & 4.4 & 4.8 & 17 & 20 \\
\hline 5 & 2.5 & 2.8 & 19 & 24 \\
\hline 10 & 1.5 & 2.0 & 24 & 32 \\
\hline 20 & 0.00 & 1.5 & -- & 43 \\
\hline
\end{tabular}

\section{DURATION OF DAILY FLOW}

Flow equaled or exceeded for indicated percentage of time (cubic feet per second)

\begin{tabular}{|c|c|c|c|c|c|c|c|}
\hline Percentage & 5 & 10 & 25 & 50 & 75 & 90 & 95 \\
\hline Flow & 1,150 & 520 & 107 & 36 & 11 & 5.8 & 4.0 \\
\hline
\end{tabular}


STATION NAME AND NUMBER—02467000 Tombigbee River at Demopolis Lock and Dam near Coatopa, AL

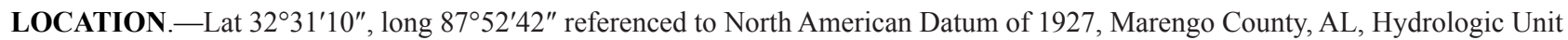
03160201, on left bank $100 \mathrm{ft}$ upstream from lock and dam, $0.5 \mathrm{mi}$ downstream from Foscue Creek, 2.5 mi west of Demopolis, 3.5 mi upstream from Hall Creek, 13 mi east of Coatopa, and at mile 171.2.

DRAINAGE AREA. $-15,385 \mathrm{mi}^{2}$.

PERIOD OF RECORD.--August 1928 to March 2014.

PERIOD OF ANALYSIS.-April 1985 to March 2014.

NUMBER OF CLIMATE YEARS IN FREQUENCY ANALYSIS.—-29

REMARKS.-Period of record analyzed includes all current regulation structures. Records since January 16, 1985, include diversions from Tennessee River basin through Bay Springs lock on Tennessee-Tombigbee Waterway. Regulated by Jamie Whitten Lock and Dam (1983), G.V. Montgomery Lock and Dam (1984), John Ranking Lock and Dam (1984), Fulton Lock and Dam (1981), Glover Wilkins Lock and Dam (1981), Amory Lock and Dam (1979), Aberdeen Lock and Dam (1981), John C. Stennis Lock and Dam (1978), Tom Bevill Lock and Dam (1978), Howell Heflin Lock and Dam (1977), and Demopolis Lock and Dam (1955).

\begin{tabular}{|c|c|c|c|c|}
\hline \multirow[t]{2}{*}{$\begin{array}{l}\text { Recurrence } \\
\text { intervals } \\
\text { (years) }\end{array}$} & \multicolumn{2}{|c|}{$\begin{array}{l}\text { Lowest average flow for } \\
\text { indicated number of } \\
\text { consecutive days } \\
\text { (cubic feet per second) }\end{array}$} & \multicolumn{2}{|c|}{$\begin{array}{l}\text { Time-sampling error } \\
\text { (in percent) }\end{array}$} \\
\hline & 1 & 7 & 1 & 7 \\
\hline 2 & 1,410 & 1,930 & 8 & 9 \\
\hline 5 & 1,010 & 1,320 & 8 & 10 \\
\hline 10 & 859 & 1,080 & 10 & 12 \\
\hline 20 & 750 & 909 & 12 & 15 \\
\hline
\end{tabular}

\begin{tabular}{|c|c|c|c|c|c|c|c|}
\hline \multicolumn{1}{|c|}{ DURATION OF DAILY FLOW } \\
\hline \multicolumn{7}{|c|}{ Flow equaled or exceeded for indicated percentage of time } \\
(cubic feet per second)
\end{tabular}


STATION NAME AND NUMBER—02467500 Sucarnoochee River at Livingston, AL

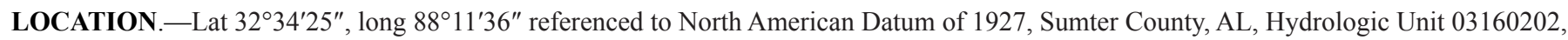
on downstream side of downstream guard rail of new bridge on U.S. Highway 11, $550 \mathrm{ft}$ upstream from Southern Railway bridge, $0.8 \mathrm{mi}$ southwest of Livingston, and 9 mi upstream from Alamuchee Creek.

DRAINAGE AREA. $-607 \mathrm{mi}^{2}$.

PERIOD OF RECORD.-October 1938 to March 2014.

PERIOD OF ANALYSIS.-April 1939 to March 2014.

NUMBER OF CLIMATE YEARS IN FREQUENCY ANALYSIS.—75

\section{REMARKS.-}

\begin{tabular}{|c|c|c|c|c|}
\hline $\begin{array}{l}\text { Recurrence } \\
\text { intervals } \\
\text { (years) }\end{array}$ & \multicolumn{2}{|c|}{$\begin{array}{l}\text { Lowest average flow for } \\
\text { indicated number of } \\
\text { consecutive days } \\
\text { (cubic feet per second) }\end{array}$} & \multicolumn{2}{|c|}{$\begin{array}{l}\text { Time-sampling error } \\
\text { (in percent) }\end{array}$} \\
\hline & 1 & 7 & 1 & 7 \\
\hline 2 & 102 & 107 & 4 & 4 \\
\hline 5 & 76 & 79 & 5 & 5 \\
\hline 10 & 66 & 69 & 5 & 5 \\
\hline 20 & 58 & 61 & 6 & 6 \\
\hline 50 & 51 & 53 & 8 & 8 \\
\hline
\end{tabular}

\section{DURATION OF DAILY FLOW}

Flow equaled or exceeded for indicated percentage of time (cubic feet per second)

\begin{tabular}{|c|c|c|c|c|c|c|c|}
\hline Percentage & 5 & 10 & 25 & 50 & 75 & 90 & 95 \\
\hline Flow & 3,260 & 2,160 & 788 & 340 & 171 & 118 & 99 \\
\hline
\end{tabular}


STATION NAME AND NUMBER—02468000 Alamuchee Creek near Cuba, AL

LOCATION.-Lat $32^{\circ} 26^{\prime} 00^{\prime \prime}$, long $88^{\circ} 20^{\prime} 00^{\prime \prime}$ referenced to North American Datum of 1927, Sumter County, AL, Hydrologic Unit 03160202, on U.S. Highway 80, 2.5 mi northeast of Cuba, and 4 mi upstream from Toomsuba Creek.

DRAINAGE AREA. $-62.3 \mathrm{mi}^{2}$.

PERIOD OF RECORD.--August 1954 to September 1967.

PERIOD OF ANALYSIS.-April 1955 to March 1967.

NUMBER OF CLIMATE YEARS IN FREQUENCY ANALYSIS.—12

REMARKS.-

\begin{tabular}{|c|c|c|c|c|}
\hline $\begin{array}{l}\text { Recurrence } \\
\text { intervals } \\
\text { (years) }\end{array}$ & \multicolumn{2}{|c|}{$\begin{array}{l}\text { Lowest average flow for } \\
\text { indicated number of } \\
\text { consecutive days } \\
\text { (cubic feet per second) }\end{array}$} & \multicolumn{2}{|c|}{$\begin{array}{l}\text { Time-sampling error } \\
\text { (in percent) }\end{array}$} \\
\hline & 1 & 7 & 1 & 7 \\
\hline 2 & 4.8 & 5.2 & 15 & 14 \\
\hline 5 & 3.2 & 3.5 & 18 & 17 \\
\hline 10 & 2.5 & 2.7 & 23 & 23 \\
\hline 20 & 2.0 & 2.2 & 31 & 30 \\
\hline
\end{tabular}

DURATION OF DAILY FLOW

Flow equaled or exceeded for indicated percentage of time (cubic feet per second)

\begin{tabular}{|c|c|c|c|c|c|c|c|}
\hline Percentage & 5 & 10 & 25 & 50 & 75 & 90 & 95 \\
\hline Flow & 246 & 124 & 51 & 18 & 9.3 & 5.8 & 4.0 \\
\hline
\end{tabular}


STATION NAME AND NUMBER—02468500 Chickasaw Bogue near Linden, AL

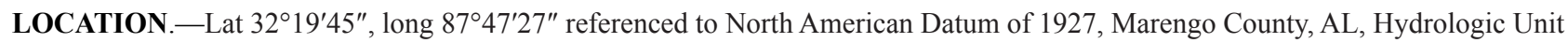
03160201, on U.S. Highway 43, 1.5 mi north of Linden, 2 mi downstream from Atkin Creek, and 11 mi upstream from mouth.

DRAINAGE AREA. $-257 \mathrm{mi}^{2}$.

PERIOD OF RECORD.- January 1944 to September 1946, October 1965 to September 1988.

PERIOD OF ANALYSIS.-April 1944 to March 1946, April 1966 to March 1988.

NUMBER OF CLIMATE YEARS IN FREQUENCY ANALYSIS.—-24

REMARKS.-

\begin{tabular}{|c|c|c|c|c|}
\hline $\begin{array}{l}\text { Recurrence } \\
\text { intervals } \\
\text { (years) }\end{array}$ & \multicolumn{2}{|c|}{$\begin{array}{l}\text { Lowest average flow for } \\
\text { indicated number of } \\
\text { consecutive days } \\
\text { (cubic feet per second) }\end{array}$} & \multicolumn{2}{|c|}{$\begin{array}{l}\text { Time-sampling error } \\
\text { (in percent) }\end{array}$} \\
\hline & 1 & 7 & 1 & 7 \\
\hline 2 & 1.9 & 2.0 & 18 & 17 \\
\hline 5 & 0.95 & 1.1 & 20 & 17 \\
\hline 10 & 0.66 & 0.81 & 24 & 19 \\
\hline 20 & 0.49 & 0.64 & 30 & 23 \\
\hline
\end{tabular}

\section{DURATION OF DAILY FLOW}

Flow equaled or exceeded for indicated percentage of time (cubic feet per second)

\begin{tabular}{|c|c|c|c|c|c|c|c|}
\hline Percentage & 5 & 10 & 25 & 50 & 75 & 90 & 95 \\
\hline Flow & 1,720 & 600 & 153 & 34 & 6.6 & 2.9 & 1.8 \\
\hline
\end{tabular}


STATION NAME AND NUMBER—02469000 Kinterbish Creek near York, AL

LOCATION.-Lat $32^{\circ} 19^{\prime} 00^{\prime \prime}$, long $88^{\circ} 10^{\prime} 00^{\prime \prime}$ referenced to North American Datum of 1927, Sumter County, AL, Hydrologic Unit 03160201, on State Highway 17, 0.8 mi north of Choctaw-Sumter Countyline, 5.5 mi downstream from Little Kinterbish Creek, and 14 mi southeast of York.

DRAINAGE AREA. $-90.9 \mathrm{mi}^{2}$.

PERIOD OF RECORD.-August 1954 to September 1967.

PERIOD OF ANALYSIS.-April 1955 to March 1967.

NUMBER OF CLIMATE YEARS IN FREQUENCY ANALYSIS.-12

\section{REMARKS.-}

\begin{tabular}{|c|c|c|c|c|}
\hline $\begin{array}{l}\text { Recurrence } \\
\text { intervals } \\
\text { (years) }\end{array}$ & \multicolumn{2}{|c|}{$\begin{array}{l}\text { Lowest average flow for } \\
\text { indicated number of } \\
\text { consecutive days } \\
\text { (cubic feet per second) }\end{array}$} & \multicolumn{2}{|c|}{$\begin{array}{l}\text { Time-sampling error } \\
\text { (in percent) }\end{array}$} \\
\hline & 1 & 7 & 1 & 7 \\
\hline 2 & 9.9 & 11 & 24 & 23 \\
\hline 5 & 5.1 & 5.7 & 29 & 29 \\
\hline 10 & 3.5 & 3.9 & 39 & 38 \\
\hline 20 & 2.5 & 2.8 & 53 & 52 \\
\hline
\end{tabular}

\begin{tabular}{|c|c|c|c|c|c|c|c|}
\hline \multicolumn{8}{|c|}{ DURATION OF DAILY FLOW } \\
\hline \multicolumn{8}{|c|}{$\begin{array}{l}\text { Flow equaled or exceeded for indicated percentage of time } \\
\text { (cubic feet per second) }\end{array}$} \\
\hline Percentage & 5 & 10 & 25 & 50 & 75 & 90 & 95 \\
\hline Flow & 336 & 198 & 88 & 37 & 19 & 11 & 6.9 \\
\hline
\end{tabular}


STATION NAME AND NUMBER—02469500 Tuckabum Creek near Butler, AL

LOCATION.-Lat $32^{\circ} 11^{\prime} 00^{\prime \prime}$, long $88^{\circ} 10^{\prime} 12^{\prime \prime}$ referenced to North American Datum of 1927, Choctaw County, AL, Hydrologic Unit 03160201, $150 \mathrm{ft}$ upstream from bridge on State Highway 17, 2.5 mi upstream from Yantley Creek, 4 mi downstream from Boguelichitto Creek, and 7 mi northeast of Butler.

DRAINAGE AREA.-115 $\mathrm{mi}^{2}$.

PERIOD OF RECORD.- October 1954 to September 1970.

PERIOD OF ANALYSIS.-April 1955 to March 1970.

NUMBER OF CLIMATE YEARS IN FREQUENCY ANALYSIS.—15

\section{REMARKS.-}

\begin{tabular}{|c|c|c|c|c|}
\hline $\begin{array}{l}\text { Recurrence } \\
\text { intervals } \\
\text { (years) }\end{array}$ & \multicolumn{2}{|c|}{$\begin{array}{l}\text { Lowest average flow for } \\
\text { indicated number of } \\
\text { consecutive days } \\
\text { (cubic feet per second) }\end{array}$} & \multicolumn{2}{|c|}{$\begin{array}{l}\text { Time-sampling error } \\
\text { (in percent) }\end{array}$} \\
\hline & 1 & 7 & 1 & 7 \\
\hline 2 & 5.2 & 5.7 & 43 & 38 \\
\hline 5 & 1.9 & 2.2 & 81 & 56 \\
\hline 10 & 0.84 & 1.1 & 84 & 71 \\
\hline 20 & 0.36 & 0.55 & 147 & 123 \\
\hline
\end{tabular}

\begin{tabular}{|c|c|c|c|c|c|c|c|}
\hline \multicolumn{7}{|c|}{ DURATION OF DAILY FLOW } \\
\hline \multicolumn{7}{|c|}{ Flow equaled or exceeded for indicated percentage of time } \\
(cubic feet per second)
\end{tabular}


STATION NAME AND NUMBER-02469550 Horse Creek near Sweetwater, AL

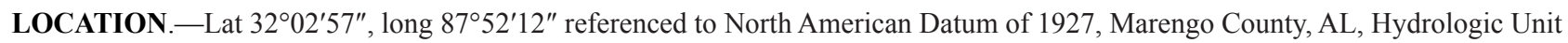
03160201, on county road 25, 0.5 mi downstream from Mill Creek, 0.8 mi south of Exmoor, 1.2 mi north of Hoboken, and 3.5 mi south of Sweetwater.

DRAINAGE AREA. $-60.4 \mathrm{mi}^{2}$.

PERIOD OF RECORD.- October 1959 to September 1970.

PERIOD OF ANALYSIS.-April 1960 to March 1970.

NUMBER OF CLIMATE YEARS IN FREQUENCY ANALYSIS.-10

REMARKS.-

\begin{tabular}{|c|c|c|c|c|}
\hline $\begin{array}{c}\text { Recurrence } \\
\text { intervals } \\
\text { (years) }\end{array}$ & \multicolumn{2}{|c|}{$\begin{array}{c}\text { Lowest average flow for } \\
\text { indicated number of } \\
\text { consecutive days } \\
\text { (cubic feet per second) }\end{array}$} & \multicolumn{2}{|c|}{$\begin{array}{c}\text { Time-sampling error } \\
\text { (in percent) }\end{array}$} \\
\hline \multicolumn{7}{|c|}{1} & 7 & 1 & 7 \\
\hline 2 & 1.4 & 1.8 & 41 & 61 \\
\hline 5 & 0.35 & 0.46 & 53 & 126 \\
\hline 10 & 0.00 & 0.19 & -- & 246 \\
\hline 20 & 0.00 & 0.08 & -- & 83 \\
\hline
\end{tabular}

\begin{tabular}{|c|c|c|c|c|c|c|c|}
\hline \multicolumn{8}{|c|}{ DURATION OF DAILY FLOW } \\
\hline \multicolumn{8}{|c|}{$\begin{array}{l}\text { Flow equaled or exceeded for indicated percentage of time } \\
\text { (cubic feet per second) }\end{array}$} \\
\hline Percentage & 5 & 10 & 25 & 50 & 75 & 90 & 95 \\
\hline Flow & 275 & 132 & 54 & 20 & 6.3 & 3.0 & 1.8 \\
\hline
\end{tabular}


STATION NAME AND NUMBER—02469700 Okatuppa Creek at Gilbertown, AL

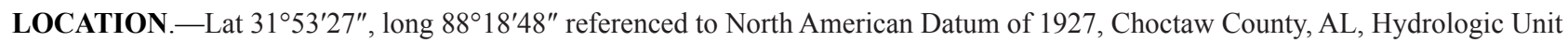
03160201, on Highway 17, 0.8 mi northeast of Gilbertown, and 1.5 mi upstream from Boguelossa Creek.

DRAINAGE AREA.-148 $\mathrm{mi}^{2}$.

PERIOD OF RECORD.- - October 1956 to September 1969.

PERIOD OF ANALYSIS.-April 1957 to March 1969.

NUMBER OF CLIMATE YEARS IN FREQUENCY ANALYSIS.-12

REMARKS.-

\begin{tabular}{|c|c|c|c|c|}
\hline $\begin{array}{l}\text { Recurrence } \\
\text { intervals } \\
\text { (years) }\end{array}$ & \multicolumn{2}{|c|}{$\begin{array}{l}\text { Lowest average flow for } \\
\text { indicated number of } \\
\text { consecutive days } \\
\text { (cubic feet per second) }\end{array}$} & \multicolumn{2}{|c|}{$\begin{array}{l}\text { Time-sampling error } \\
\text { (in percent) }\end{array}$} \\
\hline & 1 & 7 & 1 & 7 \\
\hline 2 & 7.9 & 9.1 & 38 & 38 \\
\hline 5 & 2.9 & 3.3 & 50 & 49 \\
\hline 10 & 1.5 & 1.8 & 70 & 69 \\
\hline 20 & 0.84 & 0.98 & 110 & 106 \\
\hline
\end{tabular}

\section{DURATION OF DAILY FLOW}

Flow equaled or exceeded for indicated percentage of time (cubic feet per second)

\begin{tabular}{|c|c|c|c|c|c|c|c|}
\hline Percentage & 5 & 10 & 25 & 50 & 75 & 90 & 95 \\
\hline Flow & 650 & 400 & 190 & 71 & 25 & 10 & 6.7 \\
\hline
\end{tabular}


STATION NAME AND NUMBER—02469761 Tombigbee River at Coffeeville Lock and Dam near Coffeeville, AL

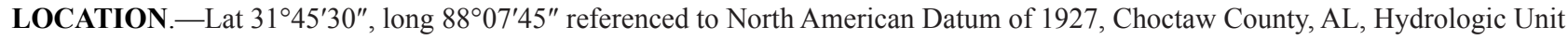
03160203, near right bank at Coffeeville Lock and Dam, 2 mi west of Coffeeville, 4 mi downstream of Turkey Creek, and at river mile 74.7.

DRAINAGE AREA. $-18,417 \mathrm{mi}^{2}$.

PERIOD OF RECORD.- October 1960 to March 2014.

PERIOD OF ANALYSIS.-April 1985 to March 2014.

\section{NUMBER OF CLIMATE YEARS IN FREQUENCY ANALYSIS. - 28}

REMARKS.-QAQC reviews indicate periods for which no flows were released for one or two days during drought periods (such as 1999). Consequently, applying tthe log-Pearson Type III distribution to the 1-day flows was determined to be questionable and therefore, frequency statistics are being provided for the 7-day flows only. Period of record analyzed includes all current regulation structures. Records since January 16, 1985, include diversions from Tennessee River basin through Bay Springs lock on Tennessee-Tombigbee Waterway. Some regulation by Jamie Whitten Lock and Dam (1983), G.V. Montgomery Lock and Dam (1984), John Ranking Lock and Dam (1984), Fulton Lock and Dam (1981), Glover Wilkins Lock and Dam (1981), Amory Lock and Dam (1979), Aberdeen Lock and Dam (1981), John C. Stennis Lock and Dam (1978), Tom Bevill Lock and Dam (1978), Howell Heflin Lock and Dam (1977), and Demopolis Lock and Dam (1955). In addition, some regulation by Smith Lake on Sipsey Fork and several locks and dams on Black Warrior River (Bankhead Lock and Dam (1914), Holt Lock and Dam (1968), Oliver Lock and Dam (1940) and Selden Lock and Dam (1958)).

\begin{tabular}{|c|c|c|c|c|}
\hline $\begin{array}{c}\text { Recurrence } \\
\text { intervals } \\
\text { (years) }\end{array}$ & \multicolumn{2}{|c|}{$\begin{array}{c}\text { Lowest average flow for } \\
\text { indicated number of } \\
\text { consecutive days } \\
\text { (cubic feet per second) }\end{array}$} & \multicolumn{2}{|c|}{$\begin{array}{c}\text { Time-sampling error } \\
\text { (in percent) }\end{array}$} \\
\hline \multicolumn{2}{|c|}{1} & 7 & 1 & 7 \\
\hline 2 & --- & 2,370 & --- & 10 \\
\hline 5 & --- & 1,620 & --- & 11 \\
\hline 10 & --- & 1,340 & --- & 13 \\
\hline 20 & --- & 1,150 & --- & 9 \\
\hline
\end{tabular}

\section{DURATION OF DAILY FLOW}

Flow equaled or exceeded for indicated percentage of time (cubic feet per second)

\begin{tabular}{|c|c|c|c|c|c|c|c|}
\hline Percentage & 5 & 10 & 25 & 50 & 75 & 90 & 95 \\
\hline Flow & 104,000 & 73,200 & 35,900 & 14,700 & 5,620 & 2,990 & 2,110 \\
\hline
\end{tabular}


STATION NAME AND NUMBER—02469800 Satilpa Creek near Coffeeville, AL

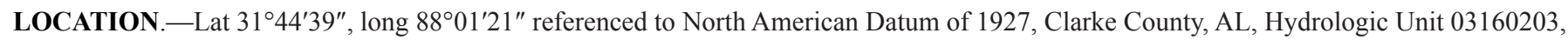
near left bank on downstream side of bridge on State Highway 12, 0.2 mi upstream of unnamed tributary, 3 mi downstream of Harris Creek, and 3.8 mi east of Coffeeville.

DRAINAGE AREA.- $-164 \mathrm{mi}^{2}$.

PERIOD OF RECORD.- October 1956 to September 1970, October 1974 to March 2014.

PERIOD OF ANALYSIS.-April 1957 to March 1970, April 1975 to March 2014.

NUMBER OF CLIMATE YEARS IN FREQUENCY ANALYSIS.—-52

\section{REMARKS.-}

\begin{tabular}{|c|c|c|c|c|}
\hline $\begin{array}{l}\text { Recurrence } \\
\text { intervals } \\
\text { (years) }\end{array}$ & \multicolumn{2}{|c|}{$\begin{array}{l}\text { Lowest average flow for } \\
\text { indicated number of } \\
\text { consecutive days } \\
\text { (cubic feet per second) }\end{array}$} & \multicolumn{2}{|c|}{$\begin{array}{l}\text { Time-sampling error } \\
\text { (in percent) }\end{array}$} \\
\hline & 1 & 7 & 1 & 7 \\
\hline 2 & 11 & 12 & 8 & 8 \\
\hline 5 & 7.1 & 7.6 & 9 & 9 \\
\hline 10 & 5.6 & 5.9 & 10 & 11 \\
\hline 20 & 4.6 & 4.8 & 13 & 14 \\
\hline 50 & 3.6 & 3.8 & 17 & 18 \\
\hline
\end{tabular}

\section{DURATION OF DAILY FLOW}

Flow equaled or exceeded for indicated percentage of time (cubic feet per second)

\begin{tabular}{|c|c|c|c|c|c|c|c|}
\hline Percentage & 5 & 10 & 25 & 50 & 75 & 90 & 95 \\
\hline Flow & 826 & 464 & 219 & 86 & 30 & 15 & 11 \\
\hline
\end{tabular}


STATION NAME AND NUMBER—02470072 Bassett Creek at U.S. Highway 43 near Thomasville, AL

LOCATION.-Lat $31^{\circ} 51^{\prime} 50^{\prime \prime}$, long 8744'50" referenced to North American Datum of 1927, Clarke County, AL, Hydrologic Unit 03160203, on downstream side of bridge on U.S. Highway 43, 3 mi south of Thomasville.

DRAINAGE AREA. $-10.5 \mathrm{mi}^{2}$.

PERIOD OF RECORD.- October 1995 to March 2014.

PERIOD OF ANALYSIS.--April 1996 to March 2014.

NUMBER OF CLIMATE YEARS IN FREQUENCY ANALYSIS._-18

\section{REMARKS.-}

\begin{tabular}{|c|c|c|c|c|}
\hline $\begin{array}{c}\text { Recurrence } \\
\text { intervals } \\
\text { (years) }\end{array}$ & \multicolumn{2}{|c|}{$\begin{array}{c}\text { Lowest average flow for } \\
\text { indicated number of } \\
\text { consecutive days } \\
\text { (cubic feet per second) }\end{array}$} & \multicolumn{2}{|c|}{$\begin{array}{c}\text { Time-sampling error } \\
\text { (in percent) }\end{array}$} \\
\hline \multicolumn{7}{|c|}{1} & 7 & 1 & 7 \\
\hline 2 & 0.04 & 0.07 & 27 & 27 \\
\hline 5 & 0.01 & 0.02 & 23 & -- \\
\hline 10 & 0.00 & 0.00 & -- & -- \\
\hline 20 & 0.00 & 0.00 & -- & 27 \\
\hline
\end{tabular}

\section{DURATION OF DAILY FLOW}

Flow equaled or exceeded for indicated percentage of time (cubic feet per second)

\begin{tabular}{|c|c|c|c|c|c|c|c|}
\hline Percentage & 5 & 10 & 25 & 50 & 75 & 90 & 95 \\
\hline Flow & 53 & 26 & 9.5 & 3.2 & 0.60 & 0.13 & 0.06 \\
\hline
\end{tabular}


STATION NAME AND NUMBER—02470100 Bassett Creek at Walker Springs, AL

LOCATION.-Lat $31^{\circ} 32^{\prime} 15^{\prime \prime}$, long 8747'24" referenced to North American Datum of 1927, Clarke County, AL, Hydrologic Unit 03160203, on county road, $1,000 \mathrm{ft}$ southeast of Walker Springs, and $2.8 \mathrm{mi}$ upstream from Rabbit Creek.

DRAINAGE AREA.- $-195 \mathrm{mi}^{2}$.

PERIOD OF RECORD.- October 1956 to September 1970.

PERIOD OF ANALYSIS.-April 1957 to March 1970.

NUMBER OF CLIMATE YEARS IN FREQUENCY ANALYSIS.—13

REMARKS.-

\begin{tabular}{|c|c|c|c|c|}
\hline $\begin{array}{l}\text { Recurrence } \\
\text { intervals } \\
\text { (years) }\end{array}$ & \multicolumn{2}{|c|}{$\begin{array}{l}\text { Lowest average flow for } \\
\text { indicated number of } \\
\text { consecutive days } \\
\text { (cubic feet per second) }\end{array}$} & \multicolumn{2}{|c|}{$\begin{array}{l}\text { Time-sampling error } \\
\text { (in percent) }\end{array}$} \\
\hline & 1 & 7 & 1 & 7 \\
\hline 2 & 27 & 29 & 14 & 15 \\
\hline 5 & 20 & 21 & 11 & 12 \\
\hline 10 & 18 & 18 & 11 & 12 \\
\hline 20 & 16 & 16 & 15 & 16 \\
\hline
\end{tabular}

\section{DURATION OF DAILY FLOW}

Flow equaled or exceeded for indicated percentage of time (cubic feet per second)

\begin{tabular}{|c|c|c|c|c|c|c|c|}
\hline Percentage & 5 & 10 & 25 & 50 & 75 & 90 & 95 \\
\hline Flow & 840 & 551 & 297 & 138 & 62 & 36 & 29 \\
\hline
\end{tabular}


STATION NAME AND NUMBER—02471001 Chickasaw Creek near Kushla, AL

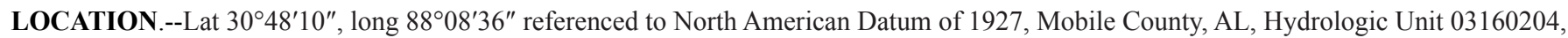
on left bank at downstream side of downstream (eastbound) highway bridge, 0.7 mi upstream of Seabury Creek, 1.4 mi southeast of Kushla, 7 mi northwest of Mobile, and at mile 12.2.

DRAINAGE AREA. $-125 \mathrm{mi}^{2}$.

PERIOD OF RECORD.-October 1951 to March 2014.

PERIOD OF ANALYSIS.-April 1952 to March 2014.

NUMBER OF CLIMATE YEARS IN FREQUENCY ANALYSIS.—62

REMARKS.-Prior to October 1968, published as 02471000, Chickasaw Creek near Whistler, AL.

\begin{tabular}{|c|c|c|c|c|}
\hline \multirow[t]{2}{*}{$\begin{array}{l}\text { Recurrence } \\
\text { intervals } \\
\text { (years) }\end{array}$} & \multicolumn{2}{|c|}{$\begin{array}{l}\text { Lowest average flow for } \\
\text { indicated number of } \\
\text { consecutive days } \\
\text { (cubic feet per second) }\end{array}$} & \multicolumn{2}{|c|}{$\begin{array}{l}\text { Time-sampling error } \\
\text { (in percent) }\end{array}$} \\
\hline & 1 & 7 & 1 & 7 \\
\hline 2 & 53 & 57 & 6 & 6 \\
\hline 5 & 36 & 39 & 7 & 7 \\
\hline 10 & 29 & 32 & 9 & 9 \\
\hline 20 & 24 & 27 & 11 & 11 \\
\hline 50 & 20 & 21 & 15 & 15 \\
\hline
\end{tabular}

\section{DURATION OF DAILY FLOW}

Flow equaled or exceeded for indicated percentage of time (cubic feet per second)

\begin{tabular}{|c|c|c|c|c|c|c|c|}
\hline Percentage & 5 & 10 & 25 & 50 & 75 & 90 & 95 \\
\hline Flow & 800 & 531 & 293 & 161 & 97 & 65 & 50 \\
\hline
\end{tabular}


STATION NAME AND NUMBER—02471065 Montilmar Creek at U.S. Highway 90 at Mobile, AL

LOCATION.--Lat 30³8'59", long 8807'34" referenced to North American Datum of 1927, Mobile County, AL, Hydrologic Unit 03160205, on U.S. Highway 90, 0.1 mi west intersection of Interstate Highway 65 in Mobile.

DRAINAGE AREA. $-7.28 \mathrm{mi}^{2}$.

PERIOD OF RECORD.- - June 1962 to September 1967, October 1974 to September 1983.

PERIOD OF ANALYSIS.-April 1963 to March 1967, April 1975 to March 1983.

NUMBER OF CLIMATE YEARS IN FREQUENCY ANALYSIS.-12

REMARKS.-

\begin{tabular}{|c|c|c|c|c|}
\hline $\begin{array}{l}\text { Recurrence } \\
\text { intervals } \\
\text { (years) }\end{array}$ & \multicolumn{2}{|c|}{$\begin{array}{l}\text { Lowest average flow for } \\
\text { indicated number of } \\
\text { consecutive days } \\
\text { (cubic feet per second) }\end{array}$} & \multicolumn{2}{|c|}{$\begin{array}{l}\text { Time-sampling error } \\
\text { (in percent) }\end{array}$} \\
\hline & 1 & 7 & 1 & 7 \\
\hline 2 & 9.9 & 11 & 13 & 12 \\
\hline 5 & 7.2 & 8.0 & 18 & 15 \\
\hline 10 & 5.7 & 6.5 & 24 & 20 \\
\hline 20 & 4.5 & 5.3 & 35 & 30 \\
\hline
\end{tabular}

\section{DURATION OF DAILY FLOW}

Flow equaled or exceeded for indicated percentage of time (cubic feet per second)

\begin{tabular}{|c|c|c|c|c|c|c|c|}
\hline Percentage & 5 & 10 & 25 & 50 & 75 & 90 & 95 \\
\hline Flow & 60 & 35 & 21 & 17 & 13 & 9.2 & 7.2 \\
\hline
\end{tabular}


STATION NAME AND NUMBER—02471078 Fowl River at Half Mile Road near Laurendine, AL

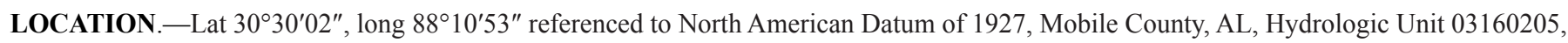
at bridge on Half Mile Road about 1 mi west of Laurendine.

DRAINAGE AREA.- $-16.5 \mathrm{mi}^{2}$.

PERIOD OF RECORD.-March 1995 to March 2014.

PERIOD OF ANALYSIS.-April 1995 to March 2014.

NUMBER OF CLIMATE YEARS IN FREQUENCY ANALYSIS.—12

REMARKS.-

\begin{tabular}{|c|c|c|c|c|}
\hline $\begin{array}{l}\text { Recurrence } \\
\text { intervals } \\
\text { (years) }\end{array}$ & \multicolumn{2}{|c|}{$\begin{array}{l}\text { Lowest average flow for } \\
\text { indicated number of } \\
\text { consecutive days } \\
\text { (cubic feet per second) }\end{array}$} & \multicolumn{2}{|c|}{$\begin{array}{l}\text { Time-sampling error } \\
\text { (in percent) }\end{array}$} \\
\hline & 1 & 7 & 1 & 7 \\
\hline 2 & 16 & 16 & 6 & 6 \\
\hline 5 & 13 & 13 & 7 & 6 \\
\hline 10 & 11 & 12 & 8 & 7 \\
\hline 20 & 10 & 11 & 10 & 9 \\
\hline
\end{tabular}

\section{DURATION OF DAILY FLOW}

Flow equaled or exceeded for indicated percentage of time (cubic feet per second)

\begin{tabular}{|c|c|c|c|c|c|c|c|}
\hline Percentage & 5 & 10 & 25 & 50 & 75 & 90 & 95 \\
\hline Flow & 78 & 50 & 33 & 25 & 20 & 16 & 14 \\
\hline
\end{tabular}


STATION NAME AND NUMBER—02479431 Pond Creek near Deer Park, AL

LOCATION.--Lat $31^{\circ} 09^{\prime} 39^{\prime \prime}$, long 88 $21^{\prime} 43^{\prime \prime}$ referenced to North American Datum of 1927, Washington County, AL, Hydrologic Unit 03170008, on county road, $1.3 \mathrm{mi}$ upstream from mouth, and 5 mi southwest of Deer Park.

DRAINAGE AREA. $-20.4 \mathrm{mi}^{2}$.

PERIOD OF RECORD.- - October 1976 to September 1999.

PERIOD OF ANALYSIS.-April 1977 to March 1999.

NUMBER OF CLIMATE YEARS IN FREQUENCY ANALYSIS.—-22

REMARKS.-

\begin{tabular}{|c|c|c|c|c|}
\hline $\begin{array}{l}\text { Recurrence } \\
\text { intervals } \\
\text { (years) }\end{array}$ & \multicolumn{2}{|c|}{$\begin{array}{l}\text { Lowest average flow for } \\
\text { indicated number of } \\
\text { consecutive days } \\
\text { (cubic feet per second) }\end{array}$} & \multicolumn{2}{|c|}{$\begin{array}{l}\text { Time-sampling error } \\
\text { (in percent) }\end{array}$} \\
\hline & 1 & 7 & 1 & 7 \\
\hline 2 & 0.27 & 0.34 & 18 & 18 \\
\hline 5 & 0.14 & 0.18 & 18 & 17 \\
\hline 10 & 0.10 & 0.14 & 20 & 19 \\
\hline 20 & 0.08 & 0.11 & 24 & 23 \\
\hline
\end{tabular}

\section{DURATION OF DAILY FLOW}

Flow equaled or exceeded for indicated percentage of time (cubic feet per second)

\begin{tabular}{|c|c|c|c|c|c|c|c|}
\hline Percentage & 5 & 10 & 25 & 50 & 75 & 90 & 95 \\
\hline Flow & 142 & 88 & 40 & 14 & 3.2 & 0.82 & 0.44 \\
\hline
\end{tabular}


STATION NAME AND NUMBER—02479500 Escatawpa River near Wilmer, AL

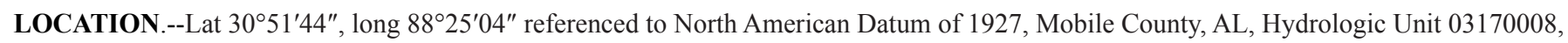
on U.S. Highway 98, 0.5 mi upstream from Rocky Creek, and 4 mi northwest of Wilmer.

DRAINAGE AREA. $-511 \mathrm{mi}^{2}$.

PERIOD OF RECORD.-October 1945 to September 1973.

PERIOD OF ANALYSIS.-April 1946 to March 1973.

NUMBER OF CLIMATE YEARS IN FREQUENCY ANALYSIS.—27

REMARKS.-

\begin{tabular}{|c|c|c|c|c|}
\hline $\begin{array}{l}\text { Recurrence } \\
\text { intervals } \\
\text { (years) }\end{array}$ & \multicolumn{2}{|c|}{$\begin{array}{l}\text { Lowest average flow for } \\
\text { indicated number of } \\
\text { consecutive days } \\
\text { (cubic feet per second) }\end{array}$} & \multicolumn{2}{|c|}{$\begin{array}{l}\text { Time-sampling error } \\
\text { (in percent) }\end{array}$} \\
\hline & 1 & 7 & 1 & 7 \\
\hline 2 & 91 & 100 & 9 & 9 \\
\hline 5 & 64 & 69 & 10 & 10 \\
\hline 10 & 53 & 57 & 11 & 12 \\
\hline 20 & 46 & 48 & 14 & 15 \\
\hline
\end{tabular}

DURATION OF DAILY FLOW

Flow equaled or exceeded for indicated percentage of time (cubic feet per second)

\begin{tabular}{|c|c|c|c|c|c|c|c|}
\hline Percentage & 5 & 10 & 25 & 50 & 75 & 90 & 95 \\
\hline Flow & 3,320 & 2,130 & 1,020 & 452 & 193 & 120 & 93 \\
\hline
\end{tabular}


STATION NAME AND NUMBER—02479560 Escatawpa River near Agricola, AL

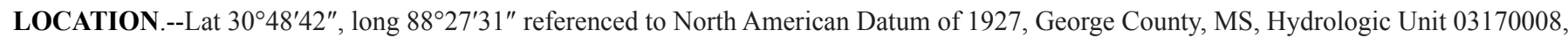
near left bank on downstream side of bridge on County Road 612, 2.5 mi west of Alabama-Mississippi State line, 3.7 mi east of Agricola, MS, $4.8 \mathrm{mi}$ downstream of old gage at Escatawpa River near Wilmer, AL, and 6.7 mi west of Wilmer.

DRAINAGE AREA. $-562 \mathrm{mi}^{2}$.

PERIOD OF RECORD.-October 1973 to March 2014.

PERIOD OF ANALYSIS.-April 1974 to March 2014.

NUMBER OF CLIMATE YEARS IN FREQUENCY ANALYSIS.—40

\section{REMARKS.-}

\begin{tabular}{|c|c|c|c|c|}
\hline $\begin{array}{c}\text { Recurrence } \\
\text { intervals } \\
\text { (years) }\end{array}$ & \multicolumn{2}{|c|}{$\begin{array}{l}\text { Lowest average flow for } \\
\text { indicated number of } \\
\text { consecutive days } \\
\text { (cubic feet per second) }\end{array}$} & \multicolumn{2}{|c|}{$\begin{array}{l}\text { Time-sampling error } \\
\text { (in percent) }\end{array}$} \\
\hline & 1 & 7 & 1 & 7 \\
\hline 2 & 129 & 134 & 6 & 7 \\
\hline 5 & 97 & 101 & 6 & 6 \\
\hline 10 & 85 & 89 & 6 & 6 \\
\hline 20 & 76 & 82 & 8 & 8 \\
\hline 50 & 68 & 75 & 10 & 11 \\
\hline
\end{tabular}

\section{DURATION OF DAILY FLOW}

Flow equaled or exceeded for indicated percentage of time (cubic feet per second)

\begin{tabular}{|c|c|c|c|c|c|c|c|}
\hline Percentage & 5 & 10 & 25 & 50 & 75 & 90 & 95 \\
\hline Flow & 3,860 & 2,550 & 1,260 & 565 & 271 & 165 & 131 \\
\hline
\end{tabular}


STATION NAME AND NUMBER—02479945 Big Creek at County Road 63 near Wilmer, AL

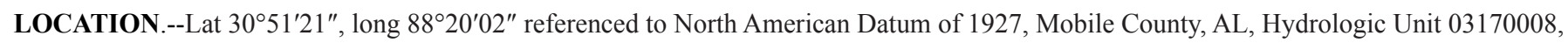
on downstream side of bridge at County Road 63, about 2.9 mi northeast of Wilmer, and 3.2 mi north of U.S. Highway 98.

DRAINAGE AREA. $-31.5 \mathrm{mi}^{2}$.

PERIOD OF RECORD.-- June 1990 to March 2014.

PERIOD OF ANALYSIS.-April 1991 to March 2014.

NUMBER OF CLIMATE YEARS IN FREQUENCY ANALYSIS.—23

REMARKS.-

\begin{tabular}{|c|c|c|c|c|}
\hline $\begin{array}{l}\text { Recurrence } \\
\text { intervals } \\
\text { (years) }\end{array}$ & \multicolumn{2}{|c|}{$\begin{array}{l}\text { Lowest average flow for } \\
\text { indicated number of } \\
\text { consecutive days } \\
\text { (cubic feet per second) }\end{array}$} & \multicolumn{2}{|c|}{$\begin{array}{l}\text { Time-sampling error } \\
\text { (in percent) }\end{array}$} \\
\hline & 1 & 7 & 1 & 7 \\
\hline 2 & 15 & 16 & 9 & 9 \\
\hline 5 & 11 & 12 & 11 & 10 \\
\hline 10 & 8.9 & 9.6 & 13 & 13 \\
\hline 20 & 7.5 & 8.1 & 17 & 17 \\
\hline
\end{tabular}

\section{DURATION OF DAILY FLOW}

Flow equaled or exceeded for indicated percentage of time (cubic feet per second)

\begin{tabular}{|c|c|c|c|c|c|c|c|}
\hline Percentage & 5 & 10 & 25 & 50 & 75 & 90 & 95 \\
\hline Flow & 180 & 118 & 59 & 36 & 23 & 16 & 13 \\
\hline
\end{tabular}


STATION NAME AND NUMBER—02479980 Crooked Creek near Fairview, AL

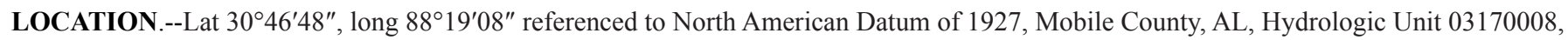
on right bank 1 mi southwest of Fairview, and 4 mi southeast of Wilmer.

DRAINAGE AREA. $-8.08 \mathrm{mi}^{2}$.

PERIOD OF RECORD.- - June 1990 to March 2014.

PERIOD OF ANALYSIS.-April 1991 to March 2014.

NUMBER OF CLIMATE YEARS IN FREQUENCY ANALYSIS.—23

REMARKS.-

\begin{tabular}{|c|c|c|c|c|}
\hline $\begin{array}{l}\text { Recurrence } \\
\text { intervals } \\
\text { (years) }\end{array}$ & \multicolumn{2}{|c|}{$\begin{array}{l}\text { Lowest average flow for } \\
\text { indicated number of } \\
\text { consecutive days } \\
\text { (cubic feet per second) }\end{array}$} & \multicolumn{2}{|c|}{$\begin{array}{l}\text { Time-sampling error } \\
\text { (in percent) }\end{array}$} \\
\hline & 1 & 7 & 1 & 7 \\
\hline 2 & 6.7 & 7.1 & 6 & 5 \\
\hline 5 & 5.4 & 5.8 & 7 & 6 \\
\hline 10 & 4.8 & 5.1 & 9 & 8 \\
\hline 20 & 4.3 & 4.6 & 11 & 10 \\
\hline
\end{tabular}

\section{DURATION OF DAILY FLOW}

Flow equaled or exceeded for indicated percentage of time (cubic feet per second)

\begin{tabular}{|c|c|c|c|c|c|c|c|}
\hline Percentage & 5 & 10 & 25 & 50 & 75 & 90 & 95 \\
\hline Flow & 39 & 23 & 15 & 11 & 8.6 & 6.9 & 6.0 \\
\hline
\end{tabular}


STATION NAME AND NUMBER—02480002 Hamilton Creek at Snow Road near Semmes, AL

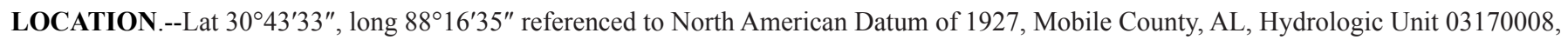
on right bank about 60 feet downstream of bridge on Snow Road, 1.0 mi north of Tanner Williams Road, and 4 mi south of Semmes.

DRAINAGE AREA. $-8.22 \mathrm{mi}^{2}$.

PERIOD OF RECORD.- June 1990 to March 2014.

PERIOD OF ANALYSIS.-April 1991 to March 2014.

NUMBER OF CLIMATE YEARS IN FREQUENCY ANALYSIS.—23

\section{REMARKS.-}

\begin{tabular}{|c|c|c|c|c|}
\hline $\begin{array}{l}\text { Recurrence } \\
\text { intervals } \\
\text { (years) }\end{array}$ & \multicolumn{2}{|c|}{$\begin{array}{l}\text { Lowest average flow for } \\
\text { indicated number of } \\
\text { consecutive days } \\
\text { (cubic feet per second) }\end{array}$} & \multicolumn{2}{|c|}{$\begin{array}{l}\text { Time-sampling error } \\
\text { (in percent) }\end{array}$} \\
\hline & 1 & 7 & 1 & 7 \\
\hline 2 & 9.8 & 10 & 4 & 4 \\
\hline 5 & 8.3 & 8.7 & 5 & 5 \\
\hline 10 & 7.6 & 7.9 & 6 & 6 \\
\hline 20 & 7.1 & 7.3 & 7 & 8 \\
\hline
\end{tabular}

\section{DURATION OF DAILY FLOW}

Flow equaled or exceeded for indicated percentage of time (cubic feet per second)

\begin{tabular}{|c|c|c|c|c|c|c|c|}
\hline Percentage & 5 & 10 & 25 & 50 & 75 & 90 & 95 \\
\hline Flow & 44 & 29 & 20 & 16 & 12 & 11 & 9.8 \\
\hline
\end{tabular}


STATION NAME AND NUMBER—03572110 Crow Creek at Bass, AL

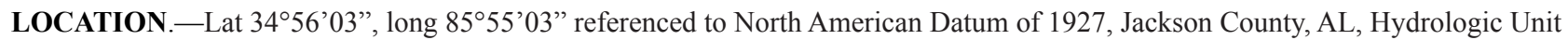
06030001, on downstream side of bridge on Alabama Highway 117, 1,500 ft northwest of Bass, 1 mi upstream from Bennett Cove Creek, 3.7 mi south of Ala-Tenn State line, river mile 15.8.

DRAINAGE AREA.-131 $\mathrm{mi}^{2}$.

PERIOD OF RECORD.-May 1975 to October 1996.

PERIOD OF ANALYSIS.-April 1976 to March 1996.

NUMBER OF CLIMATE YEARS IN FREQUENCY ANALYSIS.—-20

\section{REMARKS.-}

\begin{tabular}{|c|c|c|c|c|}
\hline $\begin{array}{l}\text { Recurrence } \\
\text { intervals } \\
\text { (years) }\end{array}$ & \multicolumn{2}{|c|}{$\begin{array}{l}\text { Lowest average flow for } \\
\text { indicated number of } \\
\text { consecutive days } \\
\text { (cubic feet per second) }\end{array}$} & \multicolumn{2}{|c|}{$\begin{array}{l}\text { Time-sampling error } \\
\text { (in percent) }\end{array}$} \\
\hline & 1 & 7 & 1 & 7 \\
\hline 2 & 4.9 & 5.8 & 17 & 17 \\
\hline 5 & 2.9 & 3.4 & 15 & 15 \\
\hline 10 & 2.3 & 2.7 & 15 & 16 \\
\hline 20 & 2.0 & 2.2 & 19 & 20 \\
\hline
\end{tabular}

\begin{tabular}{|c|c|c|c|c|c|c|c|c|}
\hline \multicolumn{7}{|c|}{ DURATION OF DAILY FLOW } \\
\hline \multicolumn{7}{|c|}{ Flow equaled or exceeded for indicated percentage of time } \\
(cubic feet per second)
\end{tabular}


STATION NAME AND NUMBER—03572900 Town Creek near Geraldine, AL

LOCATION.-Lat 34²2’42”, long 85'59'25” referenced to North American Datum of 1927, DeKalb County, AL, Hydrologic Unit 06030001, on State Highway 75, 0.3 mi downstream from Reedy Creek, 2 mi north-northeast of Geraldine, and at mile 20.4.

DRAINAGE AREA.-141 $\mathrm{mi}^{2}$.

PERIOD OF RECORD.- October 1957 to September 1980.

PERIOD OF ANALYSIS.--April 1958 to March 1980.

NUMBER OF CLIMATE YEARS IN FREQUENCY ANALYSIS.—-22

REMARKS.-

\begin{tabular}{|c|c|c|c|c|}
\hline $\begin{array}{l}\text { Recurrence } \\
\text { intervals } \\
\text { (years) }\end{array}$ & \multicolumn{2}{|c|}{$\begin{array}{l}\text { Lowest average flow for } \\
\text { indicated number of } \\
\text { consecutive days } \\
\text { (cubic feet per second) }\end{array}$} & \multicolumn{2}{|c|}{$\begin{array}{l}\text { Time-sampling error } \\
\text { (in percent) }\end{array}$} \\
\hline & 1 & 7 & 1 & 7 \\
\hline 2 & 0.49 & 0.82 & 36 & 38 \\
\hline 5 & 0.10 & 0.15 & 33 & 41 \\
\hline 10 & 0.00 & 0.03 & -- & 49 \\
\hline 20 & 0.00 & 0.00 & -- & -- \\
\hline
\end{tabular}

DURATION OF DAILY FLOW

Flow equaled or exceeded for indicated percentage of time (cubic feet per second)

\begin{tabular}{|c|c|c|c|c|c|c|c|}
\hline Percentage & 5 & 10 & 25 & 50 & 75 & 90 & 95 \\
\hline Flow & 992 & 635 & 331 & 120 & 17 & 3.0 & 1.0 \\
\hline
\end{tabular}


STATION NAME AND NUMBER—03574500 Paint Rock River near Woodville, AL

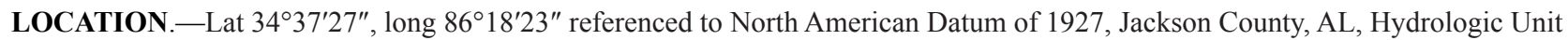
06030002, NW 1/4 sec. 10, T.5S., R.3E., on downstream side of bridge on U.S. Highway 72, 1,000 ft downstream from Southern Railway bridge, 2 mi west of Woodville, 4.1 mi upstream from Little Paint Creek, and at mile 26.6.

DRAINAGE AREA. $-320 \mathrm{mi}^{2}$.

PERIOD OF RECORD.-January 1936 to March 2014.

PERIOD OF ANALYSIS.-April 1936 to March 2014.

NUMBER OF CLIMATE YEARS IN FREQUENCY ANALYSIS.—78

REMARKS.-

\begin{tabular}{|c|c|c|c|c|}
\hline $\begin{array}{c}\text { Recurrence } \\
\text { intervals } \\
\text { (years) }\end{array}$ & \multicolumn{2}{|c|}{$\begin{array}{l}\text { Lowest average flow for } \\
\text { indicated number of } \\
\text { consecutive days } \\
\text { (cubic feet per second) }\end{array}$} & \multicolumn{2}{|c|}{$\begin{array}{l}\text { Time-sampling error } \\
\text { (in percent) }\end{array}$} \\
\hline & 1 & 7 & 1 & 7 \\
\hline 2 & 13 & 15 & 8 & 8 \\
\hline 5 & 7.6 & 8.3 & 9 & 9 \\
\hline 10 & 5.5 & 6.1 & 12 & 12 \\
\hline 20 & 4.1 & 4.6 & 16 & 15 \\
\hline 50 & 2.9 & 3.3 & 22 & 21 \\
\hline
\end{tabular}

\section{DURATION OF DAILY FLOW}

Flow equaled or exceeded for indicated percentage of time (cubic feet per second)

\begin{tabular}{|c|c|c|c|c|c|c|c|}
\hline Percentage & 5 & 10 & 25 & 50 & 75 & 90 & 95 \\
\hline Flow & 2,860 & 1,600 & 660 & 217 & 49 & 21 & 13 \\
\hline
\end{tabular}


STATION NAME AND NUMBER—0357479650 Hester Creek near Plevna, AL

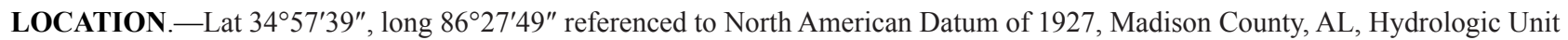
06030002, on right bank on Buddy Williamson Road, 2.7 mi east of Plevna, 3.8 mi northwest of New Market, and 2 mi south of AlabamaTennessee State line.

DRAINAGE AREA. $-33.0 \mathrm{mi}^{2}$.

PERIOD OF RECORD.- October 1998 to September 2013.

PERIOD OF ANALYSIS.-April 1999 to March 2013.

NUMBER OF CLIMATE YEARS IN FREQUENCY ANALYSIS.-14

REMARKS.-

\begin{tabular}{|c|c|c|c|c|}
\hline $\begin{array}{l}\text { Recurrence } \\
\text { intervals } \\
\text { (years) }\end{array}$ & \multicolumn{2}{|c|}{$\begin{array}{l}\text { Lowest average flow for } \\
\text { indicated number of } \\
\text { consecutive days } \\
\text { (cubic feet per second) }\end{array}$} & \multicolumn{2}{|c|}{$\begin{array}{l}\text { Time-sampling error } \\
\text { (in percent) }\end{array}$} \\
\hline & 1 & 7 & 1 & 7 \\
\hline 2 & 2.2 & 2.4 & 12 & 11 \\
\hline 5 & 1.6 & 1.8 & 12 & 11 \\
\hline 10 & 1.3 & 1.6 & 13 & 12 \\
\hline 20 & 1.2 & 1.4 & 16 & 14 \\
\hline
\end{tabular}

\begin{tabular}{|c|c|c|c|c|c|c|c|}
\hline \multicolumn{7}{|c|}{ DURATION OF DAILY FLOW } \\
\hline \multicolumn{7}{|c|}{ Flow equaled or exceeded for indicated percentage of time } \\
(cubic feet per second)
\end{tabular}


STATION NAME AND NUMBER—03575000 Flint River near Chase, AL

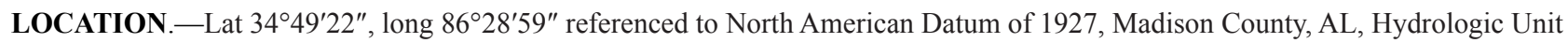
06030002, on Winchester Road, $400 \mathrm{ft}$ downstream from Brier Fork, 4.3 mi northeast of Chase, and at mile 36.2

DRAINAGE AREA.-342 $\mathrm{mi}^{2}$.

PERIOD OF RECORD.-May 1930 to September 1981, October 1982 to December 1994.

PERIOD OF ANALYSIS.-April 1931 to March 1981, April 1983 to March 1994.

NUMBER OF CLIMATE YEARS IN FREQUENCY ANALYSIS.-61

REMARKS.-

\begin{tabular}{|c|c|c|c|c|}
\hline $\begin{array}{l}\text { Recurrence } \\
\text { intervals } \\
\text { (years) }\end{array}$ & \multicolumn{2}{|c|}{$\begin{array}{l}\text { Lowest average flow for } \\
\text { indicated number of } \\
\text { consecutive days } \\
\text { (cubic feet per second) }\end{array}$} & \multicolumn{2}{|c|}{$\begin{array}{l}\text { Time-sampling error } \\
\text { (in percent) }\end{array}$} \\
\hline & 1 & 7 & 1 & 7 \\
\hline 2 & 82 & 85 & 2 & 2 \\
\hline 5 & 70 & 74 & 3 & 3 \\
\hline 10 & 64 & 69 & 4 & 3 \\
\hline 20 & 60 & 64 & 5 & 4 \\
\hline 50 & 54 & 60 & 6 & 5 \\
\hline
\end{tabular}

\section{DURATION OF DAILY FLOW}

Flow equaled or exceeded for indicated percentage of time (cubic feet per second)

\begin{tabular}{|c|c|c|c|c|c|c|c|}
\hline Percentage & 5 & 10 & 25 & 50 & 75 & 90 & 95 \\
\hline Flow & 1,840 & 1,110 & 542 & 239 & 126 & 95 & 84 \\
\hline
\end{tabular}


STATION NAME AND NUMBER—03575100 Flint River at Brownsboro, AL

LOCATION.-Lat 3444'57", long 86²6'48" referenced to North American Datum of 1927, Madison County, AL, Hydrologic Unit 06030002, on right bank on Brownsboro Road, 0.3 mi north of U.S. Highway 72, 10 mi east of Huntsville, at Brownsboro, and river mile 27.6.

DRAINAGE AREA. $-375 \mathrm{mi}^{2}$.

PERIOD OF RECORD.--October 1998 to March 2014.

PERIOD OF ANALYSIS.-April 1999 to March 2014.

NUMBER OF CLIMATE YEARS IN FREQUENCY ANALYSIS.—15

\section{REMARKS.-}

\begin{tabular}{|c|c|c|c|c|}
\hline $\begin{array}{l}\text { Recurrence } \\
\text { intervals } \\
\text { (years) }\end{array}$ & \multicolumn{2}{|c|}{$\begin{array}{l}\text { Lowest average flow for } \\
\text { indicated number of } \\
\text { consecutive days } \\
\text { (cubic feet per second) }\end{array}$} & \multicolumn{2}{|c|}{$\begin{array}{l}\text { Time-sampling error } \\
\text { (in percent) }\end{array}$} \\
\hline & 1 & 7 & 1 & 7 \\
\hline 2 & 83 & 87 & 9 & 9 \\
\hline 5 & 67 & 70 & 7 & 7 \\
\hline 10 & 61 & 64 & 7 & 7 \\
\hline 20 & 57 & 60 & 10 & 10 \\
\hline
\end{tabular}

\section{DURATION OF DAILY FLOW}

Flow equaled or exceeded for indicated percentage of time (cubic feet per second)

\begin{tabular}{|c|c|c|c|c|c|c|c|}
\hline Percentage & 5 & 10 & 25 & 50 & 75 & 90 & 95 \\
\hline Flow & 1,910 & 1,200 & 631 & 281 & 136 & 95 & 85 \\
\hline
\end{tabular}


STATION NAME AND NUMBER—03575500 Tennessee River at Whitesburg, AL

LOCATION.-Lat 34³4'18", long 86³3'29" referenced to North American Datum of 1927, Madison County, AL, Hydrologic Unit 06030002, at Whitesburg, on right bank 2,500 ft upstream from Aldridge Creek, 3,000 ft upstream from Clement C. Clay Bridge on U.S. Highway 231, $11.0 \mathrm{mi}$ south of Huntsville, $15.1 \mathrm{mi}$ downstream from Guntersville Dam, and at mile 333.9.

DRAINAGE AREA. $-26,610 \mathrm{mi}^{2}$.

PERIOD OF RECORD.- October 1924 to September 1995, October 2002 to September 2003, October 2004 to September 2005.

PERIOD OF ANALYSIS.-April 1925 to March 1936.

NUMBER OF CLIMATE YEARS IN FREQUENCY ANALYSIS.-11

REMARKS.- Since 1936, flow regulated by increasing number of reservoirs above stations. Due to uncertainty in changing regulation patterns over time, the low-flow frequency statistics are providing for the pre-regulation period only.

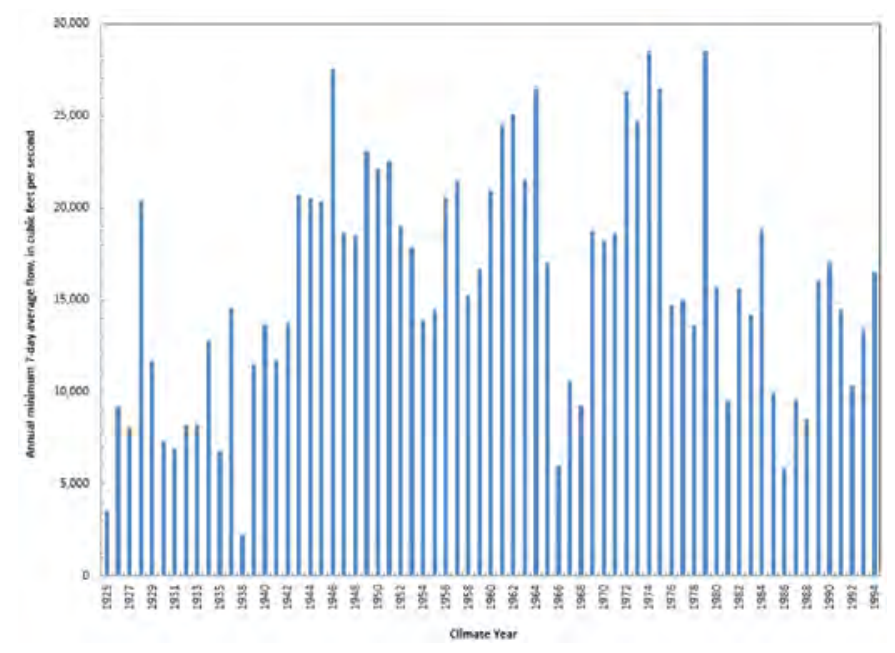

\begin{tabular}{|c|c|c|c|c|}
\hline $\begin{array}{l}\text { Recurrence } \\
\text { intervals } \\
\text { (years) }\end{array}$ & \multicolumn{2}{|c|}{$\begin{array}{l}\text { Lowest average flow for } \\
\text { indicated number of } \\
\text { consecutive days } \\
\text { (cubic feet per second) }\end{array}$} & \multicolumn{2}{|c|}{$\begin{array}{l}\text { Time-sampling error } \\
\text { (in percent) }\end{array}$} \\
\hline & 1 & 7 & 1 & 7 \\
\hline 2 & 7,930 & 8,570 & 14 & 14 \\
\hline 5 & 5,590 & 5,920 & 14 & 16 \\
\hline 10 & 4,690 & 4,880 & 16 & 18 \\
\hline 20 & 4,080 & 4,170 & 20 & 22 \\
\hline
\end{tabular}

\begin{tabular}{|c|c|c|c|c|c|c|c|}
\hline \multicolumn{1}{|c|}{ DURATION OF DAILY FLOW } \\
\hline \multicolumn{1}{|c|}{ Flow equaled or exceeded for indicated percentage of time } \\
(cubic feet per second)
\end{tabular}


STATION NAME AND NUMBER—03575830 Indian Creek near Madison, AL

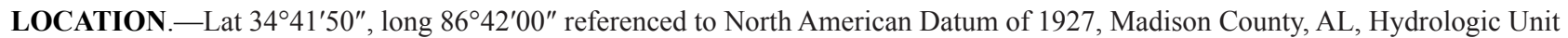
06030002, on downstream side of bridge on State Highway 20, $0.3 \mathrm{mi}$ downstream from Southern Railroad bridge, 2.8 mi east of Madison, and 5.8 mi upstream from mouth.

DRAINAGE AREA. $-49.0 \mathrm{mi}^{2}$.

PERIOD OF RECORD.- October 1959 to September 1966, October 1975 to June 2002.

PERIOD OF ANALYSIS._April 1960 to March 1966, April 1976 to March 2002.

NUMBER OF CLIMATE YEARS IN FREQUENCY ANALYSIS.—32

REMARKS.-

\begin{tabular}{|c|c|c|c|c|}
\hline \multirow[t]{2}{*}{$\begin{array}{l}\text { Recurrence } \\
\text { intervals } \\
\text { (years) }\end{array}$} & \multicolumn{2}{|c|}{$\begin{array}{l}\text { Lowest average flow for } \\
\text { indicated number of } \\
\text { consecutive days } \\
\text { (cubic feet per second) }\end{array}$} & \multicolumn{2}{|c|}{$\begin{array}{l}\text { Time-sampling error } \\
\text { (in percent) }\end{array}$} \\
\hline & 1 & 7 & 1 & 7 \\
\hline 2 & 3.7 & 4.2 & 11 & 11 \\
\hline 5 & 2.2 & 2.6 & 14 & 14 \\
\hline 10 & 1.6 & 1.9 & 19 & 18 \\
\hline 20 & 1.2 & 1.4 & 26 & 25 \\
\hline 50 & 0.84 & 1.0 & 38 & 37 \\
\hline
\end{tabular}

\section{DURATION OF DAILY FLOW}

Flow equaled or exceeded for indicated percentage of time (cubic feet per second)

\begin{tabular}{|c|c|c|c|c|c|c|c|}
\hline Percentage & 5 & 10 & 25 & 50 & 75 & 90 & 95 \\
\hline Flow & 250 & 147 & 71 & 26 & 8.6 & 5.0 & 3.8 \\
\hline
\end{tabular}


STATION NAME AND NUMBER—03576148 Cotaco Creek at Florette, AL

LOCATION.-Lat 3424'49", long 86²1'16" referenced to North American Datum of 1927, Morgan County, AL, Hydrologic Unit 06030002, on county road, 0.9 mi east of Florette, 1 mi upstream from Sixmile Creek, and 3.1 mi upstream from Wheeler Lake boundary.

DRAINAGE AREA.-136 $\mathrm{mi}^{2}$.

PERIOD OF RECORD.- - October 1965 to September 1980.

PERIOD OF ANALYSIS.-April 1966 to March 1980.

NUMBER OF CLIMATE YEARS IN FREQUENCY ANALYSIS._-14

REMARKS.-

\begin{tabular}{|c|c|c|c|c|}
\hline $\begin{array}{l}\text { Recurrence } \\
\text { intervals } \\
\text { (years) }\end{array}$ & \multicolumn{2}{|c|}{$\begin{array}{l}\text { Lowest average flow for } \\
\text { indicated number of } \\
\text { consecutive days } \\
\text { (cubic feet per second) }\end{array}$} & \multicolumn{2}{|c|}{$\begin{array}{l}\text { Time-sampling error } \\
\text { (in percent) }\end{array}$} \\
\hline & 1 & 7 & 1 & 7 \\
\hline 2 & 1.4 & 1.7 & 35 & 34 \\
\hline 5 & 0.50 & 0.65 & 40 & 38 \\
\hline 10 & 0.28 & 0.38 & 51 & 46 \\
\hline 20 & 0.18 & 0.25 & 67 & 58 \\
\hline
\end{tabular}

\section{DURATION OF DAILY FLOW}

Flow equaled or exceeded for indicated percentage of time (cubic feet per second)

\begin{tabular}{|c|c|c|c|c|c|c|c|}
\hline Percentage & 5 & 10 & 25 & 50 & 75 & 90 & 95 \\
\hline Flow & 1,130 & 715 & 304 & 96 & 15 & 3.4 & 1.7 \\
\hline
\end{tabular}


STATION NAME AND NUMBER—03576250 Limestone Creek near Athens, AL

LOCATION.-Lat 3445'06", long 86 49'24" referenced to North American Datum of 1927, Limestone County, AL, Hydrologic Unit 06030002, on downstream side of U.S. Highway 72 bridge, 9 mi east of Athens, 12 mi west of Huntsville, and at mile 17.0.

DRAINAGE AREA.-119 $\mathrm{mi}^{2}$.

PERIOD OF RECORD.- October 1939 to September 1970, October 1994 to March 2014.

PERIOD OF ANALYSIS.-April 1940 to March 1970, April 1995 to March 2014.

NUMBER OF CLIMATE YEARS IN FREQUENCY ANALYSIS.—49

\section{REMARKS.-}

\begin{tabular}{|c|c|c|c|c|}
\hline \multirow[t]{2}{*}{$\begin{array}{l}\text { Recurrence } \\
\text { intervals } \\
\text { (years) }\end{array}$} & \multicolumn{2}{|c|}{$\begin{array}{l}\text { Lowest average flow for } \\
\text { indicated number of } \\
\text { consecutive days } \\
\text { (cubic feet per second) }\end{array}$} & \multicolumn{2}{|c|}{$\begin{array}{l}\text { Time-sampling error } \\
\text { (in percent) }\end{array}$} \\
\hline & 1 & 7 & 1 & 7 \\
\hline 2 & 14 & 15 & 5 & 5 \\
\hline 5 & 11 & 12 & 5 & 5 \\
\hline 10 & 9.6 & 10 & 6 & 6 \\
\hline 20 & 8.7 & 9.3 & 7 & 7 \\
\hline 50 & 7.7 & 8.3 & 9 & 9 \\
\hline
\end{tabular}

\section{DURATION OF DAILY FLOW}

Flow equaled or exceeded for indicated percentage of time (cubic feet per second)

\begin{tabular}{|c|c|c|c|c|c|c|c|}
\hline Percentage & 5 & 10 & 25 & 50 & 75 & 90 & 95 \\
\hline Flow & 674 & 414 & 200 & 73 & 29 & 18 & 15 \\
\hline
\end{tabular}


STATION NAME AND NUMBER—03576500 Flint Creek near Falkville, AL

LOCATION.-Lat $34^{\circ} 22^{\prime} 23^{\prime \prime}$, long $86^{\circ} 56^{\prime} 01^{\prime \prime}$ referenced to North American Datum of 1927, Morgan County, AL, Hydrologic Unit 06030002, near left bank on downstream side of bridge on county road 55, 1.2 mi downstream from Robinson Creek, 1.5 mi west of Falkville, and 2.8 mi upstream from Cedar Creek.

DRAINAGE AREA. $-86.3 \mathrm{mi}^{2}$.

PERIOD OF RECORD.-August 1952 to September 1970, October 1992 to September 1999, October 2011 to March 2014.

PERIOD OF ANALYSIS.-April 1953 to March 1970, April 1993 to March 1999, April 2012 to March 2014.

NUMBER OF CLIMATE YEARS IN FREQUENCY ANALYSIS. - 25

\section{REMARKS.-}

\begin{tabular}{|c|c|c|c|c|}
\hline $\begin{array}{c}\text { Recurrence } \\
\text { intervals } \\
\text { (years) }\end{array}$ & \multicolumn{2}{|c|}{$\begin{array}{c}\text { Lowest average flow for } \\
\text { indicated number of } \\
\text { consecutive days } \\
\text { (cubic feet per second) }\end{array}$} & \multicolumn{2}{|c|}{$\begin{array}{c}\text { Time-sampling error } \\
\text { (in percent) }\end{array}$} \\
\hline \multicolumn{7}{|c|}{1} & 7 & 1 & 73 \\
\hline 2 & 0.12 & 0.18 & 34 & -- \\
\hline 5 & 0.00 & 0.00 & -- & -- \\
\hline 10 & 0.00 & 0.00 & -- & -- \\
\hline 20 & 0.00 & 0.00 & -- & \\
\hline
\end{tabular}

\begin{tabular}{|c|c|c|c|c|c|c|c|}
\hline \multicolumn{7}{|c|}{ DURATION OF DAILY FLOW } \\
\hline \multicolumn{7}{|c|}{ Flow equaled or exceeded for indicated percentage of time } \\
(cubic feet per second)
\end{tabular}


STATION NAME AND NUMBER—03585300 Sugar Creek near Good Springs, AL

LOCATION.-Lat $34^{\circ} 56^{\prime} 40^{\prime \prime}$, long $87^{\circ} 09^{\prime} 20^{\prime \prime}$ referenced to North American Datum of 1927, Limestone County, AL, Hydrologic Unit 06030004, on State Highway 99, 0.2 mi downstream from Bridgeforth Branch, 2.2 mi east of Good Springs, 2.4 mi upstream from Dobbins Branch, and at mile 8.1 .

DRAINAGE AREA. $-152 \mathrm{mi}^{2}$.

PERIOD OF RECORD.- October 1957 to September 1969.

PERIOD OF ANALYSIS.-April 1958 to March 1969.

NUMBER OF CLIMATE YEARS IN FREQUENCY ANALYSIS.-11

REMARKS.-

\begin{tabular}{|c|c|c|c|c|}
\hline $\begin{array}{c}\text { Recurrence } \\
\text { intervals } \\
\text { (years) }\end{array}$ & \multicolumn{2}{|c|}{$\begin{array}{c}\text { Lowest average flow for } \\
\text { indicated number of } \\
\text { consecutive days } \\
\text { (cubic feet per second) }\end{array}$} & \multicolumn{2}{|c|}{$\begin{array}{c}\text { Time-sampling error } \\
\text { (in percent) }\end{array}$} \\
\hline \multicolumn{7}{|c|}{1} & 7 & 1 & 7 \\
\hline 2 & 26 & 28 & 11 & 6 \\
\hline 5 & 23 & 25 & 5 & 13 \\
\hline 10 & 22 & 24 & 12 & 19 \\
\hline 20 & 22 & 24 & 18 & \\
\hline
\end{tabular}

\begin{tabular}{|c|c|c|c|c|c|c|c|}
\hline \multicolumn{7}{|c|}{ DURATION OF DAILY FLOW } \\
\hline \multicolumn{7}{|c|}{ Flow equaled or exceeded for indicated percentage of time } \\
(cubic feet per second)
\end{tabular}


STATION NAME AND NUMBER—03586500 Big Nance Creek at Courtland, AL

LOCATION.-Lat $34^{\circ} 40^{\prime} 12^{\prime \prime}$, long $87^{\circ} 19^{\prime} 02^{\prime \prime}$ referenced to North American Datum of 1927, Lawrence County, AL, Hydrologic Unit 06030005, near right bank on downstream side of bridge on County Road 25 at Courtland, and at mile 12.9.

DRAINAGE AREA.-166 $\mathrm{mi}^{2}$.

PERIOD OF RECORD.-September 1935 to September 1940, April 1945 to September 1981, March 1988 to March 2014.

PERIOD OF ANALYSIS.-April 1936 to March 1940, April 1945 to March 1981.

\section{NUMBER OF CLIMATE YEARS IN FREQUENCY ANALYSIS. - 40}

REMARKS.-Based on QAQC reviews, it appears that low-flow patterns after 1981 are different, which is likely due to anthropogenic influences in the basin. Thus, the period being analyzed here likely represents more natural conditions. A separate analysis will be done for the period after 1981 .

\begin{tabular}{|c|c|c|c|c|}
\hline $\begin{array}{c}\text { Recurrence } \\
\text { intervals } \\
\text { (years) }\end{array}$ & \multicolumn{2}{|c|}{$\begin{array}{l}\text { Lowest average flow for } \\
\text { indicated number of } \\
\text { consecutive days } \\
\text { (cubic feet per second) }\end{array}$} & \multicolumn{2}{|c|}{$\begin{array}{l}\text { Time-sampling error } \\
\text { (in percent) }\end{array}$} \\
\hline & 1 & 7 & 1 & 7 \\
\hline 2 & 2.1 & 2.6 & 17 & 17 \\
\hline 5 & 0.98 & 1.2 & 17 & 17 \\
\hline 10 & 0.67 & 0.81 & 19 & 19 \\
\hline 20 & 0.49 & 0.60 & 22 & 22 \\
\hline 50 & 0.35 & 0.43 & 30 & 29 \\
\hline
\end{tabular}

\begin{tabular}{|c|c|c|c|c|c|c|c|}
\hline \multicolumn{8}{|c|}{ DURATION OF DAILY FLOW } \\
\hline \multicolumn{8}{|c|}{$\begin{array}{l}\text { Flow equaled or exceeded for indicated percentage of time } \\
\text { (cubic feet per second) }\end{array}$} \\
\hline Percentage & 5 & 10 & 25 & 50 & 75 & 90 & 95 \\
\hline Flow & 1,200 & 599 & 225 & 65 & 14 & 4.5 & 2.3 \\
\hline
\end{tabular}


STATION NAME AND NUMBER—03586500 Big Nance Creek at Courtland, AL

LOCATION.-Lat $34^{\circ} 40^{\prime} 12^{\prime \prime}$, long $87^{\circ} 19^{\prime} 02^{\prime \prime}$ referenced to North American Datum of 1927, Lawrence County, AL, Hydrologic Unit 06030005, near right bank on downstream side of bridge on County Road 25 at Courtland, and at mile 12.9.

DRAINAGE AREA. $-166 \mathrm{mi}^{2}$.

PERIOD OF RECORD.-September 1935 to September 1940, April 1945 to September 1981, March 1988 to March 2014.

PERIOD OF ANALYSIS.--April 1988 to March 2014.

\section{NUMBER OF CLIMATE YEARS IN FREQUENCY ANALYSIS.—-26}

REMARKS.-Based on QAQC reviews, it appears that low-flow patterns after 1981 are different, which is likely due to anthropogenic influences in the basin. The period of record used in this analysis represents current conditions in the basin reflecting anthropogenic influences.

\begin{tabular}{|c|c|c|c|c|}
\hline $\begin{array}{c}\text { Recurrence } \\
\text { intervals } \\
\text { (years) }\end{array}$ & \multicolumn{2}{|c|}{$\begin{array}{c}\text { Lowest average flow for } \\
\text { indicated number of } \\
\text { consecutive days } \\
\text { (cubic feet per second) }\end{array}$} & \multicolumn{2}{|c|}{$\begin{array}{c}\text { Time-sampling error } \\
\text { (in percent) }\end{array}$} \\
\hline \multicolumn{2}{|c|}{1} & 7 & 1 & 7 \\
\hline 2 & 3.7 & 4.3 & 25 & 25 \\
\hline 5 & 1.3 & 1.6 & 29 & 36 \\
\hline 10 & 0.76 & 0.89 & 37 & 46 \\
\hline 20 & 0.47 & 0.56 & 47 & \\
\hline
\end{tabular}

\begin{tabular}{|c|c|c|c|c|c|c|c|}
\hline \multicolumn{8}{|c|}{ DURATION OF DAILY FLOW } \\
\hline \multicolumn{8}{|c|}{$\begin{array}{l}\text { Flow equaled or exceeded for indicated percentage of time } \\
\text { (cubic feet per second) }\end{array}$} \\
\hline Percentage & 5 & 10 & 25 & 50 & 75 & 90 & 95 \\
\hline Flow & 1,240 & 651 & 262 & 86 & 28 & 5.6 & 2.6 \\
\hline
\end{tabular}


STATION NAME AND NUMBER—03589500 Tennessee River at Florence, AL

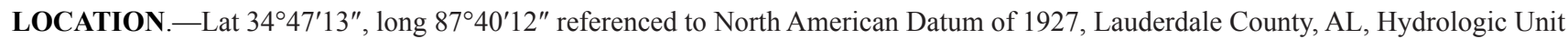
06030005, on right bank at lower end of Patton Island, $137 \mathrm{ft}$ upstream from Southern Railway bridge, $700 \mathrm{ft}$ upstream from O'Neal Bridge on U.S. Highway 72, $1.1 \mathrm{mi}$ south of Florence Post Office, $1.7 \mathrm{mi}$ upstream from Cypress Creek, 2.7 mi downstream from Wilson Dam, and at mile 256.7.

DRAINAGE AREA. $-30,810 \mathrm{mi}^{2}$, approximately.

PERIOD OF RECORD.-October 1894 to September 1997, October 1999 to September 2003, October 2004 to November 2005.

PERIOD OF ANALYSIS.-April 1895 to March 1924.

\section{NUMBER OF CLIMATE YEARS IN FREQUENCY ANALYSIS. - 29}

REMARKS.-Regulated by Wilson Lake since 1924 and by increasing number of other reservoirs since 1936. Due to uncertainty in changing regulation patterns over time, the low-flow frequency statistics are providing for the pre-regulation period only.

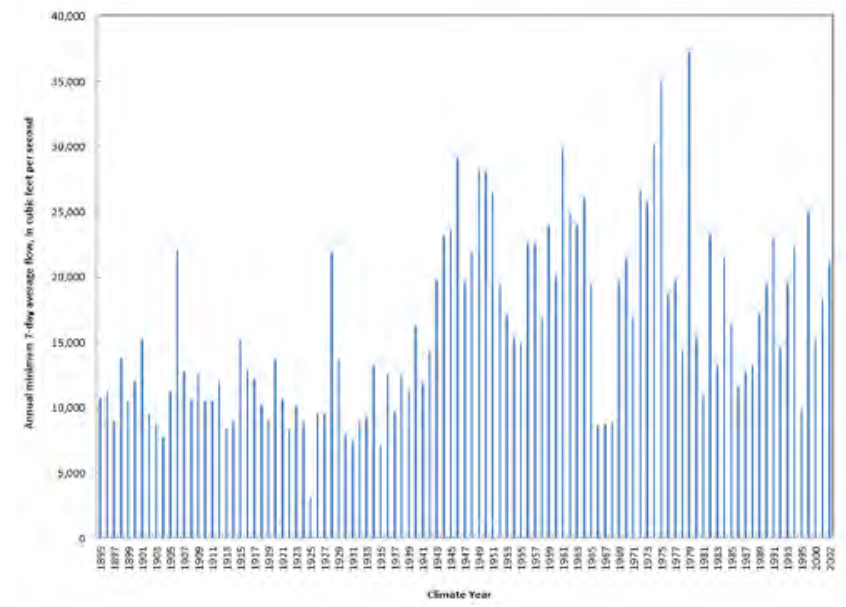

\begin{tabular}{|c|c|c|c|c|}
\hline $\begin{array}{c}\text { Recurrence } \\
\text { intervals } \\
\text { (years) }\end{array}$ & \multicolumn{2}{|c|}{$\begin{array}{c}\text { Lowest average flow for } \\
\text { indicated number of } \\
\text { consecutive days } \\
\text { (cubic feet per second) }\end{array}$} & \multicolumn{2}{|c|}{$\begin{array}{c}\text { Time-sampling error } \\
\text { (in percent) }\end{array}$} \\
\hline & 1 & 7 & 1 & 5 \\
\hline 2 & 10,400 & 10,700 & 5 & 3 \\
\hline 5 & 8,930 & 9,210 & 4 & 4 \\
\hline 10 & 8,370 & 8,650 & 4 & 5 \\
\hline 20 & 7,990 & 8,280 & 5 & 5 \\
\hline
\end{tabular}

\section{DURATION OF DAILY FLOW}

Flow equaled or exceeded for indicated percentage of time (cubic feet per second)

\begin{tabular}{|c|c|c|c|c|c|c|c|}
\hline Percentage & 5 & 10 & 25 & 50 & 75 & 90 & 95 \\
\hline Flow & 169,000 & 121,000 & 64,500 & 36,000 & 18,600 & 12,600 & 11,000 \\
\hline
\end{tabular}


STATION NAME AND NUMBER—03590000 Cypress Creek near Florence, AL

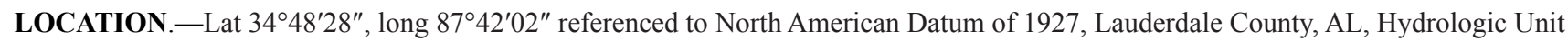
06030005, on State Highway 2, 2 mi west of Florence, 4 mi downstream from Cox Creek, and 4 mi upstream from mouth.

DRAINAGE AREA. $-209 \mathrm{mi}^{2}$.

PERIOD OF RECORD.- - June 1934 to September 1953.

PERIOD OF ANALYSIS.-April 1935 to March 1953.

NUMBER OF CLIMATE YEARS IN FREQUENCY ANALYSIS.—18

REMARKS.-

\begin{tabular}{|c|c|c|c|c|}
\hline $\begin{array}{l}\text { Recurrence } \\
\text { intervals } \\
\text { (years) }\end{array}$ & \multicolumn{2}{|c|}{$\begin{array}{l}\text { Lowest average flow for } \\
\text { indicated number of } \\
\text { consecutive days } \\
\text { (cubic feet per second) }\end{array}$} & \multicolumn{2}{|c|}{$\begin{array}{l}\text { Time-sampling error } \\
\text { (in percent) }\end{array}$} \\
\hline & 1 & 7 & 1 & 7 \\
\hline 2 & 60 & 63 & 7 & 7 \\
\hline 5 & 52 & 55 & 4 & 4 \\
\hline 10 & 49 & 52 & 6 & 6 \\
\hline 20 & 48 & 51 & 9 & 9 \\
\hline
\end{tabular}

DURATION OF DAILY FLOW

Flow equaled or exceeded for indicated percentage of time (cubic feet per second)

\begin{tabular}{|c|c|c|c|c|c|c|c|}
\hline Percentage & 5 & 10 & 25 & 50 & 75 & 90 & 95 \\
\hline Flow & 1,120 & 750 & 420 & 186 & 94 & 70 & 64 \\
\hline
\end{tabular}


STATION NAME AND NUMBER—03590500 Tuscumbia Spring at Tuscumbia, AL

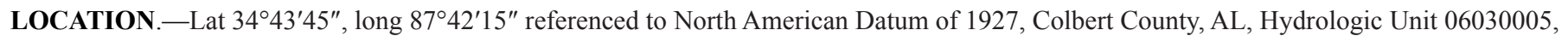
on south end of Main Street in Tuscumbia, and 0.1 mil upstream from mouth.

DRAINAGE AREA.- - Spring (indeterminate)

PERIOD OF RECORD.-December 1928 to March 1930, January 1956 to September 1965.

PERIOD OF ANALYSIS.-April 1929 to March 1930, April 1956 to March 1965.

NUMBER OF CLIMATE YEARS IN FREQUENCY ANALYSIS.-11

REMARKS.- -

\begin{tabular}{|c|c|c|c|c|}
\hline $\begin{array}{l}\text { Recurrence } \\
\text { intervals } \\
\text { (years) }\end{array}$ & \multicolumn{2}{|c|}{$\begin{array}{l}\text { Lowest average flow for } \\
\text { indicated number of } \\
\text { consecutive days } \\
\text { (cubic feet per second) }\end{array}$} & \multicolumn{2}{|c|}{$\begin{array}{l}\text { Time-sampling error } \\
\text { (in percent) }\end{array}$} \\
\hline & 1 & 7 & 1 & 7 \\
\hline 2 & 14 & 15 & 13 & 12 \\
\hline 5 & 11 & 11 & 11 & 12 \\
\hline 10 & 9.2 & 9.9 & 12 & 13 \\
\hline 20 & 8.4 & 8.9 & 14 & 15 \\
\hline
\end{tabular}

\section{DURATION OF DAILY FLOW}

Flow equaled or exceeded for indicated percentage of time (cubic feet per second)

\begin{tabular}{|c|c|c|c|c|c|c|c|}
\hline Percentage & 5 & 10 & 25 & 50 & 75 & 90 & 95 \\
\hline Flow & 121 & 112 & 93 & 66 & 31 & 21 & 17 \\
\hline
\end{tabular}


STATION NAME AND NUMBER—03591800 Bear Creek near Hackleburg, AL

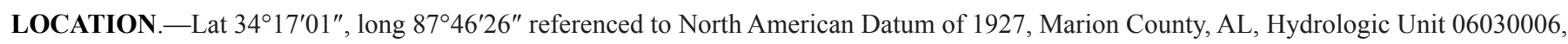
on State Highway 172, 2 mi upstream from Bluff Creek, 3.5 mi east of Hackleburg, and at mile 104.8.

DRAINAGE AREA.-143

PERIOD OF RECORD.-OCtober 1956 to September 1979, October 1980 to September 1981.

PERIOD OF ANALYSIS.-April 1957 to March 1978.

NUMBER OF CLIMATE YEARS IN FREQUENCY ANALYSIS.—-21

REMARKS.-Since 1978, regulated by Upper Bear Creek Reservoir. Frequency statistics are for pre-regulation period.

\begin{tabular}{|c|c|c|c|c|}
\hline $\begin{array}{c}\text { Recurrence } \\
\text { intervals } \\
\text { (years) }\end{array}$ & \multicolumn{2}{|c|}{$\begin{array}{c}\text { Lowest average flow for } \\
\text { indicated number of } \\
\text { consecutive days } \\
\text { (cubic feet per second) }\end{array}$} & \multicolumn{2}{|c|}{$\begin{array}{c}\text { Time-sampling error } \\
\text { (in percent) }\end{array}$} \\
\hline \multicolumn{7}{|c|}{} & 1 & 7 & 1 & 7 \\
\hline 2 & 11 & 12 & 9 & 9 \\
\hline 5 & 8.1 & 9.1 & 8 & 10 \\
\hline 10 & 7.1 & 7.8 & 9 & 12 \\
\hline 20 & 6.4 & 6.9 & 11 & \\
\hline
\end{tabular}

\section{DURATION OF DAILY FLOW}

Flow equaled or exceeded for indicated percentage of time (cubic feet per second)

\begin{tabular}{|c|c|c|c|c|c|c|c|}
\hline Percentage & 5 & 10 & 25 & 50 & 75 & 90 & 95 \\
\hline Flow & 977 & 595 & 269 & 108 & 37 & 19 & 14 \\
\hline
\end{tabular}


STATION NAME AND NUMBER—03592000 Bear Creek near Red Bay, AL

LOCATION.-Lat $34^{\circ} 26^{\prime} 38^{\prime \prime}$, long $88^{\circ} 06^{\prime} 56^{\prime \prime}$ referenced to North American Datum of 1927, Franklin County, AL, Hydrologic Unit 06030006, on State Highway 24, 1.8 mi east of Red Bay, and at mile 61.9.

DRAINAGE AREA. -263

PERIOD OF RECORD.- October 1913 to May 1920, October 1958 to September 1967, March 1969 to September 1981.

PERIOD OF ANALYSIS.-April 1914 to March 1920, April 1959 to March 1967.

\section{NUMBER OF CLIMATE YEARS IN FREQUENCY ANALYSIS.-14}

REMARKS.-Flow regulated since March 1969 by Bear Creek Reservoir and since 1978 by Upper Bear Creek Reservoir. Frequency statistics are for pre-regulation period.

\begin{tabular}{|c|c|c|c|c|}
\hline \multirow[t]{2}{*}{$\begin{array}{l}\text { Recurrence } \\
\text { intervals } \\
\text { (years) }\end{array}$} & \multicolumn{2}{|c|}{$\begin{array}{l}\text { Lowest average flow for } \\
\text { indicated number of } \\
\text { consecutive days } \\
\text { (cubic feet per second) }\end{array}$} & \multicolumn{2}{|c|}{$\begin{array}{l}\text { Time-sampling error } \\
\text { (in percent) }\end{array}$} \\
\hline & 1 & 7 & 1 & 7 \\
\hline 2 & 30 & 34 & 14 & 11 \\
\hline 5 & 20 & 24 & 17 & 12 \\
\hline 10 & 15 & 21 & 23 & 15 \\
\hline 20 & 12 & 18 & 32 & 18 \\
\hline
\end{tabular}

\begin{tabular}{|c|c|c|c|c|c|c|c|c|}
\hline \multicolumn{7}{|c|}{ DURATION OF DAILY FLOW } \\
\hline \multicolumn{7}{|c|}{ Flow equaled or exceeded for indicated percentage of time } \\
(cubic feet per second)
\end{tabular}


STATION NAME AND NUMBER—03592200 Cedar Creek near Pleasant Site, AL

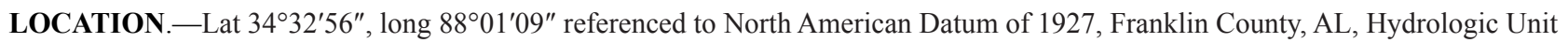
06030006, 2.6 mi east of Pleasant Site, 4.3 mi upstream from Little Bear Creek, and at mile 19.1.

DRAINAGE AREA. -189

PERIOD OF RECORD.- October 1957 to September 1977.

PERIOD OF ANALYSIS.--April 1958 to March 1977.

NUMBER OF CLIMATE YEARS IN FREQUENCY ANALYSIS.-19

REMARKS.-Flow regulated since 1979 by Cedar Creek Dam. These frequency statistics represent pre-regulation conditions.

\begin{tabular}{|c|c|c|c|c|}
\hline $\begin{array}{l}\text { Recurrence } \\
\text { intervals } \\
\text { (years) }\end{array}$ & \multicolumn{2}{|c|}{$\begin{array}{l}\text { Lowest average flow for } \\
\text { indicated number of } \\
\text { consecutive days } \\
\text { (cubic feet per second) }\end{array}$} & \multicolumn{2}{|c|}{$\begin{array}{l}\text { Time-sampling error } \\
\text { (in percent) }\end{array}$} \\
\hline & 1 & 7 & 1 & 7 \\
\hline 2 & 8.8 & 11 & 16 & 13 \\
\hline 5 & 5.0 & 7.4 & 19 & 11 \\
\hline 10 & 3.6 & 6.3 & 25 & 11 \\
\hline 20 & 2.7 & 5.6 & 33 & 14 \\
\hline
\end{tabular}

\section{DURATION OF DAILY FLOW}

Flow equaled or exceeded for indicated percentage of time (cubic feet per second)

\begin{tabular}{|c|c|c|c|c|c|c|c|}
\hline Percentage & 5 & 10 & 25 & 50 & 75 & 90 & 95 \\
\hline Flow & 1,260 & 777 & 342 & 122 & 39 & 18 & 13 \\
\hline
\end{tabular}


STATION NAME AND NUMBER—03592300 Little Bear Creek near Halltown, AL

LOCATION.-Lat $34^{\circ} 29^{\prime} 19^{\prime \prime}$, long $88^{\circ} 02^{\prime} 07^{\prime \prime}$ referenced to North American Datum of 1927, Franklin County, AL, Hydrologic Unit 06030006, 2.7 mi northeast of Halltown, and at mile 4.3.

DRAINAGE AREA.- -78.2

PERIOD OF RECORD.- - October 1957 to September 1977.

PERIOD OF ANALYSIS.-April 1958 to March 1975.

NUMBER OF CLIMATE YEARS IN FREQUENCY ANALYSIS._-17

REMARKS.-Flow regulated since 1975 by Little Bear Creek Dam. Frequency statistics are for pre-regulation period.

\begin{tabular}{|c|c|c|c|c|}
\hline $\begin{array}{c}\text { Recurrence } \\
\text { intervals } \\
\text { (years) }\end{array}$ & \multicolumn{2}{|c|}{$\begin{array}{c}\text { Lowest average flow for } \\
\text { indicated number of } \\
\text { consecutive days } \\
\text { (cubic feet per second) }\end{array}$} & \multicolumn{2}{|c|}{$\begin{array}{c}\text { Time-sampling error } \\
\text { (in percent) }\end{array}$} \\
\hline \multicolumn{7}{|c|}{1} & 7 & 1 & 7 \\
\hline 2 & 7.3 & 8.9 & 13 & 9 \\
\hline 5 & 4.8 & 6.7 & 14 & 9 \\
\hline 10 & 3.9 & 5.9 & 16 & 11 \\
\hline 20 & 3.3 & 5.4 & 19 & \\
\hline
\end{tabular}

\section{DURATION OF DAILY FLOW}

Flow equaled or exceeded for indicated percentage of time (cubic feet per second)

\begin{tabular}{|c|c|c|c|c|c|c|c|}
\hline Percentage & 5 & 10 & 25 & 50 & 75 & 90 & 95 \\
\hline Flow & 513 & 321 & 155 & 59 & 23 & 14 & 11 \\
\hline
\end{tabular}


STATION NAME AND NUMBER—03592500 Bear Creek at Bishop, AL

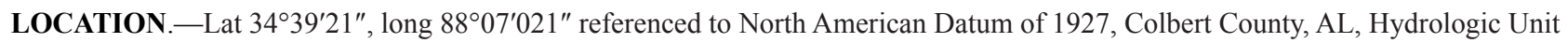
06030006, 0.5 mi downstream from Cedar Creek, 0.8 mi southwest of Bishop, and at mile 27.3.

DRAINAGE AREA.-667

PERIOD OF RECORD.- October 1926 to May 1928, March 1929 to March 1932, October 1933 to September 1979.

PERIOD OF ANALYSIS.-April 1927 to March 1928, April 1929 to March 1932, April 1934 to March 1979.

\section{NUMBER OF CLIMATE YEARS IN FREQUENCY ANALYSIS.—49}

REMARKS.-Flow regulated since March 1969 by Bear Creek Reservoir, regulated since 1975 by Little Bear Creek Dam, and since 1978 by Upper Bear Creek Reservoir, and since 1979 by Cedar Creek Dam. However, the QAQC analyses indicate that those upstream reservoirs did not substantially alter the low-flow patterns. Therefore, the complete period of record was used in the analyses.

\begin{tabular}{|c|c|c|c|c|}
\hline $\begin{array}{l}\text { Recurrence } \\
\text { intervals } \\
\text { (years) }\end{array}$ & \multicolumn{2}{|c|}{$\begin{array}{l}\text { Lowest average flow for } \\
\text { indicated number of } \\
\text { consecutive days } \\
\text { (cubic feet per second) }\end{array}$} & \multicolumn{2}{|c|}{$\begin{array}{l}\text { Time-sampling error } \\
\text { (in percent) }\end{array}$} \\
\hline & 1 & 7 & 1 & 7 \\
\hline 2 & 58 & 65 & 9 & 9 \\
\hline 5 & 35 & 40 & 10 & 10 \\
\hline 10 & 26 & 30 & 13 & 13 \\
\hline 20 & 21 & 24 & 17 & 17 \\
\hline 50 & 15 & 18 & 23 & 23 \\
\hline
\end{tabular}

\begin{tabular}{|c|c|c|c|c|c|c|c|}
\hline \multicolumn{8}{|c|}{ DURATION OF DAILY FLOW } \\
\hline \multicolumn{8}{|c|}{$\begin{array}{l}\text { Flow equaled or exceeded for indicated percentage of time } \\
\text { (cubic feet per second) }\end{array}$} \\
\hline Percentage & 5 & 10 & 25 & 50 & 75 & 90 & 95 \\
\hline Flow & 4,240 & 2,790 & 1,270 & 472 & 167 & 86 & 64 \\
\hline
\end{tabular}


Low-flow statistics for the following stations are from USGS continuous-record streamgaging stations from basins adjoining Alabama in Florida, Georgia, Mississippi, and Tennessee. 
STATION NAME AND NUMBER—02330450 Chattahoochee River at Helen, GA

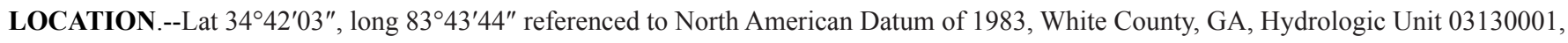
on downstream side of bridge on GA 17 and 75 at Helen, and 1.1 miles downstream from Smith Creek.

DRAINAGE AREA. $-44.7 \mathrm{mi}^{2}$.

PERIOD OF RECORD.--May 1981 to March 2014.

PERIOD OF FREQUENCY ANALYSIS.-April 1982 to March 2014.

NUMBER OF CLIMATE YEARS IN FREQUENCY ANALYSIS.-32

REMARKS.-

\begin{tabular}{|c|c|c|c|c|}
\hline $\begin{array}{c}\text { Recurrence } \\
\text { intervals } \\
\text { (years) }\end{array}$ & \multicolumn{2}{|c|}{$\begin{array}{c}\text { Lowest average flow for } \\
\text { indicated number of } \\
\text { consecutive days } \\
\text { (cubic feet per second) }\end{array}$} & \multicolumn{2}{|c|}{$\begin{array}{c}\text { Time-sampling error } \\
\text { (in percent) }\end{array}$} \\
\hline \multicolumn{7}{|c|}{1} & 7 & 1 & 7 \\
\hline 2 & 38 & 40 & 8 & 9 \\
\hline 5 & 27 & 28 & 9 & 10 \\
\hline 10 & 22 & 24 & 10 & 12 \\
\hline 20 & 19 & 20 & 12 & 15 \\
\hline 50 & 16 & 17 & 16 & \\
\hline
\end{tabular}

\begin{tabular}{|c|c|c|c|c|c|c|c|}
\hline \multicolumn{7}{|c|}{ DURATION OF DAILY FLOW } \\
\hline \multicolumn{7}{|c|}{ Flow equaled or exceeded for indicated percentage of time } \\
(cubic feet per second)
\end{tabular}


STATION NAME AND NUMBER—02331000 Chattahoochee River near Leaf, GA

LOCATION.--Lat 34³4'37", long 8338'09" referenced to North American Datum of 1927, Habersham County, GA, Hydrologic Unit 03130001, 700 feet upstream from bridge on GA 115, 3 miles from confluence with Soque River and 1.5 miles east of Leaf, GA.

DRAINAGE AREA.- $-150 \mathrm{mi}^{2}$.

PERIOD OF RECORD.-February 1940 to September 1971, October 2008 to March 2014.

PERIOD OF FREQUENCY ANALYSIS.-April 1940 to March 1971, April 2009 to March 2014.

NUMBER OF CLIMATE YEARS IN FREQUENCY ANALYSIS.-36

REMARKS.-Potentially influenced from upstream diversions (Gotvald, 2016).

\begin{tabular}{|c|c|c|c|c|}
\hline $\begin{array}{c}\text { Recurrence } \\
\text { intervals } \\
\text { (years) }\end{array}$ & \multicolumn{2}{|c|}{$\begin{array}{c}\text { Lowest average flow for } \\
\text { indicated number of } \\
\text { consecutive days } \\
\text { (cubic feet per second) }\end{array}$} & \multicolumn{2}{|c|}{$\begin{array}{c}\text { Time-sampling error } \\
\text { (in percent) }\end{array}$} \\
\hline & 1 & 7 & 1 & 6 \\
\hline 2 & 124 & 132 & 6 & 6 \\
\hline 5 & 97 & 102 & 5 & 6 \\
\hline 10 & 86 & 91 & 6 & 7 \\
\hline 20 & 79 & 83 & 7 & 10 \\
\hline 50 & 73 & 76 & 9 & 6 \\
\hline
\end{tabular}

\begin{tabular}{|c|c|c|c|c|c|c|c|}
\hline \multicolumn{1}{|c|}{ DURATION OF DAILY FLOW } \\
\hline \multicolumn{7}{|c|}{ Flow equaled or exceeded for indicated percentage of time } \\
(cubic feet per second)
\end{tabular}


STATION NAME AND NUMBER—02333500 Chestatee River near Dahlonega, GA

LOCATION.--Lat 34³1'41", long 8356'23" referenced to North American Datum of 1983, Lumpkin County, GA, Hydrologic Unit 03130001, on left bank 250 feet upstream from Bearden Bridge on GA 52, 2.0 miles downstream from Ballplay Creek, 2.5 miles east of Dahlonega, and 3.5 miles upstream from Yahoola Creek.

DRAINAGE AREA. $-153 \mathrm{mi}^{2}$.

PERIOD OF RECORD.-July 1929 to January 1932, April 1940 to March 2014.

PERIOD OF FREQUENCY ANALYSIS.-April 1930 to March 1931, April 1940 to March 2014.

NUMBER OF CLIMATE YEARS IN FREQUENCY ANALYSIS.—75

REMARKS.-

\begin{tabular}{|c|c|c|c|c|}
\hline $\begin{array}{c}\text { Recurrence } \\
\text { intervals } \\
\text { (years) }\end{array}$ & \multicolumn{2}{|c|}{$\begin{array}{c}\text { Lowest average flow for } \\
\text { indicated number of } \\
\text { consecutive days } \\
\text { (cubic feet per second) }\end{array}$} & \multicolumn{2}{|c|}{$\begin{array}{c}\text { Time-sampling error } \\
\text { (in percent) }\end{array}$} \\
\hline & 1 & 7 & 1 & 7 \\
\hline 2 & 104 & 110 & 5 & 7 \\
\hline 5 & 71 & 76 & 7 & 8 \\
\hline 10 & 57 & 61 & 9 & 11 \\
\hline 20 & 47 & 50 & 11 & 16 \\
\hline 50 & 37 & 40 & 16 & \\
\hline
\end{tabular}

\begin{tabular}{|c|c|c|c|c|c|c|c|}
\hline \multicolumn{1}{|c|}{ DURATION OF DAILY FLOW } \\
\hline \multicolumn{7}{|c|}{ Flow equaled or exceeded for indicated percentage of time } \\
(cubic feet per second)
\end{tabular}


STATION NAME AND NUMBER—02335000 Chattahoochee River near Norcross, GA

LOCATION.--Lat 3359'50", long 84¹2'07" referenced to North American Datum of 1983, Gwinnett County, GA, Hydrologic Unit 03130001, on downstream side of right bank pier of bridge on GA 141, 1.5 miles upstream from Johns Creek, 4.5 miles north of Norcross, 6.5 miles downstream from Suwanee Creek, 18.0 miles downstream from Buford Dam, and at mile 330.8.

DRAINAGE AREA.- $-1,170 \mathrm{mi}^{2}$.

PERIOD OF RECORD._-January 1903 to September 1946, October 1956 to March 2014.

PERIOD OF FREQUENCY ANALYSIS.-April 1903 to March 1946.

NUMBER OF CLIMATE YEARS IN FREQUENCY ANALYSIS. - 43

REMARKS.-The low-flow and duration analyses are based on the period of record prior to regulation by Lake Lanier.

\begin{tabular}{|c|c|c|c|c|}
\hline $\begin{array}{c}\text { Recurrence } \\
\text { intervals } \\
\text { (years) }\end{array}$ & \multicolumn{2}{|c|}{$\begin{array}{c}\text { Lowest average flow for } \\
\text { indicated number of } \\
\text { consecutive days } \\
\text { (cubic feet per second) }\end{array}$} & \multicolumn{2}{|c|}{$\begin{array}{c}\text { Time-sampling error } \\
\text { (in percent) }\end{array}$} \\
\hline & 1 & 7 & 1 & 7 \\
\hline 2 & 739 & 795 & 8 & 8 \\
\hline 5 & 497 & 570 & 11 & 11 \\
\hline 10 & 380 & 464 & 14 & 15 \\
\hline 20 & 294 & 384 & 21 & 21 \\
\hline 50 & 213 & 305 & 33 & \\
\hline
\end{tabular}

\begin{tabular}{|c|c|c|c|c|c|c|c|}
\hline \multicolumn{1}{|c|}{ DURATION OF DAILY FLOW } \\
\hline \multicolumn{1}{|c|}{ Flow equaled or exceeded for indicated percentage of time } \\
(cubic feet per second)
\end{tabular}


STATION NAME AND NUMBER—02337000 Sweetwater Creek near Austell, GA

LOCATION.--Lat 3346’35.4”, long 84³6'56.2" referenced to North American Datum of 1927, Douglas County, GA, Hydrologic Unit 03130002, on right bank 100.0 feet upstream from bridge on Interstate 20, 400.0 feet upstream from Blair Bridge, 3.0 miles southeast of Austell, and 5.5 miles upstream from mouth.

DRAINAGE AREA.- $-238 \mathrm{mi}^{2}$.

PERIOD OF RECORD.-May 1904 to December 1905, March 1937 to March 2014.

PERIOD OF FREQUENCY ANALYSIS.-April 1937 to March 2014.

NUMBER OF CLIMATE YEARS IN FREQUENCY ANALYSIS. - 77

REMARKS.-Potentially influenced from upstream diversions (Gotvald, 2016).

\begin{tabular}{|c|c|c|c|c|}
\hline \multirow[t]{2}{*}{$\begin{array}{l}\text { Recurrence } \\
\text { intervals } \\
\text { (years) }\end{array}$} & \multicolumn{2}{|c|}{$\begin{array}{l}\text { Lowest average flow for } \\
\text { indicated number of } \\
\text { consecutive days } \\
\text { (cubic feet per second) }\end{array}$} & \multicolumn{2}{|c|}{$\begin{array}{l}\text { Time-sampling error } \\
\text { (in percent) }\end{array}$} \\
\hline & 1 & 7 & 1 & 7 \\
\hline 2 & 34 & 39 & 13 & 13 \\
\hline 5 & 14 & 16 & 17 & 16 \\
\hline 10 & 7.6 & 9.4 & 23 & 22 \\
\hline 20 & 4.3 & 5.6 & 33 & 31 \\
\hline 50 & 2.2 & 3.0 & 52 & 48 \\
\hline
\end{tabular}

\begin{tabular}{|c|c|c|c|c|c|c|c|}
\hline \multicolumn{1}{|c|}{ DURATION OF DAILY FLOW } \\
\hline \multicolumn{7}{|c|}{ Flow equaled or exceeded for indicated percentage of time } \\
(cubic feet per second)
\end{tabular}


STATION NAME AND NUMBER—02337500 Snake Creek near Whitesburg, GA

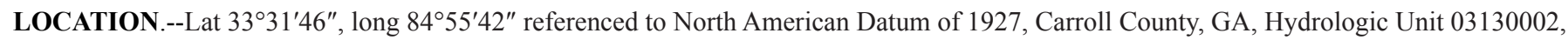
on left bank, on downstream side of former bridge pier, 50.0 feet upstream from county highway bridge, at Banning Mills, 1.6 miles north of US 27 (ALT), 3.0 miles northwest of Whitesburg, 4.0 miles downstream from Little Snake Creek, and 7.0 miles upstream from mouth.

DRAINAGE AREA.-35.5 $\mathrm{mi}^{2}$.

PERIOD OF RECORD.-September 1954 to March 2014.

PERIOD OF FREQUENCY ANALYSIS.-April 1955 to March 2000.

NUMBER OF CLIMATE YEARS IN FREQUENCY ANALYSIS. - 45

REMARKS.-Flow regulated by earthen dam (Snake Creek Reservoir) upstream of gage since 2001. Frequency and duration statistics represent pre-regulation period.

\begin{tabular}{|c|c|c|c|c|}
\hline $\begin{array}{c}\text { Recurrence } \\
\text { intervals } \\
\text { (years) }\end{array}$ & \multicolumn{2}{|c|}{$\begin{array}{c}\text { Lowest average flow for } \\
\text { indicated number of } \\
\text { consecutive days } \\
\text { (cubic feet per second) }\end{array}$} & \multicolumn{2}{|c|}{$\begin{array}{c}\text { Time-sampling error } \\
\text { (in percent) }\end{array}$} \\
\hline \multicolumn{5}{|c|}{} \\
\hline & 1 & 7 & 1 & 7 \\
\hline 2 & 16 & 16 & 11 & 13 \\
\hline 5 & 9.5 & 10 & 15 & 18 \\
\hline 10 & 6.5 & 7.3 & 20 & 26 \\
\hline 20 & 4.5 & 5.2 & 30 & 44 \\
\hline 50 & 2.8 & 3.4 & 51 & \\
\hline
\end{tabular}

\section{DURATION OF DAILY FLOW}

Flow equaled or exceeded for indicated percentage of time (cubic feet per second)

\begin{tabular}{|c|c|c|c|c|c|c|c|}
\hline Percentage & 5 & 10 & 25 & 50 & 75 & 90 & 95 \\
\hline Flow & 122 & 92 & 62 & 39 & 25 & 17 & 13 \\
\hline
\end{tabular}


STATION NAME AND NUMBER—02338660 New River at GA 100, near Corinth, GA

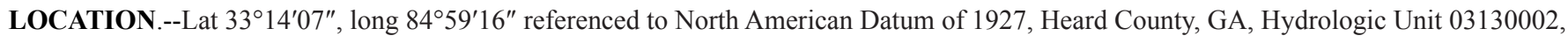
at the bridge on Georgia Highway 100, 8.1 mi upstream from confluence with Chattahoochee River, 1.7 mi downstream from confluence with Caney Creek, 3.9 mi downstream from confluence with Mountain Creek, and 2.5 mi west of Corinth.

DRAINAGE AREA. $-127 \mathrm{mi}^{2}$.

PERIOD OF RECORD.- October 1978 to March 2014.

PERIOD OF FREQUENCY ANALYSIS.-April 1979 to March 2014.

NUMBER OF CLIMATE YEARS IN FREQUENCY ANALYSIS.-35

REMARKS. - Potentially influenced from upstream diversions (Gotvald, 2016).

\begin{tabular}{|c|c|c|c|c|}
\hline $\begin{array}{c}\text { Recurrence } \\
\text { intervals } \\
\text { (years) }\end{array}$ & \multicolumn{2}{|c|}{$\begin{array}{c}\text { Lowest average flow for } \\
\text { indicated number of } \\
\text { consecutive days } \\
\text { (cubic feet per second) }\end{array}$} & \multicolumn{2}{|c|}{$\begin{array}{c}\text { Time-sampling error } \\
\text { (in percent) }\end{array}$} \\
\hline & 1 & 7 & 1 & 7 \\
\hline 2 & 5.7 & 6.9 & 29 & 33 \\
\hline 5 & 1.4 & 2.0 & 37 & 43 \\
\hline 10 & 0.63 & 0.95 & 49 & 60 \\
\hline 20 & 0.30 & 0.49 & 69 & 90 \\
\hline 50 & 0.12 & 0.22 & 109 & \\
\hline
\end{tabular}

\begin{tabular}{|c|c|c|c|c|c|c|c|}
\hline \multicolumn{1}{|c|}{ DURATION OF DAILY FLOW } \\
\hline \multicolumn{7}{|c|}{ Flow equaled or exceeded for indicated percentage of time } \\
(cubic feet per second)
\end{tabular}


STATION NAME AND NUMBER—02339000 Yellowjacket Creek near La Grange, GA

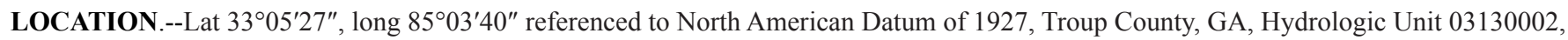
at downstream end of right bank pier of bridge on State Highway 219, 1.2 mi downstream from Beech Creek, 2 mi upstream from Jackson Creek, 4.2 mi northwest of La Grange.

DRAINAGE AREA.- $-182 \mathrm{mi}^{2}$.

PERIOD OF RECORD.-January 1951 to March 1971.

PERIOD OF FREQUENCY ANALYSIS.-April 1951 to March 1971.

NUMBER OF CLIMATE YEARS IN FREQUENCY ANALYSIS.-20

REMARKS.-Station was inundated by West Point Lake around 1974.

\begin{tabular}{|c|c|c|c|c|}
\hline $\begin{array}{c}\text { Recurrence } \\
\text { intervals } \\
\text { (years) }\end{array}$ & \multicolumn{2}{|c|}{$\begin{array}{l}\text { Lowest average flow for } \\
\text { indicated number of } \\
\text { consecutive days } \\
\text { (cubic feet per second) }\end{array}$} & \multicolumn{2}{|c|}{$\begin{array}{l}\text { Time-sampling error } \\
\text { (in percent) }\end{array}$} \\
\hline & 1 & 7 & 1 & 7 \\
\hline 2 & 29 & 33 & 17 & 16 \\
\hline 5 & 16 & 19 & 22 & 21 \\
\hline 10 & 11 & 13 & 29 & 28 \\
\hline 20 & 7.7 & 9.3 & 42 & 41 \\
\hline
\end{tabular}

\section{DURATION OF DAILY FLOW}

Flow equaled or exceeded for indicated percentage of time (cubic feet per second)

\begin{tabular}{|c|c|c|c|c|c|c|c|}
\hline Percentage & 5 & 10 & 25 & 50 & 75 & 90 & 95 \\
\hline Flow & 656 & 416 & 237 & 127 & 72 & 44 & 33 \\
\hline
\end{tabular}


STATION NAME AND NUMBER—02340500 Mountain Oak Creek near Hamilton, GA

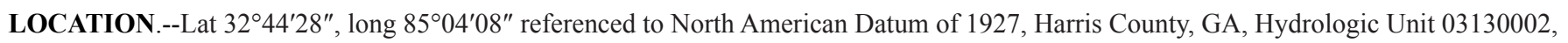
on right bank $300 \mathrm{ft}$ upstream from bridge on State Highway 103, 5 mi upstream from mouth, and 11 mi west of Hamilton.

DRAINAGE AREA. $-61.7 \mathrm{mi}^{2}$.

PERIOD OF RECORD.-December 1943 to September 1971.

PERIOD OF FREQUENCY ANALYSIS.-April 1944 to March 1971.

NUMBER OF CLIMATE YEARS IN FREQUENCY ANALYSIS.-27

REMARKS.-

\begin{tabular}{|c|c|c|c|c|}
\hline $\begin{array}{c}\text { Recurrence } \\
\text { intervals } \\
\text { (years) }\end{array}$ & \multicolumn{2}{|c|}{$\begin{array}{c}\text { Lowest average flow for } \\
\text { indicated number of } \\
\text { consecutive days } \\
\text { (cubic feet per second) }\end{array}$} & \multicolumn{2}{|c|}{$\begin{array}{c}\text { Time-sampling error } \\
\text { (in percent) }\end{array}$} \\
\hline \multicolumn{2}{|c|}{1} & 7 & 1 & 7 \\
\hline 2 & 13 & 15 & 11 & 13 \\
\hline 5 & 8.6 & 9.7 & 13 & 16 \\
\hline 10 & 6.6 & 7.5 & 16 & 22 \\
\hline 20 & 5.3 & 6.0 & 21 & \\
\hline
\end{tabular}

\begin{tabular}{|c|c|c|c|c|c|c|c|}
\hline \multicolumn{1}{|c|}{ DURATION OF DAILY FLOW } \\
\hline \multicolumn{7}{|c|}{ Flow equaled or exceeded for indicated percentage of time } \\
(cubic feet per second)
\end{tabular}


STATION NAME AND NUMBER—02341800 Upatoi Creek near Columbus, GA

LOCATION.--Lat 32²4’48”, long 8449'12" referenced to North American Datum of 1927, Chattahoochee County, GA, Hydrologic Unit 03130003, at downstream side of pier near left end of bridge on Red Arrow Road at Fort Benning, 2.0 miles downstream from Randall Creek, 2.0 miles upstream from Ochillee Creek, 8.0 miles southeast of Columbus, and 12.0 miles upstream from mouth.

DRAINAGE AREA. $-342 \mathrm{mi}^{2}$.

PERIOD OF RECORD.-April 1968 to March 2014.

PERIOD OF FREQUENCY ANALYSIS.-April 1968 to March 2014.

NUMBER OF CLIMATE YEARS IN FREQUENCY ANALYSIS.-46

REMARKS.-

\begin{tabular}{|c|c|c|c|c|}
\hline $\begin{array}{l}\text { Recurrence } \\
\text { intervals } \\
\text { (years) }\end{array}$ & \multicolumn{2}{|c|}{$\begin{array}{l}\text { Lowest average flow for } \\
\text { indicated number of } \\
\text { consecutive days } \\
\text { (cubic feet per second) }\end{array}$} & \multicolumn{2}{|c|}{$\begin{array}{l}\text { Time-sampling error } \\
\text { (in percent) }\end{array}$} \\
\hline & 1 & 7 & 1 & 7 \\
\hline 2 & 107 & 113 & 5 & 5 \\
\hline 5 & 81 & 87 & 6 & 5 \\
\hline 10 & 71 & 76 & 7 & 6 \\
\hline 20 & 63 & 67 & 8 & 8 \\
\hline 50 & 55 & 59 & 11 & 10 \\
\hline
\end{tabular}

\begin{tabular}{|c|c|c|c|c|c|c|c|}
\hline \multicolumn{1}{|c|}{ DURATION OF DAILY FLOW } \\
\hline \multicolumn{7}{|c|}{ Flow equaled or exceeded for indicated percentage of time } \\
(cubic feet per second)
\end{tabular}


STATION NAME AND NUMBER—02359000 Chipola River near Altha, FL

LOCATION.-Lat $30^{\circ} 32^{\prime} 02^{\prime \prime}$, long $85^{\circ} 09^{\prime} 55^{\prime \prime}$ referenced to North American Datum of 1927, in NW 1/4 sec.32, T.2 N., R.9 W., Calhoun County, FL, Hydrologic Unit 03130012, on right downstream side wingwall of bridge on county Road 274, 0.9 mi downstream from Holliman Branch, $3.5 \mathrm{mi}$ southwest of Altha, and $54 \mathrm{mi}$ upstream from mouth.

DRAINAGE AREA. $-781 \mathrm{mi}^{2}$.

PERIOD OF RECORD.-December 1912 to December 1913, October 1921 to September 1927, August 1929 to September 1931, March 1943 to March 2014.

PERIOD OF FREQUENCY ANALYSIS._-April 1922 to March 1927, April 1930 to March 1931, April 1943 to March 2014.

NUMBER OF CLIMATE YEARS IN FREQUENCY ANALYSIS.-77

\section{REMARKS.-}

\begin{tabular}{|c|c|c|c|c|}
\hline $\begin{array}{l}\text { Recurrence } \\
\text { intervals } \\
\text { (years) }\end{array}$ & \multicolumn{2}{|c|}{$\begin{array}{l}\text { Lowest average flow for } \\
\text { indicated number of } \\
\text { consecutive days } \\
\text { (cubic feet per second) }\end{array}$} & \multicolumn{2}{|c|}{$\begin{array}{l}\text { Time-sampling error } \\
\text { (in percent) }\end{array}$} \\
\hline & 1 & 7 & 1 & 7 \\
\hline 2 & 578 & 607 & 4 & 4 \\
\hline 5 & 442 & 465 & 4 & 4 \\
\hline 10 & 381 & 400 & 6 & 6 \\
\hline 20 & 335 & 352 & 7 & 7 \\
\hline 50 & 288 & 303 & 9 & 9 \\
\hline
\end{tabular}

\section{DURATION OF DAILY FLOW}

Flow equaled or exceeded for indicated percentage of time (cubic feet per second)

\begin{tabular}{|c|c|c|c|c|c|c|c|}
\hline Percentage & 5 & 10 & 25 & 50 & 75 & 90 & 95 \\
\hline Flow & 3,490 & 2,730 & 1,810 & 1,080 & 761 & 575 & 478 \\
\hline
\end{tabular}


STATION NAME AND NUMBER—02365500 Choctawhatchee River at Caryville, FL

LOCATION.--Lat 3046'32", long 8549'40" referenced to North American Datum of 1927, Holmes County, FL, Hydrologic Unit 03140203, near right bank on downstream side of bridge on U.S. Highway 90, $300 \mathrm{ft}$ downstream from Louisville and Nashville Railroad bridge, $0.8 \mathrm{mi}$ west of Caryville, $1.8 \mathrm{mi}$ downstream from Wrights Creek, and 64 mi upstream from mouth.

DRAINAGE AREA.-3,499 $\mathrm{mi}^{2}$.

PERIOD OF RECORD.- October 1929 to March 1995, October 1996 to March 2014.

PERIOD OF FREQUENCY ANALYSIS.-April 1930 to March 1994, April 2001 to March 2014.

NUMBER OF CLIMATE YEARS IN FREQUENCY ANALYSIS.—77

REMARKS.-

\begin{tabular}{|c|c|c|c|c|}
\hline $\begin{array}{c}\text { Recurrence } \\
\text { intervals } \\
\text { (years) }\end{array}$ & \multicolumn{2}{|c|}{$\begin{array}{c}\text { Lowest average flow for } \\
\text { indicated number of } \\
\text { consecutive days } \\
\text { (cubic feet per second) }\end{array}$} & \multicolumn{2}{|c|}{$\begin{array}{c}\text { Time-sampling error } \\
\text { (in percent) }\end{array}$} \\
\hline & 1 & 7 & 1 & 7 \\
\hline 2 & 1,160 & 1,220 & 4 & 5 \\
\hline 5 & 866 & 904 & 5 & 5 \\
\hline 10 & 747 & 777 & 5 & 6 \\
\hline 20 & 662 & 687 & 6 & 8 \\
\hline 50 & 579 & 599 & 8 & \\
\hline
\end{tabular}

\begin{tabular}{|c|c|c|c|c|c|c|c|}
\hline \multicolumn{1}{|c|}{ DURATION OF DAILY FLOW } \\
\hline \multicolumn{1}{|c|}{ Flow equaled or exceeded for indicated percentage of time } \\
(cubic feet per second)
\end{tabular}


STATION NAME AND NUMBER—02366000 Holmes Creek at Vernon, FL

LOCATION.--Lat 30³7'36", long 85² $42^{\prime} 44^{\prime \prime}$ referenced to North American Datum of 1927, Washington County, FL, Hydrologic Unit 03140203, in the center of the new bridge on upstream side on State Highway 79 at Vernon, 0.2 mi downstream from Pippin Mill Creek and 25 mi upstream from mouth.

DRAINAGE AREA. $-386 \mathrm{mi}^{2}$.

PERIOD OF RECORD.-April 1950 to May 1979, October 2005 to December 2013.

PERIOD OF FREQUENCY ANALYSIS.-April 1950 to March 1979, April 2006 to March 2013.

NUMBER OF CLIMATE YEARS IN FREQUENCY ANALYSIS.—-36

REMARKS.-

\begin{tabular}{|c|c|c|c|c|}
\hline $\begin{array}{c}\text { Recurrence } \\
\text { intervals } \\
\text { (years) }\end{array}$ & \multicolumn{2}{|c|}{$\begin{array}{c}\text { Lowest average flow for } \\
\text { indicated number of } \\
\text { consecutive days } \\
\text { (cubic feet per second) }\end{array}$} & \multicolumn{2}{|c|}{$\begin{array}{c}\text { Time-sampling error } \\
\text { (in percent) }\end{array}$} \\
\hline & 1 & 7 & 1 & 7 \\
\hline 2 & 302 & 307 & 2 & 2 \\
\hline 5 & 272 & 275 & 2 & 3 \\
\hline 10 & 257 & 260 & 3 & 3 \\
\hline 20 & 246 & 249 & 3 & 4 \\
\hline 50 & 234 & 237 & 4 & \\
\hline
\end{tabular}

\begin{tabular}{|c|c|c|c|c|c|c|c|}
\hline \multicolumn{1}{|c|}{ DURATION OF DAILY FLOW } \\
\hline \multicolumn{7}{|c|}{ Flow equaled or exceeded for indicated percentage of time } \\
(cubic feet per second)
\end{tabular}


STATION NAME AND NUMBER—02368000 Yellow River at Milligan, FL

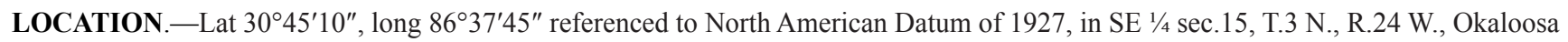
County, FL, Hydrologic Unit 03140103, near center on downstream side of bridge on U.S. Highway 90, 0.5 mi east of Milligan, 0.5 mi upstream from Trammel Creek, $6.7 \mathrm{mi}$ upstream from Shoal River, and $40 \mathrm{mi}$ upstream from mouth.

DRAINAGE AREA. $-624 \mathrm{mi}^{2}$.

PERIOD OF RECORD.-August 1938 to October 1993, August 1996 to March 2014.

PERIOD OF FREQUENCY ANALYSIS._-April 1939 to March 1993, April 1997 to March 2014.

NUMBER OF CLIMATE YEARS IN FREQUENCY ANALYSIS.-71

REMARKS.-

\begin{tabular}{|c|c|c|c|c|}
\hline $\begin{array}{c}\text { Recurrence } \\
\text { intervals } \\
\text { (years) }\end{array}$ & \multicolumn{2}{|c|}{$\begin{array}{c}\text { Lowest average flow for } \\
\text { indicated number of } \\
\text { consecutive days } \\
\text { (cubic feet per second) }\end{array}$} & \multicolumn{2}{|c|}{$\begin{array}{c}\text { Time-sampling error } \\
\text { (in percent) }\end{array}$} \\
\hline & 1 & 7 & 1 & 7 \\
\hline 2 & 261 & 274 & 5 & 5 \\
\hline 5 & 192 & 201 & 5 & 6 \\
\hline 10 & 164 & 171 & 6 & 7 \\
\hline 20 & 143 & 150 & 7 & 9 \\
\hline 50 & 123 & 129 & 10 & \\
\hline
\end{tabular}

\begin{tabular}{|c|c|c|c|c|c|c|c|}
\hline \multicolumn{1}{|c|}{ DURATION OF DAILY FLOW } \\
\hline \multicolumn{1}{|c|}{ Flow equaled or exceeded for indicated percentage of time } \\
(cubic feet per second)
\end{tabular}


STATION NAME AND NUMBER—02368500 Shoal River near Mossy Head, FL

LOCATION.-Lat 3047'45", long 86¹8'25" referenced to North American Datum of 1927, in SW 1/4 sec.36, T.4 N., R.21 W., Walton County, FL, Hydrologic Unit 03140103 , near center span on downstream side of bridge on County Road 1087, about $200 \mathrm{ft}$ downstream from Machine Branch, 3.9 mi north of Mossy Head, and 34 mi upstream from mouth.

DRAINAGE AREA. $-123 \mathrm{mi}^{2}$.

PERIOD OF RECORD.--March 1951 to October 1978, May 2000 to September 2002, October 2003 to March 2014.

PERIOD OF FREQUENCY ANALYSIS.-April 1951 to March 1978, April 2001 to March 2002, April 2004 to March 2014.

NUMBER OF CLIMATE YEARS IN FREQUENCY ANALYSIS.-38

REMARKS.- -

\begin{tabular}{|c|c|c|c|c|}
\hline $\begin{array}{c}\text { Recurrence } \\
\text { intervals } \\
\text { (years) }\end{array}$ & \multicolumn{2}{|c|}{$\begin{array}{c}\text { Lowest average flow for } \\
\text { indicated number of } \\
\text { consecutive days } \\
\text { (cubic feet per second) }\end{array}$} & \multicolumn{2}{|c|}{$\begin{array}{c}\text { Time-sampling error } \\
\text { (in percent) }\end{array}$} \\
\hline & 1 & 7 & 1 & 7 \\
\hline 2 & 67 & 70 & 7 & 8 \\
\hline 5 & 47 & 50 & 9 & 11 \\
\hline 10 & 38 & 40 & 11 & 14 \\
\hline 20 & 32 & 34 & 15 & 20 \\
\hline 50 & 25 & 27 & 21 & 7 \\
\hline
\end{tabular}

\begin{tabular}{|c|c|c|c|c|c|c|c|}
\hline \multicolumn{1}{|c|}{ DURATION OF DAILY FLOW } \\
\hline \multicolumn{7}{|c|}{ Flow equaled or exceeded for indicated percentage of time } \\
(cubic feet per second)
\end{tabular}


STATION NAME AND NUMBER—02369000 Shoal River near Crestview, FL

LOCATION.-Lat $30^{\circ} 41^{\prime} 50^{\prime \prime}$, long 86³4'15" referenced to North American Datum of 1927, in SW 1/4 sec.5, T.2 N., R.23 W., Okaloosa County, FL, Hydrologic Unit 03140103, near center of bridge on downstream side of southbound lane on State Highway 85, 3.5 mi downstream from Titi Creek, 4.2 mi south of Crestview, and 7 mi upstream from mouth.

DRAINAGE AREA. $-474 \mathrm{mi}^{2}$.

PERIOD OF RECORD.-August 1938 to March 2014.

PERIOD OF FREQUENCY ANALYSIS.-April 1939 to March 2014.

NUMBER OF CLIMATE YEARS IN FREQUENCY ANALYSIS.—75

REMARKS.-

\begin{tabular}{|c|c|c|c|c|}
\hline $\begin{array}{c}\text { Recurrence } \\
\text { intervals } \\
\text { (years) }\end{array}$ & \multicolumn{2}{|c|}{$\begin{array}{c}\text { Lowest average flow for } \\
\text { indicated number of } \\
\text { consecutive days } \\
\text { (cubic feet per second) }\end{array}$} & \multicolumn{2}{|c|}{$\begin{array}{c}\text { Time-sampling error } \\
\text { (in percent) }\end{array}$} \\
\hline & 1 & 7 & 1 & 7 \\
\hline 2 & 382 & 401 & 4 & 4 \\
\hline 5 & 295 & 310 & 4 & 5 \\
\hline 10 & 256 & 269 & 5 & 7 \\
\hline 20 & 226 & 238 & 7 & 9 \\
\hline 50 & 196 & 207 & 9 & 4 \\
\hline
\end{tabular}

\begin{tabular}{|c|c|c|c|c|c|c|c|}
\hline \multicolumn{1}{|c|}{ DURATION OF DAILY FLOW } \\
\hline \multicolumn{7}{|c|}{ Flow equaled or exceeded for indicated percentage of time } \\
(cubic feet per second)
\end{tabular}


STATION NAME AND NUMBER—02370000 Blackwater River near Baker, FL

LOCATION.-Lat $30^{\circ} 50^{\prime} 00^{\prime \prime}$, long $86^{\circ} 44^{\prime} 05^{\prime \prime}$ referenced to North American Datum of 1927, in SW 1/4 sec.22, T.4 N., R.25 W., Okaloosa County, FL, Hydrologic Unit 03140104, near left bank on downstream side of bridge on State Highway 4, 0.3 mi downstream from Red Wash Branch, 3.8 mi northwest of Baker, and 35 mi upstream from mouth.

DRAINAGE AREA. - $205 \mathrm{mi}^{2}$.

PERIOD OF RECORD.-April 1950 to November 1992, July 1996 to March 2014.

PERIOD OF FREQUENCY ANALYSIS.-April 1950 to March 1992, April 1997 to March 2014.

NUMBER OF CLIMATE YEARS IN FREQUENCY ANALYSIS.- 59

\section{REMARKS.-}

\begin{tabular}{|c|c|c|c|c|}
\hline $\begin{array}{c}\text { Recurrence } \\
\text { intervals } \\
\text { (years) }\end{array}$ & \multicolumn{2}{|c|}{$\begin{array}{c}\text { Lowest average flow for } \\
\text { indicated number of } \\
\text { consecutive days } \\
\text { (cubic feet per second) }\end{array}$} & \multicolumn{2}{|c|}{$\begin{array}{c}\text { Time-sampling error } \\
\text { (in percent) }\end{array}$} \\
\hline & 1 & 7 & 1 & 7 \\
\hline 2 & 85 & 89 & 4 & 4 \\
\hline 5 & 70 & 72 & 4 & 4 \\
\hline 10 & 63 & 65 & 4 & 5 \\
\hline 20 & 59 & 60 & 5 & 6 \\
\hline 50 & 54 & 56 & 6 & 4 \\
\hline
\end{tabular}

\begin{tabular}{|c|c|c|c|c|c|c|c|}
\hline \multicolumn{1}{|c|}{ DURATION OF DAILY FLOW } \\
\hline \multicolumn{7}{|c|}{ Flow equaled or exceeded for indicated percentage of time } \\
(cubic feet per second)
\end{tabular}


STATION NAME AND NUMBER—02370500 Big Coldwater Creek near Milton, FL

LOCATION.-Lat 3042'30", long 86 58'20" referenced to North American Datum of 1927, in SW 1/4 sec.5, T.2 N., R.27 W., Santa Rosa County, FL, Hydrologic Unit 03140104, near center channel on downstream side of bridge on State Highway 191, 3 mi upstream from mouth, and $6.5 \mathrm{mi}$ northeast of Milton.

DRAINAGE AREA. $-237 \mathrm{mi}^{2}$.

PERIOD OF RECORD.-December 1938 to June 1979, February 1980 to April 1980, July 1980 to March 1992, October 1997 to August 1999, May 2000 to February 2014.

PERIOD OF FREQUENCY ANALYSIS.-April 1939 to March 1979, April 1981 to March 1992, April 1998 to March 1999 , April 2001 to March 2013.

NUMBER OF CLIMATE YEARS IN FREQUENCY ANALYSIS. - 63

REMARKS.-

\begin{tabular}{|c|c|c|c|c|}
\hline $\begin{array}{c}\text { Recurrence } \\
\text { intervals } \\
\text { (years) }\end{array}$ & \multicolumn{2}{|c|}{$\begin{array}{l}\text { Lowest average flow for } \\
\text { indicated number of } \\
\text { consecutive days } \\
\text { (cubic feet per second) }\end{array}$} & \multicolumn{2}{|c|}{$\begin{array}{l}\text { Time-sampling error } \\
\text { (in percent) }\end{array}$} \\
\hline & 1 & 7 & 1 & 7 \\
\hline 2 & 258 & 264 & 3 & 3 \\
\hline 5 & 213 & 220 & 3 & 3 \\
\hline 10 & 192 & 199 & 4 & 4 \\
\hline 20 & 175 & 183 & 5 & 5 \\
\hline 50 & 158 & 166 & 7 & 7 \\
\hline
\end{tabular}

\begin{tabular}{|c|c|c|c|c|c|c|c|}
\hline \multicolumn{1}{|c|}{ DURATION OF DAILY FLOW } \\
\hline \multicolumn{2}{|c|}{ Flow equaled or exceeded for indicated percentage of time } \\
(cubic feet per second)
\end{tabular}


STATION NAME AND NUMBER—02370700 Pond Creek near Milton, FL

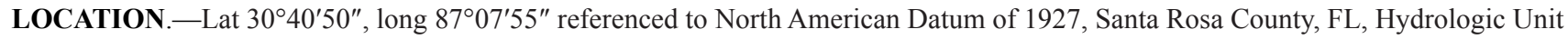
03140104, near center of span on upstream side of bridge on State Highway 191, 0.6 mi downstream from Reader Creek, 6.4 mi northwest of Milton, and $10 \mathrm{mi}$ upstream from mouth.

DRAINAGE AREA. $-58.7 \mathrm{mi}^{2}$.

PERIOD OF RECORD.- January 1958 to October 1978, April 1979 to July 1979, November 1999 to January 2003, March 2003 to September 2006 .

PERIOD OF FREQUENCY ANALYSIS.-April 1958 to March 1978, April 2000 to March 2002, April 2003 to March 2006.

NUMBER OF CLIMATE YEARS IN FREQUENCY ANALYSIS. - 25

\section{REMARKS.-}

\begin{tabular}{|c|c|c|c|c|}
\hline $\begin{array}{c}\text { Recurrence } \\
\text { intervals } \\
\text { (years) }\end{array}$ & \multicolumn{2}{|c|}{$\begin{array}{c}\text { Lowest average flow for } \\
\text { indicated number of } \\
\text { consecutive days } \\
\text { (cubic feet per second) }\end{array}$} & \multicolumn{2}{|c|}{$\begin{array}{c}\text { Time-sampling error } \\
\text { (in percent) }\end{array}$} \\
\hline \multicolumn{2}{|c|}{1} & 7 & 1 & 7 \\
\hline 2 & 44 & 45 & 6 & 7 \\
\hline 5 & 35 & 36 & 6 & 8 \\
\hline 10 & 31 & 32 & 8 & 11 \\
\hline 20 & 28 & 28 & 10 & 6 \\
\hline
\end{tabular}

\begin{tabular}{|c|c|c|c|c|c|c|c|}
\hline \multicolumn{7}{|c|}{ DURATION OF DAILY FLOW } \\
\hline \multicolumn{7}{|c|}{ Flow equaled or exceeded for indicated percentage of time } \\
(cubic feet per second)
\end{tabular}


STATION NAME AND NUMBER—02376000 Pine Barren Creek near Barth, FL

LOCATION.--Lat $30^{\circ} 47^{\prime} 55^{\prime \prime}$, long $87^{\circ} 22^{\prime} 05^{\prime \prime}$ referenced to North American Datum of 1927, Escambia County, FL, Hydrologic Unit 03140305, near right bank $10 \mathrm{ft}$ downstream from Wiggins Bridge on private road, $0.3 \mathrm{mi}$ upstream from Blue Water Creek, 4.0 mi northwest of Barth, and $7.3 \mathrm{mi}$ upstream from mouth.

DRAINAGE AREA. $-75.3 \mathrm{mi}^{2}$.

PERIOD OF RECORD.- October 1952 to September 1994.

PERIOD OF FREQUENCY ANALYSIS.-April 1953 to March 1994.

NUMBER OF CLIMATE YEARS IN FREQUENCY ANALYSIS.-41

REMARKS.-

\begin{tabular}{|c|c|c|c|c|}
\hline $\begin{array}{c}\text { Recurrence } \\
\text { intervals } \\
\text { (years) }\end{array}$ & \multicolumn{2}{|c|}{$\begin{array}{l}\text { Lowest average flow for } \\
\text { indicated number of } \\
\text { consecutive days } \\
\text { (cubic feet per second) }\end{array}$} & \multicolumn{2}{|c|}{$\begin{array}{l}\text { Time-sampling error } \\
\text { (in percent) }\end{array}$} \\
\hline & 1 & 7 & 1 & 7 \\
\hline 2 & 78 & 80 & 3 & 3 \\
\hline 5 & 67 & 69 & 3 & 3 \\
\hline 10 & 62 & 64 & 4 & 4 \\
\hline 20 & 58 & 59 & 5 & 5 \\
\hline 50 & 53 & 55 & 7 & 7 \\
\hline
\end{tabular}

\begin{tabular}{|c|c|c|c|c|c|c|c|}
\hline \multicolumn{1}{|c|}{ DURATION OF DAILY FLOW } \\
\hline \multicolumn{7}{|c|}{ Flow equaled or exceeded for indicated percentage of time } \\
(cubic feet per second)
\end{tabular}


STATION NAME AND NUMBER—02376300 Brushy Creek near Walnut Hill, FL

LOCATION.--Lat 3053'21", long 87³2'24" referenced to North American Datum of 1927, Escambia County, FL, Hydrologic Unit 03140106, near right bank on downstream side of county road bridge, 1,000 ft downstream from Rocky Creek, 2.0 mi west of Walnut Hill, and 7.9 mi upstream from mouth.

DRAINAGE AREA. $-49 \mathrm{mi}^{2}$.

PERIOD OF RECORD.-February 1958 to November 1991.

PERIOD OF FREQUENCY ANALYSIS. - April 1958 to March 1991.

NUMBER OF CLIMATE YEARS IN FREQUENCY ANALYSIS.-33

REMARKS.-

\begin{tabular}{|c|c|c|c|c|}
\hline $\begin{array}{c}\text { Recurrence } \\
\text { intervals } \\
\text { (years) }\end{array}$ & \multicolumn{2}{|c|}{$\begin{array}{c}\text { Lowest average flow for } \\
\text { indicated number of } \\
\text { consecutive days } \\
\text { (cubic feet per second) }\end{array}$} & \multicolumn{2}{|c|}{$\begin{array}{c}\text { Time-sampling error } \\
\text { (in percent) }\end{array}$} \\
\hline & 1 & 7 & 1 & 7 \\
\hline 2 & 50 & 51 & 3 & 3 \\
\hline 5 & 44 & 46 & 3 & 3 \\
\hline 10 & 42 & 43 & 3 & 4 \\
\hline 20 & 40 & 41 & 4 & 5 \\
\hline 50 & 38 & 39 & 5 & 3 \\
\hline
\end{tabular}

\section{DURATION OF DAILY FLOW}

Flow equaled or exceeded for indicated percentage of time (cubic feet per second)

\begin{tabular}{|c|c|c|c|c|c|c|c|}
\hline Percentage & 5 & 10 & 25 & 50 & 75 & 90 & 95 \\
\hline Flow & 274 & 162 & 98 & 72 & 59 & 52 & 49 \\
\hline
\end{tabular}


STATION NAME AND NUMBER—02380500 Coosawattee River near Ellijay, GA

LOCATION.--Lat 3440’30”, long 84³0’31” referenced to North American Datum of 1927, Gilmer County, GA, Hydrologic Unit 03150102, on right bank, $0.5 \mathrm{mi}$ downstream from GA Route 5, 2.2 mi downstream from confluence of Cartecay and Elijay Rivers, 6.9 mi upstream from confluence with Mountaintown Creek, and $2.0 \mathrm{mi}$ southwest of Ellijay.

DRAINAGE AREA.-236 $\mathrm{mi}^{2}$.

PERIOD OF RECORD.-October 1938 to December 1949, June 1963 to March 2014.

PERIOD OF FREQUENCY ANALYSIS.-April 1939 to March 1949, April 1964 to March 2014.

NUMBER OF CLIMATE YEARS IN FREQUENCY ANALYSIS.-60

REMARKS.-Potentially influenced by upstream diversions (Gotvald, 2016).

\begin{tabular}{|c|c|c|c|c|}
\hline $\begin{array}{c}\text { Recurrence } \\
\text { intervals } \\
\text { (years) }\end{array}$ & \multicolumn{2}{|c|}{$\begin{array}{c}\text { Lowest average flow for } \\
\text { indicated number of } \\
\text { consecutive days } \\
\text { (cubic feet per second) }\end{array}$} & \multicolumn{2}{|c|}{$\begin{array}{c}\text { Time-sampling error } \\
\text { (in percent) }\end{array}$} \\
\hline & 1 & 7 & 1 & 5 \\
\hline 2 & 154 & 162 & 5 & 7 \\
\hline 5 & 110 & 116 & 7 & 9 \\
\hline 10 & 89 & 95 & 9 & 12 \\
\hline 20 & 74 & 79 & 12 & 17 \\
\hline 50 & 59 & 63 & 17 & 7 \\
\hline
\end{tabular}

\begin{tabular}{|c|c|c|c|c|c|c|c|}
\hline \multicolumn{1}{|c|}{ DURATION OF DAILY FLOW } \\
\hline \multicolumn{7}{|c|}{ Flow equaled or exceeded for indicated percentage of time } \\
(cubic feet per second)
\end{tabular}


STATION NAME AND NUMBER—02381600 Fausett Creek near Talking Rock, GA

LOCATION.--Lat 34³4'13”, long 84²8’08”' referenced to North American Datum of 1927, Gilmer County, GA, Hydrologic Unit 03150102, on right bank 25.0 feet upstream from culvert on County Road 1011, 3.6 miles upstream from mouth, and 4.5 miles northeast of Talking Rock.

DRAINAGE AREA. $-9.99 \mathrm{mi}^{2}$.

PERIOD OF RECORD.--October 1974 to March 2014.

PERIOD OF FREQUENCY ANALYSIS.-April 1975 to March 2014.

NUMBER OF CLIMATE YEARS IN FREQUENCY ANALYSIS. - 39

REMARKS.-

\begin{tabular}{|c|c|c|c|c|}
\hline $\begin{array}{l}\text { Recurrence } \\
\text { intervals } \\
\text { (years) }\end{array}$ & \multicolumn{2}{|c|}{$\begin{array}{l}\text { Lowest average flow for } \\
\text { indicated number of } \\
\text { consecutive days } \\
\text { (cubic feet per second) }\end{array}$} & \multicolumn{2}{|c|}{$\begin{array}{l}\text { Time-sampling error } \\
\text { (in percent) }\end{array}$} \\
\hline & 1 & 7 & 1 & 7 \\
\hline 2 & 3.2 & 3.6 & 18 & 17 \\
\hline 5 & 1.3 & 1.6 & 24 & 22 \\
\hline 10 & 0.75 & 0.92 & 32 & 30 \\
\hline 20 & 0.44 & 0.56 & 47 & 43 \\
\hline 50 & 0.22 & 0.30 & 77 & 69 \\
\hline
\end{tabular}

\section{DURATION OF DAILY FLOW}

Flow equaled or exceeded for indicated percentage of time (cubic feet per second)

\begin{tabular}{|c|c|c|c|c|c|c|c|}
\hline Percentage & 5 & 10 & 25 & 50 & 75 & 90 & 95 \\
\hline Flow & 43 & 30 & 18 & 11 & 6.5 & 3.6 & 2.4 \\
\hline
\end{tabular}


STATION NAME AND NUMBER—02382000 Scarecorn Creek at Hinton, GA

LOCATION.--Lat $34^{\circ} 28^{\prime} 04^{\prime \prime}$, long 84³5'30” referenced to North American Datum of 1927, Pickens County, GA, Hydrologic Unit 03150102, on left bank $100 \mathrm{ft}$ upstream from bridge on State Highway 53, 0.2 mi west of Hinton, 1 mi upstream from Dean's Mill, and 5 mi upstream from mouth.

DRAINAGE AREA. $-21.3 \mathrm{mi}^{2}$.

PERIOD OF RECORD.-April 1939 to December 1942, May 1959 to September 1974, August 1986 to April 1991.

PERIOD OF FREQUENCY ANALYSIS._-April 1939 to March 1942, April 1960 to March 1974, April 1981 to March 1991.

NUMBER OF CLIMATE YEARS IN FREQUENCY ANALYSIS. - - 21

REMARKS.-

\begin{tabular}{|c|c|c|c|c|}
\hline $\begin{array}{l}\text { Recurrence } \\
\text { intervals } \\
\text { (years) }\end{array}$ & \multicolumn{2}{|c|}{$\begin{array}{l}\text { Lowest average flow for } \\
\text { indicated number of } \\
\text { consecutive days } \\
\text { (cubic feet per second) }\end{array}$} & \multicolumn{2}{|c|}{$\begin{array}{l}\text { Time-sampling error } \\
\text { (in percent) }\end{array}$} \\
\hline & 1 & 7 & 1 & 7 \\
\hline 2 & 3.6 & 4.2 & 19 & 18 \\
\hline 5 & 1.8 & 2.2 & 24 & 23 \\
\hline 10 & 1.2 & 1.5 & 32 & 31 \\
\hline 20 & 0.81 & 1.0 & 46 & 45 \\
\hline
\end{tabular}

\begin{tabular}{|c|c|c|c|c|c|c|c|}
\hline \multicolumn{7}{|c|}{ DURATION OF DAILY FLOW } \\
\hline \multicolumn{7}{|c|}{ Flow equaled or exceeded for indicated percentage of time } \\
(cubic feet per second)
\end{tabular}


STATION NAME AND NUMBER—02382200 Talking Rock Creek near Hinton, GA

LOCATION.--Lat 34³1'22”, long 84³6’40” referenced to North American Datum of 1983, Pickens County, GA, Hydrologic Unit 03150102, on left bank, 300 feet downstream from Scarecorn Creek, and 3.3 miles northwest of Hinton, GA.

DRAINAGE AREA.-119 $\mathrm{mi}^{2}$.

PERIOD OF RECORD.- November 1973 to March 2014.

PERIOD OF FREQUENCY ANALYSIS.-April 1974 to March 2014.

NUMBER OF CLIMATE YEARS IN FREQUENCY ANALYSIS. - 40

REMARKS.-

\begin{tabular}{|c|c|c|c|c|}
\hline $\begin{array}{l}\text { Recurrence } \\
\text { intervals } \\
\text { (years) }\end{array}$ & \multicolumn{2}{|c|}{$\begin{array}{l}\text { Lowest average flow for } \\
\text { indicated number of } \\
\text { consecutive days } \\
\text { (cubic feet per second) }\end{array}$} & \multicolumn{2}{|c|}{$\begin{array}{l}\text { Time-sampling error } \\
\text { (in percent) }\end{array}$} \\
\hline & 1 & 7 & 1 & 7 \\
\hline 2 & 34 & 38 & 18 & 17 \\
\hline 5 & 14 & 18 & 24 & 23 \\
\hline 10 & 8.1 & 10 & 32 & 31 \\
\hline 20 & 4.7 & 6.0 & 48 & 46 \\
\hline 50 & 2.3 & 3.0 & 81 & 80 \\
\hline
\end{tabular}

\section{DURATION OF DAILY FLOW}

Flow equaled or exceeded for indicated percentage of time (cubic feet per second)

\begin{tabular}{|c|c|c|c|c|c|c|c|}
\hline Percentage & 5 & 10 & 25 & 50 & 75 & 90 & 95 \\
\hline Flow & 460 & 313 & 196 & 113 & 65 & 36 & 25 \\
\hline
\end{tabular}


STATION NAME AND NUMBER—02383000 Rock Creek near Fairmont, GA

LOCATION.--Lat 34²1'32”, long 8446’46” referenced to North American Datum of 1927, Bartow County, GA, Hydrologic Unit 03150102, on right upstream wingwall of culvert on State Highway 140, 2.8 mi upstream from mouth, and 7 mi southwest of Fairmont.

DRAINAGE AREA. $-6.17 \mathrm{mi}^{2}$.

PERIOD OF RECORD.- - October 1951 to September 1974.

PERIOD OF FREQUENCY ANALYSIS.-April 1952 to March 1974.

NUMBER OF CLIMATE YEARS IN FREQUENCY ANALYSIS.-22

REMARKS.-

\begin{tabular}{|c|c|c|c|c|}
\hline \multirow[t]{2}{*}{$\begin{array}{l}\text { Recurrence } \\
\text { intervals } \\
\text { (years) }\end{array}$} & \multicolumn{2}{|c|}{$\begin{array}{l}\text { Lowest average flow for } \\
\text { indicated number of } \\
\text { consecutive days } \\
\text { (cubic feet per second) }\end{array}$} & \multicolumn{2}{|c|}{$\begin{array}{l}\text { Time-sampling error } \\
\text { (in percent) }\end{array}$} \\
\hline & 1 & 7 & 1 & 7 \\
\hline 2 & 0.70 & 0.76 & 11 & 9 \\
\hline 5 & 0.45 & 0.54 & 14 & 11 \\
\hline 10 & 0.35 & 0.44 & 18 & 13 \\
\hline 20 & 0.28 & 0.38 & 24 & 17 \\
\hline 50 & 0.22 & 0.31 & 33 & 22 \\
\hline
\end{tabular}

\section{DURATION OF DAILY FLOW}

Flow equaled or exceeded for indicated percentage of time (cubic feet per second)

\begin{tabular}{|c|c|c|c|c|c|c|c|}
\hline Percentage & 5 & 10 & 25 & 50 & 75 & 90 & 95 \\
\hline Flow & 22 & 13 & 6.8 & 3.1 & 1.6 & 1.0 & 0.86 \\
\hline
\end{tabular}


STATION NAME AND NUMBER—02383500 Coosawattee River near Pine Chapel, GA

LOCATION.--Lat 34³3'51”, long 8449'59” referenced to North American Datum of 1983, Gordon County, GA, Hydrologic Unit 03150102, on the downstream side of right bank pier of Owens Bridge on Owens Gin Road, 1.4 miles downstream from Sallacoa Creek, 8.7 miles upstream from confluence with Conasauga River, and 2.4 miles east of Pine Chapel.

DRAINAGE AREA. $-831 \mathrm{mi}^{2}$.

PERIOD OF RECORD.-November 1938 to March 2014.

PERIOD OF FREQUENCY ANALYSIS.-April 1939 to March 1973.

NUMBER OF CLIMATE YEARS IN FREQUENCY ANALYSIS. - 34

REMARKS.-Since 1974, flow regulated by Carters Lake and Carters Re-regulation Dam. Frequency and duration data represent pre-regulation conditions.

\begin{tabular}{|c|c|c|c|c|}
\hline $\begin{array}{l}\text { Recurrence } \\
\text { intervals } \\
\text { (years) }\end{array}$ & \multicolumn{2}{|c|}{$\begin{array}{l}\text { Lowest average flow for } \\
\text { indicated number of } \\
\text { consecutive days } \\
\text { (cubic feet per second) }\end{array}$} & \multicolumn{2}{|c|}{$\begin{array}{l}\text { Time-sampling error } \\
\text { (in percent) }\end{array}$} \\
\hline & 1 & 7 & 1 & 7 \\
\hline 2 & 338 & 356 & 5 & 5 \\
\hline 5 & 282 & 296 & 4 & 4 \\
\hline 10 & 259 & 273 & 4 & 4 \\
\hline 20 & 244 & 256 & 5 & 5 \\
\hline 50 & 230 & 241 & 7 & 7 \\
\hline
\end{tabular}

\begin{tabular}{|c|c|c|c|c|c|c|c|}
\hline \multicolumn{7}{|c|}{ DURATION OF DAILY FLOW } \\
\hline \multicolumn{7}{|c|}{ Flow equaled or exceeded for indicated percentage of time } \\
(cubic feet per second)
\end{tabular}


STATION NAME AND NUMBER—02387500 Oostanaula River near Resaca, GA

LOCATION.--Lat 34³4'37.6”, long 8456’30.67" referenced to North American Datum of 1983, Gordon County, GA, Hydrologic Unit 03150103, on downstream side of center pier of bridge on US 41 at Resaca, 200 feet downstream from Nashville, Chattanooga, \& St. Louis Railway bridge, 0.8 miles upstream from Camp Creek, and 3.5 miles downstream from confluence of Conasauga and Coosawattee Rivers.

DRAINAGE AREA. $-1,602 \mathrm{mi}^{2}$.

PERIOD OF RECORD.-November 1892 to March 2014.

PERIOD OF FREQUENCY ANALYSIS.-April 1893 to March 1973.

NUMBER OF CLIMATE YEARS IN FREQUENCY ANALYSIS. - 80

REMARKS.-Since 1974, flow regulated by Carters Lake and Carters Re-regulation Dam. Frequency and duration data represent pre-regulation conditions.

\begin{tabular}{|c|c|c|c|c|}
\hline $\begin{array}{c}\text { Recurrence } \\
\text { intervals } \\
\text { (years) }\end{array}$ & \multicolumn{2}{|c|}{$\begin{array}{l}\text { Lowest average flow for } \\
\text { indicated number of } \\
\text { consecutive days } \\
\text { (cubic feet per second) }\end{array}$} & \multicolumn{2}{|c|}{$\begin{array}{l}\text { Time-sampling error } \\
\text { (in percent) }\end{array}$} \\
\hline & 1 & 7 & 1 & 7 \\
\hline 2 & 480 & 505 & 4 & 4 \\
\hline 5 & 358 & 378 & 4 & 4 \\
\hline 10 & 309 & 327 & 5 & 5 \\
\hline 20 & 274 & 291 & 6 & 6 \\
\hline 50 & 241 & 256 & 8 & 8 \\
\hline
\end{tabular}

\begin{tabular}{|c|c|c|c|c|c|c|c|}
\hline \multicolumn{1}{|c|}{ DURATION OF DAILY FLOW } \\
\hline \multicolumn{1}{|c|}{ Flow equaled or exceeded for indicated percentage of time } \\
(cubic feet per second)
\end{tabular}


STATION NAME AND NUMBER—02388000 West Armuchee Creek near Subligna, GA

LOCATION.--Lat 34³4'04”, long 8509'16” referenced to North American Datum of 1927, Chattooga County, GA, Hydrologic Unit 03150103, on left bank $500 \mathrm{ft}$ downstream from bridge on county road, 1 mi upstream from Ruff Creek, and 2 mi east of Subligna.

DRAINAGE AREA. $-36.4 \mathrm{mi}^{2}$.

PERIOD OF RECORD.-April 1939 to June 1940, May 1960 to September 1981.

PERIOD OF FREQUENCY ANALYSIS.-April 1939 to March 1940, April 1961 to March 1981.

NUMBER OF CLIMATE YEARS IN FREQUENCY ANALYSIS.-21

REMARKS.-

\begin{tabular}{|c|c|c|c|c|}
\hline $\begin{array}{l}\text { Recurrence } \\
\text { intervals } \\
\text { (years) }\end{array}$ & \multicolumn{2}{|c|}{$\begin{array}{l}\text { Lowest average flow for } \\
\text { indicated number of } \\
\text { consecutive days } \\
\text { (cubic feet per second) }\end{array}$} & \multicolumn{2}{|c|}{$\begin{array}{l}\text { Time-sampling error } \\
\text { (in percent) }\end{array}$} \\
\hline & 1 & 7 & 1 & 7 \\
\hline 2 & 6.9 & 7.2 & 6 & 6 \\
\hline 5 & 5.7 & 6.1 & 6 & 5 \\
\hline 10 & 5.2 & 5.6 & 6 & 5 \\
\hline 20 & 4.8 & 5.3 & 7 & 6 \\
\hline
\end{tabular}

DURATION OF DAILY FLOW

Flow equaled or exceeded for indicated percentage of time (cubic feet per second)

\begin{tabular}{|c|c|c|c|c|c|c|c|}
\hline Percentage & 5 & 10 & 25 & 50 & 75 & 90 & 95 \\
\hline Flow & 209 & 129 & 61 & 26 & 12 & 8.6 & 7.4 \\
\hline
\end{tabular}


STATION NAME AND NUMBER—02388300 Heath Creek near Rome, GA

LOCATION.--Lat 34²1'57’, long 85¹6’17” referenced to North American Datum of 1927, Floyd County, GA, Hydrologic Unit 03150103.

DRAINAGE AREA. $-14.7 \mathrm{mi}^{2}$.

PERIOD OF RECORD.-May 1968 to September 1989.

PERIOD OF FREQUENCY ANALYSIS.-April 1969 to March 1989.

NUMBER OF CLIMATE YEARS IN FREQUENCY ANALYSIS.-20

REMARKS.-

\begin{tabular}{|c|c|c|c|c|}
\hline $\begin{array}{c}\text { Recurrence } \\
\text { intervals } \\
\text { (years) }\end{array}$ & \multicolumn{2}{|c|}{$\begin{array}{c}\text { Lowest average flow for } \\
\text { indicated number of } \\
\text { consecutive days } \\
\text { (cubic feet per second) }\end{array}$} & \multicolumn{2}{|c|}{$\begin{array}{c}\text { Time-sampling error } \\
\text { (in percent) }\end{array}$} \\
\hline \multicolumn{7}{|c|}{1} & 7 & 1 & 7 \\
\hline 2 & 1.7 & 1.7 & 12 & 14 \\
\hline 5 & 1.1 & 1.2 & 15 & 18 \\
\hline 10 & 0.83 & 0.91 & 20 & 24 \\
\hline 20 & 0.65 & 0.73 & 28 & \\
\hline
\end{tabular}

\section{DURATION OF DAILY FLOW}

Flow equaled or exceeded for indicated percentage of time (cubic feet per second)

\begin{tabular}{|c|c|c|c|c|c|c|c|}
\hline Percentage & 5 & 10 & 25 & 50 & 75 & 90 & 95 \\
\hline Flow & 102 & 59 & 24 & 7.2 & 3.0 & 2.0 & 1.6 \\
\hline
\end{tabular}


STATION NAME AND NUMBER—02389000 Etowah River near Dawsonville, GA

LOCATION.--Lat 34²2'57”, long 8403'21”' referenced to North American Datum of 1927, Dawson County, GA, Hydrologic Unit 03150104, on left bank 0.4 mi upstream from Palmer Creek, 0.5 mi upstream from bridge on State Highway 53, 1.2 mi downstream from Russell Creek, 4 mi southeast of Dawsonville, and 7.5 mi upstream from Shoal Creek.

DRAINAGE AREA. $-107 \mathrm{mi}^{2}$.

PERIOD OF RECORD.-March 1940 to September 1976.

PERIOD OF FREQUENCY ANALYSIS.-April 1940 to March 1976.

NUMBER OF CLIMATE YEARS IN FREQUENCY ANALYSIS.-36

REMARKS.-

\begin{tabular}{|c|c|c|c|c|}
\hline $\begin{array}{c}\text { Recurrence } \\
\text { intervals } \\
\text { (years) }\end{array}$ & \multicolumn{2}{|c|}{$\begin{array}{c}\text { Lowest average flow for } \\
\text { indicated number of } \\
\text { consecutive days } \\
\text { (cubic feet per second) }\end{array}$} & \multicolumn{2}{|c|}{$\begin{array}{c}\text { Time-sampling error } \\
\text { (in percent) }\end{array}$} \\
\hline \multicolumn{7}{|c|}{1} & 7 & 1 & 7 \\
\hline 2 & 91 & 95 & 6 & 6 \\
\hline 5 & 70 & 73 & 6 & 7 \\
\hline 10 & 61 & 64 & 7 & 9 \\
\hline 20 & 55 & 57 & 9 & 11 \\
\hline 50 & 49 & 50 & 11 & 6 \\
\hline
\end{tabular}

DURATION OF DAILY FLOW

Flow equaled or exceeded for indicated percentage of time (cubic feet per second)

\begin{tabular}{|c|c|c|c|c|c|c|c|}
\hline Percentage & 5 & 10 & 25 & 50 & 75 & 90 & 95 \\
\hline Flow & 599 & 470 & 323 & 212 & 139 & 104 & 88 \\
\hline
\end{tabular}


STATION NAME AND NUMBER—02390000 Amicalola Creek near Dawsonville, GA

LOCATION.-Lat $34^{\circ} 25^{\prime} 32^{\prime \prime}$, long $84^{\circ} 12^{\prime} 43^{\prime \prime}$ referenced to North American Datum of 1927, Dawson County, GA, Hydrologic Unit 03150104, at the bridge on SR 53, on left bank, 1.8 miles below Holly Creek and 6.9 miles above Etowah River.

DRAINAGE AREA. $-89.0 \mathrm{mi}^{2}$.

PERIOD OF RECORD.-April 1939 to May 1952, September 2005 to March 2014.

PERIOD OF FREQUENCY ANALYSIS.-April 1939 to March 1952, April 2006 to March 2014.

NUMBER OF CLIMATE YEARS IN FREQUENCY ANALYSIS.-21

REMARKS.-

\begin{tabular}{|c|c|c|c|c|}
\hline $\begin{array}{l}\text { Recurrence } \\
\text { intervals } \\
\text { (years) }\end{array}$ & \multicolumn{2}{|c|}{$\begin{array}{l}\text { Lowest average flow for } \\
\text { indicated number of } \\
\text { consecutive days } \\
\text { (cubic feet per second) }\end{array}$} & \multicolumn{2}{|c|}{$\begin{array}{l}\text { Time-sampling error } \\
\text { (in percent) }\end{array}$} \\
\hline & 1 & 7 & 1 & 7 \\
\hline 2 & 63 & 66 & 10 & 10 \\
\hline 5 & 43 & 46 & 12 & 12 \\
\hline 10 & 35 & 37 & 15 & 14 \\
\hline 20 & 29 & 32 & 19 & 18 \\
\hline
\end{tabular}

\section{DURATION OF DAILY FLOW}

Flow equaled or exceeded for indicated percentage of time (cubic feet per second)

\begin{tabular}{|c|c|c|c|c|c|c|c|}
\hline Percentage & 5 & 10 & 25 & 50 & 75 & 90 & 95 \\
\hline Flow & 472 & 364 & 248 & 157 & 99 & 69 & 59 \\
\hline
\end{tabular}


STATION NAME AND NUMBER—02392000 Etowah River at Canton, GA

LOCATION.-Lat 34¹4'23.4”, long 84²9’41.08” referenced to North American Datum of 1927, Cherokee County, GA, Hydrologic Unit 03150104, on left bank 100 feet downstream from bridge on GA 5 Spur and GA 140 at Canton, 0.8 mi upstream from confluence with Canton Creek, $1.8 \mathrm{mi}$ downstream from confluence with Hickory Log Creek, and $0.3 \mathrm{mi}$ northwest of Canton.

DRAINAGE AREA. $-613 \mathrm{mi}^{2}$.

PERIOD OF RECORD.- October 1896 to September 1905, October 1936 to March 2014.

PERIOD OF FREQUENCY ANALYSIS._-April 1897 to March 1905, April 1937 to March 2014.

NUMBER OF CLIMATE YEARS IN FREQUENCY ANALYSIS.- - 85

REMARKS.-Negligible amount of regulation caused by mill dams on both mainstream and tributaries. There is an intake for the Hickory Log Creek Reservior located approximately 1.75 miles upstream of the gage an at USGS gaging station 02391860-Etowah River below I-575 at Canton, GA.

\begin{tabular}{|c|c|c|c|c|}
\hline $\begin{array}{c}\text { Recurrence } \\
\text { intervals } \\
\text { (years) }\end{array}$ & \multicolumn{2}{|c|}{$\begin{array}{c}\text { Lowest average flow for } \\
\text { indicated number of } \\
\text { consecutive days } \\
\text { (cubic feet per second) }\end{array}$} & \multicolumn{2}{|c|}{$\begin{array}{c}\text { Time-sampling error } \\
\text { (in percent) }\end{array}$} \\
\hline & 1 & 7 & 1 & 7 \\
\hline 2 & 343 & 363 & 6 & 7 \\
\hline 5 & 221 & 237 & 7 & 9 \\
\hline 10 & 168 & 183 & 10 & 13 \\
\hline 20 & 131 & 145 & 14 & 18 \\
\hline 50 & 96 & 109 & 20 & 6 \\
\hline
\end{tabular}

\begin{tabular}{|c|c|c|c|c|c|c|c|}
\hline \multicolumn{1}{|c|}{ DURATION OF DAILY FLOW } \\
\hline \multicolumn{7}{|c|}{ Flow equaled or exceeded for indicated percentage of time } \\
(cubic feet per second)
\end{tabular}


STATION NAME AND NUMBER—02392500 Little River near Roswell, GA

LOCATION.-Lat 3407'09”, long 84²3'18” referenced to North American Datum of 1927, Fulton County, GA, Hydrologic Unit 03150104, on upstream side of bridge on State Highway 140, 1 mi downstream from Cooper Sandy Creek, and 7 mi north of Roswell.

DRAINAGE AREA. $-60.0 \mathrm{mi}^{2}$.

PERIOD OF RECORD._-January 1947 to September 1976.

PERIOD OF FREQUENCY ANALYSIS.-April 1947 to March 1976.

NUMBER OF CLIMATE YEARS IN FREQUENCY ANALYSIS.-29

REMARKS.-Potentially influenced by upstream diversions (Gotvald, 2016).

\begin{tabular}{|c|c|c|c|c|}
\hline $\begin{array}{c}\text { Recurrence } \\
\text { intervals } \\
\text { (years) }\end{array}$ & \multicolumn{2}{|c|}{$\begin{array}{c}\text { Lowest average flow for } \\
\text { indicated number of } \\
\text { consecutive days } \\
\text { (cubic feet per second) }\end{array}$} & \multicolumn{2}{|c|}{$\begin{array}{c}\text { Time-sampling error } \\
\text { (in percent) }\end{array}$} \\
\hline \multicolumn{7}{|c|}{1} & 7 & 1 & 7 \\
\hline 2 & 13 & 15 & 15 & 15 \\
\hline 5 & 6.9 & 8.0 & 19 & 24 \\
\hline 10 & 4.7 & 5.5 & 24 & 33 \\
\hline 20 & 3.3 & 3.9 & 33 & \\
\hline
\end{tabular}

\section{DURATION OF DAILY FLOW}

Flow equaled or exceeded for indicated percentage of time (cubic feet per second)

\begin{tabular}{|c|c|c|c|c|c|c|c|}
\hline Percentage & 5 & 10 & 25 & 50 & 75 & 90 & 95 \\
\hline Flow & 245 & 153 & 91 & 56 & 33 & 21 & 14 \\
\hline
\end{tabular}


STATION NAME AND NUMBER—02395120 Two Run Creek near Kingston, GA

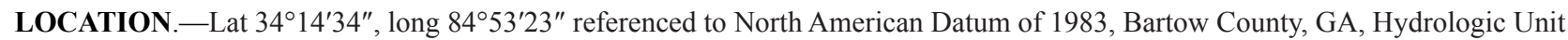
03150104, on right bank 200.0 feet upstream from bridge on GA 293, 1.9 miles upstream from Limekiln Branch, and 3.0 miles east of Kingston.

DRAINAGE AREA. $-33.1 \mathrm{mi}^{2}$.

PERIOD OF RECORD.--May 1980 to March 2014.

PERIOD OF FREQUENCY ANALYSIS.-April 1981 to March 2014.

NUMBER OF CLIMATE YEARS IN FREQUENCY ANALYSIS. - 33

REMARKS.-

\begin{tabular}{|c|c|c|c|c|}
\hline $\begin{array}{l}\text { Recurrence } \\
\text { intervals } \\
\text { (years) }\end{array}$ & \multicolumn{2}{|c|}{$\begin{array}{l}\text { Lowest average flow for } \\
\text { indicated number of } \\
\text { consecutive days } \\
\text { (cubic feet per second) }\end{array}$} & \multicolumn{2}{|c|}{$\begin{array}{l}\text { Time-sampling error } \\
\text { (in percent) }\end{array}$} \\
\hline & 1 & 7 & 1 & 7 \\
\hline 2 & 8.0 & 9.2 & 8 & 8 \\
\hline 5 & 5.7 & 6.4 & 9 & 9 \\
\hline 10 & 4.6 & 5.2 & 12 & 12 \\
\hline 20 & 3.9 & 4.3 & 15 & 17 \\
\hline 50 & 3.1 & 3.4 & 22 & 23 \\
\hline
\end{tabular}

\section{DURATION OF DAILY FLOW}

Flow equaled or exceeded for indicated percentage of time (cubic feet per second)

\begin{tabular}{|c|c|c|c|c|c|c|c|}
\hline Percentage & 5 & 10 & 25 & 50 & 75 & 90 & 95 \\
\hline Flow & 121 & 79 & 43 & 22 & 13 & 9.3 & 7.5 \\
\hline
\end{tabular}


STATION NAME AND NUMBER—02411800 Little River near Buchanan, GA

LOCATION.-Lat $33^{\circ} 47^{\prime} 51^{\prime \prime}$, long $85^{\circ} 07^{\prime} 03^{\prime \prime}$, referenced to North American Datum of 1927, Haralson County, GA, Hydrologic Unit 03150108, on right bank 150.0 feet upstream from county highway bridge, 4.3 mi east of Buchanan, and 7 mi upstream from mouth.

DRAINAGE AREA. $-20.2 \mathrm{mi}^{2}$.

PERIOD OF RECORD.- - June 1959 to September 1985.

PERIOD OF FREQUENCY ANALYSIS.-April 1960 to March 1985.

NUMBER OF CLIMATE YEARS IN FREQUENCY ANALYSIS.-25

REMARKS.-

\begin{tabular}{|c|c|c|c|c|}
\hline $\begin{array}{l}\text { Recurrence } \\
\text { intervals } \\
\text { (years) }\end{array}$ & \multicolumn{2}{|c|}{$\begin{array}{l}\text { Lowest average flow for } \\
\text { indicated number of } \\
\text { consecutive days } \\
\text { (cubic feet per second) }\end{array}$} & \multicolumn{2}{|c|}{$\begin{array}{l}\text { Time-sampling error } \\
\text { (in percent) }\end{array}$} \\
\hline & 1 & 7 & 1 & 7 \\
\hline 2 & 3.9 & 4.4 & 10 & 10 \\
\hline 5 & 2.6 & 3.0 & 11 & 11 \\
\hline 10 & 2.1 & 2.5 & 14 & 13 \\
\hline 20 & 1.7 & 2.1 & 18 & 16 \\
\hline
\end{tabular}

\section{DURATION OF DAILY FLOW}

Flow equaled or exceeded for indicated percentage of time (cubic feet per second)

\begin{tabular}{|c|c|c|c|c|c|c|c|}
\hline Percentage & 5 & 10 & 25 & 50 & 75 & 90 & 95 \\
\hline Flow & 105 & 63 & 35 & 19 & 10 & 6.4 & 4.7 \\
\hline
\end{tabular}


STATION NAME AND NUMBER—02429900 Big Brown Creek near Booneville, MS

LOCATION.-Lat 34³7’29”, long 88²6’42” referenced to North American Datum of 1983, in SW 1/4 NE 1/4 sec.27, T.5 S., R.8 E., Prentiss County, MS, Hydrologic Unit 03160101, Chickasaw Meridian, on State Highway 30, 2.5 mi upstream from Martin Creek, 8 mi east of Highway 45 at Booneville and 14.2 mi upstream from the mouth at Tombigbee River.

DRAINAGE AREA. $-27.1 \mathrm{mi}^{2}$.

PERIOD OF RECORD.--June 1973 to September 2003.

PERIOD OF FREQUENCY ANALYSIS.-April 1974 to March 2003.

NUMBER OF CLIMATE YEARS IN FREQUENCY ANALYSIS.-29

\section{REMARKS.-}

\begin{tabular}{|c|c|c|c|c|}
\hline \multirow[t]{2}{*}{$\begin{array}{l}\text { Recurrence } \\
\text { intervals } \\
\text { (years) }\end{array}$} & \multicolumn{2}{|c|}{$\begin{array}{l}\text { Lowest average flow for } \\
\text { indicated number of } \\
\text { consecutive days } \\
\text { (cubic feet per second) }\end{array}$} & \multicolumn{2}{|c|}{$\begin{array}{l}\text { Time-sampling error } \\
\text { (in percent) }\end{array}$} \\
\hline & 1 & 7 & 1 & 7 \\
\hline 2 & 0.59 & 0.73 & 20 & 22 \\
\hline 5 & 0.25 & 0.29 & 23 & 25 \\
\hline 10 & 0.16 & 0.18 & 29 & 31 \\
\hline 20 & 0.10 & 0.12 & 37 & 39 \\
\hline
\end{tabular}

\section{DURATION OF DAILY FLOW}

Flow equaled or exceeded for indicated percentage of time (cubic feet per second)

\begin{tabular}{|c|c|c|c|c|c|c|c|}
\hline Percentage & 5 & 10 & 25 & 50 & 75 & 90 & 95 \\
\hline Flow & 159 & 94 & 46 & 18 & 3.1 & 0.98 & 0.60 \\
\hline
\end{tabular}


STATION NAME AND NUMBER—02429980 Pollard Mill Branch at Paden, MS

LOCATION.-Lat 34³9'14", long 88¹4'56" referenced to North American Datum of 1983, in SW 1/4 SE 1/4 sec.9, T.5 S., R.10 E., Tishomingo County, MS, Hydrologic Unit 03160101, Chickasaw Meridian, on State Highway 30, 0.8 mi east of Paden.

DRAINAGE AREA.-2.01 $\mathrm{mi}^{2}$.

PERIOD OF RECORD.- October 1972 to May 2004.

PERIOD OF FREQUENCY ANALYSIS.-April 1973 to March 2004.

NUMBER OF CLIMATE YEARS IN FREQUENCY ANALYSIS.-31

REMARKS.-

\begin{tabular}{|c|c|c|c|c|}
\hline $\begin{array}{c}\text { Recurrence } \\
\text { intervals } \\
\text { (years) }\end{array}$ & \multicolumn{2}{|c|}{$\begin{array}{c}\text { Lowest average flow for } \\
\text { indicated number of } \\
\text { consecutive days } \\
\text { (cubic feet per second) }\end{array}$} & \multicolumn{2}{|c|}{$\begin{array}{c}\text { Time-sampling error } \\
\text { (in percent) }\end{array}$} \\
\hline \multicolumn{5}{|c|}{} \\
\hline & 2.6 & 7 & 1 & 7 \\
\hline 2 & 2.0 & 2.1 & 7 & 8 \\
\hline 5 & 1.6 & 1.8 & 12 & 10 \\
\hline 10 & 1.3 & 1.5 & 17 & 15 \\
\hline 20 & 1.1 & 1.3 & 26 & 22 \\
\hline 50 & &
\end{tabular}

\begin{tabular}{|c|c|c|c|c|c|c|c|}
\hline \multicolumn{7}{|c|}{ DURATION OF DAILY FLOW } \\
\hline \multicolumn{7}{|c|}{ Flow equaled or exceeded for indicated percentage of time } \\
(cubic feet per second)
\end{tabular}


STATION NAME AND NUMBER—02430000 Mackeys Creek near Dennis, MS

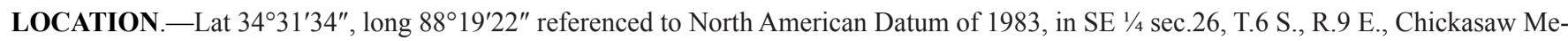
ridian, Tishomingo County, MS, Hydrologic Unit 03160101, on State Highway 4, 6.0 mi southwest of Dennis.

DRAINAGE AREA.- $66.9 \mathrm{mi}^{2}$, prior to construction of Tennessee-Tombigbee Waterway.

PERIOD OF RECORD.-October 1937 to October 1979.

PERIOD OF FREQUENCY ANALYSIS.-April 1938 to March 1973.

NUMBER OF CLIMATE YEARS IN FREQUENCY ANALYSIS.-35

REMARKS.-Since 1975, site regulated by Tennessee-Tombigbee Waterway. Frequency and duration analyses for pre-regulated conditions. End date for period of frequency analysis was set based on reviews of data.

\begin{tabular}{|c|c|c|c|c|}
\hline \multirow[t]{2}{*}{$\begin{array}{l}\text { Recurrence } \\
\text { intervals } \\
\text { (years) }\end{array}$} & \multicolumn{2}{|c|}{$\begin{array}{l}\text { Lowest average flow for } \\
\text { indicated number of } \\
\text { consecutive days } \\
\text { (cubic feet per second) }\end{array}$} & \multicolumn{2}{|c|}{$\begin{array}{l}\text { Time-sampling error } \\
\text { (in percent) }\end{array}$} \\
\hline & 1 & 7 & 1 & 7 \\
\hline 2 & 16 & 18 & 6 & 6 \\
\hline 5 & 12 & 13 & 7 & 7 \\
\hline 10 & 10 & 11 & 8 & 9 \\
\hline 20 & 9.2 & 9.6 & 10 & 12 \\
\hline 50 & 7.9 & 8.1 & 14 & 16 \\
\hline
\end{tabular}

\begin{tabular}{|c|c|c|c|c|c|c|c|}
\hline \multicolumn{1}{|c|}{ DURATION OF DAILY FLOW } \\
\hline \multicolumn{7}{|c|}{ Flow equaled or exceeded for indicated percentage of time } \\
(cubic feet per second)
\end{tabular}


STATION NAME AND NUMBER—02430085 Red Bud Creek near Moores Mill, MS

LOCATION.-Lat $34^{\circ} 28^{\prime} 00^{\prime \prime}$, long $88^{\circ} 17^{\prime} 01^{\prime \prime}$ referenced to North American Datum of 1983, in SW 1/4 SE 1/4 sec.18, T.7 S., R.10 E., Tishomingo County, MS, Hydrologic Unit 03160101, Chickasaw Meridian, near left bank on upstream side of bridge on county road, 0.18 mi south of intersection of county road and blacktop road, 2.7 mi east-southeast of Moores Mill, and 5.6 mi southwest of Belmont.

DRAINAGE AREA. $-15.7 \mathrm{mi}^{2}$.

PERIOD OF RECORD.- June 1975 to March 2014.

PERIOD OF FREQUENCY ANALYSIS.-April 1976 to March 2014.

NUMBER OF CLIMATE YEARS IN FREQUENCY ANALYSIS.-38

REMARKS.-

\begin{tabular}{|c|c|c|c|c|}
\hline $\begin{array}{c}\text { Recurrence } \\
\text { intervals } \\
\text { (years) }\end{array}$ & \multicolumn{2}{|c|}{$\begin{array}{c}\text { Lowest average flow for } \\
\text { indicated number of } \\
\text { consecutive days } \\
\text { (cubic feet per second) }\end{array}$} & \multicolumn{2}{|c|}{$\begin{array}{c}\text { Time-sampling error } \\
\text { (in percent) }\end{array}$} \\
\hline & 1 & 7 & 1 & 7 \\
\hline 2 & 2.8 & 3.1 & 7 & 6 \\
\hline 5 & 2.1 & 2.3 & 7 & 7 \\
\hline 10 & 1.8 & 2.0 & 7 & 9 \\
\hline 20 & 1.6 & 1.8 & 9 & 11 \\
\hline 50 & 1.4 & 1.6 & 12 & 6 \\
\hline
\end{tabular}

\begin{tabular}{|c|c|c|c|c|c|c|c|}
\hline \multicolumn{1}{|c|}{ DURATION OF DAILY FLOW } \\
\hline \multicolumn{7}{|c|}{ Flow equaled or exceeded for indicated percentage of time } \\
(cubic feet per second)
\end{tabular}


STATION NAME AND NUMBER—02430615 Mud Creek near Fairview, MS

LOCATION._Lat 34²3'33", long 88²1'18" referenced to North American Datum of 1983, in NW 1/4 NE 1/4 Sec.16, T.8 S., R.9 E., Itawamba County, MS, Hydrologic Unit 03160101, Chickasaw Meridian, at left bank on downstream side of bridge on county road 3.0 mi northwest of Fairview and 8.8 mi north-northeast of Fulton.

DRAINAGE AREA. - $-11.1 \mathrm{mi}^{2}$.

PERIOD OF RECORD.- June 1975 to December 2011.

PERIOD OF FREQUENCY ANALYSIS.-April 1976 to March 2011.

NUMBER OF CLIMATE YEARS IN FREQUENCY ANALYSIS.-35

REMARKS.-

\begin{tabular}{|c|c|c|c|c|}
\hline $\begin{array}{c}\text { Recurrence } \\
\text { intervals } \\
\text { (years) }\end{array}$ & \multicolumn{2}{|c|}{$\begin{array}{c}\text { Lowest average flow for } \\
\text { indicated number of } \\
\text { consecutive days } \\
\text { (cubic feet per second) }\end{array}$} & \multicolumn{2}{|c|}{$\begin{array}{c}\text { Time-sampling error } \\
\text { (in percent) }\end{array}$} \\
\hline & 1 & 7 & 1 & 7 \\
\hline 2 & 3.2 & 3.4 & 11 & 12 \\
\hline 5 & 1.9 & 2.0 & 12 & 15 \\
\hline 10 & 1.5 & 1.5 & 15 & 20 \\
\hline 20 & 1.1 & 1.2 & 19 & 27 \\
\hline 50 & 0.87 & 0.92 & 26 & \\
\hline
\end{tabular}

\begin{tabular}{|c|c|c|c|c|c|c|c|}
\hline \multicolumn{1}{|c|}{ DURATION OF DAILY FLOW } \\
\hline \multicolumn{7}{|c|}{ Flow equaled or exceeded for indicated percentage of time } \\
(cubic feet per second)
\end{tabular}


STATION NAME AND NUMBER—02430680 Twentymile Creek near Guntown, MS

LOCATION.-Lat $34^{\circ} 27^{\prime} 10^{\prime \prime}$, long 88 $34^{\prime} 38^{\prime \prime}$ referenced to North American Datum of 1983, in SW 1/4 SW 1/4 sec.21, T.7 S., R.7 E., Lee County, MS, Hydrologic Unit 03160101, Chickasaw Meridian, on downstream side of bridge on county road 2578, and 6.0 mi southeast of Baldwyn, and 6.0 mi east of Guntown.

DRAINAGE AREA.-131 $\mathrm{mi}^{2}$.

PERIOD OF RECORD.- October 1982 to March 2014.

PERIOD OF FREQUENCY ANALYSIS.-April 1983 to March 2011.

NUMBER OF CLIMATE YEARS IN FREQUENCY ANALYSIS.-31

REMARKS.-

\begin{tabular}{|c|c|c|c|c|}
\hline $\begin{array}{c}\text { Recurrence } \\
\text { intervals } \\
\text { (years) }\end{array}$ & \multicolumn{2}{|c|}{$\begin{array}{c}\text { Lowest average flow for } \\
\text { indicated number of } \\
\text { consecutive days } \\
\text { (cubic feet per second) }\end{array}$} & \multicolumn{2}{|c|}{$\begin{array}{c}\text { Time-sampling error } \\
\text { (in percent) }\end{array}$} \\
\hline & 1 & 7 & 1 & 7 \\
\hline 2 & 0.56 & 0.76 & 26 & 32 \\
\hline 5 & 0.16 & 0.23 & 30 & 40 \\
\hline 10 & 0.08 & 0.12 & 37 & 51 \\
\hline 20 & 0.03 & 0.07 & 46 & 71 \\
\hline 50 & 0.00 & 0.04 & -- & \\
\hline
\end{tabular}

\begin{tabular}{|c|c|c|c|c|c|c|c|}
\hline \multicolumn{1}{|c|}{ DURATION OF DAILY FLOW } \\
\hline \multicolumn{7}{|c|}{ Flow equaled or exceeded for indicated percentage of time } \\
(cubic feet per second)
\end{tabular}


STATION NAME AND NUMBER—02430880 Cummings Creek near Fulton, MS

LOCATION.-Lat $34^{\circ} 18^{\prime} 16^{\prime \prime}$, long $88^{\circ} 22^{\prime} 16^{\prime \prime}$ referenced to North American Datum of 1983, in SE 1/4 NE 1/4 Sec.17, T.9 S., R.9 E., Itawamba County, MS, Hydrologic Unit 03160101, in left bank, $20 \mathrm{ft}$ downstream from bridge, on county road, 3.2 mi northeast of Fulton, and 4.2 mi upstream from mouth and Tenn Tombigbee Waterway.

DRAINAGE AREA.-19.1 $\mathrm{mi}^{2}$.

PERIOD OF RECORD.-July 1975 to March 2014.

PERIOD OF FREQUENCY ANALYSIS.-April 1976 to March 2014.

NUMBER OF CLIMATE YEARS IN FREQUENCY ANALYSIS.—38

REMARKS.-

\begin{tabular}{|c|c|c|c|c|}
\hline $\begin{array}{c}\text { Recurrence } \\
\text { intervals } \\
\text { (years) }\end{array}$ & \multicolumn{2}{|c|}{$\begin{array}{c}\text { Lowest average flow for } \\
\text { indicated number of } \\
\text { consecutive days } \\
\text { (cubic feet per second) }\end{array}$} & \multicolumn{2}{|c|}{$\begin{array}{c}\text { Time-sampling error } \\
\text { (in percent) }\end{array}$} \\
\hline & 1 & 7 & 1 & 7 \\
\hline 2 & 5.8 & 6.5 & 8 & 10 \\
\hline 5 & 3.9 & 4.3 & 9 & 12 \\
\hline 10 & 3.1 & 3.4 & 12 & 15 \\
\hline 20 & 2.6 & 2.8 & 15 & 20 \\
\hline 50 & 2.1 & 2.3 & 19 & \\
\hline
\end{tabular}

\begin{tabular}{|c|c|c|c|c|c|c|c|}
\hline \multicolumn{1}{|c|}{ DURATION OF DAILY FLOW } \\
\hline \multicolumn{7}{|c|}{ Flow equaled or exceeded for indicated percentage of time } \\
(cubic feet per second)
\end{tabular}


STATION NAME AND NUMBER—02431000 Tombigbee River near Fulton, MS

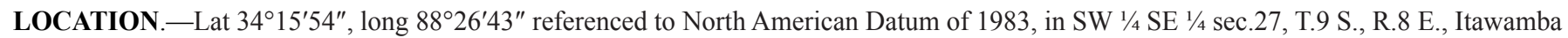
County, MS, Hydrologic Unit 03160101, Chickasaw Meridian, on left bank at downstream side of bridge on old U.S. Highway 78, 1,000 ft downstream from Twentymile-Fulton Canal, 2.2 mi west of Fulton, 6.2 mi upstream from Mantachie Creek Canal, 13.5 mi downstream from Twentymile Creek Canal, and at mile 421.8 .

DRAINAGE AREA.- $612 \mathrm{mi}^{2}$.

PERIOD OF RECORD.- October 1928 to March 2014.

PERIOD OF FREQUENCY ANALYSIS.-April 1929 to March 1984.

NUMBER OF CLIMATE YEARS IN FREQUENCY ANALYSIS. — 55

REMARKS.- - Since about 1985, flows influenced by upstream diversions and regulation. Frequency analysis represents period prior to Tennessee-Tombigbee Waterway.

\begin{tabular}{|c|c|c|c|c|}
\hline $\begin{array}{c}\text { Recurrence } \\
\text { intervals } \\
\text { (years) }\end{array}$ & \multicolumn{2}{|c|}{$\begin{array}{c}\text { Lowest average flow for } \\
\text { indicated number of } \\
\text { consecutive days } \\
\text { (cubic feet per second) }\end{array}$} & \multicolumn{2}{|c|}{$\begin{array}{c}\text { Time-sampling error } \\
\text { (in percent) }\end{array}$} \\
\hline & 1 & 7 & 1 & 7 \\
\hline 2 & 49 & 54 & 7 & 8 \\
\hline 5 & 32 & 36 & 8 & 9 \\
\hline 10 & 26 & 29 & 10 & 12 \\
\hline 20 & 21 & 24 & 13 & 15 \\
\hline 50 & 17 & 19 & 17 & 7 \\
\hline
\end{tabular}

\begin{tabular}{|c|c|c|c|c|c|c|c|}
\hline \multicolumn{1}{|c|}{ DURATION OF DAILY FLOW } \\
\hline \multicolumn{7}{|c|}{ Flow equaled or exceeded for indicated percentage of time } \\
(cubic feet per second) \\
\hline Percentage & 5 & 10 & 25 & 50 & 75 & 90 & 95 \\
\hline Flow & 3,500 & 2,080 & 1,040 & 387 & 132 & 71 & 52 \\
\hline
\end{tabular}


STATION NAME AND NUMBER—02433000 Bull Mountain Creek near Smithville, MS

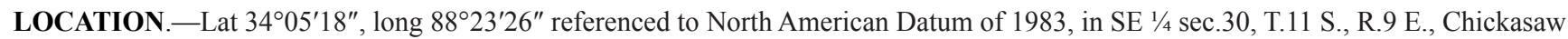
Meridian, Itawamba County, MS, Hydrologic Unit 03160101, on State Highway 25, 1.1 mi north of Smithville.

DRAINAGE AREA. $-336 \mathrm{mi}^{2}$.

PERIOD OF RECORD.- October 1940 to September 1984.

PERIOD OF FREQUENCY ANALYSIS.-April 1941 to March 1984.

NUMBER OF CLIMATE YEARS IN FREQUENCY ANALYSIS.-43

REMARKS.-

\begin{tabular}{|c|c|c|c|c|}
\hline $\begin{array}{c}\text { Recurrence } \\
\text { intervals } \\
\text { (years) }\end{array}$ & \multicolumn{2}{|c|}{$\begin{array}{c}\text { Lowest average flow for } \\
\text { indicated number of } \\
\text { consecutive days } \\
\text { (cubic feet per second) }\end{array}$} & \multicolumn{2}{|c|}{$\begin{array}{c}\text { Time-sampling error } \\
\text { (in percent) }\end{array}$} \\
\hline \multicolumn{5}{|c|}{} \\
\hline & 4 & 7 & 1 & 7 \\
\hline 2 & 35 & 51 & 6 & 7 \\
\hline 5 & 29 & 31 & 7 & 8 \\
\hline 10 & 25 & 27 & 9 & 10 \\
\hline 20 & 21 & 23 & 11 & 14 \\
\hline 50 & &
\end{tabular}

\begin{tabular}{|c|c|c|c|c|c|c|c|}
\hline \multicolumn{7}{|c|}{ DURATION OF DAILY FLOW } \\
\hline \multicolumn{7}{|c|}{ Flow equaled or exceeded for indicated percentage of time } \\
(cubic feet per second)
\end{tabular}


STATION NAME AND NUMBER—02433500 Tombigbee River at Bigbee, MS

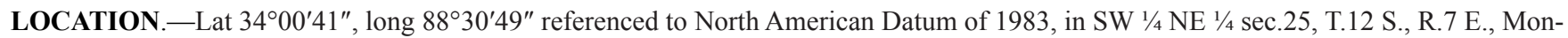
roe County, MS, Hydrologic Unit 03160101, Chickasaw Meridian, near right bank on downstream side of bridge on State Highway 6, 0.2 mi upstream from St. Louis-San Francisco Railway bridge, $0.5 \mathrm{mi}$ southeast of Bigbee, 2 mi northwest of Amory, 3.7 mi upstream from Town Creek, and at mile 383.1.

DRAINAGE AREA.-1,226 $\mathrm{mi}^{2}$.

PERIOD OF RECORD.- October 1944 to September 1946, October 1947 to September 1954, October 1963 to September 1998 , October

2001 to March 2014.

PERIOD OF FREQUENCY ANALYSIS.-April 1945 to March 1946, April 1948 to March 1954, April 1964 to March 1984.

NUMBER OF CLIMATE YEARS IN FREQUENCY ANALYSIS. - 27

REMARKS.- - Since about 1985, flows influenced by upstream diversions and regulation. Frequency analysis represents period prior to Tennessee-Tombigbee Waterway.

\begin{tabular}{|c|c|c|c|c|}
\hline $\begin{array}{c}\text { Recurrence } \\
\text { intervals } \\
\text { (years) }\end{array}$ & \multicolumn{2}{|c|}{$\begin{array}{c}\text { Lowest average flow for } \\
\text { indicated number of } \\
\text { consecutive days } \\
\text { (cubic feet per second) }\end{array}$} & \multicolumn{2}{|c|}{$\begin{array}{c}\text { Time-sampling error } \\
\text { (in percent) }\end{array}$} \\
\hline \multicolumn{7}{|c|}{} & 1 & 7 & 1 & 7 \\
\hline 2 & 139 & 151 & 9 & 7 \\
\hline 5 & 99 & 112 & 9 & 8 \\
\hline 10 & 84 & 98 & 9 & 10 \\
\hline 20 & 75 & 89 & 11 & \\
\hline
\end{tabular}

\begin{tabular}{|c|c|c|c|c|c|c|c|}
\hline \multicolumn{1}{|c|}{ DURATION OF DAILY FLOW } \\
\hline \multicolumn{7}{|c|}{ Flow equaled or exceeded for indicated percentage of time } \\
(cubic feet per second)
\end{tabular}


STATION NAME AND NUMBER—02435020 Town Creek at Eason Boulevard at Tupelo, MS

LOCATION.-Lat 34¹4'08”, long 8841'45" referenced to North American Datum of 1983, in NE 1/4 NW 1/4 sec.8, T.10 S., R.6 E., Lee County, MS, Hydrologic Unit 03160102, Chickasaw Meridian, on Eason Blvd. in Tupelo, $400 \mathrm{ft}$ upstream from Kings Creek and 2.0 mi upstream from the mouth..

DRAINAGE AREA. $-233 \mathrm{mi}^{2}$.

PERIOD OF RECORD.- October 1970 to September 2003.

PERIOD OF FREQUENCY ANALYSIS.-April 1971 to March 2003.

NUMBER OF CLIMATE YEARS IN FREQUENCY ANALYSIS.-32

\section{REMARKS.-}

\begin{tabular}{|c|c|c|c|c|}
\hline \multirow[t]{2}{*}{$\begin{array}{l}\text { Recurrence } \\
\text { intervals } \\
\text { (years) }\end{array}$} & \multicolumn{2}{|c|}{$\begin{array}{l}\text { Lowest average flow for } \\
\text { indicated number of } \\
\text { consecutive days } \\
\text { (cubic feet per second) }\end{array}$} & \multicolumn{2}{|c|}{$\begin{array}{l}\text { Time-sampling error } \\
\text { (in percent) }\end{array}$} \\
\hline & 1 & 7 & 1 & 7 \\
\hline 2 & 4.1 & 4.8 & 16 & 15 \\
\hline 5 & 2.0 & 2.5 & 19 & 18 \\
\hline 10 & 1.3 & 1.7 & 25 & 22 \\
\hline 20 & 0.90 & 1.2 & 33 & 28 \\
\hline 50 & 0.58 & 0.82 & 47 & 39 \\
\hline
\end{tabular}

\begin{tabular}{|c|c|c|c|c|c|c|c|}
\hline \multicolumn{1}{|c|}{ DURATION OF DAILY FLOW } \\
\hline \multicolumn{7}{|c|}{ Flow equaled or exceeded for indicated percentage of time } \\
(cubic feet per second)
\end{tabular}


STATION NAME AND NUMBER—02436500 Town Creek near Nettleton, MS

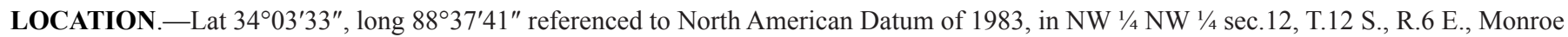
County, MS, Hydrologic Unit 03160102, Chickasaw Meridian, near right bank on downstream side of downstream bridge on U.S. Highway 45, 1.5 mi downstream from Chiwapa Creek, $2.1 \mathrm{mi}$ south of Nettleton, and 9.2 mi upstream from mouth.

DRAINAGE AREA. $-620 \mathrm{mi}^{2}$.

PERIOD OF RECORD.-October 1939 to September 1988, June 1989 to March 2014.

PERIOD OF FREQUENCY ANALYSIS._-April 1940 to March 1988, April 1990 to March 2014.

NUMBER OF CLIMATE YEARS IN FREQUENCY ANALYSIS.-71

REMARKS.-Prior to October 1970, published as West Fork Tombigbee River near Nettleton.

\begin{tabular}{|c|c|c|c|c|}
\hline $\begin{array}{c}\text { Recurrence } \\
\text { intervals } \\
\text { (years) }\end{array}$ & \multicolumn{2}{|c|}{$\begin{array}{c}\text { Lowest average flow for } \\
\text { indicated number of } \\
\text { consecutive days } \\
\text { (cubic feet per second) }\end{array}$} & \multicolumn{2}{|c|}{$\begin{array}{c}\text { Time-sampling error } \\
\text { (in percent) }\end{array}$} \\
\hline & 1 & 7 & 1 & 7 \\
\hline 2 & 17 & 19 & 10 & 12 \\
\hline 5 & 8.1 & 9.6 & 13 & 15 \\
\hline 10 & 5.3 & 6.5 & 17 & 20 \\
\hline 20 & 3.7 & 4.6 & 22 & 27 \\
\hline 50 & 2.4 & 3.1 & 31 & \\
\hline
\end{tabular}

\begin{tabular}{|c|c|c|c|c|c|c|c|}
\hline \multicolumn{1}{|c|}{ DURATION OF DAILY FLOW } \\
\hline \multicolumn{7}{|c|}{ Flow equaled or exceeded for indicated percentage of time } \\
(cubic feet per second)
\end{tabular}


STATION NAME AND NUMBER—02439400 Buttahatchee River near Aberdeen, MS

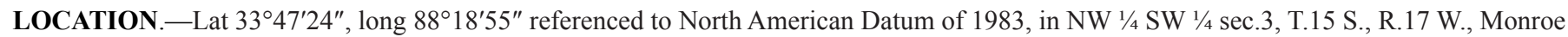
County, MS, Hydrologic Unit 03160103, Huntsville Meridian, near right bank on downstream side of bridge on county highway, 10.1 mi downstream from Sipsey Creek, $13.7 \mathrm{mi}$ southeast of Aberdeen, and $28.6 \mathrm{mi}$ upstream from the mouth.

DRAINAGE AREA. $-798 \mathrm{mi}^{2}$.

PERIOD OF RECORD.-July 1966 to March 2014.

PERIOD OF FREQUENCY ANALYSIS.-April 1967 to March 2014.

NUMBER OF CLIMATE YEARS IN FREQUENCY ANALYSIS.-47

REMARKS.-

\begin{tabular}{|c|c|c|c|c|}
\hline $\begin{array}{c}\text { Recurrence } \\
\text { intervals } \\
\text { (years) }\end{array}$ & \multicolumn{2}{|c|}{$\begin{array}{c}\text { Lowest average flow for } \\
\text { indicated number of } \\
\text { consecutive days } \\
\text { (cubic feet per second) }\end{array}$} & \multicolumn{2}{|c|}{$\begin{array}{c}\text { Time-sampling error } \\
\text { (in percent) }\end{array}$} \\
\hline & 1 & 7 & 1 & 7 \\
\hline 2 & 163 & 174 & 6 & 7 \\
\hline 5 & 118 & 126 & 7 & 8 \\
\hline 10 & 98 & 105 & 9 & 11 \\
\hline 20 & 84 & 90 & 11 & 14 \\
\hline 50 & 70 & 76 & 15 & 6 \\
\hline
\end{tabular}

\begin{tabular}{|c|c|c|c|c|c|c|c|}
\hline \multicolumn{1}{|c|}{ DURATION OF DAILY FLOW } \\
\hline \multicolumn{7}{|c|}{ Flow equaled or exceeded for indicated percentage of time } \\
(cubic feet per second)
\end{tabular}


STATION NAME AND NUMBER—02440000 Chuquatonchee Creek near Egypt, MS

LOCATION.-Lat 3350'24”, long 8845’43” referenced to North American Datum of 1983, in NW 1/4 NE 1/4 sec.27, T.14 S., R.5 E., Chickasaw County, MS, Hydrologic Unit 03160104, Chickasaw Meridian, at bridge on State Highway 8, 4.5 mi southwest of Egypt.

DRAINAGE AREA.- $-167 \mathrm{mi}^{2}$.

PERIOD OF RECORD.- October 1951 to September 1973.

PERIOD OF FREQUENCY ANALYSIS.-April 1952 to March 1973.

NUMBER OF CLIMATE YEARS IN FREQUENCY ANALYSIS.—-21

REMARKS.-

\begin{tabular}{|c|c|c|c|c|}
\hline $\begin{array}{c}\text { Recurrence } \\
\text { intervals } \\
\text { (years) }\end{array}$ & \multicolumn{2}{|c|}{$\begin{array}{c}\text { Lowest average flow for } \\
\text { indicated number of } \\
\text { consecutive days } \\
\text { (cubic feet per second) }\end{array}$} & \multicolumn{2}{|c|}{$\begin{array}{c}\text { Time-sampling error } \\
\text { (in percent) }\end{array}$} \\
\hline \multicolumn{7}{|c|}{1} & 7 & 1 & 79 \\
\hline 2 & 0.15 & 0.50 & 38 & -- \\
\hline 5 & 0.00 & 0.00 & -- & -- \\
\hline 10 & 0.00 & 0.00 & -- & -- \\
\hline 20 & 0.00 & 0.00 & -- & \\
\hline
\end{tabular}

\begin{tabular}{|c|c|c|c|c|c|c|c|}
\hline \multicolumn{1}{|c|}{ DURATION OF DAILY FLOW } \\
\hline \multicolumn{1}{|c|}{ Flow equaled or exceeded for indicated percentage of time } \\
(cubic feet per second)
\end{tabular}


STATION NAME AND NUMBER—02440500 Chuquatonchee Creek near West Point, MS

LOCATION.-Lat 33³6'26”, long 8842’33" referenced to North American Datum of 1983, in NW 1/4 NE 1/4 sec.18, T.17 S., R.6 E., Clay County, MS, Hydrologic Unit 03160104, Chickasaw Meridian, at bridge on State Highway 50, 3.0 mi west of West Point.

DRAINAGE AREA. $-505 \mathrm{mi}^{2}$.

PERIOD OF RECORD.- October 1943 to September 1946, October 1947 to September 1973, April 1996 to September 2004.

PERIOD OF FREQUENCY ANALYSIS.-April 1944 to March 1946, April 1948 to March 1973, April 1997 to March 2004.

NUMBER OF CLIMATE YEARS IN FREQUENCY ANALYSIS.-34

REMARKS.-

\begin{tabular}{|c|c|c|c|c|}
\hline $\begin{array}{c}\text { Recurrence } \\
\text { intervals } \\
\text { (years) }\end{array}$ & \multicolumn{2}{|c|}{$\begin{array}{c}\text { Lowest average flow for } \\
\text { indicated number of } \\
\text { consecutive days } \\
\text { (cubic feet per second) }\end{array}$} & \multicolumn{2}{|c|}{$\begin{array}{c}\text { Time-sampling error } \\
\text { (in percent) }\end{array}$} \\
\hline \multicolumn{7}{|c|}{1} & 7 & 1 & 48 \\
\hline 2 & 0.67 & 0.93 & 43 & -- \\
\hline 5 & 0.00 & 0.00 & -- & -- \\
\hline 10 & 0.00 & 0.00 & -- & -- \\
\hline 20 & 0.00 & 0.00 & -- & -- \\
\hline 50 & 0.00 & 0.00 & -- & \\
\hline
\end{tabular}

\begin{tabular}{|c|c|c|c|c|c|c|c|}
\hline \multicolumn{7}{|c|}{ DURATION OF DAILY FLOW } \\
\hline \multicolumn{7}{|c|}{ Flow equaled or exceeded for indicated percentage of time } \\
(cubic feet per second)
\end{tabular}


STATION NAME AND NUMBER—02443000 Luxapallila River at Steens, MS

LOCATION.-Lat 3333'37”, long 88¹8'55" referenced to North American Datum of 1983, in NE 1/4 sec.28, T.17 S., R.17 W., Lowndes County, MS, Hydrologic Unit 03160105, Huntsville Meridian, on county highway 0.2 mi southeast of Steens.

DRAINAGE AREA.-309 $\mathrm{mi}^{2}$.

PERIOD OF RECORD.-October 1943 to September 1947, October 1949 to October 1977, November 1987 to August 1989.

PERIOD OF FREQUENCY ANALYSIS.-April 1944 to March 1947, April 1950 to March 1977.

NUMBER OF CLIMATE YEARS IN FREQUENCY ANALYSIS.-30

REMARKS.- -

\begin{tabular}{|c|c|c|c|c|}
\hline $\begin{array}{c}\text { Recurrence } \\
\text { intervals } \\
\text { (years) }\end{array}$ & \multicolumn{2}{|c|}{$\begin{array}{c}\text { Lowest average flow for } \\
\text { indicated number of } \\
\text { consecutive days } \\
\text { (cubic feet per second) }\end{array}$} & \multicolumn{2}{|c|}{$\begin{array}{c}\text { Time-sampling error } \\
\text { (in percent) }\end{array}$} \\
\hline \multicolumn{5}{|c|}{1} \\
1 & 57 & 7 & 1 & 7 \\
\hline 2 & 42 & 44 & 7 & 8 \\
\hline 5 & 35 & 37 & 9 & 10 \\
\hline 10 & 31 & 32 & 11 & 12 \\
\hline 20 & 27 & 28 & 15 & 16 \\
\hline 50 & &
\end{tabular}

\section{DURATION OF DAILY FLOW}

Flow equaled or exceeded for indicated percentage of time (cubic feet per second)

\begin{tabular}{|c|c|c|c|c|c|c|c|}
\hline Percentage & 5 & 10 & 25 & 50 & 75 & 90 & 95 \\
\hline Flow & 1,730 & 1,050 & 540 & 227 & 107 & 70 & 57 \\
\hline
\end{tabular}


STATION NAME AND NUMBER—02448000 Noxubee River at Macon, MS

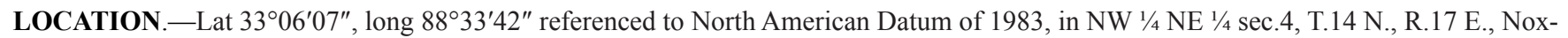
ubee County, MS, Hydrologic Unit 03160108, Choctaw Meridian, over mid-channel on the downstream side of the downstream bridge on U.S. Highway 45 at Macon, 3 miles south of the Highway 14 and Highway 45 intersection.

DRAINAGE AREA. $-768 \mathrm{mi}^{2}$.

PERIOD OF RECORD.-August 1928 to September 1932, September 1938 to September 1989, October 1990 to March 2014.

PERIOD OF FREQUENCY ANALYSIS.-April 1929 to March 1932, April 1939 to March 1989, April 1991 to March 2014.

NUMBER OF CLIMATE YEARS IN FREQUENCY ANALYSIS.-76

REMARKS.- - Since 1950, minor regulation from Bluff Lake, which is on a tributary to Noxubee River (Telis, 1991).

\begin{tabular}{|c|c|c|c|c|}
\hline $\begin{array}{c}\text { Recurrence } \\
\text { intervals } \\
\text { (years) }\end{array}$ & \multicolumn{2}{|c|}{$\begin{array}{c}\text { Lowest average flow for } \\
\text { indicated number of } \\
\text { consecutive days } \\
\text { (cubic feet per second) }\end{array}$} & \multicolumn{2}{|c|}{$\begin{array}{c}\text { Time-sampling error } \\
\text { (in percent) }\end{array}$} \\
\hline & 1 & 7 & 1 & 7 \\
\hline 2 & 51 & 54 & 4 & 4 \\
\hline 5 & 38 & 40 & 4 & 5 \\
\hline 10 & 33 & 35 & 5 & 6 \\
\hline 20 & 30 & 31 & 6 & 8 \\
\hline 50 & 26 & 27 & 8 & 4 \\
\hline
\end{tabular}

\begin{tabular}{|c|c|c|c|c|c|c|c|}
\hline \multicolumn{1}{|c|}{ DURATION OF DAILY FLOW } \\
\hline \multicolumn{7}{|c|}{ Flow equaled or exceeded for indicated percentage of time } \\
(cubic feet per second)
\end{tabular}


STATION NAME AND NUMBER—02477000 Chickasawhay River at Enterprise, MS

LOCATION.-Lat $32^{\circ} 10^{\prime} 33^{\prime \prime}$, long 88 $49^{\prime} 11^{\prime \prime}$ referenced to North American Datum of 1983, in SE 1/4 SE 1/4 NW 1/4 Sec.24, T.4 N., R.14 E., Clarke County, MS, Hydrologic Unit 03170002, Choctaw Meridian, on right bank at downstream side of right-main pie of the old Highway 513 bridge in Enterprise, at river mile 158.2, 0.5 mi downstream from confluence of Chunky River and Okatibbee Creek.

DRAINAGE AREA. $-918 \mathrm{mi}^{2}$.

PERIOD OF RECORD.-August 1938 to March 2014.

PERIOD OF FREQUENCY ANALYSIS.-April 1939 to March 1968.

NUMBER OF CLIMATE YEARS IN FREQUENCY ANALYSIS.-29

REMARKS.-Since November 1968, regulated by Okatibbee Lake. Analysis represents pre-regulation period.

\begin{tabular}{|c|c|c|c|c|}
\hline $\begin{array}{c}\text { Recurrence } \\
\text { intervals } \\
\text { (years) }\end{array}$ & \multicolumn{2}{|c|}{$\begin{array}{c}\text { Lowest average flow for } \\
\text { indicated number of } \\
\text { consecutive days } \\
\text { (cubic feet per second) }\end{array}$} & \multicolumn{2}{|c|}{$\begin{array}{c}\text { Time-sampling error } \\
\text { (in percent) }\end{array}$} \\
\hline \multicolumn{2}{|c|}{1} & 7 & 1 & 7 \\
\hline 2 & 49 & 53 & 12 & 12 \\
\hline 5 & 30 & 32 & 13 & 15 \\
\hline 10 & 23 & 24 & 15 & 18 \\
\hline 20 & 19 & 20 & 18 & \\
\hline
\end{tabular}

\section{DURATION OF DAILY FLOW}

Flow equaled or exceeded for indicated percentage of time (cubic feet per second)

\begin{tabular}{|c|c|c|c|c|c|c|c|}
\hline Percentage & 5 & 10 & 25 & 50 & 75 & 90 & 95 \\
\hline Flow & 4,910 & 2,940 & 1,130 & 392 & 132 & 71 & 49 \\
\hline
\end{tabular}


STATION NAME AND NUMBER—02477990 Buckatunna Creek near Denham, MS

LOCATION.-Lat 31 $41^{\prime} 39^{\prime \prime}$, long 88³1'09" referenced to North American Datum of 1983, in NE 1/4 NE 1/4 sec.6, T.8 N., R.5 W., Wayne County, MS, Hydrologic Unit 03170002, St. Stephens Meridian, on right bank on downstream side of bridge on county road, 3.5 mi north of Denham, and 8.0 mi east of Waynesboro.

DRAINAGE AREA. $-492 \mathrm{mi}^{2}$.

PERIOD OF RECORD.- January 1972 to September 2013.

PERIOD OF FREQUENCY ANALYSIS.-April 1972 to March 2013.

NUMBER OF CLIMATE YEARS IN FREQUENCY ANALYSIS.-41

REMARKS.-

\begin{tabular}{|c|c|c|c|c|}
\hline $\begin{array}{c}\text { Recurrence } \\
\text { intervals } \\
\text { (years) }\end{array}$ & \multicolumn{2}{|c|}{$\begin{array}{c}\text { Lowest average flow for } \\
\text { indicated number of } \\
\text { consecutive days } \\
\text { (cubic feet per second) }\end{array}$} & \multicolumn{2}{|c|}{$\begin{array}{c}\text { Time-sampling error } \\
\text { (in percent) }\end{array}$} \\
\hline & 1 & 7 & 1 & 7 \\
\hline 2 & 24 & 26 & 13 & 14 \\
\hline 5 & 12 & 13 & 15 & 18 \\
\hline 10 & 8.3 & 9.1 & 19 & 23 \\
\hline 20 & 6.0 & 6.7 & 24 & 30 \\
\hline 50 & 4.2 & 4.7 & 32 & \\
\hline
\end{tabular}

\begin{tabular}{|c|c|c|c|c|c|c|c|}
\hline \multicolumn{1}{|c|}{ DURATION OF DAILY FLOW } \\
\hline \multicolumn{7}{|c|}{ Flow equaled or exceeded for indicated percentage of time } \\
(cubic feet per second)
\end{tabular}


STATION NAME AND NUMBER—02478500 Chickasawhay River at Leakesville, MS

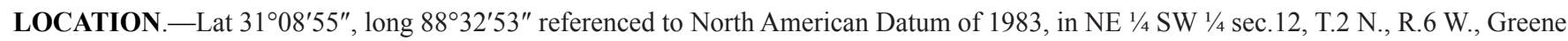
County, MS, Hydrologic Unit 03170003, St. Stephens Meridian, on left bank on downstream side of abandoned bridge $400 \mathrm{ft}$ below State Highway 63, $0.5 \mathrm{mi}$ southeast of Leaksville, $1.8 \mathrm{mi}$ upstream from Faulk Ditch, and $29.1 \mathrm{mi}$ upstream from confluence with Leaf River.

DRAINAGE AREA. $-2,690 \mathrm{mi}^{2}$.

PERIOD OF RECORD.-September 1938 to March 2014.

PERIOD OF FREQUENCY ANALYSIS.-April 1939 to March 2014.

NUMBER OF CLIMATE YEARS IN FREQUENCY ANALYSIS. - 75

REMARKS.-

\begin{tabular}{|c|c|c|c|c|}
\hline $\begin{array}{c}\text { Recurrence } \\
\text { intervals } \\
\text { (years) }\end{array}$ & \multicolumn{2}{|c|}{$\begin{array}{c}\text { Lowest average flow for } \\
\text { indicated number of } \\
\text { consecutive days } \\
\text { (cubic feet per second) }\end{array}$} & \multicolumn{2}{|c|}{$\begin{array}{c}\text { Time-sampling error } \\
\text { (in percent) }\end{array}$} \\
\hline & 1 & 7 & 1 & 7 \\
\hline 2 & 401 & 417 & 5 & 5 \\
\hline 5 & 290 & 299 & 5 & 6 \\
\hline 10 & 245 & 253 & 6 & 7 \\
\hline 20 & 215 & 220 & 7 & 9 \\
\hline 50 & 185 & 190 & 9 & \\
\hline
\end{tabular}

\begin{tabular}{|c|c|c|c|c|c|c|c|}
\hline \multicolumn{1}{|c|}{ DURATION OF DAILY FLOW } \\
\hline \multicolumn{1}{|c|}{ Flow equaled or exceeded for indicated percentage of time } \\
(cubic feet per second)
\end{tabular}


STATION NAME AND NUMBER—03543500 Sewee Creek near Decatur, TN

LOCATION.-Lat 35³4'52.48”, long 8444'53.19” referenced to North American Datum of 1927, Meigs County, TN, Hydrologic Unit 06020001, on right bank 0.3 mi downstream from bridge on State Hwy. 58, 0.5 mi downstream from Dry Fork, 5.0 mi north of Decatur, and at mile 5.7.

DRAINAGE AREA.- $-117 \mathrm{mi}^{2}$.

PERIOD OF RECORD.-June 1934 to November 1994, April 2012 to March 2014.

PERIOD OF FREQUENCY ANALYSIS.-April 1935 to March 1994, April 2012 to March 2014.

NUMBER OF CLIMATE YEARS IN FREQUENCY ANALYSIS.-61

\section{REMARKS.-}

\begin{tabular}{|c|c|c|c|c|}
\hline $\begin{array}{c}\text { Recurrence } \\
\text { intervals } \\
\text { (years) }\end{array}$ & \multicolumn{2}{|c|}{$\begin{array}{c}\text { Lowest average flow for } \\
\text { indicated number of } \\
\text { consecutive days } \\
\text { (cubic feet per second) }\end{array}$} & \multicolumn{2}{|c|}{$\begin{array}{c}\text { Time-sampling error } \\
\text { (in percent) }\end{array}$} \\
\hline & 1 & 7 & 1 & 7 \\
\hline 2 & 19 & 21 & 5 & 5 \\
\hline 5 & 15 & 16 & 5 & 5 \\
\hline 10 & 13 & 14 & 6 & 6 \\
\hline 20 & 11 & 12 & 7 & 8 \\
\hline 50 & 10 & 11 & 9 & 5 \\
\hline
\end{tabular}

\begin{tabular}{|c|c|c|c|c|c|c|c|}
\hline \multicolumn{1}{|c|}{ DURATION OF DAILY FLOW } \\
\hline \multicolumn{7}{|c|}{ Flow equaled or exceeded for indicated percentage of time } \\
(cubic feet per second)
\end{tabular}


STATION NAME AND NUMBER—03544500 Richland Creek near Dayton, TN

LOCATION.-Lat 35³0'17”, long 8501'20” referenced to North American Datum of 1927, Rhea County, TN, Hydrologic Unit 06020001, on left bank at Morgantown, 0.4 mi upstream from bridge on State Highway 30, 1.0 mi northwest of Dayton, 1.8 mi downstream from Payne Creek, and at mile 5.2.

DRAINAGE AREA. $-50.2 \mathrm{mi}^{2}$.

PERIOD OF RECORD.-July 1927 to September 1931, July 1934 to September 1955, July 1979 to December 1981.

PERIOD OF FREQUENCY ANALYSIS.-April 1928 to March 1931, April 1935 to March 1955, April 1980 to March 1981.

NUMBER OF CLIMATE YEARS IN FREQUENCY ANALYSIS.—-24

REMARKS.-

\begin{tabular}{|c|c|c|c|c|}
\hline $\begin{array}{l}\text { Recurrence } \\
\text { intervals } \\
\text { (years) }\end{array}$ & \multicolumn{2}{|c|}{$\begin{array}{l}\text { Lowest average flow for } \\
\text { indicated number of } \\
\text { consecutive days } \\
\text { (cubic feet per second) }\end{array}$} & \multicolumn{2}{|c|}{$\begin{array}{l}\text { Time-sampling error } \\
\text { (in percent) }\end{array}$} \\
\hline & 1 & 7 & 1 & 7 \\
\hline 2 & 0.10 & 0.15 & 31 & 31 \\
\hline 5 & 0.03 & 0.05 & 30 & 30 \\
\hline 10 & 0.02 & 0.02 & 33 & 33 \\
\hline 20 & 0.01 & 0.01 & 40 & 40 \\
\hline
\end{tabular}

\section{DURATION OF DAILY FLOW}

Flow equaled or exceeded for indicated percentage of time (cubic feet per second)

\begin{tabular}{|c|c|c|c|c|c|c|c|}
\hline Percentage & 5 & 10 & 25 & 50 & 75 & 90 & 95 \\
\hline Flow & 398 & 252 & 121 & 34 & 2.4 & 0.3 & 0.2 \\
\hline
\end{tabular}


STATION NAME AND NUMBER—03566420 Wolftever Creek near Ooltewah, TN

LOCATION.-Lat $35^{\circ} 03^{\prime} 43^{\prime \prime}$, long $85^{\circ} 03^{\prime}$ '59" referenced to North American Datum of 1927, Hamilton County, TN, Hydrologic Unit 06020001, on right bank at bridge on county road, 0.6 mi downstream from Southern Railway bridge, 0.85 mi south of post office at Ooltewah, and $1.65 \mathrm{mi}$ above mouth of Little Wolftever Creek.

DRAINAGE AREA.- $-18.8 \mathrm{mi}^{2}$.

PERIOD OF RECORD.-February 1964 to September 1989.

PERIOD OF FREQUENCY ANALYSIS.-April 1964 to March 1989.

NUMBER OF CLIMATE YEARS IN FREQUENCY ANALYSIS.-25

\section{REMARKS.-}

\begin{tabular}{|c|c|c|c|c|}
\hline \multirow[t]{2}{*}{$\begin{array}{l}\text { Recurrence } \\
\text { intervals } \\
\text { (years) }\end{array}$} & \multicolumn{2}{|c|}{$\begin{array}{l}\text { Lowest average flow for } \\
\text { indicated number of } \\
\text { consecutive days } \\
\text { (cubic feet per second) }\end{array}$} & \multicolumn{2}{|c|}{$\begin{array}{l}\text { Time-sampling error } \\
\text { (in percent) }\end{array}$} \\
\hline & 1 & 7 & 1 & 7 \\
\hline 2 & 2.9 & 3.1 & 10 & 10 \\
\hline 5 & 2.0 & 2.1 & 12 & 11 \\
\hline 10 & 1.6 & 1.7 & 15 & 14 \\
\hline 20 & 1.3 & 1.4 & 19 & 18 \\
\hline
\end{tabular}

\section{DURATION OF DAILY FLOW}

Flow equaled or exceeded for indicated percentage of time (cubic feet per second)

\begin{tabular}{|c|c|c|c|c|c|c|c|}
\hline Percentage & 5 & 10 & 25 & 50 & 75 & 90 & 95 \\
\hline Flow & 113 & 66 & 30 & 13 & 5.7 & 3.8 & 2.9 \\
\hline
\end{tabular}


STATION NAME AND NUMBER—03567500 South Chickamauga Creek near Chickamauga, TN

LOCATION.-Lat $35^{\circ} 00^{\prime} 50.57^{\prime}$, long 85²'23.91” referenced to North American Datum of 1927, Hamilton County, TN, Hydrologic Unit 06020001, on left bank, atop flood levee, $400 \mathrm{ft}$. upstream from bridge on U.S. Hwy 11/64 (Brainerd Rd.), 0.8 mi. downstream of confluence of West Chickamauga Creek and South Chickamauga Creek, 6.0 miles east of the city hall in Chattanooga, and at river mile 12.2 .

DRAINAGE AREA. $-428 \mathrm{mi}^{2}$.

PERIOD OF RECORD.- October 1928 to September 1978, October 1980 to September 1994, May 2012 to March 2014.

PERIOD OF FREQUENCY ANALYSIS._-April 1929 to March 1978, April 1981 to March 1994, April 2013 to March 2014.

NUMBER OF CLIMATE YEARS IN FREQUENCY ANALYSIS.- 63

REMARKS.-

\begin{tabular}{|c|c|c|c|c|}
\hline $\begin{array}{c}\text { Recurrence } \\
\text { intervals } \\
\text { (years) }\end{array}$ & \multicolumn{2}{|c|}{$\begin{array}{c}\text { Lowest average flow for } \\
\text { indicated number of } \\
\text { consecutive days } \\
\text { (cubic feet per second) }\end{array}$} & \multicolumn{2}{|c|}{$\begin{array}{c}\text { Time-sampling error } \\
\text { (in percent) }\end{array}$} \\
\hline & 1 & 7 & 1 & 7 \\
\hline 2 & 111 & 116 & 3 & 4 \\
\hline 5 & 90 & 95 & 4 & 4 \\
\hline 10 & 81 & 86 & 5 & 6 \\
\hline 20 & 73 & 78 & 6 & 7 \\
\hline 50 & 65 & 70 & 8 & 4 \\
\hline
\end{tabular}

\begin{tabular}{|c|c|c|c|c|c|c|c|}
\hline \multicolumn{1}{|c|}{ DURATION OF DAILY FLOW } \\
\hline \multicolumn{7}{|c|}{ Flow equaled or exceeded for indicated percentage of time } \\
(cubic feet per second)
\end{tabular}


STATION NAME AND NUMBER—03568933 Lookout Creek near New England, GA

LOCATION.-Lat 34 53'51", long 85²7'47" referenced to North American Datum of 1983, Dade County, GA, Hydrologic Unit 06020001, at bridge on County Road 2214, 0.4 miles downstream of Squirrel Town Creek, 2.2 miles southeast of New England.

DRAINAGE AREA.-149 $\mathrm{mi}^{2}$.

PERIOD OF RECORD.--August 1979 to March 2014.

PERIOD OF FREQUENCY ANALYSIS.-April 1980 to March 2014.

NUMBER OF CLIMATE YEARS IN FREQUENCY ANALYSIS.-34

REMARKS.-Potential influence by upstream diversions (Gotvald, 2016).

\begin{tabular}{|c|c|c|c|c|}
\hline $\begin{array}{l}\text { Recurrence } \\
\text { intervals } \\
\text { (years) }\end{array}$ & \multicolumn{2}{|c|}{$\begin{array}{l}\text { Lowest average flow for } \\
\text { indicated number of } \\
\text { consecutive days } \\
\text { (cubic feet per second) }\end{array}$} & \multicolumn{2}{|c|}{$\begin{array}{l}\text { Time-sampling error } \\
\text { (in percent) }\end{array}$} \\
\hline & 1 & 7 & 1 & 7 \\
\hline 2 & 18 & 19 & 7 & 7 \\
\hline 5 & 13 & 13 & 9 & 9 \\
\hline 10 & 11 & 11 & 12 & 11 \\
\hline 20 & 8.8 & 9.2 & 15 & 15 \\
\hline 50 & 7.1 & 7.5 & 21 & 20 \\
\hline
\end{tabular}

\section{DURATION OF DAILY FLOW}

Flow equaled or exceeded for indicated percentage of time (cubic feet per second)

\begin{tabular}{|c|c|c|c|c|c|c|c|}
\hline Percentage & 5 & 10 & 25 & 50 & 75 & 90 & 95 \\
\hline Flow & 849 & 540 & 256 & 93 & 35 & 21 & 16 \\
\hline
\end{tabular}


STATION NAME AND NUMBER—03578000 Elk River near Pelham, TN

LOCATION.-Lat 35¹7'48.19”, long 8552'11.19” referenced to North American Datum of 1927, Grundy County, TN, Hydrologic Unit 06030003, on top of left abutment at downstream side of bridge on U.S. Highway 41, 1.1 mi southeast of Pelham, 1.8 mi upstream from Caldwell Creek, and at mile 194.2.

DRAINAGE AREA. $-65.6 \mathrm{mi}^{2}$.

PERIOD OF RECORD.-December 1951 to December 1987, November 2000 to March 2014.

PERIOD OF FREQUENCY ANALYSIS.-April 1952 to March 1987, April 2001 to March 2014.

NUMBER OF CLIMATE YEARS IN FREQUENCY ANALYSIS.-48

REMARKS.-

\begin{tabular}{|c|c|c|c|c|}
\hline $\begin{array}{c}\text { Recurrence } \\
\text { intervals } \\
\text { (years) }\end{array}$ & \multicolumn{2}{|c|}{$\begin{array}{c}\text { Lowest average flow for } \\
\text { indicated number of } \\
\text { consecutive days } \\
\text { (cubic feet per second) }\end{array}$} & \multicolumn{2}{|c|}{$\begin{array}{c}\text { Time-sampling error } \\
\text { (in percent) }\end{array}$} \\
\hline & 1 & 7 & 1 & 7 \\
\hline 2 & 2.9 & 3.1 & 12 & 11 \\
\hline 5 & 1.5 & 1.8 & 14 & 13 \\
\hline 10 & 1.0 & 1.3 & 18 & 15 \\
\hline 20 & 0.73 & 1.1 & 23 & 20 \\
\hline 50 & 0.50 & 0.82 & 31 & \\
\hline
\end{tabular}

\begin{tabular}{|c|c|c|c|c|c|c|c|}
\hline \multicolumn{1}{|c|}{ DURATION OF DAILY FLOW } \\
\hline \multicolumn{7}{|c|}{ Flow equaled or exceeded for indicated percentage of time } \\
(cubic feet per second)
\end{tabular}


STATION NAME AND NUMBER—03584000 Richland Creek near Pulaski, TN

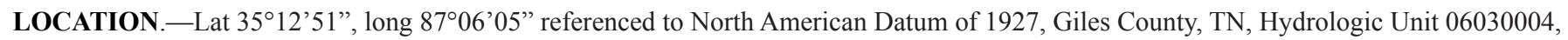
on right bank 1,200 ft upstream from bridge on U.S. Highway 64, 1 mi downstream from Weakley Creek, 4 mi west of Pulaski, and at mile 30.1 .

DRAINAGE AREA.- $-366 \mathrm{mi}^{2}$.

PERIOD OF RECORD.-May 1934 to October 1975.

PERIOD OF FREQUENCY ANALYSIS.-April 1935 to March 1975.

NUMBER OF CLIMATE YEARS IN FREQUENCY ANALYSIS.-40

REMARKS.-

\begin{tabular}{|c|c|c|c|c|}
\hline $\begin{array}{c}\text { Recurrence } \\
\text { intervals } \\
\text { (years) }\end{array}$ & \multicolumn{2}{|c|}{$\begin{array}{c}\text { Lowest average flow for } \\
\text { indicated number of } \\
\text { consecutive days } \\
\text { (cubic feet per second) }\end{array}$} & \multicolumn{2}{|c|}{$\begin{array}{c}\text { Time-sampling error } \\
\text { (in percent) }\end{array}$} \\
\hline & 1 & 7 & 1 & 7 \\
\hline 2 & 25 & 27 & 9 & 8 \\
\hline 5 & 17 & 18 & 8 & 8 \\
\hline 10 & 14 & 16 & 8 & 10 \\
\hline 20 & 13 & 14 & 10 & 14 \\
\hline 50 & 11 & 12 & 14 & 8 \\
\hline
\end{tabular}

\begin{tabular}{|c|c|c|c|c|c|c|c|}
\hline \multicolumn{1}{|c|}{ DURATION OF DAILY FLOW } \\
\hline \multicolumn{7}{|c|}{ Flow equaled or exceeded for indicated percentage of time } \\
(cubic feet per second)
\end{tabular}


STATION NAME AND NUMBER—03588000 Shoal Creek at Lawrenceburg, TN

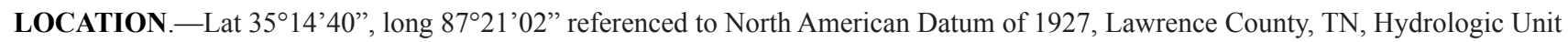
06030005, on left bank at downstream side of highway bridge, 1,500 feet upstream from Crowson Creek, 1,800 feet downstream from Little Shoal Creek, 1.1 miles west of the courthouse in Lawrenceburg, and at mile 55.6

DRAINAGE AREA. $-55.4 \mathrm{mi}^{2}$.

PERIOD OF RECORD._-July 1932 to March 1934, March 1967 to November 1991.

PERIOD OF FREQUENCY ANALYSIS._-April 1933 to March 1934, April 1967 to March 1991.

NUMBER OF CLIMATE YEARS IN FREQUENCY ANALYSIS.-25

REMARKS.-

\begin{tabular}{|c|c|c|c|c|}
\hline $\begin{array}{c}\text { Recurrence } \\
\text { intervals } \\
\text { (years) }\end{array}$ & \multicolumn{2}{|c|}{$\begin{array}{c}\text { Lowest average flow for } \\
\text { indicated number of } \\
\text { consecutive days } \\
\text { ccubic feet per second) }\end{array}$} & \multicolumn{2}{|c|}{$\begin{array}{c}\text { Time-sampling error } \\
\text { (in percent) }\end{array}$} \\
\hline \multicolumn{7}{|c|}{1} & 7 & 1 & 7 \\
\hline 2 & 22 & 23 & 5 & 5 \\
\hline 5 & 18 & 20 & 5 & 5 \\
\hline 10 & 17 & 18 & 5 & 6 \\
\hline 20 & 15 & 17 & 6 & 5 \\
\hline
\end{tabular}

\section{DURATION OF DAILY FLOW}

Flow equaled or exceeded for indicated percentage of time (cubic feet per second)

\begin{tabular}{|c|c|c|c|c|c|c|c|}
\hline Percentage & 5 & 10 & 25 & 50 & 75 & 90 & 95 \\
\hline Flow & 298 & 168 & 92 & 53 & 34 & 25 & 22 \\
\hline
\end{tabular}


STATION NAME AND NUMBER—03588400 Chisholm Creek at Westpoint, TN

LOCATION.-Lat $35^{\circ} 08^{\prime} 04^{\prime \prime}$, long $87^{\circ} 31^{\prime} 45^{\prime \prime}$ referenced to North American Datum of 1927, Lawrence County, TN, Hydrologic Unit 06030005 , on downstream side of left pier of county road bridge $0.3 \mathrm{mi}$ northeast of Westpoint, and 1.2 mi above mouth.

DRAINAGE AREA. $-43.0 \mathrm{mi}^{2}$.

PERIOD OF RECORD.-August 1962 to December 1987.

PERIOD OF FREQUENCY ANALYSIS.-April 1963 to March 1987.

NUMBER OF CLIMATE YEARS IN FREQUENCY ANALYSIS.-24

REMARKS.-

\begin{tabular}{|c|c|c|c|c|}
\hline $\begin{array}{c}\text { Recurrence } \\
\text { intervals } \\
\text { (years) }\end{array}$ & \multicolumn{2}{|c|}{$\begin{array}{c}\text { Lowest average flow for } \\
\text { indicated number of } \\
\text { consecutive days } \\
\text { (cubic feet per second) }\end{array}$} & \multicolumn{2}{|c|}{$\begin{array}{c}\text { Time-sampling error } \\
\text { (in percent) }\end{array}$} \\
\hline \multicolumn{7}{|c|}{} & 1 & 7 & 1 & 7 \\
\hline 2 & 15 & 16 & 7 & 8 \\
\hline 5 & 11 & 12 & 8 & 9 \\
\hline 10 & 9.9 & 10 & 9 & 11 \\
\hline 20 & 8.9 & 9.3 & 11 & 7 \\
\hline
\end{tabular}

\begin{tabular}{|c|c|c|c|c|c|c|c|}
\hline \multicolumn{1}{|c|}{ DURATION OF DAILY FLOW } \\
\hline \multicolumn{7}{|c|}{ Flow equaled or exceeded for indicated percentage of time } \\
(cubic feet per second)
\end{tabular}


STATION NAME AND NUMBER—03588500 Shoal Creek at Iron City, TN

LOCATION.-Lat 3501'26.54", long 87³4'44.43" referenced to North American Datum of 1927, Lawrence County, TN, Hydrologic Unit 06030005, on right downstream bank at bridge, on county road, $400 \mathrm{ft}$ downstream from Holly Creek, 1,350 ft upstream from Louisville and Nashville Railroad bridge, 1,350 ft northeast of Iron City Post Office, and at mile 22.3.

DRAINAGE AREA. $-348 \mathrm{mi}^{2}$.

PERIOD OF RECORD.- July 1925 to September 1994, October 2000 to March 2014.

PERIOD OF FREQUENCY ANALYSIS._-April 1951 to March 1994, April 2001 to March 2014.

NUMBER OF CLIMATE YEARS IN FREQUENCY ANALYSIS.- -56

REMARKS.-Prior to January 1951, diurnal fluctuation at low flow caused by powerplant near Lawrenceburg.

\begin{tabular}{|c|c|c|c|c|}
\hline $\begin{array}{c}\text { Recurrence } \\
\text { intervals } \\
\text { (years) }\end{array}$ & \multicolumn{2}{|c|}{$\begin{array}{c}\text { Lowest average flow for } \\
\text { indicated number of } \\
\text { consecutive days } \\
\text { (cubic feet per second) }\end{array}$} & \multicolumn{2}{|c|}{$\begin{array}{c}\text { Time-sampling error } \\
\text { (in percent) }\end{array}$} \\
\hline & 1 & 7 & 1 & 7 \\
\hline 2 & 119 & 125 & 4 & 5 \\
\hline 5 & 93 & 98 & 5 & 6 \\
\hline 10 & 82 & 86 & 5 & 7 \\
\hline 20 & 74 & 77 & 7 & 9 \\
\hline 50 & 65 & 68 & 9 & 4 \\
\hline
\end{tabular}

\begin{tabular}{|c|c|c|c|c|c|c|c|}
\hline \multicolumn{1}{|c|}{ DURATION OF DAILY FLOW } \\
\hline \multicolumn{1}{|c|}{ Flow equaled or exceeded for indicated percentage of time } \\
(cubic feet per second)
\end{tabular}


STATION NAME AND NUMBER—03592718 Little Yellow Creek near Burnsville, MS

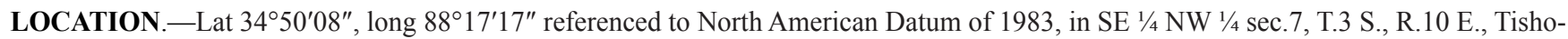
mingo County, MS, Hydrologic Unit 06030005 , Chickasaw Meridian, on right bank about $70 \mathrm{ft}$ downstream of bridge on county road $0.2 \mathrm{mi}$ northeast of Walker Siding, $2.0 \mathrm{mi}$ east of Burnsville, $0.1 \mathrm{mi}$ north of railroad and $0.25 \mathrm{mi}$ upstream of confluence of Tenn-Tom Waterway.

DRAINAGE AREA. $-24.7 \mathrm{mi}^{2}$, Tennessee Valley Authority.

PERIOD OF RECORD.--May 1973 to March 2013.

PERIOD OF FREQUENCY ANALYSIS.-April 1974 to March 2012.

NUMBER OF CLIMATE YEARS IN FREQUENCY ANALYSIS.-38

\section{REMARKS.-}

\begin{tabular}{|c|c|c|c|c|}
\hline $\begin{array}{c}\text { Recurrence } \\
\text { intervals } \\
\text { (years) }\end{array}$ & \multicolumn{2}{|c|}{$\begin{array}{c}\text { Lowest average flow for } \\
\text { indicated number of } \\
\text { consecutive days } \\
\text { (cubic feet per second) }\end{array}$} & \multicolumn{2}{|c|}{$\begin{array}{c}\text { Time-sampling error } \\
\text { (in percent) }\end{array}$} \\
\hline & 1 & 7 & 1 & 7 \\
\hline 2 & 3.7 & 4.2 & 7 & 7 \\
\hline 5 & 2.7 & 3.1 & 7 & 8 \\
\hline 10 & 2.3 & 2.7 & 9 & 9 \\
\hline 20 & 2.0 & 2.4 & 11 & 12 \\
\hline 50 & 1.7 & 2.1 & 14 & 7 \\
\hline
\end{tabular}

\begin{tabular}{|c|c|c|c|c|c|c|c|}
\hline \multicolumn{1}{|c|}{ DURATION OF DAILY FLOW } \\
\hline \multicolumn{7}{|c|}{ Flow equaled or exceeded for indicated percentage of time } \\
(cubic feet per second) \\
\hline Percentage & 5 & 10 & 25 & 50 & 75 & 90 & 95 \\
\hline Flow & 126 & 72 & 36 & 18 & 8.5 & 5.1 & 4.2 \\
\hline
\end{tabular}


Table 7. Differences between the annual minimum 7-day average streamflow with a 10-year recurrence interval published in this report and previously published values.

[USGS, U.S. Geological Survey; $\mathrm{mi}^{2}$, square mile; $\mathrm{ft}^{3} / \mathrm{s}$, cubic foot per second; N, no; Y, yes; Y (PR), currently regulated but comparison was for pre-regulation conditions; QAQC, quality assurance and quality control; SIMS, Site Information Management System]

\begin{tabular}{|c|c|c|c|c|c|c|}
\hline \multirow{2}{*}{$\begin{array}{c}\text { Site } \\
\text { index } \\
\text { number } \\
\text { (fig. 1) }\end{array}$} & \multirow{2}{*}{$\begin{array}{l}\text { USGS } \\
\text { station } \\
\text { number }\end{array}$} & \multirow{2}{*}{ Station name } & \multirow{2}{*}{$\begin{array}{c}\text { Drainage area } \\
\qquad\left(\mathrm{mi}^{2}\right)\end{array}$} & \multirow{2}{*}{ Regulated } & \multicolumn{2}{|r|}{ From Atkins and Pearman (1994) } \\
\hline & & & & & $\begin{array}{l}7010 \\
\left(\mathrm{ft}^{3} / \mathrm{s}\right)\end{array}$ & Period of record \\
\hline 2 & 02342500 & Uchee Creek near Fort Mitchell, AL & 322 & $\mathrm{~N}$ & 9.5 & October 1946 to September 1990 \\
\hline 3 & 02342933 & $\begin{array}{l}\text { South Fork Cowikee Creek near } \\
\text { Batesville, AL }\end{array}$ & 112 & $\mathrm{~N}$ & 0.8 & $\begin{array}{l}\text { October } 1963 \text { to September } 1971 \text {, } \\
\text { October } 1974 \text { to September } 1990\end{array}$ \\
\hline 8 & 02361500 & $\begin{array}{l}\text { Choctawhatchee River near } \\
\text { Bellwood, AL }\end{array}$ & 1,280 & $\mathrm{~N}$ & 225 & December 1921 to October 1925 \\
\hline 11 & 02364500 & Pea River near Samson, AL & 1,182 & $\mathrm{~N}$ & 138 & $\begin{array}{l}\text { August } 1904 \text { to August } 1913 \text {, October } 1922 \\
\text { to September } 1925 \text {, October } 1935 \text { to } \\
\text { September } 1970\end{array}$ \\
\hline 12 & 02364570 & Panther Creek near Hacoda, AL & 26.2 & $\mathrm{~N}$ & 1.1 & October 1974 to September 1990 \\
\hline 13 & 02369800 & Blackwater River near Bradley, AL & 87.7 & $\mathrm{~N}$ & 24 & October 1967 to September 1990 \\
\hline 15 & 02371500 & Conecuh River at Brantley, AL & 500 & $\mathrm{~N}$ & 31 & October 1937 to September 1990 \\
\hline 17 & 02372250 & Patsaliga Creek near Brantley, AL & 442 & $\mathrm{~N}$ & 26 & October 1974 to September 1990 \\
\hline 19 & 02372500 & Conecuh River near Andalusia, AL & 1,344 & Y & 147 & $\begin{array}{l}\text { September } 1904 \text { to December } 1919 \text {, } \\
\text { October } 1929 \text { to September 1952, } \\
\text { October } 1965 \text { to September } 1968\end{array}$ \\
\hline 33 & 02378500 & Fish River near Silver Hill, AL & 55.3 & $\mathrm{~N}$ & 40 & $\begin{array}{l}\text { July } 1953 \text { to September } 1969 \text {, October } \\
1970 \text { to September } 1971 \text {, November } \\
1986 \text { to September } 1990\end{array}$ \\
\hline 34 & 02398300 & $\begin{array}{l}\text { Chattooga River above } \\
\text { Gaylesville, } \mathrm{AL}\end{array}$ & 366 & $\mathrm{~N}$ & 94 & $\begin{array}{l}\text { January } 1959 \text { to September } 1967 \text {, } \\
\text { October } 1984 \text { to September } 1990\end{array}$ \\
\hline 38 & 02399200 & Little River near Blue Rond, AL & 199 & $\mathrm{~N}$ & 0.60 & $\begin{array}{l}\text { October } 1958 \text { to September } 1967 \text {, } \\
\text { October } 1970 \text { to September } 1990\end{array}$ \\
\hline 41 & 02400100 & Terrapin Creek at Ellisville, AL & 252 & $\mathrm{~N}$ & 62 & $\begin{array}{l}\text { October } 1962 \text { to September } 1967 \text {, } \\
\text { October } 1980 \text { to September } 1990\end{array}$ \\
\hline 44 & 02401000 & Big Wills Creek near Reece City, AL & 182 & $\mathrm{~N}$ & 31 & $\begin{array}{l}\text { October } 1943 \text { to September } 1970 \\
\text { October } 1986 \text { to September } 1990\end{array}$ \\
\hline 45 & 02401370 & Big Canoe Creek near Springville, AL & 45 & $\mathrm{~N}$ & 5.6 & October 1978 to September 1990 \\
\hline 47 & 02401470 & Little Canoe Creek near Steele, AL & 22.3 & $\mathrm{~N}$ & 1.4 & April 1982 to September 1990 \\
\hline
\end{tabular}


Table 7. Differences between the annual minimum 7-day average streamflow with a 10 -year recurrence interval published in this report and previously published values.-Continued

[USGS, U.S. Geological Survey; $\mathrm{mi}^{2}$, square mile; $\mathrm{ft}^{3} / \mathrm{s}$, cubic foot per second; N, no; Y, yes; Y (PR), currently regulated but comparison was for pre-regulation conditions; QAQC, quality assurance and quality control; SIMS, Site Information Management System]

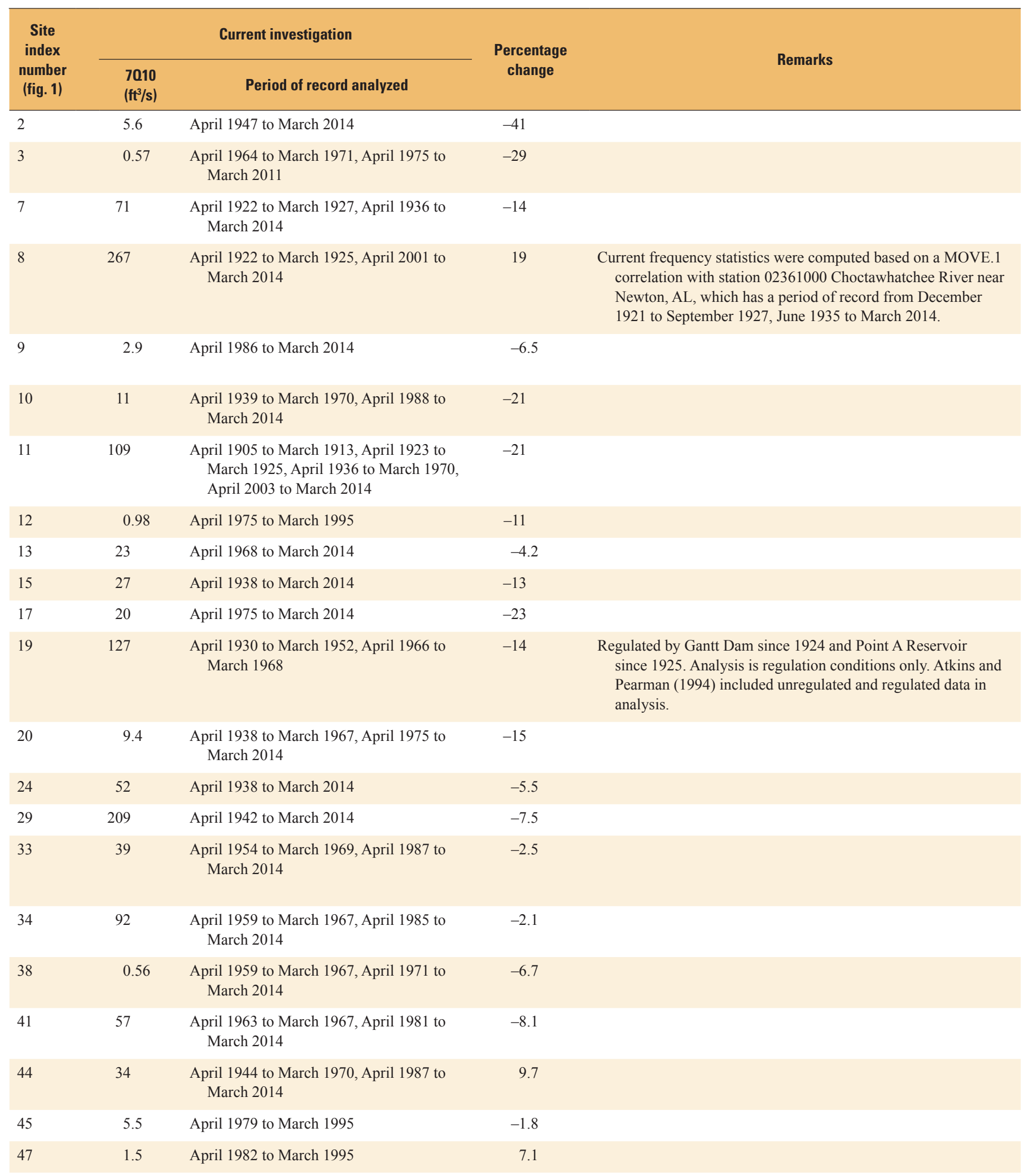


Table 7. Differences between the annual minimum 7-day average streamflow with a 10 -year recurrence interval published in this report and previously published values. - Continued

[USGS, U.S. Geological Survey; $\mathrm{mi}^{2}$, square mile; $\mathrm{ft}^{3} / \mathrm{s}$, cubic foot per second; N, no; Y, yes; Y (PR), currently regulated but comparison was for pre-regulation conditions; QAQC, quality assurance and quality control; SIMS, Site Information Management System]

\begin{tabular}{|c|c|c|c|c|c|c|}
\hline \multirow{2}{*}{$\begin{array}{c}\text { Site } \\
\text { index } \\
\text { number } \\
\text { (fig. 1) }\end{array}$} & \multirow{2}{*}{$\begin{array}{c}\text { USGS } \\
\text { station } \\
\text { number }\end{array}$} & \multirow{2}{*}{ Station name } & \multirow{2}{*}{$\begin{array}{l}\text { Drainage area } \\
\qquad\left(\mathrm{mi}^{2}\right)\end{array}$} & \multirow{2}{*}{ Regulated } & \multicolumn{2}{|r|}{ From Atkins and Pearman (1994) } \\
\hline & & & & & $\begin{array}{l}7010 \\
\left(\mathrm{ft}^{3} / \mathrm{s}\right)\end{array}$ & Period of record \\
\hline 50 & 02403500 & Coldwater Spring near Anniston, AL & Indeterminate & $\mathrm{N}$ & 34 & $\begin{array}{l}\text { July } 1944 \text { to March 1947, April } 1957 \text { to } \\
\text { September } 1990\end{array}$ \\
\hline 52 & 02404400 & $\begin{array}{l}\text { Choccolocco Creek at Jackson Shoal } \\
\text { near Lincoln, AL }\end{array}$ & 481 & $\mathrm{~N}$ & 121 & $\begin{array}{l}\text { October } 1960 \text { to September } 1967, \\
\text { October } 1984 \text { to September } 1990\end{array}$ \\
\hline 54 & 02405500 & Kelly Creek near Vincent, AL & 193 & $\mathrm{~N}$ & 1.7 & $\begin{array}{r}\text { December } 1951 \text { to September } 1970, \\
\text { October } 1986 \text { to September } 1990\end{array}$ \\
\hline
\end{tabular}

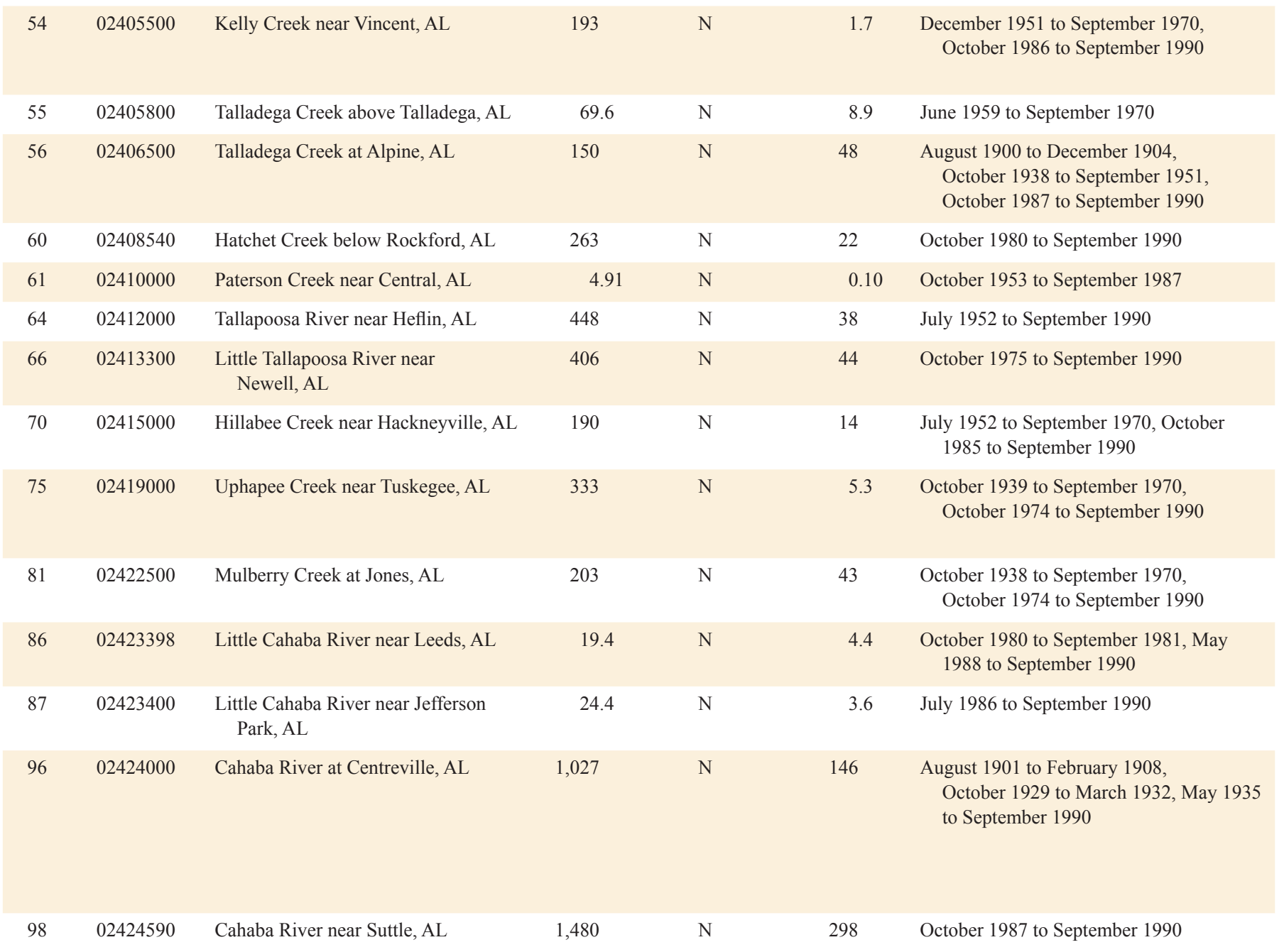


Table 7. Differences between the annual minimum 7-day average streamflow with a 10 -year recurrence interval published in this report and previously published values.-Continued

[USGS, U.S. Geological Survey; $\mathrm{mi}^{2}$, square mile; $\mathrm{ft}^{3} / \mathrm{s}$, cubic foot per second; N, no; Y, yes; Y (PR), currently regulated but comparison was for pre-regulation conditions; QAQC, quality assurance and quality control; SIMS, Site Information Management System]

\begin{tabular}{lcccc}
\hline $\begin{array}{c}\text { Site } \\
\text { index } \\
\text { number } \\
\text { (fig. 1) }\end{array}$ & $\begin{array}{c}\mathbf{7 0 1 0} \\
\left(\mathbf{f t}^{3} / \mathbf{s}\right)\end{array}$ & Period of record analyzed & $\begin{array}{c}\text { Percentage } \\
\text { change }\end{array}$ & Remarks \\
\hline 50 & 35 & April 1957 to March 1996 & 2.9 & $\begin{array}{c}\text { Atkins and Pearman (1994) stated that about 20 } \mathrm{ft}^{3} / \mathrm{s} \text { was diverted } \\
\text { by the City of Anniston. No drainage area (DA) provided in } \\
\text { NWISweb due to this being a spring. The DA shown computed } \\
\text { from StreamStats was } 1.25 \mathrm{mi}^{2} .\end{array}$
\end{tabular}

\begin{tabular}{llll}
\hline 52 & 117 & $\begin{array}{l}\text { April } 1961 \text { to March 1967, April } 1985 \text { to } \\
\text { March 2014 }\end{array}$ & -3.3 \\
\hline 54 & 2.1 & April 1952 to March 1970
\end{tabular}
from StreamStats was $1.25 \mathrm{mi}^{2}$.

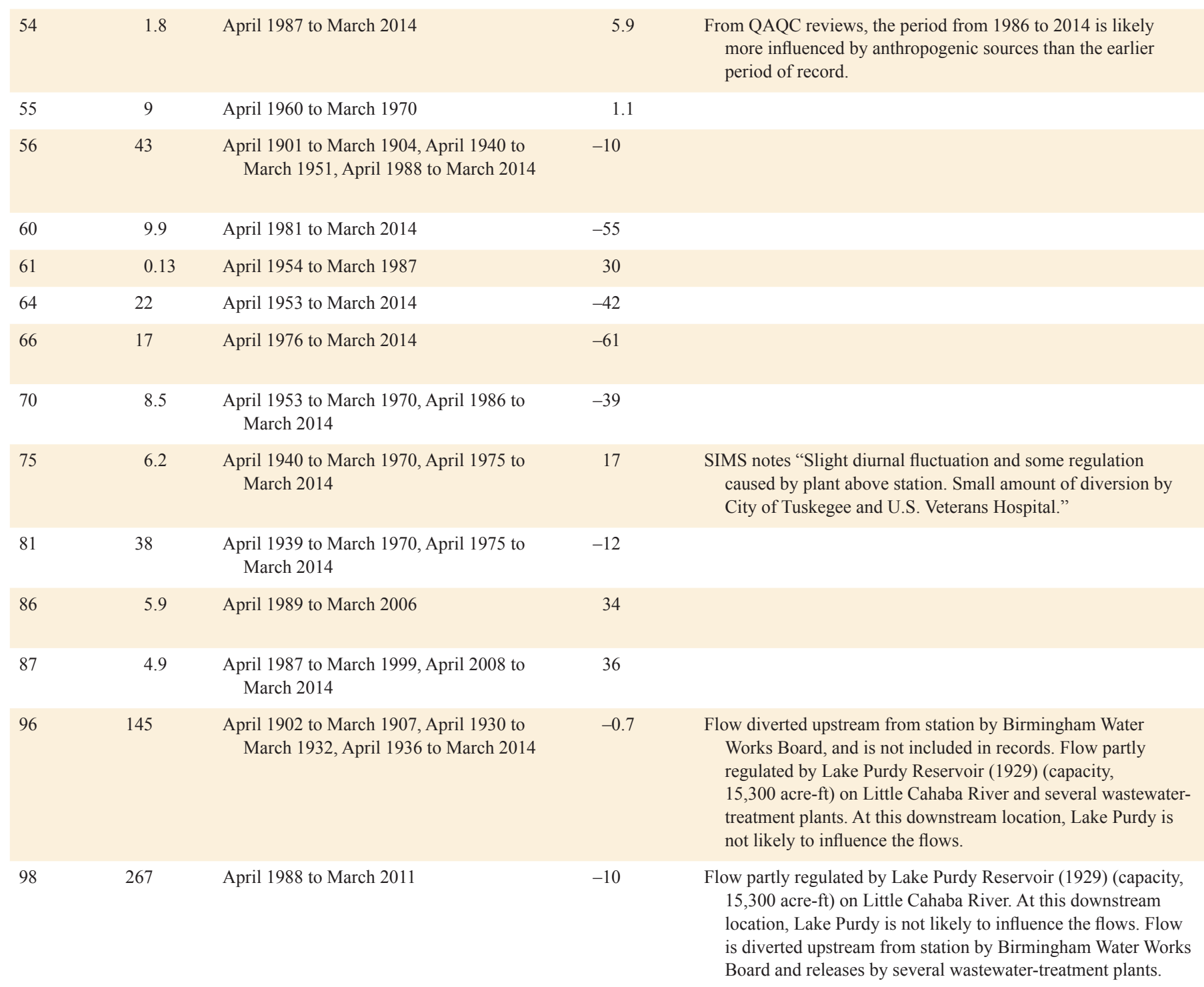
natural conditions than the latter period of record. SIMS notes "Record considered fair due to backwater from the Coosa River and overbank storage. This station is subject to high negative shifts due to beaver activity." 
Table 7. Differences between the annual minimum 7-day average streamflow with a 10-year recurrence interval published in this report and previously published values.-Continued

[USGS, U.S. Geological Survey; $\mathrm{mi}^{2}$, square mile; $\mathrm{ft}^{3} / \mathrm{s}$, cubic foot per second; N, no; Y, yes; Y (PR), currently regulated but comparison was for pre-regulation conditions; QAQC, quality assurance and quality control; SIMS, Site Information Management System]

\begin{tabular}{|c|c|c|c|c|c|c|}
\hline \multirow{2}{*}{$\begin{array}{c}\text { Site } \\
\text { index } \\
\text { number } \\
\text { (fig. 1) }\end{array}$} & \multirow{2}{*}{$\begin{array}{c}\text { USGS } \\
\text { station } \\
\text { number }\end{array}$} & \multirow{2}{*}{ Station name } & \multirow{2}{*}{$\begin{array}{c}\text { Drainage area } \\
\left(\mathrm{mi}^{2}\right)\end{array}$} & \multirow{2}{*}{ Regulated } & \multicolumn{2}{|r|}{ From Atkins and Pearman (1994) } \\
\hline & & & & & $\begin{array}{l}7010 \\
\left(\mathrm{ft}^{3} / \mathrm{s}\right)\end{array}$ & Period of record \\
\hline 100 & 02425000 & $\begin{array}{l}\text { Cahaba River near Marion } \\
\text { Junction, AL }\end{array}$ & 1,766 & $\mathrm{~N}$ & 321 & $\begin{array}{l}\text { October } 1938 \text { to September } 1954, \\
\text { October } 1968 \text { to September } 1990\end{array}$ \\
\hline 101 & 02425200 & Big Swamp Creek near Orrville, AL & 35.8 & $\mathrm{~N}$ & 0.50 & March 1972 to September 1985 \\
\hline 106 & 02427700 & Turkey Creek at Kimbrough, AL & 97.5 & $\mathrm{~N}$ & 0.60 & October 1958 to September 1990 \\
\hline 108 & 02428500 & Big Flat Creek near Fountain, AL & 247 & $\mathrm{~N}$ & 1.5 & October 1943 to September 1970 \\
\hline 112 & 02438000 & $\begin{array}{l}\text { Buttahatchee River below } \\
\text { Hamilton, AL }\end{array}$ & 277 & $\mathrm{~N}$ & 27 & October 1950 to September 1970 \\
\hline 116 & 02444000 & Coal Fire Creek near Pickensville, AL & 126 & $\mathrm{~N}$ & 6.7 & $\begin{array}{l}\text { October } 1954 \text { to September 1971, } \\
\text { October } 1974 \text { to September } 1980\end{array}$ \\
\hline 118 & 02444500 & Tombigbee River near Cochrane, AL & 5,940 & $\mathrm{Y}(\mathrm{PR})$ & 346 & October 1938 to March 1978 \\
\hline 119 & 02445500 & Sipsey River at Fayette, AL & 282 & $\mathrm{~N}$ & 12 & February 1939 to September 1959 \\
\hline 120 & 02446000 & Sipsey River at Moores Bridge, $\mathrm{AL}$ & 413 & $\mathrm{~N}$ & 33 & February 1939 to September 1951 \\
\hline
\end{tabular}

\begin{tabular}{|c|c|c|c|c|c|c|}
\hline 121 & 02446500 & Sipsey River near Elrod, AL & 528 & $\mathrm{~N}$ & 29 & $\begin{array}{l}\text { September } 1928 \text { to March } 1932, \\
\text { October } 1939 \text { to September 1971, } \\
\text { October } 1978 \text { to September } 1990\end{array}$ \\
\hline 122 & 02447000 & Sipsey River near Pleasant Ridge, AL & 769 & $\mathrm{~N}$ & 29 & February 1939 to September 1959 \\
\hline
\end{tabular}

\begin{tabular}{ccccccc}
124 & 02448500 & Noxubee River near Geiger, AL & 1,097 & N & 35 & $\begin{array}{r}\text { March 1939 to September 1940, } \\
\text { August 1944 to September 1965, } \\
\text { October 1966 to September 1990 }\end{array}$ \\
\hline 126 & 02449000 & Tombigbee River at Gainesville, AL & 8,632 & Y (PR) & 487 & $\begin{array}{r}\text { October 1938 to September 1955, } \\
\text { October 1960 to September 1978 }\end{array}$ \\
127 & 02449245 & Brush Creek near Eutaw, AL & 43.2 & N & 0.04 & June 1975 to September 1990 \\
130 & 02450000 & Mulberry Fork near Garden City, AL & 358 & N & 5.3 & October 1928 to September 1990
\end{tabular}


Table 7. Differences between the annual minimum 7-day average streamflow with a 10-year recurrence interval published in this report and previously published values.-Continued

[USGS, U.S. Geological Survey; $\mathrm{mi}^{2}$, square mile; $\mathrm{ft}^{3} / \mathrm{s}$, cubic foot per second; N, no; Y, yes; Y (PR), currently regulated but comparison was for pre-regulation conditions; QAQC, quality assurance and quality control; SIMS, Site Information Management System]

\begin{tabular}{|c|c|c|c|c|}
\hline \multirow{2}{*}{$\begin{array}{c}\text { Site } \\
\text { index } \\
\text { number } \\
\text { (fig. 1) }\end{array}$} & \multicolumn{2}{|r|}{ Current investigation } & \multirow{2}{*}{$\begin{array}{l}\text { Percentage } \\
\text { change }\end{array}$} & \multirow{2}{*}{ Remarks } \\
\hline & $\begin{array}{l}7010 \\
\left(\mathrm{ft}^{3} / \mathrm{s}\right)\end{array}$ & Period of record analyzed & & \\
\hline 100 & 303 & $\begin{array}{l}\text { April } 1939 \text { to March 1954, April } 1969 \text { to } \\
\text { March } 2014\end{array}$ & -5.6 & $\begin{array}{l}\text { Flow partly regulated by Lake Purdy Reservoir (1929) (capacity, } \\
15,300 \text { acre-ft) on Little Cahaba River. At this downstream } \\
\text { location, Lake Purdy is not likely to influence the flows. Flow } \\
\text { is diverted upstream from station by Birmingham Water Works } \\
\text { Board and releases by several wastewater-treatment plants. }\end{array}$ \\
\hline 101 & 0.54 & April 1972 to March 1985 & 8.0 & \\
\hline 106 & 0.62 & April 1959 to March 1996 & 3.3 & \\
\hline 108 & 1.5 & April 1944 to March 1970 & 0.0 & \\
\hline 112 & 30 & $\begin{array}{l}\text { April } 1951 \text { to March 1970, April } 1992 \text { to } \\
\text { March } 2014\end{array}$ & 11 & \\
\hline 115 & 48 & $\begin{array}{l}\text { April } 1955 \text { to March 1959, April } 1981 \text { to } \\
\text { March 1986, April } 2002 \text { to March } 2011\end{array}$ & 14 & \\
\hline 116 & 7.0 & $\begin{array}{l}\text { April } 1955 \text { to March 1971, April } 1975 \text { to } \\
\text { March } 1980\end{array}$ & 4.5 & \\
\hline 118 & 353 & April 1939 to March 1978 & 2.0 & Estimates for pre-regulated conditions \\
\hline 119 & 16 & April 1939 to March 1959 & 33 & $\begin{array}{l}\text { Current frequency statistics were computed based on a MOVE.1 } \\
\text { correlation with station } 02446500 \text { Sipsey River near Elrod, } \\
\text { AL, which has a period of record from September } 1928 \text { to } \\
\text { March 1932, October } 1939 \text { to September 1971, October } 1978 \\
\text { to March 2014, which represents } 69 \text { climatic years. }\end{array}$ \\
\hline 120 & 25 & April 1939 to March 1951 & -24 & $\begin{array}{l}\text { The current frequency statistics were computed based on a } \\
\text { MOVE.1 correlation with station } 02446500 \text { Sipsey River near } \\
\text { Elrod, AL, which has a period of record from September } 1928 \\
\text { to March } 1932 \text {, October } 1939 \text { to September } 1971 \text {, October } \\
1978 \text { to March } 2014 \text {, which represents } 69 \text { climatic years. }\end{array}$ \\
\hline
\end{tabular}

$\begin{array}{llll}121 & \begin{array}{l}\text { April } 1929 \text { to March 1932, April } 1940 \text { to } \\ \text { March 1971, April 1979 to March } 2014\end{array} & 10 \\ 122 & 40 & \text { April } 1939 \text { to March1959 }\end{array}$

1978 to March 2014, which represents 69 climatic years.

\begin{tabular}{|c|c|c|c|c|}
\hline & & & & $\begin{array}{l}\text { correlation with station } 02446500 \text { Sipsey River near Elrod, } \\
\text { AL, which has a period of record from September } 1928 \text { to } \\
\text { March 1932, October } 1939 \text { to September } 1971 \text {, October } 1978 \\
\text { to March 2014, which represents } 69 \text { climatic years. }\end{array}$ \\
\hline 124 & 39 & $\begin{array}{l}\text { April } 1939 \text { to March 1940, April } 1945 \text { to } \\
\text { March 1965, April } 1967 \text { to March } 2014\end{array}$ & 11 & \\
\hline 126 & 504 & $\begin{array}{l}\text { April } 1939 \text { to March 1955, April } 1961 \text { to } \\
\text { March } 1978\end{array}$ & 3.5 & Estimates for pre-regulated conditions \\
\hline 127 & 0.05 & April 1976 to March 1997 & 25 & \\
\hline 130 & 4.4 & April 1929 to March 1964 & -17 & $\begin{array}{l}\text { The flow patterns changed in the mid } 1960 \text { s with increasing } \\
\text { annual 7-day minimum average flows likely associated with } \\
\text { anthropogenic changes in the watershed. This analysis is for } \\
\text { October } 1928 \text { to March 1964, which likely represents more of } \\
\text { a natural condition than the latter period of record. }\end{array}$ \\
\hline
\end{tabular}


Table 7. Differences between the annual minimum 7-day average streamflow with a 10-year recurrence interval published in this report and previously published values.-Continued

[USGS, U.S. Geological Survey; $\mathrm{mi}^{2}$, square mile; $\mathrm{ft}^{3} / \mathrm{s}$, cubic foot per second; N, no; Y, yes; Y (PR), currently regulated but comparison was for pre-regulation conditions; QAQC, quality assurance and quality control; SIMS, Site Information Management System]

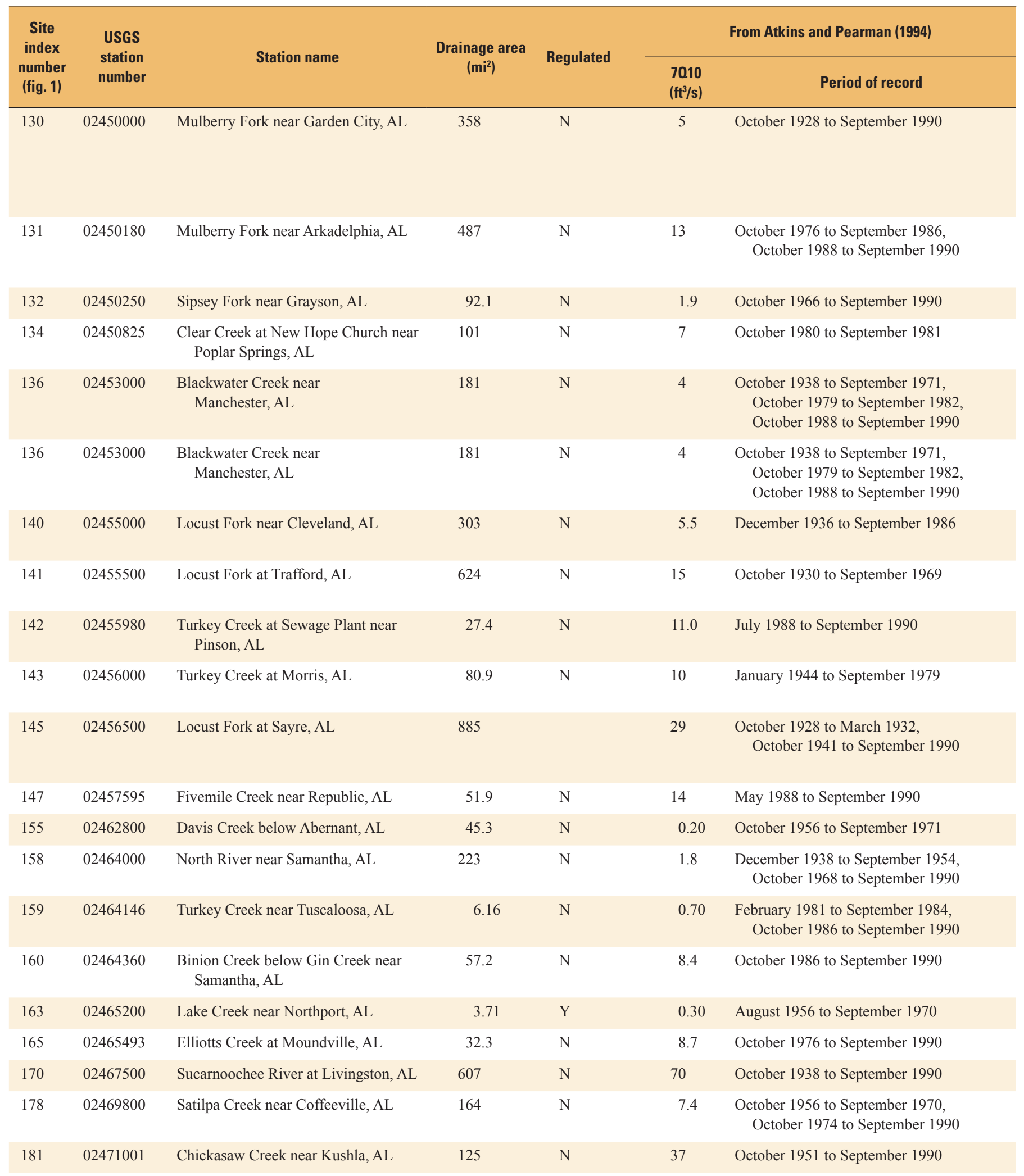


Table 7. Differences between the annual minimum 7-day average streamflow with a 10 -year recurrence interval published in this report and previously published values.-Continued

[USGS, U.S. Geological Survey; $\mathrm{mi}^{2}$, square mile; fts, cubic foot per second; N, no; Y, yes; Y (PR), currently regulated but comparison was for pre-regulation conditions; QAQC, quality assurance and quality control; SIMS, Site Information Management System]

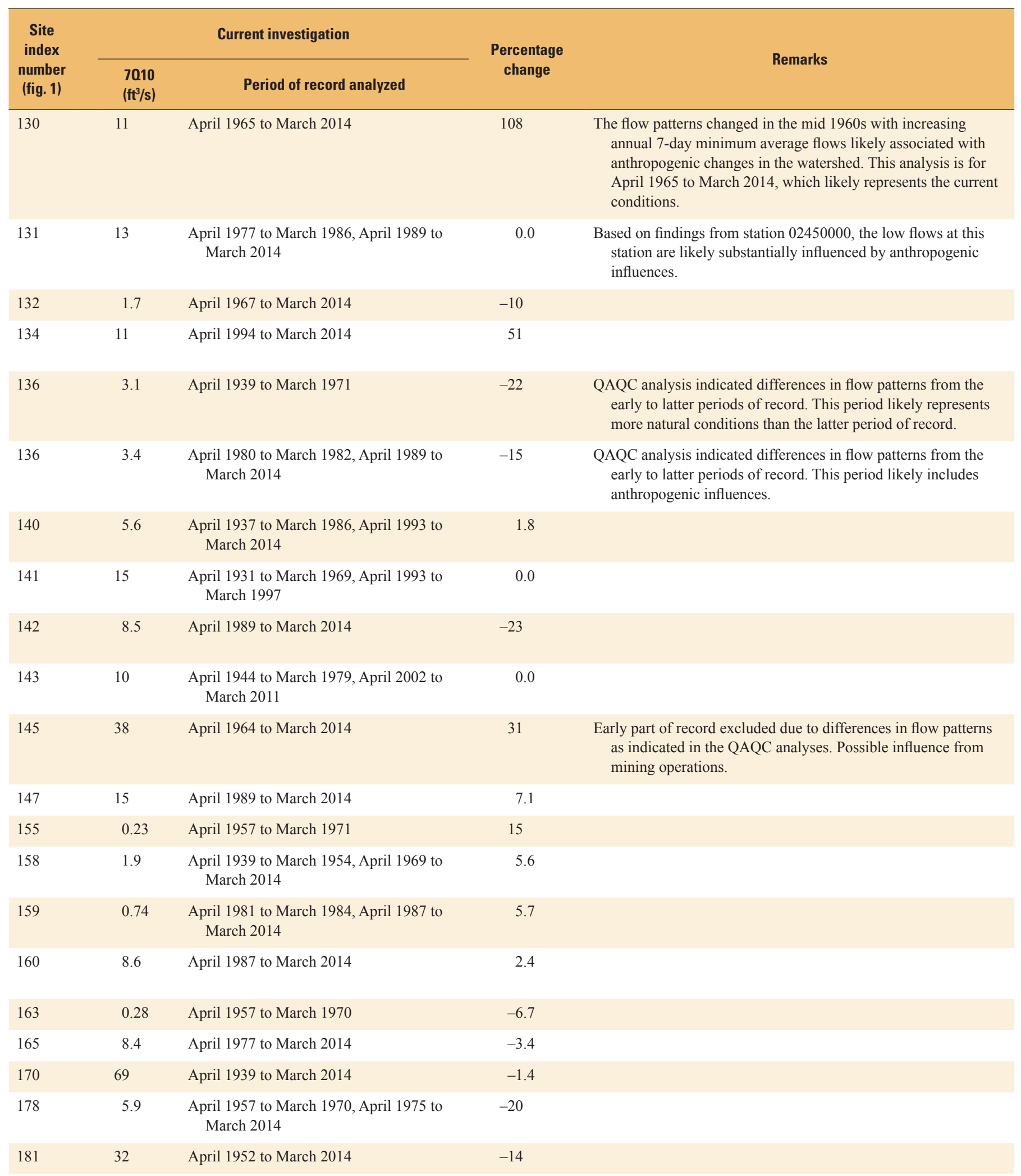


Table 7. Differences between the annual minimum 7-day average streamflow with a 10 -year recurrence interval published in this report and previously published values.-Continued

[USGS, U.S. Geological Survey; $\mathrm{mi}^{2}$, square mile; $\mathrm{ft}^{3} / \mathrm{s}$, cubic foot per second; N, no; Y, yes; Y (PR), currently regulated but comparison was for pre-regulation conditions; QAQC, quality assurance and quality control; SIMS, Site Information Management System]

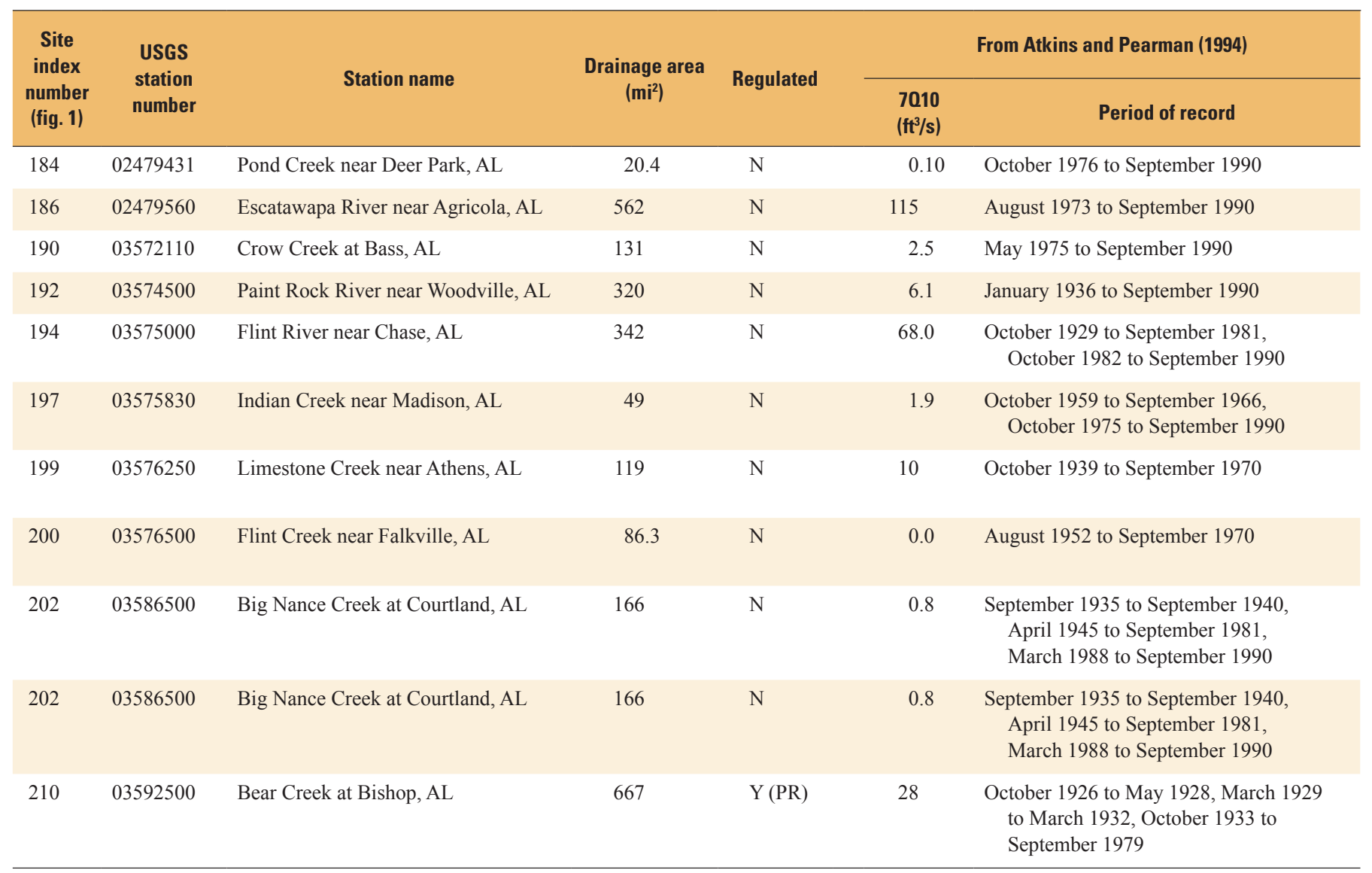


Table 7. Differences between the annual minimum 7-day average streamflow with a 10-year recurrence interval published in this report and previously published values.-Continued

[USGS, U.S. Geological Survey; $\mathrm{mi}^{2}$, square mile; $\mathrm{ft}^{3} / \mathrm{s}$, cubic foot per second; N, no; Y, yes; $\mathrm{Y}(\mathrm{PR})$, currently regulated but comparison was for pre-regulation conditions; QAQC, quality assurance and quality control; SIMS, Site Information Management System]

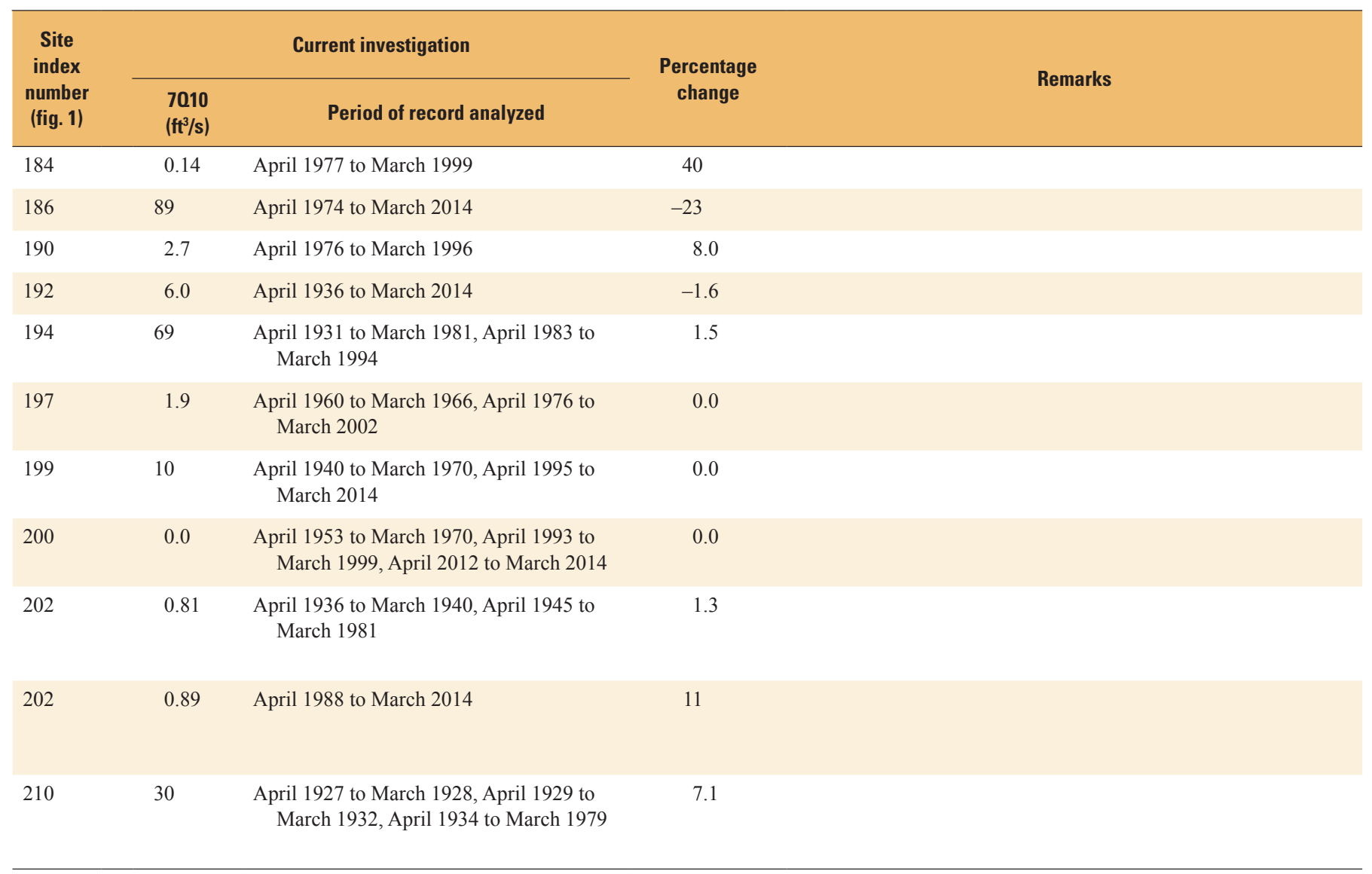


Manuscript was approved July 24, 2017

For additional information about this publication contact Director, Lower Mississippi Gulf Water Science Center U.S. Geological Survey

640 Grassmere Park

Nashville, TN 37211

Prepared by the U.S. Geological Survey Scientific Publishing Network Reston Publishing Service Center 

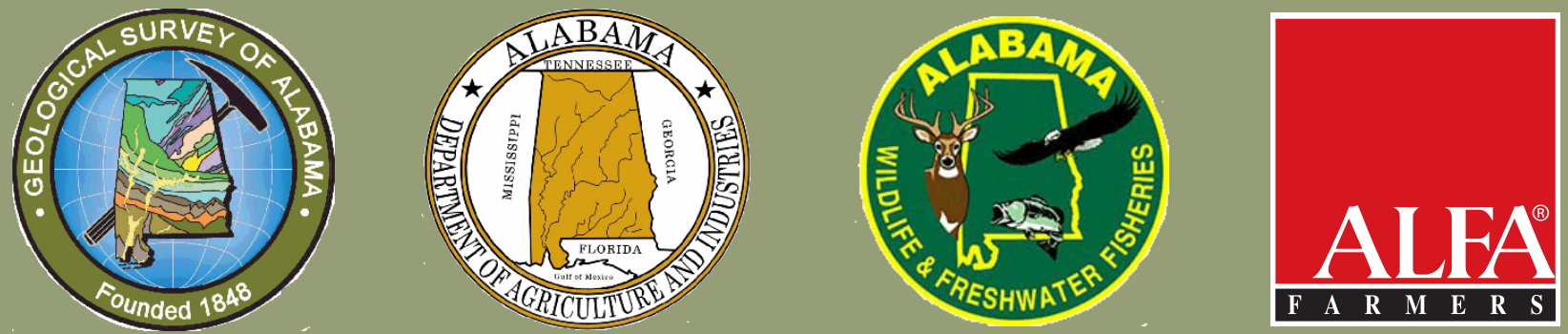

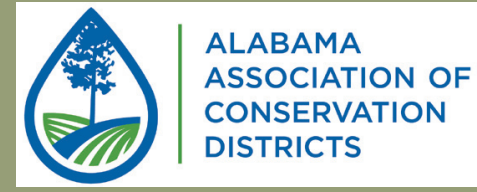

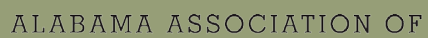

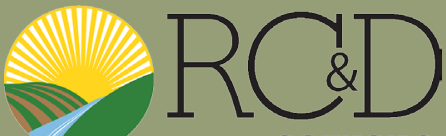

COUNCILS
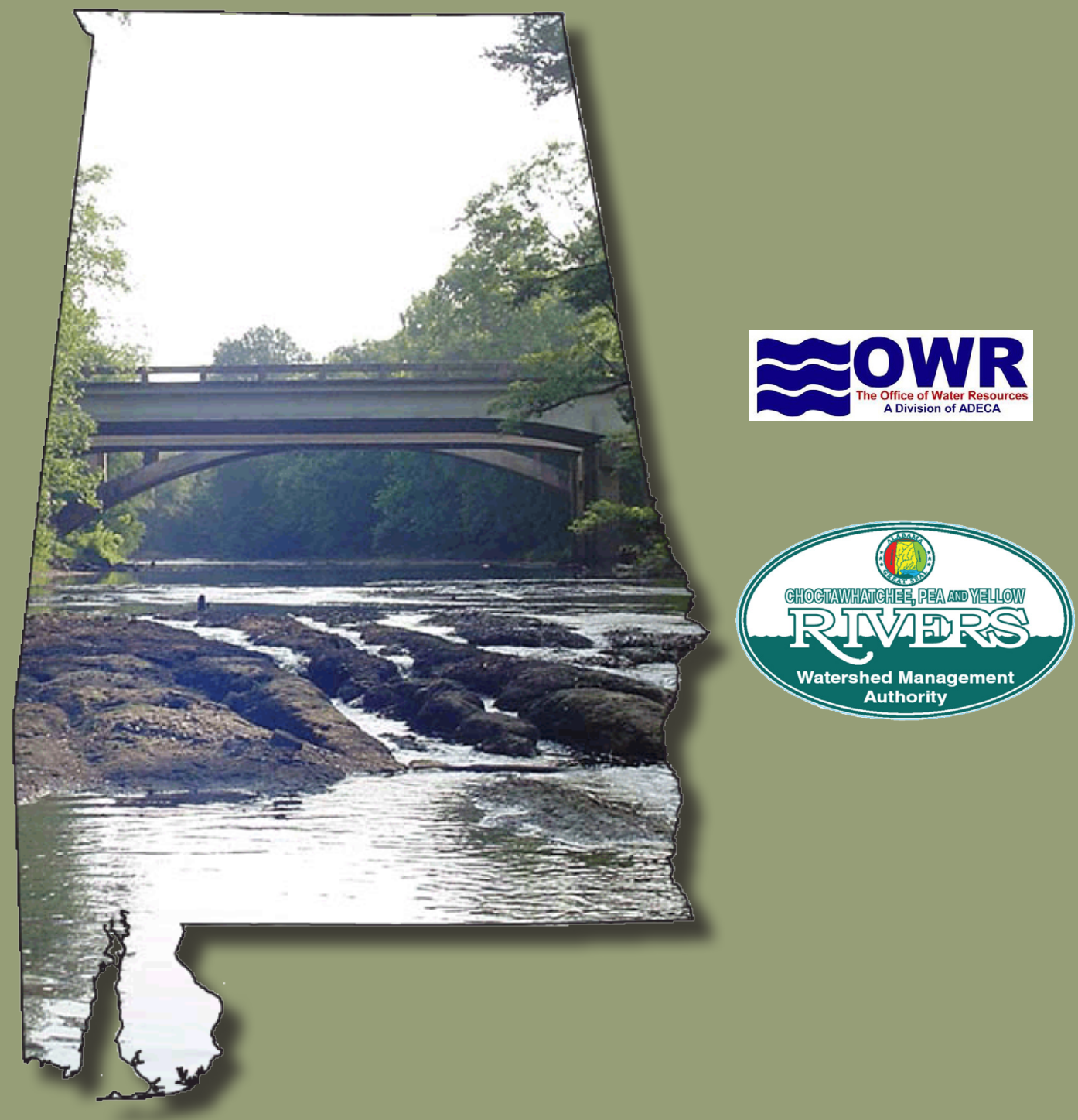